SOUS LA DIRECTION DE

Fernand Dumont ( 1927-1997) et Yves Martin

sociologues, Université Laval

(1962)

\title{
Situation de la recherche sur le Canada français
}

\author{
Premier colloque de la revue \\ RECHERCHES SOCIOGRAPHIQUES \\ du département de sociologie et d'anthropologie de l'Université Laval
}

Un document produit en version numérique par Diane Brunet, bénévole,

Diane Brunet, bénévole, guide, Musée de La Pulperie, Chicoutimi

Courriel: Brunet_diane@hotmail.com

Page web dans Les Classiques des sciences sociales

Dans le cadre de: "Les classiques des sciences sociales"

Une bibliothèque numérique fondée et dirigée par Jean-Marie Tremblay, professeur de sociologie au Cégep de Chicoutimi

Site web: http://classiques.uqac.ca/

Une collection développée en collaboration avec la Bibliothèque

Paul-Émile-Boulet de l'Université du Québec à Chicoutimi

Site web: http://bibliotheque.uqac.ca/ 


\section{Politique d'utilisation de la bibliothèque des Classiques}

Toute reproduction et rediffusion de nos fichiers est interdite, même avec la mention de leur provenance, sans l'autorisation formelle, écrite, du fondateur des Classiques des sciences sociales, Jean-Marie Tremblay, sociologue.

Les fichiers des Classiques des sciences sociales ne peuvent sans autorisation formelle:

- être hébergés (en fichier ou page web, en totalité ou en partie) sur un serveur autre que celui des Classiques.

- servir de base de travail à un autre fichier modifié ensuite par tout autre moyen (couleur, police, mise en page, extraits, support, etc...),

Les fichiers (.html, .doc, .pdf, .rtf, .jpg, .gif) disponibles sur le site Les Classiques des sciences sociales sont la propriété des Classiques des sciences sociales, un organisme à but non lucratif composé exclusivement de bénévoles.

Ils sont disponibles pour une utilisation intellectuelle et personnelle et, en aucun cas, commerciale. Toute utilisation à des fins commerciales des fichiers sur ce site est strictement interdite et toute rediffusion est également strictement interdite.

L'accès à notre travail est libre et gratuit à tous les utilisateurs. C'est notre mission.

Jean-Marie Tremblay, sociologue

Fondateur et Président-directeur général, LES CLASSIQUES DES SCIENCES SOCIALES. 
Cette édition électronique a été réalisée par Diane Brunet, bénévole, guide, Musée de La Pulperie, Chicoutimi à partir du livre de :

à partir de l’ouvrage:

Sous la direction de Fernand Dumont et Yves Martin

\section{Situation de la recherche sur le Canada français.}

Premier colloque de la revue RECHERCHES SOCIOGRAPHIQUES du département de sociologie et d'anthropologie de l'Université Laval

Québec : Les Presses de l’Université Laval, 1962, 296 pp.

M. Fernand Dumont (1927-1997), sociologue, enseignait la sociologie à l'Université Laval.

M. Yves Martin est sociologue (retraité de l'enseignement) de l'Université Laval.

[Autorisation formelle confirmée le 6 février 2006 au téléphone par M. Yves Martin et confirmée par écrit le 7 février 2006 de diffuser la totalité de ses œuvres: articles et livres]

Courriel : yves_martin@sympatico.ca

Polices de caractères utilisée :

Pour le texte: Times New Roman, 14 points.

Pour les citations : Times New Roman 12 points.

Pour les notes de bas de page : Times New Roman, 12 points.

Édition électronique réalisée avec le traitement de textes Microsoft Word 2008 pour Macintosh.

Mise en page sur papier format : LETTRE US, 8.5'’ x 11’’.

Édition numérique réalisée le 5 avril 2013 à Chicoutimi, Ville de Saguenay, province de Québec, Canada. 
SOUS LA DIRECTION DE

Fernand Dumont et Yves Martin

Sociologues, département de sociologie, Université Laval

\section{Situation de la recherche sur le Canada français.}

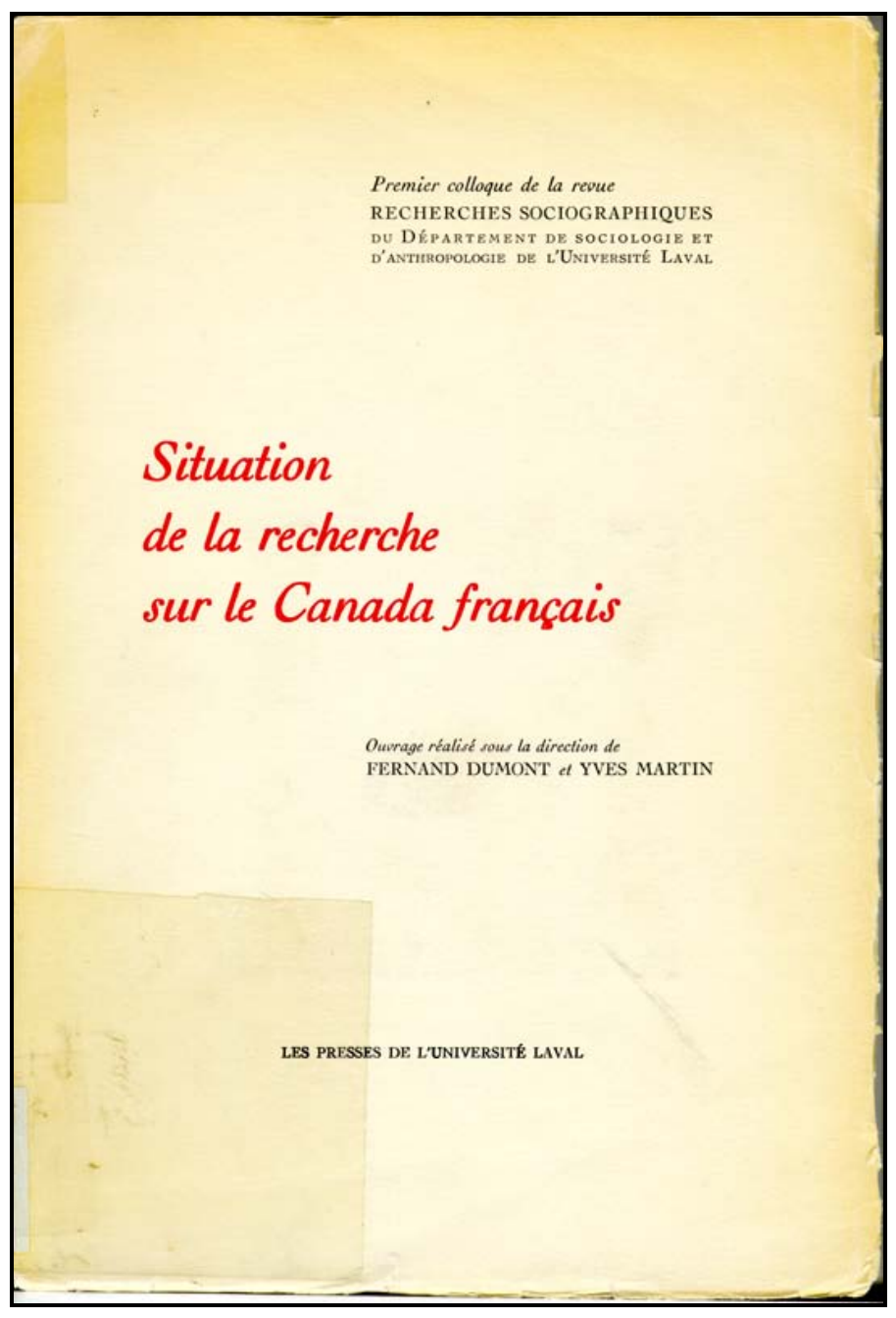

Premier colloque de la revue RECHERCHES SOCIOGRAPHIQUES du département de sociologie et d'anthropologie de l'Université Laval. Québec: Les Presses de l’Université Laval, 1962, 296 pp. 
[295]

\section{Table des matières}

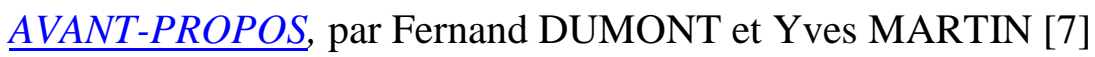

\section{Perspectives historiques}

1. André VACHON, "État des recherches sur le régime français (1632-1760)." [11]

Commentaire, par Marcel TRUDEL. [25]

2. Fernand OUELLET L'étude du XIX ${ }^{\mathrm{e}}$ siècle canadien-français." [27] Commentaire, par Michel BRUNET.” [43]

3. Albert FAUCHER, “L'histoire économique de la province de Québec jusqu'à la fin du XIX ${ }^{\mathrm{e}}$ siècle." [45] [Texte diffusé en tiré à part.]

Commentaire, par Jean HAMEL1N. [54]

4. André RAYNAULD, “Recherches économiques récentes sur la province de Québec.” [55] [Texte diffusé en tiré à part.]

Commentaire, par Charles LEMELIN. [65]

5. Jean-Charles BONENFANT, “Les études politiques.” [75]

Commentaire, par Vincent LEMIEUX. [83]

\section{Perspectives écologiques}

1. Fernand GRENIER, “L'état présent des études régionales sur le Québec.” [89]

Commentaire, par Pierre HARVEY. [102]

2. Gérald FORTIN, “L'étude du milieu rural.” [105] [Texte diffusé en tiré à part.]

Commentaire, par Napoléon LEBLANC. [117]

3. Yves MARTIN, “Les études urbaines.” [119] [Texte diffusé en tiré à part.] Commentaire, par Louis TROTIER. [129]

4. Jacques HENRIP1N, “Les études démographiques.” [133] 
Commentaire, par Yves DE JOCAS. [142]

5. Marc-Adélard TREMBLAY, "L'état des recherches sur la culture acadienne.” [145] [Texte diffusé en tiré à part.]

Commentaire, par Clément CORMIER, c.s.c. [168]

\section{Perspectives sur l'étude de la structure sociale}

1. Guy ROCHER, "Les recherches sur les occupations et la stratification sociale.” [173] [Texte diffusé en tiré à part.]

Commentaire, par Émile GOSSELIN. [185]

2. Bernard MAILHIOT, o.p., "Les recherches en psychologie sociale au Canada français (1946-1962).” [189]

Commentaire, par Jacques BRAZEAU. [205]

3. Jean-Charles FALARDEAU, "Les recherches religieuses au Canada français." [209]

Commentaire, par Norbert LACOSTE. [229]

4. Gilles-R. LEFEBVRE, “L'étude de la culture : la linguistique.” [233]

Commentaire, par Gaston DULONG. [250]

5. Luc LACOURCIÈRE, "L'étude de la culture : le folklore". [253]

Commentaire, par Benoît LACROIX, o.p. [263]

6. Marcel RIOUX, "L'étude de la culture canadienne-française : aspects microsociologiques." [267] [Texte diffusé en tiré à part.]

Commentaire, par Claude GALARNEAU. [273]

7. Fernand DUMONT, "L'étude systématique de la société globale canadienne-française.” [277] [Texte diffusé en tiré à part.]

Commentaire, par Maurice TREMBLAY. [293] 


\title{
Situation de la recherche sur le Canada français
}

\author{
AVANT-PROPOS
}

$\underline{\text { Retour à la table des matières }}$

Nous publions ici les textes des communications présentées à l'occasion du premier colloque de la revue Recherches sociographiques, organisé par le Département de sociologie et d'anthropologie de l'Université Laval et tenu à Québec, en avril 1962.

Dans le premier fascicule de Recherches sociographiques, périodique fondé en 1960 par notre département et consacré entièrement à la sociologie du Canada français, les directeurs de la revue écrivaient : « L'équipe de la revue espère... organiser, de temps en temps, des sessions de travail sur les problèmes posés par la description sociographique ». Nous avons pensé que la première de ces rencontres devait tout naturellement donner lieu à un bilan d'ensemble. D'où le choix de notre thème : Situation de la recherche sur le Canada français.

En 1952, un symposium important avait été organisé par la Faculté des sciences sociales de l'Université Laval sur les problèmes originaux posés par l'industrialisation et l'urbanisation au Canada français. L'ouvrage qui a réuni les travaux de cette rencontre - les Essais sur le Québec contemporain - a marqué une étape décisive dans l'étude de notre milieu. Nous n'avons pas cru qu'il était opportun de reprendre la même perspective. Depuis lors, des chantiers nouveaux de la recherche ont été ouverts, des vues et des discussions nouvelles ont été in- 
troduites dans les études plus anciennes. Nous nous sommes donc proposé, plutôt que de formuler des interprétations directes de tel ou tel aspect de notre milieu, d'esquisser un diagnostic de l'état des travaux dans les divers domaines de la recherche et d'indiquer les tâches prochaines qui s'offrent aux chercheurs. «Travail fait, travail à faire »; ce sous-titre d'une petite collection fondée jadis par Henri Berr définit fort bien l'objectif que nous poursuivions.

Dans ce programme d'inventaire élaboré sous les auspices de la sociologie et de l'anthropologie, on remarquera pourtant que nous avons insisté sur les aspects proprement historiques et écologiques. Comme nous ne pouvions songer a un bilan tout a fait exhaustif, il nous est apparu qu'il fallait donner une grande place à ces deux secteurs qui, d'une part, ont fait l'objet des travaux les plus nombreux et qui, d'autre part, doivent sans doute avoir la priorité dans une démarche de connaissance globale de notre milieu. La troisième partie du colloque traite de secteurs plus spécifiquement sociologiques ou anthropologiques - et qui, par contre, sont encore un peu en friche. On [8] notera facilement que la répartition des chapitres entre ces diverses parties n'a rien de systématique : à partir d'un cadre souple, d'où toute spéculation théorique inopportune avait été volontairement exclue, nous avons aussi tenu compte des orientations personnelles des chercheurs invités à participer à ce travail. De même, tout en tenant à une certaine homogénéité, nous avons laissé beaucoup de liberté aux participants : ceux-ci ont tantôt insisté sur l'aspect documentaire, tantôt sur le commentaire critique.

Nous avons voulu faire appel à des représentants de la plupart des sciences de l'homme. Nous sommes heureux que nos collègues de l'Université de Montréal nous aient accordé une collaboration très large et très spontanée. Ajoutons qu'un effort tout particulier a été fait pour que nos étudiants dans les diverses sciences de l'homme, à Montréal comme à Québec, participent activement à ces assises. De fait, plus de trois cents personnes ont suivi les travaux du colloque. Les uns et les autres ont vu dans cette manifestation, par delà les diversités locales, une sorte de symbole de l'existence d'une même université canadienne-française.

Nous nous interdirons toute généralisation à propos des Inventaires et des hypothèses proposés dans les textes réunis ici. Les orientations sont encore trop diverses, le dialogue entre les chercheurs a auquel 
nous avons voulu, pour notre part, contribuer) est encore trop lâche, bien des positions de problèmes sont encore trop nouvelles et trop polémiques par rapport aux vues conventionnelles que l'on entretenait naguère sur notre milieu pour qu'il soit possible, autrement qu'en termes très personnels, de soumettre des hypothèses à la fois précises et globales. Nous sommes davantage encore à l'âge de la coordination plutôt qu'à celui de la synthèse. Une réflexion nous paraît pourtant s'imposer. Peu de chercheurs canadiens-français, et on le constatera une fois de plus dans cette publication, situent leurs études sur leur société dans une perspective de comparaison avec d'autres milieux. C'est une troublante lacune. Il nous semble y avoir là l'indication d'une étape prochaine à franchir pour la recherche au Canada français.

$$
* * *
$$

Préparé sous notre responsabilité plus Immédiate, le colloque a été en réalité une initiative collective des professeurs du Département de sociologie et d'anthropologie de l'Université Laval. On nous permettra de remercier amicalement de leur très étroite collaboration nos collègues Jean-Charles Falardeau, Gérald Fortin, Napoléon LeBlanc et Marc-Adélard Tremblay.

Fernand Dumont

Yves Martin 
[9]

Situation de la recherche sur le Canada français

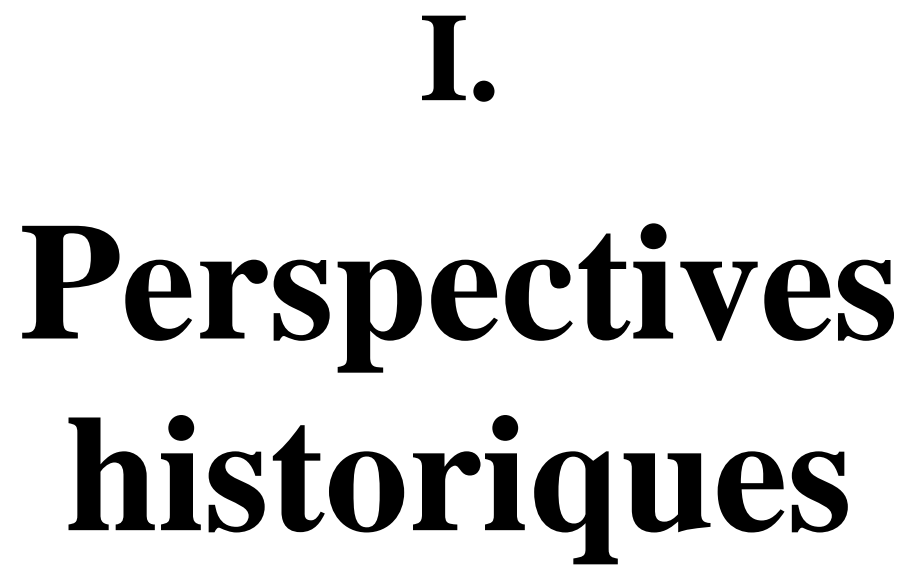

$\underline{\text { Retour à la table des matières }}$ 


\title{
Situation de la recherche sur le Canada français
}

\section{Perspectives historiques}

\author{
André Vachon \\ Directeur des Éditions, \\ Les Presses de l’Université Laval

\section{"État des recherches (1632-1760)".} \\ sur le Régime français
}

[pp. 11-24.]

$\underline{\text { Retour à la table des matières }}$

Il convient de féliciter les organisateurs de ce colloque d'avoir inscrit, en tête du programme, un exposé de l'état actuel des recherches sur le régime français. Pour comprendre la société canadiennefrançaise d'aujourd'hui, il est essentiel en effet de remonter à l'époque déjà lointaine où s'est formée cette société. Mais, par suite de l'abondance même des études consacrées à la Nouvelle-France, il n'est pas facile de faire le partage entre les connaissances acquises et les connaissances a. acquérir. Pour donner une idée exacte de la situation, il eût fallu lire une quantité énorme de volumes, d'articles de revue et de thèses manuscrites. Or, dans mon inventaire, je m'en suis tenu délibérément aux ouvrages publiés en librairie, sans toutefois négliger entièrement les articles de revue. C'est dire les limites de cet exposé, 
qui risque de ne donner, à la question posée, qu'une réponse partielle. D'autant plus que le sujet qu'on m'a confié est très vaste. Pour le traiter d'une façon un peu complète, il faut faire l'inventaire des études portant sur chacun des aspects principaux de la vie canadienne au XVII ${ }^{\mathrm{e}}$ et au XVIII ${ }^{\mathrm{e}}$ siècles : vie politique, vie économique, explorations, vie religieuse, vie sociale. Est-il nécessaire d'ajouter que ces divisions, utiles pour les fins de notre enquête, ne correspondent guère à la réalité de la vie, laquelle ne se cloisonne pas en sections étanches.

\section{La vie politique}

Dans cette première partie, j'entends parler des études portant sur les institutions administratives, sur les relations politiques de la Nouvelle-France avec les colonies anglaises d'Amérique et avec les Indiens et sur l'histoire militaire.

On a assez bien étudié jusqu'ici les institutions administratives de la fin du régime français, au moment où elles avaient en quelque sorte atteint leur plein développement. L'apport de Frégault ${ }^{1}$ et de Filteau ${ }^{2}$, en ce domaine, [12] a été considérable. Mais la plupart des travaux sur l'administration portent exclusivement sur le rôle du gouverneur général, de l'intendant et du conseil souverain : dans sa thèse sur L'administration de la Nouvelle-France, ${ }^{3}$ Lanctot s'en tient à ces trois institutions ; Du Bois Cahall ${ }^{4}$ et Delalande 5 , pour leur part, étudient le

1 Guy Frégault, La civilisation, de la Nouvelle-France (1713-1744), Montréal, Société des Éditions Pascal, [1944], 285 p., 21cm.

2 Gérard Filteau, La naissance d'une nation ; tableau du Canada en 1755, Montréal, Éditions de l'A.C.-F., [1937], 2 vol. 20.5cm. L'ouvrage le plus complet que nous ayons sur les institutions politiques, économiques, religieuses et sociales de la Nouvelle-France.

3 Gustave LANCTOT, I,'administration de la Nouvelle-France : l'administration générale, Paris, H. Champion, 1929, 169 p., $25.5 \mathrm{~cm}$.

4 Raymond DU BOIS CAHALL, The Sovereign Council of New France, New-York, Columbia University, 1915, 274 p., 24 cm.

5 J. DELALANDE, Le Conseil souverain de la Nouvelle-France, Québec, LsA. Proulx, Imprimeur du Roi, 1927, 358 p., 19 cm. L'auteur s'attache surtout à la description des fonctions du conseil souverain. Ce volume est toutefois inférieur au précédent. 
conseil souverain. Seul Filteau est allé largement au-delà de ces trois institutions. Enfin, il existe, à côté de ces études plus élaborées, quelques articles de J.-Edmond Roy, ${ }^{6}$ de P.-Georges Roy ${ }^{7}$ et d'E.-Z. Massicotte ${ }^{8}$ sur les cours de justice royales ou seigneuriales.

Mais notre connaissance des institutions administratives de la Nouvelle-France est loin d'être parfaite. Il faudrait étudier à fond le mécanisme et le fonctionnement des diverses cours de justice, le rôle des compagnies qui, même après 1674, participèrent à l'administration coloniale, le bureau du Domaine du roi, etc. Il faudrait également suivre l'évolution des différentes institutions, afin de dégager l'esprit qui a inspiré leur élaboration - ce qui doit être en définitive le but ultime de ces recherches. Enfin, il est essentiel que l'étude des institutions administratives ne se fasse pas uniquement à partir des grands documents officiels que sont les commissions des gouverneurs et des intendants, l'édit de création du conseil souverain ou la correspondance générale. Il faut que les historiens s'imposent de dépouiller et d'étudier les archives administratives pour voir comment, dans la réalité, fonctionnaient ces institutions, comment elles s'adaptaient aux besoins nouveaux. Car, des principes théoriques affirmés dans les documents officiels à l'application dans la pratique quotidienne, il y a souvent un écart considérable.

Une telle étude des institutions administratives est, à mon sens, inséparable de l'étude du droit en Nouvelle-France. Nous avons bien eu, il y a un demi-siècle et plus, quelques historiens du droit canadien, 9

$6 \quad$ Citons par exemple La justice seigneuriale de Notre-Dame-des-Anges, s.l., s.d., 23 p., 24 cm. les Mémoires de la Société royale du Canada, 1915, section I, 173-187.

7 Par exemple « Les Conseillers au Conseil souverain », dans les Mémoires de la Société royale du Canada, 1915, section I, 173-187.

8 Entre autres : « Les tribunaux et les officiers de justice de Montréal sous le régime français », Bulletin des Recherches historiques, XXXVII, 1931, 122128, 179-192, 252-256, 302-313.

9 B.-A. TESTARD DE MONTIGNY, Histoire du droit canadien, Montréal, Eusèbe Sénécal, 1869, 984 p., 23 cm.

Edmond LAREAU, Histoire du droit canadien, 2 vol., Montréal, Librairie générale de droit et de jurisprudence, A. Périard, libraire-éditeur, 18881889, 518 et 544 p., 22cm.

Rodolphe LEMIEUX, Les origines du droit franco-canadien, Montréal, C. Théoret, éditeur, 1901, XXIX + 483 p., 23 cm. 
mais leurs [13] ouvrages ont beaucoup vieilli. Voilà certainement un champ considérable d'activité pour les historiens et les juristes.

À la politique extérieure de la Nouvelle-France, on a consacré peu d'études spéciales, si l'on excepte l'article de Shortt et Chapais, « The Colony in its Political Relations " 10 publié dans Canada and its Provinces. Cette étude demeure la plus complète - elle couvre tout le régime français - et l'une des meilleures que nous ayons à ce sujet. Plusieurs historiens néanmoins ont écrit là-dessus de fort bonnes pages dans des ouvrages plus généraux : Desrosiers dans son Iroquoisie, ${ }^{11}$ Lanctot dans son Histoire du Canada, 12 Chapais dans son Jean Talon 13 et Eccles dans son ouvrage sur Frontenac 14 ont traité cette question pour le $\mathrm{XVII}^{\mathrm{e}}$ siècle : Frégault l'a fait d'une façon très complète dans la plupart de ses ouvrages 15 sur le $\mathrm{XVIII}{ }^{\mathrm{e}}$ siècle ; sans oublier la synthèse du chanoine Groulx dans les deux premiers tomes de son Histoire du Canada français. 16

D'une façon générale, la politique de la Nouvelle-France à l'endroit des colonies anglaises a été bien étudiée. Mais, dans la mesure où l'on comprendra mieux les problèmes économiques de la Nouvelle-France, la politique de la métropole française et les visées des colonies anglaises, on sera peut-être amené à nuancer ou même à rectifier certaines interprétations.

10 Adam SHORTT et Thomas CHAPAIS, « The Colony in its Political Relations ", dans Canada and its Provinces, Toronto, 1913-1914, II, 315-378.

11 Léo-Paul DESROSIERS, Iroquoisie \{1534-1646), vol. I, Montréal, Institut d'histoire de l'Amérique française [1947], 351 p., $18.5 \mathrm{~cm}$. Seul ce premier volume a paru. Desrosiers a toutefois publié un bon nombre d'articles sur le même sujet dans la Revue d'Histoire de l'Amérique française et dans les Cahiers des Dix.

12 Gustave LANCTOT, Histoire du Canada, vol. I : Des origines au régime royal, Montréal, Librairie Beauchemin, 1959, 460 p., $21.5 \mathrm{~cm}$.

13 Thomas CHAPAIS, Jean Talon, intendant de la Nouvelle-France (16651672), Québec, Imprimerie S.-A. Demers, 1904, 540 p., 23.5 cm.

14 W. J. ECCLES, Frontenac, the Courtier Governor, Toronto, McClelland and Stewart, Limited, [1959], 406 p., 24cm.

15 Voir note 1, page 11, et les notes 4, 5 et 6, page 14.

16 Lionel GROULX, Histoire du Canada français depuis la découverte, [Montréal], L'Action nationale, [1950 et 1951], 221 et 302 p., $21.5 \mathrm{~cm}$. 
Si la politique de la Nouvelle-France à l'endroit des colonies anglaises d'Amérique a été bien étudiée, je ne crois pas qu'on puisse en dire autant de la politique coloniale à l'endroit des Indiens. Un grand nombre d'historiens ont abordé cette question, mais il me semble que, Desrosiers 17 mis à part, on n'a pas suffisamment tenté de comprendre l'Indien, qu'on a trop souvent étudié comme s'il eût été un produit de la civilisation occidentale et chrétienne, et en lui prêtant des préoccupations, des réactions ou des sentiments qui n'ont jamais été les siens. Par suite de cette ignorance des Indiens, on n'a pas toujours su juger correctement la politique française à leur égard et l'on s'est trompé assez souvent sur la portée réelle de telle attitude des autorités françaises vis-à-vis des Indiens ou de ces derniers vis-à-vis des Français.

Personnellement, je déplore beaucoup l'absence de bonnes études sur les Indiens de la Nouvelle-France et le fait que les historiens ne les aient pas davantage intégrés à notre histoire. Les Indiens, il est vrai, ont été étudiés par un nombre d'ailleurs restreint d'anthropologues canadiens : Barbeau, Beaugrand-Champagne, Rousseau, Jenness. Mais trop peu d'historiens ont emprunté les méthodes ou utilisé les conclusions des anthropologues. De sorte que historiens et anthropologues continuent de s'ignorer, alors qu'ils trouveraient tellement d'avantages à collaborer. Cette collaboration nous vaudrait peut-être quelques ouvrages de la valeur de celui de Bailey, The Conflict of European and Eastern Algonkian Cultures ${ }^{18}$, qui est une contribution vraiment remarquable à l'étude de la civilisation canadienne.

Dans la politique extérieure de la Nouvelle-France, l'histoire militaire occupe une place importante. Or, dans ce domaine, le nombre des publications est considérable. Mais, à y regarder de plus près, une grande partie de ces publications consiste en éditions de textes (récits de batailles, journaux d'officiers, rapports, etc.). Les études sont moins nombreuses, et quelques-unes assez faibles, soit que l'on ait méconnu les intérêts économiques qui animaient les belligérants, ou que l'on n'ait pas suffisamment compris la mentalité des Indiens alliés ou en-

17 Voir note 2 ci-haut.

18 Alfred G. Bailey, The Conflict of European and Eastern Algonkian Cultures, 1504-1700. A study in Canadian civilization. Saint John, New Brunswick Museum, 1937, 206 p., 25 cm. 
nemis. Par contre, il y en a d'excellentes : celles de Desrosiers dans l'Iroquoisie, 19 d'Eccles dans son étude sur Frontenac 20 et de Frégault dans Iberville le Conquérant ${ }^{21}$ François Bigot ${ }^{22}$ et La guerre de la Conquête. ${ }^{23}$ Il reste néanmoins à combler certaines lacunes : les expéditions de Tracy, la guerre de Succession d'Espagne et les guerres d'Acadie mériteraient d'être mieux connues. Signalons enfin que l'armature militaire de la colonie reste à étudier dans le détail. On n'a guère là-dessus que quelques études trop brèves et trop générales, comme celles de Lanctot sur Les troupes de la Nouvelle-France ${ }^{24}$ ou de Filteau dans La naissance d'une nation. 25

Au moment de terminer ces considérations sur l'étude de la vie politique en Nouvelle-France, je veux attirer l'attention sur le fait que bien peu d'administrateurs coloniaux ont été l'objet d'études poussées, si ce n'est [15] Talon ${ }^{26}$ et Bigot ${ }^{27}$ parmi les intendants et, parmi les gouverneurs-Champlain mis à part —, Frontenac ${ }^{28}$, Vaudreuil II 29 et (à peine) La Galissonnière ${ }^{30}$. N'est-il pas significatif et inquiétant à la fois que ceux-là mêmes qui dirigèrent la politique de la NouvelleFrance soient si peu connus ? Si l'on a beaucoup étudié l'histoire poli-

19 Voir note 2, page 13.

20 Voir note 5 , page 13.

21 Guy Frégault, Iberville le Conquérant, Montréal, Pascal, [1944], 415 p., $21.5 \mathrm{~cm}$.

22 Guy Frégault, François Bigot, administrateur français, Montréal, Institut d'Histoire de l'Amérique française, 1948, 2 vol., 442 et 415 p., $21.5 \mathrm{~cm}$.

23 Guy Frégault, La guerre de la conquête, Montréal, Fides [1955], 514 p., 25 $\mathrm{cm}$.

24 Gustave Lanctot, « Les troupes de la Nouvelle-France », The Canadian Historical Association Report, 1926, 40-60.

25 Voir note 2, page 11.

26 Voir note 4 page 13.

27 Voir note 5, page 14.

28 Henri Lorin, Le comte de Frontenac; étude sur le Canada fançais à la fin du XVII siècle, Paris, A. Colin \& Cie, 1895, 502 p., 22.5 cm. Voir aussi note 5 , page 13.

29 Guy Frégault, Le grand marquis, Pierre de Rigaud de Vaudreuil et la Louisiane, Montréal, Fides, 1952, 481 p., $21 \mathrm{~cm}$.

30 Voir les articles de Roland Lamontagne dans la Revue d'Histoire de L'Amérique française des dernières années. 
tique de la Nouvelle-France, il reste encore bien des connaissances à parfaire.

\section{La vie économique}

S'il me fallait caractériser d'un mot l'état actuel des recherches sur l'économie de la Nouvelle-France, je dirais que nous n'avons pas encore d'études économiques en profondeur ou, si l'on veut, que nous avons des études économiques sans chiffres. Nous manquons en effet de travaux fondés sur le dépouillement systématique des greffes des notaires, des documents seigneuriaux, des livres de comptes des communautés et des particuliers, et des archives portuaires de France. Aussi longtemps que ces sources n'auront pas été exploitées, on devra se contenter de faire l'histoire économique d'après certaines données très générales et très partielles de la Correspondance générale ou des Ordres du Roi. Ces sources officielles, si elles donnent une idée générale de l'orientation et des caractéristiques principales de l'économie canadienne au $\mathrm{XVII}^{\mathrm{e}}$ et au $\mathrm{XVIII}^{\mathrm{e}}$ siècles, ne permettent toutefois pas d'expliquer tous les phénomènes socio-économiques et, surtout, elles manquent singulièrement de nuances.

En attendant ces études plus approfondies, sur quoi pouvons-nous compter actuellement? Il y a d'abord, dans plusieurs revues et notamment dans le Bulletin des Recherches historiques, un grand nombre de petites études de détail dont l'ensemble constitue certainement un riche apport à la connaissance de la vie économique du régime français, mais auxquelles on n'a pas suffisamment accordé d'attention. Mentionnons les articles de Suite, de P.-G. Roy et surtout de E.-Z. Massicotte. D'autres travaux, plus considérables, sont également précieux : les articles de Mgr Tessier sur les Forges du Saint-Maurice 31, de P.-G. Roy sur la construction des navires ${ }^{32}$, par exemple, et l'ouvrage bien documenté de J.-Noël Fauteux sur l'industrie [16] en Nou-

31 Voir les Cahiers des Dix, 12, 1947, 53-74 ; 13, 1948, 63-84 ; 14, 1949, $165-186$; 15, 1950, 163-184.

32 Pierre-Georges Roy, «La Construction Royale de Québec », Cahiers des Dix, 11, 1946, 141-190. 
velle-France. ${ }^{33}$ Ce dernier ouvrage, qui consacre un chapitre à chacune des industries du régime français, a le mérite d'être un inventaire très complet; on regrette toutefois que Fauteux n'ait pas jugé bon d'enrichir son étude d'une conclusion qui eût pu être une excellente synthèse de cette question.

Il faut également mentionner quelques études remarquables portant sur l'un ou l'autre des aspects importants de la vie économique de la Nouvelle-France : l'ouvrage classique de Biggar, The Early Trading Companies of New France ; 34 les ouvrages d'Innis sur la traite des fourrures 35 et les pêcheries; 36 l'article de Shortt, "The Canadian Currency and Exchange under French Rule »: 37 et les travaux de Salone 38 et de Caron ${ }^{39}$ sur la colonisation. Des recherches plus minutieuses dans des sources encore insuffisamment exploitées pourront certainement apporter des corrections à ces ouvrages, mais il reste que ce sont là d'excellentes études de base.

À côté de ces études de détail, il y a quelques synthèses, comme l'article de Shortt, « The Colony in its Economie Relations », ${ }^{40}$ publié dans Canada and its Provinces. En outre, certaines histoires économiques accordent quelques chapitres au régime français : Mary Quayle

33 Joseph-Noël Fauteux, Essai sur l'industrie au Canada sous le régime français, 2 vol., Québec, Louis Proulx, Imprimeur du roi, 1927, 572 p., 19 cm.

34 H. P. Biggar, The Early Trading Companies of New France, Toronto, 1901, 308 p., $27.5 \mathrm{~cm}$.

35 Harold A. Innis, The Fur Trade in Canada ; An Introduction to Canadian Economic History, New-Haven, Yale University Press ; Londres, H. Milford, Oxford University Press, 1930, 44 p., $24.5 \mathrm{~cm}$.

36 Harold A. Innis, The Cod Fisheries ; The History of an International Economy, New-Haven, Yale University Press ; Toronto, Ryerson Press, 1940, Xv -f- 520 p., $24 \mathrm{~cm}$.

37 Adam Shortt, «Canadian Currency and Exchange under French Rule », Journal of the Canadian Banker's Association, I, 1898-99, 147-

38 Emile Salone, La colonisation de la Nouvelle-France. Étude sur les origines de la nation canadienne-française, Paris, Guilmoto, 1906, XII + 467 p., 22 cm.233.

39 Ivanhoe Caron, La colonisation du Canada sous la domination française ; précis historique, XII + 90 p., $26 \mathrm{~cm}$.

40 Adam Shortt, "The Colony in its Economie Relations », dans Canada and its Provinces, II, 445-530. 
Innis ${ }^{41}$ consacre à la Nouvelle-France 52 pages, mais son étude est aujourd'hui dépassée par celle d'Easterbrook et Aitken, 42 beaucoup plus dense et mieux charpentée, qui consacre un peu plus de 100 pages au régime français. Il reste néanmoins que ces généralisations ont besoin d'être soumises à la vérification stricte des chiffres que peuvent fournir les greffes des notaires, les documents seigneuriaux, les livres de comptes et les archives portuaires.

C'est donc à cette étude en profondeur, basée sur le dépouillement systématique de sources aussi abondantes que riches, qu'il faut désormais s'attaquer. Car tout ce que contiennent les sources officielles a été utilisé [17] avec beaucoup de méthode et d'intelligence par quelques historiens et en particulier par Paul-Emile Renaud dans son étude très complète - elle touche à tous les secteurs de l'activité économique — sur Les origines économiques du Canada. ${ }^{43}$ Je ne crois pas qu'on puisse aller beaucoup plus loin que Renaud, à moins d'avoir recours à de nouvelles sources d'information. Et cela est possible, comme l'a prouvé Jean Hamelin dans son étude récente sur l'économie de la Nouvelle-France. ${ }^{44}$ Après avoir dépouillé les livres de comptes du Séminaire de Québec, Hamelin a pu, par exemple, tracer la première courbe du blé qui soit fondée sur une étude rigoureuse des prix. PaulEmile Renaud et Jean Hamelin ont tracé la voie : souhaitons qu'une équipe d'historiens poursuive ces recherches qui ne peuvent être que fructueuses.

41 Mary Quayle Innis, An Economic History of Canada, Toronto, The Ryerson Press, 1935, 3 éd., 1948, 363 p.

42 W. T. EASTERBROOK et Hugh G. J. AITKEN, Canadian Economic History, Toronto, The Macmillan Company of Canada, Limited, 1956, XX + 606 p., $25 \mathrm{~cm}$.

43 Paul-Émile RENAUD, Les origines économiques du Canada ; l'œuvre de la France, Marnes, Gabriel Énault, imprimeur-éditeur, 1928, 488 p., 24.5 cm.

44 Jean HAMELIN, Économie et société en Nouvelle-France, Québec, Les Presses universitaires Laval, 1960, 137 p., $25.5 \mathrm{~cm}$. (Cahiers de l'Institut d'histoire, $\mathrm{n}^{\circ}$ 3.) 


\section{Les explorations}

Arrêtons-nous un instant seulement aux études sur les explorations en Nouvelle-France. Si les voyages de Cartier et de Champlain sont bien connus maintenant, les études sur les explorations de la période 1635-1670 demanderaient d'être revues, car les travaux de Gosselin 45, de J.-E. Roy ${ }^{46}$ et de Justin Winsor ${ }^{47}$ sont aujourd'hui nettement dépassés. Toutefois, grâce à l'étude définitive de Grâce Lee Nute, ${ }^{48}$ les explorations de Radisson et de Des Groseilliers ne posent plus guère de problèmes. Grâce aussi aux travaux récents de Delanglez, ${ }^{49}$ de Frégault 50 et de Groulx, ${ }^{51}$ les connaissances sur les découvertes de Jolliet et Marquette et de La Salle ont fait de grands progrès, quoique La Salle reste encore à étudier, je crois. De même, pour le XVIII ${ }^{\mathrm{e}}$ siècle, toute l'entreprise de La Vérendrye et de ses fils mériterait d'être étudiée d'une façon plus systématique.

45 Auguste GOSSELIN, Jean Nicolet et le Canada de son temps, 1618-1642, Québec, Laflamme, 1905, 280 p., 19 cm. Le même, Jean Bourdon et son ami l'abbé de Saint-Sauveur, Québec, Dussault \& Proulx, 1904, 248 p., 19 $\mathrm{cm}$.

46 J.-Edmond ROY, Le premier colon de Lévis, Guillaume Couture, Lévis, Mercier \& Cie, 1884, 160 p., 16.5 cm. Le même, Histoire de la seigneurie de Lauzon, vol. I, Lévis, Mercier \& Cie, 1897, 202-224.

47 Justin WINSOR, éd., Narrative and Critical History of America, Boston, Mifflin \& Co., 1889, 8 vol. 27 cm.

48 Grâce Lee NUTE, Caesars of the Wilderness. Médart Chouart, sieur des Groseilliers and Pierre Esprit Radisson, 1618-1710, New-York, Appleton, [1943], 386 p., 23 cm.

49 Jean de DELANGLEZ, s.j., Life and Voyages of Louis Jolliet, 1645-1700, Chicago, Institute of Jesuit History, 1948, 289 p., 23.5 cm. Le même, Some La Salle Journeys, Chicago, Institute of Jesuit History, 1938, 103 p., 23.5 $\mathrm{cm}$.

$50 \quad$ Voir note 4, page 14.

51 Lionel GROULX. Notre grande aventure. L'empire français en Amérique du Nord, 1535-1760, Montréal, Fides, [1958], 302 p., 24.5 cm. 


\section{La vie religieuse}

L'importance des entreprises missionnaires et l'ampleur du rôle joué par l'Église sous le régime français sont si grandes qu'on ne saurait en négliger l'étude sans risquer de ne rien comprendre à l'histoire de la Nouvelle-France. Pendant longtemps (1632-1663), la mission canadienne prend le pas sur la colonie, laquelle est en quelque sorte à la remorque de la mission ; et, même après 1663, les préoccupations et le travail missionnaires conservent, au pays, une place à part. À partir de 1659, cependant, Mgr de Laval commence à organiser l'Église canadienne proprement dite et lui donne des institutions destinées à répondre aux besoins de la colonie. À cause de la grande influence qu'ils exercèrent sur leurs contemporains, ces hommes - Mgr de Laval, les Jésuites - et ces femmes aussi - Marie de l'Incarnation, Jeanne Mance, Marguerite Bourgeoys — qui sont à l'origine de notre histoire religieuse, méritent d'être mieux connus. Une excellente connaissance de leur spiritualité et de leur doctrine est en effet essentielle à la compréhension du comportement religieux de la population.

On a beaucoup parlé des entreprises missionnaires, et de celles, en particulier, de la première moitié du XVII ${ }^{\mathrm{e}}$ siècle. Mais, trop souvent, s'est-on contenté de paraphraser les Relations des Jésuites ou de laisser courir son imagination. Pour beaucoup d'écrivains, la vie des missionnaires devint un roman d'aventures. Il existe toutefois des études d'une conception plus scientifique, dont l'ouvrage considérable de Rochemonteix sur les Jésuites de la Nouvelle-France au $\mathrm{XVII}^{\mathrm{e}}$ et au $\mathrm{XVIII}^{\mathrm{e}}$ siècles ${ }^{52}$. Un peu dépassés aujourd'hui, les travaux de Rochemonteix ont été repris en partie, mais d'une façon plus rigoureuse, par quelques Jésuites canadiens : les pères Léon Pouliot, ${ }^{53}$ Florian Lari-

52 Camille de Rochemonteix, s.j., Les Jésuites et la Nouvelle-France au XVII siècle. . ., Paris, Letouzé \& Ané, 1895-96, 3 vol. 22.5 cm. Le même, Les Jésuites et la Nouvelle-France au XVIII' siècle ...., Paris, Alphonse Picard \& Fils, 1906, 2 vol. $22.5 \mathrm{~cm}$.

53 Léon POULIOT, s.j., Étude sur les Relations des Jésuites de la NouvelleFrance, 1632-1672, Paris, Desclée de Brouwer \& Cie, 1940, 319 p., 24.5 
vière, ${ }^{54}$ René Latourelle 55 et Jean Côté, 56 entre autres. D'une façon générale, toutefois, les historiens des missions, après Rochemonteix, ne se sont guère intéressés qu'à l'époque héroïque, celle des martyrs. Outre l'ouvrage de Rochemonteix, et celui — plus général — de Gosselin sur l'Église canadienne, 57 il n'y a à peu près pas [19] d'études sur les missions postérieures à la suppression des Relations des Jésuites en 1672. Il y a donc là une lacune à combler. Il faudrait également étudier les entreprises missionnaires du Séminaire des Missions Étrangères de Québec, du Séminaire Saint-Sulpice de Montréal et la mission de l'Acadie. De plus, les remarques que j'ai faites précédemment sur la nécessité de mieux connaître les Indiens s'appliquent éminemment au problème missionnaire. Jusqu'ici, les historiens des missions n'ont guère poussé leurs recherches de ce côté. Pourtant, aussi longtemps qu'on n'aura pas étudié à fond les Indiens et leur civilisation, on ne pourra émettre de jugements valables sur les résultats et la portée des efforts missionnaires. Il est déplorable, à ce point de vue, qu'un ouvrage récent comme celui de Kennedy, Jesuit and Savage in New France, 58 n'apporte guère de nouveau dans l'interprétation des problèmes missionnaires.

En somme, il faut maintenant étudier les entreprises missionnaires postérieures à 1670 — sans oublier celles des Récollets d'avant 1629,

cm. Le même, Les Saints Martyrs canadiens, Montréal, Bellarmin, [1949], 174 p., $18.5 \mathrm{~cm}$.

54 Florian LARIVIERE, s.j., La vie ardente de saint Charles Garnier, Montréal, Les Éditions Bellarmin, [1957], 212 p., 19.5 cm.

55 René LATOURELLE, s.j., Étude sur les écrits de saint Jean de Brébeuf, Montréal, Les Éditions de l'Immaculée-Conception, 1952, 2 vol. $24.5 \mathrm{~cm}$.

56 Jean CÔTÉ, s.j., "L'institution des donnés », Revue d'Histoire de l'Amérique française, $X V$, 1961-62, 344-378.

57 Auguste GOSSELIN, La mission du Canada avant Mgr de Laval (16151659), Èvreux, Imprimerie de l'Eure, 1909, 176 p., 25 cm. Le même, Vie de Mgr de Laval [....] 1622-1J08, 2 vol., Québec, Imprimerie de L.-J. Demers \& Frère, 1890, 22.5 cm. Le même, L'Église du Canada depuis Mgr de Laval jusqu'à la conquête, 3 vol., Québec, Typ. Laflamme \& Proulx, 1914-1917, $23.5 \mathrm{~cm}$.

58 J. H. KENNEDY, Jesuit and Savage in New France, New-Haven, Yale University Press, 1950, 206 p., 24 cm. 
un peu trop négligées depuis Jouve 59 — et surtout réinterpréter, grâce à une meilleure connaissance des Indiens et à une critique plus attentive des sources, tout l'effort missionnaire du régime français.

$$
* \quad * *
$$

Le travail apostolique auprès des Indiens n'est toutefois qu'un aspect de la vie religieuse sous le régime français. À plusieurs égards, la vie de l'Église canadienne est pour nous plus importante. Quel est, sur ce point, 60 l'état de nos connaissances ? Grâce aux travaux de Gosselin, nous avons une bonne vue d'ensemble de l'histoire de l'Église de la Nouvelle-France. En outre, plusieurs études de détail sur l'établissement du Séminaire de Québec et des dîmes, sur l'érection des paroisses, sur le régime des cures, complètent et précisent les travaux de Gosselin. La plupart de ces études se trouvent dans les Rapports de la Société canadienne d'Histoire de l'église catholique, organisme qui, depuis plus de vingt-cinq ans, a beaucoup contribué à la connaissance de l'histoire religieuse du régime français.

Il faudrait maintenant que l'on s'attache à étudier en profondeur certaines questions aussi importantes que l'organisation, le rôle et l'esprit du Séminaire de Québec, lequel devait, pour reprendre l'expression même de Mgr de Laval, « servir de clergé à cette nouvelle Église », par exemple, ou que les relations de l'Église et de l'État qui, malgré les travaux trop uniquement [20] narratifs d'Eastman ${ }^{61}$ ou de Delanglez, ${ }^{62}$ restent à expliquer. En outre, il faudrait étudier la vie et l'œuvre des évêques de la Nouvelle-France, qui ne nous sont guère connus qu'à travers l'histoire générale de Gosselin ou les biographies

59 Odoric-Marie JOUVE, O.F.M.. Les Franciscains et le Canada ; l'établissement de la foi, 1615-1629, Québec, 1915, 506 p., 23 cm.

60 Voir note 6, page 18.

61 Mack EASTMAN, Church and State in Early Canada, Edimbourg, 1915, 301 p., $24.5 \mathrm{~cm}$.

62 Jean de DELANGLEZ, s.j., Frontenac and the Jesuits, Chicago, Institute of Jesuit History, 1939, 296 p., 23.5 cm. 
trop sommaires de Mgr Têtu. 63 Il y a donc des lacunes à combler. Mais il y aura aussi à réinterpréter, grâce à une étude plus systématique et plus attentive des sources et avec le double éclairage de l'histoire religieuse de la France des $\mathrm{XVII}^{\mathrm{e}}$ et $\mathrm{XVIII}^{\mathrm{e}}$ siècles et de l'histoire générale de la Nouvelle-France, toute cette histoire religieuse du régime français qui, me semble-t-il, n'a été qu'à peine ébauchée.

Dans cette réinterprétation de l'histoire religieuse, on devra porter une attention spéciale à l'influence des différentes écoles de spiritualité qui, nombreuses au $\mathrm{XVII}^{\mathrm{e}}$ siècle, marquèrent profondément plusieurs des maîtres spirituels de la colonie. L'influence d'un M. de Bernières sur toute la carrière de Mgr de Laval, par exemple, ne saurait impunément être négligée. Ces grandes figures religieuses - Marie de l'Incarnation, Marguerite Bourgeoys, Jeanne Mance, Maisonneuve — qui, avec Mgr de Laval, furent si présentes aux colons, doivent être étudiées si on veut comprendre un jour la mentalité religieuse des Canadiens du régime français. À part Dom Jamet, 64 qui fit en ce sens un travail remarquable, mais trop tôt interrompu, le père Porter 65 et tout récemment le jésuite Roustang, 66 les historiens ont quasi entièrement négligé cette étude. Aussi longtemps que cette lacune n'aura pas été comblée, notre connaissance de la société du régime français restera amputée de l'un de ses éléments essentiels.

63 Mgr Henri TÊTU, Les évêques de Québec, Québec, N.-S. Hardy, 1889, 692 p., $23.5 \mathrm{~cm}$.

64 Écrits spirituels et historiques [de Marie de l'Incarnation], publiés par Dom Claude Martin [...] réédités par Dom Albert Jamet [...] avec des annotations critiques, des pièces documentaires et une biographie nouvelle. Paris, Desclée de Brouwer \& Cie, 1929-1939, 4 vol. 25 cm. Dom Albert JAMET, Les Annales de l'Hôtel-Dieu de Québec, 1636-1716, à l'Hôtel-Dieu de Québec, 1939, XLVII + 444 p., $33 \mathrm{~cm}$.

65 Fernand PORTER, o.f.m., L'instruction catéchistique au Canada, Deux siècles de formation religieuse, 1663-1833, Montréal, Les éditions franciscaines, 1949, 332 p., $24 \mathrm{~cm}$.

66 François ROUSTANG, s.j., Jésuites de la Nouvelle-France, Desclée de Brouwer, [1961], 351 p., $20 \mathrm{~cm}$. 


\section{La société}

Sous ce titre, j'entends parler brièvement de l'état des recherches sur la population, le régime seigneurial, les groupes sociaux et la vie intellectuelle et artistique du régime français.

Il y a peu d'études démographiques portant sur la Nouvelle-France. Pourtant, nombreux sont les chercheurs qui ont accumulé une masse impressionnante [21] de renseignements : Mgr Tanguay 67 et le Père Archange Godbout, 68 entre autres. Et combien d'articles précieux pour la connaissance de la population canadienne sous le régime français ont été publiés dans des revues comme le Bulletin des Recherches historiques ou les Mémoires de la Société généalogique canadiennefrançaise. Néanmoins, on ne compte guère que deux études d'ensemble, du reste très inégales en valeur. La première, due à Georges Langlois, 69 est assez faible, parce qu'elle ne repose pas sur une étude critique des chiffres fournis par les recensements ou les documents officiels. Or, je sais, pour y avoir beaucoup travaille, que les recensements de la Nouvelle-France sont très incomplets : il y manque généralement le quart, quand ce n'est pas le tiers de la population. Beaucoup plus intéressante et précieuse est l'étude de Jacques Henripin sur la population de la Nouvelle-France au XVIII ${ }^{\text {e }}$ siècle. ${ }^{70}$ Fondée sur une méthode rigoureuse, elle tient compte davantage de la marge possible d'erreurs - l'auteur utilise les données de Tanguay — et établit d'une façon beaucoup plus satisfaisante les taux de nuptialité, de fécondité et de mortalité infantile qui font l'objet de ses recherches.

67 Mgr Cyprien TANGUAY, Dictionnaire généalogique des familles canadiennes depuis la fondation de la colonie jusqu'à nos jours, 7 vol., Province de Québec, Eusèbe Sénécal, imprimeur-éditeur, 1871-1890, 27 cm.

68 Archange GODBOUT, o.f.m., Nos ancêtres au XVII siècle, publié par tranches dans le Rapport de l'Archiviste de Québec depuis 1951.

69 Georges LANGLOIS, Histoire de la population canadienne-française, $2^{\mathrm{e}}$ édition, Montréal, Albert Lévesque, 1935, 309 p. 19.5 cm.

70 Jacques HENRIPIN, La population canadienne au début du XVIII ${ }^{\mathrm{e}}$ siècle. Nuptialité, fécondité, mortalité infantile, Presses universitaires de France, 1954, 129 p., $24.5 \mathrm{~cm}$. 
Henripin a démontré la possibilité et l'utilité de telles études pour le régime français. Mais il reste beaucoup à faire. Ces recherches sur la population devraient s'étendre à l'immigration, à la composition de la société canadienne, à la densité et aux mouvements de la population. Signalons que nous avons en ce sens certains travaux de qualité : l'ouvrage de Lanctot sur les filles du roi, ${ }^{71}$ l'étude de Malchelosse sur les faux sauniers, les prisonniers et les fils de famille en NouvelleFrance, 72 les chapitres consacrés par Renaud 73 et Hamelin ${ }^{74}$ aux engagés et aux gens de métier, et plusieurs cartes sur le peuplement publiées par Marcel Trudel dans son Atlas historique du Canada. ${ }^{75}$

Il serait dommage que les études démographiques fussent négligées dans un pays où les anciens registres d'état-civil, les greffes des notaires [22] et les archives seigneuriales du régime français constituent une source unique de documentation.

La population de la Nouvelle-France vécut en grande majorité à l'intérieur du régime seigneurial, lequel fut à l'origine d'un mode de vie. C'est pourquoi il est essentiel de le bien étudier pour connaître la société du régime français. Or, sur le fonctionnement, la conception et le rôle du régime seigneurial, les bonnes études ne manquent pas. Outre l'ouvrage classique de Salone ${ }^{76}$ sur la colonisation, on a les études de Munro, ${ }^{77}$ de Heneker ${ }^{78}$ et de Morin, ${ }^{79}$ certains chapitres de Fré-

71 Gustave LANCTOT, Filles de joie ou filles du roi. Étude sur l'immigration féminine en Nouvelle-France, Montréal, Chanteclerc, 1952, 230 p., 19.5 cm.

72 Gérard MALCHELOSSE, «Faux sauniers, prisonniers et fils de famille en Nouvelle- France », Cahiers des Dix, 1944, 161-194. Autre étude dans ibid., 1946, 261-311.

73 Voir note 1 , page 17.

$74 \quad$ Voir note 2, page 17.

75 Marcel Trudel, Atlas historique du Canada français des origines à 1867. Édition remaniée. Presses de l'Université Laval, 1961. 93 cartes, 28 cm.

76 Voir note 6, page 16.

77 William Bennet MUNRO, The Seignorial System in Canada. A Study in French Colonial Policy, New-York, Longmans, Green and Co., 1907, 296 p., $23 \mathrm{~cm}$.

78 Dorothy A. HENEKER, The Seignorial Regime in Canada, Québec, Imprimerie Ls-A. Proulx, 1927, 447 p., $20 \mathrm{~cm}$.

79 Victor MORIN, Seigneurs et censitaires, castes disparues, Éditions des Dix, 1941, 104 p., 24 cm. 
gault 80 et de Filteau, 81 de même que la plaquette de Marcel Trudel 82 qui explique minutieusement le fonctionnement du système. Mais tout n'a pas été dit sur le régime seigneurial. Il reste à faire beaucoup d'études de détail, grâce au dépouillement des archives seigneuriales et des greffes des notaires, sur les conditions des concessions, les relations de seigneur à colon, les communes, la banalité, etc. Mentionnons, comme un exemple de ces travaux de détail, l'excellent article de Roland Sanfaçon, "La construction du premier chemin QuébecMontréal et le problème des corvées ». 83 Il est probable qu'à la suite de semblables études il faudra réviser certains jugements et certaines interprétations qui ont actuellement cours sur le régime seigneurial.

Mais la société du régime français déborde largement le cadre seigneurial. Comme en toute société, on y voit des fonctionnaires, des militaires, des clercs, des commerçants, des artisans. Il est extrêmement important d'étudier à fond le comportement économique et social de ces groupes, afin de découvrir leur mentalité et leurs aspirations collectives et de déterminer le rôle exact qu'ils jouèrent dans la société canadienne du régime français. Il faut bien avouer, pourtant, qu'en ce domaine on n'a guère d'études poussées, quoique, dans des ouvrages généraux de Groulx, 84 de Frégault, 85 de Munro, 86 de Colby, 87 de Jean Hamelin, 88 on trouve des données intéressantes. [23] Souhaitons que, à l'aide de la riche documentation des greffes des notaires - encore une fois ! - , on poursuive des recherches qui accroîtront notre connaissance des types sociaux de la Nouvelle-France.

$80 \quad$ Voir note 1 , page 11.

81 Voir note 2, page 11.

82 Marcel Trudel, Le régime seigneurial. Publication $n^{\circ} 6$ de la Société historique du Canada, 20 p., $21 \mathrm{~cm}$.

83 Roland Sanfaçon, «La construction du premier chemin Québec-Montréal et le problème des corvées », Revue d'Histoire de l'Amérique française, XII, 1958-59, 3-29.

$84 \quad$ Voir note 7 , page 13.

85 Voir note 1 , page 11.

86 William Bennett MUNRO, Crusaders of New France; a Chronicle of the Fleur-de-lis in the Wilderness, New-Haven, Yale University Press, 1918, 237 p., $21 \mathrm{~cm}$.

87 Charles William Colby, Canadian Types of the Old Régime, 1608-1698, New-York, H. Holt and Co., 1908, 366 p., 23 cm.

88 Voir note 2, page 17. 
Dans l'étude de la société du régime français, il importe beaucoup de s'arrêter à l'activité intellectuelle et artistique. Plusieurs auteurs se sont consacrés à cette recherche. Mentionnons les études de Gosselin, 89 de Groulx 90 et de Audet ${ }^{91}$ sur le système d'enseignement du régime français, l'ouvrage d'Antoine Roy sur Les lettres, les sciences et les arts..., ${ }^{92}$ les travaux de Morisset, ${ }^{93}$ de Traquair 94 et de Gowans 95 sur les arts. En ce domaine, les études semblent bien engagées. Souhaitons qu'elles soient poursuivies, car tout n'a certainement pas été dit sur cet aspect important de la culture canadienne.

Si l'on a beaucoup écrit, jusqu'ici, sur la société du régime français, il reste néanmoins beaucoup à faire avant de prétendre à une connaissance approfondie de cette société. La rareté des bonnes études de détail, l'absence de toute recherche en certains secteurs et le peu d'historiens qui se soient intéressés à ces questions, voilà qui explique peutêtre que nous n'ayons pas encore d'études d'ensemble sur la société du régime français. Mentionnons toutefois quelques contributions de bel-

89 Amédée Gosselin, L'instruction au Canada sous te régime français (16351760), Québec, Typ. Laflamme \&Proulx, 1911, 501 p., 23 cm.

90 Lionel Groulx, L'enseignement français au Canada, vol. I : Dans le Québec, Montréal, Granger, 1931, 22 cm.

91 Louis-Philippe Audet, Le système scolaire de la province de Québec. Tome II : L’instruction publique de 1635 à 1800, Québec, Les Éditions de l'Érable, 1950, $20 \mathrm{~cm}$.

92 Antoine Roy, Les lettres, les sciences et les arts au Canada sous le régime français. Essai de contribution à l'histoire de la civilisation canadienne. Paris, Jouve \& Cie, 1930, 292 p., $24.5 \mathrm{~cm}$.

93 Gérard Morisset, L'architecture en Nouvelle-France, Québec, 1949, 150 p., 19.5 cm. Le même, Coup d'œil sur les arts en Nouvelle-France, Québec, Charrier et Dugal, 1941, 170 p., 19 cm. Le même, La vie et l'œuvre de frère Luc, Québec, Médium, 1944, 19 cm. Dans plusieurs autres ouvrages, M. Morisset a traité des arts en Nouvelle-France. Son œuvre est un apport vraiment remarquable.

94 Ramsay Traquair, The Old Architecture of Québec ; A Study oj the Buildings Erected in New France from the Earliest Explorers to the Middle of the Nineteenth Ceniury, Toronto, MacMillan Co. of Canada, 1947, XIX + 324 p., $30 \mathrm{~cm}$. Traquair a également publié un grand nombre d'articles sur l'architecture religieuse.

95 Alan Gowans, Church Architecture in New France, Toronto, University of Toronto Press, 1955, XII + 161 p., 25 cm. 
le qualité : Aux sources de notre histoire, de Gérin ; 96 La civilisation de la Nouvelle-France 97 et La société canadienne sous le régime français 98 de Frégault ; "La Nouvelle-France », un article de Marcel Trudel ; 99 La naissance d'une nation, de Filteau ; 100 [24] et quelques chapitres de l'Histoire du Canada français, de Groulx ${ }^{101}$. Pour le moment, c'est donc à l'étude de détail des différents aspects de cette société que doivent s'attacher les historiens, afin qu'on puisse écrire un jour cette synthèse à laquelle nous rêvons tous.

\section{Conclusion}

Il est incontestable que l'on a fait, jusqu'ici, beaucoup de progrès vers la connaissance de notre régime français. Mais il reste à étudier plusieurs secteurs mal connus, que j'ai tenté d'indiquer, et à réinterpréter plusieurs aspects de notre histoire. En particulier, j'ai insisté sur la nécessité, en certains domaines, de faire un dépouillement systématique de ces sources essentielles que sont les greffes des notaires, les archives seigneuriales, les livres de comptes des institutions et des particuliers et les archives portuaires de France. Mais si les greffes des notaires sont, dans quelques districts judiciaires, accessibles et bien classés, il n'en va pas de même partout ; en outre, plusieurs institutions religieuses, qui conservent une riche documentation nécessaire à une étude approfondie du régime seigneurial ou de l'histoire des prix, ne mettent pas toujours volontiers leurs richesses à la disposition des historiens ; enfin, peu de recherches ont été faites dans les archives portuaires de France et il est très difficile, sinon tout à fait impossible, pour les chercheurs canadiens d'aller eux-mêmes explorer ces dépôts

96 Léon GÉRIN, Aux sources de notre histoire. Les conditions économiques et sociales de la colonisation en Nouvelle-France, Montréal, Fides, 1946, 274 p., $19 \mathrm{~cm}$.

$97 \quad$ Voir note 1 , page 11.

98 Guy Frégault, La société canadienne sous le régime français, Ottawa, Les brochures de la Société historique du Canada, 1954, 16 p., 21 cm.

99 Marcel Trudel, « La Nouvelle-France », Cahiers de l'Académie canadiennefrançaise, II, 23-50.

100 Voir note 2, page 11.

101 Voir note 7, page 13. 
dont on ne fait du reste que soupçonner les richesses. Ce sont là trois obstacles sérieux. Le gouvernement provincial ne pourrait-il pas, par l'intermédiaire des Archives de la Province, faire déplacer vers les grands centres les archives notariales du régime français conservées au petit bonheur dans certains districts judiciaires éloignés ? Il serait essentiel aussi que le même service des Archives provinciales fasse microfilmer, avec la permission des communautés religieuses concernées, les archives d'intérêt national qu'elles conservent, ce qui, en plus d'aider considérablement la recherche, constituerait une excellente mesure de sécurité. Enfin, une politique semblable pourrait s'appliquer aux archives portuaires, dont les documents intéressant l'histoire du Canada devraient être microfilmés. Ces trois mesures, appliquées à temps, seraient une aide fort précieuse à la connaissance du régime français. 102

\author{
André Vachon \\ Directeur des éditions, \\ Les Presses de l'Université Laval.
}

102 On comprendra que, dans les limites de cet exposé, nous ne pouvions faire état des instruments de travail et des éditions de documents relatifs à la Nouvelle-France. On nous permettra, toutefois, de rappeler les nombreuses publications (inventaires, index, bibliographies, éditions de documents, etc.) que nous devons aux Archives de la Province de Québec et dont ne saurait se passer l'historien de la Nouvelle-France. 


\title{
COMMENTAIRE
}

\author{
Marcel Trudel
}

Institut d'histoire, Université Laval.

[pp. 25-26.]

$\underline{\text { Retour à la table des matières }}$

À l'inventaire patient que vient de présenter M. Vachon de l'état des recherches sur le régime français, je n'ai pas l'intention d'ajouter des éléments qu'il aurait oubliés. Je veux m'en tenir à un commentaire marginal.

Le travail de $M$. Vachon nous a surtout rappelé qu'il restait beaucoup à faire. Notre histoire du régime français souffre de vides effarants : songeons que nous ne savons encore presque rien de l'habitant, ni du rôle qu'il a joué dans la traite des pays d'en haut (lui qu'on représente si attaché au sol) ; qu'il n'existe aucune étude sociale d'un organisme aussi imposant que le Conseil Supérieur, aucune étude sociale des troupes de la colonie; si l'on connaît assez bien le clergé de la grande époque mystique, nous savons très peu de choses du clergé du $\mathrm{XVIII}^{\mathrm{e}}$ siècle, ce clergé qui cesse d'être missionnaire pour se " fonctionnariser ». Or il est essentiel de connaître exactement le régime français et surtout le régime français du XVIII ${ }^{\mathrm{e}}$ siècle : ce sont les institutions du $\mathrm{XVIII}^{\mathrm{e}}$ siècle, enfin stabilisées après les flottements du 
$\mathrm{XVII}^{\mathrm{e}}$, qui ont fait le Canada français du $\mathrm{XIX}^{\mathrm{e}}$ et, encore en bonne partie, celui d'aujourd'hui. Je parlais tantôt de clergé : pour comprendre le clergé du XIX ${ }^{\mathrm{e}}$ siècle, c'est celui du XVIII ${ }^{\mathrm{e}}$ qu'il faut étudier ; or on ne l'a pas étudié parce qu'il n'avait pas figure de vedette comme celui de l'époque mystique.

Le régime français, et en particulier celui du $\mathrm{XVIII}^{\mathrm{e}}$ siècle, nous a laissé, au siècle suivant et plus tard, une vie religieuse conçue de telle façon, des normes déterminées, une société bâtie de telle manière. Nous ne pourrons bien nous connaître que lorsque nous connaîtrons bien ce régime français.

Et pourtant, on a souvent affirmé que, dans leurs préoccupations historiques, les Canadiens français s'en tenaient au régime d'avant 1760, négligeant en quelque sorte d'étudier ce qui s'était produit depuis. Pourquoi alors pouvons-nous, à propos de l'étude de ce régime français, parler de vides effarants ? Les explications sont nombreuses et vaudront, je pense, pour quelques-uns des champs d'enquête de ce colloque.

En excluant les conférences publiques qui se donnaient dans les universités depuis Ferland, conférences qui n'apportaient que de l'information, l'histoire n'est entrée comme discipline régulière dans nos universités de langue française qu'en 1947 ; c'est en 1947 seulement que les universités se sont mises à former des historiens : pour un groupe ethnique qui fonde tout avec acharnement sur l'histoire, c'est là un phénomène qui demeure pour moi un mystère. C'est dire qu'avant 1947, les historiens n'étaient en général que des autodidactes (médecins, notaires, avocats, membres du clergé), sauf les rarissimes exceptions qui avaient pu aller chercher outre frontières une formation historique. Dans ces conditions, notre bibliographie du régime français contient une part très lourde de travaux d'amateurs.

La fièvre de la recherche systématique n'avait pas encore saisi les universitaires ; et je dirais que c'est heureux, puisque la société d'alors n'encourageait pas la recherche: les rares chercheurs de ce temps n'ont survécu que par une force singulière de tempérament, selon la loi de la sélection naturelle; et n'entraient dans certaines salles d'archives que ceux qui avaient eu assez de vigueur pour mettre la muselière au " chien méchant ». Dans les Facultés mêmes, c'était le règne de la dissertation, survivante de l'ancienne « amplification »; il eût été gênant 
pour un étudiant de faire des [26] travaux de recherche quand le professeur n'en faisait pas. Et rappelons, toujours à propos d'université, qu'il existait entre les Facultés un cloisonnement étanche : chaque universitaire se cantonnait dans sa discipline, faisant avec amour l'élevage de ses champignons, mais ignorant (quand cette ignorance n'était pas un certain sourire) ce qui se passait l'autre côté du mur mitoyen. L'histoire, pour sa part, se trouvait ou se voulait coupée de disciplines auxiliaires qui lui sont essentielles.

Tout cela, ajouté au problème des archives dont $\mathrm{M}$. Vachon a parlé, aide à faire comprendre pourquoi, après avoir tant parlé du régime français, nous le connaissons si peu et si mal. Heureusement, il se fait un rapide progrès ; depuis quelques années, la situation de l'histoire s'améliore nettement. Le seul chemin parcouru dans l'enseignement universitaire depuis 1947 est étonnant : les candidats historiens sont plus nombreux, le personnel enseignant s'est multiplié et se livre tout entier à la recherche, la thèse est devenue un important moyen de formation de l'historien (ainsi, à l'Institut d'histoire de Laval, il faut une thèse pour la licence, une autre ensuite pour le diplôme d'études supérieures et une autre pour le doctorat : après quoi, on peut dire que l'historien a eu le temps de se faire la main) ; les étudiants reçoivent aussi un enseignement sur la recherche elle-même et la publication ; ils ont à leur disposition des outils que n'avaient pas leurs prédécesseurs, comme cet inventaire bibliographique d'histoire du Canada que nous avons à notre Institut. L'aide à la recherche est devenue facile et généreuse (ce qui n'empêche pas des incidents cocasses de se produire à la porte de certaines maisons où il y a des archives). Dans leur formation même ou dans l'exercice de leur profession, les historiens actuels ont des préoccupations plus étendues : étudiants, professeurs ou chercheurs, ils s'intéressent aux problèmes de la géographie humaine, de l'économique, de la sociologie, du juridique et d'autres disciplines, dans une vie universitaire où le cloisonnement tend à disparaître : les cours, les relations personnelles et les relations plus fréquentes d'une Faculté à une autre amènent l'historien formé ou en formation, à se situer non plus simplement dans le champ exclusif de l'histoirediscipline, mais dans un horizon plus sain des sciences de l'homme. Nous le constatons déjà dans les thèses que préparent nos candidats : par le choix du sujet et par la façon de le traiter (quoique sur ce dernier point il y ait des restrictions à faire), nos candidats montrent déjà 
qu'ils ont davantage le souci de cet horizon des sciences de l'homme. Cette génération d'historiens (et, j'espère, celles qui suivront) nous fournira certainement une connaissance plus profonde de ce régime français qui nous a faits et permettra aux ouvriers des autres sciences de l'homme d'utiliser des données historiques plus exactes et plus abondantes quand ils étudieront la société vivante.

Marcel Trudel,

Institut d'histoire, Université Laval. 


\title{
Situation de la recherche sur le Canada français
}

\section{Perspectives historiques}

\author{
Fernand Ouellet \\ Faculté de commerce, \\ Université Laval

\section{"L'étude du XIX ${ }^{\mathrm{e}}$ siècle canadien-français.”}

[pp. 25-42.]

\section{$\underline{\text { Retour à la table des matières }}$}

Que savons-nous au juste de notre XIX ${ }^{\mathrm{e}}$ siècle ? L'historien qui désire répondre à une telle question se voit confronté à un certain nombre de difficultés. Ainsi, qui peut prétendre avoir seulement parcouru toute la production historique relative au siècle dernier ? Qu'il s'agisse de l'évolution économique, sociale, démographique, politique ou religieuse, l'historien dispose maintenant d'un assez large éventail de travaux susceptibles de lui fournir une vue d'ensemble de cette époque troublée. Évidemment, parmi les historiens, des figures dominantes se dégagent d'emblée, tels Garneau, David, Suite, Chapais, Groulx, Maheux, Bruchési, Christie, Dent, l'équipe de Canada and its Provinces, Creighton, Lower, Mclnnis, Wade, Morton et Easterbrook, qui non seulement ont réalisé un effort considérable de synthèse mais qui ont aussi pour plusieurs influencé d'une façon décisive l'orientation de la recherche. Ces quelques noms ne font pas oublier les nombreux au- 
teurs d'études spécialisées, les biographes, les fabricants de monographies paroissiales ou régionales et, enfin, les généalogistes.

Suffit-il de s'être familiarisé avec les études les plus marquantes pour être en mesure de porter un jugement valable sur une masse aussi imposante de travaux ? Il faut de plus avoir pris contact avec l'ensemble des problèmes que soulève l'évolution globale du Canada français depuis l'introduction du parlementarisme jusqu'à la prise du pouvoir par Laurier. Tout cela constitue une sérieuse invitation à la prudence.

Deux principaux courants parallèles et, en très grande partie, autonomes ont marqué le développement de l'historiographie canadienne depuis Garneau jusqu'à la seconde guerre mondiale. L'un, d'inspiration essentiellement nationaliste, reflète l'unanimité idéologique des historiens canadiens-français et l'autre, d'origine anglo-saxonne, se révèle depuis la fin du $\mathrm{XIX}^{\mathrm{e}}$ siècle plus ouvert à la diversité et plus soucieux de s'appuyer sur des méthodes scientifiques. Ce n'est pas qu'une certaine influence réciproque ne se soit fait sentir - les œuvres de Chapais, De Celles, Bruchési, Lower et Mason Wade en témoignent mais il reste que, d'une façon générale, les échanges de points de vue ont été plutôt limités. Tout cela tend à donner l'impression d'une double construction historique étroitement cloisonnée.

[28]

\section{I \\ L'apport de l'historiographie canadienne-française}

Un des traits les plus frappants de l'historiographie canadiennefrançaise, c'est, derrière son étonnante unité d'interprétation, l'emprise plus ou moins absolue des valeurs nationalistes. Évidemment, tous les mouvements nationalistes ont suscité un immense intérêt pour l'histoire. Comment, en effet, justifier l'existence et la volonté d'affirmation d'une nation si cette dernière n'apparaît comme l'aboutissement d'impératifs historiques profonds ? C'est dire que, dès le moment où le Canada français devint sensible à l'appel du principe des nationalités, il éprouva le besoin d'une histoire nationale qui le reliât plus sûrement à 
son passé. Cette réaction fut d'autant plus vive que la conscience nationale canadienne-française prit naissance dans un contexte où, en conséquence de brisures dans ses structures anciennes, l'héritage traditionnel paraissait menacé d'aliénation. Cette première crise nationaliste, qui se situe au début du $\mathrm{XIX}^{\mathrm{e}}$ siècle, engendra une première vocation d'historiographe : celle de Jacques Viger qui, dès 1807, alors qu'il était rédacteur du Canadien, entreprit de recueillir la documentation pour une histoire du Canada. De cette œuvre à dessein patriotique que constitue la Saberdache, cette compilation de documents divers, se dégagent déjà les éléments d'une interprétation de l'histoire canadienne qui variera somme toute assez peu après Viger. Puis, un patriote fervent, Jacques Labrie, rédigea sa propre version du passé canadienfrançais. Malheureusement son manuscrit ne fut jamais publié. La perte de ce texte - reflet sans doute du nationalisme agressif et démocratique des années 1830 — est d'autant plus regrettable qu'elle éliminait de notre historiographie traditionnelle la seule production historique susceptible de manifester une certaine indulgence à l'endroit de la démocratie. Il faudra attendre plus d'un siècle avant qu'une telle prédilection ne réapparaisse chez nos historiens.

À la suite de l'entreprise historique du bureaucrate Michel Bibaud, on vit Garneau publier, de 1845 à 1852, son Histoire du Canada. Cette œuvre, bien documentée et éminemment politique dans sa conception, qui, au surplus, a tellement servi de guide à la plupart de nos historiens, était le produit du nationalisme des années 1825 revenu à la mode depuis la faillite du mouvement révolutionnaire. Antidémocratique et épris de conservatisme social et économique, le nationaliste Garneau avait cependant été marqué par le courant réformiste du temps. Mais ce libéralisme, bien qu'admirateur du parlementarisme britannique, ne débordait guère le domaine fort restreint des institutions politiques. Au fond n'était-ce pas surtout parce qu'elles servaient la cause nationale que notre premier historien, accepté comme tel, avait pactisé avec les idées réformistes ? Certaine lettre adressée [29] à Papineau le laisse croire. En tant qu'esprit profondément conservateur, Garneau était l'adversaire de toute attitude extrémiste. De plus, on s'étonnera, après avoir lu son œuvre, de le savoir agnostique et, sachant cela, on comprendra mal ses points de vue exclusivement cléricaux. Était-ce le fruit de son objectivité ? On doit en douter. Comme la plupart de ses contemporains, Garneau voyait dans le ca- 
tholicisme une institution nationale intangible. D'ailleurs, aurait-il voulu remettre en question un certain passé clérical que ses contemporains ne l'auraient pas accepté. Les fortes réactions devant ses petites audaces à propos de Mgr de Laval en font foi. Les hommes de l'époque avaient trop besoin d'unanimité face à l'Anglais protestant pour permettre à l'historien des écarts idéologiques. Expression du nationalisme à un moment donné et d'une conception de l'histoire qui assure la primauté du politique, l'Histoire du Canada de Garneau, du moins en ce qui concerne son interprétation du $\mathrm{XIX}^{\mathrm{e}}$ siècle, demeure l'œuvre d'un esprit modéré. Si elle ne possède pas l'éclat des fresques de David, elle manifeste cependant beaucoup plus de sérieux et de rigueur.

Plus encore que Sulte - l'auteur de l'Histoire des Canadiens français, d'une Histoire populaire du Canada et de biographies de Papineau et de Cartier — , David, par son livre sur l'Union des Canadas, représente la tendance majeure de l'historiographie canadiennefrançaise de la seconde moitié du XIX ${ }^{\mathrm{e}}$ siècle. Avec ce romantique, au reste fort conservateur, la description des faits et des personnages acquiert un éclat inconnu chez Garneau et ses disciples directs. Le mythe du grand homme, porteur d'une mission historique, anime ses biographies et portraits. On lui doit, à lui qui, dans sa jeunesse, avait respiré à retardement l'atmosphère des soi-disant jours glorieux de 1837, d'avoir réconcilié l'épopée révolutionnaire avec la problématique nationaliste. Papineau est grand : il a incarné à une époque difficile les aspirations nationales; Lafontaine l'est non moins : il a conquis le gouvernement responsable. Certes David et Sulte ont subi l'influence du courant libéral doctrinaire du temps ; mais on la décèle à peine dans leurs œuvres qui demeurent tributaires du nationalisme clérical des années 1851 à 1896.

Puis, en même temps que De Celles, qui a laissé des biographies fort intéressantes de Papineau et de Cartier et cela dans l'esprit même de la tradition de Garneau, apparaît une troisième figure dominante de notre historiographie. Chapais, qui se définissait lui-même comme une personnalité universellement conservatrice, était le disciple direct de Garneau. Beaucoup plus traditionaliste, il s'en rapprochait aussi par son sens de l'analyse. De même, on décèle chez cet historien prolifique l'influence des historiens anglo-saxons, en particulier de Charles Dent et de Kingsford. Mais on ne doit surtout pas oublier, si on veut comprendre l'interprétation de Chapais, que ce dernier n'est devenu 
historien qu'après une longue carrière [30] politique. En effet, Chapais avait milité en faveur des forces conservatrices au temps de Macdonald. Aussi avait-il été profondément marqué par l'idéologie politique dominante des années 1871 à 1896, à laquelle il était resté fidèle. D'autre part, on trouve chez lui de solides convictions ultra-montaines, celles mêmes que véhiculait le nationalisme clérical de la seconde moitié du XIX ${ }^{\mathrm{e}}$ siècle. Tout cela confère aux Cours d'Histoire du Canada de Chapais, publiés entre les années 1919 et 1934, ainsi qu'à ses études particulières sur le $\mathrm{XIX}^{\mathrm{e}}$ siècle, un caractère quelque peu anachronique pour l'époque. Certes l'interprétation générale de Chapais s'inscrivait dans la plus pure tradition du nationalisme canadienfrançais et, à ce titre, elle rejoignait aussi bien le lecteur de 1934 que celui de 1871 ; mais elle portait en plus l'empreinte du grand courant politique qui avait appelé après 1855 l'unité canadienne. C'est en ce sens que l'œuvre de Chapais comme celle de De Celles faisait figure de réaction contre l'École de David dont l'emprise se maintenait dans le contexte des luttes anti-impérialistes et de la grande crise. Il n'est donc pas étonnant que le pan-britannisme de Chapais n'ait eu qu'une résonance assez limitée dans le milieu canadien-français. Aussi Chapais fut-il, malgré le mérite incontestable de ses travaux, le plus québécois de nos historiens, tout simplement parce qu'il arrivait trop tard dans notre historiographie. Homme du $\mathrm{XIX}^{\mathrm{e}}$ siècle, il ne pouvait occuper le premier rang à l'heure du chanoine Groulx.

Avec le chanoine Groulx, l'historiographie nationaliste traditionnelle atteint son sommet. Non seulement il a opéré la synthèse des deux tendances historiographiques issues de Garneau et de David mais il est parvenu à construire une histoire qui fût exactement le reflet des aspirations nationales canadiennes-françaises à l'époque de l'entre-deux-guerres. Plus que tout autre historien avant lui, il fut parfaitement accordé à son temps. L'histoire de Garneau reproduisait le nationalisme de 1825 en ses objectifs ultimes ; celle de David, le nationalisme de 1850 et celle de Chapais, celui des années 1875. Au contraire, l'esprit qui anime la production historique du chanoine Groulx ne comporte pas un tel décalage. De là son influence extraordinaire sur ses contemporains. Doué d'un remarquable esprit de synthèse, servi par une grande intuition et écrivain de talent, il parviendra à dégager une vision cohérente de l'ensemble des problèmes du XIX ${ }^{\mathrm{e}}$ siècle. Il ne s'est pas contenté d'assumer le legs du passé ; par ses re- 
cherches originales sur les grands personnages du siècle - Papineau, Lafontaine, Cartier —, par ses recherches personnelles sur l'enseignement, sur l'histoire des idées et sur l'évolution constitutionnelle, il a accru considérablement notre connaissance. Evidemment, on contestera ses points de vue par trop cléricaux, une tendance assez manifeste à la littérature comme au lyrisme ; surtout, on verra dans son interprétation le produit d'une problématique trop rigoureusement nationaliste, comme si l'historien n'avait d'abord qu'à faire le partage des fidélités ou des infidélités. Il serait cependant [31] injuste de nier toute préoccupation scientifique à cette œuvre si riche ; au contraire, à mesure que cette dernière s'édifie, le problème d'une méthodologie se précise davantage réduisant de plus en plus la part de l'influence idéologique. Son Histoire du Canada, qui demeure une construction nationaliste, reflète cette orientation de fin de carrière.

Tels sont les principaux « chefs de file » de l'historiographie traditionnelle. Très peu d'historiens ont échappé à leur message ou au climat qui a favorisé l'édification de leur œuvre. Obligés d'acquérir euxmêmes la formation historique nécessaire à la poursuite de leurs travaux, il est normal que dans une conjoncture sensiblement différente ils fassent figure de pionniers. Est-ce à dire que leur apport soit négligeable ? Bien sûr, leur contribution comporte des lacunes importantes qu'on se doit de discuter, mais cela n'implique pas un rejet.

L'emprise presque universelle de l'idéologie nationaliste sur notre première littérature historique constitue une de ses principales limites. Certes le nationalisme est une des constantes majeures du XIX ${ }^{\mathrm{e}}$ siècle mais de là à croire qu'il est tout le siècle, il y a une marge importante. C'est en raison même de son rôle qu'il doit devenir un objet d'études et non pas servir à filtrer toute notre connaissance du passé. Dès le moment où les valeurs nationales et leur contenu furent érigés en absolus dans l'ordre scientifique, tout le réel devait se conformer, sous peine d'être dévalorisé, à ces dogmes inattaquables. L'Histoire des patriotes de Filteau en est un exemple typique. Le moins qu'on puisse dire d'une telle démarche, qui consiste à projeter systématiquement son univers sur un monde qui lui échappe en grande partie, c'est qu'elle tend à simplifier des réalités fort complexes qui le plus souvent obéissent à des forces internes. La réduction du passé en des schémas simplistes, même si elle favorise la poursuite de certains objectifs présents, n'aboutit finalement qu'à atténuer, voire même à abolir, le désir de 
comprendre. Aucun des maîtres de cette époque n'a évité suffisamment cet écueil. À plus forte raison en est-il ainsi des disciples plus ou moins talentueux. Saisir le réseau complexe de significations autour duquel se sont construits et édifiés les phénomènes historiques, n'estce pas là un des aspects essentiels du travail de l'historien ?

Tout cela résulte du fait que l'idéologie nationaliste débouchait non pas sur des hypothèses d'ordre scientifique mais sur un ensemble de préoccupations morales. De là la tendance moralisatrice voire même édifiante de notre historiographie. Ceci est vrai non seulement pour l'histoire religieuse, les monographies paroissiales et les manuels de classe mais aussi pour certains travaux plus généraux. Moins marquées chez les meilleurs historiens, ces propensions s'expriment plus librement chez la plupart des autres. Parfois cela aboutit à la puérilité pure et simple.

Il n'est pas étonnant non plus que le cléricalisme soit aussi un des traits les plus voyants de notre première construction de l'histoire. Ici [32] l'objectivité n'avait aucun droit. Si on songe à l'âpreté des critiques formulées contre les quelques audaces de Garneau et de Suite nous ne pensons pas surtout à celles de l'abbé Brasseur de Bourbourg vertement mis à sa place par l'abbé Casgrain —, on peut se rendre compte que de Garneau au chanoine Groulx une remise en question par un historien de notre passé clérical était impensable. La force contraignante de l'ultramontanisme et l'horreur du libéralisme étaient telles que seul un historien de type conservateur était possible. Notre historiographie fut trop sujette des valeurs ultramontaines pour que cela ne produise pas une justification systématique des entreprises cléricales. À cet égard le chanoine Groulx fut celui qui manifesta peutêtre la plus grande liberté d'esprit. Par contre, l'étude du Père Léon Pouliot sur Mgr Bourget et son temps, malgré son sérieux, est un exemple frappant de cette sorte de sujétion. Qu'on examine les travaux faits sur toutes les manifestations de libéralisme et on y trouvera une réprobation antérieure à tout examen objectif.

Une des conséquences les plus frappantes de cette approche limitée, c'est qu'elle tend à perpétuer les mythes édifiés dans le passé et à meubler trop grassement le panthéon des grands hommes. Enfin, on ne doit pas oublier l'aspect souvent morbide d'une histoire qui assez facilement convertit les injustices isolées en un dangereux système de persécution. Cette fidélité sans réserves à l'idéologie nationaliste et à 
ses impératifs profonds explique que derrière la richesse apparente de la production historique antérieure au dernier conflit mondial se cache une grande pauvreté. La croyance en la primauté du phénomène politique a concouru à concentrer l'attention de la majorité des historiens dans une seule direction. Pourtant le politique n'est pas le seul moteur de l'évolution historique. Une histoire politique n'a de sens que si elle fait appel à un ensemble de facteurs économiques, démographiques, sociaux ou idéologiques qui parfois se subordonnent au politique mais qui, à d'autres moments, vont l'entraîner dans leur mouvement. Tout cela exige une pondération d'éléments divers, impossible à réaliser dans une histoire purement politique. C'est là une des principales faiblesses de l'historiographie traditionnelle. À l'exception de Langlois, aucun historien ne s'est sérieusement appliqué à l'étude du mouvement démographique. Dans le domaine de l'histoire économique, la pénurie est presque complète. Il y a bien quelques livres sur l'agriculture et la colonisation (l'abbé Caron, Létourneau et Perron), le Montréal économique de Esdras Minville et quelques chapitres d'une allure parfaitement statique dans les études générales de Groulx et de Chapais ; mais on ne peut considérer ces contributions comme suffisantes. D'autre part, de nombreux motifs incitent à croire que l'historiographie religieuse serait en meilleure position ; mais, après examen, que peut-on retenir comme travaux marquants ? Quelques biographies d'évêques, quelques monographies de communautés religieuses, un nombre imposant d'études paroissiales faites par des amateurs [33] et quelques bons articles de revues. Le secteur de l'enseignement est mieux représenté avec les études exhaustives de Groulx et de Audet. Par contre, dans le domaine inexploré de l'histoire des idées, trois noms seulement retiennent l'attention : Mgr C. Roy, S. Marion et Lareau. Si, par surcroît, on se réfère à l'histoire du droit, qu'est-il de vraiment important en dehors de Lareau, De Montigny, J.-E. Roy et A. Perrault ? Enfin, dans une catégorie à part, les intéressantes et superficielles chroniques politiques de $\mathrm{M}$. Rumilly, qui dissipent à peine l'obscurité enveloppant encore la période qui suit la Confédération.

L'historiographie traditionnelle n'a pas cessé, depuis la dernière guerre, de susciter de nouveaux travaux, telle l'Histoire du Canada de $\mathrm{M}^{\mathrm{gr}}$ Tessier. Mais, depuis ce temps, de nouvelles tendances sont apparues à côté des orientations anciennes. La fondation des Instituts d'histoire de Montréal et de Québec, grâce à l'impulsion du chanoine 
Groulx et de l'abbé Maheux, a fortement contribué à inscrire une plus grande diversité dans la démarche des historiens. Il en est résulté un plus grand souci de rigueur scientifique, d'érudition et surtout des préoccupations méthodologiques plus grandes. De même le développement de la recherche dans les autres sciences humaines a agi sur les historiens et les a incités davantage à concevoir une approche plus globale du XIX ${ }^{\mathrm{e}}$ siècle.

Cette orientation nouvelle, qui a déjà produit des résultats assez féconds, demeure encore plus chargée de promesses que de réalisations. L'entreprise pionnière de M. M. Trudel, L'influence de Voltaire au Canada, en promet d'autres sur Lamennais et Veuillot de même que sur le courant libéral. L'histoire religieuse attire aussi ses chercheurs : depuis la biographie de Chiniquy par $\mathrm{M}$. Trudel et celle de $\mathrm{M}^{\mathrm{gr}}$ Laflèche par Rumilly, le frère R. Sylvain a mis au point ses études sur $\mathrm{H}$. de Courcy et Gavazzi. Il faut aussi signaler, hors la collection d'articles du rapport annuel de la Société canadienne d'Histoire de l'Eglise, les publications du Père Carrière et les textes de L.-E. Hamelin si riches en perspectives nouvelles. Dans un autre secteur, les études électorales de Hamelin et Letarte ouvrent la voie à une histoire des partis politiques. De même, après celle de E. de Nevers, des biographies de Tarte, Tardivel, Cartier, Laurier et Papineau, sont en préparation. En histoire constitutionnelle, on retiendra les travaux de G.-Lajoie, Lamontagne et Bonenfant. Quant à l'histoire économique, on pense d'abord aux textes d'Albert Faucher et à d'autres moissons en perspective. En démographie, les noms de Yves Martin et de Jacques Henripin s'imposent. En histoire du droit, on se réfère immédiatement aux écrits du professeur Baudoin et à la thèse, riche en aperçus économiques et sociaux, de A. Morel. De même on signalera une autre contribution récente de $\mathrm{A}$. Vachon à l'histoire du notariat. Enfin, le domaine de l'éducation s'est enrichi d'une étude fondamentale : celle de A. Labarrère sur le destin de l'instituteur laïque au $\mathrm{XIX}^{\mathrm{e}}$ siècle.

[34]

La problématique nationaliste elle-même n'a pas échappé à cet effort de révision. Conscient des lacunes de l'historiographie traditionnelle, le nouveau courant nationaliste déduit ses hypothèses du drame de la conquête, drame qui aurait détruit les assises matérielles et morales de la nation canadienne-française. Cette catastrophe expliquerait les faiblesses ultérieures des milieux d'affaires canadiens-français. De 
cette façon d'envisager le problème découle inévitablement la nécessité d'une étude approfondie sur l'évolution de la bourgeoisie canadienne-française, qui établisse hors de tout doute le ou les moments de la décapitation sociale. C'est là que réside le principal intérêt scientifique de la problématique nationaliste actuelle, en rupture de ban avec les schémas cléricaux, glorieux et messianiques d'autrefois. Or, les publications récentes de $\mathrm{M}$. Michel Brunet ne touchent principalement qu'aux mythes compensateurs, voire même auto-destructeurs, élaborés par les Canadiens français, prétend-il, en conséquence du traumatisme de la conquête. Quant aux articles de M. Maurice Séguin sur le régime seigneurial, ils ne contribuent que bien faiblement à démontrer le bien-fondé des postulats originels. De même, on pourra croire qu'ils tendent à produire une image déformée de ce que fut ce système dans la conjoncture particulière des années 1806 à 1854. C'est pourquoi nous attendons avec impatience cette étude décisive sur les destins successifs de la bourgeoisie canadienne-française.

\section{II \\ L'historiographie anglo-saxonne}

L'évolution de l'historiographie anglo-saxonne se présente sous un jour différent. L'ampleur même de la production historique accroît les risques d'une simplification qui en masquerait la diversité. Qu'on songe, par exemple, au nombre imposant de biographies de gouverneurs et d'hommes politiques écrites depuis la fin du siècle dernier mais qui intéressent l'histoire du Canada français. La question de l'immigration a ses études générales et spécialisées. L'histoire économique, si négligée du côté français, a reçu une attention particulière, au moins aussi prononcée que l'histoire politique. En 1930, on évaluait à 120 volumes l'apport des historiens canadiens-anglais à l'histoire économique du Canada. En histoire constitutionnelle, on inscrit d'emblée les noms de Clockie, Kennedy, Dawson, Trotter et Saywell. L'histoire sociale ellemême a inspiré des contributions appréciables. On ne saurait même enfermer cette historiographie sous l'étiquette unique de nationaliste.

Évidemment ce courant historiographique a eu au $\mathrm{XIX}^{\mathrm{e}}$ siècle ses figures pionnières : William Smith, Christie, Dent et Kingsford. Alors 
que Smith et Christie perçoivent leur époque à la lumière de l'idéologie mercantile [35] des années 1800-1850, Charles Dent et Kingsford, contemporains de David, se relient davantage, l'un dans ses biographies et portraits comme dans sa valable histoire de l'Union, l'autre dans son histoire du Canada (10 vol.), au courant libéral. Ces premières expériences aux préoccupations scientifiques forcément limitées expriment l'idéologie dominante du moment. L'ère victorienne n'a pas cessé de diffuser ses valeurs et ses attitudes. Par la suite, le nationalisme pan-canadien continuera à influencer la production historique du Canada anglais ; mais plus tôt qu'au Canada français, il cessera de dominer sans contrepoids la démarche des meilleurs historiens. Ce qui ne veut pas dire qu'il ne faille pas tenir compte de cette constante même chez les chercheurs les plus soucieux de s'en tenir à une problématique rigoureusement scientifique. Il y a des hérédités ou des façons de voir qui ne s'oublient pas aisément. Ainsi la perception de la responsabilité ministérielle par les historiens de tendances libérales conserve une forte saveur de mythe, celle même qui se retrouve du côté français. N'aboutit-elle pas à ériger un absolu là où il n'y a qu'une valeur, importante sans doute mais, en fin de compte, relative ? De même on trouvera dans les livres du professeur Creighton des attitudes qui, parfois, rappellent les réactions de Christie ou celles de la bourgeoisie capitaliste de la première moitié du $\mathrm{XIX}^{\mathrm{e}}$ siècle face au problème canadien-français. Il faut voir ici la marque de la tradition conservatrice du XIX ${ }^{\mathrm{e}}$ siècle. Mais ce n'est pas là l'essentiel de son approche scientifique.

Dès la fin du $\mathrm{XIX}^{\mathrm{e}}$ siècle, on assiste dans les milieux anglo-saxons à un étonnant réveil de la recherche historique. Des érudits, tels Shortt, Doughty, Wrong, Langhton et Grant, entreprennent, aidés des meilleurs historiens du pays, de jeter les fondements d'une histoire générale du Canada. De 1910 à 1926, trois grandes collections sont publiées : Canada and its Provinces, Chronicles of Canada et celle des Makers of Canada. Ces grandes séries de biographies et d'études systématiques, d'inégale valeur parce que souvent mal libérées des interprétations antérieures, couvraient la plupart des aspects du développement du Canada, depuis l'évolution économique jusqu'à l'histoire militaire et littéraire. En plus de leur apport respectif, ces études ouvraient la voie aux riches moissons de l'entre-deux-guerres. 
Le développement de l'enseignement de l'histoire au niveau universitaire, la fondation de revues historiques tout autant que l'influence de certains travaux antérieurs stimulèrent considérablement la recherche scientifique. Aussi les vingt années qui suivirent la première guerre mondiale furent-elles particulièrement fructueuses en travaux spécialisés. Certes l'histoire politique et constitutionnelle, comme l'histoire militaire, attire toujours beaucoup de chercheurs et elle réalise des progrès notables, mais c'est l'histoire économique qui enregistre les gains les plus remarquables. On ne peut songer à cette période sans évoquer le rayonnement de [36] Innis; mais il n'y a pas que lui. Comment oublier les noms de Martin, Munro, Brebner, Glazebrook, Tucker, Mackintosh, Masters, Lower et Creighton. En 1935, Mary Innis publiait une histoire économique du Canada. Les études de Logan sur le syndicalisme et celles de Macdonald et de Cowan sur l'immigration datent aussi de cette époque.

Par sa richesse en monographies, la période de l'entre-deux-guerres préparait le terrain à un effort de synthèse. Burt, à la suite de Bracq, avait bien publié, en 1933, son histoire de la province de Québec, mais ce n'était là qu'une entreprise encore assez réduite, prélude à des essais plus vastes. Ici les noms de Creighton, Mclnnis, Lower, Mason Wade et Easterbrook sont les plus significatifs.

En 1944, le professeur Creighton, l'auteur d'une remarquable biographie de Macdonald, publiait un livre capital, The Dominion of the North. Dans cette large synthèse, on retrouve une problématique déjà esquissée dans ses études sur l'empire commercial du Saint-Laurent et sur les fondements économiques de la Confédération. Pour la première fois un historien canadien essayait avec succès de situer l'évolution historique du Canada en fonction des oscillations à long terme de la vie économique. Cette fructueuse méthode qui vise à une délimitation plus organique des périodes, de façon à en mieux faire ressortir les tendances majeures, confère à ce livre toute sa fécondité. On déplorera toutefois l'insuffisance des aperçus sociaux. Peut-être est-ce manque d'intérêt ? Peut-être est-ce davantage le résultat d'une approche par trop qualitative et structurelle de la réalité économique ?

Par contre, on trouvera dans les travaux du professeur Lower un intérêt beaucoup plus marqué pour les réalités sociales et culturelles. Ses hypothèses en font foi. Son dernier livre, Canadians in the $\mathrm{Ma}$ king, illustre bien ces préoccupations fondamentales. Après avoir pu- 
blié en 1938 une étude exhaustive sur le commerce du bois au XIX siècle, le professeur Lower produisit en 1946 une histoire canadienne sous le titre A Colony to Nation. Euvre d'un esprit libéral, cette synthèse générale, au reste très sensible au phénomène politique, essaie de tenir compte d'un ensemble de facteurs économiques, géographiques, sociaux et culturels. A ce point de vue, elle comble, comme le livre de Mclnnis d'ailleurs, certaines lacunes importantes du livre de Creighton. En somme, ces grandes synthèses, rédigées d'un point de vue strictement canadien ou impérial, comme l'ensemble de l'historiographie anglo-saxonne, n'arrivent pas à mettre suffisamment en lumière le développement interne du Canada français. Des lacunes semblables se retrouvent dans l'histoire toute politique des Canadiens français du professeur Mason Wade. Malgré son intérêt, cet important ouvrage, écrit selon une perspective libérale, ne rejoint trop qu'un seul aspect de la vie canadienne-française. Enfin, cet effort de synthèse aura son couronnement dans une grande collection, The Canadian University Series, qui fait appel à des [37] collaborateurs canadiensfrançais. Son directeur, le professeur Morton, l'auteur de The Canadian Identity, voit dans cette série une vaste mise au point sur l'état actuel de nos connaissances.

Depuis la dernière guerre, on décèle à travers les travaux des historiens du Canada anglais des orientations nouvelles susceptibles de produire une approche différente du $\mathrm{XIX}^{\mathrm{e}}$ siècle. Déjà l'histoire économique du Canada de Easterbrook et Aitken fait état de ces moments de transition. Il en est ainsi des biographies de Brown et de Mackenzie publiées respectivement par Careless et Dale Thomson. Tout cela est plus manifeste dans certains articles parus dans la Canadian Historical Review sous les signatures de Saywell, Neatby, Cook, Parker et Fraser. Enfin, il faut mentionner comme contributions récentes, la remarquable introduction au journal de Lady Aberdeen par J. Saywell et le livre de $\mathrm{M}^{\mathrm{me}}$ Helen Taft-Manning qui analyse la révolte des Canadiens français. 


\section{Bilan et perspectives}

Que doit-on penser de cette masse de travaux maintenant disponibles sur le $\mathrm{XIX}^{\mathrm{e}}$ siècle canadien-français ? Évidemment, on pourra contester, au nom de perspectives méthodologiques actuelles, certaines orientations prises par le passé. À l'historiographie canadiennefrançaise, on a déjà reproché son excessive subordination à l'idéologie nationaliste ; à celle du Canada anglais, on reprochera son éclairage trop exclusivement canadien et impérial. Il reste cependant que les historiens d'hier ne se sont pas contentés d'établir et d'accumuler les faits ; ils ont aussi tracé les lignes de force d'une évolution. Est-ce à dire qu'on doive nier d'un seul coup toute valeur à ces cadres ou qu'on doive leur manifester sans discernement une fidélité inébranlable ? Les tendances récentes de l'historiographie canadienne autorisent une attitude critique mais positive.

Une approche plus globale de l'histoire du Canada français au $\mathrm{XIX}^{\mathrm{e}}$ siècle paraît indispensable. D'abord, mieux situer qu'on ne l'a fait jusqu'à présent cette histoire en fonction d'un contexte plus large qui en révèle la sensibilité exacte à certains impératifs internationaux, impériaux, nord-américains et canadiens, voilà un objectif à atteindre. Le Canada français du siècle dernier ne vit pas dans l'isolement complet ; d'une façon ou d'une autre, il participe à une vie de relations qui dépasse ses frontières et il est soumis à une conjoncture extérieure dont il n'est pas le moteur. Ceci n'est pas seulement vrai pour l'histoire économique mais pour tous les autres aspects de son développement. Il ne s'agit pas ici d'observer l'évolution de la société canadiennefrançaise en fonction des seules pressions extérieures; mais plutôt d'établir la nature réelle de ses rapports avec le monde. C'est à ce point de vue que l'apport de l'historiographie anglo-saxonne est le plus intéressant.

[38]

Une histoire globale du XIX ${ }^{\mathrm{e}}$ siècle est-elle possible sans l'assimilation par les historiens de certaines méthodes ou techniques utilisées dans les autres sciences de l'homme? Nous ne le croyons pas. Ainsi une histoire économique quantitative qui, dépassant le plan des struc- 
tures, débouche véritablement sur une analyse précise de la conjoncture à long, à moyen et à court terme, telle semble être la condition première d'un élargissement de la problématique historique. De la réalisation de cette entreprise, dans laquelle interviendraient tous les facteurs susceptibles de définir la conjoncture à un moment donné ou un changement de structure, dépendent plus ou moins les progrès des études démographiques, de l'histoire sociale, de l'histoire du droit, de l'histoire politique voire même de celle des mentalités. Il est incontestable aussi qu'une histoire démographique qui, en raison de sa méthode, ne ferait pas appel à un assez large éventail de données économiques, sociales ou culturelles n'aurait finalement qu'une signification assez limitée. Ces quelques exemples, tout en laissant entrevoir une orientation, partiellement engagée d'ailleurs, posent des exigences de plus en plus grandes à l'historien qui doivent l'inciter à la collaboration et non plus à l'isolement.

\section{Fernand Ouellet}

Faculté de commerce, Université Laval.

\section{BIBLIOGRAPHIE SOMMAIRE}

Nous avons laissé de côté les études faites par des intellectuels français. Elles sont fort nombreuses mais les plus remarquables restent celles de R. Blanchard, A. Siegfried et G. Vattier. Il aurait fallu, au surplus, insister longuement, en raison de leur importance méthodologique, sur les écrits des sociologues. Nous pensons à ceux de Miner, de Hughes, de J.-C. Falardeau, de F. Dumont, de M. Rioux, de M. Tremblay et de P.-E. Trudeau qui, pour la plupart, envisagent les problèmes du $\mathrm{XIX}^{\mathrm{e}}$ siècle à la lumière de " modèles idéaux » : la société traditionnelle et la société industrielle. Évidemment, ces modèles collent assez bien à l'ensemble de notre réalité ; mais l'état peu avancé de la recherche empirique complique leur utilisation. Aussi cette dernière comporte-t-elle des risques de généralisations hâtives, à moins que le sociologue ne devienne lui-même historien. Pour notre part, nous préférons, parce que plus diversifiée, la typologie du professeur E. Labrousse. Ses recherches l'ont amené à mettre en évidence trois types 
successifs de développement: $1^{\circ}$ l'ancien régime économique; $2^{\circ}$ l'économie moderne à monnaie stable ; $3^{\circ}$ l'économie contemporaine à monnaie instable. Ces trois structures fondamentales comportant chacune une hiérarchisation particulière de l'activité économique sontelles en fin de compte parfaitement significatives des grandes phases du développement du Canada français ? Certes, le Canada français n'est pas demeuré insensible face à l'évolution de l'Europe occidentale, mais il n'y a pas eu ajustement suffisant pour justifier une application intégrale de cette typologie. Une adaptation semble nécessaire. Toutefois, à l'aide de ce modèle économique, qui n'est pas entièrement économique, et du modèle sociologique, qui n'est pas complètement sociologique, il sera peut-être possible de produire une image globale du $\mathrm{XIX}^{\mathrm{e}}$ siècle canadien-français.

Cette bibliographie ne prétend pas être complète ; elle indique les principaux travaux et certaines revues indispensables à l'historien.

[39]

\section{Synthèses et collections}

SMITH, W., History of Canada, Québec, 1815.

BIBAUD, M., Histoire du Canada . . ., Montréal, 1841-44, 2 vol.

GARNEAU, F.-X., Histoire du Canada depuis sa découverte jusqu'à nos jours, Montréal, 1845-52, 4 vol.

CHRISTIE, R., A History of the Late Province of Lower Canada..., Québec, 1848-55, 6 vol.

DENT, C, The Last Forty Years, Toronto, 1881, 2 vol.

SULTE, B., Histoire des Canadiens français, Montréal, 1882, 8 vol. ; (en collaboration), A History of Québec, Montréal, 1908, 2 vol.

KINGSFORD, W., The History of Canada, Toronto, 1887-98, 2 vol.

DAVID, L.-O., L'Union des Canadas (1841-67), Montréal, 1898 ; Histoire du Canada (1867-87), Montréal, 1909. 
SHORTT and DOUGHTY (eds.), Canada and Its Provinces, Toronto, 1913-17, 23 vol.

WRONG and LANGHTON (eds.), Chronicles of Canada, Toronto, 1920, 32 vol.

GRANT (éd.), Makers of Canada, Oxford, 1926, 12 vol.

BRACQ J.-C., The Evolution of French Canada, New-York, 1924.

CHAPAIS, T., Cours d'Histoire du Canada, Québec, 1919-34, 8 vol.

BURT, The Old Province of Québec, Toronto, 1933.

BRUCHESI, J., Histoire du Canada pour tous, Montréal, 1934-35, 2 vol. ; Canada : réalités d'hier et d'aujourd'hui, Montréal, 1948.

CREIGHTON, D. G, The Commercial Empire of the St. Lawrence, Toronto, 1937 ; The Dominion of the North, Boston, 1944.

MAHEUX, A., Ton histoire est une épopée, Québec, 1941.

LOWER, A. R. M., Colony to Nation, Toronto, 1946 ; Canadians in the Making, Toronto, 1958.

McINNIS, E., Canada : A Political and Social History, Toronto, 1947.

RUMILLY, R., Histoire de la province de Québec, Montréal, 1941-62, 34 vol. ; Histoire du Canada, Paris, 1951.

GROULX, L., Histoire du Canada français depuis la découverte, Montréal, 1950ss., 4 vol.

WADE, Mason, The French Canadians (1760-1945), Toronto, 1955.

TESSIER, A., Histoire du Canada, Québec, 1958, 2 vol.

MORTON, W. L., The Canadian Identity, Toronto, 1961. 


\section{II. Études spéciales}

AITKEN, H. G. T., The Welland Canal Company : A Study in Canadian Enterprise, Cambridge, Mass. 1955.

AUDET, F.-J., auteur de nombreux articles biographiques dans les différentes revues canadiennes et en particulier dans les Mémoires de la Société royale du Canada [MSRC].

BAUDOIN, L., « Conflits nés de la coexistence juridique au Canada », dans La dualité canadienne, 106-127 ; « Le marchandage juridique de l'adultère de la femme au cours de la liquidation des intérêts pécuniaires des époux en cas de séparation de corps ", Revue du notariat, 1962, 229-249.

BONENFANT, J.-C, Les institutions politiques canadiennes, Québec, 1954.

BREBNER, T. B., North Atlantic Triangle, The Interplay of Canada, the United States and Great Britain, New-Haven, 1945.

BROUILLETTE, B., La pénétration du continent américain par les Canadiens français (1763-1846), Montréal, 1939.

BRUNET, M., Canadians et Canadiens, Montréal, 1954 : La présence anglaise et les Canadiens, Montréal, 1958.

Bulletin des Recherches historiques [BRH], publié depuis 1895.

Canadian Historical Association [CHA], rapports annuels publiés depuis 1922.

[40]

Canadian Historical Review [CHR], publiée depuis 1920.

Canadian Journal of Economies and Political Science [CJEPS], publié depuis 1935.

CARELESS, J. M. S., Brown and the Globe, Toronto, 1959.

CARON, I., La colonisation de la province de Québec (17911815), Québec, 1927. 
CARRIÈRE, G., o.m.i., Histoire documentaire de la congrégation des Missionnaires Oblats. . . dans l'Est du Canada (1841-61), Ottawa, 1957-61, 3 vol. 1942.

CLARK, S. D., The Social Development of Canada, Toronto,

CLOCKIE, H. M., Canadian Government and Politics, Toronto, 1944.

COWAN, H. I., British Immigration to British North America, Toronto, 1928.

CREIGHTON, D. G., British North America at Confédération, Ottawa, 1940 ; Rowell-Sirois Report, ap. 2 ; John A. Macdonald ; The Young Politician; The Old Chieftain, Toronto, 1955-56, 2 vol.

CURRIE, A. W., The Grand Trunk Railway of Canada, Toronto, 1957.

DAFOE, J. S., W. Laurier : A Study In Canadian Politics, Toronto, 1922.

DAVID, L.-O., Sir L.-H. Lafontaine, Montréal, 1872 ; Esquisse biographique de Sir G.-E. Cartier, Montréal, 1873 ; Biographies et portraits, Montréal, 1876 ; Les Patriotes de 1837-38, Montréal, 1884 ; Les deux Papineau, Montréal, 1896 ; Laurier et son temps, Montréal, 1905 ; Souvenirs et biographies, Montréal, 1911.

DAVIDSON, G. C, The North West Company, Berkerley, 1918.

DAWSON, R. M., The Government of Canada, Toronto, 1952.

DE CELLES, A.-D., Papineau, Montréal, 1905 ; The Patriots of 37, Toronto, 1920 ; Cartier et son temps, Montréal, 1913 ; Laurier et son temps, Montréal, 1920 ; Lafontaine et son temps, Montréal, 1922.

DENT, J. C, The Canadian Portrait Gallery, Toronto, 1881.

EASTERBROOK, W. T., and AITKEN, H. G. J., Canadian Economic History, Toronto, 1956.

FALARDEAU, J.-C, éd., Essais sur le Québec contemporain, Québec, 1953 ; "Les Canadiens français et leur idéologie », dans : Mason Wade, éd., La dualité canadienne, Québec, 1960, 20-38. 
FAUCHER, A., " The Decline of Shipbuilding at Québec in the Nineteenth Century », CJEPS, 23, 2, May 1957, 195-215; «Le fonds d'emprunt municipal ", Recherches sociographiques [RS], I, 1, janv.-mars 1960, 7-31; " Le problème financier de la Province du Canada (1841-1867) », RS, I, 3, juil.-sept. 1960, 343-362 ; « La dualité canadienne et l'économique : tendances divergentes et tendances convergentes ", dans Mason WADE, éd., La dualité canadienne, Québec et Toronto, 1960, 222-238.

FAUTEUX, A., Les Patriotes de 1837-38, Montréal, 1950.

FILTEAU, G., Histoire des patriotes, Montréal, 1938-39, 3 vol.

FLEMING, S., The Intercolonial : A History, Montréal, 1876.

FRASER, B., "The Political Career of Sir Hector Langevin », CHR, 1961, 93-132.

GALARNEAU, C, E. de Nevers, essayiste, Québec, 1960 (Cahiers de l'Institut d'histoire, $\mathrm{n}^{\circ} 2$ ).

GÉRIN-LAJOIE, P., Constitutional Amendment In Canada, Toronto, 1950.

GLAZEBROOK, G. P. de T., A History of Transportation In Canada, Toronto, 1938 ; A History of Canadian External Relations, Toronto, 1950.

GOODWIN, C. D. W., Canadian Economie Thought, Toronto, London, 1961.

GOSSELIN, A., L'Église du Canada après la conquête, Québec, 1916, 2 vol.

GRAHAM, G. S., Sea Power In British North America (17831820) ; A Study In British Colonial Policy, Cambridge, Mass., 1941.

GROULX, L., La confédération canadienne : ses origines, Montréal, 1918 ; Notre maître le passé, Montréal, 1924-44, 3 vol. ; Nos luttes constitutionnelles, Montréal, 1915-16 ; L'enseignement français au Canada, Montréal, 1933, 2 vol. ; Pourquoi nous sommes divisés, Montréal, 1943. 
HAMELIN, J., et al., «Les élections provinciales dans le Québec », Cahiers de géographie de Québec, 1960 ; Les mours électorales dans le Québec, Montréal, 1962.

HAMELIN, L.-E., Quelques matériaux de sociologie religieuse canadienne, Montréal, 1956 ; "Évolution numérique séculaire du clergé catholique dans le Québec », RS, II, 2, avril-juin 1961, 189243.

HUGHES, E., French Canada in Transition, Chicago, 1943.

HENRIPIN, J., "Aspects démographiques », dans : La dualité canadienne, 81-106.

Innis, H., History of the Canadian Pacific Railway, London, 1923 ; The Fur Trade in Canada, New-Haven, 1929 ; Problems of Staple Production in Canada, 1933 ; The Cod Fisheries, New Haven, 1940.

INNIS, M. Q., An Economie History of Canada, Toronto, 1935.

JONES, R. L., "Agriculture in Lower Canada (1702-1815) », CHR, 1946, 33-51.

KEEFER, T. C, The Canals of Canada, Montréal, 1894.

KENNEDY, The Constitution of Canada, London, 1938 ; Lord Elgin, Oxford, 1926.

KEYFITZ, N., "Population Problems », dans : Essais sur le Québec contemporain, 67-96 ; "Some Démographie Aspects of FrenchEnglish Relations in Canada », dans : La dualité canadienne, 129-149.

KINGSFORD, W., The Canadian Canals, Toronto, 1865.

LAMONTAGNE, M., Le fédéralisme canadien, Québec, 1954.

LAMONTAGNE, M., et FAUCHER, A., " History of Industrial Development », dans : Essais sur le Québec contemporain, 23-54.

LANCTOT, G., et al., Les Canadiens français et leurs voisins du sud, New-Haven, 1941.

LANGLOIS, G., Histoire de la population canadienne-française, Montréal, 1935.

LAREAU, E., Histoire du droit canadien, Montréal, 1888, 2 vol. ; Histoire de la littérature canadienne, Montréal, 1874.

LÉTOURNEAU, F., Histoire de l'agriculture, 1950. 
LEWIS, D., G. Brown, 1926.

LOGAN, H. A., The History of Trade Union Organization in Canada, Chicago, 1928.

LONGLEY, R. S., Sir Francis Hincks, A Study of Canadian Politics, Railways, and Finance in the Nineteenth Century, Toronto, 1943.

LOWER, A., The North American Assault on the Canadian Forest, Toronto, 1938.

McDIARMID, O. J., Commercial Policy in the Canadian Economy, Cambridge, 1946.

McGIBBON, D. A., The Canadian Grain Trade, Toronto, 1932.

McINTOSH, W. A., The Economie Background of DominionProvincial Relations, Ottawa, 1940, Rowell-Sirois Report, Appendix 3.

McIVOR, R. C, Canadian Monetary, Banking and Fiscal Development, Toronto, 1958.

MacNUTT, W. S., Days of Lorne (1878-83), Fredericton, 1955.

MAHEUX, A., Propos sur l'éducation, Québec, 1941 ; Pourquoi sommes-nous divisés ? Montréal, 1943.

MANNING, H. T., The Revolt of French Canada (1800-1835), Toronto, 1962.

MARION, S., Lettres canadiennes d'autrefois, Ottawa, 1939-46.

MASTERS, D. C, The Reciprocity Treaty of 1854, London, 1936.

MINVILLE, E., et al., Montréal économique, Montréal, 1943. Mémoires de la Société royale du Canada [MSRC], publiés depuis 1882.

MOREL, A., Les limites de la liberté testamentaire dans le droit civil de la province de Québec, Paris, 1960 ; « La réaction des Canadiens devant l'administration de la justice de 1764 à 1774 », Revue du Barreau, 1960, 53-63.

NEW, C. W., Lord Durham, Oxford, 1929.

OUELLET, F., Papineau, textes choisis (Cahiers de l'Institut d'histoire de l'Université Laval, $\mathrm{n}^{\circ} 1$ ) ; Papineau : un être divisé, Brochures de la Canadian Historical Association ; Histoire de la Chambre de 
Commerce de Québec, Québec, 1959 ; «L'enseignement primaire : [42] responsabilité des Églises ou de l'État (1801-36) »RS, II, 2, 1961, 171-187 ; voir aussi pour d'autres textes sur la période 1791-1860 le $B R H$, la $R H A F$, le $C H A R$ et la revue Hermès.

PARKER, G., "A New Look at Unrest in Lower Canada in the 1830's », CHR, 1959, 209-218.

PARKIN, G. R., Sir J. A. Macdonald, Oxford, 1926.

PERRON, M., Un grand éducateur agricole: Ed.-A. Barnard, 1955.

POULIOT, L., s.j., Mgr Bourget et son temps, Montréal, 1955, vol. I.

Recherches sociographiques [RS], revue publiée depuis 1960.

Revue d'Histoire de l'Amérique française [RHAF], publiée depuis 1947.

ROY, C., Essais sur la littérature canadienne, Québec, 1907 ; Nouveaux essais sur la littérature canadienne, Québec, 1914.

ROY, J.-E., Histoire du notariat, Lévis, 1899-1902, 4 vol.

RUMILLY, R., Sir W. Laurier, Paris, 1931 ; Papineau, Montréal, 1934 ; Mgr Laflèche et son temps, Montréal, 1945.

SAYWELL, J. T., The Office of Lieutenant Governor, Toronto, 1957 ; The Canadian Journal of Lady Aberdeen, Publications of Champlain Society, XXXVIII, Toronto, 1960, Introduction, p. xivlxxxiv ; "The Crown and the Politicians : the Canadian Succession Question (1891-96) », CHR, 1956, 309-337.

SAYWELL, J. T., and Neatby, H. B., " Chapleau and the Conservative Party in Québec », CHR, 1956, 1-22.

SCOTT, F., " Areas of Conflict in the Field of Public Law and Policy », dans : La dualité canadienne, 81-106.

SÉGUIN, M., "Le régime seigneurial au pays de Québec », RHAF, I, 382-402 ; 519-532.

SHIPPEE, Canadian American Relations (1849-1874), NewHaven, 1939.

SHORTT, A., Lord Sydenham, Oxford, 1926. 
SKELTON, O. D., General Economie History of the Dominion (1867-1912), Toronto, 1914 ; The Railway Builders, Toronto, 1916 ; The Life and Times of Sir A. Galt, Toronto, 1920 ; Life and Letters of Sir W. Laurier, Toronto, 1921.

Société canadienne d'Histoire de l'Église catholique [SCHEC], rapports annuels publiés depuis 1933.

STACEY, C. P., Canada and the British Army (1846-71), London, 1936.

STANLEY, C. P., Canada's Soldiers (1604-1954): The Military History of an Unmilitary People, Toronto, 1954.

SULTE, B., Mélanges historiques, Montréal, (1918-34), 17 vol. ; Sir G.-E. Cartier, Montréal, 1919 ; Histoire de la milice canadiennefrançaise, Montréal, 1897 ; Les Forges du Saint-Maurice, Montréal, 1920.

TESSIER, A., Les Forges du Saint-Maurice, Trois-Rivières, 1952.

THOMSON, D., A. Mackenzie : Clear Grit, Toronto, 1960.

TROTTER, R. G., The Canadian Confederation, Toronto, 1924.

TRUDEL, M., L'Influence de Voltaire au Canada, Montréal, 1945, 2 vol. ; Chiniquy, Trois-Rivières, 1955, $2^{\mathrm{e}}$ éd.

TUCKER, G. N., The Canadian Commercial Révolution (18451851), New-Haven, 1936.

TURCOTTE, E., Le Canada sous l'Union, Québec, 1871, 2 vol.

UNDERHILL, F. H., Ed. Blake and Canadian Nationalism, Toronto, 1939 . 


\title{
COMMENTAIRE
}

\section{L’étude du XIX ${ }^{\mathrm{e}}$ siècle canadien-français et l'interprétation de l'équipe gagnante}

\author{
Michel Brunet \\ Institut d'histoire, \\ Université de Montréal.
}

[pp. 43-44.]

$\underline{\text { Retour à la table des matières }}$

Avant d'avoir une histoire écrite, une collectivité doit d'abord exister. Les Canadiens français n'ont donc pas attendu l'arrivée des historiens pour affirmer leur survivance comme groupe distinct en Amérique du Nord.

Lorsque nous nous interrogeons sur notre $\mathrm{XIX}^{\mathrm{e}}$ siècle, il est essentiel de nous rappeler qu'il y a eu auparavant près de deux siècles d'histoire canadienne-française. Depuis six générations - cela avait commencé dès la première moitié du XVII ${ }^{\mathrm{e}}$ siècle — des hommes et des femmes qui s'appelaient Canadiens avaient conscience de participer à un destin collectif. À l'époque de Louis-Joseph Papineau comme à celle de Pierre Le Moyne d'Iberville, à l'époque de l'industrialisation naissante comme à celle de la traite des fourrures, les Canadiens, tout simplement parce qu'ils existaient, avaient la légitime ambition de se perpétuer collectivement dans la vallée du Saint-Laurent. Les circons- 
tances avaient changé. Les moyens d'action collective étaient différents. Néanmoins, l'objectif demeurait toujours le même.

Doit-on attribuer cette interprétation des faits à l'action néfaste d'un virus que certains observateurs contemporains de la société canadienne-française désignent sous l'expression barbare de "problématique nationaliste » ? Il faudrait en conclure que la "problématique antinationaliste » ou " anationaliste » donne à ceux qui s'en inspirent le privilège exclusif de saisir les réalités du passé ! Ils bénéficieraient donc de révélations particulières refusées aux chercheurs qui ont jusqu'ici écrit l'histoire du Canada français.

L'erreur de nos historiens n'a pas été de croire en l'existence d'une nation canadienne-française. Celle-ci avait pris forme plusieurs décennies avant la naissance du premier historien canadien-français. Leur faiblesse - si c'en est une - fut d'accepter intégralement l'interprétation du passé qu'avaient construite, au niveau de l'action quotidienne, les dirigeants de la collectivité entre 1760 et 1820 . Cette explication de l'évolution historique des Canadiens français était celle de l'équipe gagnante, celle des classes sociales dominantes au $\mathrm{XIX}^{\mathrm{e}}$ siècle. Or, au Canada français, deux types de dirigeants avaient émergé depuis 1760 : l'administrateur ecclésiastique et l'avocat-politicien.

Faut-il s'étonner alors que notre histoire écrite ait célébré les mérites du clergé et exalté les luttes de nos orateurs politiques ? Un peuple se donne les héros qu'il peut avoir. À l'époque de la Nouvelle-France, la moisson fut abondante : militaires, explorateurs, découvreurs, fondateurs, grands commis de la monarchie, missionnaires. Après la Conquête, les canaux de promotion ouverts aux Canadiens ayant considérablement diminué, le choix devint plus restreint. Les historiens à tendance cléricale ont spontanément donné un rôle privilégié aux hommes d'Église. Les historiens laïcs ou laïcisants ont manifesté des préférences pour les hommes politiques. Fait étonnant à retenir : l'interprétation générale est la même des deux côtés. Fait encore plus bouleversant : les historiens anglo-canadiens communient à cette interprétation lorsqu'ils daignent s'intéresser au [44] Canada français. La plupart des chercheurs contemporains en sciences sociales - même ceux qui prétendent apporter des vues nouvelles sur le passé et le présent du Canada français - ne se sont pas encore rendu compte combien ils demeurent tributaires des postulats traditionnels. 
Quelle est cette interprétation de notre XIX ${ }^{\mathrm{e}}$ siècle ? Il faut d'abord savoir qu'elle est liée à l'image globale que les Canadiens français se sont donnée de leur évolution historique. Cette vision est essentiellement optimiste et idéaliste. Elle a joué un rôle compensateur. La période coloniale française y apparaît comme une époque haute en couleur où se multiplient les actes de dévouement, les fondations pieuses et les gestes héroïques. La traite des fourrures, le commerce de l'eau de vie, les coureurs de, bois et l'intendant Bigot apportent quelques ombres à cette image d'Épinal. Quelques historiens anglo-canadiens se sont chargés d'idéaliser le coureur de bois dans lequel ils s'entêtent à voir un précurseur de la démocratie britannique au Canada. Les historiens canadiens-français d'allégeance agriculturiste ont transformé «l'habitant » en paysan. Pour les sociologues et les anthropologues contemporains, les Canadiens français ont longtemps formé une folksociety.

Tous les historiens anglo-canadiens et canadiens-français ont été longtemps d'accord pour soutenir que la Conquête n'avait pas modifié les structures ni l'orientation de la société canadienne. Les premiers soulignent la générosité du conquérant et la souplesse des institutions britanniques. Les seconds insistent sur la ténacité des Canadiens. La majorité d'entre eux ne rejette pas l'hypothèse de la survivance quasi miraculeuse des Canadiens français. Pourquoi priver ceux-ci d'un thème qui a eu l'avantage de les consoler pendant quelques générations ? Tous s'entendent pour s'incliner devant l'apothéose du responsible government.

Quand il s'agit d'interpréter la Confédération, les opinions varient quelque peu mais elles ne sont pas opposées. Tout naturellement, l'histoire économique a retenu l'attention des chercheurs anglo-canadiens qui ont décrit avec enthousiasme l'œuvre accomplie par les fondateurs du second royaume du Canada. L'histoire sociale en est encore à ses premiers balbutiements. Seuls s'en étonneront ceux qui ont la naïveté de croire que notre véritable histoire politique est connue.

L'histoire écrite de notre $\mathrm{XIX}^{\mathrm{e}}$ siècle sera bien différente le jour où toute l'évolution historique du Canada français se présentera à nous dans sa perspective globale. Les historiens doivent d'abord se libérer de l'interprétation que nous a imposée l'ancienne équipe gagnante. Celle-ci, formée des dirigeants ecclésiastiques et politiques canadiensfrançais qui se sont vus forcés de collaborer avec la bourgeoisie an- 
glo-canadienne, a commencé à retraiter devant les nouveaux porteparole de la collectivité. C'est pourquoi il est facile de prévoir que nous aurons une nouvelle histoire écrite. Quelques chercheurs en ont déjà jeté les principaux jalons.

Michel Brunet

Institut d'histoire, Université de Montréal. 
[45]

Situation de la recherche sur le Canada français

\title{
I. Perspectives historiques
}

\author{
Albert Faucher \\ Département d'économique, \\ Université Laval

\section{"L'histoire économique de la province de Québec jusqu'à la fin du XIX ${ }^{\mathrm{e}}$ siècle.”}

[pp. 45-53.]

$\underline{\text { Retour à la table des matières }}$

Cette communication envisage l'historiographie canadienne dans la perspective d'une histoire économique de la province de Québec du $\mathrm{XIX}^{\mathrm{e}}$ siècle. Nous allons donc, d'abord, nous demander ce qui a été fait, ce qui peut être considéré comme matériaux pour une histoire économique du $\mathrm{XIX}^{\mathrm{e}}$ siècle. Nous allons, deuxièmement, nous interroger sur les perspectives et les problématiques d'une telle histoire et, enfin, sur les conditions fondamentales de l'historiographie économique au Canada français. 


\section{I \\ Inventaire des matériaux}

Les matériaux pour une histoire économique de la province de Québec sont nombreux et hétéroclites à la fois. Ils appartiennent, pour ainsi dire, au genre de la petite histoire et il faut, pour les apprécier, les considérer comme éléments d'une histoire intégrale. Pourtant, on ne peut pas dire qu'ils ont été écrits dans cette perspective. On ne peut pas dire non plus qu'ils reflètent l'effort scientifique, surtout pas ceux du XIX ${ }^{\mathrm{e}}$ siècle. Plutôt, ils nous paraissent être le fruit de la spontanéité, comme la prose de monsieur Jourdain. Certains curés, par exemple, en écrivant l'histoire de leur paroisse, nous ont fourni des renseignements susceptibles d'éclairer l'histoire économique. Quelques professeurs d'université, à l'occasion de travaux de recherche paraacadémiques, qu'il fallait présenter dans une certaine rétrospective, auraient écrit quelques pages d'histoire économique. Et les chambres de commerce ou autres associations de défense ou de promotion n'y auraient-elles pas fourni des contributions appréciables ? Et tous ceux qui, à l'occasion d'un centenaire, ont publié une histoire de leur institution séculaire ? De même, certaines associations qui se sont inquiétées de l'exode rural, de l'urbanisation ou qui, en général, se sont préoccupées de l'avenir des francophones en Amérique du Nord ? Et les fonctionnaires dans leurs rapports au gouvernement, les rapports de Comités ou de Commissions [46] royales ? Au XIX ${ }^{\mathrm{e}}$ siècle, et au début du $\mathrm{XX}^{\mathrm{e}}$, les hauts fonctionnaires et les ingénieurs étaient pratiquement les seuls à réfléchir sur les problèmes économiques.

Aux fins de la présente communication, on peut réduire à quatre les centres d'intérêt qui ont polarisé les efforts de réflexion économique. Ce sont : $1^{\circ}$ Géographie et population ; $2^{\circ}$ Industrie et commerce ; $3^{\circ}$ Agriculture, colonisation, mines, chasse et pêche ; $4^{\circ}$ Etudes de caractère local ou régional.

Bien entendu, il ne s'agit pas d'un inventaire bibliographique ; un minimum de mentions suffira aux fins de la présente étude. 


\section{Géographie et population}

Sous le titre " géographie et population », il faut mentionner l'enquête de Joseph Bouchette et divers rapports d'arpenteurs pour le compte du gouvernement, le premier tome des Études de notre milieu, des Hautes Études commerciales, avec Brouillette, Tanghe, Dagenais ; et des articles, pour la plupart par des arpenteurs et ingénieurs, dans la Revue de la Société de géographie. Mentionnons l'histoire de la population canadienne-française de Langlois, quelques articles dans la $R e-$ vue canadienne, la Revue franco-américaine, la Revue trimestrielle canadienne. Le sujet le plus fréquent nous paraît être l'émigration des Canadiens français. Serait-ce l'événement majeur du XIX ${ }^{\mathrm{e}}$ siècle, et la manifestation d'un déséquilibre économique de la société canadiennefrançaise ? Le sujet a été traité dans un ouvrage publié sous la direction de Gustave Lanctot: Les Canadiens français et leurs voisins du Sud. Plus récemment, Robert Rumilly, dans son Histoire des FrancoAméricains (Montréal, 1961) esquisse le mouvement migratoire des " premières paroisses ». Il écrit notamment : "Les phénomènes économiques déterminèrent une émigration continue ».

\section{Industrie et commerce}

Sur l'industrie et le commerce, un ouvrage de Louis Strauss, Le Canada au point de vue économique, publié à Paris en 1867, traite incidemment de la province de Québec, de même que l'ouvrage de Maurice Dewavrin, Le Canada économique, publié à Paris en 1909 ; mais ces deux ouvrages touchent assez peu aux problèmes de l'évolution économique du Canada au $\mathrm{XIX}^{\mathrm{e}}$ siècle.

Horace Têtu publiait à Québec, en 1899, son Résumé historique de l'industrie et du commerce, de 1775 à 1900, un ouvrage de description et de compilation statistique. Les ouvrages de J.-M. Lemoine et de William Wood ont un caractère général et ne traitent qu'occasionnellement d'industrie à l'échelle du Québec ; de même Donald Bartlett, en ce qui regarde l'industrie métallurgique de la région de Montréal. La revue Actualité économique contient plusieurs monographies indus- 
trielles comportant des [47] esquisses historiques ; de même, la série Les manufactures de la province de Québec, publiée par le Ministère du Commerce de la province. Le même ministère publiait récemment, dans le genre économétrique, l'ouvrage d'André Raynauld, Croissance et structure économiques de la province de Québec. On y trouve une rétrospective qui nous reporte à 1870 .

Sur le commerce local, régional ou provincial, assez peu de matériaux. Il convient toutefois de mentionner Gérard Parizeau, L'assurance Incendie au Canada (Montréal, 1935), Arthur Belzile sur les sociétés mutuelles, mais il peut être hasardeux de parler de mutualité et de coopération sous la rubrique commerciale.

Le grand commerce ou les ramifications du grand commerce dans la province de Québec ont peut-être attiré plus de chercheurs ; mais les travaux de ces chercheurs ne visent pas directement la province de Québec. C'est le cas, par exemple, de certains articles dans la série Canada and its Provinces.

On reconnaîtra toutefois qu'en cette matière, et comme dans l'étude des grandes industries contemporaines, il peut être difficile de limiter la recherche aux problèmes qui touchent immédiatement, ou directement, la province de Québec. Ces types d'activité économique s'accommodent mal des frontières politiques ou ethniques. Et le chercheur est bien obligé d'en tenir compte. La délimitation ou la définition doivent donc être faites par rapport à des espaces abstraits plutôt qu'à des espaces géographiques. Les bornes spatiales du sujet qu'on traite sont imposées par le réseau des relations humaines, technologiques, financières qu'il comporte et les bornes peuvent varier à l'intérieur d'un même sujet d'étude, selon qu'on le traite du point de vue humain, du point de vue technologique ou du point de vue financier. De sorte que chaque sujet implique ses espaces spécifiques, espaces qui ne sont pas nécessairement co-extensifs aux bornes géographiques de la province de Québec et qu'il incombe au chercheur d'accepter et à l'écrivain de déterminer.

Sans contester sa raison d'être, on peut dire que la petite histoire, en économique, devient un genre de plus en plus difficile à pratiquer. Cette difficulté provient des ramifications de plus en plus vastes des tissus de l'événement qu'on aborde; ramifications vastes, plus ou moins, selon les divers aspects d'un même problème. Envisagée par 
rapport à la notion d'espace économique, cette difficulté fait comprendre pourquoi géographes et théoriciens économistes ont tant de peine à s'entendre sur une commune conception de la région économique. Pour les mêmes raisons, sont difficiles les recherches d'histoire économique centrées sur l'entreprise ou, plus exactement, sur la notion d'entrepreneurship, à laquelle on voudrait donner une connotation canadienne-française. Soyons d'accord avec F.-A. Angers qui, dans un article de la Revue d'histoire de l'Amérique française (septembre 1961), réclamait des études de cette espèce, études qui, semble-t-il dire, [48] pourraient nous éclairer sur l'origine des grandes défections nationalistes. Soyons d'accord, bien sûr, mais admettons en hypothèse que l'expansion de la grande entreprise, qui oblige l'historien économiste à travailler sur des espaces aussi vastes que variables, exposait le chef d'entreprise canadien-français à l'aliénation.

Quant à la possibilité d'éclairer l'histoire économique de la province de Québec en prenant ce thème comme point de départ, on peut en discuter. Pour le moment, poursuivons la reconnaissance du terrain historiographique.

\section{Agriculture et colonisation, chasse, pêcheries et mines}

Agriculture et colonisation, chasse, pêcheries et mines. En somme, on pourrait dire : mise en valeur et aménagement de l'espace.

Sur l'agriculture et la colonisation, nous pouvons considérer comme matériaux authentiques les travaux de l'abbé Ivanhoe Caron, Stanislas Drapeau, Vattier, Leslie Jones, J.-C. Chapais, E. Bouchette, Esdras Minville, Firmin Letourneau, Marc Perron. Sur les pêcheries : Louis Bérubé et Pierre Dagenais. Sur la chasse, Benoît Brouillette et Gérard Gardner. Sur la forêt, l'industrie forestière et les mines : Pierre Asselin, François Vézina, Arthur Lower et Harold Innis. 


\section{4. Études de caractère local ou régional}

Enfin, nous arrivons à une quatrième et dernière catégorie : les études de caractère local et régional. Même si les monographies paroissiales n'ont pas été écrites dans l'optique économique, elles n'en contiennent pas moins quelques pages utiles à l'étude du milieu économique de la province de Québec du XIX ${ }^{\mathrm{e}}$ siècle. Les monographies régionales sont toutefois, du point de vue économique, plus remarquables, tout naturellement, car on y essaie d'expliquer les événements par rapport à des espaces qui correspondent davantage aux cadres de l'activité économique. Ici, il faut mentionner l'histoire économique de Montréal, de Bruchési, les études de Conklin sur Québec et d'Atherton sur Montréal.

En terminant ce bref inventaire, il peut être bienséant de souligner le grand mérite du groupe de l'École des Hautes Études commerciales qui s'est exprimé depuis 1925, dans l'Actualité économique, et qui a produit les Études de notre milieu de 1942 à 1946. Et enfin, une mention toute particulière de Raoul Blanchard, géographe du Canada français. Il ne s'est jamais donné pour historien économiste et pourtant, dans ses enquêtes, il n'a jamais négligé la dimension temporelle des phénomènes spatiaux qu'il a étudiés. Ses ouvrages abondent en aperçus historiques. L'historien pourra le critiquer, il n'aura jamais fini de le consulter ou de le citer. Peut-être dira-t-on de lui ce que Talleyrand disait de Jeremy Bentham : on a beau le piller, il demeure riche.

[49]

En conclusion, disons que nous avons accumulé une quantité assez considérable de matériaux pour servir à une histoire économique de la province de Québec au XIX ${ }^{\mathrm{e}}$ siècle. Et cependant, il faut bien avouer que la plupart ne répondent pas aux exigences méthodologiques et techniques de l'histoire économique, que la plupart ne comportent pas ce minimum d'analyse qui les rendrait immédiatement utilisables. On ne peut pas les insérer comme tels dans un schème d'explication globale de l'économie du XIX ${ }^{\mathrm{e}}$ siècle. Ce sont des matériaux.

Pour faire une histoire économique de la province de Québec, il faudra des historiens économistes. Or cette espèce n'est pas facile à 
recruter, dans les écoles de sciences sociales du moins, non seulement au Canada, mais aussi aux États-Unis. Ou encore, ceux des économistes qui se vouent à cette discipline auront tendance à sacrifier la réalité historique à leur modèle théorique. Il demeure que nous avons un besoin urgent d'historiens économistes, surtout au niveau des sciences sociales. C'est précisément à ce niveau que nous allons maintenant nous interroger sur les perspectives et les problématiques de l'histoire économique.

\section{II \\ Perspectives et problématiques}

Il n'est peut-être pas exagéré de dire que l'histoire économique de la province de Québec est encore à faire. Est-il nécessaire d'ajouter qu'elle doit être faite, autant que possible, par des historiens économistes ? Mais alors, historiens économistes de quelle espèce ? Car il y en a deux espèces : ceux dont la formation de base est historique et ceux dont la formation de base est économique. Nous n'avons pas le choix, tellement sont rares ceux qui s'orientent délibérément vers cette discipline. L'on sait, toutefois, que les universités nord-américaines exigent de ceux qui se préparent à l'histoire économique beaucoup de théorie, ce qui veut dire passablement de mathématiques.

L'histoire économique vient à peine de se relever du choc de l'impérialisme keynesien qui l'a presque anéantie au cours de la période d'après-guerre.

Sous l'empire de la Théorie générale de Keynes, qui a même refoulé dans l'ombre le célèbre Schumpeter, on ne s'embarrassait pas de perspectives longues, non plus que de rétrospectives. Dans une telle conjoncture, il devenait difficile de recruter des candidats à l'enseignement et à la recherche, en histoire économique. Toutefois, cette discipline a survécu. Et l'historien économiste continue sa tâche, accompagné, cette fois, du théoricien du développement économique, cette espèce d'historien lui-même, qui s'applique [50] principalement à l'histoire économique de l'avenir. Avec lui l'avenir est garant du passé. Les perspectives appellent les rétrospectives. 
Ce qu'il faut souligner surtout, c'est que l'historien économiste de la renaissance post-keynesienne a pour compagnon un collègue féru de mathématique. En sa présence, s'il éprouve lui-même quelque penchant pour la mathématique, l'historien peut contracter le mal de l'imiter ; ce qui ne serait pas si grave s'il n'allait pas ainsi se soustraire au labeur purement historique en prenant la tangente du modèle théorique. Cette solution de facilité consiste à fabriquer des schèmes d'explication ou d'interprétation et à les remplir de faits propres à les étayer. Une université américaine est même en voie de pousser cette méthode au point de confier au cerveau électronique l'interprétation des quantités économiques du XIX ${ }^{\mathrm{e}}$ siècle. Tout en admettant l'efficacité de ce procédé dans la manipulation de certaines séries chronologiques, on peut se demander, avec une certaine inquiétude, si le procédé ne va pas se répandre au point d'éliminer les travailleurs manuels de l'histoire économique. Phénomène d'automatisation.

Le professeur Heaton, au congrès des historiens économistes de Philadelphie en 1960, où les théoriciens du développement économique avaient la majorité (nous ne constituons pas une profession fermée), remarquait qu'il avait vécu assez longtemps pour assister à la naissance de la cliométrie.

Tout en nous réjouissant de cette innovation, qui doit aussi réjouir les marchands d'appareils électroniques, nous pouvons nous interroger sur l'avenir de l'histoire économique, dans une conjoncture dominée par la mathématique. Remarquons que les économistes se font de plus en plus rares dans les salles des archives publiques. Nos historiens économistes cèdent-ils au démon de la tangente mathématique? Qu'on se le tienne pour dit : ce n'est pas un démon qu'on chasse par le jeûne et la pénitence. Un peu de patience, un peu d'humour l'effaroucheraient davantage.

En ce qui concerne la province de Québec, dont l'histoire économique est encore à faire, nous avons besoin de chercheurs rompus au métier d'historien. C'est un point de vue qu'on peut soutenir sans mépris pour les méthodes d'analyse et les techniques de travail les plus récentes. Aussi longtemps qu'on n'aura pas transformé toutes les pièces d'archives en cartes électroniques, et aussi longtemps qu'on n'aura pas automatisé nos bibliothèques, la province de Québec aura besoin de travailleurs manuels. Travailler au pic et à la pelle, tel me semble être le lot de l'historien, même au milieu du $\mathrm{XX}^{\mathrm{e}}$ siècle. De cette espè- 
ce archaïque de travailleurs, le Canada français ressent un extrême besoin.

À travers tous ces propos, vous avez reconnu les historiens qui travaillent en compagnie d'économistes ou de théoriciens de l'économique. Il ne faudrait pas croire, pourtant, que l'histoire économique ne peut se développer qu'à l'ombre des sciences sociales. En France, elle s'est développée, et très bien, dans les cadres d'instituts divers. Et en Angleterre, où généralement [51] l'histoire économique est logée dans les départements d'économique, le célèbre Clapham a fait sa carrière dans un département d'histoire, à Cambridge.

Où qu'ils soient, à quelque catégorie qu'ils appartiennent, les historiens économistes demeurent une espèce hybride et exposée aux réprimandes, étant donné qu'ils ont établi leur quartier au carrefour de deux disciplines majeures : l'histoire et la théorie économique. Aux uns, on peut reprocher leur insuffisance en histoire, aux autres, leur insuffisance en théorie économique. Il n'est pas improbable, cependant, qu'un jour ils occupent la pièce centrale d'un édifice qu'on appellera du nom de l'ancien temple déserté : l'économie politique.

Tout cela pour dire que l'histoire économique jouera un grand rôle au Canada français, et à certaines conditions qu'il fallait soumettre à votre critique. Ces conditions sont : $1^{\circ}$ La présence d'historiens économistes en plus grand nombre ; ce qui suppose une politique de recrutement, ou une tactique de séduction, vu que les candidats aux études supérieures n'ont pas tendance à s'orienter de ce côté-là. $2^{\circ}$ La stabilité des historiens en place. Leur métier doit demeurer l'histoire, même s'ils ont été formés aux disciplines de la science économique. Ne pas esquiver. $3^{\circ}$ Le rassemblement des historiens économistes de métier, sous une commune bannière, d'où qu'ils viennent, afin qu'ils se réconfortent mutuellement, car ils sont exposés à la sévérité du jugement, soit du côté des économistes purs, soit du côté des historiens purs. Il faut qu'ils comprennent que ce jugement ne peut pas être le dernier.

Comment orienter notre histoire économique ? Vers quels secteurs de l'activité économique doit-elle porter ses efforts?

Il semble que nous devrions, d'abord, organiser un fichier des matériaux acquis : documents, chroniques, brochures, ou autres médiums d'expression. Ce serait constituer des sources d'information générale 
sur l'économie du Québec au XIX ${ }^{\mathrm{e}}$ siècle. Un tableau général de l'économie du $\mathrm{XIX}^{\mathrm{e}}$ siècle faciliterait la pondération des événements, permettrait d'établir approximativement le poids des matériaux de la structure globale. Deuxièmement, nous pourrions colliger les quantités économiques, constituer des séries chronologiques, en particulier les prix des denrées agricoles. De là, nous pourrions préciser la chronologie des cycles. Grâce à l'information générale, grâce aussi à cette compilation des quantités économiques, l'historien pourrait plus facilement reconnaître les problèmes et les événements les plus significatifs quant aux mouvements de la conjoncture et à la transformation des structures.

Puisqu'il s'agit, ici, de besoins communs à tous les historiens économistes, pourquoi ceux-ci ne confieraient-ils pas ces tâches à un centre de recherche, c'est-à-dire à une équipe de travailleurs entraînés et bien dirigés. Certes, la recherche authentique repose sur l'effort individuel et sur la pensée [52] originale de chacun ; il n'en demeure pas moins que certaines tâches ne peuvent pas être exécutées adéquatement sans coopération et sans assistance.

Au-delà de ces tâches fondamentales, la coopération entre collègues demeure encore une possibilité, vu que la technique des références et des notes de travail qu'utilise l'historien invite au dialogue et aux échanges de vues. Est-il nécessaire de dire que l'historiographie du $\mathrm{XIX}^{\mathrm{e}}$ siècle n'a pas répondu à cette exigence de l'enquête scientifique ?

\section{III \\ Les conditions du progrès en histoire économique}

L'histoire économique a pu se développer, soit en marge de la théorie et comme spécialité de l'histoire générale, soit à l'intérieur de la théorie, ou comme substitut à la théorie, avec Schmoller, par exemple ; mais, dans l'un et l'autre cas, elle s'est préoccupée des ensembles d'abord, pour s'appliquer à des études particulières ensuite. On ne gagnerait rien à ériger l'histoire économique en spécialité si l'on confi- 
nait sa tâche à un simple choix de faits plus ou moins importants pour l'explication des structures et de leur évolution.

Le souci de synthèse a guidé les débuts de l'historiographie économique : traités d'agriculture, dissertations sur le commerce, sur la monnaie ou autres, débouchent sur des grands schèmes. Eighty Years' Progress of the United States, édité par L. Stebbins, de Hartford, Conn., en 1861, de même que son analogue Eighty Years' Progress in Canada, édité par Hind en 1881, présentent des études sur l'agriculture, les plantations, la navigation à vapeur, le transport et les manufactures. Au début du XIX ${ }^{\mathrm{e}}$ siècle, Callendar et Gommons publiaient des documents. G. S. Callendar, Sélection from the Economie History of the United States, parut en 1909 ; les dix volumes de J. R. Commons, Documentary of American Industrial Society, parurent de 1909 à 1911. En 1904, la Carnegie Institution assumait la responsabilité financière d'une série dirigée par Henry W. Farnham et qui contient Johnson sur le commerce (1915), Clarke sur les manufactures (1916), Merger et McGill sur les transports (1917), Commons sur le travail (1918), Bidwell et Falconer sur l'agriculture (1925).

Ces ouvrages ont fait sentir le besoin d'un substratum d'information statistique et, en 1922, se constituait le National Bureau of Economie Research, sous la direction de E. F. Gay et W. C. Mitchell. Au Canada, l'historiographie économique a suivi une courbe semblable. Elle s'est acquis une place dans les départements d'histoire économique grâce aux efforts de compilation documentaire de Lower et Innis, Select Documents in [53] Canadian Economie History, d'où sortit un premier traité général d'histoire économique du Canada en 1933. Taylor et Mitchell publièrent leurs contributions statistiques à l'histoire économique du Canada en 1931.

À côté de la recherche documentaire et statistique, il faut mentionner le rôle que la géographie est appelée à jouer comme discipline auxiliaire de l'histoire économique, surtout lorsqu'il s'agit de travaux portant sur des infrastructures et des aménagements de l'espace. On peut en dire autant de la démographie.

Enfin quelques réflexions finales. Nous avons voulu indiquer quelques avenues de recherche ou quelques voies de pénétration dans le champ d'une histoire économique de la province de Québec au XIX siècle, à partir d'un certain héritage historiographique. Les voies d'ac- 
cès peuvent être nombreuses, et toutes peuvent être bonnes, pourvu qu'elles débouchent sur des ensembles et qu'elles aboutissent à des aperçus sur les structures, et à des explications sur les changements économiques de la société québécoise, et pourvu qu'on y traite adéquatement les quantités économiques.

L'histoire de certaines entreprises québécoises du $\mathrm{XIX}^{\mathrm{e}}$ siècle, par rapport au rôle de l'entrepreneur, offrirait, en particulier, une bonne voie de pénétration. Mais ce serait une voie aussi difficile que les autres. La notion schumpetérienne d'entrepreneur, ou l'activité entrepreneuriale, couvre plusieurs sphères de décisions ; et sans aucun doute, toute étude d'entreprise dirigée dans cette optique obligerait à reconstituer le milieu ambiant et déboucherait sur un grand schème de recherche.

De quelque côté qu'on oriente les efforts historiographiques, il faudra organiser les archives du $\mathrm{XIX}^{\mathrm{e}}$ siècle ; mais si, en particulier, on veut orienter les efforts vers l'étude des entreprises capitalistes, il faudra dresser l'inventaire de nos archives régionales, privées et publiques, et stimuler la coordination et l'organisation des services au niveau des archives provinciales. On n'écrit pas l'histoire sans documents.

\author{
Albert Faucher \\ Département d'économique, \\ Université Laval.
}




\title{
COMMENTAIRE
}

\author{
Jean Hamelin \\ Institut d'histoire, \\ Université Laval.
}

[page 54.]

$\underline{\text { Retour à la table des matières }}$

Monsieur Faucher nous a donné une communication remarquable qui dénote non seulement un savant au courant des publications concernant son champ de recherches, mais aussi un chercheur qui a longuement réfléchi sur les vicissitudes et les limites de son métier. Il a catalogué avec justesse les travaux relatifs à l'évolution économique du Canada français au XIX ${ }^{\mathrm{e}}$ siècle, il les a situés dans leur contexte et nous en a montré les déficiences et les mérites. Il a aussi décrit avec chaleur et humour le drame de l'historien-économiste qui a planté sa tente au carrefour de deux disciplines si différentes en leurs méthodes et en leurs objectifs.

La communication de monsieur Faucher ouvre donc la porte à de nombreux commentaires. Par quel biais aborder l'évolution économique du Canada français, comment résoudre le problème de la formation des historiens-économistes, voilà quelques-unes des questions que vous aimerez sans doute discuter au moment du forum. Pour ma part, j'aimerais revenir sur l'impression d'ensemble qui se dégage de sa 
conférence. En effet, au terme de cette communication, on a l'impression que la recherche en histoire économique du Canada français si jeune, si embryonnaire soit-elle, a déjà atteint un seuil, qu'elle se trouve dans une sorte d'impasse. Je ne taxerai pas monsieur Faucher d'être pessimiste puisqu'il propose une solution : l'organisation de la recherche.

Je verrais trois étapes à parcourir pour atteindre cet objectif. D'abord, il faudrait publier un inventaire analytique des documents manuscrits et imprimés susceptibles de fournir des données concernant l'évolution économique du Canada français. Car, il faut bien l'avouer, la plupart des chercheurs n'ont aucune idée de la richesse documentaire que nous possédons. Une deuxième démarche viserait à créer un service de documentation qui grouperait, à l'aide des procédés modernes de reproduction, les matériaux relevés dans l'inventaire. La dernière étape serait la création d'un centre de recherches qui deviendrait le point de rencontre des historiens et des économistes, des chercheurs rompus au métier et des novices. C'est avec raison que monsieur Faucher voit dans un tel centre la solution d'avenir susceptible de résoudre deux graves problèmes : le recrutement des chercheurs et la fausse position de l'historien-économiste.

Voilà ! Monsieur Faucher a esquissé un bilan, il a montré la voie à suivre, il ne reste plus qu'à s'engager dans les réalisations concrètes.

Jean Hamelin

Institut d'histoire, Université Laval. 
[55]

Situation de la recherche sur le Canada français

I. Perspectives historiques

\author{
André Raynauld \\ Département de science économique, \\ Université de Montréal

\section{"Recherches économique récentes sur la Province de Québec.”}

\author{
[pp. 55-64.]
}

$\underline{\text { Retour à la table des matières }}$

Comme la Faculté des sciences sociales de Laval semble vouloir établir une tradition de colloques décennaux, j'ai choisi de limiter mes observations aux travaux qui ont été entrepris depuis dix ans. Et puisque le choix des études sur lesquelles je vais m'arrêter est un peu arbitraire, autant le définir dès le début : j'exclurai donc de mon sujet, parce que traitées ailleurs, les études portant sur les occupations, sur des industries particulières et les études régionales, non sans retenir, toutefois, parmi ces dernières, les études portant sur le Québec (!).

\title{
1. Développement économique
}

Si je repousse de quelques paragraphes la discussion sur le cas particulier des recherches associées à la Commission royale d'enquête sur les problèmes constitutionnels, j'observe d'abord que toute la littérature a porté sur le même thème, depuis dix ans, parmi les économistes. 
On est particulièrement frappé par ce phénomène quand on relit d'un coup l'ensemble des publications comme j'ai dû le faire. Ce thème consiste dans « le retard de croissance » de la province de Québec. Le problème a été formulé de plusieurs façons différentes ; il a été mesuré, évalué, ajusté à maintes reprises. Mais il apparaît sous le même jour, que ce soit dans les études relatives aux différences interprovinciales de revenus et de salaires, aux différences de structure industrielle ou que ce soit dans les études sur l'emploi et le chômage. La préoccupation est partout identique, à savoir : comment expliquer que le niveau de développement du Québec soit inférieur à celui de l'Ontario.

On nous avait pendant longtemps accoutumés à donner trois réponses à cette question, réponses qui, avec le poids du temps, sont devenues des stéréotypes: $1^{\circ}$ le déterminisme historique ; $2^{\circ}$ le déterminisme géographique ; $3^{\circ}$ le déterminisme, j'oserais dire, du système des valeurs du Canadien français. Ces trois catégories d'explication contiennent une certaine dose de fatalisme et d'irréversibilité. Dans le cas des valeurs, on a voulu [56] rationaliser et justifier l'état de fait en faisant appel à des valeurs supérieures. 103

Dans leur magistrale semonce, ici même il y a dix ans, messieurs Lamon-tagne et Faucher ont dénoncé les trois systèmes d'explications à la fois. Ils ont soutenu que les valeurs et la culture n'avaient rien à voir à l'état de développement économique de la province de Québec ; et ils ont montré, ce qui me paraît la partie la plus indiscutable de leur thèse, que la géographie et l'histoire, bien loin de sauver un peuple pour toujours ou de le condamner irrémédiablement, exercent sur l'avenir une influence dont la nature peut complètement changer suivant des circonstances plus fondamentales encore. Si le Québec apparaît comme une zone excentrique située hors des courants d'échange du continent, ce fait, en lui-même, n'est pas une réponse ; il est encore une question puisque, autrefois, le Québec n'était pas seulement le centre ou le cœur de l'activité économique, il en constituait le tout.

103 Sur le premier point, je renvoie le lecteur à Michel Brunet, La présence anglaise et les Canadiens, Montréal, Beauchemin, 1958, notamment p. 229. Sur le second, je cite plus loin les références nécessaires ; du troisième, on trouve des traces partout : citons seulement E. Minville, L'homme d'affaires, Montréal, Fides, 1945, en particulier, les pages 157 à 164, ou encore : Le citoyen canadien-français, Montréal, Fides, 1946, tome II, 329-330. 
Messieurs Lamontagne et Faucher, dans leur approche « continentale » selon leur expression, ont établi que le facteur essentiel d'explication résidait dans le caractère favorable ou défavorable du rapport entre la nature et l'évolution de la demande mondiale d'un côté et le pattem domestique des ressources de l'autre. La composition de la demande mondiale évolue à son tour suivant les changements de goûts des consommateurs et surtout suivant les changements de la technologie. Cet ordre d'explication exclut la prédétermination du destin des peuples. Il remplace avantageusement, à notre avis, les types de réponses que nous trouvions jusque là au problème du développement économique de la province de Québec.

Ceci dit, la façon dont le modèle général a été appliqué à l'évolution économique du Québec se révèle peut-être un peu rapide en ce qui concerne notamment la première vague d'industrialisation de la province au cours de la période 1896-1914. Reconnaissons d'abord à la suite de nos auteurs que la conjoncture mondiale n'a pas été favorable à la province de Québec pendant la seconde moitié du XIX ${ }^{\mathrm{e}}$ siècle parce que la technologie dominante de l'époque reposait sur des ressources qui étaient rares ou inexistantes dans la province. Il est cependant nécessaire d'ajouter au tableau que, de 1860 à 1890, le monde entier s'est trouvé dans un mouvement conjoncturel long à la baisse ; puis, que le Canada a découvert dans l'élargissement de son marché domestique un substitut à la maladaptation du pattem des ressources à la demande mondiale de l'époque. La spectaculaire mise en chantier de l'Ouest a été, pour le Québec, un vigoureux foyer de croissance pendant quinze ans. Si, enfin, on verse au dossier la Politique Nationale de 1879, qui protégeait les industries de transformation légère et invitait les [57] investissements étrangers, on enrichit considérablement, il me semble, le modèle de MM. Lamontagne et Faucher et on explique du même coup, non seulement la décadence du Québec au $\mathrm{XIX}^{\mathrm{e}}$ siècle, mais aussi le démarrage accéléré du début du $\mathrm{XX}^{\mathrm{e}}$ siècle, qui est un fait indiscutable. En ce qui concerne l'industrialisation récente, le modèle s'applique intégralement. On observe en effet depuis 1935, par contraste avec l'industrialisation légère d'avant 1914, une réorientation évidente de l'économie vers une exploitation plus intensive des ressources naturelles à l'intention du marché mondial. La demande mondiale est de nouveau favorable par rapport au pattern des ressources de la province. 
Mais peut-on exclure tout-à-fait les facteurs culturels ou ethniques ? John Dales, dans un chapitre de l'ouvrage intitulé La dualité canadienne, nous invite à réintroduire les facteurs culturels dans l'analyse. La voie par laquelle l'auteur nous y amène est cependant tortueuse pour dire le moins. Il n'est pas sûr que ce ne soit plutôt un cul-desac. Selon John Dales, l'importance globale et la composition de l'industrie manufacturière se distinguent de celles de l'Ontario par des différences dans les facteurs déterminants. Or, il existe deux seules catégories de facteurs qui diffèrent ainsi entre les deux provinces : les ressources naturelles et la culture. Si, donc, on pouvait attribuer aux ressources naturelles toutes les différences observées dans les manufactures, les explications culturelles seraient inutiles; dans le cas contraire, elles seraient indispensables. Dans les faits, il arrive que les ressources naturelles n'expliquent pas tout. Et même qu'elles expliquent fort peu, soit entre 5 et 14\% du retard du Québec dans l'industrie manufacturière. L'auteur tire donc ses conclusions : "This suggests that the resource hypothesis is inadéquate, and that the major explanation of the différences in industrial development between Québec and Ontario . . . are to be found in . . cultural différences »104 La phrase qui suit cette sitation est "So much for logic ». Je confesse que cette logique me paraît un peu courte. Acceptons un moment cette classification. Nous devons alors inclure parmi les facteurs culturels tout ce que l'économiste consomme à chaque jour : les proportions de facteurs dans les fonctions de production et le rendement du capital, notamment. Et c'est bien ce que M. Dales entend que nous fassions. Il se place à un tel niveau d'analyse que même l'abondance de la maind'œuvre eu égard au capital dans le Québec ne constitue pour lui qu'une autre manière de poser la même question : pourquoi, dans ce cas, rétorque-t-il, le capital n'a-t-il pas augmenté plus vite ? Le marché est trop limité ? Pourquoi ce marché n'est-il pas plus large ? En effet, pourquoi la population du Québec n'est-elle pas aussi élevée qu'aux États-Unis ? L'économiste n'aurait-il donc aucune contribution utile à [58] apporter dans l'explication du niveau de développement d'un pays, et du type ou de la structure de son industrialisation ? Le malheur, c'est que l'économiste, même quand il partage ces vues, essaie

104 John H. DALES, « A Comparison of Manufacturing Industry in Québec and Ontario, 1952 », dans Mason Wade, éd., La dualité canadienne, Presses Universitaires Laval, 1960, 205. 
quand même de poursuivre l'analyse. Comme pour M. Dales aucune raison valable ne peut justifier que la population totale du Québec soit inférieure à celle de l'Ontario, qu'aucune raison ne peut justifier que le nombre absolu de la main-d'œuvre soit moins élevé, que l'importance de l'industrie manufacturière se confond entièrement avec le niveau du développement et du standard de vie, il se donne pour objet de comparer et bien sûr d'expliquer (en dépit des dénégations de l'auteur) pourquoi on trouve 180,000 employés des manufactures de moins dans le Québec que dans l'Ontario en 1952. Poser ainsi le problème, et cette fois je reconnais la stricte logique de l'auteur, c'est, au départ, refuser de chercher des explications d'ordre économique.

Quant aux comparaisons inter-provinciales des industries, une à une ou groupe par groupe, comme si chaque industrie devait avoir la même importance absolue ou relative, dans les deux provinces, je n'ai jamais décelé d'autre raison que la " raison du cœur » pour s'y arrêter. M. Bonin, dans un article intitulé « La répartition régionale des investissements depuis la guerre », procède ainsi en 1960. Bien au contraire, à mon avis, si la structure économique des deux provinces doit refléter le pattern des ressources (mais pas seulement des ressources naturelles cette fois), elle doit être très différente. Non seulement l'importance relative des industries doit différer, mais les proportions des facteurs utilisés dans chaque industrie et dans chaque entreprise fabriquant le même produit doivent différer entre les deux provinces. Alors à quoi bon, dans ces conditions, observer les «déficiences » de l'investissement ou de la production dans la sidérurgie ou la fabrication des outils ? Ces soi-disant déficiences sont celles qu'une politique rationnelle devrait susciter et encourager. C'est par la spécialisation et non par l'imitation qu'un pays ou une région se développe.

Si on ne peut sans difficulté expliquer le niveau de développement du Québec à partir de l'importance globale ou de la composition du secteur manufacturier, comment, donc, aborder l'étude du problème ? Plusieurs auteurs l'ont fait par le biais des niveaux de revenus et des salaires. Messieurs Angers, Harvey et Parenteau, dans des articles cités à la fin de cet expose, représentent ce courant. Les arguments invoqués sont à peu près les mêmes. La structure industrielle, plutôt que d'être le phénomène à expliquer, devient un facteur explicatif des bas salaires du Québec, du moins chez Angers et Harvey. La raison en serait que les taux de salaires se fixent par référence «au salaire 
moyen du milieu » et que, par suite, les industries à bas salaires exercent une pression sur l'ensemble des salaires. Nous retrouvons aussi chez Angers, chez Parenteau et chez Harvey le facteur géographique dont nous avons précédemment disposé ; un autre facteur revient chez Angers et Harvey : c'est celui des différences de revenus [59] entre les milieux urbains et les milieux ruraux. Puisque la population urbaine est relativement plus élevée en Ontario, les revenus moyens doivent être plus élevés. Mais, bien sûr, les auteurs n'ont pas voulu citer ces rapports statistiques à titre d'explication proprement dite puisqu'à ce compte-là, les revenus moyens de la province devraient être plus élevés que ceux du Canada tout entier. Enfin, Harvey et surtout Parenteau invoquent à bon droit des facteurs démographiques pour expliquer l'excédent chronique de main-d'œuvre dans la province. Mais, ni les taux de fécondité, seuls, ni la structure des âges de la maind'œuvre, ni la main-d'œuvre féminine n'expliquent entièrement les différences régionales dans les taux de salaires ou les revenus. Henripin a estimé par exemple que l'âge et la main-d'œuvre féminine n'affectent les revenus moyens que dans une proportion de 4\% à 5\%. ${ }^{105}$ Reliée à ces questions, se trouve celle de la mobilité de la main-d'œuvre que P. Harvey a brillamment illustrée grâce à ses calculs des taux régionaux de chômage et aux implications importantes qu'il a dégagées quant à la politique économique visant au plein emploi.

À ce stade des recherches sur le développement économique du Québec, il me paraît un peu illusoire de rechercher des causes particulières qui contribueraient à $x$ pour cent des différences observées. C'est plutôt dans une dynamique du développement que nous identifierons suffisamment les facteurs-clefs de la croissance au Québec pour pouvoir établir les priorités que doit observer une politique économique efficace. À cet égard, mais à simple titre d'exemple, je serais porté à me tourner vers l'agriculture pour résoudre les surplus de main-d'œuvre de l'industrie manufacturière. Peut-être les modèles classiques de développement s'appliquent-ils au Québec ? Quand l'offre globale de travail est parfaitement élastique, Lewis a en effet montré que c'est dans le secteur de subsistance, soit dans l'agriculture, que la productivité doit d'abord être accrue pour affecter le surplus techni-

105 J. Henripin, «Population et main-d'œuvre », chapitre 6 de Croissance et structure économiques de la province de Québec, 262. 
que ou l'accumulation du capital, puis le prix d'offre du travail dans le secteur industriel.

Je ne saurais laisser ce sujet sans commenter brièvement un article que M. J.-C. McGee a publié en 1950 sur la production de la province. Sans visiblement ne rien connaître des tableaux économiques, $\mathrm{M}$. McGee a tenté d'en construire un sur la base des données du Recensement de 1941. Les liens interindustriels sont mesurés par l'emploi et des estimations sont présentées sur les importations en provenance des autres provinces et de l'étranger. Les secteurs utilisés sont au nombre de 7. On peut avoir une idée de la démarche par les résultats obtenus :

«Sur les $27.6 \%$ de la population active engagée dans les manufactures, $7.6 \%$ dépend de matières premières importées ; 9.3\% de produits miniers ; $4.8 \%$ de produits forestiers et $3.4 \%$ de produits agricoles. »

[60]

Si je pense à l'ensemble de la population active, la répartition finale est la suivante :

«38.7\% de la population active de la province dépend de l'industrie agricole ; $20.8 \%$ de l'industrie minière ; $17.3 \%$ de l'industrie forestière ; $12.6 \%$ d'industries important leurs matières premières. "

L'intérêt de cet article ne réside évidemment pas dans les résultats. Ils sont faux, c'est certain. Mais pour gratuit et vain qu'il fut, cet effort témoigne quand même de la justesse des préoccupations ; c'est un essai valeureux qu'il faut retenir dans notre petite histoire de la pensée.

\section{Finances publiques}

Quoique les finances publiques et les questions de centralisation et d'autonomie n'aient jamais longtemps retenu mon attention, on ne me pardonnerait pas de passer sous silence dans une circonstance comme celle-ci, les travaux considérables qui ont été effectués sur la province 
de Québec par la Commission royale d'enquête sur les problèmes constitutionnels. Il m'est pourtant impossible de faire pleinement justice aux auteurs (et à moi-même) dans le cadre étroit de cet exposé.

À s'en tenir à une conception restrictive des problèmes d'ordre économique, on peut limiter le compte rendu aux références suivantes. Du rapport proprement dit de la Commission : la $2^{\mathrm{e}}$ partie, où on fait un examen statistique des finances publiques de la province ; le chapitre 9 de la $4^{\mathrm{e}}$ partie, qui comprend une critique de la thèse " centraliste » en matière économique, sociale et fiscale ; le chapitre 11 de la $5^{\mathrm{e}}$ partie, où on trouve un estimé détaillé des besoins financiers du Québec ; enfin, les recommandations sur le partage des ressources fiscales. Parmi les annexes, citons l'excellente histoire de la pensée dans le domaine des finances publiques de $M$. Angers (annexe $n^{\circ} 5$ ), l'étude remarquable de $\mathrm{M}$. Parenteau sur l'inégalité des provinces (annexe $\mathrm{n}^{\circ}$ 8) et enfin l'annexe 11, sur la centralisation.

Je limiterai mes commentaires aux principales recommandations. Sur le fond du problème, je le dis tout net : les recommandations de la Commission royale Tremblay ne peuvent être acceptées parce qu'elles procèdent d'une fausse notion de la réalité sociale et économique. J'en appelle notamment aux deux premières recommandations : la première consiste à fixer, par entente entre les gouvernements, la limite maximum de l'impôt global en termes d'un pourcentage du produit national (qui soit le plus faible possible) en vue de sauvegarder la liberté du citoyen. La deuxième recommandation est de régler le partage des impôts directs suivant une estimation des besoins fiscaux qui corresponde exactement aux fonctions respectives des divers gouvernements, telles que la Constitution les définit. Dans ces deux cas, la réalité sociale est perçue, au pire, comme fixée pour jamais à un moment donné du temps en fonction "d'un ordre transcendant», au mieux [61] comme si les besoins évoluaient suivant un rapport stable, unique et prédéterminé avec des fonctions sociales préalablement réparties entre les divers niveaux de gouvernements. Sur le plan technique, cette position équivaut à dire que quels que soient les changements dans les fonctions assumées par les divers gouvernements, on ne devra jamais envisager de passer d'une catégorie d'impôts (directs ou indirects) à l'autre. On en arrive à la conclusion absurde que ce sont les fonctions qui doivent s'adapter aux diverses catégories disponibles d'impôts et non l'inverse. Sur le plan technique encore, la solution n'at- 
teint pas son but qui était de rendre les gouvernements provinciaux et fédéral autonomes dans l'exercice des fonctions qui leur sont respectivement dévolues par la Constitution. Si une fonction provinciale prend plus d'importance qu'une fonction fédérale, par exemple, il faudra que le fédéral abaisse ses propres impôts pour faire place aux dépenses provinciales, puisque l'impôt total est fixé à un pourcentage fixe du produit national.

La préoccupation philosophique d'ensemble qui préside à ces recommandations semble nettement abusive quand elle conduit à des distinctions aussi spécieuses que celle qui a été faite entre l'impôt direct et l'impôt indirect : l'impôt direct appartiendrait aux provinces parce qu'il touche les personnes et que la juridiction en matière culturelle et sociale incombe aux provinces ; pareillement, l'impôt indirect toucherait seulement « les biens et la circulation des biens ", tendrait « à susciter des frontières à l'intérieur du pays », exercerait une influence plus directe et plus efficace sur la conjoncture économique et, pour ces raisons, devrait relever de la responsabilité du gouvernement fédéral.

Avant d'en terminer de ce sujet, j'ajouterai que je suis en parfait accord avec la troisième série de recommandations portant sur la participation des provinces à l'élaboration et $a$. la mise en exécution de la politique économique qui est aujourd'hui réservée au Gouvernement fédéral.

\section{Programme de travail}

Quiconque dresse le bilan de la recherche économique sur la province de Québec constate, après une heure de travail, que rien n'a encore été fait. Les études individuelles que j'ai rapidement examinées ici portent toutes sur le même problème. C'en est quelque peu effarant quand on y songe. La pensée devrait au contraire s'exprimer dans une foule de voies différentes sur la base d'un matériel statistique et institutionnel varié et d'approches théoriques beaucoup plus diverses. Incidemment, le manque de travaux de recherche n'est pas entièrement dû à la déficience des statistiques. Ici de nouveau on a plutôt affaire à une certaine conception de la recherche. Je voudrais m'élever ici en parti- 
culier contre cette notion d'inventaire dont on nous accable constamment dans notre milieu. Cette façon de concevoir, [62] je ne dirais pas la recherche, mais le travail, a causé, à mon avis, un gaspillage immense d'énergies. L'inventaire, comme on sait, consiste à réunir dans les mains de quelqu'un ou dans un ouvrage tout ce qu'on est censé savoir sur un sujet donné. Mais comme aucun ordre ne préside au choix des informations, ni du point de vue statistique ni du point de vue de l'analyse proprement dite, l'accumulation des matériaux ne fait jamais avancer le travail ultérieur. Je choisirai un exemple de ce fléau au Ministère du Travail à Ottawa. Il s'agit des taux de salaires qu'on recense depuis 60 ans, dans des milliers d'occupations différentes, mais avec lesquels personne jamais n'a pu construire de séries chronologiques d'un côté ni de comparaisons inter-industrielles de l'autre, parce qu'on n'a pas pondéré les occupations concernées.

Pour éviter à l'avenir de recueillir des informations inutiles et sans intérêt, je voudrais suggérer que dans le domaine économique, on subordonne toutes les computations statistiques à l'établissement d'une comptabilité nationale intégrée et consistante de l'ensemble des activités de la province. Dans cette perspective, on devrait s'atteler sans retard à la tâche de construire des indices de prix qui reposent sur des pondérations provinciales et, par suite, sur des budgets spécifiques de consommation des personnes et des entreprises; il faudrait en outre trouver moyen de mesurer les échanges de produits et de capitaux entre la province et l'extérieur, de même que les mouvements de population. La précaution essentielle étant toutefois de fixer avec le plus grand soin un seul système de concepts et de classifications, qui soit comparable autant que possible avec celui du Bureau fédéral de la statistique. Voilà ce qui me paraît le plus urgent dans le domaine de l'information.

Du côté de l'analyse au sens strict du mot, la province de Québec ne constitue pas nécessairement le meilleur champ d'expérimentation. Pour comprendre le fonctionnement d'une économie, les études anglaises, américaines, françaises ou africaines sont aussi importantes pour la connaissance du Québec que les travaux locaux. Sur ce plan, il faut sans cesse se rappeler la mise en garde d'Édouard Montpetit: « N'exagérons pas le milieusisme ». L'idéal est d'étudier les problèmes de la province de Québec, mais avec des méthodes et des outils d'analyse universels. D'où l'importance de prendre du recul parfois pour 
mieux connaître. Il suit que, sur ce plan, aucun sujet particulier de travail n'est de soi plus urgent qu'un autre. Cette fois-ci, il faut éviter de concentrer les efforts. Au contraire, ce dont nous avons le plus besoin au stade actuel, c'est de diversité, c'est d'imagination, c'est de perspectives et d'ouverture d'esprit dans toutes les directions imaginables. Il faut relire Veblen pour s'en convaincre au besoin et cultiver comme il dit l'idle curiosity des vieux jours.

Reste un dernier point. Celui de la politique économique qui nous ramène aux préoccupations du milieu. Dans ce secteur, comme dans celui [63] de la statistique, la poursuite d'un seul objectif, suffisamment englobant, est préférable à la dispersion des travaux. Cet objectif, à mon avis, devrait être de préparer un plan quinquennal de développement économique. Une commission permanente d'experts devrait être chargée spécifiquement, d'ici 1965, de la mise en place des organes administratifs et du choix des méthodes suivant lesquelles les informations statistiques indispensables à un plan seront recueillies, classifiées et analysées. Pour être menée à bien sans gaspillage des ressources, cette tâche implique que la commission d'experts décidera, aussi avant 1965, des méthodes de programmation qui seront utilisées dans le plan lui-même et de tout l'appareil technique de préparation.

Une fois les méthodes choisies et les informations en mains, on devrait pouvoir se consacrer à la préparation immédiate du plan à partir de 1965 et le mettre en application pour célébrer le centenaire de la Confédération! 106

\author{
André Raynauld \\ Département de science économique, \\ Université de Montréal.
}

106 II n'est peut-être pas inutile de signaler que dans cette communication, nous nous sommes volontairement limité aux études qui avaient le Québec pour sujet explicite. Il est évident qu'une grande quantité d'études canadiennes existent qui se rapportent indirectement à la province. Certains travaux sur des industries particulières par exemple, comme les textiles, la pulpe et le papier sont en même temps des études régionales. Plusieurs des recherches de la Commission royale sur les perspectives économiques du Canada seraient particulièrement pertinentes à l'étude du Québec, si nous avions voulu faire la somme de tout le connu. 


\section{Bibliographie sur \\ la structure et le développement économiques du Québec, 1950-1962}

\section{A. Publications}

Allen, P., Tableau de l'activité économique de la Province de Québec, annexe 9 au Rapport de la Commission royale d'enquête sur les problèmes constitutionnels, 1955.

Angers, F.-A., " Progrès industriels du Québec ", Actualité économique (A.É.), juil-sept. 1952, 329-341.

BERgEvin, M.-A., « Évolution de la structure de l'industrie au Canada », A.É., 1953-54, 765-776.

BERGEVIN, M.-A., « Incidence du chômage sur les industries manufacturières », A.É., 1957-58, 559-571.

Bonin, M.-B., "Répartition régionale des investissements depuis la guerre », A.É., ;janv.- mars 1960, 566-596.

DALES, J. H., «A Comparison of Manufacturing Industry in Québec and Ontario ", dans Mason Wade, éd., La dualité canadienne, Québec, Les Presses Universitaires Laval, 1960, 203-222.

DAles, J. H., Hydroelectricity and Industrial Development, Québec 1808-1940, Harvard University Press, 1957, 269 p.

[64]

Durocher, R., « Pourquoi plus de faillites dans Québec que dans l'Ontario ? », A.E.., 1950-51, 705-748.

HaRvey, P., "Conjoncture et structures : les perspectives spatiales du plein-emploi auCanada », A.É., oct.-déc. 1956, 383-405.

HARVEY, P., «Les salaires dans la province de Québec », A.É., juil-sept. 1955, 292-305.

HaRvey, P., "Plein-emploi national et plein-emploi régional au Canada depuis la guerre », A.É., 1956-57, 5-26. 
HaRVey, P., "The Economy of Québec », University oj Toronto Quarterly, XXVII, 3, April 1958.

LAmONTAgne, M., "The American Economie Impact on Québec », dans Hugh G. J.

AITKEN, et al., The American Economie Impact on Canada, Durham, N. C, Duke University Press, 1959, 88-100.

Lamontagne, M., et Faucher, A., " History of Industrial Development », dans Jean-C. Falardeau, éd., Essais sur le Québec contemporain, Québec, Les Presses Universitaires Laval, 1953, 23-54.

MCGEE, J.-C, «L'importance relative des industries de la province de Québec », A.É., oct.-déc. 1950, 549-566.

MÉLANÇON, J., « Retard de croissance de l'entreprise canadiennefrançaise », A.É., janv.- mars 1956, 503-522.

OstRY, S., «Inter-establishment Dispersion of Occupation Wage Rates, Ontario and Québec, 1957 », Canadian Journal of Economies and Political Science, 26, 2, May 1960, 277-288.

Parenteau, R., «Salaires différentiels », A.E., oct.-déc. 1954, 538-551.

PARIZEAU, J., Commentaire sur « Les problèmes économiques de la province de Québec », A.É., oct.-déc. 1959, 486-492.

RAYNAUlD, A., Croissance et structure économiques de la province de Québec, Québec, Ministère de l'Industrie et du Commerce, 1961, 629 p.

RAYNAULD, A., "Les problèmes économiques de la province de Québec », A.ت̈., oct.-déc. 1959, 414-421.

\section{B. Thèses non publiées de maîtrise en économique (Université de Montréal)}

Archambault, Guy, Différentiation des salaires entre l'Ontario elle Québec, 1954 (R.I.).

Michaud, Laurent, L'influence du travail et du capital sur la production au Québec et au Canada, 1933-1957, 1948-1957, 1961. 
Rivard, J.-Y., La Politique Nationale et le développement industriel du Québec 1879-1910. Les effets de la politique tarifaire, 1960.

TUCHMAIER, D., Analyse structurelle de l'économie de la province de Québec, 1961. 


\title{
COMMENTAIRE
}

\author{
Charles Lemelin
}

Département d'économique,

Université Laval.

\author{
[pp. 65-74.]
}

Retour à la table des matières

Lorsque les organisateurs du présent colloque m'ont invité à commenter la communication de monsieur Raynauld, ma première réaction a été de répondre : "Suis-je responsable de mon frère ? » Une telle réponse eût contribué à perpétuer la fausse impression que les théoriciens ne consentent pas à sortir de leur tour d'ivoire.

Je voudrais justement montrer aujourd'hui que les théoriciens ont un rôle complémentaire mais essentiel à jouer dans la recherche empirique, que cette collaboration entre les économistes ingénieurs et les économistes théoriciens est devenue une nécessité cruciale dans le milieu québécois. Avant d'aller plus loin, je voudrais vous soumettre quelques distinctions.

Les problèmes que je soulèverai ne concernent pas la recherche à contenu économique mais qui s'inspire d'un modèle relevant d'une discipline autre que la théorie économique. Exemples : l'enquête sur les budgets familiaux patronnée par les Caisses Desjardins, l'étude de monsieur Guy Rocher sur les occupations, les études de monsieur Gérald Fortin sur les milieux agricoles. 
Deuxième distinction : beaucoup d'études empiriques consistent à traiter un contenu économique quantitatif suivant les méthodes de l'analyse statistique, soit pour établir une taxonomie ou systématique des faits, soit pour défendre une thèse quelconque. Ce genre d'étude fera l'objet de quelques remarques, mais ce n'est pas sur ce plan que porte l'essentiel de ma contribution.

Ma discussion a trait à la recherche empirique dont le contenu est économique et qui s'appuie sur un modèle de théorie économique, modèle d'allocation des ressources, modèle relié aux problèmes de la stabilité économique, de l'emploi, de l'inflation, etc., modèle de croissance, modèle de développement, modèle relatif aux structures industrielles et à leur évolution.

Une autre clarification s'impose par rapport à ce type spécifique de recherche économique. Et il faut la faire du point de vue de la profession d'économiste. Un économiste professionnel a une façon à lui de penser, qui s'inspire des schémas propres à ses théories. Devant une situation concrète, ses antennes sont orientées d'une façon particulière, ce qui fait que ses intuitions diffèrent de celles d'un spécialiste d'une autre discipline. Un tel spécialiste peut évidemment faire des options personnelles, être même politicien. Il peut agir comme conseiller des politiciens. Il peut choisir soit de former, soit d'éclairer l'opinion publique. Enfin, il peut s'adonner à la recherche. C'est sur ce dernier plan uniquement que j'entends le juger. Comme chercheur, l'économiste pourra faire des études empiriques soit à la manière de l'historien ou du journaliste, soit à la manière du statisticien, soit à la manière de l'économètre. Tous quatre font, chacun suivant sa méthode, de la recherche économique authentique dès que le modèle dont ils s'inspirent est un modèle économique. Car, à mon sens, c'est la nature du modèle choisi qui spécifie une recherche empirique. Me permettez-vous d'ajouter que l'économiste statisticien peut se contenter de harnacher les données quantitatives pour les apprêter, grand style, à une sauce d'économiste. L'économètre, au contraire, accepte par définition de quantifier son modèle théorique, de donner à l'ossature de son modèle un revêtement empirique. L'économètre établit littéralement les ponts entre le modèle abstrait et la réalité. 
Encore une fois l'historien économiste, le statisticien économiste, l'économètre, pour autant qu'ils s'inspirent de l'économique, font tous trois œuvre de recherche économique empirique au sens précis où j'entends le terme.

Ces distinctions étant faites, voici ma position vis-à-vis la question posée au début.

Je soumets que l'état des recherches empiriques véritablement économiques a été, jusqu'à très récemment, pitoyable. J'entends la recherche organisée systématiquement... et continue. Sans doute qu'on pourrait établir un catalogue intéressant des contributions individuelles, isolées et portant sur des questions parcellaires. Sans doute aussi que le groupe des chercheurs parmi les économistes canadiensfrançais a vécu des moments où la conjoncture était favorable. Je pense en particulier à l'équipe Minville et aux Études sur notre milieu 107 Je pense à la Commission royale Tremblay et aux études qu'elle a suscitées. ${ }^{108}$ Pourquoi ces efforts sont-ils restés sans lendemain ? Pour une multitude de raisons. Je voudrais en souligner une, aussi importante que chacune des autres parce qu'elle concerne les chercheurs eux-mêmes. Ces recherches sont restées sans lendemain parce que trop des auteurs dans l'équipe n'ont pas su se détacher d'un nationalisme trop immédiat, trop particulier. La recherche a besoin de respirer à l'aise pour vivre et pour être féconde.

Que dire maintenant de ce que j'appellerai, sans ironie, la recherche économique « nouvelle vague »?

Dans le Québec, la conjoncture est plus favorable que jamais à la recherche économique. Le public manifeste sans équivoque le besoin d'être éclairé sur les questions économiques. Les gouvernants sont extrêmement sympathiques à la recherche. Il ne faudrait pas que les chercheurs eux-mêmes, par manque de réflexion, contribuent aux tristes répétitions de l'histoire.

Dès avant la fin de l'« ancien régime », le gouvernement acceptait dans ses cadres plusieurs jeunes économistes. Le gros de ces effectifs

107 Études sur notre milieu, collection dirigée par Esdras MinviLLe, Éditions Fides, Montréal.

108 La Commission royale d'enquête sur les problèmes constitutionnels, province de Québec. 
a été versé à la recherche. Plusieurs économistes de ma génération se sont vus confier la tâche de diriger cette équipe nouvelle. Malgré le peu d'expérience acquise dans ce domaine, les résultats ont été étonnants. À l'occasion de la réunion de l'ACFAS en 1960, je louais sans équivoque ce travail de pionnier. En effet, les organisateurs du congrès de l'ACFAS m'avaient invité à commenter la communication de monsieur Raynauld sur l'interdépendance des industries du Québec. Depuis, le Ministère de l'Industrie et du Commerce a publié, sous la signature de monsieur Raynauld, un magnifique volume intitulé Croissance et structure économiques de la province de Québec ? 109

Je me propose, dans la deuxième partie de la présente communication, de faire la critique de ce volume.

J'ai choisi cette stratégie parce qu'elle me permet de démontrer les faiblesses de la recherche économique telle qu'actuellement organisée dans le Québec, de démontrer que ces faiblesses pourraient être évitées et que les [67] chercheurs seraient ainsi plus efficaces. Comme monsieur Raynauld me semble difficile à impressionner, je m'en tiendrai cette fois à l'aspect négatif de ma critique. Qu'il soit assuré, une fois pour toutes, que mon admiration pour lui et mes bons sentiments à son égard n'ont en rien changé depuis l'automne 1960.

Ma première critique portera sur la façon dont $\mathrm{M}$. Raynauld juge 110 le symposium de Laval de 1952. ${ }^{111}$ Je ferai remarquer que le symposium ne portait pas sur le développement du Québec. Le sujet à traiter était beaucoup plus précis. Les communications portaient sur « Les répercussions sociales de l'industrialisation sur le milieu québécois ». La première séance comportait deux communications, l'une par MM. Albert Faucher et Maurice Lamontagne, 112 l'autre par Charles Lemelin. ${ }^{113}$ En résumé, ces études établissaient une distinction entre

109 André RAYNAULD, Croissance et structure économiques de la province de Québec, Ministère de l'Industrie et du Commerce, Province de Québec, 1961.

110 Ibid., 53.

111 Jean-C. FALARDEAU, éd., Essais sur le Québec contemporain, Québec, Presses Universitaires Laval, Québec, 1953.

112 Albert FAucher et Maurice LAMONTAgne, « History of Industrial Development », Essais sur le Québec contemporain, chapitre I.

113 Charles LEMELIN, « The State of Agriculture », ibid., chapitre III. 
industrialisation et urbanisation ou développement. Le point essentiel était que les industries-clefs — ou de base — de la province de Québec étaient orientées suivant un axe à l'échelle continentale. D'autre part, la vie économique du milieu ethnique était restée jusqu'à récemment isolée de cette influence. - Monsieur Lamontagne avait démontré un peu auparavant devant un groupe de Dalhousie que le Canada n'avait pas encore, au moment de la dernière guerre mondiale, complété son industrialisation. 114 - Ces communications concluaient que les effets de l'industrialisation, ainsi entendue, sur le milieu avaient été malheureusement faibles. Il est symptomatique qu'un autre participant à la séance du lendemain, monsieur Esdras Minville 115 ne s'appuyant pas sur les mêmes définitions que l'équipe de Québec, ait inclus dans son concept d'industrialisation non seulement l'évolution de la grande industrie mais aussi l'évolution économique du milieu. Cette approche plus générale lui permettait de présenter un tableau très optimiste de l'évolution économique du Québec et de la place importante que pouvaient $\mathrm{y}$ jouer le petit et le moyen entrepreneurs canadiensfrançais. ${ }^{116}$ Ces débats avaient lieu à l'occasion du centenaire de l'Université Laval, et nous avions à Québec la visite de monsieur Raoul Blanchard. Celui-ci m'avouait que ses observations géographiques faites dans les régions du Québec pendant les années 1930117 gardaient leur valeur intrinsèque. Mais monsieur Blanchard avait, en conclusion de ses études régionales, soutenu une thèse. Pour lui, nos ressources physiques - entre autres la terre arable - et nos ressources humaines étaient suffisantes pour déclencher une demande de biens et services capable d'assurer la prospérité [68] économique de l'agriculture et de l'industrie. Cependant, en 1952, il m'avouait au cours d'un tête-à-tête qu'au moment où il publiait le résultat de ses

114 Maurice Lamontagne, «Québec : Rich Resources for Industry », Public Affairs, Dalhousie University, Halifax, Dec. 1948.

115 Esdras Minville, «Conditions de notre avenir », Essais sur le Québec contemporain, chapitre XI.

116 Voir aussi : Esdras MinviLLE, Le chef d'entreprise, étude $\mathrm{n}^{\circ}$ 6, Service de documentation économique, École des Hautes Etudes commerciales, Montréal, 1953.

117 Raoul Blanchard, L'Est du Canada français, 2 vol., Montréal, 1935 ; articles dans la Revue de Géographie alpine, 1930, 1931, 1932, 1933, 1938 et 1939 ; et «Les excédents de population et l'agriculture dans la province de Québec », L'Actualité économique, XXIV, 4, 1949. 
études québécoises, il n'avait pas encore saisi l'importance de la dépression mondiale de 1930 et que, de plus, les conclusions économiques de ses études géographiques traduisaient l'influence qu'à son insu l'opinion des leaders québécois, entre autres les agronomes, avait exercé sur son esprit.

Mais revenons à notre interprétation de la thèse LamontagneFaucher-Lemelin. Je regrette, mais dans l'ouvrage de M. Raynauld on trouve en de nombreux points de l'analyse des confirmations non équivoques de cette thèse. Je pourrais, si j'en avais le temps, citer abondamment à ce propos Croissance et structure économiques de la province de Québec. 118

Ma deuxième critique portera sur la deuxième partie du volume. Celle-ci est consacrée au problème d'interdépendance des industries du Québec et, en particulier, cherche à isoler les activités domestiques des importations venant non seulement des pays étrangers mais encore des autres provinces canadiennes. J'ai, à l'automne 1960, montré le danger que comportent les postulats relatifs aux importations quand le milieu d'origine du produit est une autre province canadienne. Je ne reviendrai pas sur ce point, car l'auteur a, dans le volume, bien explicité ses postulats et nettement indiqué les limitations inhérentes à la procédure suivie. J'aurais de nombreux commentaires à ajouter sur ce problème crucial des importations, mais il me semble préférable de reporter cette discussion à un moment plus opportun. 119

118 Voir pp. 72, 74, 75, 76, 79, et spécialement p. 95. Même confirmation aux pp. 98, 99, 105, 211.

119 Lecture faite du texte sur cette question des importations, j'éprouve encore, comme économiste théoricien, un certain malaise. En effet, j'aurais aimé que les distinctions soient plus nettement établies entre les diverses façons de traiter les importations dans un tableau économique. J'admets qu'au moment où l'ouvrage a été publié les économètres canadiens eux-mêmes n'avaient pas discuté systématiquement les diverses alternatives théoriques qui s'offrent dans le traitement des données relatives aux importations. Aujourd'hui, on sait que si les industries peuvent être définies en termes de biens physiques, alors les importations entrant en concurrence avec les facteurs domestiques de production peuvent être intégrées au système de manière que chaque coefficient d'interdépendance du tableau tienne compte des importations. C'est l'idéal recherché dans le tableau économique américain (1947). Si les diverses industries sont définies non plus sur la base d'un produit mais sur la base d'un « établissement », alors on peut exclure les importations de 
[69]

La faiblesse que je veux souligner ici, devant ce groupe de chercheurs spécialistes, me permettra d'illustrer le danger que comporte l'absence de dialogue entre théoriciens et chercheurs empiriques et les conséquences graves qui en résultent au moment de l'interprétation des résultats de l'analyse.

la production intermédiaire et compter les importations dans le groupe des facteurs primaires au même titre que les services du travail. C'est de cette façon que la dernière version des tableaux économiques canadiens a été présentée. Et comme le dit monsieur Raynauld, si une telle version avait existé au moment de la préparation des tableaux québécois, la tâche eût été beaucoup plus facile. Malheureusement, monsieur Raynauld a dû baser ses déductions québécoises sur un tableau où les importations étaient comptées en bloc comme un secteur particulier dans la production intermédiaire, au même titre, disons, que l'industrie de l'acier. Suivant cette version, qui avait d'abord été adoptée par le Bureau fédéral de la statistique, les importations se trouvent à influencer l'ensemble des coefficients du tableau économique, et non pas chacun d'entre eux comme dans la version américaine. Ajoutons enfin que la définition des industries, aussi bien que la façon de traiter les importations, dépend à la fois de la nature des informations statistiques disponibles et de l'usage auquel le tableau est destiné. À tout événement, je crois que le tableau québécois des valeurs domestiques globales de 1949 ne devrait pas être transformé en tableau de coefficients et encore moins en matrice-solution, sans que toute la question théorique sous-jacente à sa construction soit de nouveau discutée à fond.

Il faudrait, de plus, vérifier empiriquement la validité du postulat formulé comme suit par les auteurs : "Pour être appropriée, cette procédure suppose que les industries donnent une préférence aux ressources domestiques et qu'elles n'importent de l'extérieur que pour l'excédent de leurs besoins par rapport à l'offre domestique totale moins les exportations » (Croissance et structure économiques de la province de Québec, p. 161). En d'autres termes, on est forcé par les exigences de la procédure à supposer absence de substitution entre les biens de production importés et les biens de production fournis par le milieu. Cette vérification empirique peut se faire par des études complémentaires à l'édification des tableaux économiques. Elle s'impose même pour le tableau canadien, à moins que celui-ci ne soit pas utilisé pour l'étude d'années autres que l'année de base. Cette vérification s'impose quand les importations sont comptées comme un secteur spécial. Elle s'impose également, à mon avis, lorsque le modèle est ouvert de façon à ramener les importations dans la catégorie des facteurs primaires. 
La lecture de la deuxième partie du volume donne nettement l'impression que les auteurs ne connaissent pas suffisamment les propriétés du modèle qui inspire leur démarche.

En effet, en vue de choisir le critère général d'une classification des industries québécoises susceptible d'inspirer les responsables de l'orientation de l'économie québécoise, les auteurs, dans cette deuxième section de l'ouvrage, ont mesuré l'importance des achats et des ventes domestiques de chacune des industries. Or, ce critère immédiat s'appuie sur les achats domestiques directs tels que colligés au tableau des agrégats économiques de l'année 1949. Mais c'est justement de l'essence d'un modèle de Leontief de montrer l'importance non seulement des effets directs mais aussi des effets indirects de la demande sur l'ensemble de la production des industries. L'intuition nous porte à croire que les effets indirects sont moins importants, relativement, que les effets directs, mais c'est là une erreur. Par exemple, 120 en 1947, aux États-Unis, la demande finale directe pour l'acier par le secteur de la construction s'élevait à 0.876 milliard de dollars ; celle venant des unités de ménages était nulle. Si l'on tient compte des effets directs et indirects, la demande pour l'acier de la part des ménages s'élevait à 4.023 milliards de dollars contre seulement 2.867 milliards de dollars dans le cas du secteur de la construction. On voit que l'effet direct seul ne peut servir à établir l'importance d'un client et que l'effet indirect peut être de fait très supérieur à l'effet direct. 121

120 W. Duane Evans et Marvin HoffenBerg, «The Inter-industry Relation Study for 1947 », Review oj] Economies and Statistics, XXXIV, 2, 1952.

121 Pour donner un contenu concret à ces notions d'effets directs et d'effets indirects, ajoutons les explications suivantes :

En 1947, les achats directs et indirects d'acier par le secteur de la construction étaient comme suit (en milliards de dollars) :

a) Achats directs (disons une livraison de tuyaux d'acier)...

b) Achat d'acier entrant comme matière première

0.445 dans la fabrication de tuyaux et tenant également de l'industrie de l'acier (disons des plaques d'acier destinées à la fabrication de la tuyauterie)... 
Les auteurs du volume prennent la précaution de mentionner le phénomène de la diffusion des influences et minimisent l'importance de ce phénomène dans leur schéma, ce que je suis prêt à concéder. En effet, le concept de diffusion se rapporte à l'éventail des industries affectées par une demande donnée. Aussi, dans une économie fort industrialisée, l'acier entre directement ou indirectement dans la fabrication d'une multitude de biens. Dans une économie sans gadgets ménagers et centrée surtout sur la consommation, les produits naturels, laine, bois, etc., seront les vedettes sur le plan de la diffusion. Mais minimiser l'importance de la diffusion n'équivaut pas, loin de là, à minimiser l'importance des effets indirects de la demande sur les activités de production.

Je m'empresse d'ajouter que pour faire l'estimation statistique des effets indirects, il faut effectuer de nombreux calculs (près de 4,000 multiplications dans l'exemple cité plus haut) si la matrice-solution est déjà publiée. Sans cette solution générale, les calculs sont tout simplement impossibles. Or, la solution générale relative aux tableaux canadiens n'a été publiée qu'à la fin de 1960. Seul un économètre serait en mesure de dire jusqu'à quel point la solution générale canadienne peut servir des fins régionales. En tant qu'économiste théoricien, je dois avouer que j'ai été étonné de constater que, malgré cette lacune, les responsables ont continué à bâtir une classification générale des industries où l'influence des achats et des ventes sur les entre-

c) Achat d'acier sous forme, supposons, d'isolant de

0.001 coton venant du secteur agricole où le coton est produit avec de la machinerie dont la fabrication est d'acier...

d) Achat d'acier sous forme de produits finis consommés par le secteur de la construction mais venant de tous les secteurs autres que ceux de l'agriculture et de l'acier...

e) Total des effets indirects...

f) Total des effets directs et indirects... 
prises du milieu est mesurée uniquement en termes directs. C'est évidemment un métier bien particulier que celui de scruter la pensée économique contemporaine. A côté du métier d'économètre, il y a cet autre métier qui consiste à scruter les propriétés des modèles théoriques proposés aux spécialistes de la recherche empirique. Or, celle-ci accapare tellement son homme, à cause de la masse de données à harnacher, à cause des compromis que la réalisation impose, à cause des problèmes d'administration et de direction inhérents à la recherche en équipe, qu'il devient imprudent d'ignorer le spécialiste en théorie comme il serait imprudent d'ignorer l'économètre.

Ma critique, dira-t-on, fait peut-être trop grand état d'un simple accident. J'aimerais le croire, mais malheureusement le chapitre V, intitulé « Les revenus et les salaires », fournit de nouveau l'exemple d'une faille théorique importante. Sans aller dans les détails, disons que, faute d'avoir compris toutes les implications du modèle de Samuelson sur l'égalisation des prix relatifs des facteurs de production, les auteurs n'ont pas vu la nécessité d'étudier systématiquement le degré d'utilisation du capital dans les industries, et c'est là une lacune assez sérieuse du volume. 122

122 En effet, la section $\mathrm{C}$ de ce chapitre tente d'expliquer le phénomène du différentiel des salaires entre le Québec et l'Ontario. L'interprétation des chiffres analysés dans les sections précédentes du chapitre veut s'appuyer sur le théorème classique de Samuelson explicitant les conditions théoriques d'égalisation des prix des facteurs de production. À propos de ce théorème, Samuelson écrit que beaucoup d'économistes se sont évertués à prouver que la valeur absolue des salaires pouvait s'égaliser d'un milieu à l'autre : «A number of economists have tortured themselves trying to manipulate these expressions so as to result in $a=a$, etc. " (Paul A. SAMUELSON, "Price Equalization Once Again », Economic Journal, LXIX, 1949). En fait, Samuelson établit les conditions qui permettent aux prix relatifs des facteurs de s'égaliser entre deux milieux économiques isolés quant aux marchés des facteurs mais reliés l'un à l'autre via les marchés des produits finis. Le théorème, encore une fois, prouve que c'est le rapport des salaires à la rémunération du capital du milieu « $\mathrm{A}$ » qui s'égalise au rapport des salaires à la rémunération du capital du milieu « B ». - La même conclusion peut s'exprimer en disant que le rapport des salaires des deux milieux va égaliser le rapport des rémunérations du capital des deux milieux. Mais, à moins que, par exception, la valeur absolue des rémunérations du capital soit unique pour les deux milieux, on ne peut jamais conclure à l'égalité en termes absolus des salaires. Je dois dire que le modèle est très difficile à saisir. Samuel- 
Ma troisième critique portera sur le chapitre II, « La croissance des industries 1935-1955 ». Dans ce chapitre, l'analyse est conduite de main de maître. Il faut souligner en particulier l'étude des changements dans la structure industrielle du Québec et de l'Ontario. Mentionnons aussi les quelques remarques bien amenées sur la stabilité de la croissance. Cependant, tant valent les données de base tant vaut l'analyse du chapitre II. L'appendice nous fournit de façon systématique toutes les données statistiques utilisées dans le corps du volume. On y trouve exposées en détail toutes les procédures statistiques mises en œuvre pour les fins de compilation. La plupart des sources sont des sources secondaires. En d'autres termes, les analyses sont en grande partie basées sur la documentation officielle des bureaux fédéral et provincial de la statistique. ${ }^{123}$ J'aurais aimé que l'équipe responsable de la compilation statistique de l'appendice du volume publié par le Ministère du Commerce eût inclus une critique définitive, à fond, de ces séries, de façon à en établir une fois pour toute la valeur intrinsèque. Même si les résultats avaient été défavorables à la survie d'une partie des données, il est probable qu'il serait resté une quantité suffisante de matériaux valables après cet élagage. Pour qu'il soit possible que la recherche en équipe ait de la continuité, il faut bâtir ce que j'appellerai un capital d'information que chacune des recherches successi-

son, qui est pourtant un expert pédagogue, s est vu forcé d'en présenter au moins trois versions. Quoi qu'il en soit, les auteurs constatent la constance des écarts de salaires entre le Québec et l'Ontario de 1938 à 1957. Ceci est déjà, à mon sens, une certaine confirmation du modèle d'analyse choisi. Pourtant, au lieu de poursuivre dans cette direction, les auteurs écrivent : «... conformément au modèle, les deux provinces auraient dû se spécialiser... à un degré suffisant pour profiter l'une et l'autre de l'abondance relative de leurs facteurs respectifs de production et [notez bien] faire disparaître les différences de salaire... » et le texte continue : «En réalité, nous avons vu que le différentiel de salaires persiste... » Le chapitre $\mathrm{V}$ fournit donc un deuxième exemple où la démarche aurait pu prendre une autre direction si les propriétés du modèle utilisé avaient été mieux exploitées.

123 Ces rapports présentent un inconvénient sérieux : par suite de changements dans les définitions, les séries chronologiques ne sont pas toujours comparables. De plus, il est reconnu que les données annuelles nationales ne sont souvent que des intrapolations des données des recensements périodiques. Enfin, les chiffres régionaux sont souvent déduits des séries nationales par des procédés d'intrapolation plus ou moins arbitraires. 
ves contribuera à augmenter. Une étude systématique de la valeur intrinsèque des séries statistiques officielles serait la première démarche à effectuer à cette fin.

En recherche économique, les études basées sur les données chronologiques officielles resteront importantes. Aussi est-il urgent d'établir sans a priori d'aucune sorte leur valeur intrinsèque, ce qui implique plus que le travail déjà excellent de l'appendice de Croissance et structure économiques de la province de Québec.

Une étude des taux de croissance, pour être complète, exige à mon avis que les valeurs absolues qui servent de base aux calculs soient examinées systématiquement. Je crains toujours les comparaisons basées sur les pourcentages. Si l'on distribuait un dollar à chacun des assistants de cette assemblée, ceux qui ont cent dollars en poche verraient leur actif s'accroître de un pour cent. D'autre part, les assistants qui ne possèdent déjà qu'un dollar verraient leur richesse monter en flèche à 200 pour cent. J'ai, par [72] exemple, été intrigué de constater que l'industrie des provendes occupait la vedette (avec trois autres industries) du point de vue de la croissance. Par curiosité, j'ai examiné le tableau des valeurs absolues et j'ai fait la constatation suivante :

\begin{tabular}{|l|c|c|c|}
\hline \multirow{2}{*}{ INDUSTRIES } & \multicolumn{3}{|c|}{$\begin{array}{c}\text { VALEUR BRUTE } \\
\text { millions de dollars })\end{array}$} \\
\cline { 2 - 4 } & 1935 & 1955 & Changement \\
\hline Abattoirs & 22.09 & 178.00 & 8 fois \\
\cline { 1 - 1 } Provendes & .84 & 63.06 & 75 fois \\
\hline
\end{tabular}

La synthèse de Croissance et structure économiques de la province de Québec est présentée au chapitre VII. (Je considère les excellentes contributions de MM. Jacques Henripin et Jacques Saint-Laurent comme des études hors-texte.) 124

124 Chapitres VI et VIII, Croissance et structure économiques de la province de Québec (Voir Avant-propos, p. 10). 
L'idée maîtresse consiste à établir une classification québécoise des industries, compte tenu des tendances que l'analyse a dégagées. De nouveau, l'abondance de la main-d'œuvre, de l'énergie électrique est soulignée. Les caractéristiques de structure du secteur industriel québécois sont réexaminées de façon à faire ressortir les «avantages comparatifs » de la province. Puis les critères de classification des industries sont présentés systématiquement. En voici une description sommaire :

Pour occuper un rang prioritaire, une industrie doit :

a) consommer davantage les ressources existant en abondance dans la province

b) exercer une forte influence sur la croissance des autres industries.

À propos de l'utilisation des ressources, on emploie trois indices :

$\left.a^{\prime}\right)$ Salaires et gages

Valeur ajoutée

ce rapport doit être grand

b') $\underline{\text { Consommation d'électricité }}$ Valeur ajoutée

ce rapport doit être grand

$\left.c^{6}\right)$ Nombre d'heures de travail ce rapport doit être petit.

Valeur ajoutée

L'hypothèse : Le travail et le capital sont considérés comme complémentaires. " Le travail par dollar de production nette » est considéré comme un indice de «la quantité de capital par dollar de production nette ». 
Ce rapport ( $c$ ') doit être petit puisqu'il est censé refléter l'utilisation du capital et que le capital est considéré comme une ressource rare. En d'autres [73] termes, une industrie qui utilise typiquement beaucoup de capital se déclasse.

En fait ( $\left.a^{\prime}\right)$ et $\left(c^{\prime}\right)$ vous disent tout naïvement que les industries les plus souhaitables sont celles qui rapportent de gros revenus tout en exigeant peu d'heures de travail.

À propos de l'influence sur la croissance industrielle, on emploie trois autres indices :

$\left.a^{\prime}\right)$ Les ventes domestiques aux autres industries de la province.

$\left.b^{\prime}\right)$ Les achats domestiques des autres industries de la province. Plus les échanges domestiques sont considérables, plus l'industrie qui les effectue occupe un rang élevé dans la classification.

c') Le taux de croissance de l'industrie.

Un taux de croissance élevé contribue à donner une priorité à l'industrie évaluée. Ce facteur est considéré comme un indice d'adaptation à l'évolution.

Quels jugements porter sur l'ensemble de ces critères ? Ceux qui se rapportent aux influences sur les autres industries du milieu sont inacceptables par un économiste parce que, comme je l'ai expliqué à propos du tableau économique de la province, ils ne tiennent pas compte des effets indirects.

Le choix des critères relatifs à la main-d'œuvre et au capital n'est pas appuyé, à mon sens, sur une juste évaluation du rôle que joue le facteur « capital » dans le développement.

Seul le critère relatif aux taux de croissance résiste à la critique. Chose assez étrange, son importance est justement minimisée : on trouve, en effet, dans le texte des phrases comme celle-ci : "Ce que nous faisons dans cette section est un exercice de style...»

Finalement, les auteurs font remarquer que leur classification ne tient pas compte de l'aspect demande des produits. «Les conditions 
d'offre des produits et la structure manufacturière du Québec qui s'ensuit semblent mal adaptées aux conditions de la demande. L'exemple de la fonte et de l'affinage des métaux non ferreux est patent. Cette industrie repose sur une technologie à forte intensité de capital et les échanges avec les autres industries de la province sont très faibles. L'avantage relatif de l'industrie est par suite très faible... " ${ }^{125}$ En fait, cette industrie se classe $28^{\mathrm{e}}$ sur un total de 32 industries.

Et les auteurs de conclure : " Est-il excessif de déduire de cette observation que la structure manufacturière de la province de Québec a besoin d'un renouvellement profond et d'une nouvelle orientation vers des secteurs plus dynamiques de l'industrie ? Des innovations majeures s'imposent... pour transformer la nature des avantages relatifs dont la province dispose en accroissant le capital, par exemple... » 126

\section{Conclusion}

Cette réflexion me permet de revenir au point de départ de ma critique. En 1952, la thèse soutenue par Faucher, Lamontagne et Lemelin était que, [74] historiquement, les industries-clefs du milieu québécois avaient eu une influence minime sur l'évolution économique du milieu. Les auteurs soutenaient également que les politiques économicosociales avaient contribué à perpétuer ce fossé entre le secteur des économies locales et le secteur des industries de base. Pour ma part d'ailleurs MM. Faucher et Lamontagne partagent sans doute mon avis - je crois que ce fossé a complètement disparu grâce aux politiques fiscales de la dépression et de la période de guerre et grâce au phénomène d'industrialisation de la dernière guerre mondiale et des années 1950-56.

Aujourd'hui, l'orientation de l'économie québécoise pose des problèmes tout différents. D'une part, les politiques de développement économique devraient favoriser la modernisation des industries manufacturières, viser à accroître la position concurrentielle de ces industries en intensifiant la capitalisation, mais en courte période la main-

125 Croissance et structure économiques de ta province de Québec, 301.

126 Ibid., 302. 
d'œuvre en souffrira. D'un autre côté, les politiques de développement doivent viser à équilibrer l'offre et la demande sur le marché du travail, par exemple, en prolongeant la période de fréquentation scolaire, en favorisant l'éducation. Comment résoudre ce dilemme que pose la conjoncture ? La recherche économique peut certainement contribuer à éclairer les hommes politiques sur ce point.

Face à l'opinion publique, doit-on étatiser les industries de base ? Cette question définit un second sujet de recherche aussi stratégique que le premier. Dans quelle mesure les fonds requis à cette fin vont-ils concurrencer les fonds requis pour les investissements sociaux ? Une telle étude est d'une urgence aiguë.

Les recherches sont-elles suffisantes dans le Québec actuellement pour permettre à l'État de négocier de plain-pied avec la grosse industrie ? Voilà encore un domaine où la recherche est urgente.

Comme on peut le constater, le choix des projets de recherche relève plus de jugements, de la connaissance de l'histoire et de la conjoncture que de l'ordre de priorité indiqué par la classification des industries telle que la propose cette étude sur la croissance de l'économie québécoise.

Charles Lemelin

Département d'économique, Université Laval. 


\title{
Situation de la recherche sur le Canada français I. Perspectives historiques
}

\section{Jean-Charles Bonenfant}

Département de science politique, Université Laval

Bibliothèque de la Législature, Québec

\section{"Les études politiques."}

\author{
[pp. 75-82.]
}

\section{$\underline{\text { Retour à la table des matières }}$}

Science de carrefour et science de synthèse, dont l'objet est l'autorité, les gouvernants et le pouvoir, la science politique peut être tentée par l'impérialisme. Aussi, pour bien en délimiter les frontières et pour ne pas empiéter sur le domaine d'autrui, j'ai cru devoir adopter comme rubriques fondamentales de mon étude, celles qu'ont précisées un groupe d'experts réunis par l'Unesco à Paris, en 1948, soit : la théorie politique, les institutions politiques, les partis, les groupes et l'opinion publique, et finalement les relations internationales. ${ }^{127}$ À la demande des organisateurs du colloque, j'ai ajouté à ces rubriques le droit. Ce n'est pas une hérésie complète puisque, à la rigueur, une bonne partie du droit peut être incluse dans les institutions politiques ; c'est en mê-

127 La science politique contemporaine. Contribution à la recherche, la méthode et l'enseignement, Unesco, 1950, 4. 
me temps pour la science politique une douce vengeance vu que, jusqu'ici, dans notre province surtout, elle s'est trop souvent confondue avec le droit constitutionnel.

Lorsqu'il y a une quinzaine d'années, Burton G. Keirstead et Frederick M. Watkins préparèrent pour l'Unesco un bilan de la science politique au Canada, ils ne purent citer qu'un seul nom canadien-français, celui de Henri Bourassa comme représentant de ce qu'ils appelaient " quelques pamphlétaires et quelques journalistes inspirés » dont, ajoutaient-ils « on ne saurait ranger (les) écrits passionnés parmi les ouvrages scientifiques ». ${ }^{128}$ Les deux universitaires canadiens-anglais ne furent pas injustes à notre égard, car nous verrons que le bilan de notre science politique est plutôt mince.

La première rubrique de l'Unesco est donc la théorie politique à laquelle on a joint l'histoire des idées politiques. C'est un double domaine qu'on n'a guère abordé au Canada français. $\mathrm{Si}$, pour utiliser les mots de Jean Meynaud, 129 « la théorie forme un ensemble articulé de définitions, d'assomptions et de propositions couvrant un sujet déterminé » et "doit permettre de présenter des relations susceptibles de vérification », elle n'a [76] pas beaucoup été pratiquée ici. Lorsqu'on s'est intéressé à des phénomènes comme le nationalisme, le fédéralisme, le bicaméralisme, on l'a fait dans le concret, le plus souvent même dans une atmosphère de polémique. Il y a tout de même de la théorie politique dans ce livre de précurseur que publia à Paris, en 1896, Edmond de Nevers, sous le titre de L'avenir du peuple canadienfrançais ; on en trouve dispersée dans les écrits de l'abbé Wilfrid Morin, de M Esdras Minville, du R. P. Richard Arès et dans les livraisons de l'Action française de 1918 à 1928 et de l'Action nationale de 1933 à nos jours. Le plus loin où l'on ait osé se rendre en ce domaine est peut-être contenu dans le volume II du Rapport de la Commission royale d'enquête sur les problèmes constitutionnels où on retrouve, malgré l'anonymat de la rédaction, les principales idées de M. Minville et du R. P. Arès sur l'État et sur le fédéralisme.

128 Ibid., 178.

129 Jean MEYnAud, Introduction à la science politique, Paris, Librairie Armand Colin; 1959, 18 (Cahiers de la Fondation nationale des sciences politiques, 100). [Livre disponible dans Les Classiques des sciences sociales. JMT.] 
Quant à l'histoire des idées politiques, elle n'a même pas été esquissée. Nous ne possédons qu'une chronique des événements dans les trente-trois tomes de l'Histoire de la province de Québec, de Robert Rumilly, et une synthèse plus concise avec quelques jugements de valeur dans The French Canadians 1760-1945, de Mason Wade.

Au chapitre des institutions politiques, qui comprend d'abord l'étude de la constitution et celle des relations entre le gouvernement central et les gouvernements locaux, nous avons été un peu plus féconds pour autant que les problèmes étaient envisagés sous un éclairage juridique. Les Pères de la Confédération, que ce soient les quatre Canadiens français ou leurs vingt-neuf collègues canadiens-anglais, n'étaient pas de profonds penseurs ; ils ne citaient pas, comme leurs prédécesseurs américains de 1787, Locke et Montesquieu. Ce n'étaient que des praticiens de la politique à la recherche de solutions immédiates et on leur a prêté, au Canada français surtout, de belles idées auxquelles ils n'ont jamais songé. Aussi, faut-il attendre à 1883 et 1884 pour que soient publiées trois lettres du juge T.-J.-J. Loranger sur L'interprétation de la constitution fédérale, lettres qui constituent le premier commentaire canadien-français ayant quelque valeur sur l'Acte de l'Amérique du Nord britannique. En 1889, le futur juge P.-B. Mignault publiait son Manuel de droit parlementaire dont le second titre Cours élémentaire de droit constitutionnel reflète davantage le contenu qui n'est pas sans une certaine valeur. Et les années passèrent sans que s'enrichisse substantiellement notre bibliothèque constitutionnelle jusqu'au moment où la menace que le Statut de Westminster permette au pouvoir central d'être entièrement maître de l'Acte de l'Amérique britannique du Nord et de ses modifications vienne en quelque sorte magnifier la théorie du pacte. Cette théorie fut énoncée officiellement en septembre 1930 par le premier ministre d'Ontario, G. H. Ferguson, mais c'est surtout dans le Québec qu'elle fut ensuite utilisée. Si on examine les nombreuses références que le R. P. Arès a recueillies à ce sujet, soit dans Dossier sur le pacte fédératif publié en 1941 [77] ou dans La Confédération, pacte ou loi ? publié quelques années plus tard, on s'aperçoit que, malheureusement, nos constitutionalistes manquèrent d'imagination ou se montrèrent paresseux, se contentant le plus souvent d'invoquer en faveur de la théorie du pacte des arguments d'autorité. D'ailleurs, à propos de la nature de notre constitution formelle, nous avons manqué une belle occasion de nous débarrasser 
de notre conception primitive, presque charismatique du droit et le plus souvent stérile en n'approfondissant pas davantage la théorie de l'institution. Quelques Canadiens français l'esquissèrent, comme Léopold Richer dans Notre problème politique 130 et Edouard Laurent dans un des premiers Cahiers de l'École des sciences sociales, politiques et économiques de Laval, intitulé Quelle est la nature de l'Acte de 1867 ? Le R.P. J.-T. Delos, grand spécialiste de la question, en causa avec professeurs et élèves pendant son séjour au Canada, mais les praticiens même les plus évolués, comme $\mathrm{M}^{\mathrm{e}}$ Antonio Perrault, se scandalisèrent de ces intrus qui venaient ébranler les cadres traditionnels du droit. 131

Lorsque fut créée par Ottawa, en 1937, la Commission royale des relations entre le Dominion et les provinces, événement décisif dans le développement de la science politique au Canada, nous n'avions guère étudié notre constitution et nous étions en ce domaine en retard sur les Canadiens anglais. Chez eux, selon le témoignage de Kirstead et Watkins, " la science politique... (était) demeurée dans une large mesure une branche subalterne de la science économique ». ${ }^{132}$ Mais ils avaient tout de même eu un Stephen Leacock, qui fut autre chose qu'un humoriste, et un O.D. Skelton dont l'essai sur le socialisme avait été jugé digne de figurer dans les quelque cinquante livres qu'on plaça dans le tombeau de Lénine. Les Canadiens anglais possédaient surtout un nombre considérable d'universitaires qui purent participer au travail de la Commission Rowell-Sirois. Les Canadiens français n'y furent représentés momentanément que par le juge Thibaudeau Rinfret, qui était un civiliste, et ensuite par un notaire d'ailleurs intelligent et sympathique mais qui n'enseignait que par à côté le droit constitutionnel à Laval.

Parmi les auteurs des nombreuses études qui furent préparées pour la Commission, on ne trouve que deux noms canadiens-français, celui de $\mathrm{M}$. Esdras Minville et celui de $\mathrm{M}^{\mathrm{e}}$ Léon Mercier-Gouin. En résumé, nous ne pouvions offrir à la Commission un Robert Alexander Mac-

130 Léopold RICHER, Noire problème politique, Montréal, Éditions de l'A.CF., 1938, 122 et suiv.

131 Revue du Barreau, 1942, 355-359.

132 La science politique contemporaine. Contribution à la recherche, la méthode et l'enseignement, Unesco, 1950, 178. 
Kay, professeur de science politique à l'Université Dalhousie, un Henry Forbes Angus, professeur d'économie politique à l'Université de la Colombie britannique, et un grand journaliste politique comme John W. Dafoe, qui, en définitive, [78] préparèrent le Rapport de la Commission et orientèrent ainsi pour longtemps le fédéralisme canadien.

C'est tout de même jusqu'à un certain point des idées énoncées par la Commission Rowell-Sirois que devait naître, en 1954, un de nos premiers livres de science politique véritable. Le fédéralisme canadien, de Maurice Lamontagne. Enfin, au Canada français, on abordait l'étude du fédéralisme en dehors des strictes œillères juridiques. Certes, M. Lamontagne s'est fait des adversaires pour bien d'autres raisons, mais inconsciemment ceux qui le combattirent le firent parce qu'ils sentaient que le fédéralisme échappait à leur étude exclusive et pouvait être examiné en tenant compte d'éléments autres que les textes sacro-saints des lois ou des décisions du Comité judiciaire du Conseil privé.

Puis ce fut, en 1953, la création d'une autre commission, québécoise cette fois, la Commission royale d'enquête sur les problèmes constitutionnels, la commission Tremblay. Quelques-uns des mémoires qui lui furent soumis, son rapport publié en 1956 et au moins une des annexes à ce rapport, celle de M. François-Albert Angers, sur La centralisation et les relations fédérales-provinciales, ont contribué à l'enrichissement de notre science politique. Je note aussi que les commissaires, ayant sans doute souffert eux-mêmes de l'absence de tant de monographies qui leur auraient été utiles, recommandèrent à l'Etat provincial de favoriser dans toute la mesure du possible l'organisation de la recherche dans les universités et les centres d'enseignement supérieur dans toutes les branches du savoir et particulièrement dans certaines disciplines qu'ils énumèrent, dont « la science politique ». 133

Il est d'ailleurs intéressant de constater que ce sont peut-être jusqu'ici les enquêtes des commissions royales qui, dans le Québec, ont fait accomplir le plus de progrès à la science politique si bien qu'en 1957, M. Gérard Bergeron a pu intituler Problèmes politiques du Québec un répertoire bibliographique des commissions royales d'en-

133 Rapport de la Commission royale d'enquête sur les problèmes constitutionnels, vol. III, tome II, 324. 
quête, présentant un intérêt spécial pour la province de Québec de 1940 à 1957.

Sous la rubrique des institutions politiques, la classification de l'Unesco inclut l'administration publique. En cette matière, nous n'avons rien produit. Nos hommes politiques ont beaucoup disserté sur les bienfaits ou les dangers du développement de l'action de l'État, mais jamais nous n'avons étudié scientifiquement ce qui constitue, à mon sens, un des problèmes les plus aigus de la science politique moderne, c'est-à-dire le contrôle de l'acte administratif, la façon de protéger l'individu en face d'un État qui devient nécessairement de plus en plus puissant.

La troisième rubrique de l'Unesco traite des partis, des groupes et de l'opinion publique. C'est un domaine passionnant de la science politique où elle ne se distingue pas toujours très bien de la sociologie «si d'ailleurs, [79]comme dirait malicieusement Duverger, elles se distinguent autrement que par les départements ». ${ }^{134}$ C'est un domaine qui s'est développé considérablement en France et en Angleterre ces dernières années et c'est un domaine dans lequel nous commençons à balbutier. L'histoire de nos partis politiques n'a pas été écrite ; nous avons un peu pratiqué la sociologie électorale et, récemment, les partis politiques semblent avoir compris qu'il $y$ avait pour eux-mêmes un intérêt immédiat à sonder scientifiquement l'opinion publique. Je signale en particulier Les électeurs québécois, Attitudes et opinions à la veille de l'élection de 1960, rapport d'un groupe de recherches sociales, de Montréal, derrière lequel agissait le parti libéral. Je n'oublie pas non plus l'intéressante étude de Jean Hamelin, Jacques Letarte et Marcel Hamelin, publiée en 1960, dans les Cahiers de géographie de Québec, dont les auteurs eux-mêmes ont admis que ce n'était pas un essai de science politique mais uniquement un instrument de travail.

Enfin, sous la quatrième rubrique de l'Unesco, relations internationales, les travaux canadiens-français ont été plutôt rares car pendant longtemps il fut même élégant de se moquer des organismes internationaux.

134 Maurice DuVERGER, dans le Traité de sociologie, publié sous la direction de Georges Gurvitch, Presses Universitaires de France, tome second, 1960, 3. 
On me reprochera peut-être de ne pas avoir dressé un inventaire méthodique et complet de nos richesses. C'était impossible et j'en ferai d'ailleurs, dans un instant, l'objet d'une recommandation. Mais même si on peut me citer quelques ouvrages oubliés, je pense que nous admettons tous notre pauvreté en science politique. Pour nous en convaincre par des arguments indirects, je vous invite à quelques petites expériences de recherche auxquelles par besoin vous vous êtes peut-être d'ailleurs livrés. Ouvrez par exemple la bibliographie que le gouvernement canadien a publiée en 1951 des Thèses des gradués canadiens dans les humanités et les sciences sociales de 1921 à 1946 ; parcourez les chapitres consacrés à la science politique et aux sciences connexes et vous verrez que l'apport canadien-français est assez maigre. Prenez les deux index décennaux du Canadian Journal of Economies and Political Science qui couvrent une période allant de 1935 à 1954 et qui permettent de connaître directement ou indirectement, par la bibliographie, tout ce qui s'est publié de valable en science politique au Canada depuis environ un quart de siècle et vous constaterez que nous n'avons pas fourni grand-chose. Enfin, lorsque en science politique nous voulons pratiquer l'humilité, nous songeons à toutes les belles études qui ont été publiées dans la série Canadian Government par University of Toronto Press, études parmi lesquelles nous pouvons au moins nous enorgueillir de l'ouvrage de M. Paul Gérin-Lajoie sur les amendements à la constitution. Nous pensons aussi aux quelque dix ouvrages consacrés à l'arrière-plan et au développement du crédit social en Alberta. Et nous pensons à tout ce que nous pourrions faire !

[80]

Il me reste à dire un mot du droit. Dieu sait si nous l'avons pratiqué. Nous avons produit ce que nous appelons de savants juges et d'habiles avocats qui se sont contentés de faire de l'interprétation et qui, très rarement, ont témoigné d'imagination juridique. Trois traités généraux de droit civil ont été publiés, celui de Langelier, celui de Mignault et celui qui se termine actuellement chez Wilson \& Lafleur. Ce sont trois ouvrages qui, en France, seraient classés parmi ceux de l'école classique dite de l'exégèse, une école qui, depuis la fin du siècle dernier, a été remplacée par l'école dite moderne, celle dont les tenants, pour employer les termes du doyen Léon Julliot de la Morandière, " ne se contentent plus du commentaire logique de la loi » mais « font intervenir dans leur exposé et leur critique des solutions légales, 
des considérations économiques, sociales » et "se préoccupent du droit comparé, des exemples donnés par les législations étrangères et surtout prennent conscience du rôle de la coutume, de la place essentielle de la jurisprudence ». 135

À vrai dire, nous n'avons qu'un ouvrage général de ce genre. C'est la synthèse que le professeur Louis Baudoin, de McGill, a publiée en 1953, sous le titre Le droit civil de la province de Québec, modèle vivant de droit comparé.

Au lendemain de 1930, nous eûmes tout de même une polémique juridique qui opposa les causalistes et les anti-causalistes. En 1936, $\mathrm{M}^{\mathrm{e}}$ Marcel Faribault eut l'originalité d'appliquer la théorie de l'institution à la fiducie dans un livre, intitulé Traité théorique et pratique de la fiducie ou trust du droit civil dans la province de Québec, qui fut cité à l'étranger. En 1941, fut fondée la Revue du Barreau, dans laquelle $\mathrm{M}^{\mathrm{e}}$ Antonio Perrault et après lui quelques praticiens osèrent critiquer les arrêts de tribunaux. En 1934, 1939, 1952 et 1958, les réunions au Canada français de juristes du monde entier groupés dans l'Association Henri-Capitant donnèrent à quelques avocats et notaires la tentation de voir le droit en dehors des dossiers de leurs cabinets. Enfin, ces dernières années, une génération de jeunes professeurs est née dans nos Facultés de droit et nous offre maintenant des ouvrages originaux que je n'ai pas le temps d'énumérer ici, mais dont le livre de $\mathrm{M}^{\mathrm{e}}$ André Morel, Les limites de la liberté testamentaire dans le droit civil de la province de Québec, publié à Paris en 1960, me semble le plus bel exemple. Il reste que notre droit s'est développé au hasard de la jurisprudence et des amendements des parlements et qu'il est terriblement en retard sur les faits.

Le bilan que je viens d'esquisser est assez pauvre en science politique comme en droit, mais les chercheurs ne peuvent qu'en être heureux car ils éprouvent une joie professionnelle à constater que leur champ de travail n'est pas encore défriché. Mais il y a tant à faire qu'il faut, surtout [81] dans une petite société comme la nôtre où le nombre des travailleurs sérieux demeurera longtemps restreint, l'accomplir avec méthode et coopération.

135 Léon JUlLIOT DE LA MORANDiÈRE, Introduction à l'étude du droit civil français, Introduction à l'étude du droit, Paris, 1951, 241. 
Pour éviter de répéter inutilement le travail déjà accompli et pour utiliser tous les matériaux disponibles, il faut d'abord dresser l'inventaire complet, définitif et analytique des travaux et des documents. Il est urgent que nous ayons pour les publications gouvernementales du Québec de 1867 à nos jours une bibliographie analogue à celles qui ont été soigneusement rédigées pour les provinces des Prairies, la Colombie britannique et le Nouveau-Brunswick. Souhaitons aussi que le gouvernement du Québec multiplie ses publications et les mette à la disposition de tous. Ottawa nous offre depuis une quinzaine d'années un excellent service de documents variés. À Québec, c'est la pauvreté et le désordre parfait. Pour ma part, avec toute la prudence d'un fonctionnaire, je réclamais déjà en 1956 dans une communication devant la Société canadienne d'éducation des adultes un meilleur système de distribution des publications du Québec. Je pense que c est toujours urgent. Si le rapport de la Commission Tremblay a été jadis aussi difficile à obtenir qu'un exemplaire de l'Amant de Lady Chatterley avant le jugement de la Cour suprême, ce n'est pas surtout parce que le premier ministre de l'époque en redoutait inconsciemment le contenu, mais c'est parce qu'il n'existait pour une telle publication aucun système normal de distribution. Si, depuis deux ans, vous avez eu besoin de quelques publications qui, à mon sens, sont indispensables aux politicologues du Québec, voici, si vous aviez la chance d'être bien renseignés, ce que vous avez dû faire. Pour obtenir le Rapport des élections de 1960, vous avez écrit au Président général des élections ; pour obtenir L'Annuaire statistique de la Province, vous vous êtes adressés au directeur du Bureau des statistiques ; c'est au bureau du Premier ministre qu'on distribuait L'Étude préliminaire à la révision de la carte électorale de la province de Québec et c'est au bureau de la distribution de l'Assemblée législative que vous pouviez obtenir le Bill 15, la Loi électorale de Québec. Et je pourrais pendant longtemps continuer la promenade.

Nous avons aussi besoin de plus de coopération entre les Universités et même entre les Facultés, les instituts, et il faut songer à une distribution du travail entre tous ceux qui, au Canada, s'intéressent à la science politique et au droit. Au niveau de l'enseignement supérieur, notre petite société ne peut se donner le luxe de la concurrence. Nous pouvons même souhaiter qu'un certain dirigisme permette d'orchestrer 
le travail de recherche, d'éviter la duplication et d'orienter les étudiants vers les monographies dont nous avons le plus besoin.

Enfin, il nous faut surtout des professeurs, pas des improvisateurs autodidactes, comme ceux de ma génération, mais des professeurs auxquels je souhaite avec envie une formation canadienne, américaine, britannique [82] et française, car notre science politique et notre droit doivent être plus qu'ailleurs le fruit d'une synthèse.

Ne croyons pas toutefois que le développement de la science politique transformera radicalement notre société. Comme l'a écrit Jean Meynaud, même si l'on consacrait à la science politique des sommes égales à celles affectées à la physique nucléaire ou à la chimie, on n'arriverait pas à des résultats de même portée et il en découle que « la modestie demeurera longtemps l'une des vertus les plus nécessaires au spécialiste en science politique. » 136

Tout de même, dans une société où l'on est porté à croire que les changements s'opèrent surtout par les hommes, il est peut-être temps que l'on songe à modifier les institutions, et pour cela la connaissance et l'utilisation des données de la science politique sont évidemment nécessaires.

Jean-Charles Bonenfant

Département de science politique, Université Laval ;

Bibliothèque de la Législature, Québec. 


\title{
COMMENTAIRE
}

\author{
Vincent Lemieux \\ Département de science politique, \\ Université Laval.
}

[pp. 83-85.]

$\underline{\text { Retour à la table des matières }}$

Je ne m'emploierai pas ici à relever les études oubliées, volontairement ou non, par $\mathrm{M}$. Bonenfant. Ou plutôt je n'en relèverai qu'une : Les institutions politiques canadiennes 137 de qui vous savez. M. Bonenfant m'en voudra peut-être, mais je soutiendrai quand même qu'en attendant l'ouvrage complet qu'il nous prépare, ce petit volume d'entretiens doit être intégré à l'inventaire de nos quelques produits originaux.

Ceci dit, je me limiterai, durant les quelques minutes qui me sont allouées, à l'une des quatre rubriques posées par les experts de l'Unesco, celle des partis, des groupes politiques et des opinions publiques. Certains travaux déjà faits ou entrepris, mais qui sont encore peu connus, retiendront mon attention, et aussi certains travaux à faire, dont vous vous doutez bien qu'ils sont nombreux. À la fin, je serai amené à déborder sur le secteur des institutions, ainsi que sur celui de la théorie et de l'histoire des idées, ce qui montrera, si besoin en est, que le développement dans un secteur des sciences politiques ne pourra que mettre en question, puis en branle, d'autres secteurs.

137 Jean-Charles Bonenfant, Les institutions politiques canadiennes, Québec, Les Presses Universitaires Laval, 1954, 204 p. 
Comme vient de le dire $\mathrm{M}$. Bonenfant, nous avons un peu pratiqué la sociologie électorale. En fait, nous commençons de la pratiquer. À côté de l'étude de Jean et de Marcel Hamelin et de Jacques Letarte, il faut mettre les thèses inédites de Paul Cliché et de Harold Angell, restreintes la première à la période 1927-1956, la seconde aux élections provinciales des années vingt, qui, par le débrouillage qu'elles ont accompli devraient permettre des travaux plus poussés. Au niveau des circonscriptions électorales, nous possédons aussi quelques essais de mise en ordre. La première étude a porté sur la circonscription de Lévis, une autre porte actuellement sur celle de Chicoutimi, et des calculs statistiques sont disponibles sur les circonscriptions de Beauce, de Portneuf et de Saint-Maurice. Toutefois, il n'existe pas, à ma connaissance, de travaux comparables sur les élections fédérales. Quant aux quelques recherches entreprises sur les élections et référendums municipaux, elles ont surtout montré des antagonismes internes aux municipalités : antagonismes entre quartiers à Sherbrooke, antagonismes entre vieux résidents et « immigrés » à Saint-Romuald.

Des premiers travaux portant sur les circonscriptions provinciales, certaines constantes se dégagent. Ainsi, les traditions électorales des localités composant une circonscription sont très diverses, mais les variations du vote d'une élection à l'autre sont beaucoup moins originales : si, par exemple, l'Union Nationale en reprend dans l'ensemble de la circonscription, elle en reprend aussi généralement dans à peu près toutes les localités, bien loin que de forts gains dans les localités «bleues » viennent effacer de moins fortes pertes dans les localités « rouges ». Un autre phénomène remarquable, qui se produit fréquemment dans Portneuf, est l'opposition dans une même localité entre le village et la paroisse, entendez par celle-ci le milieu plus proprement rural. C'est ainsi qu'une forte majorité « rouge » dans le village peut s'accompagner d'une non moins forte majorité «bleue » dans la paroisse, et vice versa. Je signalerai enfin un autre phénomène assez étonnant, que j'ai remarqué dans Lévis, phénomène d'ailleurs disparu : soit l'appui quasi unanime que donnaient des petites localités à l'un ou l'autre parti.

[84]

Donc, au niveau de la province et des circonscriptions, l'enquête est commencée. Au niveau des individus aussi, où le rapport du Groupe de recherches sociales, sur les opinions et attitudes des électeurs 
québécois à la veille des élections de 1960, a proposé de premières hypothèses. À Montréal, des étudiants de science politique ont entrepris une recherche sur le militantisme politique de certains partisans libéraux. Mais entre les deux niveaux, c'est l'inconnu. Je suis pourtant convaincu, confirmé en cela par la lecture des meilleurs travaux français, anglais et américains, que c'est au niveau des localités, des petites collectivités politiques, qu'il y a le mieux à faire.

Des phénomènes politiques municipaux ont été étudiés à Beauport, à Lévis, à Sherbrooke, mais que savons-nous, par exemple, des connexions entre affiliations politiques et affiliations de parenté ? Pas beaucoup plus que ce qui se trouve dans Miner. Sur cette question précise, tout est à faire, car si nos sociologues ont beaucoup discuté de la famille canadienne-française, ils ne nous ont donné que quelques études superficielles des réseaux de parenté et des attitudes qui les sous-tendent. Personne n'a suffisamment montré comment la parenté chez nous s'est instituée contre la société politique et sa coordination propre. Nous ne savons scientifiquement rien de l'organisation provinciale et locale de nos partis politiques, des techniques qu'ils emploient. Tout au plus un premier inventaire et une première interprétation de leur propagande ont-ils été tentés récemment par un étudiant.

Pourtant nous ne pourrons pas prétendre à une sociologie un peu rigoureuse du patronage politique, sociologie qui viendrait à son heure, tant que ces faits d'affiliation, d'organisation et de propagande partisanes n'auront pas été éclairés. Et comme c'est lors d'une campagne électorale que ces faits se manifestent le mieux, il faudrait aller les saisir à ce moment. Pourquoi ne pourrait-on pas considérer ces campagnes comme un composé de potlach et d'interrègne, une espèce de phénomène politique total où sont renouvelées les alliances et réévalués les prestiges, où de fortes sommes sont dépensées en quelques jours, où des prestations et contre-prestations sont échangées : votes contre promesses, quand ce n'est pas contre boissons fortes ou appareils de télévisions ? Lisez avec cette hypothèse en tète Les mœurs électorales dans le Québec de Jean et Marcel Hamelin, et vous verrez apparaître la dimension sociale du problème, trop souvent ignorée sous la dimension morale.

Des candidats à ces élections, des députés élus, que savons-nous? Voilà un autre champ de sociologie politique qui est bien peu développé et pas seulement chez nous. Pourtant une brève enquête, faite 
par un de mes étudiants, révèle un fait aussi étonnant que celui-ci : de 1867 à aujourd'hui, 10\% des députés fédéraux nouvellement élus dans l'Ontario ont moins de 35 ans, mais dans le Québec cette proportion est de 22\%. Pourquoi ? Dans une certaine mesure parce que les nouveaux députés libéraux sont en moyenne plus jeunes que les nouveaux députés conservateurs, et que ces nouveaux députés libéraux sont proportionnellement plus nombreux dans le Québec que dans l'Ontario ; mais, sans doute, d'autres facteurs jouent aussi. Voilà une piste pleine de promesses pour qui voudrait évaluer notre apport à la Confédération canadienne.

M. Bonenfant remarquait avec raison que nous n'avons rien produit en science de l'administration publique. Je signalerai toutefois qu'une enquête est en cours sur certaines caractéristiques de quelque 200 hauts fonctionnaires de la province de Québec, sans qu'on puisse encore voir ce qui [85] en sortira. Non seulement nous n'avons jamais étudié scientifiquement le problème du contrôle de l'acte administratif, mais l'histoire de nos différents ministères, leur organisation, leur fonctionnement n'ont pas encore tenté les chercheurs. C'est d'autant plus regrettable que ceux-ci disposent aujourd'hui d'une bien vivante sociologie de la bureaucratie, équipée des outils conceptuels fournis par les théories modernes de l'organisation, du pouvoir, de l'information et de la décision. On se doute bien que des expériences fort importantes et fort passionnantes se déroulent actuellement dans certains secteurs de l'administration provinciale, mais il ne se trouve personne pour les observer scientifiquement. Vous me direz qu'on ne permettrait pas à des intrus de faire l'étude de tout cela, mais a-t-on seulement tenté l'aventure?

Nous sommes encore plus pauvres, si c'est possible, en études sur les groupes de pression, alors qu'aux États-Unis, en France et en Angleterre ces études sont de plus en plus nombreuses. Dans ce domaine aussi l'observation n'est pas facile, mais la difficulté devrait être davantage un stimulant qu'un empêchement. Nous continuons de penser notre système politique en termes de pouvoir exécutif, de pouvoir législatif, et de pouvoir judiciaire, et dans ce schéma la bureaucratie, les groupes de pression, les opinions publiques s'intègrent mal ou pas du tout. De même, comme l'indiquait $\mathrm{M}$. Bonenfant, nous continuons de penser le fédéralisme canadien en des termes juridiques qui recouvrent mal ou pas du tout les phénomènes sociaux, économiques et 
même politiques qui se produisent réellement. Nous ne serons sauvés de ces mauvaises habitudes, qui se traduisent parfois en mauvaise politique, que par des théories nouvelles, partielles ou globales, qui rendront compte de tous les faits visés, en les expliquant les uns par les autres.

C'est aussi à un traitement par des méthodes nouvelles d'analyse du contenu qu'il faudrait soumettre nos idéologies politiques et leurs différents avatars, les considérant comme des mythes propres surtout à réduire des contradictions inscrites dans nos structures sociales. Mais une telle approche présuppose une connaissance très poussée des structures sociales et de la culture, aussi bien matérielle qu'immatérielle : elle dépend donc des progrès qui seront faits dans d'autres domaines.

Au terme de ce commentaire je n'ajouterai rien aux pertinentes remarques de $\mathrm{M}$. Bonenfant sur la stratégie de nos recherches. Je crois avec lui qu'un certain dirigisme est nécessaire, et qu'il faut, pour une plus grande richesse, concilier en nous les apports américain, anglais et français. J'ajouterai seulement que, déjà, des départs peuvent être pris, en séminaire, autour d'un professeur. J'en ai fait une première expérience, durant le semestre qui s'achève. Une quinzaine d'étudiants sont allés en éclaireurs dans diverses directions et ils ont rapporté de riches découvertes, dont quelques-unes furent signalées ici. Ces premières explorations ouvrent la voie à des enquêtes mieux orientées où nos quelques chercheurs pourront poser aux faits des questions plus précises.

Souhaitons enfin que ces quelques chercheurs se multiplient, non pas pour établir l'hégémonie de nos disciplines sur les autres, mais pour que notre contribution, qui est de montrer comment sont coordonnées les relations sociétales, ait sa place dans la connaissance plus exacte que nous aurons demain du Canada français.

Vincent Lemieux

Département de science politique, Université Laval. 
[87]

Situation de la recherche sur le Canada français

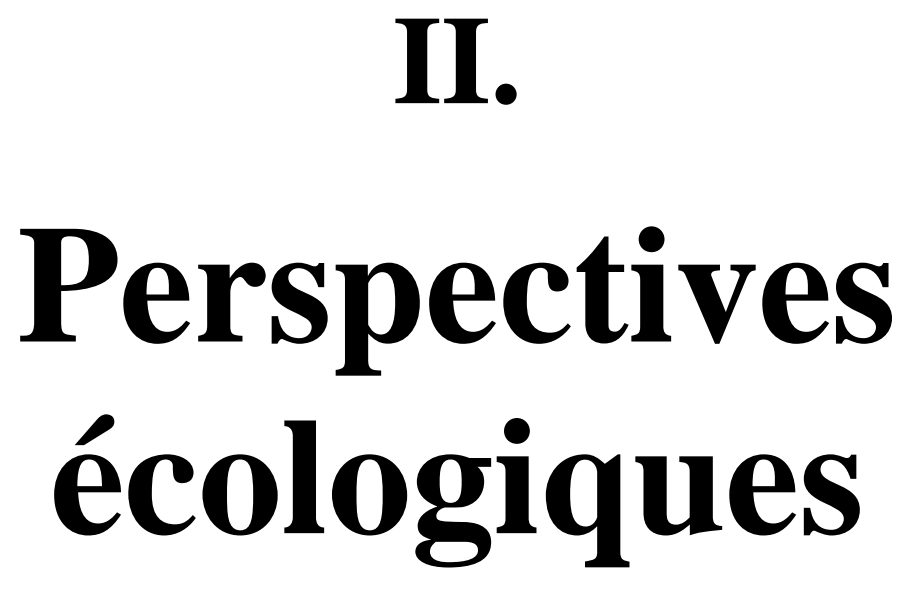

$\underline{\text { Retour à la table des matières }}$

[88] 
[89]

\title{
Situation de la recherche sur le Canada français \\ II. Perspectives écologiques
}

\author{
Fernand Grenier \\ Institut de géographie, \\ Université Laval

\section{"L'état présent des études régionales \\ sur le Québec”. *}

\author{
[pp. 89-101.]
}

Retour à la table des matières

Pourquoi les responsables de l'organisation de ce colloque sur «L'état présent des recherches sur le Canada français » ont-ils demandé à un géographe de dresser le bilan des études régionales effectuées jusqu'ici sur le Québec et d'indiquer ce que peuvent être, selon lui, les perspectives de la recherche dans ce domaine ? Vous me permettrez de signaler quelques raisons qui ont pu, consciemment ou non, guider les responsables du colloque.

Disons d'abord qu'il faut peut-être donner à cette invitation une signification symbolique. Discrètement, nos collègues sociologues auraient-ils voulu nous rappeler que nous aussi, géographes, nous prati-

* Étude préparée avec la collaboration de Pierre Cazalis, professeur de géographie à l'Université de Sherbrooke. 
quons une discipline qui, par certains côtés, est bel et bien, ou devrait être, une science " sociale » qui pourrait normalement apporter une contribution de plus en plus importante à la connaissance du Canada français, des groupes humains qui l'habitent et des régions qui le composent ? C'est fort possible. S'il est encore trop tôt pour régler définitivement les rapports qui doivent exister entre la géographie et les sciences de l'homme, il était, en tous les cas, grand temps que nous nous trouvions tous ensemble à discuter de nos problèmes communs de description, de recherche et d'analyse du contexte québécois. L'organisation de ce colloque fait honneur à l'équipe de la revue Recherches sociographiques et il me plaît de le reconnaître avec gratitude.

En me demandant cet exposé sur les études régionales, peut-être mes collègues ont-ils voulu tenir compte de cette prétention souvent affirmée par des géographes, mais, je dois dire, rarement admise par les autres, prétention suivant laquelle les études régionales constituent la chasse-gardée des géographes et, à la limite, disent certains, la justification ultime de la position de leur discipline à mi-chemin entre les sciences naturelles et les sciences de l'homme ? Est-il besoin d'ajouter que cette position n'est pas toujours très confortable ni très enviable ? Pour ma part, en tous les cas, je ne la recommande à personne et j'estime que les géographes ne se trompent pas trop quand ils considèrent la région comme le cadre privilégié de [90] leurs recherches et quand ils cherchent à améliorer les méthodes susceptibles de faire avancer les études régionales.

Nos collègues des Recherches sociographiques ont donc simplement voulu reconnaître, je crois, l'importance quantitative des efforts déployés jusqu'ici par les géographes en vue de l'établissement d'une première description régionale du Québec. Il me reste le redoutable honneur d'ébaucher un jugement d'ensemble sur les travaux de mes collègues et sur les miens, ce que je vais tenter de faire avec un très grand souci d'objectivité. 


\section{Nature de l'étude régionale}

Une première difficulté se présente immédiatement que nous ne saurions éliminer. Qu'est-ce au juste qu'une étude régionale? Dans quel sens faut-il parler ici des « études régionales »?

Il est évident que presque toutes les recherches en sciences sociales et naturelles s'appuient sur une portion plus ou moins étendue de l'espace terrestre où sont observés les phénomènes. Cela ne signifie sûrement pas que toutes ces recherches constituent des études « régionales ».

On pourrait peut-être appeler « régionales » les études générales, physiques ou humaines qui, après l'exposé d'une théorie d'ensemble, s'appliquent à situer et à délimiter des aires de répartition de phénomènes identiques par leur nature mais présentant, par exemple, des inégalités de densité dans leur distribution géographique. C'est ainsi que l'on peut définir des régions géologiques, pédologiques, climatiques ou végétales aussi bien que des régions de manufactures, de phénomènes démographiques ou de décès attribuables à la tuberculose, par exemple. À notre avis, ces études se rattachent à la géographie générale et non à la géographie régionale. Il ne faut pas négliger pour autant ces études générales qui peuvent avoir une très grande importance lorsqu'on veut aborder l'étude régionale proprement dite, et il est évident qu'il faut souhaiter leur développement.

On sait que la tradition des études régionales a été inaugurée, en France du moins, par Vidal de la Blache, puis illustrée depuis le début du $\mathrm{XX}^{\mathrm{e}}$ siècle par Demangeon et Blanchard et, plus récemment, par des géographes tels que Julliard, de Strasbourg. Il serait utile, mais un peu long, d'exposer tous les cheminements suivis par les géographes pour arriver à fixer, d'une façon qui est encore très discutée, la notion de région. Contentons-nous de signaler que les géographes, américains aussi bien qu'européens, reconnaissent maintenant deux types fondamentaux de régions. Dans les deux cas, il s'agit d'un cadre territorial pourvu d'une certaine unité, ou, si l'on veut, d'un espace organisé, structuré d'une façon originale qui permet de le distinguer d'un ensemble spatial voisin. L'unité spécifique d'une région peut provenir de 
deux séries de faits, soit: $1^{\circ}$ de l'homogénéité d'un [91] éventail de facteurs, qui ne sont pas forcément les mêmes d'ailleurs d'une région à l'autre ; soit : $2^{\circ}$ de la cohérence des facteurs propres à la région, cohérence qui peut être attribuable, par exemple, au rôle intégrateur d'une ville ou d'une métropole régionale. On parle ainsi couramment maintenant soit de régions homogènes soit de régions nodales (ou polarisées, ou cohérentes). Il est bien inutile de prolonger cet exposé théorique car, force nous est de constater, ainsi que nous le verrons tout à l'heure, que presque toutes les études régionales faites jusqu'ici dans le Québec se situent en marge de ces préoccupations méthodologiques. C'est là sûrement une explication générale, si l'on veut, mais fort valable de la faiblesse des études régionales sur le Québec.

Tout le temps consacré par certains géographes du Québec à définir des régions soi-disant économiques, à partir, d'ailleurs, de critères qui n'étaient ni économiques ni vraiment géographiques, eût été mieux utilisé, croyons-nous, à poser plus vigoureusement le cadre méthodologique de la définition puis de l'analyse des structures géographiques régionales.

Dans le sens le plus strict, les études régionales faites jusqu'ici sur le Québec engloberaient à peine une dizaine d'ouvrages, y compris la "somme » de Raoul Blanchard dont notre collègue Louis-Edmond Hamelin a d'ailleurs montré qu'elle se rattachait peut-être plus à la géographie générale qu'à la géographie régionale. Il faut donc, aux fins de cet exposé, étendre le qualificatif « régional » et utiliser comme matériel de base la plupart des études d'envergure élaborées dans une « perspective » régionale.

Ces études pourraient se classer en quatre catégories :

— études de géographie physique régionale,

- études de géographie régionale se rapprochant du type le plus pur,

- études régionales du peuplement et de la population,

— études économiques régionales. 
Nous n'avons retenu, de fait, que les études physiques présentant quelque importance pour la compréhension des faits humains des zones envisagées.

Il va sans dire, en outre, que la rigueur de ces cadres est toute relative et que ceux-ci n'ont pour but que d'apporter un peu de clarté à l'analyse qui suit ; il est des titres, en effet, que l'on pourrait ranger dans l'une ou l'autre de ces catégories.

\section{Bref inventaire des études régionales sur Le Québec}

Nous reprenons, pour la clarté de l'inventaire, les quatre classes mentionnées plus haut.

[92]

$1^{\circ} \quad$ Études essentiellement physiques :

Thèse de doctorat 1

Thèses de maîtrise $\quad 8$

Articles de revues $\quad 13$

Rapports divers $\quad \underline{4}$

26 au total, soit $24 \%$ de l'ensemble.

$2^{\circ} \quad$ Études de géographie régionale "pure " :

Thèses de doctorat

4 (présentant plus d'intérêt que ce

Thèses de maîtrise qui suit)

Ouvrages et manuels

7

Articles et rapports

6

$\underline{18}$

35 au total, soit 31\% de l'ensemble. 
$3^{\circ} \quad E ́ t u d e s$ régionales de peuplement et population :

Thèse de doctorat 1

Thèses de maîtrise 8

Articles de revues $\underline{3}$

12 au total, ou $10 \%$ de l'ensemble.

$4^{\circ} \quad$ Études régionales économiques :

Thèses de doctorat 3

Thèses de maîtrise 10

Articles de revues et rap- $\quad \underline{25}$ ports géologiques d'intérêt économique

38 au total, ou 35\% de l'ensemble.

Notre analyse s'appuie donc sur 111 titres ainsi répartis :

\begin{tabular}{|l|r|c|c|}
\hline Nature des études & Nombre & Consultées & Lues dans le détail \\
Thèses de doctorat & 9 & 6 & 3 \\
Thèses de maîtrise & 33 & 14 & 3 \\
Ouvrages et manuels & 6 & & 6 \\
Articles et rapports & 63 & 26 & 37 \\
\cline { 2 - 4 } & 111 & 46 & 65 \\
\hline
\end{tabular}




\section{Les zones étudiées du Québec}

Les ouvrages généraux et Les manuels s'attachent essentiellement à l'étude des zones peuplées du Québec ; ils n'enrichissent nullement les connaissances sur les zones situées au delà du $50^{\mathrm{e}}$ degré Nord. À l'exception [93] des monumentales Etudes canadiennes de Blanchard, ces ouvrages ou manuels (Putnam, Robinson et autres, Taylor, Beston...) n'apportent que de fort rapides études descriptives des éléments géographiques classiques : structure du relief, sols et végétation, population, activités économiques... Nous en tirons une connaissance globale du Québec méridional.

Par leur nature même, les articles ne couvrent que des aspects limités et procèdent à l'analyse de facteurs géographiques limités eux aussi : activités minières, utilisation des sols, etc. Certains, embrassant en une vingtaine de pages des zones assez vastes, n'en livrent qu'une analyse superficielle, condensée ; d'autres ne prétendent qu'à faire œuvre vulgarisatrice dans d'agréables présentations de zones pittoresques: Gaspésie, Île-aux-Coudres (M. Barbeau). Rares sont les articles de géographie régionale typique : la plupart, en dépit souvent de leur titre, se limitent à un facteur, et rejoignent ainsi la catégorie des articles à objet spécifique dont il a été question au début : ils couvrent ensemble des lambeaux de territoire fort disséminés.

Les thèses présentent incontestablement plus d'intérêt. Leurs auteurs portent en général un grand intérêt aux vallées des principaux cours d'eau du Québec : Richelieu, Ottawa, Saguenay, Saint-Laurent... Il est vrai que dans la plupart des cas le problème des Limites de la région choisie ne se pose guère ; est-ce pour la même raison que les îles du Québec : Îles-de-la-Madeleine, Île d'Orléans, Île-aux-Coudres, Île de Montréal, Île Bizard, entre autres, sont fort en faveur auprès des étudiants en quête de sujet de thèse ?

Peu nombreuses sont les régions qui n'ont fait l'objet d'aucune étude, dans la partie peuplée du Québec tout au moins ; mais certaines sont privilégiées, celles particulièrement qui entourent les grands centres : Québec, Montréal surtout ; d'autres, telles le sud-est du Québec ne sont qu'effleurées ; la Côte Nord n'intéresse le géographe que de- 
puis fort peu de temps. La plupart, toutefois, ne sont connues que dans le cadre d'études qui les survolent (même lorsqu'elles ont connu les faveurs des Études canadiennes de Blanchard, parfois) ou qui n'en retiennent qu'un aspect (géologie, morphologie, climat, population, mines...). Quelques études d'ensemble sont, enfin, d'une qualité si douteuse qu'il serait abusif de les retenir.

Précisons d'ailleurs les caractères de l'ensemble de ces études fort diverses.

\section{La nature des études}

L'inventaire des études régionales révèle, en gros, une double orientation dans les recherches :

- une première orientation vers les études de géographie physique, climatique et morphologique plus particulièrement, orientation très nette chez les chercheurs de langue anglaise (il est à noter qu'un bon nombre [94] d'études géographiques régionales ont été présentées comme thèses à McGill ou dans les universités américaines) ;

- une seconde orientation vers des recherches de géographes très spécifiques : géographie économique (recherches sur les transports, le tourisme, les ensembles industriels...), géographie de la population et du peuplement (géographie historique des auteurs de langue anglaise).

On constate aisément, ainsi qu'il a été dit au début, que les auteurs s'aventurent fort peu dans le domaine complexe des analyses de type vraiment régional.

\section{Le contenu des études}

Un plan de recherches ne saurait être immuable, identique dans toutes les études, de quelque nature qu'elles soient; ce plan doit s'adapter à la région étudiée et à la nature de l'étude, traduire, en les mettant nettement en évidence, les caractères majeurs du lieu. 
Or, il est fort étonnant de retrouver dans chacune des études analysées - et dans les thèses surtout — un plan marmoréen semblant faire fi des problèmes et des caractéristiques locaux :

— situation générale de la région,

— structure et géologie générale,

— relief,

— sols,

— végétation,

— peuplement et habitat,

— activités économiques...

Il arrive même que l'on découvre deux thèses de nature différente (géographie physique générale et géographie de la population par exemple) construites sur le même plan ; seule, la longueur des chapitres diffère.

On voit immédiatement les conséquences de cette anomalie : la région n'est pas « comprise »; on n'en discerne pas la personnalité.

Il serait abusif, toutefois, de condamner tout ce qui a été écrit jusqu'à maintenant : le défaut relevé ici ne concerne pas toutes les études ; lors même qu'il se manifeste, il n'entache pas complètement les résultats d'une recherche qui peut offrir plusieurs autres points d'intérêt : toutes les thèses que nous avons analysées fournissent d'abondants et précis éléments descriptifs fondamentaux pour la connaissance de toute région :

— éléments géologiques, puisés en général dans d'excellents rapports géologiques des ministères des Mines ;

- phénomènes morphologiques (que les auteurs ne distinguent parfois que lorsqu'ils ont été signalés par Blanchard) ;

— éléments pédologiques (que l'on tire souvent de rapports ministériels) ; 
[95]

— éléments démographiques, dont on fait d'habiles synthèses à partir des documents habituels : recensements du Canada, archives paroissiales ; il semble d'ailleurs que les chercheurs québécois aient des dispositions et des affinités particulières pour les études de population. Serait-ce parce que, de fait, elles sont les moins difficiles ?

— éléments économiques divers : retenons ici les structures industrielles et leur évolution, les structures agraires, les transports...

\section{Les méthodes}

Il y a sur ce plan beaucoup à contester.

En gros, l'analyse scientifique peut se décomposer en trois étapes : description, explication, preuve.

Or, bien peu d'études géographiques dépassent le stade de la description ; les quatre-cinquièmes des œuvres dépouillées sont infimes : deux membres fondamentaux du processus scientifique leur font défaut ; une description complète et parfaite ne remplace jamais les deux démarches suivantes, qui lui sont intimement liées.

La lacune a des conséquences fondamentales : elle ôte à l'analyse toute valeur scientifique. Quelles sont ses causes ? Il semble qu'il faille les chercher au niveau de la formation des chercheurs.

\section{Les auteurs}

Nous parlerons surtout des auteurs de thèses, des jeunes chercheurs. La lecture des thèses révèle deux sortes de défauts dans la formation des étudiants :

- ceux-ci, en premier lieu, méconnaissent les principes de base de la science expérimentale ; nous venons de voir que, limitant, en fait, leur recherche à une description, ils se condamnent à une œuvre incomplète, de niveau pré-scientifique ; 
- mais cette lacune n'est-elle pas le fait de faiblesses dans leur formation pratique ? Il paraît incontestable maintes fois que l'étudiant ne peut " pousser » une explication pourtant assez aisée en raison de l'insuffisance de ses connaissances en géographie générale.

Auteurs jeunes, donc ; auteurs peu préparés à la recherche, aussi. Il n'y a d'ailleurs pas lieu de s'étonner de ce phénomène : certains travaux ne reposent-ils pas sur un maximum de deux années d'études supérieures ? C'est nettement insuffisant.

\section{Orientation pour de futures recherches}

1. Il conviendrait, bien sûr, d'accorder quelque attention à des zones délaissées depuis longtemps, ou plus exactement jamais abordées.

[96]

Les zones de peuplement ancien ont été jusqu'ici les plus choyées, et c'est normal : plaine de Montréal, vallée du Richelieu, Québec.

De nombreux impératifs économiques, sociologiques ou purement géographiques invitent aujourd'hui à se tourner vers des zones "stagnantes » au peuplement ancien, Haute-Chaudière et Est des Cantons de l'Est, ensemble des Hautes-Terres du Saint-Laurent, Bas SaintLaurent, Saint-Maurice, Charlevoix ... ou vers des zones au peuplement récent mais au développement fort déséquilibré : Abitibi, Saguenay, Côte Nord (qui commence, d'ailleurs, à attirer les chercheurs). L'ensemble du « Nord » québécois devrait aussi naturellement attirer les chercheurs.

2. Il conviendrait surtout de définir une philosophie ou, au moins, de fixer une fin aux recherches. Les perspectives régionales traditionnelles nous semblent sinon dépassées, du moins stériles (ou stérilisantes dans le contexte québécois actuel).

L'aménagement du territoire offrirait peut-être à la recherche géographique future ces fins dont il a été question plus haut, l'orientant vers la formulation de problématiques régionales dont l'absence explique aujourd'hui, dans de nombreux cas, la banalité des résultats. 


\section{Quelques conditions de cette orientation nouvelle}

Ces conditions sont multiples :

- il s'agit, bien sûr, de favoriser matériellement de telles recherches, d'apporter à l'étudiant et à tout autre chercheur l'aide technique et financière qui lui permettra de mener à bien les études dans les zones « stagnantes » ou " déséquilibrées » dont nous parlions plus haut ; une telle assistance est d'autant plus utile que ces recherches porteront sur des zones éloignées des centres universitaires ;

— mais la condition la plus impérative nous semble être l'amélioration de la formation académique générale des chercheurs : multiplier dans certains cas les années d'études (trois sont un minimum après le diplôme de fin d'études du secondaire : B. A. ou autre) ; apporter une formation épistémologique à des gens qui doivent connaître les exigences théoriques (mentales, morales, techniques...) de la recherche avant de l'aborder.

Soyons toutefois réalistes : la formation d'une génération de chercheurs est œuvre de longue haleine ; elle commence dès les débuts mêmes de la vie scolaire du jeune ; peut-être faudra-t-il attendre l'avènement d'un enseignement homogène à tous les niveaux (primaire, secondaire, technique) pour voir arriver à l'Université des promotions entières de jeunes aptes à la recherche, capables d'absorber et d'assimiler un enseignement à cette fin. Orienter nos efforts vers la formation de professeurs de géographie au secondaire nous paraît la condition première d'un développement futur, quantitatif et qualificatif, de la recherche géographique. 


\section{CONCLUSIONS}

Me permettra-t-on, au terme de cet exposé, d'ajouter quelques remarques qui élargiront peut-être un peu le cadre de cet examen de conscience qui, jusqu'ici, a surtout mis en cause les géographes et leurs travaux?

Au cours des trois ou quatre dernières années, certaines recherches régionales ont été entreprises, non plus par des individus isolés, mais par des équipes relativement bien nanties sur le plan financier aussi bien que sur le plan du personnel.

C'est ainsi qu'il faudrait signaler l'enquête économique sur la région du Bas Saint-Laurent dont Yves Dubé, directeur du Département d'économique à la Faculté des sciences sociales de Laval, a assuré la coordination générale. La population, les sols, les transports et l'économie de la région ont formé les grandes rubriques du travail. Voici ce qu'écrivait monsieur Dubé dans les Recherches sociographiqu.es (1960, $n^{\circ} 2$, p. 223) au sujet de cette enquête :

«Il existe dans la province de Québec beaucoup de monographies locales et même régionales. Dans ces travaux, on trouve cependant très peu d'analyses. Le travail que nous avons entrepris constitue un essai dans ce sens. Nous espérons qu'il sera mené à bien, qu'il sera poursuivi dans d'autres directions et même imité dans d'autres régions. On n'en arrivera pas autrement à un aménagement rationnel de notre territoire. »

Je suis personnellement tout à fait d'accord avec ce qu'écrivait monsieur Dubé et je me permets d'insister sur l'urgence des études régionales détaillées qui constituent une pièce de base dans l'élaboration des politiques régionales.

En plus de cette étude sur le Bas Saint-Laurent, il y aurait lieu de signaler les travaux présentement en cours aux services de recherches du Ministère de l'Industrie et du Commerce sur la région de la Mauri- 
cie. Nous attendons avec impatience et intérêt les premiers résultats de ces recherches.

Ces études régionales d'envergure mobilisent un personnel nombreux, qui travaille pendant de longs mois et souvent de longues années avant qu'on soit en mesure de publier des premiers résultats. Ceci, bien entendu, doit nous inviter à un peu d'indulgence à l'égard de ces malheureux géographes qui avaient essayé, jusqu'ici, d'entreprendre généralement seuls et sans moyens financiers adéquats de difficiles études régionales.

Résumons-nous. La recherche régionale se heurte présentement à l'absence cruelle de certains matériaux de base parmi lesquels il convient de signaler les cartes géologiques, les cartes pédologiques, les données climatiques, les données démographiques (notamment celles qui permettraient d'étudier adéquatement les migrations régionales), les archives inorganisées, la pauvreté des bibliothèques, des cartothèques et des photothèques, etc. Les conditions les plus fondamentales de la recherche font cruellement défaut dans tous les secteurs précédemment énumérés.

[98]

Ajoutons que la recherche régionale n'a pas eu grand-chose à tirer jusqu'ici des résultats obtenus par certaines disciplines connexes, telles que l'histoire économique et je dirais même l'histoire tout court, la sociologie (bien que, dans ce domaine, certains travaux récents aient une importance capitale), la démographie, l'analyse économique régionale, etc. Il en est de même de la géologie qui, jusqu'ici, s'est à peu près uniquement intéressée aux gîtes miniers ; de la climatologie, qui est une science pratiquement inexistante chez nous ; de la cartographie où nous en sommes encore aux premiers balbutiements. Et je pourrais, bien entendu, allonger cette liste en signalant, entre autres, la pédologie.

Faut-il ajouter que la régionalisation de notre territoire n'a évidemment pas atteint le même degré partout ? Que les géographes doivent courir le risque de l'excommunication de la part des historiens locaux s'ils osent toucher à des limites régionales plus ou moins mythiques. On connaît le cas de la région dite du Saguenay qui, suivant certains qui se fondent sur des textes remontant à Jacques Cartier mêmement, comprendrait presque toute la côte nord du Saint-Laurent 
et des espaces considérables des territoires du Nouveau-Québec. Enfin, faut-il signaler qu'une conception un peu rigoureuse du concept de région nous amènerait peut-être à considérer l'ensemble du Québec comme une seule et unique région polarisée autour de l'agglomération montréalaise ? Sur le plan méthodologique, cette constatation aurait une importance capitale puisqu'elle suggérerait l'existence dans le reste du territoire simplement de sous-régions dont l'étude et l'analyse devraient éclairer à la fois l'intégration en fonction du pôle principal, Montréal, et les interrelations de type orbital entre les sous-régions. Il faudrait tout un colloque comme celui-ci pour discuter de ce seul problème.

Je vous laisse à ces réflexions qui expliquent, je crois, amplement la maigreur du bilan que j'ai présenté aussi bien que les raisons profondes qui justifient la difficulté des études régionales.

Jusqu'ici les chercheurs « régionaux » n'ont pas eu d'autres motivations que des motivations abstraitement scientifiques et personnelles. Il est grand temps, me semble-t-il, que la région soit reçue dans notre milieu comme une réalité politiquement valable. La recherche scientifique, qu'il faudra bien songer à organiser un jour en y incluant les sciences de l'homme, trouvera dans les études régionales un champ d'élection pour ses efforts et ses octrois. La politique, avant de s'engager trop dans l'improvisation des politiques régionales, devra prendre le temps de consulter et de réfléchir sur la région, cadre fondamental de la structuration de notre société, comme d'ailleurs de toute société.

Fernand Grenier

Institut de géographie, Université Laval. 


\section{NOTES BIBLIOGRAPHIQUES}

La documentation sur les études régionales est extrêmement dispersée et il est impossible de reproduire ici une bibliographie exhaustive, en cours de préparation toutefois et qui sera publiée sous peu. Nous signalons donc un choix d'ouvrages dont l'étude a justifié l'article qu'on vient de lire et qui permettent de poser au moins les problèmes de la géographie régionale du Québec, sinon ceux de tous les autres types d'études régionales.

\section{A. Ouvrages manuscrits (thèses)}

La source essentielle est constituée par les thèses de géographie préparées dans les universités : McGill, Montréal et Laval (Québec). Ces thèses ont été rarement publiées au complet, certains auteurs en ayant toutefois tiré quelques articles. Aussi faut-il consulter les exemplaires dactylographiés (quand ils existent !). Nous en signalons quelques-unes parmi celles qui utilisent le plus les méthodes de la géographie régionale.

Tous les ouvrages cités sont dactylographiés. Nous utilisons les abréviations suivantes : $\mathrm{D}$, pour une thèse de doctorat ; DES, pour une thèse de diplôme d'études supérieures ; $M$, pour une thèse de maîtrise. Chaque thèse comporte cartes, graphiques, tableaux et, en général, bibliographie.

BANKs, M. B., The Isle of Orléans : A Study of Influence of a River Island on the Life of People, M, Worcester, Clarke University, 1944.

Beauregard, Ludger, La vallée du Richelieu, D, Montréal, 1957, $349 \mathrm{p}$.

Bellabon, M. B., A Régional Study of the Richelieu Valley, M, McGill, 1952. 
Bertrand, Lucien, La région de Donnacona, M, Laval, 1960, 109 p.

FALAISE, Noël, Les Îles-de-la-Madeleine. Étude géographique, D, Montréal, 1954, 217 p. GLendinNing, Robert M., The Lake SaintJean Lowland, D, Michigan, 1933, 154 p.

GrenIER, Fernand, La Beauce. Étude de géographie régionale, DES, Paris, 1955, 224 p.

HÉROUX, R. P. Valbert, o.f.m.. Le bassin de la Sainte-Anne-deBeaupré. Étude de géographie régionale, M, Laval, 1962, 135 p.

MacKay, J. Ross, The Régional Geography qf the Lower Ottawa Valley, D, Montréal, 1949, 405 p.

PÉPIN, Pierre-Yves, La région du rebord sud de l'estuaire, DES, Montréal, 1958, 200 p.

PERRON, Jean-Roch, La basse vallée de la rivière du Gouffre, DES, Laval, 1959, 174 p.

Pochopien, K. M., The District of Brome, M, McGill, 1952, 155 p.

UREN, Philip E., The Historical Geography of the St. Maurice Valley, M, McGill, 1950, 133 p.

Signalons qu'on trouve aussi un grand nombre de thèses d'intérêt régional, mais limitées soit aux aspects physiques (géomorphologie, climat), soit à certains aspects humains ou économiques (démographie, tourisme, ressources, etc.).

\section{B. Problèmes de méthodes et régionalisation du Québec}

Sur ces questions fondamentales, la bibliographie est très maigre. L'article de Benoît BROUILLETTE, "Les régions géographiques et économiques de la province de Québec » (dans les Cahiers de géographie de Québec, n 6 : Mélanges Blanchard, avril-sept. 1959, 65-83) pose le problème des limites des régions en fonction principalement de l'utilisation des statistiques. Depuis quelques années, de nombreux rapports ont été préparés dans les cadres de l'administration provincia- 
le par divers experts. Malheureusement, ces travaux ne sont pas connus en dehors des milieux du Gouvernement.

\section{Enquêtes et travaux d'équipe}

Depuis une dizaine d'années, des enquêtes ont été entreprises sur diverses régions de la province par des équipes relevant du gouvernement de la province, de comités régionaux [100] d'expansion économique, d'autorités diocésaines et des universités. La plupart du temps, ces enquêtes ne sont pas encore terminées et certains rapports sont en cours de publication. Nous mentionnons plus bas quelques-uns des travaux publiés (rubriques « $\mathrm{E} »$ et « $\mathrm{F} »$ ).

\section{Périodiques}

Un grand nombre d'études régionales ont été publiées jusqu'ici dans les revues et notamment dans les suivantes :

L'Actualité économique (Montréal, École des Hautes études commerciales) ;

Cahiers de géographie de Québec (Québec, Institut de géographie, Université Laval) ;

Le Géographe canadien - Canadian Geographer (Ottawa et Toronto, Association canadienne des géographes);

Recherches sociographiques (Québec, Département de sociologie et d'anthropologie, Faculté des sciences sociales, Université Laval) ;

Revue canadienne de géographie (Montréal, la Société de géographie de Montréal et l'Institut de géographie de l'Université de Montréal). 


\section{E. Bibliographie}

Les périodiques cités en « $\mathrm{D}$ » publient régulièrement des comptes rendus et des notices bibliographiques. On trouve des énumérations plus systématiques dans la Bibliographie choisie d'ouvrages de géographie canadienne publiée chaque année par la Direction de la géographie. Ministère des Mines et des Relevés techniques, Ottawa. Ce répertoire, commode mais incomplet, peut s'amplifier par la consultation des listes bibliographiques de la revue trimestrielle Culture, publiée à Québec par les Pères Franciscains.

\section{F. Sélection d'ouvrages et d'articles}

BLANCHARD, Raoul, L'ensemble de son œuvre canadienne dont on trouvera l'inventaire dans les Mélanges géographiques canadiens offerts à Raoul Blanchard (dans Cahiers de géographie de Québec, $\mathrm{n}^{\circ}$ 6, avril-sept. 1959, 35-45).

Brouillette, Benoît, "L'habitat et la population au Saguenay », L'Actualité économique, janvier 1947, 646-671.

Brouillette, Benoît, "La région des Appalaches », L'Actualité économique, 1941, 1-28. Broulllette, Benoît et Dagenais, Pierre,

«Quelques aspects de l'économie actuelle du Saguenay — Lac SaintJean », L'Actualité économique, janvier 1948, 654-691.

CAMU, Pierre et GRENIER, Fernand, (( La région du Lac-Saint-Jean et du Saguenay ", dans : Recueil de mémoires . . . Conférence sur les Ressources et notre avenir, Ottawa, 1961, tome 1, 493-500.

CAMu, Pierre, Problèmes des transports dans la région du Bas Saint-Laurent, [Rimouski], Le Conseil d'orientation économique du Bas Saint-Laurent, 1960, xi + 121 p. Miméo.

DAGENAIS, Pierre, «La région des Laurentides ", L'Actualité économique, 1941, 101-125.

DE Jocas, Yves, «L'étude des migrations dans l'analyse régionale », Recherches sociographiques, II, 2, avril-juin 1961, 245-251. 
DUBÉ, Yves, " L'enquête économique sur la région du Bas SaintLaurent », Recherches sociographiques, I, 2, avril-juin 1960, 220223.

Grenier, Fernand et Dorion, Henri, « Québec, région économique », Commerce, (Montréal)

février 1961, 55-71 et mars 1961, 51-69.

HAMELIN, Louis-Edmond, « La Beauce canadienne dans le Québec méridional », Cahiers de géographie de Québec, avril 1957, 207-211.

MARTin, Yves, Étude démographique de la région du Bas SaintLaurent, [Rimouski], Le

Conseil d'orientation économique du Bas Saint-Laurent, 1959, 128 p. Miméo.

[101]

MINGASSON, Christian, "Évolution récente de l'Ile d'Orléans », Cahiers de géographie de Québec, octobre 1956, 55-84.

PÉPIN, Pierre-Yves, «La symbiose progressive des régions Gaspésie - Rive Sud et Côte Nord », L'Actualité économique, janvier-mars 1960, 626-658.

PÉPIN, Pierre-Yves, La mise en valeur des ressources de la région Gaspésie - Rive Sud, Québec, Ministère de l'Industrie et du Commerce, Bureau de recherches économiques, 1962, 360 p.

PhlipponneAu, Michel, L'avenir économique et social des Cantons de l'Est. Un problème type de planification régionale de la province de Québec, Québec, Ministère de l'Industrie et du Commerce, Service de géographie, Publication $n^{\circ} 2,1960,219 \mathrm{p}$.

RAYNAUlD, André, Croissance et structure économiques de la province de Québec, Québec, Ministère de l'Industrie et du Commerce, [1961], 657 p. 


\title{
COMMENTAIRE
}

\author{
Pierre Harvey \\ École des Hautes Études Commerciales, \\ Montréal.
}

[pp. 102-104.]

$\underline{\text { Retour à la table des matières }}$

N'étant pas géographe, mes remarques sur la communication de M. Grenier devront forcément se borner à ceux des problèmes du " régionalisme » qui sont communs aux géographes et aux économistes. Il est alors évident que c'est surtout la méthodologie qui doit retenir notre attention.

Le problème «régional » a surgi dans la littérature économique avec la prise de conscience des phénomènes de localisation. Ce type de préoccupation n'a cependant d'abord concerné que la firme avant de s'étendre à la "région ». Sous une forme ou sous l'autre il est resté marginal. Et si on excepte le faible courant de pensée qui relie Thünen à Isard, la dimension spatiale de la vie économique a été à peu près totalement négligée par la théorie économique qui a alors abandonné tout ce domaine aux géographes. Ce qui explique que ceux des économistes qui se sont intéressés aux conditions réelles de fonctionnement de l'économie de la province ont toujours dû faire de lourds emprunts aux géographes. Ces économistes se sont alors trouvés impliqués dans les querelles d'école qu'a soulevées chez les géographes le découpage de la province en un certain nombre de « régions ». 
Sur ce point, M. Grenier rappelle quelles modifications profondes les géographes surtout ont fait subir au concept de "région », depuis Vidal de la Blache jusqu'aux toutes dernières analyses en termes de zones polarisées. Il admet que " presque toutes les études régionales faites jusqu'ici dans le Québec se situent en marge de ces préoccupations méthodologiques », ce qui expliquerait « la faiblesse des études régionales sur le Québec ». Ce qui l'amène à conclure que «tout le temps consacré par certains géographes du Québec à définir des régions soi-disant économiques (...) eût été mieux utilisé (...) à poser plus vigoureusement le cadre méthodologique de la définition puis de l'analyse des structures géographiques régionales ».

Dans une certaine mesure, la communication de M. Grenier illustre elle-même l'importance de cette réflexion méthodologique. Pour le montrer, je m'attacherai à un passage du texte de $\mathrm{M}$. Grenier, passage qui, d'ailleurs, touche de très près les intérêts des économistes. M. Grenier déclare, en effet : "Une conception un peu rigoureuse du concept de région nous amènerait peut-être à considérer l'ensemble du Québec comme une seule et unique région polarisée autour de l'agglomération montréalaise (...) Cette constatation (...) suggérerait l'existence dans le reste du territoire simplement de sous-régions (...)»

Ce que M. Grenier évoque alors, c'est, en fait, un modèle spatial d'analyse économique, modèle dans lequel la province de Québec dans sa totalité serait considérée comme une partie (région) d'un ensemble plus vaste. Par ailleurs, cette province (région) serait ellemême composée d'un groupe de "sous-régions » intégrées surtout par le pôle montréalais.

Pour l'économiste, une telle représentation de l'économie de la province est pleine de promesses. Sous l'angle du marché du travail, un modèle de ce type a déjà été utilisé à l'École des Hautes études commerciales, à diverses occasions depuis quelques années, et il sert actuellement de cadre à des travaux de recherche. Mais la formulation qu'en fait $\mathrm{M}$. Grenier révèle qu'on est encore loin d'avoir pleinement pris conscience des problèmes méthodologiques soulevés par l'approche régionale.

En décrivant les grandes lignes du modèle qu'il suggère, M. Grenier parle en effet d'une " conception rigoureuse » de la notion de région qui [103] "nous amènerait » à considérer la province comme 
une seule région. On peut douter d'abord qu'une conception quelconque puisse avoir ce caractère de nécessité. Si, en effet, on raisonne en termes d'homogénéité comme le suggère $\mathrm{M}$. Grenier, ce qui est d'ailleurs valable, on pourrait bien trouver commode pour certaines fins, de considérer par exemple comme une même " région », toute la frange du Bouclier canadien qui donne lieu à l'implantation de l'industrie de la pâte et du papier. Le cadre régional déborderait alors les frontières provinciales qui seraient elles-mêmes vides de sens, sous l'angle choisi. Et ce n'est là qu'un exemple entre plusieurs.

Il ne semble pas possible non plus qu'une quelconque « conception rigoureuse " nous force à ne considérer qu'une véritable région et des sous-régions. Quelle différence fera-t-on entre la région et la sousrégion? $\mathrm{Si}$, en effet, la province fait elle-même partie d'un tout plus vaste, ce qui est implicite dans la formulation du modèle, cet ensemble plus vaste pourrait, à un autre niveau, être considéré comme une région, ce qui ferait de la province une sous-région, selon les termes de $\mathrm{M}$. Grenier. C'est à partir d'hypothèses de ce genre, plus ou moins explicitées d'ailleurs, que certains économistes nient l'existence même de régions dans la province, au grand scandale des géographes les plus attachés à la lignée de Vidal de la Blache.

Mais ces derniers sont-ils seuls en cause ? Je ne crois pas : l'affirmation, par le géographe, de la possibilité d'une définition quasi nécessaire de la région n'est possible que dans une optique consciemment ou inconsciemment "vidalienne ", optique qui se profile en arrière-plan des énoncés de $M$. Grenier lorsqu'il parle d'une « définition rigoureuse » ou qu'il mentionne l'existence d'une " personnalité » pour une région quelconque. Il semble donc que nos géographes n'aient pas encore pleinement réussi à transposer, dans leur vocabulaire et leur conceptualisation, les éléments les plus valables de l'évolution méthodologique signalée par M. Grenier.

Par ailleurs, nos économistes se partagent en deux groupes, en ce qui concerne les questions régionales. D'une part, il y a ceux qui excluent la dimension spatiale de leurs analyses, selon cette tradition anglo-saxonne dont Isard, dans Location and Space-Economy 138 indique bien le démarquage net par rapport aux problèmes de l'espace.

138 Walter IsARD, Location and Space-Economy, New-York, Technology Press, M.I.T., John Wiley and Sons, 1956. 
Le second groupe, formé de ceux que les problèmes régionaux ont préoccupés, a reçu purement et simplement sa notion de "région » des géographes ; mais les analyses n'ont pu alors déborder le cadre étroit des phénomènes de structures : cette approche nous a donné, par exemple, en termes de marché du travail, ce partage net de l'espace en un ensemble «normal » d'une part et en une collection de « zones de dépression structurelle » d'autre part.

Pour aller au-delà de ces deux attitudes, les économistes doivent, avec la collaboration des géographes, apprendre à raisonner en termes de modèles spatiaux articulés. Dans une telle démarche, il faudra procéder à un partage des tâches. On peut bien affirmer que la région se définit par une aire d'homogénéité, mais il faut localiser les frontières de ces aires et mesurer l'« étanchéité » de ces frontières. Il s'agit manifestement là d'un travail de géographe surtout. Par ailleurs, pour l'intégration de ces aires en un modèle explicatif cohérent, on doit faire appel à des concepts qui relèvent de "l'outillage » de l'économiste : taux de substitution et coûts de transfert, par exemple. Mais, à leur tour, ces concepts n'ont un sens [104] que par rapport à une question à élucider, question dont le degré de signification doit être élaboré avec le géographe. L'analyse régionale sous ses aspects économiques ne peut donc se faire sans une étroite collaboration des géographes et des économistes.

Jusqu'ici, dans la province, les économistes ou se sont totalement isolés des géographes en éliminant la dimension spatiale de leurs analyses, ou ils se sont au contraire soumis totalement aux géographes pour se laisser alors enfermer dans de simples descriptions de structures. La réflexion méthodologique en commun devrait chercher à éliminer ces obstacles et viser surtout à élaborer des modèles spatiaux intégrés, soit en termes de fonctionnement de l'économie de la province dans son ensemble, soit en termes de croissance. Les impératifs de l'aménagement du territoire ne sont pas nécessaires à cette démarche, même si l'on doit admettre qu'ils peuvent fournir une forte motivation.

Pierre Harvey

École des Hautes Études Commerciales, Montréal. 
[105]

Situation de la recherche sur le Canada français

II. Perspectives écologiques

Gérald Fortin

Département de sociologie et d'anthropologie,

Université Laval

\title{
“L’étude du milieu rural”.
}

\author{
[pp. 105-116.]
}

Retour à la table des matières

Pour la sociologie de la connaissance, il est sans doute très significatif qu'au Canada français la sociologie soit née sous le signe du milieu rural. Longtemps même, c'est-à-dire jusqu'aux années 1940, c'est l'analyse de ce milieu qui a surtout retenu l'attention des sociologues et des anthropologues. Plusieurs facteurs peuvent expliquer cette orientation première de la recherche sociologique. Il n'est certes pas de notre propos de présenter ici une sociologie de notre sociologie ; je tiens cependant à souligner deux faits qui sont plus directement liés à notre sujet d'analyse. Le premier de ces faits est d'ordre démographique : en 1886, lorsque Gérin entreprit ses premières monographies, 75\% de la population canadienne-française de la province de Québec habitait le milieu rural. Cette prépondérance numérique de la population rurale devait durer jusqu'en 1931. ${ }^{139}$ Sur le plan idéologique,

139 Voir : Agriculture and the Farm Population, McGill University, Social Research Bulletin No. 1, 1938. 
cette fois, la pensée sociale des élites les plus dynamiques de 1900 à 1945 a été fortement ruraliste sinon agriculturiste. ${ }^{140}$ Fondée sur la foi et la langue, la culture canadienne-française a été définie par cette pensée sociale comme se réalisant de la façon la meilleure dans le cadre rural.

Il semble tout à fait naturel que ce soit ce milieu numériquement majoritaire et idéologiquement primordial qui ait attiré l'attention de la sociologie naissante. On peut même comprendre qu'il y ait eu tendance chez les pionniers à généraliser les résultats de leur recherche à l'ensemble de la société canadienne-française, à identifier notre culture traditionnelle à la culture observée dans un petit nombre de paroisses rurales.

Pour hâtive que soit cette généralisation, il est très difficile, faute de données, de la rejeter complètement. Ajoutons qu'il est aussi difficile (pour les mêmes raisons) de l'accepter. Nous ne possédons aucune étude de la culture de la population urbaine du début du siècle (nous n'en possédons [106] même que très peu de la population contemporaine). Sans doute l'histoire nous fournit-elle quelques renseignements sur la bourgeoisie de cette époque mais que savons-nous de la majorité ouvrière des villes ? De plus, comme nous le verrons à l'instant, les quelques monographies rurales que nous possédons indiquent clairement que le milieu rural était loin d'être aussi homogène qu'on se plaît parfois à se le représenter. Déjà, dans son ensemble, la société de la fin du XIX ${ }^{\mathrm{e}}$ siècle semble présenter l'aspect d'une société complexe où plusieurs sous-cultures co-existent. Il est toutefois possible qu'un système de valeurs commun ait coiffé ces diverses sous-cultures. C'est là un problème trop peu étudié pour qu'on puisse se prononcer.

C'est donc strictement en lui-même que nous voulons examiner le milieu rural et non en tant que représentatif du milieu traditionnel canadien-français. L'image que nous en retrouverons sera une image complexe à la fois dans le temps et dans l'espace.

140 Voir: Maurice Tremblay, "Orientations de la pensée sociale », dans: Jean-C. Falardeau, éd., Essais sur le Québec contemporain, Québec, Les Presses Universitaires Laval, 1953, 193-215 ; Gérald FoRTIN, The Analysis of a French-Canadian Nalionalist Ideology, 1917-1954, thesis presented for the degree of Doctor of philosophy, Cornell University, 1956. 
En 1862, un disciple de LePlay, Gauldrée-Boileau, visite SaintIrénée, comté de Charlevoix, et écrit une monographie de la famille d'Isidore Gauthier. ${ }^{141}$ Il constate un attachement profond de la population de Saint-Irénée à l'agriculture, un esprit communautaire et familial très fort, la transmission intégrale du domaine à un seul fils. Il croit ainsi avoir trouvé la famille-souche qui constituait pour LePlay le prototype de la vie familiale.

Quelque vingt ans plus tard, en 1886, Léon Gérin, cédant aux instances de son maître Edmond Demolins, entreprend lui aussi de décrire le milieu rural québécois à l'aide de la méthode monographique. Par suite d'observations personnelles échelonnées de 1886 à 1930, il a reconstitué l'histoire de plusieurs familles rurales de divers milieux.

La plus célèbre des monographies de Gérin est sans doute celle de la famille Casaubon, de Saint-Justin. ${ }^{142}$ L'image que présente Gérin de cet habitant casanier est devenue classique. Pratiquant une agriculture essentiellement vivrière et mixte, le cultivateur de Saint-Justin ne peut survivre que grâce à l'étroite solidarité de sa famille. Chacun de ses membres ayant un rôle précis dans l'œuvre commune, la famille vit en étroite dépendance de la nature. Elle s'appuie sur la nature plus qu'elle ne cherche à la transformer. C'est la famille qui demeure la cellule sociale de base de l'habitant de Saint-Justin ; vient ensuite le rang. Le village et la paroisse sont des centres d'intégration moins importants pour l'individu. Mise à part la prédominance sociale du curé, la stratification est presque inexistante. On a affaire à une simple juxtaposition de familles à peu près [107] égales qui se suffisent à ellesmêmes. Comme à Saint-Irénée, on retrouve le désir de transmettre intact le patrimoine familial à un seul des enfants, quitte à établir ailleurs les autres enfants.

Parce que Gérin a lui-même cédé à la tentation de généraliser les résultats de cette monographie, en particulier cet aspect du « domaine plein », et aussi peut-être parce que l'habitant casanier correspondait presque parfaitement au type d'habitant défini par l'idéologie ruraliste,

141 GauldrÉE-Boileau, « Paysan de Saint-Irénée », dans : Frédéric LePlay, Ouvriers des deux Mondes, tome V, cité par Léon GÉRIN, Le type économique et social des Canadiens, Montréal, Éditions de l'A.C.F., 1938, 13.

142 Léon GÉRIN, L'habitant de Saint-Justin, Mémoires et comptes rendus de la Société Royale du Canada, série II, tome IV, 1898. 
on a eu tendance à donner trop d'importance à cette étude et à oublier les résultats de trois autres monographies contemporaines de celle de Saint-Justin.

Bien qu'il ait visité Saint-Justin en 1886, ce n'est qu'en 1890 que Gérin a commencé ses observations systématiques auprès de la famille Casaubon. Entretemps, de 1887 à 1890, il avait résidé dans une famille de Saint-Dominique près de Saint-Hyacinthe, visité les Cantons de l'Est, acheté un lot à Clifton et embauché un fermier. De ces diverses expériences, il nous a laissé trois monographies qui présentent une image du milieu rural qui est très différente de celle de SaintJustin. 143

Alors que Saint-Justin était un univers fermé par rapport à l'industrie et au commerce, à Saint-Dominique Gérin perçoit « la lente pénétration du milieu rural par le commerce ». La solidarité de la famille a été touchée par ce phénomène. Il observe un début de séparation entre l'atelier et la famille, une plus grande indépendance des membres de la famille les uns vis-à-vis les autres, un changement d'attitude à l'égard de la transmission du bien, un désir plus grand de faire " instruire » les enfants. Certaines industries se sont installées dans la paroisse et l'agriculture elle-même tend vers la commercialisation, vers la spécialisation. La famille, le rang et la paroisse demeurent les trois institutions de base, mais la situation est plus complexe qu'à Saint-Justin. La famille n'a plus autant de stabilité et de cohésion, les relations de voisinage sont moins étroites, la paroisse n'a plus autant de prestige ni d'autorité. On a brisé avec la tradition pour s'orienter vers le progrès.

En 1887, les vieilles paroisses du pays-bas de la rive sud sont déjà surpeuplées. Les jeunes qui désirent s'installer sur une terre doivent reculer vers les hautes terres des Cantons de l'Est. Ayant décidé de devenir cultivateur, Gérin les imitera et s'installera à Clifton. Dans ces paroisses de colonisation, il va rencontrer un type d'habitant plus évolué encore qu'à Saint-Dominique. Faisant face à des conditions nouvelles, le colon doit souvent renoncer aux solutions traditionnelles et innover. De plus, il est en contact avec des gens qui viennent de différents milieux. Il est ainsi forcé à confronter ses normes et ses valeurs. De cette confrontation, une attitude plus dynamique a émergé. S'appuyant sur leur esprit communautaire, sur les traditions familiales et

143 Léon GÉRIN, Le type économique et social des Canadiens, op. cit. 
sur la paroisse, les nouveaux [108] colons ont su résister à l'assimilation aux Loyalistes et instaurer eux-mêmes une révolution économique et sociale : l'industrie laitière.

Tous les jeunes des vieilles paroisses n'ont pas pu cependant émigrer vers les hauts tributaires de la Saint-François. Faute de capital, un grand nombre d'entre eux ont dû aller chercher aux États-Unis un moyen de gagner leur vie. L'émigrant déraciné, que décrit Gérin, semble avoir été un type assez répandu à la fin du XIX ${ }^{\mathrm{e}}$ siècle. Restant attaché à la terre mais incapable d'y prospérer, il fait la navette entre l'usine et l'agriculture. Parce que cette migration s'est faite en groupes familiaux et que le clergé a accompagné les émigrants pour leur conserver leur cadre paroissial, les Canadiens français ont pu résister à l'influence de la ville et de l'usine, et rester attachés à l'agriculture. Nombre d'entre eux sont revenus s'installer dans la région montagneuse du sud.

Même si Gérin constate que la famille, le rang et la paroisse demeurent les unités de base des localités rurales qu'il a étudiées, ses observations sont suffisantes pour illustrer clairement que, dès le début du $\mathrm{XX}^{\mathrm{e}}$ siècle, le milieu rural québécois était déjà très diversifié. Selon les régions et selon, comme il l'indique, le degré de pénétration de l'industrie et du commerce et aussi le degré de pénétration de l'influence des villes, les comportements et les normes traditionnels ont plus ou moins de force.

Tout en continuant à rechercher les traits communs sous-jacents aux diverses formes de la vie rurale, il aurait fallu établir de façon précise la complexité de notre monde rural, déterminer les types caractéristiques de chaque région ainsi que les facteurs pouvant expliquer la diversité. De même qu'au sud de Montréal on pouvait distinguer entre les paroisses de la vallée, des hautes terres et des régions montagneuses, des distinctions semblables auraient pu être faites au sud de Québec, dans la région du bas du fleuve, du nord de Montréal, etc.

Malheureusement, l'idée de complexité et de diversité a cédé la place à celle d'homogénéité. En 1920-29, Gérin retournait à SaintIrénée pour vérifier les résultats de Gauldrée-Boileau. ${ }^{144}$ Dès ses 
premiers contacts, il se rend compte qu'il doit abandonner l'idée de famille-souche et même de domaine plein. Cependant, ses observations lui rappellent tellement celles de Saint-Justin qu'il tend à minimiser les différences pour accentuer l'homogénéité. La culture est familiale, vivrière et traditionnelle ; la famille demeure le centre d'intégration ; le rôle du curé est prédominant. Gérin note toutefois une action lente et dissolvante du commerce. Même si l'argent est rare, les étoffes traditionnelles ont fait place aux étoffes manufacturées. " Dès les premières atteintes de cette force nouvelle qui s'exerce du dehors, une notable partie de la population se trouve ébranlée, instabilisée ». 145

[109]

En 1936, Horace Miner entreprend une analyse anthropologique de la paroisse de Saint-Denis-de-Kamouraska ${ }^{146}$ Employant des techniques différentes de celles de Gérin, l'image qu'il nous présente de cette population rurale est très proche de celle de Saint-Justin : rôle prépondérant de la famille et du rang, attachement à une agriculture vivrière. Miner remarque cependant que les contacts avec la culture urbaine tendent à changer les patterns traditionnels. Cependant, il lui apparaît que la culture traditionnelle à Saint-Denis est assez forte pour intégrer les éléments nouveaux. "Les nouveaux traits sont modifiés et rendus compatibles avec les anciens patterns culturels ». ${ }^{147}$ Généralisant lui aussi à partir d'un seul cas qu'il n'a pas situé typologiquement dans l'ensemble, Miner conclut à une culture canadienne-française traditionnelle qui évoluera lentement par suite de l'influence urbaine mais qui demeure et demeurera sans doute très vivante.

L'image de Saint-Justin, Rioux la retrouvait lui aussi à l'Île Verte durant les années 40. ${ }^{148}$ Nous-même, nous l'avons retrouvée en essayant de reconstituer la vie sociale de Sainte-Julienne (Dorchester),

\footnotetext{
145 Ibid., 44.

146 Horace Miner, Saint-Denis, A French-Canadian Parish, University of Chicago Press, 1939. [La version française du livre est disponible dans Les Classiques des sciences sociales. JMT.]

147 Ibid., 241.

148 Marcel Rioux, Description de la culture de l'Île Verte, Musée national du Canada, Bulletin n 133, Ottawa, 1954.
} 
durant les années 30. ${ }^{149}$ Sans doute, à Sainte-Julienne, la forêt joue un rôle très important que les auteurs n'avaient jamais signalé. Mais, malgré une économie à la fois agricole et forestière, l'organisation sociale de même que le système de valeurs restent semblables à ceux de Saint-Justin ou de Saint-Denis.

Cette image du milieu rural sinon de la culture traditionnelle canadienne-française, qui avait été de plus en plus admise jusqu'aux années 1950, a été fortement mise en doute par la suite. Déjà en 1949, Miner retournant à Saint-Denis remarque que l'évolution de la culture a été plus rapide qu'il ne l'avait prévu. ${ }^{150}$ Philippe Garigue retournant à Saint-Justin en 1957 constate que la famille et le rang ne sont pas les milieux homogènes qu'avait décrits Gérin. ${ }^{151}$ Il y trouve des écoles, des automobiles, des téléviseurs, des appareils électriques de toutes sortes, des industries. L'agriculture y est spécialisée, le domaine plein n'est pas une pratique courante. De toutes ces observations, il conclut que Gérin n'a pas présenté une image exacte de Saint-Justin.

[110]

Nous-même, à Sainte-Julienne, nous faisons en 1956 des observations similaires à celles de Garigue. ${ }^{152}$ Une seule différence, l'agriculture ne s'est pas spécialisée, elle a disparu. À la même date, Rioux fait les mêmes observations à Belle-Anse, village de pêcheurs de la Gaspésie, tout en constatant toutefois une forte cohésion communautaire. ${ }^{153}$ Gosselin, Tremblay et moi-même constatons, en étudiant le comportement des travailleurs forestiers, que l'attachement à l'agriculture est très faible dans une quarantaine de paroisses rurales. 154

149 Gérald FoRTIN, « Les changements socio-culturels dans une paroisse agricole ", Recherches sociographiques, II, 2, avril-juin 1961, 151-170.

150 Horace Miner, «A New Epoch in Rural Quebec », The American Journal of Soclology, 56, 1, 1-16.

151 Philip Garigue, «St. Justin : A Case Study in French Canadian Rural Organization », The Canadian Journal of Economies and Political Science, 22, 3, August 1956, 301-318.

152 Gérald FoRTIN, « Les changements socio-culturels dans une paroisse agricole », op. cit.

153 Marcel Rioux, Belle-Anse, Musée national du Canada, Bulletin n 138, Ottawa, 1938.

154 Gérald FORTIN et Émile GossELIN, « La professionnalisation du travail en forêt », Recherches sociographiques, I, 1, janvier-mars 1960, 33-61. 
Tremblay et moi-même vérifions qu'en 1959 les normes de consommation de la population rurale non agricole sont exactement les mêmes que celles de la population de Montréal. 155

Devant les contradictions évidentes entre les études récentes et les études antérieures, il est trop facile de conclure à une erreur magistrale des pionniers. Il faut plutôt supposer que le milieu rural s'est transformé. Cette transformation qui a sans doute pris place de façon accélérée durant les quinze dernières années n'est toutefois pas nouvelle. Déjà les monographies de Gérin la laissaient pressentir. Le cultivateur de Saint-Dominique de 1887 n'est déjà plus l'habitant de Saint-Justin de 1920. Gérin lui-même nous en a indiqué la raison : l'infiltration du mode de vie de la ville et de la mentalité urbaine. Après le dernier conflit mondial cette infiltration est devenue massive, même dans les coins les plus reculés, bouleversant toute la structure sociale du monde rural. Il s'agit là d'une accélération d'un processus plutôt que d'un processus nouveau.

$\mathrm{Si}$, au lieu de retourner à Saint-Justin et à Saint-Irénée, Gérin avait continué l'étude de Saint-Dominique et de Clifton ; si Miner avait étudié Saint-Anselme de Dorchester, Terrebonne ou même Saint-Didace, l'image qu'ils nous auraient laissée du milieu rural canadien-français serait tout autre. Elle n'en serait pas pour autant plus valable. Car même si les indications sont minces, on peut facilement faire l'hypothèse que notre milieu rural était dès 1900 très hétérogène. Le type de SaintDominique, comme celui de Clifton, comme celui de l'émigrant, était aussi représentatif que le type de Saint-Justin ou de Saint-Irénée. Sans doute d'autres types existaient aussi, qu'il faudrait déterminer.

$\mathrm{Si}$, dans la recherche de ces types, le degré d'infiltration urbaine (l'isolement relatif) est un facteur important, il est un autre facteur qui a été négligé et qui, lui aussi, peut permettre une différenciation. Je veux parler [111] de la situation de la municipalité rurale dans l'histoire de la colonisation. Le développement agricole de la province est un processus historique très long qui vient à peine de se terminer (s'il l'est). Plusieurs de nos vieilles paroisses étaient des colonies, il y a 60 ans. Et le fait que ces colonies aient été situées dans la plaine, sur le

155 Marc-Adélard TREMBLAY et Gérald FORTIN, Étude des conditions de vie des familles salariées, tome I, Centre de recherches sociales, Université Laval, 1962 (miméo). 
premier plateau ou sur la montagne a eu une influence sur le type d'habitants qu'on y trouvait. Ainsi, on peut facilement faire l'hypothèse que la dernière vague de colonisation en pays de montagne a produit des bûcherons plutôt que des cultivateurs. Cette dernière vague, elle date de 1890 dans le comté de Joliette 156 et de 1935 dans le comté de Matane.

Ainsi, à un moment donné, au lieu d'une grande homogénéité on trouve une grande complexité à la fois au point de vue du stade de développement sur des territoires différents et au point de vue de l'infiltration de la mentalité urbaine.

À cause des transformations rapides que subit actuellement le milieu rural, il devient urgent d'entreprendre des recherches dans cette direction. Dans ce qu'il y a "à faire », c'est peut-être ce qu'il y a de plus urgent : connaître la culture, le système de valeurs et de normes, l'organisation sociale du plus grand nombre possible de municipalité rurales les plus disparates - cette connaissance ne couvrant pas seulement le présent mais aussi, sinon plus, le passé. L'urgence de telles études ne vient pas surtout de leur utilité mais plutôt de la disparition rapide des témoins oculaires du passé. L'anthropologie, en effet, est essentielle à une telle reconstitution du passé. Sans doute, historiens, géographes et démographes ont à apporter une contribution importante. Des études comme celles de Blanchard, de Louis-Edmond Hamelin, de Yves Martin sur l'histoire du peuplement d'une région constituent un point de départ indispensable. ${ }^{157}$ Ce secteur d'analyse est malheureusement trop peu développé. Là aussi tout est à faire avant que nous puissions connaître vraiment notre milieu rural. Il n'en reste

156 Louis-Edmond HAMELIN, « La marche du peuplement à l'intérieur du diocèse de Joliette ", texte présenté au dix-septième Congrès annuel de la Société canadienne d'Histoire de l'Église catholique, Joliette, septembre 1950. Publié dans le Rapport du Congrès, Hull, 1951, 4-13.

157 Voir par exemple : Raoul BlanCHARD, L'Est du Canada français, Paris et Montréal, 1935, tome I ; Raoul BLANCHARD, L'Ouest du Canada français, Montréal 1953, I, 86 ; Yves MARTIN, Étude démographique de la région du Bas Saint-Laurent, [Rimouski], Le Conseil d'orientation économique du Bas Saint-Laurent, 1959 ; Louis-Edmond HAMELIN, « Émigration rurale à l'échelon paroissial », Le géographe canadien, 5, 1955 ; Louis-Edmond HAMELIN, "Le rang à Saint-Didace de Maskinongé », Notes de Géographie (Université Laval), 3, mai 1953. 
pas moins que c'est à l'anthropologue secondé par le folkloriste qu'il appartient surtout de découvrir toutes les facettes de la culture traditionnelle du milieu rural et de montrer les cheminements de son évolution. Pour l'anthropologie, le milieu rural québécois est un monde inexploré où tout reste à découvrir, surtout dans sa dimension temporelle.

\section{[112]}

Même si j'ai mis l'accent, à propos de ce premier groupe de recherches à faire, sur la diversité et la complexité, je ne veux pas exclure $a$ priori l'hypothèse d'une certaine culture rurale traditionnelle commune à tous les types de communautés rurales d'une époque donnée. Cependant, si je n'exclus pas a priori cette hypothèse, je ne l'accepte pas non plus. Si cette culture existe ou a existé, elle transparaîtra d'elle-même à travers la diversité.

Un phénomène particulièrement important à étudier par rapport à la nature de la culture rurale traditionnelle est celui de la migration. Contrairement au stéréotype courant de l'habitant casanier, rivé à sa paroisse, il semble que la population rurale, ou du moins une partie considérable de cette population, ait été extrêmement mobile géographiquement. L'histoire de la colonisation en est une preuve. L'émigration vers les États-Unis en est une autre. Ce phénomène de mobilité de la population rurale à l'intérieur du milieu rural et vers les villes est encore assez mal connu. Gérin y fait souvent allusion : la famille Gauthier, famille-souche de Saint-Irénée s'est établie dans les Cantons de l'Est ; les colons de Clifton viennent de tous les coins de la province ; l'émigrant est un de ses types. L'histoire du peuplement nous fait aussi soupçonner une grande mobilité. Louis-Edmond Hamelin, dans une étude sommaire du diocèse de Juliette, estime à près de 230,000 le nombre des personnes qui sont sorties des paroisses de ce diocèse entre 1850 et 1950. 158 De ce nombre, 30,000 étaient des colons ; les autres auraient émigré aux Etats-Unis ou vers la ville. C'est dire que parmi les personnes nées dans cette région, près de la moitié l'ont quittée. Des contacts ont quand même continué à exister entre les émigrants et la population rurale. Quels effets ont eu ces contacts sur la culture de ceux qui restaient ? Joliette est-il représentatif des autres

158 Louis-Edmond HAMELIN, « La marche du peuplement à l'intérieur du diocèse de Joliette », op. cit. 
diocèses ou régions ? Autant de questions sans réponses tant que des études précises n'auront pas été entreprises sur l'origine, l'intensité et la destination des grands courants migratoires qui ont agité la population rurale. Quant à l'influence de ces migrations sur la culture rurale, il sera probablement impossible de jamais l'évaluer complètement surtout en ce qui concerne les migrations passées.

Jusqu'ici je me suis surtout attaché à l'aspect historique de l'évolution de notre milieu rural, négligeant ainsi les caractéristiques contemporaines de ce milieu. Car peu importe ce qu'a été le monde rural au début du siècle ou même il y a vingt ans : ses caractéristiques actuelles ne sont plus les mêmes. Trois faits peuvent suffire à illustrer cette affirmation. Premièrement, la population agricole (c'est-à-dire vivant sur une ferme) n'est plus l'élément de base du milieu rural. Depuis 1956, la population rurale non agricole est en majorité dans les municipalités rurales. Le village a ainsi remplacé les rangs comme centre de la vie rurale. Deuxièmement, la proportion [113] de la maind'œuvre active qui travaille à l'agriculture est en août 1962 de 4.2\%. ${ }^{159}$ En dix ans cette proportion est passé de 18\% à 4.2\%. Du point de vue de la main-d'œuvre, la province de Ouébec est actuellement la moins agricole de toutes les provinces du Canada. Troisièmement, en dépit de la diminution de la main-d'œuvre agricole, la production s'est maintenue et a même légèrement augmenté ; il y a donc eu augmentation très rapide de la productivité grâce à la mécanisation et même à l'automation dans le cas de la production animale (poulet de gril, par exemple). Cette augmentation de la productivité a été rendue possible dans certains cas par des contrats intégrés de production qui ont réduit le cultivateur à un rôle à peine plus responsable que celui de l'opérateur dans une chaîne de production.

Globalement, on assiste à une émigration non seulement des surplus de population rurale mais aussi à un abandon de l'agriculture par les cultivateurs eux-mêmes. Parallèlement, ceux qui veulent continuer leur métier d'habitants doivent, pour survivre, abandonner les techniques traditionnelles et devenir de véritables chefs d'entreprise. Enfin, les travailleurs qui ont abandonné l'agriculture ne se dirigent pas nécessairement vers la ville mais cherchent plutôt à demeurer dans le

159 D'après les données de l'enquête mensuelle sur la main-d'œuvre (Bureau fédéral de la statistique). 
milieu rural en s'établissant au village. D'où une possibilité d'encombrement du marché du travail rural et une possibilité de chômage chronique.

Il s'agit là toutefois d'une vue globale qui demeure largement intuitive. Pour arriver à cerner toutes les dimensions de la situation actuelle, des études générales aussi bien que des monographies détaillées sont nécessaires. Le genre de recherches nécessaires diffère toutefois selon que celles-ci devront servir à procurer une connaissance du milieu ou servir a préparer des plans d'action, même si ces deux fins ne sont nullement exclusives l'une de l'autre.

Sur le plan de la connaissance de notre milieu, on pourrait noter les questions suivantes :

$1^{\circ}$ Jusqu'à quel point la transformation radicale qui apparaît au niveau global est-elle généralisée à toutes les régions et à tous les types de municipalités ? Quels sont les secteurs de forte résistance et quels sont les secteurs de faible résistance ? Quelles sont les modalités de la transformation selon les secteurs?

Une classification socio-économique sommaire des municipalités m'a permis d'élaborer à ce sujet un certain nombre d'hypothèses par rapport à trois types de municipalités rurales. ${ }^{160}$ Ces hypothèses n'ont été vérifiées que pour le cas des municipalités où l'agriculture est « moyenne ». Cette vérification ne s'appuie d'ailleurs que sur une seule monographie et sur des [114] observations rapides d'une quarantaine de municipalités de la région de Québec et du Bas Saint-Laurent. La classification employée reste provisoire et ne tient pas compte, par exemple, des régions et du niveau de revenu des cultivateurs. Mettre au point une typologie plus valable et déterminer pour chaque type la situation réelle, cela reste à faire.

$2^{\circ}$ Pourquoi cette transformation s'est-elle produite si rapidement ? L'étude de Sainte-Julienne permet de croire que la non-rentabilité de l'agriculture n'est pas une cause suffisante et que le facteur le plus important est la pénétration de la mentalité urbaine dans le milieu rural, cette mentalité s'exprimant surtout par le désir d'un niveau de vie su-

160 Gérald ForTIN, «Une classification socio-économique des municipalités agricoles du Québec », Recherches sociographiques, I, 2, avril-juin 1960, 207-216. 
périeur. Une autre condition de la transformation serait la possibilité pour le rural de s'adonner à des occupations non agricoles offrant un revenu supérieur à celui de l'agriculture. Il s'agit là d'une hypothèse qu'ont confirmée l'étude de Sainte-Julienne et l'étude de la mobilité des travailleurs forestiers, mais qui reste à vérifier dans d'autres milieux. Ces deux études ont permis de préciser les circonstances concrètes qui avaient facilité la pénétration de la mentalité urbaine et l'orientation en masse de la main-d'œuvre vers des activités non agricoles. Ces circonstances varient cependant selon les régions et les types de municipalités et il est important de les connaître.

$3^{\circ}$ Quelles sont et seront les conséquences de cette transformation sur la culture rurale? L'étude sur les conditions de vie des familles salariées montre que les familles rurales non agricoles ont exactement les mêmes normes de consommation et les mêmes aspirations économiques que les familles urbaines. On constate même que les familles rurales sont plus attirées que les familles urbaines par les nouvelles normes définies par la publicité de masse. Sans doute, il ne s'agit là que d'un niveau de la vie culturelle, mais on peut faire l'hypothèse que ce niveau prend de plus en plus d'importance dans le système culturel de la population rurale. À Sainte-Julienne, nous avons constaté des répercussions dans tous les aspects de la vie sociale : structure de la famille, définition du mode de vie idéal, stratification sociale, orientation vers le présent plutôt que vers le passé, etc. Les autres monographies récentes (Rioux, Garigue, Miner) montrent des phénomènes semblables. Il semble toutefois que l'évolution culturelle n'est pas achevée et que la majorité rurale soit encore attachée aux valeurs traditionnelles tout en vivant selon de nouvelles valeurs et tout en commençant à accepter ces nouvelles valeurs. Elle semble souffrir de cette forme d'ambivalence culturelle que décrit Merton en parlant d'anomie. Encore là, ce sont autant d'hypothèses que trop peu de faits viennent confirmer. Pour connaître ce qu'est et ce que sera la culture rurale comme pour connaître ce qu'elle a été, de très nombreuses monographies seront nécessaires. Ces monographies, qui devront tenir compte de la diversité et de la complexité de notre milieu rural, devront avoir un caractère beaucoup plus historique que la plupart des monographies existantes. Pour saisir l'évolution [115] du milieu rural, il ne suffit pas de connaître ce qu'il est maintenant, il faut aussi savoir ce qu'il a été. 
Cette connaissance de l'évolution de notre milieu rural, elle sera très utile non seulement au plan théorique mais aussi au plan de l'action. Cependant l'action pose sur le milieu rural un certain nombre de questions plus précises. Je veux n'en signaler que quelques-unes en guise de conclusion.

Une première série de questions se rapporte à l'émigration rurale. Oui sont les émigrants par rapport à ceux qui demeurent dans le milieu ? M.-Adélard Tremblay et Régis Lessard ont montré que dans Kamouraska les émigrants étaient mieux instruits que ceux qui héritaient le bien paternel. ${ }^{161}$ Est-ce vrai partout ? Est-ce vrai aussi des cultivateurs qui abandonnent actuellement l'agriculture ? Si oui, les possibilités de renouveau rural sont d'autant compromises. Que deviennent les émigrants ruraux dans la grande ville au point de vue professionnel et au point de vue familial ? Bien des préjugés existent sur ce point, mais aucune étude sérieuse. Pourtant on parle de plus en plus de la nécessité de vider certaines paroisses rurales - sans savoir où orienter leur population et comment la préparer au changement.

Dans un autre ordre d'idées, il semble bien clair que l'agriculture, pour survivre chez nous, doit cesser d'être un mode de vie pour devenir une industrie rationnelle. Il va donc falloir que cette agriculture soit adaptée aux possibilités du sol et aux exigences du marché. L'inventaire pédologique de notre province n'est même pas complété, l'inventaire des marchés l'est encore moins. La gestion de la ferme prendra aussi de plus en plus d'importance. Mais avant de vouloir changer les techniques de gestion de nos cultivateurs, il faudrait savoir quelles sont les techniques courantes. Encore là, l'inventaire des travaux sérieux est vite fait : une étude de Charles Lemelin, une de Ferdinand Ouellet, quelques-unes de Maurice Carel. ${ }^{162}$ Sans doute, les agronomes de comté possèdent-ils une somme importante de connaissances empiriques à ce sujet, mais aucun effort d'explicitation de ces connaissances n'a été fait.

161 Régis LESSARD, La ferme familiale de Saint-Alexandre de Kamouraska, thèse de maîtrise, Université Laval, Québec, 1950 ; M.-Adélard TREMBLAY, La ferme familiale du comté de Kamouraska, thèse de maîtrise, Université Laval, Québec, 1950.

162 Maurice CAREL, «Exemple d'étude de groupes », Agriculture, XVII, 1, janvier 1960. 
Il serait possible ici de continuer à multiplier les questions et d'accumuler les absences de réponses. J'aimerais soulever seulement une dernière question, qui est peut-être la question capitale par rapport à l'avenir même du milieu rural. Comment la population rurale non agricole (c'est-à-dire la majorité de la population rurale) va-t-elle pouvoir s'assurer des revenus suffisants pour conserver le niveau de vie qu'elle désire ? En d'autres termes, quelle est la structure du marché du travail dans le milieu rural ?

\section{[116]}

Jusqu'ici la forêt constituait la base de ce marché. Il n'est pas sûr qu'il en soit toujours ainsi. Déjà la mécanisation a été introduite dans cette industrie et des expériences d'automation sont actuellement conduites avec succès. Le problème de l'aménagement rural et régional se pose dès maintenant de façon aiguë. Le prolongement des tendances sur le plan des occupations et de la satisfaction au travail laisse prévoir un exode total du village de Sainte-Julienne dans une ou deux générations si les conditions actuelles persistent et dans dix ans, si le marché du travail rural se restreint. La connaissance du marché du travail rural et la restructuration de ce marché s'imposent dès maintenant, si l'on veut qu'en l'an 2000 le milieu rural québécois soit autre chose qu'un objet de musée ou une banlieue de Montréal.

Gérald FORTIN

Département de sociologie et d'anthropologie, Université Laval. 


\title{
COMMENTAIRE
}

\author{
Napoléon LeBlanc \\ Doyen de la Faculté des sciences sociales, \\ Université Laval.
}

[pp. 117-118.]

$\underline{\text { Retour à la table des matières }}$

Monsieur Fortin a choisi d'examiner le milieu rural en lui-même, et non en tant que représentatif du milieu canadien-français, pour nous en fournir une image complexe qui déroute nos rationalisations à l'appui de la vocation rurale de notre population.

Nous avons sûrement mal interprété Léon Gérin en projetant sur toute la province son habitant de Saint-Justin comme le type idéal de la famille rurale. J'en trouve un indice dans l'essai de M. Gérard Filion, "Le paysan et ses institutions sociales », publié dans le volume Agriculture de la collection « Notre Milieu ».

«C'est le plus Américain des Américains et cependant ce n'est pas un Américain, écrit-il, c'est un paysan de tradition française qui pense et agit comme un paysan français, mais sur des choses et dans un milieu qui ne sont pas français ". Il en voit une preuve évidente dans sa vie sociale bien différente de celle de l'entrepreneur agricole américain et de celle du paysan français. "C'est un type original, conclut-il, que les sociologues dissèquent avec un extrême plaisir. » La liste des travaux que donne Fortin indique que ces dissections n'ont pas été tellement fréquentes. 
Parmi les éléments originaux de la vie sociale en milieu rural, $\mathrm{M}$. Filion inscrit en premier lieu la famille : « Cette famille communautaire, écrit-il, a solidement résisté à tous les agents de désagrégation qui se sont ligués contre elle. Encore en 1942, continue M. Filion, elle forme un tout pour lequel chacun se sacrifie avec ou sans espoir de récompense. »

Les quelques études récentes, décrites par G. Fortin, laissent entrevoir que des familles rurales sont moins résistantes et qu'elles ne forment plus « ce tout pour lequel chacun se sacrifie avec ou sans espoir de récompense ».

Aujourd'hui le milieu rural ne jouit plus du splendide isolement d'autrefois. Si nous devons l'étudier en lui-même, pour mieux le connaître tant dans le temps que dans l'espace, nous ne pouvons cependant négliger de $\wedge$ 'étudier dans ses relations dynamiques avec le milieu urbain et industriel. À ce sujet, G. Fortin nous indique les études complémentaires et variées que postule une saisie cohérente du milieu rural actuel si nous ne voulons pas qu'il devienne « un objet de musée ou une banlieue de Montréal ». Il aurait pu mentionner l'étude de Claude Morin et de M.-A. Tremblay portant sur l'introduction d'une agence de service social en milieu rural. ${ }^{163}$

Invoquant les transformations rapides que subit le milieu rural, Fortin souligne l'urgence d'entreprendre des recherches qui nous permettront de connaître la culture, le système de valeurs et de normes, l'organisation sociale du plus grand nombre possible de municipalités rurales les plus disparates. Cependant, ces recherches devraient être complétées par d'autres études qui nous permettraient d'apprécier la qualité du leadership du milieu rural à différents moments de l'histoire. Ces recherches pourraient rejoindre les études de Taylor consacrées aux Farmers' Movements (1620-1920) aux États-Unis. ${ }^{164}$

En effet, des études de cette nature nous permettraient de mieux connaître l'action des groupements d'agriculteurs qui ont existé dans le [118] passé. Dans quelle mesure ces mouvements rencontreraient-ils

163 Claude Morin et M.-Adélard TREMBLAY, « Intégration à la communauté : étude d'une agence rurale de service social », Service social, 9, 1, janvier 1960, 6-24.

164 Carl C. TAYlor, The Farmers' Movement, New-York, American Book Co., 1953. 
la définition qu'en donne Taylor : «Un type de conduite affectant un large segment de la population non satisfaite des conditions culturelles, sociales, économiques et politiques qui lui sont faites. »

Que savons-nous des circonstances qui ont inspiré l'œuvre de l'abbé Pilote, à Sainte-Anne-de-la-Pocatière, de l'influence de ce dernier auprès des élites agricoles ? Que savons-nous également des cercles agricoles qui existèrent entre 1870 et 1896 ? Pourtant, ces cercles, réunis en association nationale, ont manifesté un dynamisme remarquable jusqu'au jour où ils s'en remirent au patronage du gouvernement. Peut-être que cette mise en tutelle révélait une anémie résultant soit d'une conjoncture socio-économique favorable, soit d'un leadership inapproprié qui s'imposa de l'extérieur.

Il en est de même des débuts du mouvement coopératif. La fin du $\mathrm{XIX}^{\mathrm{e}}$ siècle et le début du $\mathrm{XX}^{\mathrm{e}}$ siècle marquent un effort intense pour renflouer l'agriculture. Rappelons Desjardins et sa caisse populaire, l'abbé Allaire et la fondation des premières coopératives agricoles. Ces derniers, sûrement, ont été des innovateurs après s'être inspirés des expériences belges, françaises, allemandes et italiennes. Après avoir traversé des périodes difficiles, le mouvement coopératif a atteint aujourd'hui une dimension imposante. Toutefois, dans quelle mesure pouvons-nous le considérer comme un agent de changement socio-culturel ?

Plus près de nous, l'histoire de l'évolution de l'Union catholique des cultivateurs pourrait nous instruire sur l'évolution de la mentalité des ruraux.

Cette étude des mouvements agricoles nous permettrait de retracer les normes et les valeurs qui ont inspiré et continuent peut-être d'inspirer le comportement des ruraux. Nous y trouverions peut-être une explication à l'hypothèse de G. Fortin selon laquelle « l'évolution culturelle n'est pas achevée et la majorité rurale (est) encore attachée aux valeurs traditionnelles tout en vivant selon de nouvelles valeurs et tout en commençant à accepter ces nouvelles valeurs ». Encore faudrait-il identifier les oppositions entre les valeurs traditionnelles et les valeurs nouvelles.

Enfin, une étude du leadership actuel en milieu rural s'impose. Le leadership rural est forcément dispersé. À cause des problèmes qui retiennent son attention, le leader rural travaille nécessairement à 
l'échelon local et régional. Le plus souvent engagé dans le traitement de situations d'urgence, il risque de ne pas accéder à cette vision de la société globale que postule le devenir du milieu rural.

Napoléon LEBLANC

Doyen de la Faculté des sciences sociales, Université Laval. 


\title{
Situation de la recherche sur le Canada français II. Perspectives écologiques
}

\author{
Yves Martin \\ Département de sociologie et d'anthropologie, \\ Université Laval \\ “Les études urbaines
}

[pp. 119-128.]

$\underline{\text { Retour à la table des matières }}$

La délimitation du domaine des études urbaines pose un problème particulier. Dans la mesure où le phénomène de l'urbanisation se confond avec la diffusion de la culture caractéristique de la société technologique, 165 toute recherche portant sur l'un ou l'autre aspect de cette culture pourrait être inscrite au chapitre des études urbaines. Même si, par ailleurs, l'on se propose de ne retenir, sous cette rubrique, que des travaux consacrés à l'étude des villes ou des populations urbaines, on se trouve confronté à une nouvelle difficulté : celle de définir les réalités concrètes auxquelles renvoient les notions de ville ou de population urbaine.

À partir de telles considérations, les sociologues — les sociologues américains en particulier, mais non pas exclusivement - remettent en cause aujourd'hui le statut de la sociologie urbaine en tant que disci-

165 Voir, sur ce thème, l'excellent exposé de Georges FrIEDMANN, « Milieu technique et milieu naturel », dans: Georges Friedmann (sous la direction de), Villes et campagnes, Paris, Colin, [1953], 401-416. 
pline particulière et s'interrogent sur le bien-fondé de la distinction classique entre sociologie urbaine et sociologie rurale. ${ }^{166}$ Le débat ne saurait être ramené à de simples questions d'étiquettes ; il indique bien plutôt qu'on reconnaît la nécessité de définir des problématiques nouvelles pour l'étude de milieux différents à l'intérieur d'une même culture globale. ${ }^{167}$ C'est à cette perspective générale que se rattache le point de vue que nous adoptons dans le présent exposé.

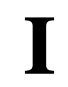

Pour établir l'inventaire de la documentation existante, nous avons défini le secteur des études urbaines d'une façon peut-être un peu stricte, [120] peut-être aussi insuffisamment rigoureuse ; notre examen a porté sur les travaux expressément consacres à l'étude de milieux urbains particuliers ou à l'étude générale du phénomène de l'urbanisation dans la province de Québec.

Considérons tout d'abord les études particulières ; elles sont nombreuses. Au terme d'une enquête très incomplète, nous avions recueilli quelque deux cents titres d'ouvrages et d'articles monographiques. Plus de la moitié de ces monographies portent sur les deux principales agglomérations de la province, Montréal et Québec, mais rares sont les villes qui n'ont fait l'objet d'aucune étude — au moins par un historien local... Pour les fins de cet exposé, nous répartirons ces études particulières en quatre grandes catégories.

Les monographies historiques forment la catégorie quantitativement la plus importante. L'histoire locale, on le sait, constitue une tradition bien établie dans notre milieu. La plupart des villes de la pro-

166 Voir, par exemple, à ce sujet : E. MANHEIM, « Theoretical Prospects of Urban Sociology in an Urbanized Society », American Journal of Sociology, LXVI, 3, November 1960, 226-229 ; Louis ChEVALIER, « Le problème de la sociologie des villes », dans : Georges Gurvitch (sous la direction de), Traité de sociologie, Parie, Presses Universitaires de France, tome premier, 1958, 292-314.

167 C'est sous cet angle que, par exemple, Henri MENDRAs cherche à définir l'orientation de la sociologie rurale dans: "Sociologie du milieu rural », dans le même Traité de sociologie, tome premier, 315-331. 
vince ont eu leur historien, sinon leurs historiens. Il s'agit presque toujours d'amateurs, travaillant sans méthode bien précise, trop souvent soucieux avant tout de rehausser le prestige de leur localité à la veille d'un cinquantenaire ou d'un centenaire... Si l'on consulte ces travaux en y cherchant des réponses à des questions précises, on y trouvera sûrement beaucoup d'enseignements, mais rarement ces histoires de villes offrent-elles par elles-mêmes un intérêt immédiat pour l'étude de l'urbanisation dans notre milieu. Retenons, à titre d'exemple, la toute récente Histoire de Louiseville, 1665-1960, du R. P. Germain Lesage. ${ }^{168}$ Cet ouvrage de quelque 450 pages rend compte de longs et patients dépouillements d'archives ; la vie et l'œuvre des divers curés y tiennent une place importante, de même que les listes de " vocations " issues de la paroisse, mais on ne trouve que de brèves allusions à l'évolution de la population et aux migrations, très peu d'indications sur les changements dans la structure des occupations, aucun essai d'analyse un peu approfondie des conséquences de l'apparition de l'industrie dans la petite ville en question (sur un événement aussi significatif que la grève de 1952-53 à Louiseville, l'auteur s'en tient à une page de renseignements très généraux). Les monographies de ce type, et même de beaucoup moins bonnes, rendent d'incontestables services aux chercheurs. Il faut souhaiter que des historiens compétents abordent le secteur de l'histoire urbaine ; les érudits locaux pourraient ainsi s'inspirer de modèles sérieux plutôt que de leur seule bonne volonté.

À la même catégorie d'études nous pouvons rattacher certains travaux d'histoire économique : mentionnons tout particulièrement l'article d'Albert Faucher sur les chantiers navals de Québec au XIX ${ }^{\mathrm{e}}$ siècle, 169 celui de [121] D.C. Masters sur la «rivalité » entre Montréal et Toronto en tant que métropoles financières 170 et l'ouvrage de Fernand Ouellet, Histoire de la Chambre de commerce de Québec. ${ }^{171}$

Une deuxième catégorie d'études urbaines comprend des travaux de géographes. Ici, il faut surtout insister sur l'apport considérable de

168 Presbytère de Louiseville, 1961, 450 p

169 Albert FAucher, «The Decline of Shipbuilding at Québec in the Nineteenth Century », Canadian Journal of Economics and Political Science, 23, 2, May 1957, 195-215.

170 D. C. MASTERs, « Toronto vs Montréal. The struggle for financial hegemony, 1860-1875 », Canadian Historical Review, 22, 1941, 133-146.

171 Québec, Faculté de commerce, Université Laval, 1959, 104 p. 
Raoul Blanchard. 172 Ses « esquisses de géographie urbaine » de Montréal et de Québec sont en tout point remarquables. Ce sont des œuvres non pas définitives, bien sûr, mais fondamentales : ce sont les premières sources que doit consulter le chercheur qui entreprend une étude historique, démographique, écologique ou sociologique sur l'une ou l'autre des deux agglomérations principales de la province. Les synthèses que propose Blanchard sont d'ailleurs construites à partir d'un examen à peu près exhaustif de la documentation existant au moment où il poursuivait ses recherches, à l'exception toutefois des fonds d'archives. Les mêmes observations vaudraient, en fait, pour toutes les principales villes de la province : à chacune, Blanchard a consacré une monographie - plus ou moins longue suivant les cas qui peut généralement servir de point de départ à des recherches plus poussées. Depuis Blanchard, les géographes ne nous ont pas encore donné d'autres travaux d'envergure sur les milieux urbains de la province. On relève cependant un bon nombre de thèses de géographie urbaine préparées aux Instituts de géographie des universités de la province, à l'École des hautes études commerciales de Montréal ou à la Faculté de commerce de l'Université Laval. ${ }^{173}$ Dans les revues canadiennes de géographie, on trouve aussi, de temps à autre, des monographies urbaines; 174 très rares sont toutefois les géographes de

172 Raoul Blanchard, L'Est du Canada français, Paris, Librairie Masson \& Cie, Montréal, Librairie Beauchemin, Limitée, 1935, 2 vol., 366 et 336 p. ; Le Centre du Canada français, Montréal, Librairie Beauchemin, Limitée, 1947, 577 p. ; L'Ouest du Canada français. Tome premier : Montréal et sa région, Montréal, Librairie Beauchemin, Limitée, 1953, 401 p. Tome deuxième : Les pays de l'Ottawa — L'Abitibi-Témiscamingue, Montréal, Librairie Beauchemin, Limitée, 1954, 334 p.

173 Un bon nombre de ces thèses sont mentionnées dans : Philip GARIGUE, $A$ Bibliographical Introduction to the Study of French Canada, Montréal, McGill University, Department of Sociology and Anthropology, 1956, 133 p.

174 Parmi les articles récemment publiés, mentionnons ceux de : Pierre BIAYs, « Une ville d'Abitibi : Senneterre », Cahiers de géographie de Québec, II, 3, octobre 1957, 63-74 ; Pierre CAMU, «Le paysage urbain de Québec », Geographical Bulletin, 10, 1957, 23-35 ; Robert GARRY, «Chibougamau, ville minière ", Revue canadienne de géographie, 9, 1, janvier-mars 1955, 4752 ; G. Humphrys, "Schefferville, Québec : a New Pioneering Town », Geographical Review, 48, 2, April 1958, 151-166. 
notre milieu qui ont fait des études urbaines le domaine principal de leurs recherches.

Les études écologiques et sociologiques forment une troisième catégorie de recherches urbaines. La majorité de ces études ont été effectuées dans les agglomérations de Montréal et de Québec, tout simplement là où [122] sont situées les institutions d'enseignement de la sociologie. L'exception importante est la monographie de Drummondville «Cantonville » par Hughes. 175 D'autres petites villes ont été étudiées par des sociologues à l'occasion de recherches portant sur certaines régions de la province : par exemple, les villes de SaintJérôme, de Sainte-Thérèse, de Lachute et de Terrebonne, au moment de l'enquête sur la structure sociale du diocèse de Saint-Jérôme, par Fernand Dumont et nous-même ; 176 ou encore les villes de Rivièredu-Loup et de Montmagny étudiées à l'occasion d'une analyse sociologique du diocèse de Sainte-Anne-de-la-Pocatière par les chercheurs du Centre de recherches en sociologie religieuse de l'Université Laval. ${ }^{177}$ Les villes nouvelles ont déjà retenu l'attention de quelques sociologues : mentionnons les articles de Derbyshire et de Garigue sur Schefferville. 178

La plupart des travaux écologiques ou sociologiques portant sur l'agglomération montréalaise ou sur l'agglomération québécoise sont inédits. Il s'agit le plus souvent de thèses de maîtrise. Ainsi, à l'Université McGill, les étudiants en sociologie ont préparé plusieurs thèses sur les « aires naturelles » de la ville de Montréal et, surtout, sur les

175 Everett C. Hughes, Rencontre de deux mondes. La crise d'industrialisation du Canada français. Traduit de l'anglais par Jean-Charles Falardeau. Montréal, Éditions Lucien Parizeau, s.d., 388 p.

176 Fernand Dumont et Yves MARTin, L'analyse des structures sociales régionales, étude sociologique de la région de Saint-Jérôme, à paraître aux Presses de l'Université Laval.

177 Rapports non publiés. Le même Centre a aussi poursuivi des recherches sur la ville d'Alma.

178 Edward DeRbyshiRE, « Notes on the Social Structure of a Canadian Pioneer Town », The Sociological Review, 8, 1, July 1960, 63-75 ; Philippe GARIGUE, « Une enquête sur l'industrialisation de la province de Québec : Schefferville », L'Actualité économique, XXXIII, 3, octobre-décembre 1957, 419436. 
problèmes de relations entre groupes ethniques dans la métropole. 179 À l'Université Laval, on trouvera, parmi les thèses portant sur Québec, quelques monographies de paroisses, quelques études sur les conditions de vie des familles ou sur le logement. ${ }^{180}$ Entre 1944 et 1950, des professeurs et des étudiants de la Faculté [123] des sciences sociales de l'Université Laval ont réalisé un ensemble de recherches, d'ordre écologique pour la plupart, sur l'agglomération québécoise. Les résultats de ces enquêtes ont été consignés dans des rapports inédits ou dans des articles. Soulignons plus particulièrement l'Étude générale de la ville de Québec (1949), de Jean-C. Falardeau ; 181 « A Survey of Québec City Families » (1947) ${ }^{182}$ et « Délimitation d'une banlieue

179 Parmi les titres indiqués dans Philip GARIGue, A Bibliographical Introduction to the Study of French Canada (op. cit.), nous avons retenu les suivants : Ronald ARELANO and others, Pontville, a Socio-Economie Study of a French Canadian Suburban Community, thèse de maîtrise, McGill, 1955 ; C. M. BAYLEY, The Social Structure of the Italian and Ukrainian Immigrant Communales in Montréal, 1939 ; Jacques BrAzEAU, The French Canadian Doctor in Montréal, 1951 ; Wilfred Harold BROWN, The Slovakian Community in Montréal, 1927 ; Mary DAVIDSON, The Social Adjustment of British Immigrant Families in Verdun and Pointe-Saint-Charles, 1933 ; Stuart M. JAMIESON, French and English in the Institutional Structure of Montréal, 1938 ; J.L. PyE, Point Saint-Charles, an Ecological Study, 1939 ; L.C. RENNIE, The Ethnie Division of Labour in Montréal, 1953; Nigel A. RICHARDSON, A Study of the Retationship between Ecological and Nonecological Factors in the Development of Natural Areas of Montréal, 1954 ; Aileen Ross, The French and English Social Elites of Montréal, 1941 : Léo ZAKUTA, The Natural Areas of the Montréal Metropolitan Community, 1948.

180 Quelques thèses de maîtrise présentées à la Faculté des sciences sociales de l'Université Lava], sur l'agglomération québécoise : Colette BEAUDET, Banlieue réelle et ville de Québec, 1947 ; L. BRISSON, La paroisse SaintSauveur au service du peuple, 1947 ; Gaétan CHARBONNEAU, Étude sur les revenus des familles dans la ville de Québec en 1939 et en 1945, 1947 ; Jeanne C. DESROCHERS, Les conditions de logement aux huttes des Covefields à Québec, 1950 ; Pierre LAPORTE, Les changements sociaux et institutionnels d'un milieu paroissial de la ville de Québec (Paroisse Notre-Damede-la-Garde), 1960 ; Marthe PAPILlON, Étude des familles de la ville de Québec, 1946 ; Fernand ROCHETTE, Monographie du quartier SaintSauveur, 1952.

181 Faculté des sciences sociales, 1949, 179 p. (dactylographiée).

182 Canadian Journal of Public Health, 38, 11, November 1947, 515-527. 
de grande ville » (1951), 183 deux articles du même auteur ; « The Life Cycle of French-Canadian Urban Families » (1947), article rédigé en collaboration par Maurice Lamontagne et Jean-C. Falardeau. ${ }^{184}$ Plus récemment, l'enquête entreprise sur le logement à Québec, sous la direction de James Hodgson, a donné lieu à un nouvel ensemble d'études sur la ville. Ces études sont encore inédites, exception faite d'un article de Gabriel Gagnon sur « Les zones sociales de l'agglomération de Québec ». 185 Signalons, par ailleurs, toujours à propos de l'agglomération québécoise, les voies nouvelles de recherche ouvertes par les travaux de Simone Paré sur la participation d'une population de banlieue aux associations volontaires 186 et par ceux de Gérard Lapointe et Vincent Lemieux sur le pouvoir municipal dans deux localités de la banlieue. 187

Parmi les études publiées sur l'agglomération métropolitaine de Montréal, retenons plus spécialement celle de Jean de Laplante sur « La communauté montréalaise » (1952), 188 celle du Groupe de recherches sociales sur La situation des immigrants à Montréal (1959) 189 et l'ouvrage de l'abbé Norbert Lacoste sur Les caractéristiques sociales de la population du Grand Montréal. Élude de sociologie urbaine (1958). 190 On trouvera, dans cet ouvrage de l'abbé Lacoste, d'abondantes compilations statistiques, mais [124] l'auteur, il faut

183 Revue canadienne d'urbanisme, I, 1, 1951, 16-22.

184 Canadian Journal of Economics and Political Science, 13, 2, May 1947, 233-247.

185 Recherches sociographiques, I, 3, juillet-septembre 1960, 255-267.

186 Simone PARÉ, «Participation d'une population de banlieue à ses groupes de famille, de parenté, d'amitié et de voisinage », Service social, 9, 1, janvier 1960, 25-47 ; " Participation aux associations volontaires dans une paroisse de la banlieue de Québec », Service social, 10, 1, mai-juin 1961, 24-42.

187 Gérard LAPOINTE, Le pouvoir municipal : essai sur les fonctions municipales à Beauport, thèse de maîtrise, Laval, 1958, 157 p. ; Vincent LEMIEUX, La Cité de Lévis : essai de sociologie municipale, essai de maîtrise, Département de science politique, Université Laval, 1959, 97 p.

188 Contributions à l'étude des sciences de l'homme, 1, 1952, 57-105.

189 La situation des immigrants à Montréal, étude sur l'adaptation occupationnelle, les conditions résidentielles et les relations sociales, réalisée par le Groupe de recherches sociales, Inc., sous les auspices du Conseil des Euvres de Montréal, Montréal, s.éd., 1959, vu + 376 p. (miméo.).

190 Montréal, Faculté des sciences sociales, Université de Montréal, 1958, 267 p. 
le regretter, n'a pas suffisamment cherché à en dégager au moins les éléments d'une synthèse sur la structure sociale de l'agglomération ; pour notre part, nous aurions préféré que l'auteur nous invite à réfléchir sur une telle synthèse, même provisoire, ou sur des hypothèses, plutôt qu'à le suivre tout au long de patients exercices de corrélation statistique dont l'utilité n'est pas toujours clairement établie (pp. 173208)... Sans doute faudrait-il évoquer bien d'autres travaux qui ont contribué, selon des perspectives très diverses, à nous éclairer sur la structure sociale de la ville ou de la zone métropolitaine de Montréal ; nous citerons, à titre d'exemple, les analyses de Jacques Henripin sur les facteurs socio-économiques des variations de la mortalité infantile d'un secteur à l'autre de la ville. 191

Une quatrième catégorie d'études urbaines comprend les travaux d'urbanistes et les dossiers urbains préparés avec la collaboration d'économistes ou de sociologues en vue de la mise au point de plans directeurs d'urbanisme. Il s'agit d'une documentation souvent originale, mais à caractère essentiellement descriptif, comme le sont d'ailleurs la plupart des études que nous avons énumérées jusqu'à présent. Les travaux que nous rangeons dans cette quatrième catégorie d'études urbaines sont déjà relativement nombreux, si l'on tient compte du fait que l'urbanisme vient à peine de naître chez nous ; les documents existants se rapportent aux villes de Montréal, Québec, Sherbrooke, TroisRivières, Granby, Rimouski, Nicolet, Sainte-Marie de Beauce et à quelques autres. 192

191 Jacques HENRIPIN, « L'inégalité sociale devant la mort : la mortinatalité et la mortalité infantile à Montréal », Recherches sociographiques, II, 1, janviermars 1961, 3-34.

192 Parmi ces documents, mentionnons : A. COUSINEAU, Urbanisation de Montréal. Plan directeur. Rapport préliminaire. Montréal, Service d'urbanisme, 1944, 60 p. ; Jacques GRÉBER, Edouard FISET, Roland BÉDARD, Projet d'aménagement de Québec et de sa région, Québec, s.éd., 1956, 72 p. (miméo.) ; Jean-Claude LA HAYE, Le plan directeur de la Cité de Sherbrooke, Sherbrooke, s.éd., 1960, 3 vol. ; Agence d'urbanisme BÉGIN et ROBERT, " plan directeur de la cité des Trois-Rivières », Architecture, 16, 184, août 1961, 38-50 ; Benoît-J. BÉGIN et Georges ROBERT, «Résumé du rapport d'accompagnement du Plan directeur d'urbanisme de la ville de Granby », Architecture, 15, 173, septembre 1960, 288-293 ; Yves DuBÉ et Yves MARTIN, "Rimouski : population et économie ; évolution et perspectives », Recherches sociographiques, I, 3, juillet-septembre 1960, 269-308 ; André 
Nous n'avons pas à insister sur l'importance que l'on doit attacher au phénomène de l'urbanisation comme facteur fondamental de transformation de notre société. Il s'agit tout simplement de se rendre à l'évidence : au moins les trois-quarts de la population de la province habitent des localités urbaines, et bien peu de secteurs échappent à l'influence dominante des centres urbains. Or, il est assez étonnant de constater l'absence presque totale d'études d'ensemble sur un aspect quelconque de l'urbanisation de notre milieu. Même sur le plan strictement démographique, le processus de la concentration de la population n'a pas encore fait l'objet d'analyses systématiques. Chez les géographes, les essais de synthèse ou les [125] travaux de portée générale sont rares. Raoul Blanchard, le seul chercheur qui ait étudié de près toutes les villes de la province, propose une synthèse très décevante dans le chapitre intitulé " Villes et campagnes » de son récent ouvrage sur Le Canada français. ${ }^{193}$ Les recherches que poursuit actuellement Louis Trotier sur le réseau urbain de la province 194 combleront une lacune importante. On ne trouve pas non plus beaucoup d'études d'ensemble parmi les travaux de sociologues. Dans plusieurs de ces travaux - ceux de Everett C. Hughes, 195 de Jean-C. Falardeau, 196 de Philippe Garigue, 197 de Marcel Rioux, 198 et d'autres chercheurs, -

RoBitAille et autres, Sainte-Marie de Beauce, études d'urbanisme, Québec, Les Presses Universitaires Laval, 1955, 24 p.

193 Raoul Blanchard, Le Canada français. La province de Québec, Paris et Montréal, Librairie Arthème Fayard, 1960, 247-283.

194 On trouvera quelques résultats de ces recherches dans: Louis TROTIER, «Some Functional Characteristics of the Main Service Centers of the Province of Québec », dans : Mélange. géographiques canadiens offerts à Raoul Blanchard, Québec, Les Presses Universitaires Laval, 1959, 243-259.

195 Everett C. Hughes, Rencontre de deux mondes, op. cit.

196 Voir, en particulier, les textes suivants de Jean-C. FALARdEAU: "The Changing Social Structures », dans Jean-C. Falardeau, éd., Essais sur le Québec contemporain, Québec, Les Presses Universitaires Laval, 1953, 101-122 ; «Les Canadiens français et leur idéologie », dans : Mason Wade (sous la direction de), La dualité canadienne, Québec, Les Presses Universitaires Laval ; Toronto, University of Toronto Press, 1960, 20-38.

197 Voir, en particulier : Philip GARIGUE, « French Canadian Kinship and Urban Life ", American Anthropologist, 58, 6, December 1956, 1090-1101 ; Philippe GARIGUE, «The French-Canadian Family », dans : Mason Wade (sous la direction de), op. cit., 181-200. 
on trouverait évidemment des éléments d'interprétation du phénomène de l'urbanisation au Canada français, mais cela serait vrai aussi de bien d'autres études relevant d'autres disciplines.

Même si nous avons défini de façon assez restrictive le champ des études urbaines et même si notre inventaire n'a sûrement pas été vraiment exhaustif, nous nous sommes toutefois trouvé devant une masse assez considérable de matériaux - de nature et de qualité très diverses, bien entendu. Pour rendre compte de façon entièrement satisfaisante du «travail fait » en ce secteur de la recherche, il nous aurait fallu procéder à une analyse systématique de chacun des documents inventoriés ; nous avons dû nous en tenir à l'examen d'un échantillon, probablement assez représentatif, de ces documents.

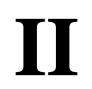

Il n'y a pas au Canada français, à proprement parler, une tradition de sociologie "urbaine ». Aux yeux du sociologue tout au moins, il s'agit moins de songer à une réorientation qu'à une orientation, en ce qui concerne la recherche urbaine dans notre milieu. Afin d'en arriver à formuler quelques propositions en ce sens, nous pouvons prendre appui, nous semble-t-il, sur une double constatation. D'une part, de plus en plus fréquemment - [126] à juste titre, d'ailleurs, — les sociologues et aussi divers essayistes proposent des hypothèses générales sur l'urbanisation en tant que facteur fondamental de la transformation de la société canadienne-française. ${ }^{199}$ D'autre part, il existe déjà

198 Marcel Rioux, « Kinship Recognition and Urbanization in French Canada », Contributions to Anthropology, 1959, Bulletin n 173, 1961, 1-11.

199 Nous pourrions citer ici tous les essais écrits sur le Canada français, surtout depuis une dizaine d'années. C'est évidemment l'un des thèmes centraux des Essais sur le Québec contemporain, op. cit. Mentionnons aussi, parmi bien d'autres travaux : Gérard PELletiER, «D'un prolétariat spirituel », Esprit, 20, 8-9, août-septembre 1952, 190-200 ; Pierre Elliott TRUDEAU, « La province de Québec au moment de la grève », dans : Pierre Elliott Trudeau (sous la direction de), La grève de l'amiante, Montréal, Les Éditions de Cité Libre, 1956, 1-91 ; Fernand DuMONT et Guy RocHER, "Introduction à une sociologie du Canada français », dans : Le Canada français, aujourd'hui et 
un bon nombre d'études, assez disparates à vrai dire, portant sur les milieux urbains de la province de Québec. Entre les hypothèses générales et les études particulières, les liens sont toutefois rarement explicites : c'est là du moins l'impression d'ensemble qui se dégage du bilan schématique que nous venons de présenter.

Ce bilan nous révèle que l'on a beaucoup écrit sur nos villes. Pourtant, l'un des auteurs dont nous avons mentionné le nom, Jean-C. Falardeau, observait récemment : « Nous connaissons encore mal ce qu'a été pour des milliers et des milliers de Canadiens français le choc graduel ou brutal engendré par leur émigration vers les villes et tout spécialement vers l'agglomération montréalaise qui contient à elle seule plus de la moitié de la population urbaine québécoise. » 200 C'est là, en réalité, poser le problème général de notre connaissance du processus de l'urbanisation et de ses conséquences particulières dans notre milieu, de notre connaissance de la culture qui est en voie de devenir celle de l'ensemble de la société canadienne-française.

Pour le sociologue, les études urbaines doivent-elles, dès lors, se confondre avec les études sociologiques générales sur notre milieu ? En un sens, oui. C'est essentiellement à travers l'analyse de milieux urbains que nous pourrons arriver à connaître un peu en profondeur les traits actuels de notre société et, en particulier, le contenu de la culture qui se diffuse à partir des foyers urbains.

Défini de cette façon, le « travail à faire » paraîtra immense. Il l'est en réalité. La première tâche doit consister en un effort pour établir un ordre de priorité entre les problèmes à étudier ou, tout au moins, pour discerner certains problèmes spécifiques dont l'analyse incomberait à ceux qu'intéresse plus immédiatement la recherche urbaine. Nous ne soumettrons ici, dans cette optique, que de brèves suggestions.

Même sur l'urbanisation vue strictement comme processus de concentration de la population, nos connaissances sont encore très limitées. Pour la province de Québec, une description systématique des changements dans la répartition territoriale de la population reste à faire. Nulle part, on ne [127] peut trouver un exposé un peu complet

demain, Paris, Librairie Arthème Fayard, 1961, 13-18 (Recherches et débats, cahier $n^{\circ} 34$ ).

200 «Les Canadiens français et leur idéologie », dans : Mason Wade (sous la direction de), op. cit., 36-37. 
sur la croissance des divers types de localités (municipalités rurales, villages, villes, banlieues, etc.) dans la province. Une telle description constituerait une entreprise assez facilement réalisable ; à la rigueur, il pourrait suffire d'exploiter minutieusement les données des recensements. On peut mentionner, à ce sujet, que le Bureau fédéral de la statistique a prévu une étude de révolution urbaine au Canada parmi les travaux de recherche qui seront préparés à partir des résultats du recensement de 1961.

Sur le plan de l'analyse, l'un des principaux objectifs des chercheurs devrait être, nous semble-t-il, de préciser le rôle des villes dans la dynamique du peuplement de notre province, de même que dans le processus global d'intégration et de structuration du territoire et de la société elle-même. Cette démarche permettrait de mettre en lumière la hiérarchie des villes selon les fonctions qu'elles exercent et la contribution qu'elles apportent à l'aménagement du territoire comme à l'aménagement de l'ensemble de la structure sociale. On voit tout de suite que la liaison ne peut être que très étroite entre les études urbaines et les études régionales. La délimitation des régions suppose, en effet, la même démarche.

La prédominance de Montréal dans l'ensemble du réseau urbain de la province n'a pas, bien entendu, à être démontrée. Il faut toutefois considérer comme fondamentale, du point de vue de la recherche urbaine, l'analyse de ce phénomène et de ses implications. Guy Rocher a déjà formulé la même proposition dans le premier fascicule des Contributions à l'étude des sciences de l'homme, paru en 1952 :

«Si l'on prend en considération, écrivait-il, l'influence que la région métropolitaine de Montréal, principal centre de diffusion de la culture urbaine dans la province de Québec, peut avoir dans le tableau de la culture canadienne-française et sur les changements sociaux et culturels que l'on ne peut manquer de constater dans cette province, il semble de grande importance d'entreprendre une étude attentive et détaillée de la culture urbaine qui s'est créée ou se crée à Montréal et se répand hors de Montréal. (...) Une analyse de Montréal serait basique en ce qu'elle servirait à déterminer la zone ou les zones d'influence de ce centre de diffusion, les canaux de communication qu'emprunte cette diffusion, et enfin par opposition elle 
aiderait à dégager les autres aires culturelles qui semblent exister dans cette province. » 201

Une meilleure connaissance de la différenciation des milieux urbains, selon leurs fonctions et leur influence en tant que pôles dans l'aménagement de l'espace et de la société, permettrait ainsi de situer par rapport à un cadre général d'interprétation les recherches proprement monographiques portant sur l'une ou l'autre des agglomérations de la province. De façon générale, ces études particulières seront fructueuses dans la mesure où elles contribueront à nous éclairer sur les caractéristiques du phénomène de l'urbanisation [128] dans notre milieu et, en même temps, sur le type nouveau de société dans laquelle nous vivons. À cette fin, on devra surtout attacher plus d'importance qu'on ne l'a fait jusqu'à présent, dans les monographies urbaines, à l'étude de l'organisation sociale et à l'étude du " mode de vie » ou de la culture. Il serait souhaitable que des sociologues, des anthropologues et des psychologues sociaux entreprennent des recherches approfondies, sur ces éléments de la réalité sociale, dans un échantillon d'agglomérations ou de zones sociales à l'intérieur de grandes agglomérations. Idéalement, un tel échantillon devrait représenter les divers types d'habitat urbain dans la province. Les moyens qu'on peut mettre en œuvre étant limités, il faut songer à des projets moins ambitieux. Pour notre part, nous soulignerons l'intérêt tout spécial qu'offrirait l'étude des banlieues nouvelles qui se multiplient autour de nos grandes villes. Comme dans une sorte de laboratoire, on trouverait là des conditions particulièrement favorables pour l'étude sociologique des modes d'organisation sociale et des modes de comportement caractéristiques du stade actuel de l'urbanisation dans notre milieu.

En terminant, sans plaider pour une discipline particulière, nous voudrions insister sur l'importance que l'on devrait attacher à l'intensification de la recherche portant sur les milieux urbains de la province. C'est par là, essentiellement, nous l'avons souligné, que nous arriverons à connaître la société canadienne-française d'aujourd'hui. En même temps, et c'est aussi une exigence qu'il ne faut pas perdre de

201 Guy RochER, «Industrialisation et culture urbaine. Note préliminaire à l'étude de la région métropolitaine de Montréal », Contributions à l'étude des sciences de l'homme, 1, 1952, 167. 
vue, nous contribuerons à une tâche urgente, celle de l'aménagement des cadres de la vie sociale dans les agglomérations où habite maintenant la plus grande partie de la population. Déjà, des problèmes sont posés aux chercheurs, par les urbanistes et d'autres responsables du développement des collectivités urbaines. Ceux-ci comptent à bon droit, pour résoudre ces problèmes, sur l'apport des chercheurs.

Yves Martin

Département de sociologie et d'anthropologie, Université Laval. 


\title{
COMMENTAIRE
}

\author{
Les études urbaines : \\ le point de vue géographique
}

Louis Trottier

Institut de géographie, Université Laval.

[pp. 129-131.]

$\underline{\text { Retour à la table des matières }}$

Après avoir dressé le bilan général des études urbaines dans le Québec, monsieur Martin s'est efforcé, dans son exposé, d'orienter la recherche urbaine dans notre milieu, du point de vue sociologique. Je me contenterai, pour ma part, de faire quelques suggestions sur les études urbaines à faire, du point de vue géographique. Le point de vue géographique, c'est essentiellement le point de vue planétaire, de sorte que, en définitive, le but de l'étude géographique des villes, c'est la description et l'explication du fait urbain à l'échelle mondiale. D'autre part, pour le géographe, la ville est à la fois un centre de relations et un paysage complexe, formé de multiples combinaisons dont les éléments sont reliés entre eux plus ou moins étroitement ; le géographe étudie donc les phénomènes urbains, non pour eux-mêmes, mais dans leurs rapports avec les autres phénomènes spatiaux. C'est dans cet esprit que devront être entreprises les études de géographie urbaine au Québec. 
Monsieur Martin a dit tout à l'heure qu'il n'y a pas au Canada français, à proprement parler, une tradition de sociologie urbaine ; il n'y a pas non plus chez nous une tradition de géographie urbaine. Dans un sens, les travaux de monsieur Blanchard, qui ne débouchent pas sur des problèmes, ont peut-être rendu aux géographes canadiens-français un mauvais service, en leur donnant l'impression que tout était dit, et cela pas seulement sur les problèmes urbains. Au fond, les géographes sont peut-être encore plus mal partagés que les sociologues, car ils ont très peu d'hypothèses qui pourraient servir à orienter leurs recherches. C'est pourquoi je devrai me contenter d'un tour d'horizon beaucoup trop théorique et beaucoup trop général des recherches géographiques à faire sur les villes du Québec.

Parmi ces recherches, les plus importantes, pour les géographes comme pour les sociologues, sont les recherches d'ensemble, portant sur l'organisation urbaine du Québec. Ces études géographiques du réseau urbain du Québec devront comporter essentiellement l'étude comparative des fonctions des villes qui en sont les éléments, afin de comprendre les relations de domination, de complémentarité ou de concurrence qui existent entre elles ; l'étude de la localisation des villes dans la région; et celle de l'organisation de l'espace urbanisé. Il s'agit en somme de voir d'abord comment les villes se sont hiérarchisées pour exercer ces fonctions de relations qui sont leur raison d'être. Ces recherches impliquent donc non seulement l'analyse du fondement économique des villes, mais aussi l'étude de leurs zones d'influences respectives, sur les plans économique, administratif et culturel. Jusqu'à quel point le réseau urbain du Québec, composé d'une multitude de petites villes, d'une ville moyenne et d'une grande métropole, est-il hiérarchisé ? Des réseaux secondaires, dominés par des centres intermédiaires, des villes-relais de la métropole, se sont-ils individualisés dans les diverses parties de la province ? Dans quels domaines la domination par Montréal est-elle la plus importante ? Quels sont les facteurs qui ont situé les villes à un endroit plutôt qu'à un autre ? Y avait-il, avant l'industrialisation de la province, un réseau de villes déjà constitué ? Dans quelle mesure l'industrie a-t-elle été la cause d'un reclassement de la situation de ces villes ? Quels sont, d'autre part, les facteurs qui expliquent les caractéristiques de [130] l'organisation de l'espace, à l'intérieur des villes du Québec ? Quelle est la part de l'influence de l'ancienneté, de la fonction originelle, du site, de 
la situation, de la dimension, des fonctions actuelles sur les structures internes des villes ? Voilà les principaux problèmes, me semble-t-il, que ces études géographiques doivent résoudre. Le but ultime de ces études, c'est la comparaison du réseau urbain du Québec aux autres réseaux urbains du monde, et en particulier à ceux qui appartiennent à la même grande série de villes, celle de l'Amérique du Nord. En somme, les géographes québécois doivent arriver à replacer les phénomènes urbains du Québec dans des classifications géographiques universelles.

Les études d'ensemble sur les villes de la province de Québec doivent être menées parallèlement à des études portant sur des villes particulières, car les deux groupes de travaux s'éclairent les uns les autres. Les études géographiques, qu'elles portent sur les fonctions ou sur l'organisation de l'espace de l'agglomération urbaine ou d'un secteur de l'agglomération, doivent être des « contributions à la définition de l'ensemble qui est la ville ou le réseau tout entier. » Les monographies géographiques des villes du Québec, monsieur Martin l'a dit il y a quelques minutes, sont beaucoup plus nombreuses que les études d'ensemble. Cependant ces monographies, de valeur d'ailleurs inégale, celles de Blanchard étant de loin les meilleures, sont sans cesse à refaire car la réalité, et la réalité urbaine surtout, est mouvante. Parmi les études géographiques à entreprendre, il faut évidemment placer au premier rang celles portant sur l'agglomération montréalaise. Bien que, grâce à Blanchard, la métropole canadienne soit assez bien connue des géographes, il est assez extraordinaire que pas un seul géographe canadien-français n'ait encore publié une étude importante sur ce complexe géographique le plus important du Canada français qu'est Montréal. Un des aspects géographiques les plus importants de l'agglomération montréalaise, et qui mérite d'attirer de plus en plus l'attention des géographes aussi bien que des sociologues, c'est ce phénomène d'expansion périphérique qui se fait à un rythme extrêmement rapide. Les conséquences géographiques de cette expansion sur les régions rurales ou semi-rurales et sur les petites villes entourant Montréal m'apparaissent devoir être signalées comme sujet d'étude prioritaire. On peut également signaler, parmi les études particulières les plus intéressantes à faire, celle de la conurbation que constituent Chicoutimi, Arvida et Jonquière-Kénogami, étude qui a d'ailleurs déjà été amorcée. 
Valables en elles-mêmes, dans la mesure où elles constitueront un apport à la géographie universelle, ces études géographiques sur les villes du Québec seront sans doute utiles aux historiens, démographes, sociologues, économistes et urbanistes du Canada français, car seules des recherches collectives peuvent mener à une connaissance globale des villes. Mais c'est surtout, me semble-t-il, dans la mesure où elles faciliteront la compréhension des problèmes régionaux que ces études de géographie urbaine constitueront un apport à la connaissance globale du Canada français. En effet, la seule région véritable et par conséquent le cadre normal de l'aménagement du territoire, c'est la région fonctionnelle, cohérente, dynamique, celle qui est organisée par la ville. Monsieur Grenier a montré plus tôt ce matin les difficultés qu'éprouvent les chercheurs à définir des régions au Québec. Il les a expliquées en particulier par l'insuffisance des recherches faites jusqu'à maintenant, qui, trop souvent, ont été de simples inventaires, plutôt que de véritables analyses. Je crois que ces difficultés résultent [131] peut-être aussi du fait que la régionalisation des structures québécoises ne s'est pas encore faite, et qu'au fond, la véritable région, c'est le Québec tout entier, dont le réseau urbain est l'épine dorsale et Montréal le centre nerveux. C'est une hypothèse que des recherches géographiques sur les villes québécoises devraient permettre de vérifier.

Enfin, j'ignore dans quelle mesure ces recherches de géographie urbaine seront utiles à ceux qui cherchent à décrire notre société canadienne-française. Ce n'est pas dans cette perspective, en tous cas, que se feront la plupart de ces recherches géographiques. La géographie est science humaine, bien sûr, car c'est l'homme qui est la mesure de l'importance des phénomènes en géographie, mais, comme l'a dit Vidal de la Blache, la géographie n'est pas la science des hommes, c'est la science des lieux.

Louis Trotier

Institut de géographie, Université Laval. 
Situation de la recherche sur le Canada français

II. Perspectives écologiques

\author{
Jacques Henripin \\ Département de science économique, \\ Université de Montréal

\section{“Les études démographiques”.}

[pp. 133-141.]

$\underline{\text { Retour à la table des matières }}$

Des commentaires qui vont suivre, assez peu seront consacrés aux recherches passées et davantage à des rêves sur l'avenir aux charmes desquels je souhaite vous faire participer. Cette disproportion entre le passé et l'avenir est d'ailleurs une image bien atténuée de la disproportion qui existe entre ce qui a été fait et ce qui devrait être fait dans le domaine des études démographiques sur le Canada français.

\title{
1. Les premières études : de 1934 à 1952
}

L'acte de naissance de la recherche démographique sur le Canada français date de 1934, année de parution du livre de Georges Langlois, Histoire de la population canadienne-française. Ce livre constituait une importante contribution à la connaissance de l'évolution de cette population, depuis son origine. Avec ce livre, on a su que l'étonnant bourgeonnement de la population canadienne-française — elle s'est 
multipliée par 90 entre 1760 et 1960 alors que la population du monde se multipliait par 4 seulement — n'était pas un miracle, mais s'expliquait, à partir d'une immigration faible, par des taux de natalité et de mortalité qui n'avaient rien de surnaturel. Avec ce livre, la démographie des Canadiens français passait de la mythologie à la connaissance empirique.

Apparemment, la petite enfance de cette démographie a été fort peu tapageuse : peu d'études ont apporté des éléments nouveaux jusqu'à 1953 environ. Il y en eut, tout de même, et qui méritent d'être mentionnées. Je ne peux, ici, en faire une liste complète, mais j'en signalerai quelques-unes, ne serait-ce que pour montrer le caractère presque fortuit de leur apparition.

En 1942, George Sabagh a publié, dans l'American Journal of Sociology, une étude sur la fécondité des couples canadiens au XVII ${ }^{\mathrm{e}}$ siècle. C'est d'ailleurs très souvent de l'étranger que nous viennent les travaux faisant appel à des techniques d'analyse un peu élaborées.

En 1943, Léon E. Truesdell, directeur des statistiques de population au Bureau of the Census des États-Unis, publiait The CanadianBorn in [134] the United States. 202 On y retrouve plusieurs informations concernant les Canadiens français émigrés aux États-Unis, pour la période 1890-1930.

La même année, $\mathrm{M}^{\mathrm{e}}$ Ross livrait une étude sur les effets culturels des mouvements de population dans les Cantons de l'Est 203 et $\mathbf{M}^{\text {me }}$ Charles écrivait un article sur la fécondité différentielle au Canada. 204 Ce dernier auteur devait, en 1948, apporter une contribution importante à la connaissance de la fécondité des Canadiens français, à l'occasion de la monographie de recensement The Changing Size of the Family In Canada. ${ }^{205}$ Exploitant avec minutie les informations particulières recueillies à l'occasion du recensement de $1941, \mathrm{M}^{\text {me }}$ Charles arrivait à estimer l'influence, sur la fécondité de certains groupes culturels, de facteurs comme la religion, l'instruction, l'habitat, la profession. Les résultats de son analyse permettaient en outre de compa-

202 New-Haven, Yale University Press, et Toronto, The Ryerson Press.

203 Canadian Journal of Economics and Political Science, November 1943, 447-462.

204 Canadian Journal of Economics and Political Science, May 1943, 175-218.

205 Ottawa, Imprimeur du Roi, 1948. 
rer le rôle de ces facteurs chez les Canadiens français et chez d'autres groupes culturels. Mais il faut ajouter que les renseignements les plus intéressants se rapportaient à des couples formes avant 1920.

Deux autres contributions intéressantes nous viennent de France. En 1947, les démographes Henry et Vincent estiment la fécondité des couples du Québec vers 1850 ; 206 cependant, leur objectif n'est pas de connaître cette fécondité pour elle-même, mais de trouver une mesure de la fécondité naturelle d'une population humaine. Un autre Français, Pierre Dumareau, est l'auteur d'un article de L'Actualité économique, paru en 1952, où l'avenir démographique des Canadiens français est supputé. 207

Je signalerai enfin une autre étude, aussi intéressante qu'inattendue pour celui qui, après coup, essaie de dresser un tableau de ces efforts sporadiques: il s'agit du travail de Falardeau et Lamontagne, "The Life Cycle of the French-Canadian Urban Families ». ${ }^{208}$ Les auteurs y estiment l'évolution comparée des revenus et des charges économiques des familles urbaines du Canada français, au cours de la vie de ces familles.

Même si cette énumération est incomplète, je crois qu'on ne trouverait pas beaucoup d'autres études démographiques du Canada français, ayant un caractère analytique, avant 1953.

\section{L'essor de la dernière décennie}

À partir de 1953, un certain essor de la recherche démographique est décelable. Rien encore de systématique, mais le feu est plus nourri. [135] Entendons-nous : il s'agit plus du tir occasionnel de chasseurs qui, sporadiquement, mettent en joue des objectifs démographiques, que de l'envol d'une escadrille balayant systématiquement de larges espaces de l'univers démographique. Cet essor, je le ferais

206 « Rythme maximum d'accroissement d'une population stable », Population, 2, 4, octobre-décembre 1947, 663-680.

207 «L'aspect et l'avenir démographiques du Canada français », L'Actualité économique, 28, 1, avril-juin 1952, 5-26.

208 Canadian Journal of Economics and Political Science, 1947, 233-247. 
volontiers débuter avec le recueil de textes édité par Falardeau : Essais sur le Québec contemporain. ${ }^{209}$ Keyfitz y faisait une analyse de quelques problèmes majeurs de la population du Québec : croissance régionale de la population, proportion des Canadiens français, migrations rurales-urbaines, structure de la population active et fécondité des couples vivant dans divers milieux.

Je ne veux pas m'engager dans l'énumération des travaux qui ont suivi. On y trouve quelques études où la préoccupation démographique est nettement dominante ; mais dans la plupart des cas, les phénomènes de population sont abordés à l'occasion d'études plus générales : monographies régionales, ou encore travaux à caractère plus pratique nécessitant la prise en considération de faits de population : les perspectives de population scolaire en sont un exemple.

Mais cette moisson n'est pas abondante. Elle n'a pas réussi, par exemple, à justifier l'existence d'une rubrique "démographie » ou " population » dans la « Revue des revues » des Recherches sociographlques.

Nous savons maintenant que la fécondité des Canadiens français tend à se rapprocher de celle des autres Canadiens ; qu'elle a beaucoup diminué dans certains milieux, à Montréal en particulier. Mais des instruments d'analyse beaucoup plus raffinés seraient nécessaires pour apprécier la signification de ce phénomène.

Par contre, la nuptialité des Canadiens français semble s'écarter de plus en plus de celle des autres Canadiens : les premiers ont participé beaucoup moins que les autres à l'accroissement de la nuptialité qu'on a observé au Canada; et le Québec est probablement la province où l'on se marie le moins.

On sait aussi que la mortalité infantile du Canada français présente un retard considérable par rapport au reste de l'Amérique du Nord. Il en va de même pour la qualité professionnelle de la population active. On a aussi mesuré l'intensité des transferts linguistiques pour chaque province, de même que la capillarité sociale.

Enfin des perspectives ont été faites pour la population des régions du Québec, mais elles négligent les migrations, facteur important.

209 Québec, Les Presses Universitaires I.aval, 1953. 
Si l'on ajoute à cela un certain nombre d'études régionales qui ont fait une place à la population - je pense, par exemple à celles d'Yves Martin sur l'Île-aux-Coudres et le Bas Saint-Laurent — on a à peu près toute la récolte des dix dernières années. Ce n'est pas négligeable, mais ce n'est pas abondant.

[136]

\section{Petit agenda des études démographiques}

Si l'on se tourne vers les tâches à accomplir, il me semble que les plus importantes peuvent se diviser en deux catégories : les travaux d'analyse démographique proprement dite et les études de certains problèmes importants dans lesquels les phénomènes de population interviennent soit comme élément d'explication, soit comme conséquence. Remarquons que cette distinction, qui me paraît commode, ne peut être respectée rigoureusement.

Examinons d'abord le premier type de travaux. Il s'agit surtout de connaître les transformations de structure de la population et les principaux facteurs de cette évolution : fécondité, nuptialité, mortalité, migrations. Ce sont là les facteurs habituellement pris en considération. Mais, pour les Canadiens français, il faut en ajouter un autre, qu'on appelle d'habitude l'assimilation, et que je préfère appeler moins élégamment, il est vrai, et à défaut d'un meilleur terme - les transferts linguistiques. Deux remarques sur ce dernier phénomène, avant d'aborder les autres : d'abord, ce facteur prendra probablement un poids de plus en plus important dans l'évolution future de la population canadienne-française ; deuxièmement, l'évolution de la proportion des Canadiens français transfuges a manifesté, au cours des trente ou quarante dernières années, une étonnante régularité, laissant ainsi soupçonner des mécanismes d'explication qu'il serait intéressant d'explorer et utile de connaître, si l'on voulait juguler cette saignée dont les perspectives semblent alarmantes, du moins en ce qui concerne la survie du fait français hors du Québec.

Voyons les autres facteurs de variation de la population. J'insisterai davantage sur la fécondité, parce qu'on trouve dans l'étude de ce phé- 
nomène une source abondante de questions conduisant à l'exploration de réalités culturelles importantes.

Si la fécondité des Canadiens français a pu être mesurée, elle n'a pas encore été étudiée profondément, depuis que cette population est entrée dans l'ère de l'infécondité volontaire, du moins si l'on fait exception de l'étude de $\mathrm{M}^{\text {me }}$ Charles, dans le cadre du recensement de 1941. Mais dans un domaine aussi mouvant, des connaissances relatives aux couples formés autour de l'année 1915 ne peuvent guère expliquer la réalité actuelle. Le dernier recensement sera probablement accompagné d'une étude importante de la fécondité au Canada et cette étude permettra sans doute de connaître beaucoup de choses sur la fécondité des couples canadiens-français. On pourra savoir comment la fécondité varie d'un milieu géographique à l'autre, de même qu'en fonction de la religion, de l'instruction, de la profession du père, etc. Cependant, ce type d'analyse est loin de répondre à toutes les questions qu'on peut se poser à propos de la fécondité. Cette analyse ne nous dit rien ni sur les moyens employés pour planifier la famille, ni sur l'attitude des couples à l'égard du nombre de leurs enfants.

[137]

Or ce sont là des problèmes beaucoup plus riches, beaucoup plus essentiels, beaucoup plus près de la vie des hommes, que ceux qu'on se plaît le plus souvent à associer à la démographie. Il y a là une série de questions d'ordre psycho-sociologique, dont l'étude apporterait des lumières fort appréciables, non seulement sur certains phénomènes essentiels (comme la perception de la charge des enfants, les obstacles que leur nombre pourrait constituer à la qualité de leur formation), mais aussi sur des attitudes, des objectifs personnels. D'une part, ces attitudes et ces objectifs reflètent la place qu'occupe la fécondité dans l'échelle des valeurs, et il serait intéressant de savoir où nous en sommes à ce point de vue ; d'autre part, ces attitudes et ces objectifs personnels peuvent révéler des conflits probablement violents entre les aspirations, les intérêts individuels ou familiaux et les préceptes moraux traditionnellement acceptés et respectés.

À cet égard, il serait intéressant de savoir par quels moyens les Canadiens français limitent leur fécondité et quelle est leur attitude à l'égard de la morale qu'impose à la plupart d'entre eux la religion catholique. Dans le même ordre d'idées, il serait souhaitable de pouvoir 
apprécier, à propos de la fécondité, la rupture entre les intérêts des couples et l'intérêt national (ou nationaliste), qui a toujours associé l'objectif des familles nombreuses au salut de la nation.

Sur l'infécondité volontaire des Canadiens français, d'autres questions se posent qui méritent examen : par quel cheminement ou par quel mécanisme une grande partie des couples canadiens-français en sont-ils venus à adopter un comportement restrictif quant aux enfants «que le Bon Dieu voulait leur envoyer »? Et, dans ce cheminement, quel a été le pouvoir de résistance de l'adhésion à la religion catholique ? C'est là une question fort délicate, mais si l'on pouvait connaître quelque chose là-dessus, on y découvrirait peut-être des éléments fort intéressants - peut-être rebutants, aussi, — à propos de l'emprise de la morale catholique sur l'un des aspects les plus déterminants des conditions de vie des familles.

Autre question : quel écart y a-t-il entre le nombre souhaité d'enfants et le nombre obtenu ? Plus particulièrement, quelle est l'efficacité des moyens adoptés - quand il y en a - pour limiter le nombre des enfants ? Cette question - comme beaucoup d'autres d'ailleurs que j'ai déjà mentionnées - a un intérêt certain au point de vue de l'analyse purement démographique, car elle permet de se faire une idée de la dimension qu'auraient les familles si les couples devenaient plus efficaces en ce qui concerne la réalisation des objectifs qu'ils se fixent. Cette question rejoint une préoccupation qui commence à se faire jour et qui concerne l'ensemble du monde contracepteur : comment assurera-t-on le maintien des populations, le jour où les couples disposeront d'un contraceptif parfait et consentiront à l'employer ? L'humanité passera peut-être beaucoup plus vite qu'on ne l'imagine de la crainte du surpeuplement à celle du dépeuplement.

\section{[138]}

Je ne peux pas me permettre de m'étendre aussi longtemps sur les autres phénomènes qui entrent d'emblée dans le champ de l'analyse démographique : mortalité, nuptialité et migrations. Sur la mortalité de l'ensemble de la population, nous savons fort peu de choses, sauf que les Canadiens français résistent moins facilement aux assauts de la mort que les autres Canadiens. Il faudrait savoir pourquoi et, en particulier, si des facteurs génétiques sont en jeu, comme cela semble probable pour la mortalité des enfants de moins d'un an. En ce qui 
concerne la population canadienne-française, tout le domaine de la génétique de population est en friche. Il ne concerne d'ailleurs pas que la mortalité, mais aussi certaines maladies ou difformités. La qualité des registres paroissiaux et la documentation généalogique de l'Institut Drouin devraient faciliter grandement de telles recherches. M. Yves Martin a d'ailleurs écrit là-dessus quelques pages fort pertinentes, que je ne reprendrai pas. 210

Cela impliquerait une étude de la nuptialité, sur laquelle nous ne savons rien, sauf ce qu'a apporté l'étude de $\mathrm{M}^{\mathrm{me}}$ Charles. Il serait intéressant de connaître les causes de l'infériorité de la nuptialité des Canadiens français par rapport à celle des autres Canadiens, phénomène qui est apparu vers 1920 et qui semble aller en s'accentuant.

Il faudrait aussi savoir à quels phénomènes conjoncturels la nuptialité est sensible, quels facteurs influent sur le choix des conjoints ; il faudrait savoir si les unions ont tendance à devenir plus hétérogènes ou plus homogènes, aux points de vue de l'origine ethnique, de la religion, de l'appartenance géographique ou sociale.

La synthèse de ces connaissances des éléments démographiques fondamentaux permettrait d'établir des perspectives ou des prévisions sur la masse et la structure de la population future, beaucoup mieux assises que ce qu'on peut faire en prolongeant des tendances passées. Ces connaissances permettraient aussi d'établir un schéma de ce que les Anglo-Saxons appellent le family cycle, schéma montrant, pour une famille typique, la durée du mariage, du veuvage et les variations de la charge des enfants, des besoins et des ressources économiques, au cours de la vie des couples. Ces deux résultats de l'analyse démographique pure (prévisions démographiques et schéma des phases de la vie familiale) seraient de la plus haute utilité pour une société en voie de socialisation, comme le Québec. De telles données sont en effet à la base de toute politique de sécurité sociale et d'économie concertée ou dirigée.

Les migrations, elles aussi, constituent un élément important de la politique économique. Les migrations interrégionales doivent intervenir, d'ailleurs, dès qu'on veut faire des prévisions de population par

210 «Sociologie, démographie et génétique de population », Recherches sociographiques, II, 2, avril-juin 1961, 257-260. 
région. [139] Même si les migrations futures sont difficiles à estimer, il peut être utile de voir ce que donne un éventail d'hypothèses vraisemblables.

\section{Quelques problèmes démo-sociaux en friche}

Les problèmes que je viens de mentionner débordent, bien sûr, les frontières du domaine purement démographique. Il en va d'ailleurs à peu près toujours ainsi, avec cette curieuse discipline qui n'a que bien peu de prise sur les mécanismes qui expliquent les phénomènes qui l'intéressent. Mais j'ai essayé de me confiner le plus possible aux phénomènes sur lesquels, par principe ou par habitude, la démographie s'interroge. Il est bien d'autres problèmes, cependant, qui, tout en appartenant par priorité à d'autres disciplines, n'en sont pas moins liés à des faits de population qui peuvent en modifier la physionomie ou apporter à leur sujet des éléments d'explication. L'influence de la dimension des familles sur la qualité de la formation des enfants et sur leur niveau intellectuel, la prise en considération nécessaire des perspectives de population dans toute politique économique, sociale ou scolaire, en sont des exemples évidents.

Autre exemple : je ne sais pas à quelle discipline appartient surtout l'élaboration d'une politique d'aide familiale ou d'allocations familiales — probablement à aucune plus particulièrement —, mais il est évident que la très grande inégalité des charges imposées par les enfants, tant d'un individu à l'autre, d'une classe sociale à l'autre, que d'une période à l'autre au cours de la vie d'un même individu, met en cause des évaluations d'ordre démographique, pour autant qu'on veuille estimer l'effet correcteur de telle ou telle mesure envisagée.

On pourrait facilement allonger la liste des problèmes sociaux faisant intervenir des phénomènes de population. Je me permettrai seulement d'insister sur l'un d'entre eux : le chômage. Résultat d'une inadaptation entre l'offre et la demande de population active, la structure de celle-ci aussi bien que son mouvement doivent intervenir, soit pour expliquer le chômage, soit pour le prévenir. C'est presque un truisme, mais ce truisme a l'avantage de replacer les choses dans un cadre plus général que celui dans lequel le problème est habituellement posé : le 
chômage évoque très couramment l'idée d'un excès de population active, du moins d'une certaine catégorie de population active ; mais il est peut-être tout autant le résultat d'une insuffisance d'autres catégories de travailleurs ou de population. C'est tout le problème de la prévision économique et de la planification de l'enseignement qui est évoqué ici, à propos du chômage structurel.

Le chômage cyclique comporte aussi de multiples implications démographiques, dont je ne signalerai que deux aspects. Le premier concerne les variations de la population active. À cause des irrégularités de la pyramide des âges, le nombre des entrées et des sorties de la population active [140] peut entraîner des variations relativement importantes du taux de croissance de l'effectif des travailleurs et rien n'assure que les variations de la demande de travail s'adapteront à ces fluctuations. L'autre point névralgique des relations entre la population et le chômage concerne l'influence des facteurs démographiques sur la demande de logements. Les variations de la mortalité et de la nuptialité exercent une influence sensible sur les besoins de logements. Or, ceux-ci constituent une part importante des investissements et le rôle dominant de ces derniers dans l'explication des variations du revenu national est généralement accepté par les économistes. Ce ne sont là que quelques aspects des relations entre le chômage et les faits de population. Peut-être suffisent-ils à suggérer l'intérêt qu'il y aurait à aborder l'étude de cet obsédant problème en faisant une large place aux facteurs démographiques. 


\section{CONCLUSION}

Si, pour me résumer, je tentais de caractériser l'intérêt des tâches à accomplir par la démographie à propos du Canada français, il me semble que cet intérêt, on le verrait apparaître facilement autour de deux types principaux de contributions de cette discipline à la connaissance de notre milieu. D'une part, on trouve des travaux dont les résultats constituent des éléments importants d'explication ou de prévision de phénomènes plus généraux. D'autre part, les préoccupations démographiques débouchent sur des problèmes fondamentaux de civilisation et de culture à propos desquels la démographie peut poser des questions fécondes. C'est précisément le cas de l'étude de la fécondité.

Il n'est pas besoin de longue démonstration pour établir qu'à l'heure présente, la recherche démographique, dans notre milieu, est importante surtout par les besoins qu'on en a et les tâches qui restent à accomplir. Il existe fort peu d'études du milieu canadien-français dont l'objet principal est l'exploration de phénomènes de population. Ce qui a été fait dans ce domaine l'a été, le plus souvent, soit incidemment à l'occasion d'un problème d'un autre ordre, soit à l'occasion de quelques monographies régionales.

Il existe aussi fort peu de chercheurs. Cela pose un sérieux problème quant à la réalisation des études fondamentales et les perspectives, de ce côté, sont déterminées par des conditions dont il ne sera probablement pas facile de s'affranchir. Je m'explique. La recherche sociale a été faite surtout, jusqu'à maintenant, par des professeurs d'université. Dans une discipline donnée, le nombre de ceux-ci dépend du nombre de cours à donner et du nombre d'étudiants. Or, ces deux facteurs sont et resteront probablement très limités : je ne connais pas d'université où se donnent plus de 300 heures de cours de démographie. Cela ne fait pas beaucoup de professeurs. 
Je vois cependant trois moyens de surmonter cette difficulté :

a) créer des organismes de recherche assurant l'étude des problèmes de population ; il faudrait alors, au moins au début, faire appel à des chercheurs étrangers ;

b) inciter les étudiants à étudier ces problèmes ; mais cela reste limité ;

c) faire en sorte que les chercheurs qui, plus ou moins directement, s'intéressent à ces problèmes, se rencontrent, se concertent, afin que les ressources disponibles soient agencées le mieux possible. Il peut arriver, par exemple, que ce qui n'est possible, ni à tel chercheur isolé, ni à tel ministère, devienne réalisable avec la collaboration de ces deux parties, qui possèdent des ressources complémentaires.

Jacques Henripin

Département de science économique, Université de Montréal. 


\title{
COMMENTAIRE
}

\author{
Yves de Jocas \\ Bureau des recherches économiques, \\ Ministère de l'Industrie et du Commerce, Québec.
}

\author{
[pp. 142-144.]
}

\section{$\underline{\text { Retour à la table des matières }}$}

Si l'on s'en rapporte à l'exposé de monsieur Henripin, on est justifié, je crois, de se demander comment il se fait que les études de population en général, et les études démographiques en particulier, n'ont pas connu, au Canada français, dans le passé et même actuellement, un essor plus considérable. Il me semble que plusieurs facteurs favorisaient l'éclosion de ces études éminemment sociales.

Pour ne mentionner que deux raisons favorables à la naissance de cette discipline, on peut faire appel à l'importance du sujet et aussi à l'accessibilité de l'information.

L'importance du sujet ne fait aucun doute, comme en témoigne l'exposé que nous venons d'entendre. Si la démographie s'intéresse immédiatement à la croissance des populations fixées aux extrémités par les naissances et les décès, on ne peut oublier qu'entre ces deux pôles s'est jouée la vie d'un individu et qu'en lui et par lui s'est aussi jouée une tranche de vie d'une collectivité. Dans un milieu donné, le nombre des naissances et des décès de même que la composition par âge et par sexe sont, en très grande partie, la résultante d'actes qui, bien que posés individuellement, n'en demeurent pas moins condi- 
tionnés par le climat de l'organisation communautaire existante. Aussi, l'indice démographique est une des mesures puissantes du fonctionnement de l'organisation sociale. À ce seul titre, la démographie aurait dû avoir une place privilégiée au sein des disciplines des sciences sociales.

Il me semble qu'en plus de l'importance du sujet, la démographie aurait dû connaître un essor plus considérable si l'on songe à l'abondance et à l'accessibilité de l'information. En ce qui concerne le Canada, les recensements fédéraux datent de 1851. Les lacunes de ces inventaires de la population ne les rendaient pas pour autant dépourvus de toute utilité, loin de là. D'ailleurs ces lacunes auraient tout probablement été comblées en bonne partie, si l'on avait su tirer profit de l'information existante.

Plusieurs raisons peuvent expliquer ce retard du développement de la démographie au sein de nos Facultés universitaires et même au sein des administrations gouvernementales. Je ne crois pas qu'il faille l'attribuer à un manque d'intérêt pour le sujet en cause. Son caractère d'inventaire, requérant chiffres et taux et référant à la mathématique et à la statistique, a pu alourdir son départ. Le manque d'appareils techniques ou mécanographiques nécessaires à l'analyse a certainement contribué à ralentir son essor.

Comme raison première, cependant, j'invoquerai la jeunesse de la science sociale en général et au Canada français en particulier. S'il est vrai que les structures démographiques influencent la vie sociale, il est tout aussi vrai et peut-être plus vrai que la vie communautaire commande le déroulement démographique. Or, on connaissait trop peu de l'homme social pour pouvoir mettre à profit son dénombrement, sauf, bien entendu pour quelques fins bien spécifiques, comme en cas de guerre, de rationnement, ou d'élection. En effet, une pyramide des âges, bien qu'elle soit une synthèse du passé, du présent et de l'avenir, nous dit très peu, à moins que l'on connaisse l'organisation sociale passée, présente et à venir. Ce serait, autrement, une démographie désincarnée. Je crois, cependant, que cette [143] faiblesse des sciences sociales aurait pu être plus vite surmontée si les étudiants de la vie des sociétés humaines avaient fait un usage plus rationnel des inventaires existants sur les populations. 
D'ailleurs, trop d'étudiants en sciences sociales ont considéré l'étude des populations comme un champ spécialisé, comparable à la sociologie, à l'économie et, même à la statistique. S'il y a du juste dans cette conception, elle ne vaut, pour ma part, que pour la démographie pure et non pour l'étude des populations. L'étude démographique pure, c'est l'étude des mécanismes de la reproduction des populations. Ainsi, peut-on prévoir des modèles de démographie pure, hypothétiquement désincarnés, comme on le fait en statistique ou en économique. L'étude des populations ne saurait être aucunement désincarnée, elle porte directement sur la vie concrète. Malheureusement, l'étude des populations ne saurait se faire sans une connaissance des mécanismes démographiques.

Ce dont nous avons manqué et manquons le plus, ce n'est peut-être pas de démographes, mais de sociologues démographes, d'économistes démographes, de psychologues démographes qui pourraient passer de l'inventaire de notre population à la connaissance de notre milieu, pouvant ainsi mieux relier le monde de la théorie et de l'hypothèse au monde réel d'aujourd'hui et de demain.

À cet effet, l'exposé de monsieur Henripin porte peu à des commentaires. Ce texte, en effet, nous livre un état de faits que je crois indiscutable, à savoir : les lacunes importantes de recherches dans un champ auquel on ne saurait refuser le qualificatif de « vital ».

Je risquerais, cependant, trois commentaires sur cet exposé.

1. Si, dans l'ensemble, le texte de monsieur Henripin a su dégager l'importance du sujet traité, il a trop peu insisté, à mon sens, sur les besoins présents de la recherche démographique extra-universitaire dans les cadres d'organismes gouvernementaux ou autres; le besoin de ces recherches démographiques se fait sentir tant sur le plan mondial, comme d'ailleurs monsieur Henripin nous l'a laissé voir à l'occasion d'une série d'émissions télédiffusées, que sur le plan national et provincial. On ne peut, sur le plan national et provincial, parler de « nos » ressources et de "notre » avenir, de planification et d'aménagement, sans poser, à la base, la nécessité de l'information et de la recherche démographiques. Ici, je crois très important que les recherches démographiques entreprises en milieux administratifs et académiques soient en étroite liaison. 
2. En second lieu, j'aurais aimé trouver dans l'exposé de monsieur Henripin des exemples plus concrets quant à l'urgence du besoin d'études démographiques pour le Canada français. Monsieur Henripin fait partie des rares chercheurs en démographie s'intéressant au Canada français. Il s'est intéressé, en outre, aux problèmes de la fécondité, de la mortalité infantile, du transfert linguistique, de la comparaison du fait démographique du Canada français et du Canada anglais. S'il a omis d'inclure ses propres ouvrages dans son exposé, j'aurais aimé qu'il montre par des exemples concrets comment les points qu'il soulevait se présentaient au Canada français. En somme, j'aurais aime connaître si l'étude démographique avait une importance particulière pour le Canada français, en plus de l'importance qu'on doit lui accorder quel que soit le pays où l'on se trouve.

\section{[144]}

3. Je présenterai mon dernier commentaire sous forme d'une question, à savoir : par quel programme d'actions et de recherches peut-on, de façon concrète, répondre aux besoins urgents de la recherche démographique ? Que doit-on considérer comme recherches fondamentales et comment peut s'établir la collaboration entre les diverses personnes et institutions intéressées aux données et aux conclusions démographiques?

Yves de Jocas

Bureau des recherches économiques, Ministère de l'Industrie et du Commerce, Québec. 
Situation de la recherche sur le Canada français

II. Perspectives écologiques

\author{
Marc-Adélard Tremblay \\ Département de sociologie et d'anthropologie, \\ Université Laval

\section{"L'état des recherches sur la culture acadienne.” *}

\author{
[pp. 145-167.]
}

\title{
I. Introduction
}

$\underline{\text { Retour à la table des matières }}$

Lorsque les Acadiens désignent leur groupe, en tant que configuration culturelle distincte de toutes les autres, ils utilisent les expressions : « le peuple acadien », ou « la nation acadienne ». Ils signifient par là qu'ils ont les mêmes caractéristiques socio-culturelles, qu'ils communient aux mêmes traditions, qu'ils s'inspirent des mêmes sources idéologiques et qu'ils partagent les mêmes aspirations. Mais ce peuple, à la suite d'une cruelle expérience que nous rappellerons brièvement plus loin, vit en petits groupes sur un très vaste territoire qui s'étend au-delà des frontières provinciales et même nationales. Il existe, en effet, dans l'État de la Louisiane des communautés d'Acadiens — que l'on appelle là-bas du terme pittoresque de Cajuns — qui ont

Nous tenons à remercier Renée Carette, Yvan Ferland, Camille Richard et le Révérend Père Albert Whilelmy, s.j., qui nous ont aidé à constituer l'inventaire bibliographique. Émile Gosselin et Camille Richard ont aussi enrichi notre exposé de précieux commentaires. 
conservé quelques traditions ancestrales et qui se rattachent, par leur origine, à l'ancienne province d'Acadie. De plus, plusieurs générations d'Acadiens, venant des provinces maritimes, ont émigré vers les États de la Nouvelle-Angleterre où ils vivent, soit dans de petites villes ou soit encore dans des centres métropolitains comme Boston. Tous ces émigrés exercent des métiers urbains et vivent dans des paroisses qu'ils partagent avec les Américains d'origine québécoise et les Irlandais catholiques. Ces Franco-Américains s'identifient encore, pour un bon nombre, à la « patrie » d'origine. La déportation agit alors comme pôle d'attraction dans les mécanismes d'identification ethnique. Une grande proximité géographique qui permet des visites régulières en Nouvelle-Écosse et au Nouveau-Brunswick ainsi que l'existence de liens de parenté particulièrement resserrés - tant dans les milieux de provenance que dans les milieux de résidence — ont limité le caractère contraignant des puissantes normes d'assimilation de la culture américaine.

Un bilan exhaustif des études acadiennes devrait, bien entendu, inclure les groupements états-uniens. C'est là un objectif qui demeure [146] très lointain, tant par l'envergure de la tâche que par la nature des ressources que celle-ci exigerait. Il n'est donc pas question de nourrir une telle ambition dans le cadre de cet article. Au demeurant, le territoire considéré inclut l'Ile du Prince-Edouard, la NouvelleEcosse, le Nouveau-Brunswick, les Îles-de-la-Madeleine et la péninsule gaspésienne. Ce territoire est d'ailleurs trop vaste pour que notre bilan soit fondé sur un inventaire complet des ouvrages pertinents à une sociologie de l'Acadie. Nous devrons restreindre encore les objectifs à l'intérieur de cet espace géographique. Plusieurs circonstances nous obligent à présenter une vue « à vol d'oiseau » de ces travaux, plutôt qu'une analyse en profondeur.

Précisons, en dernier lieu, le biais par lequel nous abordons ce bilan. Depuis juin 1950, nous avons entrepris en Nouvelle-Ecosse plusieurs voyages d'études anthropologiques dans le cadre d'une recherche multi-disciplinaire en psychiatrie sociale. Nous serons alors enclin à accorder une importance particulière à ces travaux. Notre travail reflétera de plus une plus grande familiarité avec les travaux portant sur la Nouvelle-Écosse, « le berceau de l'Acadie ». Cette lacune sera heureusement compensée par la présence d'un commentateur bien qualifié et venant, par surcroît, d'une université acadienne du Nouveau- 
Brunswick. Ayant eu à négliger les documents primaires, les articles parus dans les journaux et les revues de vulgarisation, les conférences prononcées devant les publics les plus divers, je suis conscient que plusieurs travaux de grande importance ont été négligés. Il ne faut pas l'oublier, nous n'avons pas pour l'Acadie d'Essais à la manière de ceux qui existent pour le Québec. ${ }^{211}$ Nous en sommes encore à la période des premiers balbutiements. Souhaitons que toutes ces lacunes bibliographiques et que la pénurie des travaux à caractère explicatif ayant été mise à jour par notre vue panoramique soient un puissant stimulant pour instituer un Centre de la documentation acadienne où seraient compilés, pour fins de classification et d'analyse, l'universalité des documents et données disponibles sur les différents groupes acadiens. Je sais que l'Université Saint-Joseph de Moncton et le Collège SainteAnne de la Pointe-de-1'Église, en Nouvelle-Ecosse, ont déjà amorcé un tel projet. Mais sa complète réalisation nécessitera des ressources financières et humaines très considérables.

\section{Le bilan des études acadiennes}

Un rapide examen de la bibliographie que nous avons dressée fait ressortir le grand nombre de travaux centrés sur l'histoire acadienne. Plusieurs de ces ouvrages accordent beaucoup d'importance au « Grand [147] Dérangement ». Quelques auteurs font de la dispersion le thème de leur exposé. On sait qu'à ce propos les vues sont divergentes chez les historiens : cela s'explique pour une part par les perspectives théoriques à partir desquelles s'effectue la reconstruction historique et aussi par les sentiments que l'on éprouve vis-à-vis l'une ou l'autre des deux ethnies en présence.

Tous les autres travaux peuvent être regroupés dans huit catégories distinctes, directement reliées à la survivance acadienne dans les provinces de l'Atlantique. Ces catégories sont les suivantes : A. La survivance de la «nation »; B. Les Conventions nationales ; C. Missionnaires, clergé et paroisses d'Acadie ; D. Le parler franco-acadien ; E.

211 Jean-C. FALARDEAU, éd., Essais sur le Québec contemporain, Québec, Les Presses Universitaires Lava], 1953. [Livre disponible dans Les Classiques des sciences sociales. JMT.] 
Éducation et écoles acadiennes ; F. Communautés et culture acadiennes; et G. Les relations inter-ethniques et l'acculturation des Acadiens.

\section{Les travaux historiques}

Il serait prétentieux, dans le cadre de ce rapide tour d'horizon, d'effectuer une analyse critique des travaux historiques centrés sur l'Acadie. Nous laissons cette tâche aux historiens. L'observateur éclairé ne peut toutefois s'empêcher d'être mal à l'aise et confus devant la variété des interprétations de l'histoire acadienne et, plus particulièrement, de la dispersion. Les historiens "anglais », d'une part, et les historiens " français », d'autre part, ont leur version et leur interprétation des faits. Ces interprétations ont entre elles très peu de points de rapprochement.

À ce propos, signalons deux historiens, Brebner [17] * et Frégault [46] qui ont redonné, il me semble, à la déportation une très grande authenticité. Tous les deux, par des cheminements analogues, ont démontré que la déportation fut une décision longuement mûrie, qu'elle s'élabora à partir des exigences locales d'une colonisation qui s'avérait de plus en plus difficile et menacée dans son fondement même ; et qu'enfin, elle fut fortement appuyée par le Board of Trade and Plantations, de Londres. La mise à exécution d'un tel plan s'échelonna sur une période de huit années (soit de 1755 à 1763) et demeurait inachevée au moment de la signature du Traité de Paris. L'historien Frégault établit bien à partir de quelles séries de facteurs s'élabora la décision d'expulser les Acadiens de la Nouvelle-Écosse. La grande tragédie de la Nouvelle-Écosse c'est d'avoir été cédée par la France à l'Angleterre sans qu'il y ait eu de véritable conquête militaire. Après plusieurs années d'occupation anglaise, la province avait gardé son caractère français. Voici comment se sont déroulées les étapes successives qui ont abouti à la déportation. 212

[148]

\footnotetext{
* Voir la bibliographie à la fin de l'article.

212 Guy Frégault [46], 322-331.
} 
a) Les titres de propriété des Acadiens ne sont pas reconnus par l'Angleterre, à moins que ces derniers deviennent sujets de Sa Majesté en prêtant un serinent d'allégeance à la couronne britannique ;

b) Si cette prise de position acquiert un statut juridique par la décision d'un magistrat colonial, il sera alors légal de déloger ou de déposséder les Acadiens de leurs terres ;

c) Il n'y a aucune colonisation anglaise possible au NouveauMonde tant que subsistera "l'Acadie française ». C'est alors qu'on envoie, le 24 septembre 1754, un corps expéditionnaire dans le but de protéger les intérêts anglais en Acadie et de préparer la voie à la colonisation intégrale ;

d) Le gouverneur Lawrence demande 2,000 soldats de la NouvelleAngleterre afin de réduire le pivot de « l'Acadie française » (le fort de Beauséjour) ;

e) Convergence dans les politiques acadiennes du gouverneur Lawrence et la politique impériale du Board of Trade and Plantations ;

f) Après la conquête militaire des forts de Beauséjour et de Gaspareau (16 et 17 mai 1755), les événements se précipitent avec une extrême rapidité et aboutissent à la décision d'expatrier tous les Acadiens.

La décision de déplacer les Acadiens fait d'ailleurs partie d'une opération colonisatrice comportant trois phases : le déplacement, la dispersion et le remplacement. Comme le dit Frégault dans le même article,

« ... toute l'histoire de l'Amérique en est alors une de rivalités coloniales. Tous les colonisateurs se ressemblent. L'affreux épisode de la dispersion des Acadiens ne se conçoit que dans le cadre d'un conflit de colonisation ; précisons, bien que ce soit superflu : de colonisations qui entrent en conflit précisément parce qu'elles visent, en même temps, les mêmes objectifs. » 213

213 Ibid., 333. 
À mon sens, cette analyse de Frégault situe les événements tragiques de l'histoire acadienne dans des cadres plus vastes que ceux auxquels nous étions habitués. Un fait demeure. Quelle qu'ait été la nature des événements et des motifs qui ont abouti à la dispersion des Acadiens, ces derniers ont vécu une expérience traumatisante qui transparaît aujourd'hui dans leur caractère national. L'histoire de la déportation constitue un puissant mécanisme d'identification, oriente les générations présentes vers une certaine perception et compréhension de leur passé et, en dernier ressort, met l'accent sur la nécessité de la survivance. Le thème de la survivance va nous servir d'idée-maîtresse dans l'organisation des autres matériaux compilés.

Signalons, auparavant, que quelques travaux historiques récents sont centrés sur la région, en tant qu'aire territoriale et culturelle distincte. [149] Ces histoires régionales s'inspirent d'une tradition d'allure descriptive et vont permettre de constituer une précieuse documentation dans l'élaboration d'une histoire d'Acadie qui tiendra davantage compte des différences socio-culturelles entre les divers sous-groupes.

\section{IV. les travaux centrés sur la survivance}

La survivance du fait français en Acadie est expliquée par l'extraordinaire force spirituelle et morale des ancêtres, par l'intervention de la divine providence et par l'isolement social, c'est-à-dire par le fait que les communautés acadiennes ont été durant longtemps tenues à l'écart des grands courants économiques et commerciaux dominés par les entrepreneurs anglo-saxons. La langue des communications sur les plans affectif, familial, religieux et économique demeura la langue française. Aujourd'hui, les communications de masse ont rompu cet équilibre et l'élite acadienne est consciente que le plein épanouissement de la culture française ne sera possible et réalisé que si certaines exigences préalables viennent contrebalancer et freiner les nouvelles forces d'assimilation. Plusieurs générations ont été sacrifiées durant le processus de la survivance. Les leaders savent que l'Acadie ne peut plus subir de telles saignées des effectifs démographiques sans que soit compromise la perpétuation de cette aspiration nationale. On peut, il me semble, généraliser à l'Acadie tout entière les mécanismes préconisés chez les Acadiens du sud-ouest de la Nouvelle-Ecosse [Trem- 
blay, 221] par l'élite locale pour établir sur des assises permanentes les éléments de la culture. Une première série de facteurs réfèrent à la tradition et sont des mécanismes de repliement sur soi tandis que les autres sont des mécanismes d'évolution progressive :

a) La langue française et la foi catholique sont des éléments indissociables ;

b) Le mariage entre Acadiens est une condition essentielle à la conservation des traditions familiales ;

c) On doit se protéger contre tout ce qui est étranger afin d'imperméabiliser la culture acadienne contre les influences du groupe dominant ;

d) La religion est le plus important moyen de contrôle du comportement de tous les membres ;

e) Les liens de parenté resserrent la cohésion du groupe ; les intérêts du groupe ont priorité sur les aspirations personnelles ;

f) L'essor de l'élite acadienne est vu comme un moyen de renforcer la solidarité du groupe et d'assurer son progrès ;

g) L'instruction est valorisée comme moyen d'effectuer un retour éclairé aux traditions acadiennes et de permettre l'accession à des postes de commande dans tous les secteurs ; et,

[150]

h) Il est nécessaire de planifier la survivance sur une longue période afin d'assurer sa réalisation.

On aperçoit à travers ces divers mécanismes une double intention : celle de se défendre contre les influences du groupe majoritaire et celle de tracer, en tenant compte des ressources autochtones, un schéma dynamique de la survivance à l'échelle de la « nation tout entière ». Ce schéma implique aussi l'exercice d'une action importante au niveau des structures économiques, de la structure du pouvoir, à celui de la refrancisation des communications de masse et de l'établissement de tout un réseau d'institutions d'enseignement. 
Cette stratégie de la survivance et du progrès de la «nation acadienne » a été conçue par l'intelligentsia. Elle doit être perçue, comprise, assimilée par les cadres et par la masse tout entière des Acadiens avant de devenir une réalité qui aura à la fois un caractère formel et une très grande persistance.

Vue dans cette perspective, la survie posera peut-être un problème quant au choix des moyens. Si l'instruction est valorisée, si l'élite devient ouverte au progrès, y aura-t-il lieu de se protéger contre les étrangers ? L'alliance langue-foi demeurera-t-elle indissociable?

Caractérisons très brièvement les études qui ont pour thème la survivance avant d'aborder, par la suite, les diverses autres catégories qui peuvent s'y rattacher.

\section{a) La survivance de la " nation »}

Ces études établissent le bilan de la survivance acadienne dans les Maritimes à partir de l'examen systématique d'un ensemble de critères comme le rôle du clergé et de la paroisse, le rôle de l'école et de la famille et celui de la langue parlée. Mais ces différents paliers ne sont pas suffisamment examinés en tenant compte des interrelations et du faisceau des interdépendances fonctionnelles qui existent entre chacun d'eux. Les descriptions demeurent ainsi trop statiques, elles sont faites à partir de découpages trop nombreux et atteignent rarement le niveau de l'interprétation et de l'explication sociologiques. Dans un article qui vient de paraître [Tremblay, 122], nous avons voulu reconstituer dans une perspective globale l'histoire de la survivance acadienne et montrer comment, à travers l'évolution économique et sociale, s'est précisée la configuration culturelle du groupe. La survivance des traditions n'est pas seulement menacée par les contacts inter-culturels et l'exposition à une culture de masse étrangère, elle est également compromise par la transformation des sociétés historiques sous l'impact de l'évolution technologique et du progrès. Cette transformation de la petite communauté et sa participation de plus en plus étroite à la société technique moderne sont autant d'éléments à examiner et à pondérer dans une analyse compréhensive de la survivance. 


\section{b) Les Conventions nationales}

Il s'est tenu, à la fin du $\mathrm{XIX}^{\mathrm{e}}$ siècle, trois Conventions nationales acadiennes ; une dans chacune des provinces maritimes. Ces Conventions ont joué un rôle de premier plan dans l'éveil d'une conscience nationale. Elles sont à l'origine même du désir de la survivance acadienne. En effet, ces assises ont noué des relations très étroites entre les participants et ont fait naître chez eux le sentiment d'appartenance à une même communauté idéologique. Déjà, à ce moment-là, ce « jeune peuple » veut conserver son identité propre en se distinguant des autres Canadiens d'expression française et en mettant sur pied les institutions qui lui permettront de conserver son identité. Dans sa thèse de maîtrise, Camille Richard [132], par une analyse de contenu des différents discours prononcés durant la première Convention nationale, retrace les principaux courants d'idées et identifie les centres d'influence. Dans une analyse bien menée, il attire l'attention sur le rôle du clergé acadien en tant que "définisseur de situation » sur le plan d'une vision spiritualiste du monde et sur celui des exigences fonctionnelles d'une mentalité acadienne différente de toutes les autres.

\section{c) Missionnaires, clergé et paroisses d'Acadie}

L'ensemble des travaux d'histoire religieuse portent sur les missionnaires, la paroisse et le clergé et visent à définir le rôle des premiers missionnaires dans le rétablissement de la tradition française en Acadie, à reconstituer le climat de la vie paroissiale en tant que cadre d'activités religieuses et sociales et à définir, enfin, le rôle du clergé (en particulier, des différentes congrégations religieuses) dans la prise de conscience du groupe acadien, dans la revendication de ses droits et dans la protection de ses intérêts les plus chers. Notons enfin le rôle prépondérant des évêques acadiens dans le long cheminement de la survivance. $\mathrm{M}^{\mathrm{gr}}$ Leménager, évêque de Yarmouth, fut le dernier à accéder à la direction d'un diocèse acadien. Son élection à la veille des célébrations des fêtes du bicentenaire de la déportation confirmait 
d'une manière éclatante l'intention formelle de l'Église d'aider les Français du sud-ouest de la Nouvelle-Écosse dans la poursuite de leurs objectifs nationaux.

\section{d) Le parler franco-acadien}

Les travaux portant sur la langue acadienne se divisent en deux groupes : a) ceux qui traitent la langue comme un fait d'observation important et significatif en soi, c'est-à-dire comme le véhicule d'une pensée, comme médium de communication et comme symbole de culture (au sens anthropologique du terme) ; et, $b$ ) ceux qui considèrent la langue comme [152] " gardienne de la foi », c'est-à-dire comme une forme culturelle à contenu idéologique.

Dans les quelques études du premier groupe, on vise à démontrer que le parler franco-acadien est une langue parlée et non écrite et que, par conséquent, elle est transmise par la tradition orale de génération en génération et qu'elle est sujette à des transformations plus rapides. Le parler franco-acadien est aussi distinct selon les différentes régions de l'Acadie. Finalement, le parler franco-acadien se distingue de la langue parlée des Canadiens d'expression française surtout par les caractéristiques suivantes : la construction de la phrase, la conjugaison des verbes, l'accent, l'utilisation d'archaïsmes et d'expressions nautiques et l'utilisation plus fréquente d'anglicismes et de termes anglais. Les travaux de Pascal Poirier [203 et 204] sont de première importance et fournissent tous les éléments de base pour la compréhension de la langue. Mais ils devront être revisés et mis à jour comme l'a fait le linguiste Dulong pour Chéticamp [200], car l'évolution du parler acadien est rapide. Cette transformation est attribuable, pour une large part, à l'isolement géographique et culturel de la communauté acadienne, au faible niveau d'instruction de base des Acadiens et à leur connaissance limitée de la langue française écrite.

Quant aux travaux de la seconde catégorie, ils se rattachent à une vigoureuse tradition nationaliste canadienne-française. ${ }^{214}$ Dans cette

214 Voir Henri BouRASSA, La langue gardienne de la foi : traditions nationales et religieuses des Canadiens français, Montréal, Bibliothèque de l'Action française, 1919. 
perspective idéologique, la langue est conçue comme le véhicule par excellence des croyances religieuses et ne peut se dissocier de la foi. Un affaiblissement de l'identité ethnique entraînera du même coup un affranchissement correspondant des valeurs religieuses traditionnelles et un relâchement dans l'utilisation du parler franco-acadien. À partir de nos observations limitées effectuées dans le grand Portsmouth [Tremblay, 212 et 221], nous trouvons que quatre catholiques fervents sur cinq ont conservé leur langue tandis que seulement un catholique tiède sur trois parle encore le français. Ces résultats empiriques donnent donc du poids à l'idée-maîtresse de l'association étroite languereligion chez les Acadiens.

\section{e) Éducation et écoles acadiennes}

L'ensemble de ces travaux mettent l'accent sur l'importance de l'instruction formelle dans le processus de la survivance. En tant que groupe minoritaire dans des provinces anglaises, les Acadiens ont dû, pour faire reconnaître leurs droits, mener des luttes scolaires nombreuses, plus ou moins fructueuses selon les époques et les provinces. Aujourd'hui, différentes législations scolaires tiennent compte du fait français et des exigences particulières qui s'ensuivent. Plusieurs associations sont nées [153] dont le principal objectif était de défendre les droits des élèves acadiens. Avec la reconnaissance graduelle du fait français à l'école, ces groupes ont élargi leurs objectifs, ont cessé d'être uniquement des groupes de pression auprès des pouvoirs politiques pour s'intéresser davantage à hausser les niveaux d'instruction de la niasse et à orienter les étudiants acadiens vers les différentes carrières professionnelles disponibles. Ces niveaux accrus d'instruction posent un nouveau défi à la survivance acadienne en ce sens que les économies locales devront s'enrichir de nouvelles industries et de nouvelles occupations pour attirer et absorber une main-d'œuvre dont les niveaux de qualification professionnelle seront haussés et permettront l'accès à de nouvelles carrières. Mais les jeunes seront-ils intéressés à retourner en Acadie ? Préféreront-ils plutôt émigrer et faire leur vie ailleurs où les chances de succès sont plus nombreuses et certaines ? Notons, en dernier lieu, que, par les travaux du père Le Gresley [189], nous possédons pour l'Acadie tout entière l'histoire de l'en- 
seignement du français dans les écoles publiques. Ses travaux ont été mis à date pour la Nouvelle-Écosse pour la période 1926-1949 par Joseph-Édouard Comeau [184] et par nous-même [122] pour la décade 1950-1960.

\section{f) Communautés et culture acadiennes}

Ces travaux s'inscrivent au niveau de la communauté, c'est-à-dire au niveau d'unités sociales fonctionnelles. Notons ici les travaux de Ganong [52 et 53] et plus récemment celui du père Chiasson [205]. La plupart des autres études d'importance sur la culture acadienne ont été entreprises par le groupe de recherches en psychiatrie sociale du comté de Stirling (Nouvelle-Écosse). Ces études monographiques, en plus de s'inspirer de la tradition ethnographique en anthropologie (par la description de l'adaptation de l'homme à son milieu, de l'organisation sociale et des attitudes et systèmes de valeurs), ont été menées selon des exigences conceptuelles et méthodologiques précises. L'étude du comté de Stirling fut entreprise avec l'intention d'examiner le rôle de l'environnement social dans l'étiologie des troubles mentaux. Parmi les facteurs du milieu social susceptibles de créer un impact défavorable sur l'hygiène mentale des individus, nous avons privilégié les phénomènes de désintégration sociale. ${ }^{215}$ Cette orientation particulière s'appuie sur trois postulats :

a) un milieu désintégré entrave le développement affectif normal de l'enfant ;

b) un milieu désintégré réduit non seulement la satisfaction des besoins et des tendances les plus essentiels mais favorise aussi les bouleversements affectifs ;

[154]

c) un milieu désintégré diminue sensiblement les possibilités de guérison de l'individu atteint d'une maladie somatique ou

215 Pour un exposé détaillé du cadre conceptuel de l'étude, voir : Alexander H. LEIGHTON, My Name is Legion, New-York, Basic Books, Inc., 1958. 
souffrant d'un désordre psychologique puisque les ressources dont il aurait besoin sont inexistantes ou difficilement accessibles.

Cette hypothèse fondamentale de l'influence du milieu dans les déséquilibres émotifs devait nécessairement se vérifier à deux niveaux différents : sociétaire et individuel.

a) L/épidémiologie des maladies mentales. L'équipe psychiatrique s'est intéressée tout autant à la maturité affective et au processus d'équilibre émotif qu'au dépistage et à l'étiologie des désordres psychiatriques.

b) L'intensité de la désintégration sociale. L'équipe anthropologique a non seulement analysé le processus de désintégration des communautés locales, mais aussi leur processus de croissance et de développement. On a ainsi étudié des communautés intégrées et des communautés désintégrées. Un ensemble de quatorze variables différentes sont utilisées pour définir les niveaux d'intégration/désintégration des communautés.

People of Cove and Woodlot, de Hughes, Tremblay et associés [207], décrit et analyse le milieu social du comté de Stirling. Une communauté acadienne bien intégrée que j'ai appelée L'Anse-desLavallée y est étudiée et comparée aux communautés angloprotestantes intégrées et aux communautés mixtes désintégrées. Les contrastes sont des plus intéressants tant au point de vue du fonctionnement des cultures que du point de vue de l'intégration sociale des groupements. Dans l'étude de L'Anse-des-Lavallée, la configuration culturelle acadienne est tracée en utilisant le concept de sentiments qu'ont élaboré William McDougall et Adolph Meyer. ${ }^{216}$ L'anthropo-

216 Adolph Meyer, The Collected Papers of Adolph Meyer (Eunice E. Winters, éd.), Baltimore, Johns Hopkins Press, 1951 et Psychobiolooy, a Science of Man (textes recueillis par Eunice E. Winters et Anna Mae Bowers), Springfield, III., Thomas, 1957, 85. Voir également : W. McDougALL, An Introduction to Social Psychology, $23^{\mathrm{e}}$ édition, Londres, Methuen, 1936, 104110, 137, 140, 150-196, 305, 308 et 431-433. Pour une reconstitution du 
logue Marcel Rioux a élaboré le profil culturel acadien du NouveauBrunswick par une analyse de contenu d'un ensemble de documents historiques [211]. Les résultats des deux études sont sensiblement les mêmes. Cette convergence, qui peut difficilement être imputable au hasard, souligne la très grande homogénéité de la culture acadienne des provinces de l'Atlantique.

Notons, aussi, les articles de Gosselin et Tremblay portant sur « Le continuum pauvreté-prospérité » [213] et sur « Loomervale » [206]. Le premier de ces articles élabore un cadre conceptuel dans le but d'utiliser la pauvreté comme indicateur de désintégration sociale, tandis que l'autre illustre ces principes théoriques par le truchement de l'approche monographique. [155] Mentionnons, en dernier lieu, l'excellente étude de Macmillan et Leighton, « People of the Hinterland » [209], sur une communauté bi-ethnique et économiquement déprimée.

\section{g) Relations inter-ethniques et acculturation des Acadiens 217}

Ces travaux examinent les relations entre les Acadiens et les Anglais et les influences du groupe dominant sur le groupe minoritaire (l'acculturation des Acadiens). La plupart de ces études sont de date récente et ont été effectuées par des spécialistes des sciences de l'homme. Elles revêtent ainsi un caractère de très grande importance. Les unes portent sur l'ensemble des rapports Français-Anglais [Beaudry, 214 ; Chance, 216 ; Steward et Blackburn, 219 et Thoburn, 220], tandis que d'autres examinent en profondeur le processus d'acculturation des Acadiens [Deveau, 217 et Tremblay, 212 et 221]. Les unités territoriales choisies par ces derniers sont des centres semi-urbains mixtes du comté de Stirling. Puisque le temps ne nous permet pas de résumer ces études de l'acculturation, nous sommes tenté de citer dans

profd culturel des Acadiens de la "Baie française », voir : People of Cove and Woodlot [207], 135-164.

217 Nous venons de préparer un exposé théorique qui permet d'étudier et de mieux comprendre les étapes de l'anglicisation acadienne. Cet article paraîtra dans un prochain numéro d'Anthropologica, sous le titre suivant : «Le transfert culturel : fondement et extension dans le processus d'acculturation ». 
son entier la conclusion générale à notre article sur les « Niveaux et dynamismes d'acculturation des Acadiens de Portsmouth » [221]. Cette conclusion souligne la force et la faiblesse de l'approche utilisée tout en la caractérisant suffisamment bien pour nos fins.

« Cet article, disions-nous, a permis de systématiser certaines de nos connaissances sur le processus d'acculturation et de poursuivre, par la suite, certaines opérations qui nous ont permis de fixer le processus individuel d'acculturation, de l'évaluer quantitativement et de le mettre en relation avec certaines caractéristiques de l'individu et de son milieu. Nous avons été en mesure d'utiliser une situation nord-américaine (les FrancoAcadiens de la Nouvelle-Écosse), de la caractériser dans ce qu'elle avait de spécifiquement acculturante et conservatrice par une approche structurelle-fonctionnelle. Au terme de cet exposé nous nous devons de suggérer que des études de ce genre au niveau macro-sociologique de la structure sociale devront être complétées par des études cliniques de l'acculturation afin de mieux saisir les différents éléments dynamiques et idiosyncratiques de ce processus chez les individus.

« Conscient des déficiences de notre approche nous avons quand même l'impression d'avoir défini quelques jalons et précisé certaines dimensions universelles du processus. À notre point de vue, cette étape devait précéder une analyse plus dynamique et psychologique du phénomène. C'est ainsi que nous satisferons de plus en plus aux exigences conceptuelles et opératoires essentielles à l'élaboration d'une théorie du comportement humain dans une situation de contact. »

\section{V. Évaluation des études acadiennes}

Si on essaie d'évaluer l'ensemble des travaux portant sur l'Acadie, la société et la culture acadiennes, on peut distinguer deux sources d'inspiration, [156] deux types de travaux. Les premiers, d'inspiration idéologique plus ou moins consciente et affichée ; les seconds, à caractère plus scientifique et où les explications sont élaborées à partir de faits d'observation recueillis systématiquement.

L'ensemble des travaux du premier type sont marqués par la dispersion, le rôle de certaines lignes de force dans le processus de la survivance, les dangers qui la menacent, etc.... Les travaux du second type sont moins nombreux, il est évident, mais de très grande impor- 
tance pour une conception dynamique du renouveau acadien contemporain à l'échelle de la nation. On pourra ainsi aborder objectivement la question de la survivance acadienne.

Cette conclusion intermédiaire soulève le dernier point que nous voulions examiner: Quelles études devrions-nous entreprendre en Acadie?

\section{Les études à entreprendre}

Avant de commencer cet inventaire, j'avais l'impression qu'à la suite du nombre restreint des études acadiennes, je serais obligé de mettre l'accent sur l'élaboration « d'un plan de recherche pour l'Acadie ». $\mathrm{Au}$ terme de cette communication, cependant, c'est l'inverse qui se produit. Ayant accordé beaucoup d'importance au bilan, je devrai, en conséquence, réduire le plan que je voulais proposer. Puis, à bien y songer, ce plan de recherche devrait être si vaste - le terrain est encore vierge, du moins si l'on se place dans l'optique des sciences humaines - qu'il consisterait en une simple énumération de tous les sujets d'importance dans l'étude de la société et de la culture. Indiquons quand même quelques lacunes graves.

A. Établir un bilan des effectifs démographiques acadiens tenant compte de la répartition territoriale des populations et de leurs principales caractéristiques socio-culturelles.

B. Analyser les effets des changements technologiques et de l'urbanisation sur les communautés acadiennes isolées, sur les populations acadiennes vivant soit dans des milieux à prédominance anglaise ou dans les milieux mixtes et sur la société acadienne prise comme un tout.

C. Examiner d'une manière systématique les problèmes du sousdéveloppement en Acadie. L'Acadie partage les problèmes de sousdéveloppement des provinces de l'Atlantique et sa population en subit de très graves conséquences par suite de son statut de minorité. On devrait s'intéresser à des questions comme celles-ci : 
$1^{\circ}$ La planification de l'exploitation des ressources naturelles afin d'éviter leur inutilisation ou leur gaspillage ;

[157]

$2^{\circ}$ La découverte de nouvelles industries afin d'absorber les surplus de main-d'œuvre et d'empêcher l'émigration des jeunes ;

$3^{\circ}$ Comment concurrencer sur les marchés nationaux les produits des industries manufacturières fortement équipées et localisées à proximité des marchés de consommation?

$4^{\circ}$ Comment accroître les niveaux de vie et stabiliser les sources de revenu?

$5^{\circ}$ Comment accroître la participation acadienne à des institutions financières comme les coopératives, les Caisses populaires, les sociétés immobilières acadiennes, etc. . . .

$6^{\circ}$ Comment hausser les niveaux de qualification professionnelle?

$7^{\circ}$ Étude de l'évolution de la structure des occupations utilisée comme indice de transformation de la société acadienne.

D. Les mouvements de population. L'Acadie, dans son ensemble, a perdu des effectifs démographiques importants à la suite de vagues successives d'émigration vers les provinces centrales canadiennes. Quelles régions et quels segments de la population ont été les plus vulnérables à cette force centrifuge ? Comment freiner l'émigration?

E. Étudier l'organisation sociale acadienne dans le but de mieux connaître les éléments fonctionnels et dysfonctionnels de la société prise globalement et de susciter des processus d'adaptation s'il y a lieu. On pourrait entreprendre des études de ce type :

$1^{\mathrm{c}} \quad$ La famille acadienne, sa structure et ses fonctions. Liens de parenté, de solidarité et de coopération. Mécanismes d'apprentissage. 
$2^{\circ}$ Une étude exhaustive du système scolaire et de l'enseignement à tous les niveaux.

$3^{\circ}$ Évaluation de la paroisse en tant que cadre de la pratique religieuse dans une société en voie d'urbanisation. Influences des mécanismes d'acculturation sur les croyances, les attitudes et la pratique religieuses.

$4^{\circ} \quad$ Étude du sous-emploi et de la structure des occupations.

$5^{\circ}$ Étude de la différenciation sociale (statuts, rôles, principes de stratification, etc.).

$6^{\circ} \quad$ Étude des comportements d'épargne et de consommation.

$7^{\circ}$ La structure des élites : rôle des élites traditionnelles, étude du pluralisme et du mouvement vers la diversification.

$8^{\circ} \quad$ Les organisations de loisirs.

$9^{\circ} \quad$ Les communications et systèmes d'échanges intergroupes.

$10^{\circ}$ Les mécanismes de contrôle social.

$11^{\circ}$ Les études sur le comportement politique.

$12^{\circ}$ Les études des associations acadiennes.

F. Les études sur les valeurs et la culture acadiennes. De tous les champs mentionnés à date, celui-ci est, sans aucun doute, le plus vaste et parmi les plus difficiles. Car on ne peut l'aborder sans avoir au préalable un apprentissage dans les disciplines humaines. Aucune politique d'action à long terme, soit par rapport au développement économique et social, soit encore par rapport à la survivance, ne saurait réussir sans être fondée [158] sur une connaissance approfondie des valeurs, des attitudes et de la culture des Acadiens des différentes régions.

Nous arrêtons ici notre énumération. La tâche est vraiment colossale tant par les très nombreuses avenues inexplorées et la pénurie de chercheurs que par la nécessité de continuer et d'enrichir ces premières ébauches. Il faudra d'ici peu plusieurs équipes de chercheurs, afin d'amorcer les travaux les plus essentiels en histoire, en linguistique, en économique, en sociologie, en psychologie et en anthropologie culturelle. C'est là un objectif qui s'impose si les Acadiens veulent mieux se 
connaître et, par voie de conséquence, prévenir l'action englobante de la société majoritaire anglo-saxonne et s'adapter aux conditions imposées par la société technologique.

Marc-Adélard TREMBLAY

Département de sociologie et d'anthropologie, Université Laval.

\section{INVENTAIRE BIBLIOGRAPHIQUE}

La classification adoptée, dans cet inventaire bibliographique, correspond à des catégories analytiques dont le choix est justifié dans le texte de l'article. Les deux principales catégories sont les suivantes : I. Les travaux historiques; II. Les études dont le thème général est la "survivance » du groupe acadien, ces études étant regroupées sous huit rubriques plus particulières: survivance de la «nation"; Conventions nationales; missionnaires, clergé et paroisses d'Acadie ; éducation et écoles acadiennes; le parler franco-acadien ; relations inter-ethniques et acculturation des Acadiens ; communautés et culture acadiennes ; divers.

\section{Travaux historiques}

1. AleXANDER, Sir James E., L'Acadie or Seven Years Exploration in British America, Londres, 1849, 2 vol.

2. AlBert, abbé Thomas, Histoire du Madawaska, Québec, Imprimerie Franciscaine Missionnaire, 1920.

3. AtKinson, Rev. Chas., A Historical and Statistical Account of New-Brunswick with Advice to Emigrants, Édimbourg, Anderson \& Bryce, 1844.

4. AUCOIN, Edmond-D., Le Pays d'Évangéline, Montréal, Le Pays laurentien, 1917. 
5. BEAUDRY, René, CS.C, «Aux sources de l'histoire de l'Acadie et des provinces maritimes », The Canadian Historical Association Report, 1955, 62-68.

6. —u «Un témoin de la dispersion acadienne: l'abbé Le Guerne », Revue d'Histoire de l'Amérique française, VII, 1, juin 1953, 32-44.

7. BERnARD, Antoine, c.s.v., "À qui ce pays acadien ? ", Relations, 147, mars 1953, 74-77.

8. — « Choses d'Acadie », Revue d'Histoire de l'Amérique française, $I, 1$, 39-48.

9. —_ Histoire de l'Acadie, Moncton, L'Évangéline Ltée, 1939.

10. _ _ «L'Acadie se souvient », Relations, 136, avril 1952, 9295.

11. BERnARD, Antoine, C.S.V., Le drame acadien depuis 1604, Montréal, Les Clercs de Saint-Viateur, 1936.

12. Blanchard, J.-H., Acadiens de l'île du Prince-Édouard, Charlottetown, Imprimerie Acadienne, Ltée, 1956.

13. — Histoire des Acadiens de l'Île du Prince-Édouard, Moncton, Imprimerie de l'Évangéline, 1927.

14. BIRD, J. Brian, « Settlement Patterns in Maritime Canada, 16871786 », Geographical Review, July 1955, 385-404.

15. Bourgeois, Ph.-F., c.s.c, Petit résumé de l'histoire du Nouveau-Brunswick depuis 80 ans, Moncton, N.-B., 1913.

16. Bourque, Rév. P.-A.-T., Chez les anciens Acadiens : causeries du grand-père Antoine, Moncton, L'Évangéline, 1911.

17. BREBNER, John Bartlett, New England's Outpost : Acadia before the Conquest of Canada, New-York, Columbia University Press, 1927.

18. Brown, Georges S., Yarmouth, N.S. : A Sequel to Campbell's History, Boston, Rand Avery Co., 1888.

19. Buckingham, J. S., Canada, Nova Scotia, New Brunswick and the Other British Provinces in North America, Londres, 1843. 
20. Campbell, Duncan, Nova Scotia in its Historical, Mercantile and Industrial Relations, Montréal, John Lovell, 1873.

21. Campbell, G. G., The History of Nova Scotia, Toronto, Ryerson, 1948.

22. Campbell, Rev. John Roy, A History of the County of Yarmouth of Nova Scotia, Saint John, N.-B., J. \& A. McMillan, 1876, xvi + 200 p.

23. CASGRAIN, abbé Henri-R., « Les Acadiens après leur dispersion, 1755-1775 », Transactions of the Royal Society of Canada, V, 1, 1887, 15-92.

24. —U Un pèlerinage au Pays d'Évangéline, Québec, L.-J. Demers \& Frères, 1888.

25. —_ Une seconde Acadie : l'Île Saint-Jean — Île du PrinceÉdouard sous le régime français, Québec, L.-J. Demers \& Frères, 1894.

26. CHAmpris, Gaillard de, Images du Canada français, Paris, Ed. de Flure, 1947. («En Acadie », p. 117-133).

27. Clarke, Andrew Hill, Three Centuries and the Island ; A Historical Geography of Settlement and Agriculture in P. E. I., Canada, Toronto, University of Toronto Press, 1959.

28. ClarKe, George Frederick, Expulsion of the Acadians, Fredericton, Brunswick Press, 1955.

29. CORMIER, Clément, « En route pour l'Acadie », Relations, 127, 1951, 178-180.

30. Couillard-DesprÉs, abbé A., "Aux sources de l'histoire de l'Acadie », Mémoires de la Société Royale du Canada, $3^{\mathrm{e}}$ série, XXVII, Ottawa, Imprimerie de la Société Royale, 1933.

31. _ Charles de Saint-Étienne de la Tour, Gouverneur, Lieutenant-Général en Acadie et son temps \{1593-1666), Arthabaska, Imprimerie d'Arthabaska, 1930.

32. __ En marge de «La tragédie d'un peuple» de M. Émile Lauvrière, Bruges, Desclée, de Brouwer \& Cie, 1925.

33. — Histoire des seigneurs de la Rivière du Sud et de leurs alliés canadiens et acadiens, Saint-Hyacinthe, La Tribune, 1912. 
34. — Observation sur l'« Histoire de l'Acadie française » de M. Moreau, Paris, Arbour et Dupont, 1919.

35. Dagnaud, P.-M., Les Français du Sud-Ouest de la NouvelleÉcosse, Besançon, Librairie Centrale, 1905.

[160]

36. D'ArLEs, Henri (pseud. de l'abbé Beaudet), La Déportation des Acadiens, Montréal, Bibliothèque de l'Action française, 1918

37. DiÈreville, Sieur de. Relation of the Voyage to Port Royal in Acadia or New France, Toronto, The Champlain Society, 1933.

38. Desrosiers, A., La race française en Amérique, Montréal, Beauchemin, 1910.

39. Deveau, Alphonse, Le journal de Cécile Murat, Baie-SainteMarie, 1961.

40. Doughty, Sir Arthur George, The Acadian Exiles, Toronto, Brook et Co., 1916.

41. DugRÉ, Alexandre, S.J., « Agonie de l'Acadie », Relations, 175, juillet 1955, 176-179.

42. _ _ «Le premier siècle de l'Acadie », Relations, 169, janvier 1955, 4-7.

43. FonTAInE, L.-U., Cent trente-cinq ans après ou la renaissance acadienne, Montréal, Imprimerie Gebhardt-Berthiaume, 1890.

44. _ $\_$Voyage du Sieur de Dièreville en Acadie, Québec, A. Côté \& Cie, 1885.

45. FréGAult, Guy, «Francis Parkman », Amérique française, février 1943, 27-31.

46. —La déportation des Acadiens », Revue d'Histoire de l'Amérique française, 8, 3, déc. 1954.

47. GANONG, William Francis, The History of Caraquet and Pokemouche, Saint John, New-Brunswick Muséum, 1948.

48. _ _ The History of Miscou and Shippegan, Saint John, NewBrunswick Muséum, 1946.

49. — « The History of Neguac and Burnt Church », Acadiensis, VIII, 1908, 267-287. 
50. 138-161.

51. 313-332. «The History of Shippegan », Acadiensis, VIII, 1908, «The History of Tabusintac », Acadiensis, VII, 1907,

52. , « The History of Tracadie », Acadiensis, VI, 1906, 185200.

53. séum, 1945.

54. GAUDET, Placide, Le Grand Dérangement; sur qui retombe la responsabilité de l'expulsion des Acadiens ? Ottawa, Ottawa Printing Co. Ltd., 1922.

55. GRANDMAISON, Léonce de, L'histoire pathétique du peuple acadien, Paris, Études, 1923.

56. GroulX, abbé Lionel, L'histoire acadienne, Montréal, Société Saint-Jean-Baptiste, 1917.

57. — "L'orientation fatale », L'Action nationale, XXVIII, 1954, 91-100.

58. Haliburton, Thomas C, An Historical and Statistical Account of Nova Scotia, Halifax, 1829, 2 vol.

59. Hannay, James, The History of Acadia from its First Discovery to its Surrender to England by the Treaty of Paris, Saint John, N.-B., J. \& A. McMillan, 1879.

60. HaRVey, D. C, The French Régime in Prince Edward Island, New-Haven, Yale University Press, 1926.

61. Hubert, Paul, Les Îles-de-la-Madeleine et les Madelinois, Rimouski, Imprimerie générale, 1926.

62. JÉGO, J.-Bte, Le drame du peuple acadien, Reconstitution historique en neuf tableaux et une pose plastique de la déportation des Acadiens, Paris, Imprimerie Oberthur, 1932.

63. Jules-Émile, Frère, Historique de l'Acadie, Saint-Jean, Que., Éditions du Richelieu, 1954. 
64. LANCTOT, Gustave, « L'Acadie et la Nouvelle-Angleterre, 16031763 (suite) », Revue de l'Université d'Ottawa, 11, 1941, 349370 .

65. LAUVRIÈRE, Emile, Brève histoire tragique du peuple acadien, Paris, Adrien Maisonneuve, 1947.

66. _ _ Deux traîtres d'Acadie et leur victime, les La Tour père et fils et Charles d'Aulnay, Montréal, Granger Frères, 1932.

67. _ La tragédie d'un peuple, Paris, Editions Bossard, 1923, 2 vol.

68. Lawson, J. I., and MacCullum, S.J., This is New Brunswick, Toronto, Ryerson Press, 1951.

[161]

69. LeBlanc, D.-J., The True Slory of the Acadians, Lafayette, Tribune Publishing Co., 1937.

70. Malchelosse, Gérard, «Deux tournants de l'histoire d'Acadie, 1713 et 1755 », Cahiers des Dix, 5, 1940, 107-120.

71. MARQUIS, L.-S.-D., Monographie des Îles-de-la-Madeleine, Québec, Imprimerie Commerciale, 1927.

72. MASSÉ, Pierre « Destinées acadiennes, Marie-Joseph Guillot et sa métairie ", Revue d'Histoire de l'Amérique française, $X, 1$, juin 1956, 104-114.

73. Massignon, Y.-L.-F., Au Canada ; la haute vallée du SaintJean \{Madawaska) et l'avenir franco-américain, Paris, Maisonneuve, 1943.

74. Maxwell, Lilian M., An Outline of the History of Central N. B. to the Time of Confederation, Sackville, Tribune Press, 1937.

75. McLean, C. H., " Prominent People of New Brunswick in the Religious, Political, Educational, Professional, Commercial and Social Activities », Biographical Society of Canada, 1937.

76. McLennan, J. S., Louisbourg-From its Foundation to its Fall, 1713-1758, Londres, MacMillan, 1918.

77. Michaud, Joseph-D., ptre, Notes historiques sur la Vallée de ta Matapédia, Val-Brillant, 1922. 
78. Michaud, Marguerite, «Caraquet», Revue d'Histoire de l'Amérique française, XI, juin 1957, 47-55.

79. Moreau, M., Histoire de l'Acadie française, Paris, Léon Techener, 1873.

80. Morse, W. L, Acadiensis Nova (1598-1779), Londres, Bernard, Ltd., 1935, 2 vol.

81. MURDOCK, Beamish, A History of Nova Scotia or Acadie, Halifax, James Barnes, 1865, 2 vol.

82. OsGood, H. L., The American Colonies in the Eighteenth Century, New-York, 1924, 4 vol.

83. Parkman, Francis, France and England in North America, Boston, Little, Brown and Co., 1865-1894, 9 vol.

84. Poirier, Arthur P., Histoire de Saint-Louis, comté de Kent, Moncton, Imprimerie Nationale, Ltée, 1943.

85. PoirIER, Pascal, « Des Acadiens déportés à Boston en 1755 », Transactions of the Royal Society of Canada, IV, 1, 1908, 125180.

86. — Origine des Acadiens, Montréal, Sénécal, 1874.

87. QuinPOol, John, First Things in Acadia, Halifax, First Things Publishers, 1936.

88. RAMEAU DE SAINT-PÈre, E., La France aux colonies : étude sur le développement de la race française hors de l'Europe (Acadiens et Canadiens), Paris, A. Jouby, 1859.

89. _ Une colonie féodale en Amérique : l'Acadie, 1604-1881, Paris, Plon, 2 vol., 1889.

90. RICHARD, Édouard, Acadie, reconstruction d'un chapitre perdu de l'histoire d'Amérique, Québec, Laflamme, 2 vol., 1916 et 1918.

91. RoqJJEBRune, Robert de, «Le Grand Dérangement », Revue des Deux-Mondes, juillet 1956, 51-67.

92. Rumilly, Robert, Histoire des Acadiens, Montréal, Fides, 1955, 2 vol. 
93. , Les Îles-de-la-Madeleine, Montréal, les Éditions Chanteclerc, 1951.

94. SAUNDERS, R. M., " History and French Canadian Survival », The Canadian Historical Association Report, 1943, 25-34.

95. Sulte, Benjamin, Mélanges historiques : l'Acadie française, Montréal, Éditions Édouard Garand, 1930.

96. Watson, Brooks, «The Acadian French ", Nova Scotia Historical Society Report and Collections, vol. 2, Halifax Morning Herald Office, 1881, 129-160.

97. WeEkeS, Mary, Acadian Betrayal, Toronto, Burns \& MacEachern. 1955.

[162]

\section{II. Études se rattachant au thème général de la survivance}

\section{A. Survivance de la «nation»}

98. ANGERS, François-Albert, "Un vote de race », L'Action nationale, XIX, 1941, 299-312.

99. AnONYME, " Surge Acadia Mea », L'Action nationale, XLIV, 6, février 1955, 471-472.

100. Arsenault, J.-E., «Les Acadiens de l'Île du PrinceEdouard », L'Action nationale, XXXI, mai 1948, 362-372.

101. BERNARD, Antoine, c.s.v., Histoire de la survivance acadienne, 1755-1935, Montréal, Les Clercs de Saint-Viateur, 1935.

102, L'Acadie vivante, Montréal, Éditions du Devoir, 1945.

103. __ La renaissance acadienne au $X X^{\prime}$ siècle, Québec, Le Comité de la survivance française, 1949.

104. Chauvin, F.-X., " Les Canadiens français d'Essex et de Kent », Relations, 72, 1946, 365-369.

105. Collins, Charles W., The Acadians of Madawaska, Maine, Boston, Press of Thomas-Whales, 1902. 
106. DugRÉ, Alexandre, s.j., « Acadie constructive », Relations, 36, décembre 1943, 311-314.

107. 207-209.

108. _ _ «En Acadie ressuscitée », Relations, 34, octobre 1943, 263-265.

109. __ _ «'Acadie 1755-1955 », Relations, 168, 1954, 334-337.

110. , «L'Acadie de demain », Relations, 180, décembre 1955, 321323.

111. EDWARDS, C.-E., La survivance de la culture française en Nouvelle-Écosse, thèse de maîtrise, McGill University, 1946.

112. Gosselin, Gilberte, "La liaison française en Acadie ", Vie française, 10, 1955, 11-23.

113. GroulX, abbé Lionel, «Langage et survivance », L'Action nationale, IV, 1, 1934, 46-63.

114. LÉGER, Antoine-L., Les grandes lignes de l'histoire de la Société L'Assomption, Québec, Imprimerie Franciscaine Missionnaire, 1933.

115. MaLtais, Armand, « Notre merci aux Acadiens », L'Action nationale, $X L V$, 1, septembre 1955, 9-16.

116. Plante, Albert, s.j., « Merci pour votre survivance », Relations, 178, octobre 1955, 269-272.

117. Revue trimestrielle canadienne, "L'évolution de la race française en Amérique », Montréal, Librairie Beauchemin, 1921.

118. Robichaud, Norbert, "Radio-Canada en Acadie », Vie française, 8, 1954, 456-463.

119. SAvoie, Adélard, "L'Acadie fête sa résurrection ", L'Action nationale, XXIV, octobre 1954, 161-175.

120. SloAt, Prudence, La survivance française au NouveauBrunswick, thèse de maîtrise, McGill University, 1946.

121. TAILlON, Léopold, Au service de la culture française en Acadie, 1938-1952, Montréal, Fides, 1952. 
122. Tremblay, M.-Adélard, «Les Acadiens de la Baie française : l'histoire d'une survivance ", Revue d'Histoire de l'Amérique française, $X V$, 4, mars 1962, 526-555.

123. Turgeon, Onésiphore, Un tribut à la race acadienne, Mémoires, 1871-1927, Montréal, G. Ducharme, 1928.

124. VerRetTE, Adrien, "Le bicentenaire acadien », Bulletin de la Société historique franco-américaine, nouvelle série, 1, 1955.

125. Vocelles, James T., The Triumph of the Acadians: A True Story of Evangeline's People, s. l., s. éd., 1930.

\section{B. Conventions nationales}

126. Chouinard, H.-J., Fête nationale des Canadiens français célébrée à Québec en 1880, Québec, A. Côté \& Cie, 1881.

127. Congrès national acadien, Church Point et Grand-Pré, N.-E., 1921, Livre-souvenir et programme, Moncton, Imprimerie L'Acadien, 1921.

128. Convention nationale acadienne de l'Î.-P.-É., Saint-Jacques d'Egmont Bay, 1951.

129. Deuxième congrès de la langue française au Canada, Québec, Imprimerie de l'Action Sociale, 1938.

130. En collaboration, Conventions nationales acadiennes, Shédiac, Imprimerie du Moniteur Acadien, 1907.

131. Premier congrès de la langue française au Canada, compte rendu, Québec, Imprimerie de l'Action Sociale, 1913.

132. RICHARD, Camille, L'idéologie de la première Convention nationale acadienne, thèse de maîtrise, Université Laval, Québec, 1960, 124 p. 


\section{Missionnaires, clergé et paroisses d'Acadie}

133. AlBERT, Révérend Père, o.f.m.. Son Excellence $M^{g r}$ L.-J.-Arthur Melançon, 1879-1941, Moncton, N N.- B., Cathédrale de Notre-Dame-de-l'Assomption, 1941.

134. Anonyme, Album-souvenir du cinquantenaire de la paroisse Saint-Michel de Drummond, N.-B., 1890-1940, Drummond, 1940.

135. Anonyme, Cathédrale Notre-Dame de l'Assomption, Moncton, 1956.

136. AnOnyme, Congrégation des Filles de Marie de l'Assomption, Campbellton, N.-B., Québec, L'Action sociale, 1925.

137. AnONYME, Église Saint-Bernard, Nouvelle-Écosse, Livresouvenir, Yarmouth, The Lawson Publishing Company, 1942

138. AnOnyme, Mémoire sur les missions de la Nouvelle-Écosse, du Cap-Breton et de l'île du Prince-Édouard de 1760 à 1820, Québec, C. Darveau, 1895.

139. AnONyMe, Mère Maillet (Marie-Alphonsine Ranger) fondatrice de l'Hôtel-Dieu de Saint-Joseph de Saint-Basile, N.-B., 1846-1934, Edmundston, N.-B., Le Madawaska, 1934.

140. BlanCHARD, J.-H., Rustico, une paroisse acadienne de L'Île du Prince-Edouard, s. l. s. éd., 1938.

141. BourgeoIs, Marie-Louise, «La paroisse acadienne de Chéticamp ", Journal of Education for Nova Scotia, 6, March 1935, 191-200.

142. BourgeoIs, Ph.-F., c.s.c, Les anciens missionnaires de l'Acadie devant l'histoire, Shédiac, Moniteur Acadien, 1910.

143. Calasanz, J., O.F.M., Aperçu historique de Pointe-à-la-Garde et de sa mission, juin 1941.

144. CANDIDE DE NANT, Pages glorieuses de l'épopée canadienne: une mission capucine en Acadie, Paris, Librairie Saint-François, 1927. 
145. Casgrain, abbé Henri-R., Les Sulpiciens et les Prêtres des Missions étrangères en Acadie, 1676-1762, Québec, Pruneau et Kirouac, 1897.

146. _ Mémoire sur les missions en Nouvelle-Écosse, 17701820, Québec, Darveau, 1895.

147. ChOuinard, Rév. E.-P., Histoire de la paroisse de Saint-Joseph de Carleton : 1775-1906, Rimouski, Imprimerie générale, 1906.

148. Daigle, Cyriaque, Histoire de Saint-Louis de Kent : Cent cinquante ans de vie paroissiale française en Acadie, Moncton, L'Imprimerie Acadienne, 1948.

149. _ Les anciens missionnaires de l'Acadie, Saint-Louis de Kent, 1956.

150. DAVID, P.-Albert, Les missionnaires du séminaire du SaintEsprit à Québec et en Acadie au XVIII siècle, Paris, Bariel Enault, 1926.

[164]

151. De Valigny, Pacifique, O.F.M., Chroniques des plus anciennes églises d'Acadie, Montréal, Écho de Saint-François, 1944.

152. D'Entremont, H., Leander, "Father Jean-Mande Sigogne, 1799-1844 », Nova Scotia Historical Society, Report and Collections, Halifax, The Imperial Publishing Company, vol. 23, 1936, 103-115.

153. De la CotardiÈre, Georges, C.J.M., La Congrégation de Jésus et Marie \{Eudistes) au Canada, 1890-1940, Besançon, Jacques \& Demontrand, 1946.

154. DouCET, Antonio (Père Camille, o.c.r.), À l'ombre du petit Rocher, 1797-1947, La Trappe, 1947, 203p.

155. DubÉ Joseph-Guy, Centenaire de Saint-François de Madawaska, N.-B., 1859-1959, Edmunston, Le Madawaska, 1959.

156. DuBois, Émile, ptre, Chez nos frères les Acadiens, Montréal, Bibliothèque de l'Action française, 1920.

157. En collaboration, L'Acadie, ses missionnaires, Montréal, Les Éditions du Devoir, 1925. 
158. Eudistes, RR. PP., Centenaire de la mort du Père Jean-Mandé Sigogne, missionnaire de la Baie Sainte-Marie, 1844-1944, Pointe-de-1'Église, N.-É., 1944.

159. Gallien, Arthur, ptre, Album souvenir, Église Saint-Bernard, Néguac, N.-B., Montréal, Thérien Frères, 1948.

160. GaGnON, $\mathrm{M}^{\mathrm{gr}}$ C.-O., Lettre de l'abbé Le Guerne, missionnaire de l'Acadie, Québec, Côté, 1889.

161. Gaudet, Gustave, Biographies de Memramcook, s.l, s. éd., 1955.

162. Godin, Edgar, ptre. Monseigneur Thomas Cooke, missionnaire de la Baie des Chaleurs, 1817-1823, Bathurst, N.-B., Imprimerie Leclerc, 1953.

163. L'ArCheVÊQue, Rév. Jos.-A., Histoire de la paroisse SaintJacques-le-Majeur, Scoudouc, N.-B., 1932.

164. LeBlanc, Henri-P., La paroisse Notre-Dame-de-l' Assomption de Moncton, Moncton, N. B., 1958.

165. LÉGER, Désiré, F.-X., ptre. Historique de la paroisse SaintLouis-de-France, Lewisville, Moncton, diocèse de Saint-Jean, N.-B., 1925.

166. __ L L L'histoire de la paroisse de Saint-Pierre de Cocagne, diocèse de Saint- Jean, N.-B., Moncton, L'Évangéline, 1920.

167. LEMAY, Hugolin, o.f.m., Les Récollets missionnaires en Amérique, Lévis, s. éd., 1912.

168. MacMillan, Rev. John C, The Early History of the Catholic Church in P. E. L, Québec, L'Événement Printers Co., 1905.

169. _ The History of the Catholic Church in P.E.I. from 1855 till 1891, Québec, L'Événement Printers Co., 1913.

170. McLaughlin, Rev. Charles S., Early Missionary Foot Prints in Acadie, 1604-1904, 1932.

171. McLeod, Rogers, Pioneer Missionaries in the Atlantic Provinces, Toronto, The Ryerson Press, 1930.

172. MELANÇON, abbé Arthur, Vie de l'abbé Bourg, premier prêtre acadien, Rimouski, « Le Chez-Nous », 1921. 
173. MERCIER, Jean, Les fondateurs de l'Église canadienne et l'Acadie, Québec, Le Comité des Fondateurs de l'Église canadienne, 1955.

174. Michaud, Marguerite, «Le musée de la Cathédrale de Moncton ", Revue d'Histoire de l'Amérique française, VIII, 1954, 236-242.

175. _ Le Père F.-X.-S. Michaud, grand curé bâtisseur et organisateur, Bouctouche, N.-B., Hull, Imprimerie Leclerc, 1954.

176. O'Brien, Cornélius, Memoirs of the Right Révérend Edmund Burke, Ottawa, Thoburn Co., 1894.

177. PlESSIS, $\mathrm{M}^{\text {gr }}$ J.-D., Journal des visites pastorales de 1815 à 1816, Québec, Imprimerie Franciscaine Missionnaire, 1903.

[165]

178. PoIRIER, Pascal, Le Père LeFebvre etl'Acadie, Montréal, Beauchemin, 1898.

179. Rochemonteix, Camille de, s. j., Les Jésuites et la NouvelleFrance au XVII siècle,

Paris, Letouzey et Ané, 1895.

\section{D. Éducation et écoles acadiennes}

180. AnONyme, «The Acadian Commission », Journal of Education for Nova Scotia, 6, April 1909, 54-57.

181. BERNARD, Antoine, c.s.v., «Quelques victoires dans les écoles », Relations, 3, mars 1941, 76 et suiv.

182. Blanchard, J.-H., « Les écoles acadiennes de l'Ïle du PrinceEdouard », Relations, 18, juin 1942, 156-158.

183. BREAUlt, Arthur, Éducation française en Acadie, thèse de maîtrise, Université d'Ottawa, 1933.

184. COMEAU, Joseph-Edouard, L'enseignement du français dans les écoles publiques de la Nouvelle-Écosse depuis 1900, thèse de doctorat, Université de Montréal, 1949. 
185. Frecker, G., Education in the Atlantic Provinces, Toronto, W. S. Gage, 1956.

186. Godin, Théo., "Les origines de l'Association acadienne de l'éducation: Note historique sur la question scolaire au Nouveau-Brunswick ", Revue d'Histoire de l'Amérique française, $V$, 2, septembre 1951, 186-192.

187. Hubert, A., «Écoles acadiennes de Nouvelle-Écosse », Relations, 14, février 1942, 44-46.

188. LAPLANTE, Léopold, c.j.m., « Un collège acadien en NouvelleÉcosse », Relations, août 1942, 213-220.

189. Le Gresley, Orner, L'enseignement du français en Acadie, (1604-1926), Paris, Enault. 1926.

190. MacNauGHTOn, K. F. C, The Development of the Theory and Practice of Education in N.-B., University of N.-B., Fredericton, 1947 (Historical Studies, $\mathrm{n}^{\circ}$ 1).

191. MALChElosse, Gérard, « La bibliothèque acadienne ", Cahiers des Dix, 19, 1954, 263-286.

192. Michaud, Benoît, "Écoles françaises d'Acadie », Relations, 9, sept. 1941, 227-229.

193. Plante, Albert, s.j., "Retour d'Acadie », Relations, 129, septembre 1951, 243-246.

194. RICHARD, François, s.j., «La vie française au NouveauBrunswick », Relations, 92, août 1948.

195. Tremblay, Marcel, c.j.m., Cinquante ans d'éducation catholique et française en Acadie : Caraquet 1899 - Bathurst 1949, Université du Sacré-Cœur, 1949.

\section{E. Le parler franco-acadien}

196. BAstiEn, Hermas, « Le bilinguisme dans les Provinces Maritimes ", Action française, 13, 1925, 250-268.

197. ChAPAIs, Thomas, «La langue, gardienne de la foi, des traditions, de la nationalité », Premier Congrès de la langue fran- 
çaise au Canada : compte rendu, Québec, Imprimerie de l'Action sociale, 1913, 445-453.

198. CharbonneAu, Hector, «Les archaïsmes du parler madelinois », Transactions of the Royal Society of Canada, XXXIX, 1, 1945, 19-38.

199. DoIRON, Léo-Martin, La langue française en Nouvelle-Écosse, thèse de maîtrise, Université de Montréal, 1946.

200. DulOng, Gaston, «Chéticamp, îlot linguistique du CapBreton », dans : Contributions to Anthropology, 1959, Bulletin 173, Ottawa, National Muséum of Canada, 1960, 12-41.

201. GedDES, James, Study of an Acadian-French Dialect Spoken on the North Shore of the Baie-des-Chaleurs, Halle, Niemayer, 1908.

[166]

202. Gosselin, P.-E., « La langue, gardienne de la foi », Canada français, 24, 4, 1936, 301-309.

203. PoIRIER, Pascal, "Comment une langue évolue », Transactions of the Royal Society of Canada, XXI, 1, 1927, 239-245.

204. _ Le parler franco-acadien et ses origines, Québec, Imprimerie Franciscaine Missionnaire, 1928.

\section{F. Communautés et culture acadiennes}

205. Chiasson, Père Anselme, cap., Chéticamp : histoire et traditions acadiennes, Moncton, Éditions des Aboîteaux, 1961, 317 p.

206. Gosselin, Émile et Tremblay, Marc-Adélard, "Loomervale : un cas de désintégration sociale », Recherches sociographiqucs, I, 3, juillet-septembre 1960, 309-342.

207. Hughes, C. C, Tremblay, M.-Adélard, Rapoport, R. N., et Leighton, A. H., People of Cove and Woodlot : Communities from the Viewpoint of Social Psychiatry, New-York, Basic Books, Inc., 1960. 
208. LAGACÉ, Anita, Grand-Sault, hier et aujourd'hui, s. 1., s. éd., 1946.

209. MacMillan, Allister M., et Leighton, Alexander H., « People of the Hinterland, Community Interrelation in a Maritime Province of Canada », dans : Edward H. SPICER, éd., Human Problems in Technological Change, New-York, Russel Sage Foundation, 1952, 225-243.

210. Michaud, Marguerite, La reconstruction française au Nouveau-Brunswick; Bouctouche, paroisse-type, Fredericton, Les Presses Universitaires, 1955, 223 p.

211. Rioux, Marcel, « Rapport préliminaire de l'étude sur la culture acadienne du Nouveau-Brunswick », Extrait du Bulletin n 147, Rapport annuel du Musée national 1955-1956, Ministère du Nord canadien et des Ressources nationales, 62-64.

212. Tremblay, M.-Adélard, The Acadians of Portsmouth, A Study in Culture Change, Ph. D. dissertation, Cornell University, Ithaca, N. Y., 1954.

213. Tremblay, M.-Adélard et Gosselin, Émile, « Le continuum pauvreté-prospérité ; son utilité en tant qu'indicateur de désintégration sociale », Service social, 9, 3, nov.-déc. 1960, 3-28.

\section{G. Relations inter-ethniques et acculturation des Acadiens}

214. BEAUDRY, René, c.s.c, « Les rapports ethniques dans les Provinces Maritimes », dans : La dualité canadienne (ouvrage réalisé par Mason Wade), Québec, Les Presses Universitaires Laval, 1960, 374-382.

215. Bond, Donald et alii, "Anglo-French and Franco-American Studies, a Current Bibliography », Romantic Revient, Oct. 1945, 161-190.

216. CHANCE, Norman A., Portsmouth : The Study of a Bi-Cultural Community under Stress, Ph. D. dissertation, Cornell University, Ithaca, N. Y., 1957. 
217. Deveau, Alphonse, Patterns of Acculturation of Acadian Descent People in Bristol [nom d'emprunt], thèse de maîtrise, Université Laval, 1953.

218. Moore, William Henry, Le Choc (The Clash), étude de nationalités, traduit de l'anglais par Ernest Bilodeau, Montréal, Librairie Beauchemin, Limitée, 1920.

219. SteWARD, J., et BlackBURn, S., «Tensions between EnglishSpeaking and French-Speaking Canadians », Contributions à l'étude des sciences de l'homme, 3, 1956, 145-168.

220. THORBURN, Hugh, "Biculturalism in the Maritime Provinces », dans : La dualité canadienne (ouvrage réalisé par Mason Wade), Québec, Les Presses Universitaires Laval, 1960, 383391.

221. Tremblay, M.-Adélard, " Niveaux et dynamismes d'acculturation des Acadiens de Portsmouth » [nom d'emprunt], Anthropologica, III, 2, 1961, 202-251.

[167]

H. Divers

222. Anonyme, Place Names of Prince-Edward Island with Meanings, Ottawa, King's Printer, 1925.

223. __ Place Names on Magdalen Islands, Que., Ottawa, King's Printer, 1922.

224. Bishop, Olga Bernice, Publications of the Governments of N.S., P.-E.-I., N.-B. : 1758-1952, Ottawa, National Library of Canada, 1957.

225. BRASSARD, François-J., "La chanson acadienne », L'Action nationale, XIX, 1941, 245-246.

226. Chapin, Miriam, Atlantic Canada, Toronto, Ryerson Press, 1956.

227. Clare, Nouvelle-Écosse, Chez les Acadiens, Yarmouth, R. H. Davis \& Co., 1952. 
228. CoRrivault, Blaise, Bibliographie analytique de l'histoire d'Acadie, thèse de bibliothéconomie, Church Point, N.-É., 1950.

229. DOUCET, Alain, La littérature orale de la Baie-Sainte-Marie, thèse de maîtrise. Collège Sainte-Anne, Pointe-de-1'Église, N.É., 1961, $129 \mathrm{p}$.

230. Douglas, R., La nomenclature géographique des Îles-de-laMadeleine, Ottawa, Imprimeur du Roi, 1923.

231. En collaboration. Problèmes économiques des Acadiens, Conférences données à la Journée d'études économiques organisée à l'Université Saint-Joseph, le 3 décembre 1960, Moncton, 1960.

232. GAUDET, Laura-C, Songs of Acadia, New-York, Broadcast Music, Inc., 1945.

233. HUBERT, Jean, L'Évangéline au service de l'Acadie : exposé de l'aspect rédactionnel de notre ouvre de presse catholique et française, Moncton, février 1962.

234. JACK, D. R., " Acadian Magazines », Royal Society of Canada, $2^{\text {nd }}$ Séries, vol. IX, section II, 1903-1904, Ottawa, J. Hope \& Sons, 1903.

235. LemaÎtre, Georges, Vie économique de la Nouvelle-Écosse, Paris, Presses Universitaires de France, 1931.

236. MlCHAUD, Marguerite, "L'Acadie dans la littérature », L'Action nationale, XXIX, avril 1947, 273-284 et XXX, octobre 1947, 112-126.

237. Nicolet, Jean, « Un sénateur pour les Acadiens de l'Île », L'Action nationale, XXIII, 1953, 151-152.

238. Roy, Carmen, Contes populaires gaspésiens, Montréal, Fides, 1952. 


\title{
COMMENTAIRE
}

\author{
Clément Cormier, c.s.c. \\ Recteur de l'Université Saint-Joseph, \\ Moncton, N.-B.
}

[pp. 168-170.]

$\underline{\text { Retour à la table des matières }}$

M. Tremblay a intitulé son travail : «L'état des recherches sur la culture acadienne ». Nous aussi, nous parlons de l'Acadie, mais, à moins que ce ne soit au figuré, je me demande si nous avons raison. L'Acadie fut autrefois une entité géographique et politique, avec son gouverneur propre, autonome autant qu'une colonie pouvait l'être. Mais cette entité politique a cessé d'exister ! Peut-on alors visiter — au sens propre - l'Acadie ?

Quant aux Acadiens, c'est autre chose. Descendants de l'ancienne colonie fondée sur les rives de la baie Française, ils sont répandus un peu partout, comme le laisse entendre M. Tremblay. Que je voudrais avoir plus de temps à ma disposition pour en arriver à définir les caractéristiques qu'il faut avoir pour s'appeler « un Acadien »!

Je veux vous dire ma très grande satisfaction de voir s'ébaucher un programme d'études sociologiques sur les Acadiens. Avec la thèse de 
maîtrise de $\mathbf{M}$. Camille-Antoine Richard et les recherches de $\mathbf{M}$. Tremblay, nous entrons dans une ère nouvelle, j'en suis sûr, et je m'en réjouis, car nous en avons un immense besoin.

On ne doit pas s'attendre à une critique du travail de M. Tremblay. Je constate qu'il apprend à nous connaître, à nous observer avec l'objectivité d'un chercheur consciencieux et la compétence d'un professionnel ; il a pris connaissance d'un grand nombre d'ouvrages publiés chez nous et sur nous ; et il trace un sérieux programme de recherches.

Le travail qui vient d'être présenté m'intéresse vivement. Je pourrais peut-être différer d'opinion sur certains points de détail ; peut-être pourrais-je apporter ici ou là quelque suggestion comme complément à l'exposé. Mais j'attache peu d'importance à ces questions de détail.

Comme l'a souligné $M$. Tremblay, on a beaucoup écrit sur les Acadiens. La liste bibliographique qu'il fournit, bien qu'imposante, est loin d'être complète, surtout si l'on devait inclure tous les articles de revue et toutes les brochures. Évidemment, ce n'est pas la quantité des pages écrites qui intéresse un auditoire du calibre de celui auquel je m'adresse ; j'aurais souhaité qu'on sélectionne mieux les travaux qui méritent d'être signalés dans un colloque de haute tenue universitaire.

M. Tremblay a insisté sur l'importance, au moins quantitative, des ouvrages historiques sur les Acadiens. La classification qu'il a adoptée est significative. Il a partagé la documentation sur les Acadiens en deux grandes classes : d'une part, les travaux d'histoire ; d'autre part, tout le reste, divisé en huit catégories - je ne suis pas convaincu de la justesse de la subdivision, mais qu'importe ! Je voudrais ajouter que même " tout le reste ", nous aimons l'étudier dans la perspective de l'histoire, que ce soit la paroisse, les missionnaires, l'éducation, etc.

Les Acadiens sont avides d'histoire. Nous avons des histoires de toutes les nuances : histoires savantes, histoires superficielles, histoires objectives, histoires partiales, histoires à intention apologétique, histoires générales, histoires locales. Sans prétention, je me qualifie d'historien amateur, et je me présente à vous comme présidentfondateur d'une société historique très vivante établie il y a deux ans ; c'est donc dire que j'aime l'histoire, et je prêche la fidélité au passé, parce qu'il contient les lignes de force du présent et de l'avenir. Je vous prie de vous rappeler [169] ce que je viens de dire quand vous entendrez ce qui va suivre. J'ai pris cette précaution oratoire parce que 
je veux en arriver à soutenir un peu en paradoxe qu'il faut aussi savoir rompre avec le passé ; une collectivité peut se vouer à la stagnation en s'attachant trop servilement à des formules qui vieillissent. Il ne suffit pas d'étudier le passé. Il est tout aussi important de suivre l'évolution dynamique d'un groupe social ; et c'est pourquoi les recherches sur le comportement d'une communauté dans le présent sont aussi indispensables que l'histoire pour préparer l'avenir. Or, les Acadiens ont beaucoup étudié leur histoire ; quant aux recherches scientifiques sur leur comportement présent, $M$. Tremblay a raison de dire que « nous en sommes à la période des premiers balbutiements ».

Permettez-moi d'insister. M. Tremblay prétend que la dispersion occupe une très grande place dans nos études historiques. Je suis d'avis qu'il exagère, mais pour lui donner un peu raison, je commence ce nouvel exposé en parlant de dispersion. Voici. Un siècle après la dispersion commençait le mouvement de la Renaissance acadienne. C'est un phénomène sociologique du plus haut intérêt. Les pionniers du mouvement avaient comme point de départ une population pauvre, illettrée, dispersée, sans chefs, et qui avait développé un écrasant complexe d'infériorité ; ce sont ces infortunés qu'il fallait sortir de la léthargie, auxquels il fallait faire prendre conscience des possibilités d'une vie collective organisée. L'équipe des chefs a réussi ce tour de force. Perspicaces, ils ont su découvrir et exploiter les thèmes et les procédés susceptibles d'éveiller dans l'âme de la population entière des sentiments de fierté, d'optimisme et de confiance. Sous leur inspiration, les Acadiens se sont mis à trouver leur passé glorieux, leurs traditions riches, leurs ancêtres héroïques. L'initiative a été génératrice de vie sociale ; elle a révélé une communauté de besoins et d'aspirations. Il en est résulté : la définition d'un bien commun, la convergence des énergies dans des entreprises communautaires, l'établissement de cadres sociaux et le choix d'emblèmes d'un puissant symbolisme : fête nationale, patronne, drapeau distinctif, hymne national. Dans sa thèse de maîtrise, c'est cette période d'élaboration de la "mentalité acadienne » qu'analysait Camille-Antoine Richard. L'action de l'équipe a très profondément influencé la pensée collective des Acadiens. Je regrette que le temps ne permette pas de nous étendre, et qu'il faille passer si brusquement, sans transition, à un autre point.

Mon impression, c'est que les Acadiens restent trop fidèlement attachés à des formules d'un passé décadent, alors que, depuis un quart 
de siècle surtout, les conditions de vie ont évolué de façon quasiincroyable. Voyez l'armature présente des institutions acadiennes: journal quotidien, radio et télévision françaises, écoles modernes, organisation diocésaine dépassant les espérances de la génération précédente (création de l'archidiocèse de Moncton ; transport du siège épiscopal de Chatham à Bathurst, création des diocèses d'Edmundston et de Yarmouth) ; voyez aussi ces nouvelles caractéristiques de psychologie collective chez les Acadiens : diminution d'un type de patriotisme sentimental alimenté autrefois par l'éloquence pathétique dont les foules étaient friandes ; une plus grande maturité intellectuelle qui permet un examen plus scientifique des problèmes ; une diminution de l'attitude isolationniste qui caractérisait les relations des Acadiens avec les Anglo-Canadiens aussi bien qu'avec les Canadiens français du Québec ; enfin, ajoutez une participation assez confortable à la prospérité économique contemporaine.

[170]

Au congrès de la Société nationale des Acadiens en 1960, j'avais exposé ces mêmes idées, insistant sur l'importance de repenser nos positions pour adapter davantage notre action collective aux exigences de la vie contemporaine. Comme les anciens ont fait preuve de clairvoyance et de sens d'adaptation, il nous faut aujourd'hui être aussi perspicaces et versatiles qu'ils l'ont été en adaptant nos théories, nos objectifs et nos procédés aux conditions présentes. Ce qui implique une sérieuse étude des conditions présentes.

Pour reprendre l'expérience de l'École de la Renaissance dont je vous parlais, pour arriver à redéfinir le bien commun avec le même sens d'adaptation dont nos prédécesseurs ont fait preuve au siècle dernier, il faut commencer par poursuivre une analyse scientifique du comportement de la collectivité acadienne - lequel comportement est infiniment plus complexe qu'il ne l'était il y a 75 ou 100 ans. Il faudrait une enquête approfondie sur les courants d'opinions et de sensibilité, sur les éléments dynamiques de la vie acadienne, sur l'efficacité ou l'inefficacité des cadres existants et les besoins de structures nouvelles, et surtout sur les causes de défection - car il y en a.

Un dernier commentaire : M. Tremblay a choisi comme centre de ses recherches un modeste milieu de la Nouvelle-Écosse. Il s'est dit heureux d'avoir comme commentateur un néo-brunswickois. On com- 
prendra que le milieu où j'évolue est bien différent. On y trouve les plus forts tronçons de la population acadienne, nos plus imposantes institutions, les principaux foyers d'influence. Mon objectif inavoué était d'insinuer auprès de $\mathrm{M}$. Tremblay que là aussi — là surtout ! — il était important de poursuivre un programme de recherches sociographiques.

Clément Cormier, c.s.c.

Recteur de L'Université Saint-Joseph, Moncton, N.-B. 
[171]

Situation de la recherche sur le Canada français

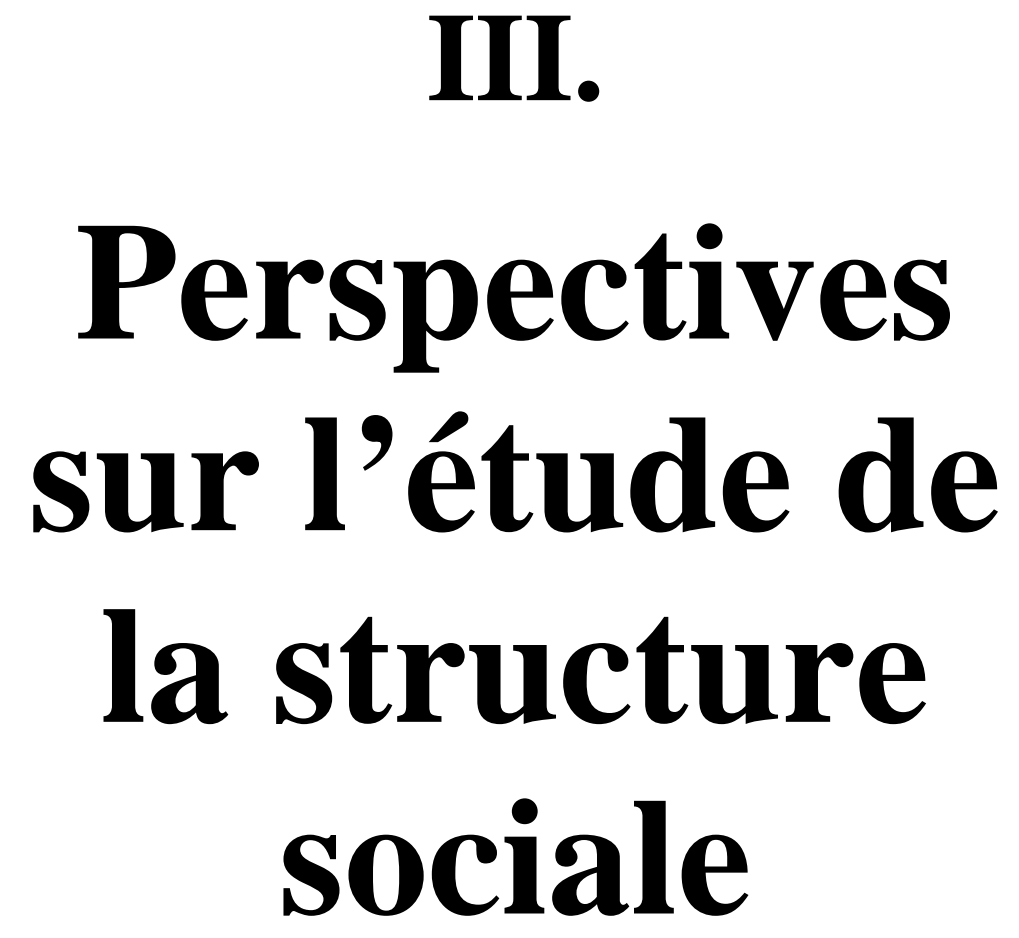

$\underline{\text { Retour à la table des matières }}$

[172] 
Situation de la recherche sur le Canada français

\title{
III. Perspectives sur l'étude de la structure sociale
}

\author{
Guy Rocher \\ Département de sociologie, \\ Université de Montréal

\section{"Les recherches sur les occupations et la stratification sociale”.}

[pp. 173-184.]

$\underline{\text { Retour à la table des matières }}$

Le titre même de cette communication indique dès le départ deux lacunes majeures des recherches sociologiques sur le Canada français. Il est en effet bien symbolique que l'on doive traiter comme un même sujet les études sur les milieux de travail et l'analyse de la stratification sociale. Ceci indique combien nous avons encore peu développé ces deux domaines de recherche. Nous croyons pourtant qu'une connaissance plus précise de la division de notre société en classes et en strates sociales apporterait l'armature centrale à presque toutes nos recherches sociographiques. Il est frappant de constater, en parcourant notre littérature sociologique, combien nos chercheurs déplorent souvent cette lacune. L'appartenance à une strate ou à une classe sociale constitue en effet une des variables les plus communément utilisées en recherche sociologique. Le fait que l'on ne puisse s'appuyer sur un ensemble de travaux dans ce domaine impose donc toujours de sérieuses limitations à nos recherches. 
De même, dans une société industrielle et technologique qui vient tout juste de succéder à une société de type agricole et artisanal, les milieux de travail sont des objets d'étude privilégiés pour le sociologue. L'évolution globale des occupations, les transformations des conditions de travail dans un grand nombre d'emplois, l'adaptation à de nouveaux modes et à de nouveaux milieux de travail, la mobilité professionnelle, voilà autant de problèmes qui se présentent dans l'usine, dans l'entreprise commerciale, dans le bureau, dans l'école ou l'université, etc. C'est dans ces cadres en évolution que se façonne une partie de la culture et de la mentalité de la population. Les traditions marxiste et durkheimienne à la fois nous ont appris à ne pas minimiser - sinon à privilégier - l'impact de l'infrastructure de la division du travail sur l'ensemble socio-culturel.

C'est donc sous le signe de cette double lacune que se situe le présent exposé. Ceci n'est cependant pas dit pour diminuer l'importance des travaux déjà faits. Les jalons posés prennent au contraire une plus grande valeur du fait qu'ils indiquent une première orientation pour les recherches que l'on peut espérer dans un avenir prochain.

En analysant ces travaux, il m'a semblé qu'on pouvait les regrouper sous trois thèmes principaux: l'évolution historique de la structure occupationnelle, [174] la répartition des groupes ethniques dans les différentes catégories d'emplois et les études de stratification et de mobilité sociales. Ce sont ces trois thèmes que je me propose de présenter successivement.

\section{L'évolution historique du travail}

Il nous manque une étude historique du travail au Canada, comme il en a été publié une récemment en France. ${ }^{218}$ Ce sera sans doute une œuvre de longue haleine, qui nécessitera au préalable des études plus locales et couvrant des secteurs restreints. On trouve un exemple d'une telle étude restreinte dans La grève de l'amiante 219 en ce qui concer-

218 Histoire générale du travail, sous la direction de Louis-Henri PARIAS, Paris, Nouvelle Librairie de France, 4 vols.

219 En collaboration, sous la direction de Pierre-Elliott TRUDEAU, La grive de l'amiante, Montréal, Éditions de Cité Libre, 1953. Voir en particulier le cha- 
ne ce type d'industrie, bien que j'aurais aimé la voir développée de façon beaucoup plus considérable. On peut en effet se demander si l'histoire du syndicalisme de l'amiante de Fernand Dumont n'aurait pas été enrichie si elle avait pu se situer dans le contexte de l'évolution technologique esquissée par Jean Gérin-Lajoie.

L'étude historique d'ensemble la plus importante dans ce domaine de travail est celle de François-Albert Angers et Patrick Allen, Évolution de la structure des emplois au Canada. Cette étude est entièrement basée sur un dépouillement minutieux de tous les renseignements sur les occupations contenus dans les recensements canadiens de 1665 à 1941. De cette analyse, les auteurs dégagent trois grandes périodes de transformations de l'emploi en fonction des changements technologiques et économiques. La première s'étend de 1760 à 1851. Elle est marquée par le développement de petites entreprises industrielles de type artisanal pour répondre à la commercialisation des besoins qui concurrence de plus en plus la production domestique. Pour cette période, la nomenclature utilisée par le recenseur paraît correspondre « à une étape où la division du travail n'existe pas encore, où le fabricant ou faiseur fait lui-même en entier l'objet qui le caractérise ». ${ }^{220}$ Les fabricants peuvent travailler isolément ou dans de petites fabriques ou boutiques dans lesquelles l'employeur travaille lui-même avec quelques salariés ou apprentis.

La deuxième période, allant de 1851 à 1891, en est une de transition où l'entreprise artisanale continue à se multiplier en même temps que se développe la petite entreprise de type plus nettement capitaliste et manufacturier. L'employeur tend à se distinguer de plus en plus du personnel ouvrier, à être un patron capitaliste, et la petite manufacture se peuple d'ouvriers sans métiers - les anciens journaliers généraux - qui occupent [175] maintenant des emplois définis. Ce nouveau type d'ouvriers de manufacture représentait 15\% des personnes engagées dans les emplois manufacturiers en 1891, comparativement à 5\% en $1881 .{ }^{221}$ C'est la première apparition d'une nouvelle classe ouvriè-

pitre II, « Histoire financière de l'industrie de l'amiante », par Jean GÉRINLAJOIE.

220 F.-A. Angers et P. Allen, Évolution de la structure des emplois au Cana$d a$, Montréal, Service de documentation économique, École des Hautes Études Commerciales, 1954, 15.

221 Ibid., 23. 
re en croissance, dans le cadre d'une industrie encore très largement artisanale. Le recenseur de 1891 est d'ailleurs frappé par la spécialisation considérable des entreprises durant la dernière décennie, par exemple la confiserie qui se distingue maintenant de la boulangerie. Il vaut la peine de rapporter ici un texte prophétique de ce recenseur cité par Angers et Allen :

« Le mouvement tendant à spécialiser ou séparer les industries, explique en grande partie l'augmentation considérable dans le nombre des établissements industriels durant la dernière décade. Il n'y a aucun doute que ces petites industries ont augmenté considérablement durant les dix années en question. Il en a surgi dans tout le pays. Elles ont pris racine, et l'avenir dira si elles ont suivi ici la règle suivie par elles dans d'autres pays, en se développant en établissements plus considérables. Comme trait de la vie industrielle du peuple canadien, cette révélation du recensement mérite d'être mentionnée. C'est un fait important qui ne devrait pas être ignoré. Il est enregistré dans les archives du recensement, afin que plus tard, quand le développement en de grandes industries se sera accompli, le peuple canadien puisse jeter un regard en arrière sur la période des " petites et faibles choses ", et montrer de quels humbles commencements la vie industrielle du pays est sortie. » 222

Nous sommes de ceux à qui il est précisément donné aujourd'hui de « jeter un regard en arrière sur la période des petites et faibles choses ». Ce recenseur prophétique avait vraiment le sentiment de vivre une période marquante de l'histoire industrielle canadienne. De fait, Angers et Allen considèrent cette date de 1891 comme constituant « le point de rupture entre les exigences de la vieille tradition et celles de la révolution industrielle ». ${ }^{223}$ C'est à ce moment que, selon eux, la révolution industrielle s'inscrit dans les archives de nos recensements et que s'ouvre une troisième période de transformations industrielles allant de 1891 à 1941. Cette période portera plus particulièrement les traits suivants : $1^{\circ}$ les métiers de type artisanal qui avaient été en expansion dans les périodes précédentes, connaissent maintenant un " déclassement général ", c'est-à-dire un ralentissement ou même une nette diminution quand il ne s'agit pas d'une quasi-disparition ; $2^{\circ}$

222 Recensement de 1881, vol. III, cité par ANGERS et AlLEN, ibid., 23.

223 Ibid., 35. 
l'ouvrier industriel devient de plus en plus dominant, sa figure se précise en se diversifiant. De décennie en décennie, il est intéressant de voir les recenseurs successifs aux prises avec des problèmes de définition et de catégorisation toujours plus complexes ; $3^{\circ}$ une nouvelle classe de travailleurs apparaît, celle des contremaîtres, symboles du passage de la petite à la grande entreprise industrielle mécanisée ; $4^{\circ}$ les cadres de l'entreprise [176] homogène éclatent, les métiers et les emplois industriels se diffusent dans tous les secteurs d'activité économique. C'est alors que commence à apparaître dans nos recensements la distinction entre la classification des industries et la classification des occupations, cette dernière recoupant la première ; $5^{\circ}$ enfin, se développe une nouvelle classe d'employés, les collets-blancs du commerce, de la finance, des bureaux et les employés des services, indice des transformations à la fois de la structure économique et du niveau de vie.

Cette étude d'Angers et Allen a été complétée par Patrick Allen d'après des renseignements fournis par le recensement de 1951. L'auteur conclut que « les tendances générales mises en relief dans l'étude (d'Angers et Allen) s'affirment avec plus de netteté encore de 1941 à 1951 ». 224 Il faut espérer que des analyses aussi bien amorcées seront continuées à la lumière du dernier recensement. Peut-être y lira-t-on la transition vers une quatrième période et pourra-t-on verser au dossier une autre envolée prophétique !

La troisième période dégagée par Angers et Allen a été analysée plus en détail par Larkin et Allen dans une publication intitulée Tendances occupationnelles au Canada 225 et dans un article plus récent de Patrick Allen dans L'Actualité économique 226 S'inspirant du cadre d'analyse développé par Jean Fourastié, ces auteurs retrouvent dans l'évolution des occupations pour l'ensemble du Canada de 1891 à 1951 les mêmes mouvements que Fourastié avait décelés dans d'autres pays industrialisés : dégonflement du secteur primaire, stabilisation du

224 Patrick ALLEN, « La structure des emplois au Canada, 1941-1951 », L'Actualité économique, XXXII, 2, 329.

225 William LARKIN et Patrick AlLEN, Tendances occupationnelles au Canada, Montréal, Service de documentation économique. École des Hautes Études Commerciales, 1951.

226 Patrick ALLEN, « Tendances récentes des occupations au Canada », L'Actualité économique, XXXII, 1, 27-65. 
secteur secondaire et croissance du secteur tertiaire. Entre les provinces canadiennes, cependant, s'affirment des différences très nettes. Pour 1951, Allen a pu les regrouper en trois classes, selon l'importance proportionnelle des trois secteurs : les groupes $\mathrm{T}-\mathrm{S}-\mathrm{P}, \mathrm{T}-\mathrm{P}-\mathrm{S}$, et $\mathrm{P}$ - T - S. C'est dans le premier que se situe le Québec avec $45 \%$ de sa main-d'œuvre dans les occupations tertiaires et $41 \%$ dans les industries tertiaires, $28 \%$ dans les occupations secondaires et 38\% dans les industries secondaires, 23\% dans les occupations primaires et $19 \%$ dans les industries primaires.

Si l'on veut maintenant tirer une conclusion de ce que l'on peut espérer être les premiers jalons d'une histoire générale du travail, disons que ces études ne portent pas sur le Canada français comme tel. Ou peut-être plus exactement devrait-on dire qu'elles situent l'évolution du travail au Canada français dans le contexte de la société globale canadienne. Sans doute des études plus minutieuses découvriront-elles des caractéristiques locales de ce mouvement, dues à la fois aux structures occupationnelles traditionnelles [177] déjà existantes et au mode d'industrialisation propre à notre région. Des recherches historiques, du genre de celle d'André Labarrère sur les enseignants, 227 constitueront des étapes essentielles dans cette démarche. Mais toute étude d'ensemble sur l'histoire du travail au Canada français devra se faire en fonction du contexte non seulement canadien, mais nord-américain et même occidental.

\section{Répartition des groupes ethniques dans la structure occupationnelle}

Si l'on passe maintenant de ces études de caractère historique aux recherches plus proprement sociologiques qui ont été menées depuis une vingtaine d'années sur le travail au Canada français, on doit immédiatement faire une double constatation. La première, c'est qu'elles ont été très largement dominées par un thème majeur, celui de la distribution différentielle des groupes ethniques dans la structure des

227 André Labarrère-Paulé, Tes instituteurs et Institutrices laïcs catholiques au Canada français, 1835-1900, thèse de doctorat, Université Laval, 1961, $1,234 \mathrm{p}$. 
emplois. En second lieu, ce sont surtout les sociologues anglophones, particulièrement ceux de l'Université McGill, qui ont développé ce thème et l'ont abondamment illustré. Ce second fait est d'autant plus paradoxal que toutes ces recherches ont montré l'une après l'autre la position privilégiée de la population anglophone dans le Québec et le statut défavorisé des Canadiens français. D'un strict point de vue politique, nos collègues de langue anglaise n'avaient sûrement aucun intérêt à établir une telle preuve !

C'est sans doute le nom d'Everett Hughes qu'il faut ici mentionner au point de départ de ces recherches. Son étude à Cantonville 228 a été la première à mettre en lumière l'impact de l'industrialisation massive sur les relations ethniques dans le monde du travail. Hughes a montré comment les Canadiens français étaient comprimés aux échelons inférieurs de l'entreprise industrielle et, surtout, combien les entrepreneurs et les cadres supérieurs américains ou anglais étaient profondément conscients de ce clivage et savaient le rationaliser.

La monographie de Hughes a ouvert la voie à toute une série d'études plus globales, particulièrement sous forme de thèses de maîtrise à l'Université McGill. Ici, sans doute, faut-il associer à celui de Hughes les noms de C.A. Dawson et d'Oswald Hall, l'intérêt de Hall pour la sociologie des occupations ayant déjà été éveillé à l'Université de Chicago où il avait fait sa thèse de doctorat sur la pratique médicale. 229 Dawson et Hall ont servi de mentors à plusieurs des jeunes chercheurs de McGill. Il faudrait [178] ici mentionner les thèses de W.J. Roy 230 et de S. Jamieson, 231 deux travaux de pionniers sur la distribution des Canadiens français et des Canadiens d'expression anglaise dans différents secteurs du travail ; puis celles de Rex Lucas sur les aspirations

228 Everett C. Hughes, French Canada in Transition, Chicago, University of Chicago Press, 1943. [La version française du livre est disponible dans Les Classiques des sciences sociales. JMT.]

229 Oswald Hall, The Informal Organization of Medical Practice in an American City, Unpuhlished Ph. D. thesis, Chicago University, 1944.

230 W. J. RoY, The French-English Division of Labor in the Province of Québec, Unpublished M. A. thesis, McGill University, 1935.

231 S. JAMIESON, French and English in the Institutional Structure of the Province of Québec, Unpublished M. A. thesis, McGill University, 1958. 
professionnelles des élèves de high school 232 dans une ville biethnique de l'Ontario et de Frank Jones sur les travailleurs dans l'industrie de l'acier de construction. ${ }^{233}$ Plus récemment, D. L. C. Rennie a continué les grandes études de Roy et Jamieson sur la base des renseignements contenus dans les recensements de 1931, 1941 et 1951 touchant la répartition des groupes ethniques dans la structure des emplois pour la ville de Montréal. ${ }^{234}$ Enfin, Bernard Blishen a fait de même pour l'ensemble du Canada, en utilisant les données du recensement de 1951. ${ }^{235}$ Utilisant comme critères le revenu moyen et le nombre d'années de scolarité, Blishen a construit une échelle occupationnelle de sept classes et il a ensuite reporté les principaux groupes ethniques canadiens dans ces sept classes. Ses conclusions méritent d'être citées :

« Les Britanniques, avec 49.0\%, sont sous-représentés dans les deux classes inférieures et généralement sur-représentés dans les autres classes plus élevées. Les Français, qui représentent 27.5\%, sont sur-représentés dans les deux classes inférieures et généralement sous-représentés dans les classes supérieures. En d'autres termes, la proportion des Britanniques s'accroît de façon générale quand on passe des classes inférieures aux classes supérieures tandis que c'est l'inverse pour les Français. Le groupe juif suit le même mouvement que les Britanniques tandis que les Canadiens d'autres origines suivent le modèle français. »

Il est en effet remarquable, si l'on analyse de près le tableau de Blishen, que les Français soient représentés dans les sept classes à peu près exactement de la même façon que les Italiens et les Polonais et même légèrement moins bien que les Scandinaves et les Allemands.

232 Rex A. LuCAS, Occupational Orientation of High School Entrants in a BiEthnic Railroad Town, Unpublished M. A. thesis, McGill University, 1950.

233 Frank E. JonES, Work Organization in the Structural Steel Industry : A Study of Industrial Organization and of Ethnic Relations Among Structural Steel Workers, Unpublished M. A. thesis, McGill University, 1950.

234 D. L. C. RENnIE, The Ethnic Division of Labour in Montréal from 19311951, Unpublished M.A. thesis, McGill University, 1953.

235 Bernard R. Blishen, « The Construction and Use of an Occupational Class Scale ", The Canadian Journal of Economics and Political Science, 24, 4, November 1958, 519-531. 
Dans la même veine, il faudrait mentionner les études de John Porter sur l'élite économique et l'élite politique au Canada. Sur 202 hauts fonctionnaires du gouvernement canadien, 27 seulement, soit 13.4\%, sont Canadiens français. Parmi les 700 personnes constituant une élite et un [179] pouvoir économique, Porter n'a identifié que 51 Canadiens français, soit $6.7 \%$. ${ }^{236}$

Revenons au paradoxe mentionné plus haut. Devant de telles conclusions, n'est-il pas étonnant que nous n'avions pas encore mentionné un seul nom de sociologue canadien-français ? Faut-il davantage s'étonner de l'absence de sens politique des sociologues - qu'ils soient français ou anglais — ou admirer plutôt leur impartiale objectivité ? Pourtant, un sociologue canadien-français s'est d'une certaine façon intéressé à ce problème. Jacques Brazeau, dans sa thèse de doctorat et un article récent, a mis en lumière les problèmes psychosociologiques des recrues canadiennes-françaises dans l'aviation canadienne, celle-ci étant définie comme une organisation essentiellement anglaise. 237

Dans son étude comparative des hommes d'affaires de langue française et de langue anglaise du Québec, Norman Taylor a décrit avec une grande richesse d'illustrations les différences de mentalité qui séparent ces deux types d'industriels. Son étude met en évidence la timidité, le besoin de sécurité des hommes d'affaires canadiens-français, leur sens familial, l'importance qu'ils accordent aux relations de type primaire. 238 Je suis prêt à accorder une grande valeur descriptive à l'étude de Taylor, mais je crois que, pour être valable et ne pas tourner

236 John PorTer, « The Economic Elite and the Social Structure in Canada ", The Canadian Journal of Economics and Political Science, 23, 5, August 1957, 376-394 et « Higher Public Servants and the Bureaucratie Elite in Canada », ibid., 24, 4, November 1958, 483-501.

237 Jacques BrazeAu, The Training of French-Canadian Ground Crew Personnel in the Royal Canadian Air Force (1953-1957), Unpublished Ph. D. thesis, Chicago University, 1961 ; aussi «Language Differences and Occupational Experience », The Canadian Journal of Economics and Political Science, 24, 4, November 1958, 532-540.

238 Norman W. TAYLOR, A Study of French Canadians as Industrial Entrepreneurs, Unpublished Ph. D. thesis, Yale University, 1957 ; «French Canadians as Industrial Entrepreneurs ", Journal of Political Economy, LXVIII, 1, February 1960, 37-52 ; "L'industriel canadien-français et son milieu », Recherches sociographiques, II, 2, avril-juin 1961, 123-150. 
à la tautologie, cette "mentalité » doit s'expliquer dans le contexte global décrit dans les études précédentes. Je crois qu'en particulier la thèse de doctorat de Jacques Brazeau fournirait des indications précieuses sur une mentalité qui, plus que le résultat ou le reflet d'une structure sociale du Canada français, serait plutôt un ensemble d'attitudes de repli, de recroquevillement en face d'un univers linguistique et culturel étranger, partiellement impénétrable et peut-être perçu par surcroît comme menaçant ou méprisant.

On peut dire, en conclusion de cette section, que toutes ces études débouchent ou peuvent déboucher sur une analyse des classes sociales au Canada. À l'occasion d'un colloque récent tenu à l'Université Carleton, John Porter et à sa suite Marcel Rioux et Jacques Dofny, s'appuyant sur les études que je viens de résumer, ont cru pouvoir parler du Canada français comme d'une " classe ethnique » dans le système de classes de la société [180] globale canadienne. ${ }^{239}$ Comme le disait Porter, le groupe français et le groupe britannique ont sans doute chacun leur système de classes; mais ces deux groupes sont en même temps imprégnés dans un réseau de relations économiques et politiques dans lequel les Canadiens français font figure d'une classe ethnique défavorisée au même titre que les autres groupes ethniques de Néo-Canadiens, à l'exception des Juifs. Cette notion de classe ethnique s'intégrant dans le système de classes de la société globale canadienne apportera-t-elle plus de lumière que de confusion? J'avoue mon hésitation à l'utiliser étant donné les réseaux de communication existants - et qu'il ne faut pas minimiser — entre les groupes ethniques à différents niveaux de classe sociale ou de strate : dans la classe ouvrière par le syndicalisme, dans la classe professionnelle et d'affaires par les associations volontaires ou professionnelles de différents ordres. Dans quelle mesure en effet peut-on dire que l'ensemble du Canada français se pense lui-même en tant que classe ethnique, comme on peut dire des ouvriers de certains pays qu'ils se pensent en tant que classe ouvrière ? Par exemple, le syndicalisme canadien, même canadien-français, ne va-t-il pas à l'encontre d'une telle représentation collective ? Je serais porté à croire qu'il faut plutôt penser en termes d'un double système de classes, s'imprégnant l'un dans l'autre à cer-

239 John PORTER, «Class in Canadian Social Structure », texte manuscrit ; Jacques DofNy et Marcel Rioux, "Les classes sociales au Canada français », Revue française de sociologie, III, 3, juillet-septembre 1962. 
tains niveaux, mais relativement autonomes l'un par rapport à l'autre. Que la représentation proportionnelle de la population ne soit pas la même aux différents paliers dans les deux systèmes ne fait pas que l'un des deux doive être conçu comme une classe ethnique, au sens où l'on parle de classe sociale.

\section{Stratification et mobilité sociales}

Nous sentons cependant ici combien nous sommes hésitants à poursuivre cette discussion du fait de l'absence de recherches assez importantes sur nos classes sociales et notre stratification sociale. Comment en effet parler de classes sociales ou de strates sociales au Canada français dans l'état actuel de nos connaissances ? Comme je le disais au début de cet exposé, c'est là, à mon sens, une lacune majeure et très grave dans nos recherches sociographiques sur le Canada français.

La question a cependant été abordée sous différents angles. Dans des "Réflexions sur nos classes sociales », Jean-Charles Falardeau proposait il y a quelques années l'hypothèse d'une double échelle d'évaluation sociale. ${ }^{240}$ La première, qui aurait été élaborée dans notre milieu et en refléterait les valeurs traditionnelles dominantes, serait constituée par les jugements populaires sur les occupations ou les groupes d'occupations, d'après leur [181] niveau de fortune et l'instruction requise. La seconde, plutôt nord-américaine d'origine et plus ou moins surajoutée à la première, serait fondée sur une appréciation du succès économique d'après le chiffre de la fortune. Mal intégrées l'une à l'autre, ces deux échelles seraient en conflit dans la conscience collective, conflit plus ou moins aigu selon les milieux et selon les moments de la vie des personnes.

On peut dire que cette hypothèse d'un conflit dans l'évaluation sociale, tout à fait plausible, dans ces termes ou dans d'autres, n'a été ni confirmée ni infirmée par les études subséquentes. Il aurait fallu pour cela étudier la stratification sociale dans son aspect subjectif, sonder la

240 Jean-Charles FALARDEAU, «Réflexions sur nos classes sociales », Nouvelle revue canadienne, $I$, $3,1-9$. 
conscience de classe et en cerner les fondements. Une telle entreprise reste encore au programme des recherches futures.

Une étude récente apporte cependant certains renseignements dans ce sens. Dans le cadre de l'enquête sur la persévérance scolaire sous la direction d'Arthur Tremblay et Pierre Bélanger, on a cherché à construire une échelle de stratification sociale afin de mesurer l'influence qu'aurait l'appartenance à une strate sociale sur la persévérance et les aspirations scolaires. Les auteurs de cette recherche ont utilisé cinq critères pour construire leur index de stratification : l'occupation du père, son degré de scolarité, la scolarité de la mère, le nombre de pièces par personne et la nature des biens mobiliers. ${ }^{241}$ Dans une communication aux journées d'étude de l'Association d'éducation du Québec sur « Les aspirations scolaires et la stratification sociale », Claude Gousse a montré que l'analyse factorielle de ces cinq variables a dégagé trois facteurs : le premier est constitué par l'occupation et la scolarité du père, le second par le nombre de pièces par personne, le troisième par la scolarité de la mère et les biens mobiliers. Ces résultats laissent un peu rêveur. En particulier, comment expliquer ce troisième facteur, surtout que, comme dit Gousse, les deux variables sont reliées en sens opposé, l'acquisition des biens étant inversement proportionnelle à la scolarité de la mère. Nous nous serions plutôt attendu à voir les variables portant sur le nombre de pièces par personne et la possession des biens mobiliers se regrouper pour constituer un facteur définissant la position économique de la famille. D'ailleurs, les auteurs ont comparé leurs résultats avec ceux obtenus aux États-Unis par Joseph A. Kahl et James A. Davis dans une recherche utilisant des techniques statistiques assez semblables. ${ }^{242}$ Or, l'étude américaine avait précisément permis de dégager assez nettement d'une part le facteur de l'occupation et de la scolarité et d'autre part le facteur de la fortune. Les auteurs de l'étude de l'A.É.Q. paraissent avoir [182] été surtout frappés par la similarité entre l'échelle américaine et l'échelle du Canada français. Je me demande si les différences ne méritent pas autant

241 Claude Gousse, « Aspirations scolaires et stratification sociale », communication présentée à la session d'étude de l'Association d'éducation du Québec, décembre 1961 (texte polycopié).

242 Joseph A. KAHL and James A. DAVIS, « A Comparison of Indexes of Socioeconomic Status », American Sociological Review, 20, 3, June 1955, 317325. 
d'attention que les similarités indéniables. Peut-être retrouverionsnous ici ce conflit posé en hypothèse par Falardeau concernant en particulier la place des symboles de fortune dans la stratification sociale. On peut, en effet, se demander si cette étude n'indique pas au Canada français une attitude moins claire, moins ferme à ce sujet que celle révélée pour les États-Unis par l'étude de Kahl et Davis.

L'étude des occupations et de la stratification a cependant été abordée dans une autre perspective, celle de la mobilité professionnelle et sociale. De Jocas et moi avons tenté une analyse assez globale du phénomène en comparant les occupations des pères et des fils à environ vingt années de distance à partir d'un échantillon provincial. 243 Les quatre principales conclusions qui se sont dégagées de cette étude sont les suivantes. Tout d'abord, c'est de l'occupation de la ferme à l'occupation urbaine que le passage se fait le plus souvent. Seulement un fils de cultivateur sur quatre est demeuré cultivateur au moment de son mariage. Mais ce qui est plus grave, c'est que la majorité de ceux qui ont quitté la ferme se retrouvent dans des occupations de manœuvres et d'ouvriers non spécialisés. L'impréparation du jeune cultivateur à effectuer le passage au marché du travail industriel s'avère ici dans tout ce qu'elle a de dramatique. En second lieu, cette étude a montré que la mobilité ascendante se faisait par paliers successifs : le fils de cultivateur qui change d'occupation devient ouvrier non spécialisé ; le fils d'ouvrier non spécialisé a des chances plus grandes de devenir ouvrier spécialisé ; le fils d'ouvrier spécialisé peut devenir petit colletblanc et le fils de petit collet-blanc peut devenir professionnel ou gérant. En troisième lieu, ce mouvement d'ascension par paliers paraît assez comparable à ce qui avait déjà été observé aux États-Unis, notamment par Nathalie Rogoff, 244 avec cependant moins de flexibilité ici. En particulier, les fils de cultivateurs américains se répartissent plus également dans les différentes strates d'occupations et les fils d'ouvriers américains ont plus de chances de devenir collets-blancs qu'au Canada français. Enfin, quatrièmement, une certaine mobilité descendante est nettement observable dans les classes supérieures

243 Yves DE JocAs et Guy RocHER, « Inter-generation Occupational Mobility in the Province of Québec », The Canadian Journal of Economics and Political Science, 23, 1, February 1957, 57-68.

244 Nathalie Rogoff, Récent Trends in Occupational Mobility, Glencoe, III., Free Press, 1952. 
d'occupations, un fils de professionnel ou de semi-professionnel ouvrier pouvant assez souvent être ouvrier spécialisé ou même non spécialisé.

Dans cette même ligne, une étude monographique plus limitée, mais poussée beaucoup plus en profondeur fut réalisée par Gérald Fortin, Émile Gosselin, M.-Adélard Tremblay et Louis-M. Tremblay sur le travailleur en [183] forêt. ${ }^{245}$ Dans une série d'articles, ces auteurs ont étudié un des aspects du passage de l'occupation agricole à une occupation qui prend de plus en plus un caractère industriel, l'occupation de bûcheron. Cette étude, replacée dans le contexte du milieu agricole particulier où se recrutent généralement les bûcherons, a mis en lumière la relation entre le travail en forêt et la pratique ou la nonpratique de l'agriculture, la mobilité professionnelle inter- et intragénération dans ces milieux et les stress psychologiques attachés à ce genre de travail et aux conditions dans lesquelles il se pratique. Plus peut-être encore qu'une étude de mobilité professionnelle (car elle est plus large que cela), on peut considérer cette recherche comme un premier jalon de sociologie industrielle chez nous. Et il est heureux qu'elle ait été faite dans une perspective plus nettement sociologique et structurelle que dans la tradition américaine des human relations.

Je voudrais mentionner, pour terminer, une autre étude que l'on pourrait classer dans le même courant, bien qu'elle ait porté sur une occupation qui se situe à l'autre extrême de l'échelle sociale : il s'agit de la thèse de maîtrise de Jacques Brazeau sur la pratique médicale. ${ }^{246}$ Brazeau a en effet analysé les différents types de pratiques médicales chez les Canadiens français de Montréal. Comme il le dit luimême, c'est en tant que constituant une élite professionnelle et sociale que Brazeau considère le médecin et qu'il analyse les voies d'accès à cette profession. De ce point de vue, sa recherche constitue la contribution la plus remarquable à l'analyse des élites canadiennes-

245 Gérald ForTin et Émile Gosselin, « La professionnalisation du travail en forêt ", Recherches sociographiques, I, 1, janvier-mars 1960, 33-60 ; MarcAdélard TREMBLAY, «Les tensions psychologiques chez le bûcheron ; quelques éléments d'explication », ibid., I, 1, janvier-mars 1960, 61-89 ; Gérald ForTin et Louis-Marie TREMBLAY, "Les changements d'occupations dans une paroisse agricole ", ibid., I, 4, octobre-décembre 1960, 467-495.

246 Jacques Brazeau, The French Canadian Doctor in Montréal : A Study of Careers in a Profession, Unpublished M.A. thesis, McGill University, 1951. 
françaises, en même temps d'ailleurs que l'analyse la plus fouillée d'une occupation en termes de rôle social.

\section{Conclusion}

Au terme de cette vue à vol d'oiseau de certains travaux de recherche dans le secteur des occupations et de la stratification sociale nous espérons n'avoir pas omis d'étude importante - il nous semble que nous pourrions faire quelques suggestions pour guider les recherches futures.

En premier lieu, il est évident qu'il nous manque une sociologie des classes sociales du Canada français. Nous avons déjà souligné cette lacune. Les études dans ce domaine seront extrêmement difficiles à faire à cause de la rapide évolution en cours et à venir. Nous formulons en effet l'hypothèse que le Canada français du XIX ${ }^{\mathrm{e}}$ siècle a vécu, en matière de classe sociale, dans une mentalité du type féodal, c'està-dire que l'on reconnaissait et acceptait une hiérarchie établie, relativement stable et définitive. C'est [184] cette mentalité qu'est venue bouleverser l'industrialisation en modifiant à la fois l'état de fait et l'état des esprits. De nouvelles catégories sociales sont apparues et ont proliféré (en particulier les ouvriers industriels et les employés colletsblancs) et de nouvelles valeurs se sont imposées. Le Canada français n'a jamais participé au credo américain de la société sans classe. Il a plutôt vu le système de stratification qu'il acceptait se modifier profondément sans pouvoir en retrouver un autre aussi cohérent dans la nouvelle société industrielle. J'avoue ici être particulièrement fasciné par l'hypothèse du conflit de valeurs et de conscience avancée par Jean-C. Falardeau. Mais de nouveaux éléments entrent aujourd'hui en ligne de compte, dont le principal me paraît être la généralisation d'un niveau plus élevé d'instruction. Ce que l'on peut appeler la démocratisation de l'enseignement, en élargissant l'accès aux études supérieures et en imposant pratiquement la scolarité secondaire à l'ensemble de la population, me paraît apporter une révolution sociale plus profonde encore en développant de nouvelles aspirations, en ouvrant à tous le principal canal de mobilité qu'est l'éducation et en modifiant les lignes de clivage social. 
Dans ce contexte de transformation rapide, il me semble que l'étude des élites (élite syndicale, élite rurale, élite technicienne, élite professionnelle) constituera un point stratégique de saisie des aspirations, des prises de conscience, des définitions collectives, en même temps qu'un palier d'analyse des structures en évolution. C'est dans et par ces élites que seront définis les nouvelles images et les nouveaux symboles de classe de la société future. À travers ces élites, peut-être obtiendrons-nous une meilleure perception de la conscience de classe, si elle existe chez nous, que par une approche trop globale et qui risquerait finalement la confusion.

En second lieu, je souhaite des études plus nombreuses des milieux de travail, soit à partir d'occupations individuelles soit à partir de secteurs d'activités industrielles. Si la sociologie ne veut pas perdre contact avec le monde qui s'élabore rapidement autour de nous, elle doit plonger à fond dans l'analyse des transformations qui s'opèrent dans ces milieux. L'étude des travailleurs de la forêt illustre bien l'apport de ce genre de recherche à une connaissance plus profonde des processus d'organisation autant que de désorganisation sociale.

Enfin, en troisième lieu, étant donné les conséquences prévisibles de la démocratisation de l'enseignement dont je viens de parler, il me semble que les études de mobilité professionnelle verticale et horizontale prendront une importance considérable. Elles permettront de suivre de plus près les changements de structure et de mentalité apportés par ce nouveau canal de mobilité et d'en mesurer la portée.

Guy Rocher

Département de sociologie, Université de Montréal. 


\title{
COMMENTAIRE
}

\author{
Émile Gosselin \\ Département de science politique, \\ Université Laval.
}

[pp. 185-188.]

$\underline{\text { Retour à la table des matières }}$

Je voudrais féliciter monsieur Guy Rocher pour son exposé franc, dépouillé d'artifice et honnête. S'abstenant de vouloir tout dire, il a assombri, peut-être à dessein, le bilan des recherches sur les occupations et la stratification sociale. Monsieur Rocher s'est surtout attaché à démontrer les lacunes de la recherche en certains domaines, parfois même son absence totale. Il nous indique plusieurs thèmes à explorer en vue d'approfondir nos connaissances sur les occupations et la stratification sociale.

Je tomberai immédiatement d'accord avec l'auteur sur la nécessité, d'abord, de vérifier l'hypothèse d'une discordance entre une double échelle de valeurs, celle dont parle Jean-C. Falardeau et, ensuite, de situer le conflit qui en résulterait, au plan de la conscience collective et à celui de la conscience individuelle. Cette recherche devrait, à mon avis, porter sur le fait même de la discordance, sur ses origines et ses effets. 
Il serait particulièrement intéressant de tracer en premier lieu un parallèle entre l'étude de Léon Gérin, Le type économique et social des Canadiens, 247 qui pourrait constituer un point de départ, et les travaux de Horace Miner, St. Denis, A French-Canadian Parish, 248 et ceux de Everett C. Hughes sur « Cantonville ». Il faudrait ensuite comparer les travaux de Gérald Fortin sur les "Attitudes à l'égard des occupations dans une paroisse agricole » 249 avec les études sur les problèmes socio-économiques des travailleurs en forêt, ${ }^{250}$ pour aboutir finalement aux analyses poursuivies dans les milieux acadiens français ou mixtes, en Nouvelle-Écosse, par M.-Adélard Tremblay, principalement, études auxquelles j'ai apporté quelque contribution. 251

À la suite d'études comparées de divers milieux, nous pourrions nous poser la question suivante : y a-t-il des constantes dans les réactions du Canadien français à l'endroit des changements technologiques ou socio-économiques rapides ? Cette double échelle de valeurs, dont parle Jean-C. Falardeau, est-elle propre à notre milieu ? Ou bien, une étude comparative de comportements de minorités nous ferait-elle aboutir à la constatation que, dans de mêmes circonstances, les minorités tendent à posséder des échelles doubles de valeurs et à se comporter de la même façon?

Monsieur Rocher déplore à juste titre l'absence d'une histoire du travail au Canada. Ce qu'il y a d'aussi déplorable, et peut-être plus encore au stade présent des recherches, c'est que les travaux épars qui ont été effectués sur divers aspects du travail n'aient pas été colligés et

247 Montréal, Éditions de l'A.C.F., 1938.

248 University of Chicago Press, 1939.

249 Recherches sociographiques, II, 1, janvier-mars 1961, 35-54.

250 Études poursuivies par Émile Gosselin, Gérald Fortin, M.-Adélard , Tremblay et Charles Lemelin, professeurs à l'Université Laval. Voir: Gérald FORTIN et Émile GOSSELIN, «La professionnalisation du travail en forêt », Recherches sociographiques, I, 1, janvier-mars 1960, 33-60 et M.-Adélard TREMBLAY, «Les tensions psychologiques chez le bûcheron : quelques éléments d'explication », ibid., 61-89.

251 Voir, en particulier : C. C. Hughes, M.-Adélard TRemblay, R. N. RapoPORT, and A. H. LeIGHTON, People of Cove and Woodlot: Communities from the Viewpoint of Social Psychiatry, New-York, Basic Books, Inc., 1960 ; Émile Gosselin et M.-Adélard TREMBLAY, « Loomervale : un cas de désintégration sociale ", Recherches sociographiques, $I$, 3 , juillet-septembre 1960, 309-342. 
annotés de façon à ce que l'on puisse s'y retrouver facilement. Certains progrès ont été [186] accomplis en vue d'une recherche dans le domaine du syndicalisme et de la main-d'œuvre par l'abbé Gérard Dion, Roger Chartier et leurs collègues du Département des relations industrielles et par d'autres collègues de la Faculté des sciences sociales de Laval qui, je le crois, méritent une juste place dans une anthologie sur le monde du travail.

Je déplorerai surtout le fait que les travaux d'histoire économique ne soient pas suffisamment à jour pour nous indiquer comment les diverses régions économiques du pays ont évolué. Il nous faudrait mieux connaître les liens d'interdépendance qui se sont créés entre les régions du pays et, plus particulièrement en ce qui nous concerne, la place qui a été faite au Québec dans le développement de nos ressources matérielles et humaines. Que connaissons-nous des conséquences que ces transformations ont entraînées au plan des industries, des occupations et des institutions de la province ? Les Essais sur le Québec contemporain constituaient une heureuse initiative dans cette direction.

Peut-on tout expliquer au Québec par le fait de notre appartenance à tel groupe ethnique plutôt qu'à tel autre ? Possédons-nous des caractéristiques dont nous aurions seuls le monopole et qui expliqueraient à la fois nos gains et nos reculs ? Qu'il y ait des conflits de valeurs, soit ! Nous ne sommes pas les seuls à les subir. Tous les pays en voie de transformations rapides, toutes les régions affectées par ces transformations et qui en subissent les contrecoups, font face à des problèmes sensiblement les mêmes. Je verrais ici un grand avantage à entreprendre une étude de milieux culturellement divers et une analyse comparative de leurs comportements organisés ou désorganisés lors de ces transformations.

Même si monsieur Rocher s'excuse d'avoir à traiter dans la même étude de milieux de travail et de stratification sociale, je crois qu'il s'agit là d'une honnête et fort heureuse faute. Je voudrais l'avoir commise personnellement. En effet, traiter séparément d'échelles d'occupations, de stratifications sociales, d'échelles de valeurs dans un milieu pluraliste où les choix dans les domaines économiques et sociaux au niveau des régions et des individus sont loin d'être cohérents et parfois contradictoires, m'apparaît une tâche extrêmement difficile, sinon impossible. Il nous faudrait, en conséquence, pousser parallèlement 
trois types d'analyses qui convergeraient vers le but même de l'étude que propose le conférencier.

La première analyse qui s'impose doit porter sur la demande de travail, c'est-à-dire sur la répartition régionale de divers types d'industries, époque par époque. Cette étude devrait comporter un examen des mécanismes économiques et politiques qui ont entraîné les transformations au niveau de la demande. Ainsi, nous posséderions, à la fois, tant le tableau et la structure des industries et des occupations qu'une interprétation économique valable des transformations de la demande, donc de diverses échelles d'occupations selon les régions.

Une deuxième démarche devrait analyser les valeurs dominantes et les institutions sociales qui ont facilité ou retardé l'adaptation sociale, collective ou individuelle, à chaque phase ou époque de l'évolution économique.

En troisième lieu, il faudrait procéder à une étude comparée des comportements de divers groupes sociaux ou ethniques au sein de contextes industriels semblables et même identiques. Il y aurait grand avantage à faire précéder une telle étude de monographies portant sur des milieux déterminés.

[187]

Avant de procéder à de telles études, sociologues et économistes auraient grand intérêt à fouiller certains travaux préparés à la demande la Commission Gordon, particulièrement l'étude à laquelle a collaboré Yves Dubé, directeur du Département d'économique de Laval, sur L'habitation et Le capital social. ${ }^{252}$ Soulignons de façon toute spéciale l'analyse préparée par la Division de la recherche économique du Ministère du travail à Ottawa, portant sur la main-d'œuvre professionnelle et spécialisée au Canada. Cette étude, présentée au Comité spécial du Sénat sur l'emploi et la main-d'œuvre, était accompagnée de

252 Yves DubÉ, J. E. Howes et D. L. McQueEn, L'habitation et le capital social, Ottawa, Commission royale d'enquête sur les perspectives économiques du Canada, 1957. 
travaux remarquables dont ceux de F. T. Denton 253 et W. R. Dymond. 254

Il y a trois études qui m'apparaissent tout à fait pertinentes au sujet que nous traitons et qui, encore aujourd'hui, peuvent nous fournir nombre d'hypothèses, même si elles ont été faites au cours de la crise économique de 1930.

Je voudrais mentionner tout particulièrement des travaux de pionniers : les deux ouvrages de L. C. Marsh, Canadians In and Out of Work 255 et Employment Research, 256 et le volume de George V. Haythorne, Land and Labour. ${ }^{257}$ Dans Employment Research, on trouve l'un des premiers jalons valables d'une recherche en sociologie industrielle entreprise dans un milieu québécois. Déjà dans ce travail, où il est question de la répartition de la main-d'œuvre montréalaise par occupation, industrie et groupe ethnique et où l'on compare le Canada, le Québec et l'Ontario selon divers facteurs, une analyse qui me paraît des plus justes est faite de la facilité ou de la relative difficulté de divers milieux de travail à s'adapter à la demande d'emplois. Premièrement, remarque l'auteur, il y a le problème des influences institutionnelles et autres qui facilitent l'adaptation sociale et économique. En deuxième lieu, il y a le problème de la polyvalence de la maind'œuvre. Un transfert de population signifie une réadaptation au niveau des conditions de travail, du rang social, des exigences requises. C'est à ce moment que vont jouer l'expérience culturelle, linguistique, pratique, la tradition reçue, etc. Une question fort pertinente se pose : l'immigration offre-t-elle à l'industrie canadienne une main-d'œuvre mieux préparée sur le plan psychologique, mental, technique et possédant une meilleure expérience que les candidats aux emplois que nous formons nous-mêmes dans nos divers types d'écoles?

Je voudrais terminer par une vue prophétique que je puise dans une citation de L. C. Marsh, qui indique bien la relation ou, mieux, cette

253 Comité spécial du Sénat sur l'emploi et la main-d'œuvre, Ottawa, 30 novembre 1960.

254 Comité spécial du Sénat sur l'emploi et la main-d'œuvre, Ottawa, le $1^{\text {er }}$ février 1961.

255 Oxford University Press, 1940.

256 Oxford University Press, 1935.

257 Oxford University Press, 1941. 
absence relative de relation entre emploi, rang social et groupe ethnique :

« Il n'y a pas d'image plus complexe que celle qui reflète la présence de divers groupes ethniques et raciaux au sein des divers secteurs d'une structure occupationnelle déterminée. À première vue, la recherche d'un lien semble contraire à toute logique. Des différences de classes existent au sein de tous les groupes ethniques et raciaux. Par exemple, chaque groupe ethnique d'immigrants se retrouve aux divers niveaux de l'échelle des occupations, et ainsi de suite. Par contre, certains groupes raciaux montrent des préférences ou prédispositions [188] professionnelles ; d'autre part, on peut démontrer une prédominance ou une absence de certains groupes ethniques dans certaines classes sociales et, du moins superficiellement, l'on peut en faire une caractéristique de ces mêmes classes sociales. Mais, l'appartenance ethnique, en tant que telle, est-elle le facteur déterminant ? Il est facile de confondre les différences ethniques et les divers facteurs économiques propres à chaque milieu et qui se sont avérés des facteurs de conditionnement autrement plus puissants que l'appartenance à un groupe ethnique déterminé. À quoi servent des préférences pour certaines occupations, si les possibilités d'accéder à ces occupations n'existent pas ? Les prédispositions de l'intelligence ou des tempéraments requièrent, à tout le moins, quelque entraînement ou tradition de métier. L'on doit donc continuellement interpréter par le recours à d'autres facteurs le rôle que joue la race ou la nationalité. Au sein de chaque groupe ethnique, il faut reconnaître des branches diverses. ... Appartenir à un même pays d'origine n'entraîne pas des chances égales d'emploi pour tous — par exemple, pour les Canadiens d'origine rurale ou urbaine. La tradition rurale de nombre de Canadiens français les place devant les mêmes difficultés, sur le plan des occupations, que nombre d'immigrants «étrangers »... D'une façon quelconque, les couches sociales et les caractéristiques sociales sont importées, même si le processus de leur assimilation dans un nouveau pays peut varier considérablement.

« Ce qui détermine la représentation des divers lieux d'origine aux divers paliers occupationnels ne peut s'expliquer par le facteur ethnique uniquement. Les facteurs culturels, éducatifs et économiques ont également leur importance. »

L'auteur poursuit en affirmant que certains groupes dominent parce qu'ils se sont implantés les premiers. D'autres, moins actifs, ont suivi la loi du moindre effort : 
«Dans plusieurs secteurs d'occupation, l'affinité de race n'est pas la cause première de supériorité, mais on l'invoque et on la met à profit en vue de défendre certaines chapelles économiques contre toute intrusion ... D'autre part, certains groupes économiquement minoritaires se sont réfugiés dans la petite entreprise pour mieux se protéger. » 258

\section{Et l'auteur de conclure :}

« L'étude de l'établissement successif et des caractéristiques des groupes ethniques constituera une variable essentielle dans toute planification sociale au Canada et cela, pour des années à venir ; mais l'étude de ces groupes différents devra être faite parce qu'elle complique le problème des inégalités sur le plan des occupations et non pour nous faire oublier les facteurs déterminants de ces inégalités ». 259

\section{Émile Gosselin}

Département de science politique, Université Laval.

258 L. C. MARsh, Canadians In and Out of Work, op. cit., 161-162.

259 Ibid., 163. 
Situation de la recherche sur le Canada français

III. Perspectives sur l'étude de la structure sociale

\section{Bernard Mailhiot, o.p.}

\section{"Les recherches en psychologie sociale au Canada français (1946-1962).”}

[pp. 189-202.]

$\underline{\text { Retour à la table des matières }}$

En plus de vouloir être une nomenclature exhaustive des travaux de recherche en psychologie sociale dans notre milieu et sur notre milieu, cet exposé voudrait tenter de dégager à la fois les orientations et les préoccupations dominantes qui ont marqué l'évolution de la psychologie sociale au Canada français. Nous procéderons en premier lieu à l'examen des recherches faites à l'Université Laval, à la Faculté des sciences sociales, pour terminer par l'évaluation des travaux achevés à l'Université de Montréal. À l'Université McGill, plusieurs recherches sont en cours sous la direction du professeur Wallace E. Lambert. Elles se concentrent toutes sur le même problème : «l'apprentissage d'une langue seconde ». De ce qui a été publié à date, aucun résultat ne comporte pour le moment d'implications spécifiques pour le psychologue social. Ce n'est que plus tard que le professeur Lambert et ses assistants comptent tenter de définir opérationnellement le niveau minimum de socialisation requis pour l'apprentissage 
d'une langue seconde. Il en va de même de l'Université d'Ottawa ; aucun travail relevant à proprement parler de la psychologie sociale n'est à signaler présentement.

Notre inventaire se limitera donc aux seules recherches entreprises aux universités Laval et de Montréal.

\section{Université Laval}

Onze thèses de maîtrise présentées à la Faculté des sciences sociales de 1946 à 1962 peuvent être considérées à des titres divers comme des travaux de psychologie sociale. Trois de ces thèses constituent des études théoriques, de mérite variable, à savoir :

Pierre Boucher, Introduction à une étude de la foule, 1946 [8] *.

Suzanne McLaren, Le concept d'anomie : essai de clarification conceptuelle, 1961 [46].

Michel CÔTÉ, Le leadership : situation d'un concept sociologique, 1962 [22].

[190]

Les huit autres thèses relèvent plutôt de la psychologie appliquée. Si l'on s'en tient aux titres, trois portent sur des problèmes de délinquance, à savoir :

Thérèse Clapperton, Facteurs individuels et sociaux décelés dans l'étude de cinquante-six cas de vol du Centre psycho-social, 1952 [15].

Roland Provençal, p.m.é., Les méthodes de rééducation au Mont SaintAntoine de Montréal, 1953 [50].

Gilbert Gagnon, Facteurs psycho-sociaux de la délinquance, 1959 [29].

Par ailleurs deux autres thèses ont tenté de dégager quelques implications de la psychologie sociale au niveau de la pédagogie. Ce sont :

Voir la bibliographie à la fin de l'article. 
Denise MORRISSETTE, Initiation des enfants à la vie sociale, 1951 [48].

Pierre BÉLANGER, Profil culturel des maîtres de $4^{e}$ à $7^{e}$ année : approche typologique, 1960 [2].

Les trois autres thèses portent sur les rapports et les affinités de certaines données de la psychologie sociale avec les thèmes en faveur dans d'autres sciences sociales, à savoir :

André DESPRÉs, L'opinion publique et les relations industrielles, 1946 [24].

Simone PARÉ, Expériences de formation de chefs, 1947 [49].

Madeleine DALMÉ, La formation des groupes et le travail social, 1948 [23].

La psychologie sociale ne constituant pas un département autonome au sein de la Faculté des sciences sociales et n'étant l'objet que de quelques séries de cours, l'on s'explique que très peu d'étudiants aient choisi, au moment de leur thèse, d'explorer un problème qui relève spécifiquement de cette discipline.

\section{Université de Montréal}

La recherche en psychologie sociale à l'Université de Montréal débute en 1950, mais elle ne prend vraiment son essor qu'à partir de 1952, avec la fondation du Centre de recherches en Relations humaines qui dès ce moment devient le laboratoire de psychologie sociale de l'Institut de psychologie. Dans les dix ans qui suivront, la recherche en psychologie de groupe connaîtra trois phases. 


\section{Psychologie des relations interethniques}

Influencées très fortement par les travaux de Kurt Lewin sur la psychologie des minorités discriminées, les premières recherches tentaient [191] d'éclairer un problème fort complexe : Le Canadien français se perçoit-il comme un minoritaire discriminé. ?

Un premier travail de déblaiement fut entrepris non sans courage et avec un excellent sens critique par madame Monique Lortie-Lussier dans le but de préciser et d'établir ce que les travaux antérieurs sur la sociologie et la psychologie de notre groupe ethnique avaient pu révéler. Les résultats de cette enquête furent publiés dans le premier numéro des Contributions à l'étude des sciences de l'homme, sous le titre : "Les relations bi-culturelles au Canada » [16]. Grâce à ce bilan, nos premières recherches purent s'orienter en continuité avec ce que $\mathrm{M}^{\text {me }}$ Lussier avait réussi à dégager comme acquis sur la psychologie du Canadien français. C'est à cette fin que s'amorcèrent une série de sondages, opérés d'abord par MM. Guy Dubreuil et Dollard Cormier, et continués par $M$. Jean-Marie Grandbois et $M^{\text {lle }}$ Lydia Landry sur « Les attitudes de la population canadienne de Montréal concernant la politique du gouvernement fédéral en matière d'immigration ». Deux hypothèses de travail nous décidèrent alors à choisir le problème de l'immigration comme symptomatique et propre à nous révéler la dynamique des divers groupes ethniques vivant au Canada. Ces hypothèses étaient :

A. C'est dans les moments où leur survivance est menacée que les groupements humains nous révèlent le mieux leur psychologie.

B. La psychologie des minorités n'est intelligible que par référence aux attitudes collectives de la majorité et vice versa. Aussi, à la lumière de ces deux hypothèses, nous postulions que chaque groupe ethnique à Montréal percevrait l'immigration comme une menace plus ou moins sérieuse à sa survivance. Nous anticipions que les Montréalais, s'ils étaient interrogés sur l'immigration, n'en discuteraient qu'en empruntant les clichés collectifs les plus en faveur dans leur groupe eth- 
nique respectif. Bien plus, ils ne le feraient que par référence aux autres groupes ethniques.

Au terme de ces sondages, opérés d'abord à Montréal, puis en divers secteurs de la province, et finalement à l'intérieur de la population étudiante de l'Université de Montréal, nous avons vu se confirmer l'hypothèse que les trois groupes ethniques prédominants à Montréal, à savoir les Canadiens anglais, les Canadiens français et les Canadiens juifs, avaient tous trois tendance à percevoir l'immigration comme une menace à leur statut privilégié ou à leur survivance. D'où chez les trois groupes ethniques des attitudes défensives similaires s'inspirant de l'ethnocentrisme le plus exclusif. M. Guy Dubreuil, dans un article intitulé : «L'immigration et les groupes canadiens », et publié dans les Contributions à l'étude des sciences de l'homme [17], présente en 1953 une synthèse de l'ensemble de ces résultats [192] qui avaient successivement fait l'objet de quatre thèses de licence en psychologie :

Guy DuBReuil, Psychologie de groupe de la population de Montréal, touchant le problème de l'immigration : expérimentation et analyse statistique, 1951 [27].

Dollard CORMIER, Psychologie de groupe de la population de Montréal, touchant le problème de l'immigration: inférences et vérification, 1951 [20].

Jean-Marie GRANDBOIS, Étude sur la réceptivité des étudiants canadiens-français de l'Université de Montréal à l'égard des étudiants immigrés, 1953 [35].

Lydia LANDRY, Recherche sur ta réceptivité d'un groupe d'étudiantes canadiennes-françaises envers les immigrés, 1953 [41].

En dépit de tâtonnements nombreux, ces premiers travaux nous avaient convaincus que la psychologie du Canadien français ne nous deviendrait intelligible qu'à la lumière d'une psychologie adéquate des relations interethniques. Celle-ci était à bâtir de toutes pièces, contrairement aux prétentions de certains chercheurs dont les théories étaient alors considérées comme définitives. Aussi nous sommes-nous vus très tôt obligés de repenser pour notre compte, en des concepts qui soient vraiment opérationnels, les multiples problèmes de méthodolo- 
gie que pose au psychologue l'exploration scientifique des phénomènes de groupe. Conséquemment, les travaux des trois années subséquentes poursuivront un double objectif :

$1^{\circ}$ dégager les options méthodologiques et à titre complémentaire mettre au point les techniques de recherche les plus fécondes en possibilités d'expérimentations au niveau de la dynamique des groupes ;

$2^{\circ}$ explorer et évaluer les possibilités d'évolution des relations interethniques ainsi que déterminer les conditions optima de cette évolution.

Montréal continuait de nous paraître un laboratoire idéal, nous présentant un cas de relations interethniques, à la fois symptomatique de ce que nous cherchions à éclairer et facilement accessible à une observation systématique de notre part. L'hypothèse de base que nous cherchions alors à vérifier avait été empruntée à Gordon W. Allport [12]. Elle se formulait comme suit :

« Pour amorcer et établir des échanges positifs entre groupes ethniques en conflit, il importe de ménager des rencontres fréquentes entre ceux de leurs membres respectifs qui sont soit du même niveau socio-économique ou appartiennent au même secteur occupationnel, »

Notre premier effort consista à mettre au point des instruments valides d'observation. De 1954 à 1957, huit thèses de licence en psychologie eurent pour objet la validation des instruments de recherches dont nous avions besoin pour procéder à une expérimentation rigoureuse des possibilités d'évolution d'un phénomène de groupe d'une telle complexité. Les instruments requis au plan de l'analyse du contenu verbal et non-verbal [193] des échanges interethniques, que nous comptions observer en des situations contrôlées, furent mis au point par les trois travaux suivants : 
Léo DoRAIs, Analyse du contenu à l'aide des catégories « a priori ». Essai méthodologique, 1956 [25].

Denise TOURILLON, Les jugements de valeur : leurs répercussions sur les échanges dans un petit groupe de discussion, 1956 [61].

Aimée LEDUC, Élaboration d'un système de catégories pour l'étude des relations interethniques, 1957 [42].

Puis, simultanément, furent présentés les résultats de travaux sur les techniques nous permettant d'observer à l'aide de catégories opérationnelles les structures formelles de ces échanges :

Antonin BoIsVert, L'apport d'une technique d'observation dans la discussion de groupe,1954 [6].

Justin CiAle, Conformity to Rides in a Small Face to Face Group during a Discussion, 1956 [14].

Toujours simultanément, les trois thèses suivantes vinrent préciser les conditions de validité de l'emploi de la technique de Bales sur les processus d'interaction aux fins de notre recherche :

Maurice GaUthIER, Validité et fiabilité des indices de statut tels que définis par Bales, 1954 [32].

Denise TÉTREAULT, Relations entre différentiation des rôles et solidarité des participants au cours d'une discussion de groupe, 1954 [59].

Louise GAMACHE, Validité des hypothèses de Bales relatives aux tendances dynamiques du processus d'interaction, 1955 [31].

Une dernière thèse fut présentée durant cette même période dans le but de dégager les aspects complémentaires entre quelques-unes de ces techniques d'observation :

Soucy GAGNÉ, Complémentarité de deux techniques dans l'analyse des phénomènes de groupe, 1956 [28].

À partir de 1957, une fois terminée la validation de nos instruments de recherche, nous fûmes en mesure de déterminer expérimentalement les conditions optima d'évolution des relations interethniques. Trois 
thèses de licence et une thèse de doctorat furent consacrées à l'interprétation des résultats alors obtenus :

Fernand Roussel, Le râle et les fonctions du meneur non-directif, 1957 [54].

Janine GAGNON, Les indices d'auto-dépréciation d'un groupe minoritaire, 1957 [30].

Robert Blain, Le bilinguisme comme indice d'adaptation socio-ethnique, 1958 [4].

Robert Blain, A Comparison Between English and French Canadian Students in Terms of Social and Personal Desirability Perceptions, 1960 [5].

Deux articles de synthèse parurent par la suite dans les Contributions à l'étude des sciences de l'homme, qui tentèrent de dégager l'apport spécifique [194] de ces cinq années de recherches à la psychologie des relations interethniques :

Bernard MAILHIOT, o.p., " La psychologie des relations interethniques à Montréal », Contributions à l'étude des sciences de l'homme, 3, 1956 [18].

Bernard MAILHIOT, o.p., "L'intégration interethnique : ses déterminants psychoculturels », Contributions à l'étude des sciences de l'homme, 4, 1959 [19].

\section{Les inaptes au travail d'équipe}

Au cours de nos recherches sur les relations interethniques, nous avions soumis à une observation systématique six groupes de 27 participants, comprenant un nombre égal de représentants des trois groupes ethniques, i.e. de Canadiens anglais, de Canadiens français et de Canadiens juifs, à savoir, 9 participants de chaque groupe ethnique. À l'occasion de rencontres périodiques, de trois heures chaque fois, couvrant en tout une durée moyenne d'au moins cinquante heures d'échanges pour chaque groupe, quatre de ces groupes ont évolué selon un rythme croissant. Ce sont : le groupe des instituteurs de l'ensei- 
gnement primaire, celui des finissants des Facultés de droit des universités McGill et Montréal, celui des membres de l'Action politique universitaire et celui des membres des syndicats ouvriers. Dans ces quatre cas, à peu de variantes près, les participants semblent d'abord avoir été obsédés d'accommodation. Durant cette première phase, ils ont paru préoccupés d'éviter tout thème de discussion de nature à les forcer à verbaliser les tensions et les conflits qui les opposaient. Au plan de la procédure, cette phase a été marquée par une dose exagérée de conformisme et d'obséquiosité devant les moindres demandes du meneur. Mais tôt ou tard cette phase d'échanges artificiels entre participants fut suivie d'abord par une phase de décharge agressive, à peine contenue, faisant place à une phase, la plus prolongée, de conflits de prestige. Dans chaque cas, ces deuxième et troisième phases furent marquées par un besoin explicite de la part de chacun des sousgroupes ethniques d'affirmer ses différences culturelles respectives. Aussi, après avoir apparemment fait groupe, au cours des premières rencontres, régressèrent-ils, dès la deuxième phase, pour nous présenter le phénomène de trois sous-groupes, affichant verbalement l'ethnocentrisme le plus outré.

L'échéance des adieux semble dans chaque cas avoir déclenché ou précipité une phase de bon-ententisme à tout prix. Ce n'est qu'au cours de ces toutes dernières rencontres, que nous pûmes repérer des indices d'une intégration embryonnaire des participants au niveau de leurs valeurs professionnelles ou de leurs intérêts de classe.

Les différences ethniques, les barrières psychologiques nombreuses, créées par les préjugés ambiants, expliquent en partie que ces quatre groupes n'aient pas évolué selon le rythme souhaité et n'aient pu s'intégrer que très lentement. Deux groupes toutefois firent exception : le groupe des avocats de carrière et celui des membres des clubs sociaux. En raison [195] même des préoccupations officielles de ces deux groupes, qui, dans le premier cas, sont de faire prévaloir la même loi pour tous et dans le second de promouvoir l'esprit civique, nous nous étions attendus à une intégration rapide de leurs participants. De fait, au terme de plusieurs semaines de rencontres, ils n'avaient pas dépassé la toute première phase d'accommodation. Leurs échanges étaient demeurés neutres, rigidement conformes aux bons usages de la politesse, mais dénués de tout désir d'explorer les incidences concrètes 
de l'ethnocentrisme qui fausse présentement nos relations communautaires à Montréal.

Pour expliquer ces deux cas nous avions suggéré provisoirement comme étant la plus plausible l'interprétation suivante [17 et 18]. Contrairement aux participants des quatre premiers groupes, nous en étions venus à considérer nombre des participants des deux derniers groupes comme des individus socialement " non-éducables ». Il nous avait semblé que chez plusieurs de ceux-ci, une cristallisation de leurs schémas d'adaptation sociale les avait rendus inaptes à des échanges interethniques. Leur socialisation n'ayant jamais été achevée, ils avaient développé à la périphérie de leur moi des attitudes sociales à la fois conformistes et stéréotypées. En profondeur, cependant, leurs attitudes à l'égard des autres groupes ethniques semblaient être devenues insensibilisées à toute polarisation altruiste. Même si en surface ils consentaient à des échanges sociaux avec les membres d'autres groupes ethniques, ils demeuraient le plus souvent incapables d'échapper à leurs intérêts égotistes, ou de se défaire d'une sorte de narcissisme collectif quand ils retrouvaient les membres de leur propre groupe.

Mais très tôt cette interprétation nous avait paru négative et peu concluante. Comment définir, dynamiquement, en termes de mobiles et de motifs, le "socialement éducable » ? Non plus en termes de dynamique personnelle, mais en termes de dynamique des groupes. Quels seraient les conditionnements psychologiques à assurer en pareils cas, pour que des individus même peu socialisés puissent faire équipe ? Ou faudrait-il convenir qu'à moins d'avoir atteint un niveau minimum de socialisation, lequel devrait être défini opérationnellement, un individu est incapable de faire équipe ? Les émotions de groupe que Fritz Redl considère comme constitutives [36], i.e. comme essentielles aux processus de formation de groupe, ne peuvent-elles être ressenties et éprouvées que par des individus d'un niveau $x$ de socialisation?

Il y avait là un problème, signalé à plusieurs reprises par l'équipe des psychologues du Research Center in Group Dynamics de l'Université de Michigan, mais qui n'avait pas encore reçu de solution adéquate. Ces derniers s'avouaient incapables de définir en termes satisfaisants les exigences psychologiques du travail d'équipe. Ils avaient pu constater, sans pouvoir jamais se l'expliquer tout à fait, que parmi 
les participants de [196] leurs workshops à Bethel, Maine, un certain nombre d'individus chaque année s'avéraient incapables de s'intégrer aux équipes de travail et de discussion. Plusieurs tentatives d'exploration systématique de ce phénomène n'avaient pas encore réussi à apporter une définition opérationnelle de ce que Leland Bradford appelle trainibility In Group Dynamics [9]. Ce groupe de chercheurs convenait qu'il y avait là plus qu'un problème de structures de personnalité qui rendaient certains individus aptes au travail d'équipe et d'autres pas. Mais aussi un problème de climat psychologique, sans quoi nous n'aurions pas de phénomène de groupe.

Si ces diverses tentatives n'avaient pas donné les résultats attendus, il fallait l'attribuer, à notre avis, aux préoccupations excessives de leurs auteurs à vouloir observer ces phénomènes en laboratoire, en des situations rigidement contrôlées [12]. De même que les théories les plus fécondes sur les dynamismes de la personnalité normale ont été élaborées par des psychologues cliniciens à partir d'observations faites sur des patients réels en des situations thérapeutiques, ainsi, nous semblait-il, devions-nous orienter la recherche sur ce problème. C'est sur des groupes réels, en mal d'organiser leur travail d'équipe, qui accepteraient le secours d'experts en dynamique des groupes que nous aurions le plus de chances d'apprendre quels sont les prérequis essentiels à un travail d'équipe efficace. Un article de Stanley Schachter et de Harvey Burdick, dans le numéro 3, volume 50, du Journal of Abnormal and Social Psychology, avait démontré de façon concluante comment certains schémas d'expérimentation en psychologie sociale doivent être abandonnés. Ces deux auteurs avaient tenté de vérifier sur des groupes réels les lois de la transmission de la rumeur, telles que formulées par G. W. Allport et L. Postman [33], et cela par référence à une rumeur réelle, qu'ils n'avaient pas eux-mêmes déclenchée. Ils avaient découvert, à leur grande surprise, que la rumeur s'était transmise selon des lois très différentes de celles dégagées par Allport et Postman en laboratoire. Dans ce dernier cas, les mécanismes de déformation, mis à jour par Postman et Allport, étaient vraisemblablement des mécanismes de défense individuels, utilisés par les sujets qui avaient consenti à cette expérience, en réaction beaucoup plus contre la situation de laboratoire qu'à l'égard de la rumeur elle-même déclenchée par les expérimentateurs. De fait, il est plus que probable que ces individus n'aient jamais fait groupe, le climat psychologique du labo- 
ratoire étant beaucoup trop artificiel, et qu'à aucun moment il n'y ait eu véritablement une dynamique de groupe en cause. Aussi, à la lumière de cette expérience, nous avait-il paru plus valide, pour l'étude des phénomènes de groupe qui nous occupaient, de recourir à des field-experiments ou à des études sur le terrain, i.e. à une vérification d'hypothèse de travail sur des groupes dont l'évolution obéirait à des motivations authentiques, et non pas à des manipulations du dehors contre lesquelles leurs membres ne pourraient que se défendre.

\section{[197]}

À cette fin nous avons tenté successivement de mettre au point trois instruments de diagnostic qui nous permettraient d'opérer le décalage le plus net entre les aptes et les inaptes au travail d'équipe. Le premier test construit et validé par nous fut le test des groupes antécédents. Grâce à ce test, nous avons pu établir que les comportements actuels en groupe sont, dans beaucoup de cas, conditionnés au sein de la famille par les premières identifications aux figures parentales, perçues comme des modèles d'autorité. Après trois ans d'utilisation de ce test, nous pouvons conclure que les inaptes au travail d'équipe sont toujours des individus dont les comportements actuels en groupe sont demeurés très déterminés par leurs tout premiers schémas de relations avec l'autorité, l'autorité dans chaque cas ayant été perçue comme un pouvoir tyrannique et arbitraire. Et cela quelles que soient les structures du groupe auquel ils appartiennent ou dans lequel ils travaillent, quels que soient le style de leadership exercé en ces groupes ou les réseaux de communication qui y prévalent.

Les deux autres tests, le « R.G.S.T. » (Reaction to Group Situation Test, de Thelen) [58] et le test des rôles que nous avons nous-mêmes construit et validé, nous ont permis de caractériser les inaptes au travail d'équipe par référence à la fois au leadership et à l'empathie. Ce que nous avons appris à date, grâce à ces deux tests, est très convergent. Les inaptes au travail d'équipe se montrent très contredépendants au point d'être des saboteurs à l'égard de l'autorité et compulsivement agressifs à l'égard de leurs coéquipiers lorsqu'ils sont membres d'un groupe. Par contre, ils deviennent très autocratiques et abusifs dans l'exercice du pouvoir lorsqu'on leur confie le leadership d'un groupe. Quant à l'empathie, soit l'auto-empathie ou l'allo-empathie, ils en sont dépourvus complètement. Ils entretiennent à leur propre sujet, i.e. quant au degré où ils sont acceptés ou rejetés par leurs coéquipiers, les 
plus flatteuses illusions. Dans leurs perceptions d'autrui et d'euxmêmes, ils font systématiquement appel au mécanisme de projection. D'où notre conviction à ce stade que l'inaptitude chronique au travail d'équipe constituerait un type de névrose caractérielle, une sorte de paranoïa sociale.

Le rationnel de ces trois tests et les résultats obtenus grâce à eux ont fait l'objet de trois thèses de licence en psychologie, de 1959 à 1961 :

Frère Louis-Maurice RICHARD, S.C, Influence des groupes antécédents sur le groupe actuel, 1959 [52].

Lise RoQuet, Analyse d'une échelle d'attitudes à l'égard du besoin d'affection, 1960 [53].

André CARRIÈRE, Statut sociométrique et mode de comportement interpersonnel, 1961 [11].

\section{Relations d'autorité dans le milieu familial}

Les données obtenues par le test des "groupes antécédents » nous laissent entrevoir que nous ne réussirons à comprendre tout à fait la psychologie du saboteur en groupe ou de l'inapte caractériel au travail d'équipe [198] que lorsque nous aurons pu éclairer le problème fondamental de l'exercice du pouvoir tant au plan des relations conjugales que des relations parentales. C'est dans la famille, semble-t-il, à observer ses parents prendre des décisions, à se soumettre à eux ou à se révolter contre eux, que l'enfant apprend très tôt à accepter l'autorité ou à la refuser. Ainsi s'édifient ses premiers modèles et ses premiers schémas de relations avec l'autorité. Par la suite ces premiers conditionnements imposent des déterminismes à la socialisation de l'enfant. Aussi nous n'espérons pas trouver réponse à nombre de questions posées par la dynamique des micro-groupes depuis le tout début de nos recherches tant que nous n'aurons pas réussi à définir de façon phénoménologique l'exercice de l'autorité en milieu familial. Nous avons donc décidé de nous consacrer à cette recherche fondamentale comme étape subséquente à nos travaux antérieurs. Nous espérons alors être en mesure de déterminer enfin de façon opérationnelle les types de 
leadership et de membership les plus fonctionnels dans les diverses activités humaines en petits groupes de travail, tant aux plans institutionnel, éducationnel qu'industriel. Pour le moment nous sommes très conscients d'avoir à nous contenter d'hypothèses plus ou moins gratuites, reposant le plus souvent sur des extrapolations idéologiques !

Voici comment s'articulent les orientations et les objectifs que nous comptons donner à cette phase la plus récente de nos recherches en psychologie sociale.

Le nombre considérable d'études concernant l'exercice du pouvoir et les relations d'autorité sont un indice certain de l'importance que l'on accorde à ce type particulier de relations interpersonnelles. Cependant, si quelques-uns de ces travaux nous ont éclairés sur les effets produits par différents styles de leadership (en termes d'efficacité ou de rendement dans la réalisation des objectifs poursuivis, de satisfaction ou de "morale » dans les relations interpersonnelles, ou encore d'évolution ou de progrès dans les processus de groupe), il y a relativement peu de recherches significatives portant spécifiquement sur le leadership, considéré comme situation vécue et chargée de signification pour le meneur et ses subordonnés. Nous ne mettons pas en doute la validité et l'intérêt des études sur le leadership conçu comme l'exercice de fonctions sociales objectivement définies, mesurées et évaluées. Cependant, les relations psychologiques avec les figures d'autorité constituent véritablement un nœud de problèmes où souvent s'arrêtent les observations et les descriptions pour faire place aux théories et même aux idéologies.

Il est fort probable qu'un retour à l'expérience vécue, à la signification donnée au phénomène par les sujets eux-mêmes dans une situation de vie réelle puisse nous aider à clarifier le jeu des facteurs psychologiques propres à l'exercice du pouvoir et aux relations d'autorité.

[199]

Il s'agit donc d'une recherche fondamentale (basic research), préalable à toute théorisation, sur la phénoménologie du leadership. Déjà, dans ce travail, nous pourrons nous appuyer sur des conceptualisations qui se sont avérées fécondes par ailleurs. Soulignons en particulier la théorie du champ de K. Lewin [44] et de ses successeurs, la théorie de la forme et ses applications à la perception sociale de T.S. Brunner [36], la théorie du rôle dans ses rapports au self de G. Mead et de Th. 
Sarbin [12] et, enfin, le travail classique de R. B. MacLeod sur la phénoménologie en psychologie sociale [36].

La question que nous nous posons au point de départ est donc la suivante. Comment l'individu, engagé dans une relation d'autorité, perçoit-il et ressent-il cette relation, considérée ici comme une région de son champ psychologique ou de son espace vital ? Il s'agit moins « d'expliquer » un comportement que de tenter l'aventure de comprendre et de décrire ce qui se passe en termes de facteurs et de forces psychologiques.

Ce sont nos travaux antérieurs sur la socialisation qui nous ont confirmés dans la nécessité d'aborder ce problème à son niveau le plus fondamental. Il nous est apparu en effet que l'intégration de l'individu à une équipe de travail (et vraisemblablement, à tout groupe orienté vers la réalisation d'une tâche) est largement fonction de la qualité et du nombre des identifications qu'il a établies antérieurement avec des figures d'autorité. Tout se passe comme si la perception de la figure d'autorité actuelle (par exemple, le meneur de cette équipe de travail) était altérée par la perception actuelle de figures d'autorité antérieures (par exemple, les professeurs en milieu scolaire, le père et la mère en milieu familial, surtout). Il semble donc, d'après nos données de recherches, que l'hypothèse de « la continuité relationnelle » soit valide.

Par ailleurs, nous avons pu constater la difficulté inhérente à toute catégorisation de nos sujets par rapport à cette dimension particulièrement vitale que constitue l'établissement de relations satisfaisantes avec des figures d'autorité. Nous avons déjà amorcé quelques tentatives dans ce sens (tests de leadership) qui nous ont révélé l'ampleur de la tâche. Notons seulement quelques points. Il faut distinguer avec soin, chez l'individu, sa réaction spontanée à une figure d'autorité, et celle que lui dicte sa «théorie personnelle » sur l'exercice de l'autorité. Il faut savoir également identifier les personnes que tel individu considère comme une autorité réelle pour lui, indépendamment de leurs rôles officiels et déclarés.

Enfin, notre travail concret auprès de groupes réels (tout particulièrement dans cette situation d'apprentissage privilégiée que constitue le Groupe de formation), nous a convaincus que les réactions de dépendance et de contredépendance, de rébellion et de soumission, de contrôle actif ou passif, à l'égard des figures d'autorité sont non seu- 
lement des indices révélateurs du système de relations interpersonnelles chez l'individu, mais aussi une source de conflits et de blocages dans la vie du groupe lui-même.

[200]

Nos recherches sur l'influence des groupes antécédents nous ont également révélé que le milieu familial est véritablement le lieu où s'édifie en quelque sorte le prototype des relations interpersonnelles, et tout spécialement des relations d'autorité. C'est dans ce milieu que certains types privilégiés de relations sont intériorisés et deviennent, semble-t-il, des traits permanents de la personnalité sociale de l'individu. Les lois gouvernant le principe de la continuité relationnelle sont mal connues. Mais, en interrogeant nos sujets sur leurs perceptions actuelles des groupes antécédents (i.e. en obtenant une mesure de l'influence du passé psychologique dans l'espace vital actuel), nous avons été convaincus que la famille est un groupe de référence particulièrement déterminant de la conduite relative aux figures d'autorité.

Les rapports que l'enfant établit avec son père et sa mère (ou leurs substituts) sont sans doute des déterminants majeurs dans la formation des dimensions proprement sociales de sa personnalité. Notre recherche ne vise pas à vérifier des hypothèses génétiques (par exemple, le comportement actuel serait déterminé exclusivement par les expériences infantiles), mais s'appuie davantage sur le principe de la contemporanéité, i.e. le comportement actuel est fonction de la situation totale actuelle (y compris les perceptions du passé et du futur). Dans cette perspective, nous croyons que le milieu familial, ici et maintenant, est un champ d'étude privilégié pour analyser l'exercice du pouvoir et les relations d'autorité.

Notons que dans cette perspective les relations entre époux et épouse sont très souvent des relations d'influence, de pouvoir, de contrôle ou d'autorité. À ce titre, les relations conjugales comme telles méritent d'être étudiées. Le choix du milieu familial et des relations conjugales comme champ d'exploration repose de plus sur un certain nombre de considérations de première importance :

A) En premier lieu, la famille ou le couple conjugal est un groupe, avec toutes les propriétés qu'on attribue habituellement aux groupe- 
ments humains : des buts communs aux partenaires; des cadres et des procédures définis en partie par la société, en partie par les partenaires eux-mêmes ; des systèmes de membership et de leadership, des réseaux de communication, un climat, etc. ...

« La situation conjugale est une situation de groupe et, comme telle, possède les caractéristiques générales de la vie en groupe. C'est dans cette perspective qu'il faut considérer les problèmes auxquels doivent faire face les partenaires dans le mariage : ces problèmes sont suscités par les relations qui s'établissent entre l'individu et son groupe. »

(Kurt LewIN, dans : Resolving Social Conflicts [43]).

B) Beaucoup d'études sur l'autorité et le pouvoir sont décevantes parce qu'elles concluent à des généralisations qui n'ont rien à voir avec les situations de vie réelles. En éliminant les cas de pure théorisation, [201] on peut malgré tout constater que, dans une large mesure, ou bien on étudie des groupes disparates dont on ne peut contrôler tous les facteurs agissant ici et maintenant, ou bien on crée de toutes pièces des groupes plus ou moins artificiels.

Le groupe familial n'est pas bâti pour fins d'expérimentation. Il existe pour lui-même et constitue un groupe « naturel » optimum ; les deux partenaires principaux se sont choisis mutuellement, et leur interdépendance est plus grande que dans tout autre groupe. On peut parler ici de groupe " primaire » dans la mesure où, pour l'individu, la famille constitue son groupe premier dans l'ordre chronologique, où les liens d'interdépendance sont plus affectifs qu'utilitaires.

Ce type de groupe possède des caractéristiques spécifiques. En outre de celles que nous avons notées précédemment, soulignons la singularité du groupe familial et conjugal : il est de dimensions réduites, il atteint les régions centrales de la personne et il implique des relations d'intimité entre les partenaires. Ajoutons que, dans notre milieu, de fortes pressions s'exercent pour lui conserver son caractère d'indissolubilité. De plus, parce que la société le considère comme son fondement, ses possibilités d'organisation et de réorganisation sont limitées, sa forme est stable, même si la constellation des facteurs à l'intérieur de ses cadres est soumise à des variations innombrables. 
Enfin, le groupe familial et conjugal offre des facilités d'expérimentation et d'observation qu'on ne trouve pas dans les groupes plus nombreux ou à structure plus complexe.

Notre recherche sur l'exercice du pouvoir et les relations d'autorité dans le milieu familial se situe dans un contexte culturel particulier : le Canada français. Les Canadiens français constituent un groupe ethnique dont malheureusement nous connaissons assez peu les caractéristiques psycho-sociologiques. En nous appuyant cependant sur quelques données sociologiques, sur des considérations historiques et sur des observations quotidiennes, il est légitime de penser que nous avons là une culture distincte qui détermine dans une certaine mesure le comportement, les perceptions et les attitudes des groupes ou des individus. Rappelons seulement que des transformations récentes aux plans institutionnel, industriel, syndical, éducationnel manifestent une volonté de démocratisation des cadres existants et une évolution dans le système de valeurs du milieu canadien-français. Dans notre recherche, non seulement nous faut-il tenir compte de ce facteur culturel, mais nous croyons que cette recherche apportera quelque lumière sur la psycho-sociologie du milieu canadien-français en ce qui concerne l'exercice du pouvoir et les relations d'autorité.

Trois thèses de doctorat en psychologie, actuellement en voie de préparation, porteront spécifiquement sur les divers aspects complémentaires de ce projet.

[202]

\section{Conclusions}

Cet inventaire permet de mettre en relief le fait que la recherche en psychologie sociale, tant à Laval qu'à Montréal, s'est au départ fortement enracinée dans des problèmes locaux dont les implications immédiates et le caractère urgent pour notre groupe ethnique ont servi d'appât ou d'attrait pour les chercheurs, tentés d'éclairer de leur lanterne et si possible d'orienter le devenir culturel de notre milieu. Peu à peu la recherche semble avoir réussi partiellement à se dégager de cette phase d'hypertrophie des préoccupations ethnocentriques. Présentement, la plupart des psychologues sociaux conviennent que leur ef- 
fort scientifique doit se porter, ici comme ailleurs, sur des problèmes plus fondamentaux et ne plus se restreindre aux secteurs de la psychologie appliquée. S'ils persévèrent dans cette voie, ils pourront contribuer, pour leur modeste part, à rendre intelligibles les lois essentielles de la communication humaine. Pour le moment, la dynamique des groupes ne présente pas aux chercheurs d'énigmes plus difficiles à déchiffrer !

Bernard MAILHIOT, o.p.

Institut de psychologie, Université de Montréal.

\section{Bibliographie}

1. BALes, Robert, F., Interaction Process Analysis, Cambridge, Addison-Wesley Press, 1951.

2. BÉLANGER, Pierre, Profil culturel des maîtres de $4^{e}$ à $7^{\circ}$ année : approche typologique, thèse de maîtrise, Université Laval, 1960.

3. Berlo, David K., The Process of Communication, New-York, Holt, Rinehart and Winston, Inc., 1960.

4. BlaIn, Robert, Le bilinguisme comme indice d'adaptation socioethnique, thèse de maîtrise, Université de Montréal, 1958.

5. Blain, Robert, A Comparison Between Engtish and French Canadian Students in Terms of Social and Personal Desirabitity Perceptions, thèse de doctorat, Université de Montréal, 1960.

6. BoISVERT, Antonin, L'apport d'une technique d'observation dans la discussion de groupe, thèse de maîtrise, Université de Montréal, 1954.

7. Bonner, H., Group Dynamics, Principles and Applications, New-York, The Ronald Press Co., 1959.

8. BOUCHER, Pierre, Introduction à une étude de la foule, thèse de maîtrise, Université Laval, 1946. 
9. Bradford, L. P., French, J. R., " The Dynamics of the Discussion Group », The Journal of Social Issues, IV, 2, 1948.

10. Buber, Martin, La vie en dialogue, Paris, Aubier, 1959.

11. CARrIÈre, André, Statut sociométrique et mode de comportement interpersonnel, thèse de maîtrise, Université de Montréal, 1961.

12. Cartwright, D., Zander, A. F., (Eds.), Group Dynamics : Research and Theory, Evanston, III, Row, Peterson, 1953. [203]

13. Cartwright, D., Studies in Social Power, Ann Arbor, University of Michigan, 1959.

14. Ciale, Justin, Conformity to Rules in a Small Face to Face Group During a Discussion, thèse de maîtrise, Université de Montréal, 1956.

15. Clapperton, Thérèse, Facteurs individuels et sociaux décelés dans l'étude de cinquante-six cas de vol du Centre psycho-social, thèse de maîtrise, Université Laval, 1952.

16. Contributions à l'étude des sciences de l'homme, cahier $\mathrm{n}^{\circ} 1$, 1952. Centre de recherches en Relations humaines, Montréal.

17. Contributions à l'étude des sciences de l'homme, cahier $\mathrm{n}^{\circ} 2$, 1953.

18. Contributions à l'étude des sciences de l'homme, cahier $n^{\circ} 3$, 1956.

19. Contributions à l'élude des sciences de l'homme, cahier $\mathrm{n}^{\circ} 4$, 1959.

20. CORMIER, Dollard, Psychologie de groupe de la population de Montréal, touchant le problème de l'immigration : inférences et vérification, thèse de maîtrise. Université de Montréal, 1951.

21. CoRnE AU, Yvon, Les accidents en industrie, thèse de maîtrise, Université de Montréal, 1952.

22. CÔTÉ, Michel, Le leadership : situation d'un concept sociologique, thèse de maîtrise ; Université Laval, 1962. 
23. DALMÉ, Madeleine, La formation des groupes et le travail social, thèse de maîtrise, Université Laval, 1948.

24. DESPRÉs, André, L'opinion publique et les relations industrielles, thèse de maîtrise. Université Laval, 1946.

25. DorAIs, Léo, Analyse du contenu à l'aide de catégories « a priori », Essai méthodologique, thèse de maîtrise, Université de Montréal, 1956.

26. Dubal, Georges, Moi et les autres, Neuchâtel, Delachaux et Niestlé, 1960.

27. Dubreuil, Guy, Psychologie de groupe de la population de Montréal, touchant le problème de l'immigration : expérimentation et analyse statistique, thèse de maîtrise, Université de Montréal, 1951.

28. GAGNÉ, Soucy, Complémentarité de deux techniques dans l'analyse des phénomènes de groupe, thèse de maîtrise, Université de Montréal, 1959.

29. Gagnon, Gilbert, Facteurs psycho-sociaux de la délinquance, thèse de maîtrise, Université Laval, 1959.

30. Gagnon, Janine, Les indices d'auto-dépréciation d'un groupe minoritaire, thèse de maîtrise, Université de Montréal, 1957.

31. GAmACHE, Louise, Validité des hypothèses de Bales relatives aux tendances dynamiques du processus d'interaction, thèse de maîtrise, Université de Montréal, 1955.

32. GauthIER, Maurice, Validité et fiabilité des indices de statut tels que définis par Bales, thèse de maîtrise, Université de Montréal, 1954.

33. GIROD, Roger, Attitudes collectives et relations humaines, Paris, Presses Universitaires de France, 1953.

34. Gordon, T., Group-Centered Leadership, New-York, Houghton, Mifflin Co., 1955.

35. GRANDBOIS, Jean-Marie, Étude sur la réceptivité des étudiants immigrés, thèse de maîtrise, Université de Montréal, 1953.

36. Hare, A. P., Borgatta, E. F., Bales, R. F., (Eds.), Small Groups, New-York, A. A. Knopf, 1955. 
37. HEIDER, Fritz, The Psychology of Interpersonal Relations, NewYork, John Wiley and Sons, Inc., 1958.

38. Hesnard, A., Psychanalyse du lien interhumain, Paris, Presses Universitaires de France, 1957.

39. Jennings, Eugène E., An Anatomy of Leadership : Princes, Heroes and Supermen, New-York, Harper and Bros., 1960.

40. KeltneR, John W., Group Discussion Processes, Toronto, Longmans, Green and Co., Volume I, 1957 ; Volume II, 1959.

[204]

41. LANDRY, Lydia, Recherche sur la réceptivité d'un groupe d'étudiantes canadiennes-françaises envers les immigrés, thèse de maîtrise, Université de Montréal, 1953.

42. LEDUC, Aimée, Élaboration d'un système de catégories pour l'étude des relations interethniques, thèse de maîtrise, Université de Montréal, 1957.

43. Lewin, K., Resolving Social Conflicts: Selected Papers on Group Dynamics, New-York, Harper Bros., 1945.

44. LEwin, K., LiPPITT, R., " An Experimental Approach to the Study of Autocracy and Democracy : A Preliminary Note », dans : Hare, P., Borgatta, E. F., Bales, R. F., (Eds.), Small Groups, New York, Knopf, 1955.

45. Lortie, Monique, Possibilités et limites d'une technique d'analyse du processus d'interaction, thèse de maîtrise, Université de Montréal, 1952.

46. MCLAREN, Suzanne, Le concept d'anomie : essai de clarification conceptuelle, thèse de maîtrise, Université Laval, 1961.

47. MiLLS, C. Wright, The Power Elite, New-York, Oxford University Press, 1956.

48. MoRrissetTe, Denise, Initiation des enfants à la vie sociale, thèse de maîtrise, Université Laval, 1951.

49. PARÉ, Simone, Expériences de formation de chefs, thèse de maîtrise, Université Laval, 1947. 
50. ProvençAL, Roland, P.M.É., Les méthodes de rééducation au Mont-Saint-Antoine de Montréal, thèse de maîtrise, Université Laval, 1954.

51. ReYmond-RIVIER, Berthe, Choix sociométriques et motivations, Neuchâtel, Delàchaux et Niestlé, 1961.

52. RICHARD, Frère Louis-Maurice, s.c, Influence des groupes antécédents sur le groupe actuel, thèse de maîtrise, Université de Montréal, 1959.

53. RoQuet, Lise, Analyse d'une échelle d'attitudes à l'égard du besoin d'affection, thèse de maîtrise, Université de Montréal, 1960.

54. Roussel, Fernand, Le rôle et les fonctions du meneur nondirectif, thèse de maîtrise, Université de Montréal, 1957.

55./SCHAAR, John H., Escape from Authority, New-York, Basic Books, Inc., 1961.

56. SchuTZ, W. C, Firo, A Three-Dimensional Theory of Interpersonal Behavior, New York, Rinehart and Co., 1958.

57. SÉVIGNY, Rohert, Le cadre interne de référence et le sentiment $d^{\prime}$ 'être compris» dans les relations interpersonnelles entre clercs et laïcs, thèse de maîtrise, Université de Montréal, 1961.

58. STOCK, D., Thelen, H., Emotional Dynamics and Group Culture, New-York, National Training Laboratories, 1958.

59. TÉtReAult, Denise, Relations entre différentiation des rôles et solidarité des participants au cours d'une discussion de groupe, thèse de maîtrise, Université de Montréal, 1954.

60. Thelen, H., Dynamics of Groups at Work, University of Chicago Press, 1954.

61. Tourillon, Denise, Les jugements de valeur ; leurs répercussions sur les échanges dans un petit groupe de discussion, thèse de maîtrise, Université de Montréal, 1956. 


\title{
COMMENTAIRE
}

\author{
Jacques Brazeau
}

Département de sociologie, Université de Montréal.

[pp. 205-208.]

$\underline{\text { Retour à la table des matières }}$

Préparer une conférence de synthèse, c'est pour le conférencier faire face à plusieurs dilemmes. En guise de commentaire sur la conférence que nous venons d'entendre, je voudrais en premier lieu rendre explicites quelques-uns de ceux-ci dans le cas présent.

L'auteur avait le choix entre deux vastes sujets - la recherche en psychologie sociale au Canada français et la recherche portant sur le Canada français. Il a choisi le premier, la perspective la plus étendue.

Immédiatement après, il lui fallait choisir entre différentes définitions de la recherche en psychologie sociale - celle où le chercheur aborde un sujet psychologique avec des préoccupations de rigueur méthodologique ou, encore, les recherches où en partant de préoccupations descriptives et théoriques en ethnologie, en science politique, en histoire ou en sociologie, un auteur rencontre des phénomènes psychologiques, formule des hypothèses à leur sujet et tire des conclusions. C'est la recherche en psychologie sociale limitée à des enquêtes utilisant la méthodologie propre à cette discipline qui nous a été pré- 
sentée ce matin. Ici, le choix du conférencier est celui d'un secteur limité, car tout ce qu'on a dit d'intérêt pour le psychologue social sur le Canada français n'a pas été dit par des psychologues sociaux. Bon nombre de chercheurs, sans parler de nombreux essayistes, se qualifient d'être lins psychologues. C'est à la psychologie sociale, je crois, d'examiner la finesse et la justesse de leurs assertions nombreuses et je regrette que le conférencier de ce matin n'ait pas examiné les courants principaux de cette pensée dans les études récentes qui portent sur le Canada français.

Le rôle de commentateur d'une communication est rendu facile par les dilemmes qui se sont posés au conférencier. Il est fortement tenté de faire le choix contraire à celui qui parla le premier et dont il examina le texte à loisir. C'est ce que je vais faire un peu, prendre une position contraire. Ceci, pas uniquement parce que je suis à la poursuite de la facilité. Je suis en désaccord avec l'idée que le progrès de la psychologie sociale soit assuré par son dégagement de préoccupations ethnocentriques, par une recherche sur les problèmes plus fondamentaux que nos problèmes particuliers. Si je crois que le psychologue se doit d'être au courant des préoccupations théoriques contemporaines et d'utiliser, quand elle lui est utile, la méthodologie universelle de la discipline mondiale de la psychologie sociale, je crois aussi qu'il doit formuler de nouvelles hypothèses, et de plus originales, à partir de l'observation phénoménologique du milieu dans lequel il vit et des observations faites par d'autres sur ce milieu.

Mon désaccord bien amical avec le conférencier, et une critique que je formulerai de la psychologie sociale canado-américaine, vous amèneront peut-être à conclure que je suis un sociologue qui s'en prend à une discipline-sœur. Je ne voudrais pas inviter mes collègues à dire si je suis sociologue ou psychologue social, les sachant trop gentils pour qu'ils m'affirment que je ne suis ni l'un ni l'autre. Sans vouloir m'éterniser sur une note personnelle, je me permets de vous indiquer que je ne sais guère si mes travaux de recherche ont été sociologiques ou socio-psychologiques. Ayant élevé cette bien faible défense, basée sur l'ignorance de mon statut sinon sur l'ignorance du rôle que je m'accorde, je formule deux observations. 
Il me semble malheureux que la psychologie sociale ne se soit pas attardée plus longtemps au stade descriptif que j'appellerai celui de la monographie psychologique. Sans doute, l'ethnologie et la sociologie ont progressé au delà de ce stade. Mais, dans chaque nouveau milieu qu'elles étudient, ces disciplines continuent d'accumuler un matériel descriptif touffu. Ce matériel oblige à jouer avec un nombre trop grand de variables et à formuler des hypothèses à la douzaine. L'acquisition d'un tel matériel permet de descendre dans la rue et de rapporter chez soi un fac-similé de la cacophonie humaine. En raison de la multiplicité croissante des situations sociales créées par la transformation de la division du travail, le sociologue sent le besoin de décrire ce qu'est l'organisation de divers milieux, d'observer les comportements qui y sont typiques, de découvrir les processus sociaux qui y opèrent et de se demander comment ceci prend place de façon plus ou moins bien intégrée à l'intérieur d'une société globale. Je vois un besoin non moins considérable d'une description empirique des aspects psychologiques des relations intrapersonnelles et interpersonnelles dans une grande variété de situations de notre vie sociale particulière. Au niveau que j'ai appelé celui de la monographie en psychologie sociale, je voudrais que l'on s'attarde à étudier les gratifications, les concurrences, les conflits de notre société ainsi que leurs structurations typiques dans diverses circonstances communes. Il me semble que c'est à la suite de ces travaux que nous en viendrons, plus ou moins adéquatement, à découvrir comment une synthèse se fait qui résout pour divers éléments de notre population les problèmes psychologiques que la vie leur présente.

Maintenant, ma deuxième observation. On dira sans doute que le psychologue social étudie aussi des situations réelles, tout comme l'ethnologue et le sociologue. À ceci je répondrai que l'étudiant de psychologie sociale est repêché trop vite de la mare, avant de s'y être débattu assez longtemps seul. Il en sort grâce à l'emprunt d'une hypothèse ou de quelques hypothèses chez un grand psychologue au nom prestigieux. Je vois pour l'étudiant, dans cette vérification d'hypothèses fournies par les écrits psychologiques, l'impossibilité presque totale de faire avancer le savoir. Je souhaiterais que plus communément les hypothèses viennent d'une autre source, qu'elles soient formulées par le chercheur comme résultantes de l'ensemble de ce qu'il connaît et peut découvrir sur la situation dont il fait l'étude. 
Voilà donc mes reproches adressés à la psychologie sociale canado-américaine : d'escamoter le stade descriptif de la vie sociale réelle et d'avoir un culte excessif : $1^{\circ}$ de la vérification de l'hypothèse formulée par les maîtres, grands et petits ; et, $2^{\circ}$ du modèle de recherche dénommé scientifique.

Maintenant, je voudrais en venir à ce que mes observations peuvent permettre de conclure au sujet de la recherche sociopsychologique au Canada français et sur le Canada français. Ce qui a été fait chez nous semble plus considérable si nous acceptons une définition large des mots " recherches » et " psychologie » que si nous employons une définition restrictive de ces termes. Les communications qui vous ont été présentées depuis trois jours, sous les auspices de diverses disciplines, en sont une indication. Mais acceptons pour l'instant une définition restrictive. Il ne semble pas nécessaire pour moi de faire part de l'intérêt des études, dont nous a parlé le Révérend Père Mailhiot, sur les relations ethniques, les petits groupes et la famille. Alors, permettez-moi plutôt d'envisager l'avenir.

[207]

Il y a quelques années, j'assistais à Toronto à une réunion de sociologues canadiens où le professeur $\mathrm{S}$. D. Clark invitait ses jeunes collègues à considérer le besoin d'une planification de la recherche afin que les problèmes sociaux du Canada reçoivent l'attention des sociologues canadiens. La réaction fut vive ! Chacun voulait faire de la sociologie avec un S majuscule, faire avancer la science dans le domaine de ses intérêts personnels - sur la famille, la bureaucratie, la stratification, etc. Peu nombreux étaient ceux qui admettaient que la planification puisse être désirable. Le mal que je décris n'existe donc pas qu'en psychologie sociale.

En psychologie sociale, je veux bien qu'il y ait au Canada français des psychologues sociaux de pure race, dont les préoccupations seraient méthodologiques et théoriques et qui feraient surtout des contributions au savoir universel. Je crois, cependant, que les contributions seront majorées par l'effort de ces gens pour faire un travail original à partir des observations faites dans leur milieu. Je voudrais aussi, cependant, que, comme on l'a fait au Centre de recherches en Relations humaines, on continue de s'intéresser aux problèmes de notre milieu. J'ajouterais que je souhaite que se diversifient les recher- 
ches à partir des hypothèses suggérées par ceux qui étudient notre milieu et qui ne sont pas des psychologues. Je souhaite qu'on utilise en psychologie une méthodologie moins élaborée, et même moins sûre, pour en arriver à tracer des esquisses de notre vie psychologique. Je préfère une esquisse dont quelques lignes sont douteuses à un dessin trop fin de sections trop infimes du paysage.

Ces croquis à grands traits ont commencé à nous être fournis par des psychologues analystes, des psychiatres et des ethnologues. Un frère enseignant s'est mis de la partie avec le succès que l'on sait. Il me semble que l'heure de la planification est arrivée chez nous pour les psychologues sociaux aussi bien que pour les représentants des autres disciplines des sciences de l'homme.

Nous voulons des données sur la famille et ses transformations afin de découvrir comment elle s'intègre avec ses membres parmi les autres institutions de notre société particulière. Nous désirons comprendre le phénomène religieux — au niveau psychologique — afin de découvrir le sens de l'activité religieuse chez nous. Les aspirations des membres de divers secteurs de notre population — quant à l'éducation, quant à la profession - nous intéressent vivement. Il en est de même des transformations de la personne à l'âge adulte qui s'opèrent par l'acculturation et l'assimilation en raison des exigences du système socio-économique pluraliste qui prévaut dans le Québec urbain. Il y a quelque dix ans qu'à Québec même, le professeur Hughes suggérait une étude des malaises psychologiques chez les fils des bourgeois devant l'industrialisation et ses exigences d'acculturation.

Si nous ne pouvons souhaiter l'étude immédiate et concurrente de tous ces problèmes - en plus de ceux de la psycho-linguistique, de la démographie, des communications de masse, de la réclame, des propagandes nationalistes et anti-nationalistes, des relations interethniques - la nomenclature indique quand même que les psychologues sociaux ont beaucoup à faire pour répondre à ce que l'on attend d'eux.

Ce que, personnellement, j'attends de nous ce sont des recherches où nos hypothèses nous seraient fournies par ce que l'on sait, encore fort mal, ou ce que l'on suppose des conséquences psychologiques des faits sociaux observables chez nous. Je m'attendrais à ce que nos conclusions en psychologie [208] sociale soient orientées de manière 
à infirmer ou à confirmer des suppositions psychologiques faites par des chercheurs d'autres disciplines.

Je me place dans une position extrémiste en disant : en psychologie sociale, plus de Canadiana et moins de théorie générale au niveau de la recherche. Je prends cette position, premièrement, afin que ses mérites soient débattus, si vous le jugez bon, et, deuxièmement, afin de vous communiquer mon souci que l'on passe à la synthèse des conclusions sur notre société auxquelles sont arrivées les diverses disciplines des sciences humaines. La poursuite d'une cohérence interne quant à ce que l'on découvre par la recherche et par la réflexion sur le Canada français m'apparaît la chose la plus importante en ce moment. Je crois que ce but doit guider les sciences sociales, dont la psychologie sociale, dans la planification de leurs recherches.

\section{Jacques Brazeau}

Département de sociologie, Université de Montréal. 
[209]

Situation de la recherche sur le Canada français

III. Perspectives sur l'étude de la structure sociale

Jean-Charles Falardeau

Département de sociologie et d'anthropologie, Université Laval

\section{“Les recherches religieuses au Canada français.”}

[pp. 209-228.]

$\underline{\text { Retour à la table des matières }}$

Seuls ceux qui sont peu sociologues s'étonneront du fait que le Canada français, si solidement encadré par des structures ecclésiastiques et si impérieusement influencé par des contrôles religieux, possède une littérature scientifique encore si hésitante sur ces questions. Peutêtre ceci est-il causé par cela ?... Nous reviendrons sur cette interrogation. Établissons auparavant le bilan des études religieuses de caractère scientifique ou d'intérêt durable sur notre milieu. Est-il besoin de préciser que nous n'inclurons, dans cet inventaire, que les études empiriques ? Seront donc écartés de notre champ de vision les mandements officiels de la hiérarchie, les travaux doctrinaux et apologétiques, les exposés pastoraux, les innombrables écrits d'intention morale et moralisante ayant eu comme objectif de redresser des torts. Si nous parlons surtout du présent, c'est que notre passé scientifique date au plus d'avant-hier. 


\section{INVENTAIRE}

\section{Essais}

Une abondante proportion des écrits sur les problèmes religieux se rangent sous la rubrique que, faute d'un terme plus précis, j'appellerai les « essais ». Nous groupons dans cette catégorie des études aussi diverses que les communications présentées annuellement aux Semaines sociales du Canada, des brochures de l'Institut social populaire, des articles publiés dans les revues Culture, Relations, Cité Libre ou Ad Usum Sacerdotum (devenue, depuis 1960, Perspectives sociales), des réflexions d'observateurs étrangers, des propos de réformateurs et des témoignages autobiographiques. Cette littérature se situe entre l'histoire, la théologie, l'éthique, l'apologétique et une sociologie impressionniste.

Rappelons en tout premier lieu que toutes les études d'ensemble sur le Canada français ont, en général, une importante incidence religieuse.

\section{[210]}

C'est le cas des fameuses et déjà anciennes monographies de Bracq [1], * de Vattier [2] et, plus près de nous, d'André Siegfried [3], dont les vigoureux chapitres comparant les institutions et les attitudes religieuses des Canadiens anglophones et francophones n'ont malheureusement servi d'inspiration à aucun chercheur subséquent.

Des essais par C.-J. Eustace [4] et le P. Irénée Beaubien, s.j. [5] ont cependant esquissé des traits des comportements catholiques et protestants et le pluralisme religieux de notre société. Le R. P. Gérard Hébert, s.j., a attentivement observé les Témoins de Jéhovah [6] et diverses sectes évangéliques et pentecôtistes [7]. Dans des chapitres du récent volume d'essais sur La dualité canadienne, Watson Kirckconnell [8] et le R.P. L.-M. Régis, o.p. [9], ont exposé comment protestants et catholiques canadiens définissent doctrinalement et idéologi-

Voir la bibliographie à la fin de ce travail. 
quement leur religion respective et se perçoivent les uns les autres. Cité Libre a consacré un numéro spécial aux protestants canadiens [10].

Il est superflu de rappeler l'importance qu'a eue la paroisse comme structure sociale dans le Canada français rural du XIX ${ }^{\mathrm{e}}$ siècle [11]. La copieuse littérature dont elle a été l'objet s'étage à trois paliers. Au niveau dont nous traitons, notons des essais de caractère lyrique, apologétique ou purement descriptif comme ceux de $\mathrm{M}^{\text {gr }}$ Landrieux [12] ou du R.P. M.-A. Lamarche, o.p. [13], et plusieurs des communications présentées en 1953 à la $\mathrm{XXX}^{\mathrm{e}}$ session des Semaines sociales du Canada [14].

La psychologie religieuse des Canadiens français a sollicité la curiosité d'un nombre croissant d'essayistes, de chrétiens engagés, tout autant que de prêtres préoccupés de pastorale et de renouveau évangélique. Les propos sur ce thème se sont multipliés dans les revues locales et dans des numéros spéciaux consacrés au Canada français par des revues françaises. Notons les brèves analyses de Jacques Perrault [15], du R.P. Adélard Dugré, s.j. [16], du R.P. Richard Ares, s.j. [17], et le grave document publié par le T.R.P. P.-M. Gaudrault, o.p., au moment de la retentissante polémique entre Jésuites et Dominicains sur la non-confessionnalité des coopératives [18]. Dans Cité Libre, Jean Lemoyne a brossé un impitoyable tableau de " L'atmosphère religieuse au Canada français » [19]. Gérard Pelletier, dans Esprit, a décrit les composantes historiques des crises religieuses contemporaines de la conscience canadienne-française, " conscience adolescente et conscience prolétarienne » [20]. Claude Ryan, dans la Chronique sociale de France, a analysé le caractère " abstrait et spéculatif » du catholicisme canadien et son universalisation récente " par en haut et par en bas » [21]. Louis O'Neill, dans Recherches et débats, a repris pour son compte un inventaire des caractéristiques de la religion des Canadiens français [22]. Ces essais sont l'expression d'une conscience de plus en plus vive des conditions [211] de plus en plus troublantes de la vie religieuse au Canada français. Plusieurs sont sociologiques d'intention ou de perspective. Ouelquefois sujets à caution, tout au moins à vérification, ils constituent un nécessaire réservoir de la recherche systématique qui peut y puiser une riche documentation, de précieuses hypothèses ou d'irremplaçables intuitions. 


\section{Études historiques}

De toutes les sciences de l'homme, l'histoire est celle que les chercheurs canadiens-français ont cultivée la première, - longtemps d'ailleurs avant qu'il ne fut question des sciences de l'homme. Les bibliographies de l'historiographie canadienne sont bien connues et faciles à repérer. Tous les travaux d'histoire générale du Canada français, depuis Garneau et Parkman, traitent inévitablement de l'Eglise catholique et de ses relations avec le pouvoir séculier des diverses époques. Ainsi en est-il des récentes synthèses historiques de Gustave Lanctot [23], de l'abbé Groulx [24], de Guy Frégault [25], de Jean Bruchési [26], de A.R.M. Lower [27], de Mason Wade [28], de plusieurs volumes de Robert Rumilly.

À un second plan, nous retiendrons les travaux portant sur l'histoire ecclésiastique proprement dite. Celle-ci a été vivement stimulée par la fondation, en 1933, de la Société canadienne d'Histoire de l'Église catholique. Les Rapports de ses congrès annuels [29], comme aussi la Revue d'Histoire d'Amérique française et les Cahiers des Dix [30], témoignent du zèle croissant des historiens professionnels ou épisodiques en ce domaine. Les sources documentaires de notre histoire religieuse sont systématiquement inventoriées et divulguées [31]. Aux ouvrages déjà anciens de $\mathrm{M}^{\mathrm{gr}}$ Gosselin [32], de Rochemonteix [33], de W.A. Riddell [34] et de L.-O. David [35], se sont ajoutées les monographies de Marcel Trudel sur L'Église canadienne sous le régime militaire [36] et de Arthur Legge sur l'Église anglicane de Trois-Rivières [37]. On a écrit l'histoire de congrégations et d'ordres religieux, tels les Oblats [38], les Sœurs de l'Assomption [39], et Marius Barbeau a réveillé de l'oubli la Confrérie de Sainte-Anne des anciens maîtrescharpentiers québécois [40]. Des biographes et des hagiographes ont évoqué des personnages prestigieux ou illustres du passé ancien ou récent : $\mathrm{M}^{\mathrm{gr}}$ de Laval [41], Marie de l'Incarnation [42], Monseigneur Bourget [43], Chiniquy [44], auxquels il faut ajouter le monumental Papineau, en préparation, de Fernand Ouellet [45].

Groupons dans une troisième catégorie hétérogène des études sur divers sujets particuliers d'histoire religieuse [46]. En tout premier lieu, sur la paroisse. M. Antoine Roy dressait, en 1936-38, l'inventaire 
de plusieurs centaines de monographies de paroisses locales [47]. Le nombre de celles-ci n'a cessé d'augmenter, quelquefois aussi leur qualité, telles une histoire de Saint-Viateur d'Outremont du P. Hector Tessier [48] et une chronique de la [212] paroisse Notre-Dame des SeptAllégresses de Trois-Rivières du R.P. Gonzalve Poulin, o.f.m. [49]. Marcel Trudel a publié un guide méthodique proposant «comment écrire l'histoire d'une paroisse » [50] et l'étude du R. P. Gonzalve Poulin, o.f.m., sur « l'évolution historico-juridique de l'institution paroissale », en 1936, a ouvert la voie à des études contemporaines plus proprement sociologiques [51]. La Société canadienne d'Histoire de l'Église catholique a consacré un de ses congrès annuels (1939-40) aux Problèmes religieux dans le Canada au lendemain de la conquête [52] ; un autre (1947-48) à la paroisse [53] ; un troisième (1949-50), à l'Histoire du diocèse de Joliette [54]. M. Gustave Lanctot a décrit «La situation politique de l'Église canadienne sous le régime français » [55] et l'abbé Groulx « La situation religieuse au Canada français vers 1840 » [56]. Le P. Conrad Morin, o.f.m. [57], et M. Olivier Maurault, p.s.s. [58], ont évoqué la naissance de l'Église canadienne et les épisodes de son histoire ; l'abbé Albert Tessier s'est intéressé à la Compagnie du Saint-Sacrement [59] et le P. Léon Pouliot, s.j., à l'Institut Canadien [60]. Mentionnons enfin le tableau panoramique des mouvements sociaux catholiques contemporains que Gérard Lemieux a présenté dans la Chronique sociale de France [61] et une récente synthèse, par Laurier LaPierre, des relations entre l'Église et l'État au Canada français [62].

\section{Études juridiques}

Il y a lieu de se demander pourquoi les juristes canadiens se sont si peu orientés vers la recherche. Il y a d'ailleurs lieu de se demander d'abord pourquoi, au Canada français où il y a eu tant d'avocats, il y a eu si peu de juristes... La bibliographie contemporaine des travaux sur la structure juridique de l'Église et de ses institutions diffère relativement peu de celle d'il y a vingt ou trente ans. Les œuvres maîtresses demeurent les grands travaux de $\mathrm{M}^{\mathrm{gr}} \mathrm{L}$.-A. Paquet sur Le droit public de l'Église [63], les traités de droit paroissial de Mignault [64] et de J.- F. Pouliot [65], et des études aussi complètement oubliées qu'elles 
avaient marqué une génération, telle l'étude du juge Pagnuelo sur la liberté religieuse au Canada [66]. La paroisse a continué d'être l'objet d'essais qui tiennent à la fois du droit et de l'histoire, de la part de l'abbé Roméo Lemelin [67], de l'abbé Honorius Provost [68] et de $\mathrm{M}^{\mathrm{e}}$ Louis-Philippe Pigeon [69]. Un moment, on a espéré que les monographies présentées à la Faculté de droit canonique qui a existé à l'Université Laval de 1939 jusque vers 1948, donneraient naissance à des travaux originaux sur les modalités canadiennes du droit canon. L'influence de l'école de pensée juridique qui a commencé à se révéler à cette époque est cependant visible dans les études de $\mathrm{M}^{\mathrm{e}}$ GeorgesMichel Giroux. Celui-ci a minutieusement redéfini le statut légal des fabriques et des corporations épiscopales [70]. Il a tenté de déduire toutes les conséquences [213] juridiques découlant du fait qu'à la conquête anglaise l'Église catholique au Canada est passée du statut d'Église d'État à celui d'association volontaire, — postulat qui était déjà implicite dans l'œuvre de Pagnuelo [71]. Le droit public du vainqueur introduit en notre pays par la conquête a éliminé les privilèges antérieurs de l'Église et a radicalement transformé la nature des relations de celle-ci avec les pouvoirs civils. C'est dans une perspective analogue que se situent une analyse de «La liberté des cultes au Canada », par $\mathrm{M}^{\mathrm{e}}$ Jean-François Pouliot [72], ainsi que la ré-évaluation récente, par $\mathrm{M}^{\mathrm{e}}$ Marc Lalonde, des «Relations juridiques Église-État au Québec » [73]. $\mathrm{M}^{\mathrm{e}}$ Lalonde analyse les corollaires du régime de séparation de l'Église et de l'État qui prévaut officiellement au Canada et souligne un certain nombre d'exceptions que la pratique a fait subir à ces principes. "Il est permis de se demander, conclut-il, si, au Québec, ces principes ne comportent pas tellement d'exceptions que c'est leur faire trop d'honneur que de leur attribuer la désignation de « principe fondamental de notre politique civile » que la législature de 1852 leur accordait » [74].

\section{Études sociographiques}

(Ethnographie, Géographie, Écologie, Démographie)

Les progrès de l'étude objective des phénomènes religieux ont suivi le rythme de l'évolution de l'enseignement supérieur et des recherches institutionnelles dans les sciences de l'homme. La plupart des 
travaux sont encore de caractère descriptif et analytique. Très peu s'élèvent au niveau de préoccupations théoriques. Dans une large mesure, leur degré de qualité scientifique dépend du degré d'ancienneté et d'expérience des centres, des instituts ou des départements universitaires qui en ont déterminé l'élaboration et dont aucun ne date de beaucoup plus de vingt ans : Départements de sociologie de Laval et de Montréal ; Instituts de folklore et de géographie de Laval ; Centre de recherches en Relations humaines de Montréal ; Institut d'études amérindiennes d'Ottawa ; Centre de recherches en sociologie religieuse de Laval ; etc.

Les recherches folkloriques, ethnographiques ou anthropologiques n'ont, en général, traité des phénomènes religieux que d'une façon indirecte ou implicite. Néanmoins, Horace Miner à Saint-Denis de Kamouraska [75] et Sœur Marie-Ursule, à Sainte-Brigitte-de-Laval [76], ont observé l'importance des fêtes et des cérémonies religieuses, les étapes de la vie de l'individu, le parallélisme entre le calendrier liturgique et le calendrier social. Madeleine Doyon a décrit les « rites de la mort » dans la Beauce [77], Marcel Rioux, la «noce » à SainteBrigitte-de-Laval [78] et Luc Lacourcière le "syndic des âmes » à l'île-aux-Coudres [78). Les répertoires de proverbes et de dictons, de chansons et de complaintes, de contes, de légendes et de superstitions, foisonnent de mythes, de symboles, de thèmes ou de pratiques à contenu religieux. Nous devons attendre des analyses plus poussées qui, [214] scrutant ces mythes, ces symboles et ces thèmes, en dégageront la signification profonde et les relieront à d'autres traits des mentalités collectives dans notre société traditionnelle.

Comme l'a rappelé Louis-Edmond Hamelin [79], le premier type d'investigation dont les membres du clergé se soient eux-mêmes préoccupés a été de caractère démographique, soit : le problème du recrutement sacerdotal. Faisant écho à de plus anciens travaux de l'abbé Noiseux [80], de $\mathrm{M}^{\text {gr }}$ Cyprien Tanguay [81] et du chanoine J.-B. Allaire [82], l'abbé Camille Poisson a remis à jour, en 1948, un opuscule qu'il avait publié en 1919 sur Les vocations sacerdotales au Canada français [83], auquel a fait suite une étude analogue, par un auteur anonyme, sur le diocèse de Québec, en 1949 [84]. Le P. Aurélien Giguère a inventorié l'évolution des effectifs chez les Oblats de 1927 à 1955 [85] et Hamelin lui-même a entrepris la première étude méthodique de l'évolution démographique séculaire du clergé québécois 
[86]. Hamelin est aussi le premier qui ait analysé le délai entre la naissance et le moment du baptême dans une paroisse de Rouyn [87].

Jusqu'à ces dernières années, les recherches d'intention sociologique exécutées sur le terrain avaient limité leur unité territoriale d'observation à la paroisse. C'est le cas de plusieurs thèses préparées à l'Université Laval [88]. Le champ d'enquête s'est maintenant élargi aux frontières du diocèse ou de la totalité d'une zone urbaine. Notons, à titre d'exemples, une monographie de Hamelin sur le diocèse de Trois-Rivières [89] ; les premières enquêtes du Centre de recherches en sociologie religieuse de Laval dans les diocèses de Québec et de Sainte-Anne de la Pocatière et dans la région d'Alma [90] et une enquête menée par le Centre de recherches socio-religieuses du diocèse de Saint-Jean. Ces enquêtes sont de caractère démographique et écologique. Elles ambitionnent principalement d'analyser la structure de la population et des occupations, les institutions de la vie économique, les éléments d'organisation sociale. Elles sont consciemment et délibérément pré-sociologiques. À ces vastes investigations diocésaines se rattachent deux enquêtes, l'une sur la mentalité chrétienne, dirigée par l'abbé Lambert Bovy, avant la " grande mission » dans l'archidiocèse de Montréal durant le Carême de 1960, l'autre sur la pratique religieuse, dirigée par l'abbé Norbert Lacoste, en novembre 1961, comme aussi le sondage sur la pratique dans une paroisse urbaine rapporté par Fernand Dumont et Gérald Fortin [91].

Pour compléter cet inventaire de la littérature sociographique, signalons la brève description qu'a donnée le R. P. Gilles Bélanger, o.p., de l'Église dans ses rapports avec les organismes socio-économiques [92]; les thèses de trois étudiants montréalais sur des groupements particuliers : l'Action catholique dans le diocèse de Saint-Hyacinthe [93], la Centrale diocésaine de Joliette [94], et la J.O.C. dans la ville de Jacques-Cartier [95] ; enfin, deux thèses d'étudiants en psychologie, l'une sur la formation apostolique [215]de l'adolescent au niveau secondaire [96], l'autre sur la contribution de l'orienteur professionnel au recrutement sacerdotal [97]. 


\section{Études psychologiques, sociologiques}

La sociologie religieuse est à peine établie dans notre milieu [98]. Elle date d'à peine dix ans. C'est imperceptiblement que l'on est passé des travaux sociographiques à la réflexion sociologique. Prenons-en, à titre d'exemples, les rares études sur les groupes non catholiques, telles la monographie du R. P. Gérard Hébert, s.j., sur les Témoins de Jéhovah [99], et deux thèses d'étudiants montréalais : une analyse de la conversion basée sur une enquête auprès des convertis du Forum catholique (Catholic Inquiry Forum) [100] et une étude de la secte de " Frères chrétiens » de Francoville près de Trois-Rivières [101]. Des très nombreux travaux du Centre de recherches en Relations humaines de Montréal sur la dynamique des groupes, très peu portent sur les comportements religieux. De ceux-ci, un seul, en définitive, a trait à notre milieu : une enquête du R. P. Mailhiot, o.p., sur la perception que se font de Dieu des enfants d'âge pré-scolaire [102]. Une thèse de Robert Sévigny, à l'Institut de psychologie de Montréal, a aussi traité de la perception des problèmes religieux chez de jeunes professionnels par suite de leurs contacts avec le clergé [103]. Marcel Rioux, dans deux de ses enquêtes ethnologiques, s'est arrêté à l'étude des attitudes religieuses. À l'Île Verte, cherchant à cerner la " personnalité de base " de la collectivité locale, il a noté un décalage entre le petit nombre de comportements religieux directement observables et les manifestations profondes de la religion dans la structure de la mentalité [104]. À Saint-Vallier, utilisant des " autobiographies d'avenir » rédigées par des adolescents, il a observé chez ceux-ci l'importance des thèmes de la vieillesse et de la religion ainsi que le haut degré de verbalisation des modèles culturels associés à l'enseignement et à la langue écrite [105, 106].

Au Département de sociologie de l'Université Laval, on s'est préoccupé d'observer les traits de la paroisse rurale canadienne de jadis et les avatars contemporains des paroisses en milieux urbains, et on a tente d'accéder à une sociologie générale de l'institution paroissiale en tant que micro-structure de l'Église catholique. Cette préoccupation a été aiguillonnée à la fois par l'exemple de Léon Gérin, par les exhortations plus directes de Everett C. Hughes dans son Programme de re- 
cherches sociales pour le Québec [107], et par la constatation de l'importance centrale de la paroisse comme cadre local d'intégration sociale dans le Canada français traditionnel.

Léon Gérin, dans ses monographies sur quelques types de familles rurales et de communautés villageoises, avait souligné le caractère paroissial des comportements collectifs des ruraux canadiens-français. Il avait aussi exigé un recours à l'histoire pour expliquer l'ambivalence " profane-religieux » dans la vie villageoise [108]. Une brève monographie sur [216] Les paroisses de France et de NouvelleFrance au XVII siècle a tenté de décrire le prototype de la paroisse canadienne hérité de France et graduellement transposé dans la campagne canadienne [109]. La quasi-totalité des innombrables monographies soi-disant paroissiales qui ont épuisé ce que Louvigny de Montigny appelait notre "martyrologe géographique » sont, à la vérité, des monographies de "villages ». Si, historiquement, la paroisse a résorbé le village canadien-français ou s'y est même substituée, ces deux réalités doivent être distinguées comme appartenant à deux ordres institutionnels spécifiquement différents. Il n'est pas de l'essence de la paroisse catholique de s'alourdir d'une caisse populaire, d'une patinoire publique ou d'une coopérative. Elle est un microcosme de l'Église universelle et, à ce titre, elle doit être considérée, comme l'Église dans sa totalité, à la fois en tant que communauté de fidèles et en tant que système de relations sociales axé sur l'administration et l'usage des instruments de salut [110].

À l'Université Laval aussi, une thèse de M. Yvan Labelle, p.m.é., a analysé la structure de l'institut religieux auquel il appartient, la Société des Missions Étrangères, son évolution, les définitions successives de son idéologie, ses champs d'action et la typologie sociale et psychologique de ses membres. Plus récemment, une équipe dirigée par Fernand Dumont a exécuté, durant les années 1956-57, dans le diocèse de Saint-Jérôme, une double enquête qui, tant par son souci de méthode que par l'ampleur de l'information recueillie, se recommandera d'elle-même longtemps comme modèle [112]. Cette étude en deux étapes a d'abord analysé l'ensemble de la société locale que constitue un diocèse, abordant les phénomènes religieux comme une variable de la structure sociale ; une seconde étape, centrée sur les phénomènes religieux, a analysé l'Église diocésaine en tant qu'aménagement de la structure sociale. Cette étude s'est, en outre, préoccupée des élites re- 
ligieuses dont elle a cherché à connaître les mécanismes de sélection. Utilisant des observations de cette enquête, Fernand Dumont a publié, depuis, deux pénétrantes études : l'une sur l'idéologie de la L.O.C., qu'il interprète " comme une tentative d'équilibre temporaire entre les thèmes culturels hérités et des problèmes nouveaux » [113] ; l'autre, sur les mouvements religieux en général [114]. C'est à Dumont aussi que nous devons les premiers essais sociologiques sur la fonction du prédicateur et sur la pastorale des ensembles dans nos milieux « déshomogénéisés » [115].

Il était inévitable qu'à la suite des historiens et des juristes, les sociologues méditent à leur tour, pour l'expliciter et l'expliquer davantage, devant le phénomène dominant de la société canadiennefrançaise : les relations entre notre société comme structure globale et l'Église catholique. Une des études du numéro spécial de la revue Esprit esquissait «le rôle et l'importance de l'Église au Canada français » [116]. Guy Rocher, dans sa thèse de doctorat encore inédite, réinterprète les deux premières phases des relations entre l'Église et le pouvoir temporel en Nouvelle-France : relations [217] de coopération durant l'époque « dévote » du « comptoir »; relations de conflit durant l'époque «gallicane » de la « colonie » [117]. Marcel Rioux, reconsidérant l'évolution socio-culturelle du Canada français, estime que 1763 a marqué le début d'un nouveau Moyen Age. Une nation canadienne n'a pu se créer à partir de ce moment que par un retour vers la "folk-société »: ce fut, durant le $\mathrm{XIX}^{\mathrm{e}}$ siècle, la fonction de l'Église de théocratiser, de sacraliser ce type de culture canadienne-française [118]. Rocher et Dumont, de leur côté, récapitulant notre récent passage d'une conscience unitaire à la reconnaissance de la pluralité, ont analysé le conflit « religieux-profane » comme l'un de nos trois désarrois idéologiques caractéristiques [119]. Certains des chapitres du volume consacré à La grève de l'amiante ont tenté d'établir le rôle exact de l'Église au cours de cette grève fameuse et d'évaluer jusqu'à quel point celle-ci a déterminé une re-structuration des relations entre quatre institutions détentrices de pouvoir : l'Église, l'État, l'entreprise industrielle, le syndicalisme [120]. De même, la Conférence de l'automne 1961 de l'Institut canadien des Affaires publiques a donné lieu à d'originales dissertations sur les rapports de notre société et de l'Église dans le domaine de l'éducation, sur la « façon dont doivent évoluer les rapports de l'Église et de l'État dans le Québec », et, de la part de Fer- 
nand Dumont, à une vigoureuse réflexion sur les facteurs qui ont déterminé, à la fin du XIX ${ }^{\mathrm{e}}$ siècle, la consolidation des liens entre nos croyances religieuses collectives et une structure particulière de la société canadienne-française [121].

\section{COMMENTAIRES}

Ce tableau laisse l'impression d'une grande hétérogénéité dans les types de préoccupations qui ont motivé les travaux sur les problèmes religieux de notre milieu, d'une grande discontinuité dans les entreprises de recherche, d'une grande variété dans les perspectives et les méthodes utilisées. J'ai délibérément laissé à cette rétrospective son caractère strictement documentaire, en y insérant le minimum de commentaires critiques. Procéder autrement eût été, me semble-t-il, injuste pour les chercheurs des époques antérieures à qui il serait pédant de reprocher de n'avoir pas connu des disciplines intellectuelles autres que celles de leur temps, et injuste pour les observateurs contemporains non sociologues que l'on ne pourrait, non plus, sans impertinence, blâmer de n'avoir pas été élèves des Facultés de sciences sociales de Laval ou de Montréal. Aux uns et aux autres, il faut laisser le mérite de leur initiative, de leur audace, tout au moins de leur bonne volonté.

Nous venons d'identifier aussi fidèlement que possible quels matériaux documentaires la littérature existante met à la disposition des sociologues contemporains; nous savons quels sont ceux qui manquent et que nous [218] avons à colliger par notre propre industrie ou avec la collaboration de nos collègues historiens, juristes, démographes, géographes et psychologues. Il nous reste à établir comment procéder, c'est-à-dire, à quelles conditions nous pourrons introduire, dans nos recherches sur les problèmes religieux, un ordre qui en assurera à la fois la validité et la continuité.

Les curiosités et les labeurs des chercheurs actuels reflètent l'effort d'une société qui, sous le choc des perturbations industrielles et urbaines qu'elle a fini par ressentir massivement, commence à se voir et à se penser d'une façon nouvelle. "La situation présente de notre société, ont écrit Dumont et Rocher, est marquée par nos difficultés à nous 
ré-inventer un visage collectif » [122]. Notre société s'interroge sur sa nouvelle identité et, dans la perspective de cette inquiète conscience d'elle-même, elle pose aussi à son passé des interrogations inédites. La sociologie, au Canada français, naît au moment où la société canadienne-française prend conscience d'elle-même et parce que notre société prend conscience d'elle-même. La sociologie, est -il besoin de le rappeler, avant d'être connaissance de la société est conscience de la société. Aussi bien, la sociologie religieuse naît ici au moment où notre société commence à ne plus prendre son catholicisme traditionnel comme un fait qui va de soi, au moment où la religion devient un problème sur lequel on s'interroge et que l'on éprouve le besoin d'étudier.

Jusqu'à une époque toute récente, les observateurs étrangers ou indigènes du Canada français ont envisagé le catholicisme de notre société comme un phénomène " naturel ». Les études les plus révélatrices à ce sujet, après celles des historiens, sont celles des folkloristes et des ethnographes. Aucune monographie ethnographique, nous l'avons noté, ne dissocie les phénomènes religieux de l'ensemble des coutumes, des traditions ou des pratiques de la civilisation traditionnelle, pour en faire un objet spécifique d'investigation. Une telle attitude était plus que légitime. Elle correspondait fidèlement à la réalité canadienne-française, c'est-à-dire à un milieu humain où ont été inextricablement mêlées, depuis ses débuts, structures politiques et structures ecclésiastiques, idéologies temporelles et idéologies spirituelles, attitudes électorales et attitudes religieuses.

Or, c'est à partir de ce phénomène culturel global que doivent se formuler encore maintenant et pour un long temps à venir les hypothèses des recherches psychologiques, anthropologiques et sociologiques qui ambitionneront d'étudier les composantes religieuses de la société canadienne-française. Ces recherches, en particulier celles de la psychologie sociale, ne déboucheront sur les conditionnements profonds des mentalités et des conduites religieuses que si elles s'intéressent d'abord et principalement aux phénomènes sociaux globaux. Il y a une indissolubilité historique de la culture canadienne française et de la religion catholique. S'il est vrai, comme le rappelle Fernand Dumont, que " l'unanimité religieuse de notre peuple est assez récente » [123], elle est néanmoins survenue comme résultat [219] de facteurs depuis longtemps à l'œuvre et dont les effets persistent indéfiniment, nonobstant la diversification accentuée de notre caractère national. 
D'après Marcel Rioux, c'est dès après la conquête que cette homogénéisation de la culture canadienne-française a commencé à se produire, sous l'action de l'Église. « La religion, écrit-il, sera (après 1763), le lien qui unira cette société et qui servira de dénominateur commun à toutes (ces) petites sociétés rurales qui formeront la plus grande partie du peuple canadien-français » [124]. La religion catholique détermine les traits dominants de la mentalité canadienne-française. C'est par l'Église catholique que le Canadien français a été principalement socialisé. C'est de l'Église qu'il a reçu sa vision du monde, sa perception de lui-même et d'autrui. Jusqu'à ces derniers temps, c'est par rapport à une appartenance religieuse qu'il était entraîné à définir " les siens » et qu'il traçait la frontière qui le séparait des « autres ». Le « nous » canadien-français est un "nous » catholique dont le rayon détermine l'envergure du réseau des relations sociales. Ce sont des normes et des principes religieux qui, dans l'enseignement implicite et explicite de la famille et de l'école, ont raison de valeurs suprêmes. La psychologie du Canadien français est, sous plus d'un rapport, théologique - et j'ai déjà ajouté que c'est une psychologie de confessionnal.

À la différence de plusieurs sociétés occidentales contemporaines où l'Église catholique et ses institutions sont dans la société et non de la société et où la pratique de la religion constitue pour les individus un secteur dissocié des autres types de comportements sociaux, au Canada français, les attitudes et les conduites religieuses imbibent en quelque sorte l'ensemble de l'existence. Il n'y a pas, d'une part, les valeurs et les attitudes religieuses et, d'autre part, les valeurs et les attitudes temporelles. Il y a une existence globale de l'individu qui est formé et informé par les valeurs et par les normes religieuses. Même si, comme l'a observé Rioux chez les jeunes de Saint-Vallier, les comportements religieux directement observables sont peu nombreux, c'est à un niveau plus profond, dans la structure de la mentalité même, que se manifeste la religion.

Si ces postulats sont justes, on ne pourra donc, dans notre milieu, dissocier les études « religieuses » des études sur la culture, sur l'ensemble de la société, ou sur quelque mouvement particulier que ce soit. Ce ne sont pas des sondages ou des enquêtes sur la pratique religieuse comme on en poursuit en France, par exemple, qui nous engageront bien avant dans la connaissance des attitudes religieuses. Si l'on veut cerner celles-ci, ce ne pourra être qu'à partir d'études sur les 
attitudes globales. En définitive, toute recherche sérieuse de psychologie ou de sociologie conduira d'elle-même à des phénomènes religieux. Peu importe que nous étiquetions nos recherches de « religieuses ». Elles le seront malgré nous si elles sont convenablement entreprises et exécutées. Pour tout dire, nous aurons une psychologie et une sociologie religieuses à condition de ne pas en faire...

[220]

Cet apparent paradoxe ne doit pas nous faire oublier que les recherches à venir vaudront ce que vaudront notre perspective historique et notre information du passé. Or, nous connaissons encore assez mal celui-ci, particulièrement notre $\mathrm{XIX}^{\mathrm{e}}$ siècle. Nous attendons des monographies qui nous renseigneraient davantage sur la mentalité et les idéologies de nos classes sociales aux époques marquantes de ce $\mathrm{XIX}^{\mathrm{e}}$ siècle, particulièrement durant la période se terminant vers 1840, laquelle semble bien avoir été, plus encore peut-être que 1763, décisive pour notre destin. Il reste à déterminer avec précision les concours de circonstances qui ont rendu possible et durable, durant la seconde moitié du XIX ${ }^{\mathrm{e}}$ siècle, la coagulation d'un caractère national canadienfrançais à dominante religieuse. Il y aurait aussi à déterminer, au plan juridique et politique, le jeu et le contre-jeu de forces qui ont entraîné le décalage décrit par Marc Lalonde entre le régime officiel de séparation théorique de l'Église et l'État et les exceptions déformantes à ce régime dans les relations concrètes. Il y aurait probablement (et je livre cette réflexion, de nouveau, aux historiens) à ré-écrire l'histoire de notre Église au XIX ${ }^{\mathrm{e}}$ siècle, ou plus exactement de nos Églises, entendant par là les idéologies diverses qui, à l'intérieur de l'Église se sont affrontées, opposées, souvent combattues aux différentes époques, et les définitions diverses que l'Église canadienne a données de sa mission dans la collectivité et de sa relation avec celle-ci. Ce sont, enfin, des analyses de caractère historique qui permettraient de mieux cerner les conflits idéologiques esquissés par Dumont et Rocher et, par là, d'évaluer les causes d'un autre décalage caractéristique dans notre société, - le divorce entre la verbalisation uniformément généralisée des normes et des idéologies, et d'autre part, les conduites effectives de plus en plus empiriques des individus et des groupes.

En effet, si nous avons évoqué le caractère unitaire de notre société traditionnelle comme une persistance dans notre société actuelle, ce dénominateur commun est sous-jacent à une différenciation et à un 
éclatement de plus en plus manifestes des structures sociales. La société canadienne-française est devenue, sous plusieurs rapports, semblable aux sociétés occidentales évoluées, — industrielle, hétérogène, pluraliste. Pour autant, si nos recherches religieuses doivent, à leur point de départ, s'inspirer du modèle sociologique qui sert à caractériser les sociétés de type traditionnel, elles doivent tout autant, dans une seconde saisie, emprunter leurs postulats, leurs définitions des problèmes et leurs schémas analytiques au modèle qui sert à identifier les sociétés de type industriel et urbain.

Dans les sociétés catholiques traditionnelles, il y a, en quelque sorte, « une parenté pseudo-naturelle entre communauté profane et communauté surnaturelle » [125]. Il y a continuité ou coïncidence entre religion et tradition, entre les institutions de l'Église et les structures sociales. Ainsi, la conception idéale de la paroisse comme structure ultime d'organisation [221] territoriale de l'Église encadrant une communauté homogène de fidèles, correspond à des conditions qui se sont historiquement réalisées dans les sociétés de foi unitaire et de civilisation traditionnelle. La paroisse coïncidait alors avec la communauté locale homogène. Dans les milieux urbains hétérogènes, les communautés profanes n'existent plus. La paroisse tend à devenir un cadre fictif superposé à des groupes segmentaires socialement dissociés. Ainsi en est-il aussi du diocèse. Si l'on pouvait dire de celui-ci qu'il « définit et organise » une société de type rural, il n'est plus, dans des régions industrialisées et socialement mouvantes, qu'une structure administrative parmi plusieurs autres, inapte à intégrer des populations dont l'identification et les allégeances dominantes se définissent par des critères bien autres que celui de chrétiens.

En dernière analyse, les recherches sociologiques sur la vie religieuse se heurtent dorénavant à la dualité Religion-Société. Pour ne pas faillir à leur objectif, ces recherches doivent se situer au niveau d'une sociologie globale du catholicisme. Etre catholique, ne l'oublions pas, signifie être citoyen du monde. Le sociologue du catholicisme ne peut s'empêcher d'élargir sa vision et sa saisie des problèmes humains à l'échelle planétaire. Le sociologue canadien-français ne peut se contenter d'observer le seul catholicisme canadien-français. Il est entraîné, par les exigences mêmes de son sujet, à saisir celui-ci dans sa réalité universelle, inter- et supra-nationale. C'est là, pour le sociologue canadien-français, une heureuse obligation qui le forcera à 
sortir de l'étroite perspective culturelle qui a été la constante tentation des recherches en sciences sociales dans notre milieu.

Or, comme l'a encore rappelé Fernand Dumont, le phénomène général qui confronte notre société est celui de « la décomposition de la Chrétienté » [126]. " L'axe essentiel d'une sociologie du catholicisme, écrit Dumont, paraît se situer au cœur de la crise séculaire que provoquent, au sein du monde chrétien, à la fois la liquidation de la chrétienté médiévale et sa survivance comme problème à résoudre » [127]. L'Église et l'ensemble de ses micro-institutions est une structure incarnée dans les sociétés concrètes "déshomogénéisées ». Si elle est une des « variables » de ces sociétés temporelles, elle est aussi, essentiellement, une communauté surnaturelle qui les transcende toutes. Or, cette communauté qui, jadis, était, en quelque sorte, un donné historique et existentiel des sociétés humaines, est maintenant un " problème à poser et à résoudre ». La communauté chrétienne est à construire. Si la réalisation de cet objectif évangélique résume l'onéreux labeur des ministres de la Cité de Dieu, ce doit être le souci des sociologues de rendre compte de ses mystérieux conditionnements dans la cité des hommes.

Jean-Charles Falardeau

Département de sociologie et d'anthropologie, Université Laval. 


\section{BIBLIOGRAPHIE}

1. BRACQ, Jean-Charlemagne, L'évolution du Canada français, Paris, Plon, 1927, 457 p.

2. VATTIER, Georges, Essai sur la mentalité canadienne-française, Paris, Librairie ancienne Honoré Champion, 1928, 384.

3. SIEGFRIED, André, Le Canada : les deux races ; problèmes politiques contemporains, Paris, Armand Colin, 1906, $415 \mathrm{p}$. [Livre disponible dans Les Classiques des sciences sociales. JMT.]

4. EUSTACE, C.J., « Catholics and Protestants in a Secularized World », Culture, 21, mars 1960, 12-31.

5. Beaubien, Irénée, L'unité chrétienne au Canada, Montréal, Éditions Bellarmin, 1955, 79 p.

6. HÉBERT, Gérard, s.j., «Les Témoins de Jéhovah », Relations, 238, octobre 1960, 259-262.

7. — _ « Les sectes évangéliques et pentecôtistes », Relations, 239, novembre 1960, 282-285.

8. KiRKCONNELL, Watson, «Religion and Philosophy: An English-Canadian Point of View », dans : La dualité canadienne, ouvrage réalisé par Mason Wade, University of Toronto Press, Presses Universitaires Laval, 1960, 41-55.

9. RÉGIS, Louis-M., o.p., « La religion et la philosophie au Canada français », dans : La dualité canadienne, ouvrage réalisé par Mason Wade, University of Toronto Press, Presses Universitaires Laval, 1960, 56-77.

10. Cité Libre, nouvelle série, XII, 39, août-septembre, Montréal, 1961. 
11. Comme sources bibliographiques, consulter: Jean-C. FALARDEAU, "Les recherches de sociologie religieuse au Canada », Lumen Vito (Bruxelles), VI, 1-2, 1951, 127-142 ; " Parish Research in Canada », dans The Sociology of the Parish, Edited by C. J. Nuesse and Thomas J. Harte, Milwaukee, The Bruce Publishing Company, 1950, Appendix A, 323-332.

12. LANDRIEUX, $\mathrm{M}^{\mathrm{gr}} \mathrm{M}$., La paroisse canadienne dans la province de Québec, Dumois-par-Ouges, 1926.

13. LAMARChE, M.-A., o.p., « La paroisse, gardienne de la vie sociale », " La paroisse, gardienne de la vie agricole », Notre vie canadienne : Études et discours, Montréal, A. Ménard, 1929, ch. V, VI.

14. Semaines sociales du Canada (Section française), «La paroisse, cellule sociale », $\mathrm{XXX}^{\mathrm{e}}$ session, Edmundston, 1953, Secrétariat des Semaines sociales du Canada, Institut social populaire, Montréal, 1953.

15. PERRAULT, Jacques, « La religion et notre société canadiennefrançaise », Culture, VI, 1945, 294-306.

16. DugRÉ, Adélard, s.j., « La religion des Canadiens français », Relations, 92, 1949, 226-229.

17. ARÈS, Richard, S.J., « Note sur la « crise religieuse » au Canada français », Relations, 184, 1956, 88-91.

18. Gaudrault, P.-M., o.p., Neutralité, non-confessionnalité et l'École sociale populaire, Montréal, Éditions du Lévrier, 1946, $63 \mathrm{p}$.

19. LEMOYNE, Jean, "L'atmosphère religieuse au Canada français », Cité Libre, 12, 1955, 1-14 ; reproduit dans Convergences, Montréal, Éditions HMH, 1961, 46-66 ; voir aussi : PierreE. TRUdEAu et Roger Rolland, « Matériaux pour servir à une enquête sur le cléricalisme », Cité Libre, 7, 1953, 29-43.

20. Pelletier, Gérard, « D'un prolétariat spirituel », Esprit, $20^{\mathrm{e}}$ année, 193-194, août-septembre 1952, 190-200 ; «La société canadienne-française et l'Église », Le Magazine Maclean, I, 11, 7 septembre 1961, 37-40. 
21. RYAN, Claude, «L'Église catholique et l'évolution spirituelle du Canada français », Chronique sociale de France, $65^{\mathrm{e}}$ année, Cahier 5, 15 septembre 1957 \{Le Canada français entre le passé et l'avenir), 443-457.

22. O'NeILL, Louis, "Vie de l'Église au Canada français », Recherches et débats, Paris, Librairie Arthème Fayard, Cahier $n^{\circ}$ 34, mars 1961 (Le Canada français aujourd'hui et demain), 8997.

23. LANCTOT, Gustave, Histoire du Canada, I, Des origines au régime royal, Montréal, Librairie Beauchemin, Limitée, 1959, $460 \mathrm{p}$.

24. GroulX, Lionel, Histoire du Canada français depuis la découverte, Montréal, L'Action Nationale, 1950-1952, 4 vols.

25. FrÉGAULT, Guy, La civilisation de la Nouvelle-France \{17131744), Montréal, Société des Éditions Pascal, 1944, 285 p.

26. BruChÉsi, Jean, Histoire du Canada, Montréal, Beauchemin, 1951, 682 p.

27. Lower, A. R. M., Colony to Nation : A History of Canada, Toronto, Longmans, 1946, XIII + 600 p. ; Canada : Nation and Neighbour, Toronto, Ryerson, 1952, VIII + 202 p.

28. WADE, Mason, The French-Canadian Outlook, A Brief Account of the Unknown Americans, New-York, The Viking Press, 1946, 182 p. ; The French Canadians, A History, 1760-1945, Toronto, The Macmillan Company of Canada, Limited, 1955, 1136 p.

29. Rapports de la Société canadienne d'Histoire de l'Église catholique (fondée le 3 juin 1933) ; vingt-six rapports annuels ont été publiés jusqu'à l'année 1959 inclusivement.

30. Les Cahiers des Dix : 25 volumes ont été publiés de 1936 à 1960 inclusivement ; le volume 25 contient une « Liste des articles parus dans les vingt-cinq premiers Cahiers des Dix (19361960) ».

31. Voir en particulier : M. R. Bonin, p.s.s., " Les archives sulpiciennes, source d'histoire ecclésiastique », Rapport 1934-35, La Société canadienne d'Histoire de l'Église catholique, 39-50 ; ab- 
bé IVANHOE CARON, "Les archives de l'archevêché de Québec », Rapport 1934-35, La Société canadienne d'Histoire de l'Église catholique, 65-73 ; "Inventaire de documents concernant l'Église du Canada sous le régime français ", Rapport de l'Archiviste de la province de Québec, 1939-40,1940-41, 194142 ; Léon RoY, "Registres et archives de paroisses », Rapport 1947-48, La Société canadienne d'Histoire de l'Église catholique, 87-92 ; abbé Arthur MAHEuX, "Où en sommes-nous en fait d'Histoire de l'Église canadienne ? », Rapport 1940, La Société canadienne d'Histoire de l'Église catholique, 13-18. Gaston CARRIÈRE, O.M.I., "Sources de notre histoire religieuse : les archives ", Recherches sociographiques, I, 2, avril-juin 1960, 189-206.

32. Gosselin, abbé Auguste, La Mission du Canada avant $M^{g r}$ de Laval (1615-1659), Évreux, Imprimerie de l'Eure, 1909 ; Vie de $M^{g r}$ de Laval, premier évêque de Québec et apôtre du Canada, Québec, Impr. de L.-J. Demers et Frère, 1890 ; L'Église du Canada depuis Monseigneur de Laval jusqu'à la conquête, Québec, Typ. Laflamme et Proulx, 1911-12-14, 3 vols ; L'Église du Canada après ta conquête, $2^{\mathrm{e}}$ partie, 1775-1789, Québec, Imprimerie Laflamme, 1917.

33. Rochemonteix, Camille de. Les Jésuites et la NouvelleFrance au XVII' siècle, Paris, Letouzey et Ané, 1896, 3 vols.

34. RIDDELL, Walter Alexander, The Rise of Ecclesiastical Control in Québec, Columbia University Studies, vol. 74, n I, New-York, 1916.

35. DAVID, L.-O., Le clergé canadien, sa mission, son œuvre, Montréal, 1896.

36. TRUDEL, Marcel, L'Église canadienne sous le Régime militaire, 1759-1764 : les problèmes, Les Études de l'Institut d'histoire de l'Amérique française, 1956, XXXIII + 362 p. ; L'Église canadienne sous le régime militaire, 1759-1764 ; vol. II, Les institutions, Québec, Les Presses Universitaires Laval, 1957. 
37. Legge, Arthur E. E., The Anglican Charch in Three Rivers, Canada\{1768-1956), Trois-Rivières et Russell, Ont., 1956, $192 \mathrm{p}$.

38. CARRIÈRE, Gaston, O.M.I., Histoire documentaire de la Congrégation des Missionnaires Oblats de Marie-Immaculée dans l'Est du Canada, $\mathrm{l}^{\mathrm{re}}$ partie : De l'arrivée au Canada à la mort du Fondateur \{1841-1861), Éditions de l'Université d'Ottawa, tome I, 1957, 378 p. ; tome II, 1959, 344 p.

39. Lesage, Germain, O.M.I.. Les origines des Sours de l'Assomption de la sainte Vierge, Nicolet, Éditions A.S.V., 1957, 344 p.

40. Barbeau, Marius, « La Confrérie de Sainte-Anne », Mémoires de la Société royale du Canada, $3^{\mathrm{e}}$ série (1945), XXXIX, mai 1945, 1-18.

41. BÉGIN, abbé Emile, François de Laval, Québec, Les Presses Universitaires Laval, 1959, 222 p.

42. BEAUMIER, Chanoine J.-L., Marie Guyard de l'Incarnation, fondatrice des Ursulines au Canada, 1599-1672, TroisRivières, Éditions du Bien-Public, 1959, 270 p.

43. Pouliot, Léon, S.J., Monseigneur Bourget et son temps, tome I, Les années de préparation, Montréal, Éditions Beauchemin, 1955, 209 p. ; tome II, L'évêque de Montréal, première partie : l'organisation du diocèse de Montréal \{1840-1846), 1956, $278 \mathrm{p}$.

44. Trudel, Marcel, Chiniquy, Éditions du Bien-Public, 1955, XXXVIII + 339 p.

45. Ouellet, Fernand, Louis-Joseph Papineau : un être divisé, Publication de la Société historique du Canada, Brochure historique $\mathrm{n}^{\circ} 11,1960,24 \mathrm{p}$.

46. Pour fins purement documentaires, on pourra consulter : $\mathbf{M}^{\text {gr }}$ Paul Bernier, La situation présente du catholicisme au Canada, Montréal, École sociale populaire, 1946, $\mathrm{n}^{\circ} 384$; aussi, P. Dominique de S.-Denis, capucin, L'Église catholique au Canada, The Catholic Church in Canada, Montréal, Les Éditions Thon, Couvent des Capucins, La Réparation, 1956, 4 éd., $269 \mathrm{p}$. 
47. Roy, Antoine, « Bibliographie des monographies et histoires de paroisses », Rapport de l'Archiviste de la province de Québec pour 1937-38, Québec, R. Paradis, 1938, 254-364.

48. TessiER, Hector, c.s.v., Saint-Viateur d'Outremont, Presbytère Saint-Viateur, Outremont, Montréal, 1954, 675 p.

49. Poulin, Gonzalve, o.f.m., Notre-Dame des Sept-Allégresses, 1911-1961 (Un demi-siècle de vie paroissiale). En vente à Notre-Dame-des-Sept-Allégresses, 1285, Saint-François-Xavier, Trois-Rivières, 1961, 93 p.

50. TRUdeL, Marcel, "Comment écrire l'histoire d'une paroisse », Revue d'Histoire de l'Amérique française, III, 1949-50, 485492.

51. Poulin, Gonzalve, o.f.m.., « L'Évolution historico-juridique de l'institution paroissiale au Canada français », Nos Cahiers, Montréal, Studium franciscain, I, 1936, 144-155, 165-186, 298315 ; II, 1937, 97-122, 203-217.

52 Rapport 1939-40, La Société canadienne d'Histoire de l'Église catholique.

53. Rapport 1947-48, La Société canadienne d'Histoire de l'Église catholique.

54. Rapport 1949-50, La Société canadienne d'Histoire de l’Église catholique.

55. LANCTOT, Gustave, « Situation politique de l'Église canadienne sous le régime français », Rapport 1940-41, La Société canadienne d'Histoire de l'Église catholique, 35-56.

56. GroulX, abbé Lionel, «La situation religieuse au Canada français vers 1840 », Rapport 1941-42, La Société canadienne d'Histoire de l'Eglise catholique, 51-76.

57. Morin, Conrad, o.f.m., "La naissance de l'Église au Canada », Revue d'Histoire de l'Amérique française, I, 2, 1947-48, 243256 ; 3, 331-341.

58. Maurault, Olivier, p.s.s., "L'Église au Canada », Revue d'Histoire de l'Amérique française, III, 2, 1949-50, 227-233. 
59. Tessier, abbé Albert, «La Compagnie du Saint-Sacrement (1627-1665) », Les Cahiers des Dix, 7, 1942, 27-43.

[225]

60. Pouliot, Léon, s.j., «L'Institut Canadien de Montréal et l'Institut National », Revue d'Histoire de l'Amérique française, $X I V, 481-486$.

61. LEMIEUX, Gérard, Notes sur le mouvement social catholique », Chronique sociale de France, Cahier 5, 65 année, 15 septembre 1957, 459-474.

62. LAPIERRE, Laurier L., « Les relations entre l'Église et l'état au Canada français : aperçu historique », L'Église et le Québec, Montréal, Éditions du Jour, 1961, ch. 2, 41-46.

63. Paquet, $\mathrm{M}^{\mathrm{gr}}$ L.-A., Droit public de l'Église, $2^{\mathrm{e}}$ édit., Québec, Laflamme, 1920, vol. II, L'organisation religieuse et le pouvoir civil.

64. Mignault, P.-B., Le droit paroissial, Montréal, C.-O. Beauchemin et Fils, 1893.

65. Pouliot, J.-P., Traité de droit fabricien et paroissial, Montréal, Wilson et Lafleur, 1936.

66. Pagnuelo, S., Études historiques et légales sur la liberté religieuse au Canada, Montréal, C.-O. Beauchemin et Valois, 1872.

67. Lemelin, abbé Roméo, «L'érection des paroisses en Canada, sous le régime français, » Rapport 1947-48, La Société canadienne d'Histoire de l'Église catholique, 65-72.

68. Provost, abbé Honorius, « Le système des cures au Canada français », Rapport 1947-48, La Société canadienne d'Histoire de l'Église catholique, 19-30 ; «Le régime des cures au Canada français (L'inamovibilité) », Rapport 1954-55, La Société canadienne d'Histoire de l'Église catholique, 85-103.

69. PigEon, Louis-Philippe, «Législation civile des paroisses », Rapport 1947-48, La Société canadienne d'Histoire de l'Église catholique, 93-99. 
70. GIROUX, Georges-Michel, « Le pouvoir des fabriques d'acquérir des immeubles », Revue du Notariat, février-mars 1945 ; "Les corporations épiscopale romaines du Québec », Revue du Notariat, 56, 1954.

71. — _ «a situation juridique de l'Église catholique dans la province de Québec », Revue du Notarial, 48, 3,4, octobre, novembre 1945.

72. Pouliot, Jean-François, «La liberté des cultes au Canada », Rapport 1953-34, Société canadienne d'Histoire de l'Église catholique, 68-79.

73. LALONDE, Marc, « Les relations juridiques Église-État au Québec », L'Église et le Québec, Montréal, Les Éditions du Jour, 1961, 77-100.

74. Ibid., 99-100.

75. Miner, Horace, Saint-Denis : A French-Canadian Parish, Chicago, University of Chicago Press, 1938, ch. V-X. [La version française du livre intituée Saint-Denis : un village québécois, est disponible dans Les Classiques des sciences sociales. JMT.]

76. Marie-Ursule, Sœur, C.S.J., Civilisation traditionnelle des Lavalois, Les Archives de Folklore, 5-6, Québec, Les Presses Universitaires Laval, 1951, ch. II, 67-135.

77. Doyon, Madeleine, « Rites de la mort, dans la Beauce », Journal of American Folklore, 6-7, 264, 137-146.

78. Rioux, Marcel, Notes sur l'urbanisation d'une communauté québécoise, Extrait du Bulletin $\mathrm{n}^{\circ} 142$, Annual Report of the National Muséum for the Fiscal Year 1954-55, Canada, Ministère du Nord canadien et des Ressources nationales, 114-120. 78a. À ces essais folkloriques, il convient d'ajouter deux thèses qui s'y rattachent intimement bien qu'elles ne portent pas sur des régions de la province de Québec: Arthur LEBLANC, $L a$ Chandeleur chez les Acadiens de l'Île-du-Cap-Breton, thèse pour la maîtrise es arts, Faculté des lettres, Université Laval, 1954 ; Anselme CHIAsson, Chéticamp : Histoire et traditions acadiennes, Moncton, Éditions des Aboîteaux, 1961, 317 p. 
79. Hamelin, Louis-Edmond, Hamelin, Colette L., Quelques matériaux de sociologie religieuse canadienne, Montréal, Les Éditions du Lévrier, 1956, 31-33.

80. NoiseuX, François, Liste chronologique du clergé, 1833.

81 TANGUAY, Cyprien, Répertoire général du clergé canadien par ordre chronologique depuis la fondation de la colonie jusqu'à nos jours, Québec, 1868, 321 p. ; $2^{\mathrm{e}}$ éd., 1893.

[226]

82. Allaire, J.-B.-A., Dictionnaire biographique du clergé canadien-français, Saint-Hyacinthe, 1908-1934, 6 vols, 3,497 p.

83. Poisson, abbé Camille, Les vocations sacerdotales au Canada français, Montréal, Institut social populaire, 1948, 24 p.

84. AnONYME, Manque-t-on de prêtres au diocèse de Québec? Québec, 1949, 43 p.

85. GiguÈre, Aurélien, O.M.I.. Recherches sur les effectifs des Pères et des Frères Oblats de Marie-Immaculée de 1927 à 1956, six cahiers ronéotypés, chez l'auteur, Ottawa.

86. HAMELIN, Louis-Edmond, « Contribution aux recherches sociales du Québec par une étude des variations régionales du nombre des vocations sacerdotales ", Cahiers de géographie de Québec, 3, nov. 1957, 5-37 ; «Évolution numérique séculaire du clergé catholique dans le Québec », Recherches sociographiques, II, 2, avril-juin 1961, 189-242.

87. « Nombre de jours entre la naissance et le baptême dans une paroisse de Rouyn », Ad Usum Sacerdotum, 14, 3, mars 1959, 7376.

88. Voir, en particulier: René CroteAu, Saint-Antoine de Tilly, thèse pour la maîtrise en sciences sociales. Faculté des sciences sociales. Université Laval, Québec, 1946 ; Pierre LAPORTE, Étude monographique sur les changements institutionnels d'un milieu paroissial de la ville de Québec, thèse présentée pour la maîtrise au Département de sociologie, Faculté des sciences sociales, Université Laval, Québec, mai 1960.

89. HAMELiN, Louis-Edmond, « Réflexions sur la structure sociale de l'Église trifluvienne », Ad Usum Sacerdotum, II, 3, déc. 
1955, 70-79 ; repris dans «Quelques aspects sociaux de l'apostolat paroissial », Ad Usum Sacerdotum, Supplément, I, 1956, 34-43 ; reproduit de nouveau sous le titre «Industrialisation et structure de l'Église dans le diocèse de Trois-Rivières : Dynamique des milieux sociaux », The Canadian Geographer, Le Géographe canadien, 7, Ottawa 1956, 35-46.

90. DoYOn, Roland, "Le Centre de recherches en sociologie religieuse de l'Université Laval », Recherches sociographiques, I, 1, janvier-mars 1960, 103-104 ; Gérard LAPOINTE, « Étude sociologique du diocèse de Sainte-Anne-de-la-Pocatière », Recherches sociographiques, I, 2, avril-juin 1960, 219-220; Marc-A. LESSARD, "Sociographie de l'Archidiocèse de Québec », Recherches sociographiques, II, 1, janvier-mars 1961, 105-106.

91. DumONT, Fernand, et ForTin, Gérald, « Un sondage de pratique religieuse en milieu urbain », Recherches sociographiques, I, 4, octobre-décembre 1960, 500-502.

92. BÉlAngER, Gilles, o.p., «L'Église et les organismes socioéconomiques », L'Église et le Québec, Montréal, Les Éditions du Jour, 1961, 131-140.

93. Phaneuf, abbé Georges-Étienne, Étude sur l'action catholique du diocèse de Saint-Hyacinthe, dissertation soumise à la Faculté des sciences sociales économiques et politiques (Section pastorale) de l'Université de Montréal, telle que requise comme condition préalable à l'obtention de la maîtrise en sciences sociales, économiques et politiques, Montréal, 1955 ; Le diocèse de Saint-Hyacinthe : étude sociologique du milieu et des institutions, Montréal, Institut social populaire (Les Cahiers de l'Institut social populaire, $\mathrm{n}^{\circ} 3$ ), 1957, $52 \mathrm{p}$.

94. SimARD, abbé Real, Les centrales diocésaines de Joliette, dissertation soumise à la Faculté des sciences sociales, économiques et politiques (Section pastorale) de l'Université de Montréal, telle que requise comme condition préalable à l'obtention de la maîtrise en sciences sociales, économiques et politiques, Montréal, 1955. 
95. BeAuvais, abbé Joseph, Étude sur une section de J. 0. C, mémoire soumis à la Faculté des sciences sociales, économiques et politiques (Section spéciale pour les prêtres) de l'Université de Montréal, tel que requis comme condition préalable à l'obtention de la maîtrise en sciences sociales, économiques et politiques, Montréal, 1956.

96. PÉPIN, abbé Paul, La formation apostolique de l'adolescent, thèse de licence en pédagogie, École normale secondaire, Université de Montréal, 1952.

[227]

97. DuVAL, abbé Roch, Une contribution de l'orientation professionnelle au recrutement sacerdotal, thèse de licence en orientation professionnelle présentée à l'École de pédagogie et d'orientation, Université Laval, 1949, 203 p.

98. DuMONT, Fernand, «La sociologie religieuse au Canada français ", Sociologie religieuse, sciences sociales, Conférence internationale de sociologie religieuse. Actes du $\mathrm{IV}^{\mathrm{e}}$ Congrès international, Paris, Les Éditions ouvrières, Économie et humanisme, 1955, 150 ; Louis-Edmond HAMELIN, Colette HAMELIN, Matériaux de sociologie religieuse, 29-30 ; Jean-C. FALARDEAU, « Les recherches de sociologie religieuse au Canada », Lumen Vitœ, 130.

99. HÉBERT, Gérard, s.j., Les Témoins de Jéhovah : essai critique d'histoire et de doctrine, Montréal, Les Éditions Bellarmin, 1960, $341 \mathrm{p}$.

100. Dagenais, Marcel, Analyse sociologique de la conversion au catholicisme: étude auprès des convertis de l' "Inquiry Forum » à Montréal, dissertation soumise à la Faculté des sciences sociales, économiques et politiques (Section de sociologie) de l'Université de Montréal, telle que requise comme condition préalable à l'obtention de la maîtrise en sociologie, 1958.

101. RAINVILLE, Jean-M., Monographie sur la secte des Frères chrétiens de Francoville; une étude d'une secte protestante canadienne-française : type de secte, sa direction interne et ses rapports avec la société ambiante, thèse présentée pour l'obtention de la maîtrise en sociologie. Université de Montréal, 1960. 
102. Mailhiot, Bernard, o.p., «Et Dieu se fit Enfant: réactions d'enfants et de groupes d'enfants à l'âge préscolaire », Cahier Lumen Vitoe de psychologie religieuse, II, 115-127.

103. SÉVIGNY, Robert, Le cadre interne de référence et le sentiment "d'être compris » dans les relations inter-personnelles entre clercs et laïcs, thèse présentée comme complément aux conditions d'obtention de la licence en philosophie (psychologie), Université de Montréal, Institut de psychologie, 1961.

104. Rioux, Marcel, "Sur le sens de l'évolution socio-culturelle de l'Île-Verte », Bulletin n ${ }^{\circ} 128$, Annual Report of the National Museum for the Fiscal Year 1951-52, 1-13.

105. _ _ Remarques sur les valeurs et les attitudes des adolescents d'une communauté agricole du Québec », Contributions à l'étude des sciences de l'homme, 3, Montréal 1956, 133-143.

106. Pour rendre cet inventaire aussi complet que possible mentionnons trois études de caractère plus général : Jacques PoHIER, o.p., Essai d'épistémologie comparée de la pensée religieuse et de la pensée de l'enfant, thèse présentée pour l'obtention du doctorat en philosophie (Montréal, s.éd.), Université de Montréal, Faculté de philosophie, 1959 ; Walter de BonT, o.p., La crise d'identité du novice : exploration d'une société close, thèse présentée comme complément aux conditions d'obtention du doctorat en philosophie (psychologie), (Montréal, s. éd.), Université de Montréal, Institut de psychologie, 1960 ; une partie substantielle de cette thèse a été reproduite, « La crise d'identité du novice », Vie Spirituelle, Supplément, $\mathrm{n}^{\circ} 58,3^{\mathrm{e}}$ trimestre 1961, 295-325 ; Hervé CARRIER, Psycho-sociologie de l'appartenance religieuse, Rome, Presses de l'Université Grégorienne, 1960, 314 p.

107. HUGHES, Everett C, Programme de recherches sociales pour le Québec, Cahiers de l'École des sciences sociales, politiques et économiques de Laval, vol. 2, n 4, Québec, 1943, 41 p.

108. Voir, Jean-C. FALARDEAU, " Léon Gérin : une introduction à la lecture de son œuvre », Recherches sociographiques, I, 2, avriljuin 1960, 123-160. 
109. Falardeau, Jean-C, Paroisses de France et de NouvelleFrance au XVII siècle, Cahiers de l'École des Sciences sociales, politiques et économiques de Lava], vol. 2, n 7, Québec, 1943, 38 p.

[228]

110. Falardeau, Jean-C, "The Parish as an Institutional Type », The Canadian Journal ot Economics and Political Science, 15, 3, August 1949, 353-367.

111. LABELlE, Yvan, p.m.é., Essai pour une sociologie d'un institut missionnaire, thèse présentée au Département de sociologie de la Faculté des sciences sociales de l'Université Laval pour l'obtention de la maîtrise en sociologie, Québec, 1959, VII + 246 + $\mathrm{xxx} 1 \mathrm{p}$.

112. DumonT, Fernand, "Sociologie d'un diocèse canadien : aspects méthodologiques », Rapport 1956-57, La Société canadienne d'Histoire de l'Église catholique, 31-38.

113. __ « Structure d'une idéologie religieuse ", Recherches sociographiques, I, 2, avril-juin 1960, 161-188.

114. , « Recherches sur les groupements religieux », texte inédit, à paraître dans Social Compass, 1962.

$115 . ـ$ _ « Une antinomie : propagande et proclamation de la parole de Dieu », Cahiers de Pastorale, 7, Sixième session d'étude sur la prédication, L'annonce missionnaire du message chrétien, Montréal, Institut dominicain de Pastorale, 1961, 2-6 ; « La pastorale des ensembles: une exigence particulière à notre temps ", Cahiers de Pastorale, 5, Cinquième session d'étude sur la prédication, La prédication dans une pastorale d'ensemble, Québec, Institut dominicain de Pastorale, 1960, 23-29.

116. FALARDEAU, Jean-C, « Rôle et importance de l'Église au Canada français », Esprit, 20 année, 193-194, août-septembre 1952, 178-229.

117. Rocher, Guy, The Relations between Church and State in New France during the Seventeenth Century: A Sociological Interprétation, a thesis presented to the Department of Social Relations in partial fulfillment of the requirements for the degree of 
Doctor in Philosophy in the subject of Sociology, Harvard University, Cambridge, Massachusetts, December 1, 1957.

118. Rioux, Marcel, « Sur le développement socio-culturel du Canada français ", Contributions à l'étude des sciences de l'homme, 4, Montréal, 1959, 144-162.

119. Dumont, Fernand, et Rocher, Guy, "Introduction à une sociologie du Canada français ", Recherches et débats, Paris, Librairie Arthème Fayard, Cahier 34, mars 1961 \{Le Canada français aujourd'hui et demain), 13-38.

120. La grève de l'amiante, une étape de la révolution industrielle au Québec, en collaboration, sous la direction de Pierre-Elliott TrudeAu, Montréal, Les Éditions Cité Libre, 1956 ; Jean-C. FAlARDEAU, "Préface », XI-XVIII ; Pierre-E. TRUdeAU : «La province de Québec au moment de la grève », chapitre 1, 3-91 ; "Épilogue », 369-404 ; Gérard Dion, « L'Église et le conflit de l'amiante », 240-262 ; Réginald BOISVERT, «La grève et le mouvement ouvrier », 345-378.

121. Dumont, Fernand, "Réflexions sur l'histoire religieuse du Canada français », L'Église et le Québec, Montréal, Les Éditions du Jour, 1961, 47-65.

122. Ibid., 13.

123. "Réflexions sur l'histoire religieuse du Canada français », L'Église et le Québec, 57.

124. Rioux, Marcel, " Sur le développement socio-culturel du Canada français ", Contributions à l'étude des sciences de l'homme, 4, 1959, 154.

125. Dumont, Fernand, « La pastorale des ensembles : une exigence particulière à notre temps ", Cahiers de Pastorale, 3, Cinquième session d'étude sur la prédication, Québec, Institut dominicain de Pastorale, 1960, 27.

126. Dumont, Fernand, "Structure d'une idéologie religieuse ", Recherches sociographiques, I, 2, avril-juin 1960, 165.

127. Ibid., 162. 


\title{
COMMENTAIRE
}

\author{
Norbert Lacoste \\ Département de sociologie, \\ Université de Montréal.
}

[pp. 229-231.]

$\underline{\text { Retour à la table des matières }}$

L'exposé que vient de faire monsieur Falardeau est précis et bien charpenté. Notre intervention reprendra donc les divisions de son méticuleux travail, ajoutant nos commentaires et ceci, surtout dans la perspective de recherches futures. Monsieur Falardeau a regroupé sous cinq chefs les travaux de sociologie religieuse : les essais, les études historiques, les études juridiques, la sociographie et la sociologie.

Ma première remarque portera sur les essais : comme l'a noté l'auteur, ils ont été multipliés ces dernières années dans notre milieu et l'on peut prévoir qu'ils se multiplieront encore dans le futur. Je me suis livré, pour la préparation d'un séminaire il y a quelques mois, à une analyse comparée du contenu des principaux essais et il est intéressant de suivre l'évolution de leur contenu idéologique. Il me semble important, désormais, de mettre en lumière la date de ces divers essais et de noter l'évolution de leur contenu.

Au sujet des études historiques : il me semble important pour les prochains travaux dans ce domaine d'utiliser nos travaux historiques et de les interpréter dans une perspective plus sociologique. On a parlé, au début de ce colloque, de l'effort inestimable de Léon Gérin dans son ouvrage Aux sources de notre histoire ; la méthode qu'il a utilisée me paraît encore féconde. Dans ce domaine, la religion ou l'idéologie peut facilement s'y mêler ; seule une perspective sociologique évitera des interprétations abusives. 
Au chapitre des études juridiques, comme l'a souligné hier monsieur Bonenfant, la méthode institutionnelle me semblerait féconde. Si les faits précèdent le droit, dans une période de planification sociale, se servir comme paramètre juridique d'un texte datant de l'époque libérale de 1852 me semble en effet dépassé pour définir le statut juridique actuel.

Pour ce qui a trait aux études sociographiques, notons que beaucoup de ce qu'on s'est plu à appeler sociologie religieuse, ici comme en Europe, n'a été, que la découverte de la réalité empirique profane par les institutions d'Église. Cette découverte, au moment de notre évolution industrielle et urbaine, me semble capitale mais insuffisante. Elle deviendra même rapidement stérile si elle fixe uniquement l'attention des quelques sociologues de la religion dans notre milieu au niveau de la structure profane alors que le contenu religieux en aura été déplacé.

Enfin, monsieur Falardeau, sur son dernier point, rappelle les études plus profondes dans notre milieu. Il faudrait ajouter à la liste l'ouvrage remarquable d'un de nos compatriotes professeur à l'Université grégorienne, le R. Père Hervé Carrier, s.j., auteur de l'ouvrage Psychosociologie de l'appartenance religieuse, publié en 1960. Je me plais également à souligner ici la qualité remarquable des travaux de Fernand Dumont dont la réputation est maintenant internationale. Falardeau et Dumont, les deux premiers directeurs du Département de sociologie de Laval ont tous les deux développé, chacun à sa manière, un intérêt pour la sociologie religieuse. Voilà un domaine où on voit maintenant poindre quelques fruits.

Passons maintenant à la seconde partie du travail de monsieur Falardeau.

[230]

Une première remarque de monsieur Falardeau me paraît juste : il demande d'interpréter notre sociologie religieuse dans une perspective globale même s'il remarque par ailleurs que notre population devient hétérogène. C'est ici en effet où la valeur de la sociologie devient appréciée, c'est de rendre compte au niveau d'une théorie scientifique de la diversité des observations empiriques, soit de même ordre comme dans un continuum rural-urbain soit d'ordres différents comme dans 
les diverses fonctions d'une même société. En effet, on ne peut pas isoler le facteur religieux d'une société de son contexte social.

Ainsi, par exemple, l'Église primitive ne se comprend que dans le cadre de l'Empire romain, l'Église du Moyen Âge que dans le contexte de la société médiévale et l'Église contemporaine que dans le cadre de nos sociétés modernes. Là où je divergerais d'opinion avec Falardeau c'est lorsqu'il note : " Toute recherche sérieuse de psychologie ou de sociologie conduira d'elle-même à des phénomènes religieux. Peu importe que nous étiquetions nos recherches « religieuses », elles le seront malgré nous si elles sont convenablement entreprises et exécutées. Pour tout dire nous aurons une psychologie et une sociologie religieuses à condition de ne pas en faire ". La perspective de monsieur Falardeau me semble possible dans une société et dans une sociologie peu diversifiées. Je vois mal une monographie rurale du Québec qui ne traite pas du point de vue religieux. Cependant, dans une société urbaine, on peut multiplier les études sur le travail ou les classes sociales sans jamais aborder l'étude de la dimension spécifiquement religieuse.

Ma seconde remarque veut relever une certaine contradiction dans le texte de monsieur Falardeau : il note : "Si nos recherches religieuses doivent, à leur point de départ, s'inspirer du modèle sociologique qui sert à caractériser les sociétés de type traditionnel, elles doivent tout autant dans une seconde saisie emprunter leurs postulats, leurs définitions des problèmes et leurs schémas analytiques au modèle qui sert à identifier les sociétés de type industriel et urbain ». Or, à la page précédente, il avait noté : " Ce ne sont pas des sondages ou des enquêtes sur la pratique religieuse comme on en poursuit en France, par exemple, qui nous engageront bien avant dans la connaissance d'attitudes religieuses ». Nous admettons volontiers que dans une petite localité une étude de pratique religieuse ne serait pas significative, mais dans une agglomération comme Montréal, si un simple pourcentage peut être intéressant, des taux différentiels par âge, sexe, scolarité, occupation, origine ethnique et lieu de naissance peuvent devenir significatifs et fournir le cadre de référence nécessaire pour des études ultérieures d'attitudes religieuses.

Je terminerai mes remarques en attirant votre attention sur des recherches qui me semblent intéressantes à poursuivre pour le développement futur de notre sociologie religieuse. 
Un premier point à souligner, c'est la nécessité de faire d'abord des études comparées à l'intérieur d'une même confession religieuse. Souvent les études sont faites avec des schémas théoriques ou des techniques de recherches différents et les comparaisons ultérieures ne sont pas possibles. Que donnerait un même questionnaire rempli à Paris, à Québec, à Montréal ou à Chicoutimi, Lauzon et Gaspé par des chrétiens d'un milieu assez semblable ? Un second type de comparaison pourrait porter sur la mobilité : l'attitude religieuse du débardeur montréalais est-elle la même lorsqu'il est [231]

dans le port que lorsqu'il est en vacances dans son village natal de Gaspésie ? Quelles sont les variantes de ce modèle ?

En troisième lieu, il me semblerait nécessaire de comparer dans un même milieu les différentes attitudes religieuses. Celles de la hiérarchie officielle, celles des pasteurs, des prêtres du milieu, celles de la majorité et celles d'indifférents ou d'hostiles. La sociologie de l'agnosticisme et de l'anti-cléricalisme reste encore à faire.

Enfin, à l'intérieur d'un même milieu, il faudrait poursuivre des études comparées à travers les divers groupes religieux ou culturels. Quelles sont les différences institutionnelles entre les paroisses catholiques françaises et anglaises à Montréal ou encore quelles sont les différences dans les attitudes religieuses d'un catholique, d'un protestant ou des juifs de langue française vis-à-vis tel événement très précis comme la mort, le travail, la souffrance, l'argent ? Je termine en vous rappelant que nous avons fondé, il y a trois ans, une Société canadienne de sociologie religieuse pour grouper des chercheurs qui ont ce centre d'intérêt. Fernand Dumont en a été le premier président et Robert Sévigny en est actuellement le secrétaire. S'il y avait des chercheurs qui étaient au travail dans ce domaine, nous serions bien heureux d'en être informés.

Norbert LACOSTE, prêtre

Département de sociologie, Université de Montréal. 


\title{
Situation de la recherche sur le Canada français \\ III. Perspectives sur l'étude de la structure sociale
}

\author{
Gilles-R. Lefebvre \\ Département de linguistique, \\ Université de Montréal

\section{“L’étude de la culture : la linguistique."}

[pp. 233-249.]

\section{$\underline{\text { Retour à la table des matières }}$}

Notre étude se propose de démontrer la possibilité d'une description objective de la langue française canadienne en faisant appel au principe socio-culturel, c'est-à-dire en considérant la langue comme un des éléments les plus importants de la culture d'une collectivité en même temps qu'une institution sociale de nature privilégiée. Nous croyons, en d'autres mots, que la description de la langue d'une entité ethnique acquiert valeur de diagnostic de son statut socio-culturel, tandis que, à l'inverse, toute étude linguistique devra tenir compte des facteurs socio-culturels d'un groupe humain dont on se propose de décrire l'organe d'expression et de communication.

Afin d'atteindre notre but, nous avons divisé le présent travail en trois sections principales : la première section, traitant des aspects généraux et théoriques de notre sujet, à savoir : la détermination - au point de vue statique - de l'objet et des tâches de la linguistique en 
tant que science sociale d'observation. Toujours dans cette première section, nous distinguerons ensuite entre linguistique interne et linguistique externe, la linguistique interne se préoccupant d'études structurelles à divers niveaux et à un titre souvent théorique ; la linguistique externe décrivant les subdivisions et les faces diverses de l'organe linguistique selon la nature de l'association et des contacts entre sujets parlants : proximité, cohésion, influences. C'est un fait admis chez la plupart des linguistes qu'à chaque groupe social correspond un moyen propre d'expression-communication, et inversement. En rapport avec la linguistique externe, nous amorcerons une solution au problème de la caractérisation de notre parler, par l'introduction d'une notion objective de dialecte à laquelle se rattachera l'esquisse d'une théorie des contacts socio-culturels - par exemple, statut minoritaire, voisinage égalitaire, coexistence de plusieurs langues usuelles, rapports de dominant à dominé, etc. - applicable aux états linguistiques du Canada qu'on appelle français. Il s'agit moins de savoir si nous parlons un patois, un dialecte, une langue autonome mais bien un idiome " pluraliste " dont les différentes couches correspondraient à une structure sociale en grande partie modelée par des contacts culturels, économiques (et linguistiques, bien sûr) avec l'anglais.

\section{[234]}

La deuxième section de notre exposé, à la lumière des principes généraux déjà dégagés, présentera en place propre une définition socio-culturelle du parler canadien-français. C'est ainsi qu'on abordera le problème du « joual » à la fois comme mythe et comme symptôme de notre statut socioculturel. Un bref rappel des origines situera le problème au point de vue diachronique.

La troisième section, enfin, montrera les efforts de description objective de notre langue déjà accomplis dans une perspective sociologique et ethnologique. En conclusion de cette dernière section, nous essaierons de montrer quelques-unes des tâches qui incombent au socio-linguiste dans le domaine de la description de l'idiome canadienfrançais. Le premier résultat à escompter d'une description socioculturelle de notre organe de communication-expression serait de monter à l'assaut des préjugés traditionnels d'un subjectivisme béat consistant à confondre la langue avec l'idée qu'on se fait du peuple qui la parle ou, qui pis est, l'idée qu'on voudrait lui imposer de ce qu'il est ou devrait être. En somme, la linguistique sociologique, en « dépas- 
sionnant » le débat de la langue chez nous, et tout en l'éloignant des querelles grammaticales et puristes, nous semble détenir la clé de la solution au problème de ce que nous appellerons de façon provisoire "le parler canadien-français ». Les quelques considérations qui suivent poseront et délimiteront le problème qui nous préoccupe tous à des titres divers.

\section{GÉNÉRALITÉS}

Les méthodes et les tâches de la linguistique comme science sociale et science de l'homme peuvent revêtir deux aspects : l'un pratique, c'est-à-dire d'analyse et de description des organes de communication et d'expression des collectivités humaines. Le but et les démarches sont de nature d'abord pédagogique (par exemple, l'École de phonologie mécaniste des Nord-Américains). Il s'agit de la description systématique des signes externes sans rapport avec le contenu, le mouvement interne des mots. L'autre aspect est de nature spéculative, plutôt épistémologique, puisque son but est la connaissance d'une structure mentale humaine se manifestant de façon naturelle et spontanée à travers la langue et dans le mot, phénomènes psychologiques en même temps que techniques hautement spécialisées. La linguistique, qu'elle soit d'aspect pratique ou spéculatif, nous montre sous forme de doctrine le rôle social de la langue, son potentiel expressif, ses niveaux, ses concrétisations locales. C'est avant tout son caractère systématique qu'elle dégage de la recherche patiente et omniprésente (= langage ; langue(s) ; parole ; style) ; la langue ne devient un objet de science qu'au moment où elle se révèle comme phénomène social et socialisateur.

[235]

La linguistique nous rappelle qu'avant d'être de nature esthétique - ainsi que la critique littéraire ou les fanatiques du "bon » usage nous le laisseraient croire - , le langage (faculté) et la langue (concrétisation particulière du langage dans la société $x$ ) sont fonctionnels, c'est-à-dire correspondent à un besoin fondamental de communication entre les hommes organisés en société, à un impératif de survie mentale. C'est en même temps une forme de communication, un mode de 
saisie et de verbalisation du réel perçu à travers une culture. Plus exactement, c'est une synthèse de l'univers enfermée dans les structures de communication et d'expression d'une collectivité donnée. Même si, apparemment, la langue d'un groupe est la même que celle d'un autre groupe, la partie invisible, la somme des contenus de cette langue variera sous l'impulsion d'une culture différente. Nous pensons ici à nombre de mots français dont les acceptions et les nuances sont fortement différentes de celles qui ont cours en France. Une étude valable de la langue canadienne-française devra tenir compte, non seulement des mots de création morphologique uniquement canadienne, mais aussi des mots français dont les significations ne sont pas les mêmes au Canada qu'en France. Pour réussir une pareille entreprise, il faudrait enquêter sur l'ensemble du vocabulaire français dans son application au Canada. On sait déjà que les institutions n'étant pas les mêmes dans beaucoup de domaines de la vie collective - politique, économique, culturel, etc. —, les mêmes mots ne pourront pas être affectés aux mêmes usages dans deux sociétés différentes. Pour avoir la même langue qu'en France, nous devrions saisir l'univers de la même façon, avoir les mêmes expériences politiques, les mêmes structures économiques, le même sol, etc.

Au point de vue interne, la linguistique nous enseigne que la faculté de langage se concrétise en de nombreuses langues qui résolvent de façon originale le problème de la communication et de l'expression dans des grammaires particulières. Mais elles ont toutes ceci de commun qu'elles sont structurées en niveaux qui régissent le matériel phonétique et grammatical que les sociétés de sujets ont hérité de leurs ancêtres. Sans le langage et les divers états de langue, il serait bien difficile de concevoir comment une société humaine parviendrait à exister et à fonctionner normalement, car, sans communication organisée, il lui serait impossible d'intégrer ses membres. Au contraire de la grammaire traditionnelle, laquelle n'est souvent qu'un reflet des préjugés philosophiques ou logicistes d'une société qui tend à se perpétuer spirituellement et à glorifier ses ancêtres spirituels, la linguistique ne procède pas de la lettre et du texte littéraire, de nature essentiellement artificielle et sélective. Elle observe les manifestations de la langue parlée actuelle d'abord, comme étant révélatrices des mécanismes spontanés de communication et d'expression du groupe. La langue naturelle n'est pas un agrégat de phénomènes anarchiques, mais une 
hiérarchie d'éléments très structurés et intégrés (phonétique, phonologie, morpho-syntaxe), une [236] " hiérarchie de systèmes », comme l'écrit André Martinet dans ses Éléments de linguistique générale. 260

Méthodologiquement parlant, le linguiste s'abstient de considérer que telle ou telle forme de langue est «bonne" (= correcte) ou « mauvaise » (= incorrecte). Un fait de langue, un organe linguistique, une locution ne sont ni «bons ni « mauvais »; ils sont ou ils ne sont pas. Ils servent adéquatement ou pas à la communication et à l'expression d'un groupe social. La linguistique enregistre tout ce qu'elle observe et entend, et sa tâche est d'être fidèle à tous les usages et non de flatter l'élite et les prétentions de cette dernière à faire croire que son dialecte est meilleur en soi parce qu'il est obligatoire pour les gueux de s'en servir afin de survivre socialement. Tout au plus pouvons-nous considérer à part le langage de l'« élite » comme marginal au même titre que l'argot des voleurs. Notons en passant que la notion de « qualité » dans la langue est arbitraire et relève d'un critère étranger à la structure, se rattachant plutôt au code de politesse dans les coutumes. Il s'agit du «bon » ou du " mauvais » usage, de l'imposition ou du rejet temporaires de telle ou telle forme de la langue commune, et souvent parce que les sujets parlants parviennent au prestige ou sont des ratés sociaux. C'est le travail d'une certaine classe de grammairiens de nous apprendre que «moue » est une forme «vicieuse », tandis que « moua » est seul acceptable. Dans le domaine de la politesse socioverbale, ils ont entièrement raison, mais c'est quand ils essaient de trouver une base absolue et une valeur intrinsèque à leurs préceptes, qu'ils ont tort. À les entendre statuer doctoralement sur telle ou telle « tournure » ou forme de langage, on a l'impression nette qu'ils ignorent que la forme " moua » fut autrefois condamnée et rejetée comme barbare et que ses usagers étaient méprisés. Je ne parle pas des critères moraux que certains voudraient accoler à la notion de " mauvais langage ». De toutes façons, l'évolution d'une langue nous enseigne brutalement que, si nous voulons nous en tenir à l'absolu, beaucoup de formes actuelles sont des grossièretés de l'âge classique et préclassique. Un petit exemple : notre noble mot de «tête », en français, est le descendant d'un terme de latin parlé qui signifiait argotiquement « citron, cafetière, bouille, fraise, etc. », et qui, pour des raisons struc-

260 André MARTINET, Éléments de linguistique générale, Paris, Colin, 1960. 
turales, internes, a remplacé le terme neutre et général de caput. Inutile d'ajouter que l'interprétation qualitative n'est pas susceptible de nous éclairer sur le fonctionnement intime des éléments de la langue. On pourrait dire, de façon paradoxale peut-être, qu'une liste de formes soi-disant incorrectes est le seul vestige d'objectivité chez les grammairiens traditionnels. Elles nous apprendraient énormément de choses sur l'état naturel et spontané, sur les conditions réelles d'une langue arbitrairement châtrée (nécessairement peut-être ?), mais injustement étouffée par l'académisme [237] et l'usage officiel, lesquels sont inévitablement partiels et partiaux et privent une couche considérable des sujets parlants ( «le bas peuple ») de son organe naturel et propre de communication et d'expression. Ceux qui, aujourd'hui, parlent des " patois » et des "dialectes » ont souvent donné — du moins leurs ancêtres - le meilleur de leur substance verbale au fond commun de la langue. Mais un ensemble de règles dirigées par une idéologie aristocratique les ont arrêtés à un certain niveau de transmission. C'est bien une classe dominante qui a opéré le choix des termes ou formes grammaticales d'origine " provinciale » qui sont passés à la langue. Le reste du matériel linguistique provincial a été, non seulement rejeté, mais on l'a écrasé de mépris ; il s'est réfugié dans les ténèbres extérieures : voilà pourquoi on a maintenant des " patois », des " dialectes », et autres expressions et concepts de discrédit linguistique.

Il est intéressant de suggérer l'hypothèse de leur existence chez les peuples à différences sociales très marquées et qui présentent une hypertrophie de la conscience aristocratique. On sait pertinemment que les couches populaires, forcément moins perméables à l'influence académique et puriste, recèlent des indices éloquents des tendances structurales de la langue commune. En conséquence, quand on étudie la langue, phénomène social, on doit le faire dans toutes ses manifestations, si on désire obtenir des résultats complets et concluants. Sur le caractère social de la langue, on a vu des témoignages venus de secteurs idéologiques apparemment étrangers à la science. Par exemple, Joseph Staline, se sentant soudain engagé dans la grande querelle de la linguistique soviétique, a violemment pris parti pour le caractère social de la langue et a souligné l'importance d'une « saine » conception de la langue dans le corps de doctrine marxiste (stalinienne) : 
«Question: Quelles sont les caractéristiques de la langue ? - Réponse : La langue est un phénomène social qui fonctionne grâce à l'existence de la société. Elle naît et se développe avec la naissance et le développement de la société. La langue meurt avec la société. Pas de société pas de langue. En conséquence, la langue et les lois de son développement ne peuvent être comprises qu'en stricte relation avec l'histoire de la société, l'histoire de la collectivité dont on décrit la langue, et qui en est le créateur et le dépositaire. » 261

L'École marxiste aussi bien que l'École bourgeoise reconnaissent que « la langue est une technique, un instrument à l'aide duquel les membres d'une société communiquent les uns avec les autres, échangent des pensées et se comprennent ». En rapport étroit avec la pensée, la langue représente matériellement et fixe de manière symbolique en $1^{\circ}$ phonèmes : unités sonores, dénuées de signification propre mais servant à distinguer les "mots" les uns des autres; $2^{\circ}$ mots : unités plus ou moins longues et arbitraires (rien, en soi, ne prédispose les sons du mot « chaise » à signifier le meuble en question); $3^{\circ}$ dans des mots combinés en phrases les mouvements de [238] l'esprit qui perçoit le réel et les réactions de l'individu qui verbalise son expérience. Ajoutons ici quelques considérations d'ordre dynamique concernant l'influence du facteur socio-culturel sur l'évolution d'une langue. Disons plutôt quelques réserves. Selon Marcel Cohen, reprenant une remarque de André-G. Haudricourt et Georges Granai, 262

« Le langage est intérieur à la société. Inclus dans la société évoluante, il évolue non certes de manière indépendante, puisqu'il appartient à cette société, mais en raison des conditions propres de la «chose » qu'il est... Autant il est désirable d'examiner comment tous les faits concernant les langages s'encadrent dans les histoires des faits sociaux, ceci en visant à constituer un riche répertoire à allure de petite encyclopédie, autant il serait illégitime dans l'état présent des études linguistiques, psychologiques et sociologiques, de prétendre à donner un manuel de sociologie linguistique. » 263

261 Marxism and Problems of Linguistics, Moscou, 1954, 29-30.

262 Voir : A.-G. HAudricourT et G. GRANAI, « Linguistique et sociologie », Cahiers internationaux de sociologie, juillet-décembre 1955.

263 Marcel CoHEN, « Structure sociale et structure linguistique, » Diogène, 15, juillet 1956, 47-48. 
Les comparaisons entre structures linguistiques (caractéristiques internes : phonologie, morpho-syntaxe, constitution des mots) et structures sociales (répartitions de groupes : familles, clans, tribus ; systèmes de préséance et de commandement, etc.) ne peuvent, à l'heure actuelle, livrer de conclusions d'un parallélisme étroit du type rêvé par Wilhelm Schmidt lequel reliait la construction grammaticale — nom déterminant (= complément) précédant le nom déterminé (quelqu'un-maison) — à une société de type archaïque, c'est-à-dire dénuée d'agriculture et de gouvernement déterminé. Une autre théorie, celle de Marr postulant les "types de langues stadiaux » reliés à des types archaïques de sociétés - et dont nos langues garderaient des vestiges - , est tombée victime de la grande querelle linguistique soviétique, où Staline et à sa suite les linguistes de l'U.R.S.S. ont mis un point final à une doctrine qui, apparemment, contredisait les dogmes sociologiques de la pensée soviétique. ${ }^{264}$

En conclusion, c'est surtout dans le domaine du vocabulaire, de l'emprunt de termes étrangers tout aussi bien que dans les changements internes des mots et les divers stades de bilinguisme (mixture) - et non en morphosyntaxe d'abord - que se manifeste une relation entre faits sociaux — spécialement de contact — et des modifications de structure linguistique.

La distinction entre linguistique interne et linguistique externe a pour avantage de détacher les phénomènes proprement grammaticaux et structuraux caractérisant les langues vis-à-vis des types " sociaux » de langues ou subdivisions linguistiques résultant d'une action socioculturelle. Quant aux phénomènes internes, ils sont essentiellement des phénomènes de structure. Nous définirons brièvement la structure comme « l'ensemble des signes externes et des mouvements de l'esprit qui leur correspondent, les [239] dirigent dans leurs fonctions et leur évolution ». Marcel Cohen la définit comme

« un agencement de certains systèmes compris dans le total... Exemple de solidarité entre le système phonémique de la langue et le système gramma-

264 The Soviet Linguistic Controversy, New-York, Columbia University (Department of Slavic Languages), 1951. 
tical : si l'évolution phonétique est telle que les finales des mots sont affaiblies jusqu'à confusion ou disparition de certaines voyelles et consonnes, les distinctions morphologiques [grammaticales] ne peuvent pas se faire au moyen de finales différentes. [Ce qui peut provoquer un chambardement général tel qu'on l'a vu à partir du latin classique vers le français moderne : passage de la structure synthétique avec syntaxe libre à la structure analytique-isolante dont la syntaxe est obligatoire et omniprésente]. Le vocabulaire lui-même n'est pas amorphe ; on doit parler de systèmes lexicaux, sujet encore mal étudié ; ainsi beaucoup de langues n'admettent que des mots d'une longueur strictement limitée, ce qui ressortit en partie de la phonétique ; en conséquence, des mots d'emprunt trop longs ne peuvent être acceptés qu'avec abrègement. » 265

La structure d'une langue évolue, dans son ensemble, beaucoup plus lentement que la collectivité qui l'utilise. Encore selon Staline, 266 la langue, en tant qu'ensemble global de phénomènes structuraux, survit à la lutte des classes ainsi qu'à la liquidation de la base. Le système grammatical, il est vrai, ne porte que très peu de marques apparentes d'un bouleversement social. Au contraire de la thèse marxiste, qui ne semble pas suffisamment reconnaître l'influence de la révolution sur le niveau lexical, nous croyons que le bouleversement peut être considérable au niveau sémantique, c'est-à-dire celui du contenu des mots. Le fait que les enveloppes phonétiques changent très peu dans une génération ne doit pas faire oublier les transformations internes - celles de signification - qui sont souvent radicales. Comparons, à titre d'échantillon, les deux contenus sémantiques totalement différents à éclairage idéologique divergent — de la même enveloppe " démocratie » en France et en U.R.S.S. Personne n'osera soutenir que résonances du même mot "démocratie », au Canada et en France, sont les mêmes...

La linguistique externe identifie, les uns par rapport aux autres, des phénomènes tels que dialecte, patois, parler; langue mixte, créole, jargon, argot. Les trois premières étiquettes ne relèvent pas essentiellement d'un fait de contact, tandis que le reste ressortit à un problème de contact entre deux groupes ou deux classes sociales, entraînant des types de langues ou parlers plus ou moins homogènes, intégrés, ou 
mixtes. Les contacts peuvent avoir lieu entre deux ou plusieurs communautés étrangères au point de vue linguistique ; mais une même collectivité, par exemple, peut utiliser — selon la nature et le volume des contacts subis par ses diverses couches- plusieurs types de langues, sans compter les divers argots techniques ou populaires.

[240]

Par rapport à la langue, réalité générale de nature essentiellement structurale, la réalité dialecte fait d'abord appel à la notion externe de subdivision linguistique. Au contraire de l'anglais usuel dialecte ce terme ne possède pas en linguistique la moindre connotation péjorative. Le dialecte représente un stade de fragmentation, dans les axes diachronique (historique) ou synchronique, d'une langue commune. Nous pouvons reconstituer comme suit, à partir de l'indo-européen (environ 3,000 ans avant J.-C), une fragmentation linguistique diachronique jusqu'au français du Canada.

\begin{tabular}{|c|c|c|}
\hline TYPOLOGIE & EXEMPLES & $\begin{array}{l}\text { DATES AP- } \\
\text { PROXIMATIVES }\end{array}$ \\
\hline grande famille ou stock (cf. Sapir) & = indo-européen & -3000 \\
\hline famille & $=$ italo-celtique & $-1200 ?$ \\
\hline sous-famille & = italique & $-900 ?$ \\
\hline grand groupe & $=$ latin & -300 \\
\hline groupe & $=$ roman & +300 \\
\hline sous-groupe & = gallo-roman & +600 \\
\hline langue & $=$ français & +900 \\
\hline dialecte & $\begin{array}{l}=\text { français du Nord } \\
\text { (standard) }\end{array}$ & +1500 \\
\hline sous-dialecte ou parler régional & français du Canada & \\
\hline $\begin{array}{l}\text { idiolectes ou parlers lo- } \\
\text { caux/individuels }\end{array}$ & $\begin{array}{l}\text { québécois, montréa- } \\
\text { lais, etc. }\end{array}$ & \\
\hline
\end{tabular}

Le processus de dialectisation est spontané, inévitable et nécessaire, car, si deux individus ne possèdent jamais les mêmes habitudes phonétiques ou linguistiques, à plus forte raison une langue tendra à 
posséder autant de dialectes ou de sous-dialectes qu'elle servira à des groupes socio-culturels différents et occupera des régions distinctes. La recherche des dialectes et de leurs caractéristiques différentielles relève de la linguistique descriptive externe. Nous pouvons donc maintenant tenter une définition objective du dialecte. Il s'agit d'une entité linguistique se rattachant synchroniquement et spatialement à un groupe plus ou moins grand d'autres entités linguistiques représentant diachroniquement (sur l'axe temporel) une fragmentation plus ou moins diversifiée d'une (grande) famille linguistique, et partageant toutes une homogénéité structurale aux niveaux phonétique, phonologique, morpho-syntaxique et, dans une certaine mesure, lexicologique. Les dialectes sont nettement « apparentés » les uns aux autres et ne se distinguent mutuellement que par des divergences mineures à certains niveaux de structure. L'intelligibilité mutuelle n'est empêchée, à des degrés divers, que par les divergences mineures de structure. Considérés sous cet angle, il est bien évident que l'acadien et le francocanadien (surtout du Québec), même s'ils ne se comprennent pas toujours, se reconnaissent comme des «parents ». Ils sont tous deux des dialectes d'une même langue, le français du Nord-Ouest - synchroniquement — ou, au point de vue diachronique, des sous-dialectes du français standard. De même, le célèbre et encombrant Parisian French, en tant qu'idiome populaire ou général du bassin parisien, [241] n'est qu'un sous-dialecte du français universel, au même titre que le français du Canada et ses subdivisions franco-canadienne et acadienne. Ce qui tend réellement à s'imposer comme français international n'est pas le parisien caractéristique, mais bien une moyenne supra-dialectale à dominance dialecte du Nord-Ouest de la France (où Paris se trouve compris). D'autre part, le vrai « parisien » n'est souvent qu'un parler populaire mêlé d'argot ou d'éléments parasitaires. En terminant, il faut dire à la décharge des dialectes que ces derniers tendent à disparaître, nivelés par le rouleau compresseur des communications rapides et faciles favorisant une langue commune par tout le territoire national. Une littérature dialectale, d'autre part, paraît de plus en plus vouée au sort d'une curiosité archéologique, objet d'étude et occupation des seuls érudits ou lettrés de cabinet. Le sort le plus heureux qui puisse échoir à un dialecte est de devenir une "langue » en accédant au statut d'organe d'expression et de communication de quelque nouveau groupe socio-politique. Peut-être verrons-nous, dans la prochaine décade, quelques idiomes africains acquérir le rang de langue nationa- 
le. On verra alors se renouveler le phénomène historique d'un dialecte qui « réussit », c'est-à-dire devenant une langue.

Selon Dauzat, un patois est le produit socialement et littéralement « déchu » d'un dialecte autrefois prépondérant dans une collectivité, mais actuellement abandonné par l'élite dirigeante de cette même collectivité au profit d'une grande langue commune de civilisation et majoritaire, ordinairement le dialecte d'une capitale et de sa périphérie. $\mathrm{Au}$ contraire de la notion de dialecte, celle de patois est subjectivement entachée d'une forte connotation péjorative en même temps qu'elle revêt une grande valeur affective chez les usagers et les lettrés qui l'étudient. En fait, c'est un dialecte organiquement semblable aux autres, mais sociologiquement infériorisé parce qu'il sert ordinairement une collectivité rurale et se manifeste le plus souvent au niveau purement oral. Presque tous les grands dialectes sont devenus des « patois » au profit d'un seul dialecte-frère - celui de la région politiquement victorieuse - devenu langue commune.

Un parler peut être défini comme un organisme linguistique fortement régional reproduisant la langue commune mais avec un accent marqué résultant d'un substrat dialectal estompé affleurant surtout au niveau de la phonétique et du vocabulaire. En France, l'exemple typique d'un parler régional est l'idiome marseillais, qui n'est pas le patois marseillais (aujourd'hui disparu), mais du français puissamment marqué par une prononciation, un rythme et un vocabulaire d'origine méridionale. C'est une espèce de langue mixte (voir ce terme plus bas) rappelant son équivalent nord-américain, le montréalais, où l'élément substratique allogène est l'anglais au lieu de la langue d'oc. On peut affirmer que dans les deux cas (marseillais-montréalais) nous avons affaire à du français commun (standard) fortement régionalisé ou même patoisant, mais tout de même du français.

[242]

Étudions maintenant, successivement, chacun des organismes linguistiques résultant d'un phénomène de contact. D'abord, une langue mixte. C'est une langue qui a absorbé plus qu'il n'en fallait d'éléments de vocabulaires étrangers. En effet, plus de 60\% quelquefois de son lexique est d'origine allogène. Le cas de l'anglais est typique d'une langue mixte, mais c'est encore une langue germanique, car les autres niveaux de la structure sont clairement identifiés comme germaniques. 
La morphologie et la syntaxe demeurent indigènes. Pour cela, l'anglais n'est donc pas une langue romane. Nous attirons l'attention sur un certain type de canadien-français, soit du prolétariat montréalais ou nordontarien, langue mixte provenant d'un contact intime et continu avec des éléments anglo-saxons. C'est aussi le résultat d'un « bilinguisme » fatal où l'illettré finit par s'assimiler totalement à la langue seconde, faute de posséder suffisamment la langue première. En conclusion, on peut dire (1) que le vocabulaire seul ne suffit pas à caractériser une langue ni n'en constitue l'essentiel ; (2) dans la langue mixte, les deux sources composantes apparaissent, mais les origines demeurent.

Quant à une langue créolisée, c'est un idiome passablement homogène résultant du contact souvent indirect et osmotique de deux collectivités très hétérogènes, comme, par exemple, les maîtres franconormands de Haïti et les esclaves importés d'Afrique occidentale. L'une des deux sources linguistiques est disparue. Comme il y a eu impossibilité, pour le maître ou l'indigène, d'apprendre la langue opposée, la langue du maître - au niveau du vocabulaire — s'est infiltrée dans une structure grammaticale africaine qui l'a lentement et harmonieusement assimilée, digérée, au point d'en faire une langue nouvelle dont les éléments sont indissolublement intégrés les uns aux autres. Ce phénomène nous rappelle un certain type de langue populaire - le " joual » - extrêmement homogène où l'on ne pourrait pas enlever les éléments d'origine anglaise sans détruire l'idiome. Il nous semble que le véritable " joual » — véritable créole — sévit en tant qu'organe d'un prolétariat urbain ayant eu, au niveau de l'industrie, des contacts de type inférieurs-supérieurs avec l'Anglo-Saxon. C'est probablement aussi l'organe de nombreux Franco-Américains ayant appris l'anglais par osmose et formant la plus grande partie des émigrants illettrés ayant trouvé à s'employer dans les factories de coton de la Nouvelle-Angleterre. Comme on ne pouvait pas apprendre de façon régulière la langue des maîtres, on s'est formé, pour communiquer avec eux, une espèce de langue de type enfantin, et souvent la langue du foyer a fini par se teinter de cet idiome invertébré.

Le pidgin ou sabir, comme le créole, provient du contact entre langues et civilisations très hétérogènes ; mais, au contraire du créole, il est resté simplement une langue véhiculaire, un organe de communication, commercial ou culturel, qui n'a pas supplanté la langue maternelle des indigènes. [243] Au point de vue structural, c'est souvent une 
simplification par les indigènes d'une langue de civilisation, tant au niveau de la grammaire (morpho-syntaxe) qu'au niveau du vocabulaire. Le pidgin finit par former un idiome fortement original possédant une structure propre, comme par exemple le pidgin anglo-chinois. Il ne s'agit pas, comme l'argot ou le jargon, d'une langue secrète, parasitaire, résultant d'une défiguration systématique et prévisible d'une langue de civilisation ; l'argot et le jargon ont à peu de choses près la même grammaire que la langue de civilisation. Cette dernière prête souvent son vocabulaire auquel on ajoute ou retranche des syllabes, auquel on accole des suffixes particuliers. L'argot, le jargon ne sont pas des langues premières ou maternelles, mais peuvent être qualifiés d'idiomes spécialisés d'intercommunication entre les membres soit d'une société hétérogène ou secrète.

Pour résumer les principes de linguistique externe contextuelle, on pourrait énoncer l'hypothèse suivante : " À un faisceau de contacts socioculturels déterminés correspond un éventail d'alternatives linguistiques de nature à la fois structurale et externe " (Rémy de Gourmont). De sorte que, si l'on connaît exactement les types de contacts socio-culturels subis à l'intérieur de la collectivité canadiennefrançaise, on peut caractériser de manière satisfaisante les types et les « niveaux » de notre parler. C'est une façon objective d'échapper au monolithisme en matière de langue.

En conclusion de notre esquisse des phénomènes de contact, nous aimerions citer ici l'opinion d'un lettré qui s'est intéressé de façon étroite à la question de la langue :

«... ou bien, résignons-nous ; laissons faire et considérons les premiers mouvements d'une formation linguistique nouvelle. Un patois européen sera peut-être la conséquence inévitable d'un état d'esprit européen, et aucun idiome n'étant assez fort pour dominer, ayant absorbé tous les autres, un jargon international se façonnera, mélange obscur et rude de tous les vocabulaires, de toutes les prononciations, de toutes les syntaxes. Déjà, il n'est pas très rare de rencontrer une phrase qui se croit française et dont plus de la moitié des mots ne sont pas français. C'est peut-être un avantgoût de la langue de l'avenir... » (Rémy de GOURMONT). 


\section{II. ÉLÉMENTS D'UNE DÉFINITION DU PARLER CANADIEN-FRANÇAIS}

Au recensement de 1951, la population française du Canada s'élevait à 4318217 âmes. Notons : population française, c'est-à-dire population de langue française, car on peut supposer encore plus nombreux les descendants des quelque 7, 000 colons venus en grande majorité du nord-ouest de la France entre 1608 et 1700 . Dès les débuts de la colonie, la coexistence de patois du Nord-Ouest et la nécessité de créer une langue commune à base de français central ont joué en faveur d'une homogénéité linguistique [244] plus grande qu'en France où les populations sont restées plus longtemps et plus fortement provincialisées qu'en Amérique. Dès cette époque reculée, les observateurs notent un parler français à teinte dialecte plutôt normannopoitevine, et non un patois, chose impossible étant donné la multiplicité des substrats dialectaux et les nécessités impérieuses de la communication. Dès les débuts, cependant, nous nous organisons en collectivité fermée, acquérant des caractéristiques qui iront s'accentuant après 1700 , une fois le flot de l'émigration française très ralenti. Nous avons développé des caractères institutionnels originaux, une mentalité très conservatrice et déjà une attitude de peuple soumis. Il est curieux de noter que, malgré le petit nombre d'Anglais établis dans la colonie vers 1792, une tendance nette vers l'anglicisation s'implantait chez nous. On note aussi que notre collectivité n'avait pas à cette époque son agressivité linguistique et son complexe national, tel qu'on le verra s'enfler dès 1830 . Un fait demeure certain, c'est que le parler canadien se trouvait déjà fortement assis et caractérisé vers la fin du XVIII $^{\mathrm{e}}$ siècle (cf. le travail du Père Potier en 1743). Au point de vue phonologique : $1^{\circ}$ on peut remarquer une articulation à traits flous, relâchés ; $2^{\circ} l^{\prime}$ « accent » semble résulter de la fusion parfaite de systèmes phonétiques provinciaux nombreux, mais à prédominance vaguement normande. Mais c'est quand même quelque chose de très différent du normand, c'est du canadien. En somme, l'homogénéité est un phénomène normal dans une collectivité linguistique où les membres, peu nombreux, sont soumis à des contacts intimes et fréquents. Selon Brunot, " deux ou plusieurs langues (ou patois) distinctes ont moins 
de force de résistance qu'une langue unique parlée par une population homogène. Aucun patois, ou forme patoise, déracinés, n'ont pu demeurer tels quels, à l'état pur ». ${ }^{267}$ Dans le franco-canadien, le lexique recèle des formes patoises, mais la structure grammaticale (morphosyntaxe) coïncide assez bien avec le français commun. Comparons cet état de langue avec l'acadien qui possède non seulement un vocabulaire différent, non seulement une prononciation fortement différente et dialectale, mais aussi une structure grammaticale particulière la rapprochant d'un certain type de normand.

Après cette incursion dans le domaine diachronique, nous allons maintenant tenter de délimiter les principales couches socioculturelles du parler régional de type français du Nord-Ouest qu'est le canadien-français. À l'heure actuelle, nous reconnaissons au moins trois sous-dialectes canadiens-français dans l'est du Canada (surtout au Québec) : 268

1. Celui de l'élite professionnelle, artistique, instruite : sauf dans le monde artistique et dans un secteur restreint des professions, où l'on tend fortement à se modeler sur un français international sans accent, l'accent est [245] nettement marqué, la grammaire et le vocabulaire se rapprochent du français commun. On trouve cependant des anglicismes de métier sous forme de calques (traductions littérales de l'anglais). La langue littéraire est souvent artificielle.

2. Celui de la population rurale : la langue de la population rurale, très stable et légèrement archaïque, teinte tous les niveaux de la population canadienne-française. Cette population rurale, de plus en plus minoritaire, colore notre parler de façon indélébile. Elle n'est pas patoisante au sens européen du mot.

3. Celui du prolétariat urbain : surtout, ce prolétariat est instable, neuf, inquiet. Seulement, il faut distinguer par zones urbaines (Québec, Montréal) afin de caractériser son parler. Il se ressemble plus ou moins partout et les différences nous semblent plus de quantité que de nature. Montréal étant majoritaire, nous la choisirons comme base de comparaison, comme critère de description. C'est ici qu'on assiste à la

267 Ferdinand Brunot, Histoire de la langue française, Paris, Colin, t. I, 25.

268 Nous laissons de côté le cas spécial de l'acadien. 
formation d'un patois, si patois il y a, et non à la campagne. C'est ici que le contact avec l'anglais, subi à des titres divers et selon les types différents, donnera au moins deux types de parlers, tous teintés d'archaïsmes et de dialecte français. C'est au niveau populaire, dans les villes surtout, qu'on peut observer à loisir des phénomènes de dynamisme linguistique se résumant dans un processus de créolisation plus ou moins achevé.

Le premier type de parler, un français mixte, combine une structure française (morpho-syntaxique) quasi-intacte avec un vocabulaire en partie anglais, surtout en ce qui a trait aux termes usuels et aux termes de métier. Cependant, il semble possible de décanter l'anglais par rapport au français et les gens disent qu'ils parlent le français ou « canadien » (ce qui est ici la même chose). Ce type de langue appartient, semble-t-il, à une couche populaire mais socialement aisée ; en d'autres termes, c'est la langue de la classe moyenne et modérément instruite. Elle est assez influente. Son contact avec l'anglais est un contact au moins d'égal à égal, c'est la classe des épiciers et des fonctionnaires, qui jouissent d'une certaine autonomie technique et industrielle en face des anglophones. Ici, la créolisation semble arrêtée, mais on pressent des signes d'une évolution prochaine soit vers l'anglo-américain, soit vers le français plus conforme à la norme internationale. Il n'en est pas de même pour le deuxième type de parler populaire, celui du véritable prolétariat où le processus de créolisation est en pleine activité, où l'anglicisation linguistique et culturelle est à un stade avancé. On se rend bien compte qu'il s'agit d'une réalité linguistique bien différente du français, et qui n'a pas fini de nous réserver des surprises. On pourrait définir le phénomène de façon plus précise en disant qu'il s'agit ici de la symbiose de l'anglais avec un parler d'origine paysanne française multiple, lui-même en processus d'urbanisation ultra-rapide. Nous sommes sûrs de retrouver au bout de la métamorphose autre chose que du français, même [246] paysan : ce qu'on appelle quelquefois du « franglais »; ce qui est bien un patois, puisque c'est un parler socialement infériorisé. C'est le « joual », tel qu'on a coutume de le dénommer depuis la pittoresque intervention du Frère Un Tel.

Le « joual », qu'un esprit mythique voudrait voir partout comme le symbole de notre soi-disant « veulerie » linguistique, est un phénomè- 
ne agaçant, certes, pour les puristes, mais c'est une « maladie » extrêmement intéressante dont les ramifications sont surtout sociologiques. Son existence est sûrement réelle, mais les dimensions mythiques qu'il a prises sont symptomatiques de notre métabolisme socio-culturel. Avant d'être une langue - car ses limites sont très floues - il risque d'être une maladie mentale se propageant à la manière d'une épidémie. Nous croyons que le grand battage de publicité malsaine et subjective — voir les articles dans les journaux — autour de ce phénomène risque d'implanter ce type de parler là où il n'existait pas. Il deviendra, à la façon d'un argot, une langue auxiliaire à nuance fortement sentimentale, et grâce à laquelle les Canadiens français pourront s'identifier. Si on ne le parle pas, on l'apprendra : nous pensons aux nouveaux dictionnaires de « joual » qui voient et qui verront le jour.

À notre avis, c'est mal poser le problème que de le limiter à un type extrême de langue, tandis que nos énergies précieuses devraient s'employer à enquêter sur les bases socio-culturelles de nos parlers, à entreprendre de façon méthodique l'enregistrement exhaustif de nos parlers régionaux qui s'en vont rejoindre les vieilles lunes. Ce qu'on peut dire du statut structural de notre langue populaire extrême, de nos parlers prolétariens, c'est qu'il se trouve en équilibre extrêmement instable, équilibre qui sera bientôt rompu soit en faveur de l'angloaméricain, soit en faveur du français international, directions qui seront dictées par les impératifs socio-économiques. Un groupe politique chauvin, accréditant le « jouai » comme parler national distinctif, contribuerait à la longue à l'anglicisation totale d'un petit peuple qui ne voudrait sûrement pas garder un patois comme organe d'expression à la face du monde entier. Enfin, la polémique du « joual », n'ayant pas à sa disposition d'arguments linguistiques très forts, s'avère pourtant très révélatrice sur le terrain socio-culturel. Elle manifeste un malaise fondamental chez un groupe humain qui se cherche une identité propre, et qui tend à rejeter celle qu'on lui impose, selon lui. Elle est un signe certain de révolte et de réelle désaffection envers nos valeurs idéologiques. Au point de vue littéraire, les gens répugnent à écrire un français trop artificiel et trop éloigné de la langue parlée pour qu'on s'y sente à l'aise. Le décalage donne aussi mauvaise conscience ; la mauvaise conscience engendre l'agressivité, de là les polémiques masochistes, les velléités sporadiques de suicide national chez certains évolués. Comme les composantes socio-culturelles de notre langue 
parlée sont trop éloignées de la langue idéale et patriotique qu'on [247] nous présente à l'école et qu'on "vénère » officiellement, le peuple, pour échapper au vertige du vide, se crée une langue mythique, une illusion linguistique, en un mot, une langue à lui. Mais on ne lui a pas appris qu'une langue, pour exprimer un peuple, n'a pas besoin d'être matériellement différente de celle du voisin. La réalité est sémantique, elle réside dans le sens des mots, dans leurs nuances et leurs emplois. À lire les articles sur le « joual » et notre parler en général, on a nettement l'impression d'avoir affaire à un peuple qui se hait luimême et qui souffre. Le peuple souffre d'insécurité idéologique et d'ambivalence : d'abord, on nous a imbus de notre supériorité messianique, mais en Nord-Américains pratiques, nous nous rendons vite compte que la réalité économique et culturelle dément de pieux mensonges. Nous sommes censés détester l'Anglais, mais sa langue, sa mentalité, son prestige économique nous entrent dans la peau. On nous a dit qu'il fallait aimer nos origines catholiques et françaises, mais notre vieux complexe de coloniaux exploités nous saute à la figure ; de plus, on nous a dit que la France moderne est " laïque ", c'est-à-dire athée. Il nous est possible de reconstituer, à l'aide de ces éléments de psychologie collective, le substrat mental collectif des Canadiens français : plus de France, pas d'Angleterre. Le Canada ? C'est pour nous une réalité menaçante et instable. Serons-nous enfin " Canadiens » tout court? "Canadian »? «Canayens »? On ne sait pas encore, car ces étiquettes s'attachent à des concepts inachevés ou douteux. Alors, que nous reste-t-il ? - À nous régénérer. - Comment ? - En repartant à neuf sur la base du français moderne ou de l'anglo-américain. Dilemme linguistique et culturel que nous résoudrons bientôt par nos options définitives. En conclusion, notre langue, à quelque niveau que ce soit, porte les marques, les blessures de nos combats, de nos ambivalences, de notre instabilité, bref, de nos traumatismes. Voilà pourquoi il est passionnant d'étudier son visage entier, et en rapport avec notre odyssée culturelle et sociale. Linguistes aussi bien que sociologues trouveront un intérêt et un avantage indéniables à se consacrer à cette recherche ; elle leur livrera le vrai visage de ce groupe ethnique dont la première caractéristique est de s'opposer - pour un temps encore - à la vague socio-culturelle du continent nord-américain. 


\section{TRAVAIL ACCOMPLI ET TRAVAIL À FAIRE}

Il convient de saluer ici les efforts et les résultats solides qui ont été enregistrés dans le domaine de la dialectologie tout d'abord. Nous connaissons les travaux d'Adjutor Rivard et de la Société du parler français, à Québec ; les acquisitions et les découvertes de l'École de Québec, sous [248] l'égide de Rivard, ont servi de base à une vaste compilation des parlers franco-canadiens, s'ajoutant à la série déjà impressionnante des glossaires des patois et parlers de la vieille France. L'École de Québec, par les recherches de Luc Lacourcière en folklore, a imprimé un progrès considérable à nos connaissances de linguistique régionale et sociologique, puisque la littérature orale, représentée par les traditions populaires, les vieux contes, les légendes, nous a fait entendre les parlers spontanés et naturels de régions et collectivités que leur humilité aurait fait sombrer dans l'oubli. À côté de la recherche folklorique, dont le but premier n'est pas la linguistique, nous voyons un gigantesque travail de linguistique géographique mis en chantier par M. Gaston Dulong et ses assistants, et qui continue la plus pure tradition des études françaises et romanes. Le résultat de cette recherche sera de nous révéler la physionomie réelle des sociétés canadiennes-françaises, en explorant un contenu culturel représenté par les expressions populaires, les métiers et leur terminologie, en décrivant leur mentalité profonde et les structures linguistiques qui la symbolisent. Quant au domaine de la phonétique, celui du système de prononciation, des travaux nous ont déjà appris, avec les études de Charbonneau (Montréal), Gendron (Québec), la relative homogénéité de notre provenance sociale ; la recherche nous donnera une plus juste répartition de cadres sociaux s'exprimant par des prononciations bien caractérisées. Enfin, nous aimerions mettre en relief deux écoles de pensée originales : d'abord, celle de linguistique structurale ou de psycho-mécanique du langage, laquelle, sous la direction de $\mathrm{M}$. Roch Valin (Québec), pourra nous éclairer sur la genèse d'une mentalité sociolinguistique, sur les principes sous-jacents à l'édification d'un organe d'expression et de communication d'une collectivité culturelle. Ensuite, je m'en voudrais de passer sous silence la seconde école linguisti- 
que, celle de la stylistique comparée qui, sous l'impulsion de M. JeanPaul Vinay (Montréal), a montré, d'une part, de façon non équivoque les rapports qui existent entre une mentalité linguistique et les procédés structuraux qu'elle utilise dans le domaine de l'expression ; d'autre part, la méthode de la stylistique comparée, rejoignant d'une certaine manière la pensée de Whorf et de Sapir, autorise l'espoir d'établir un jour une formule de rapports entre un groupe culturel et une grammaire expressive. Nous avons appris, à cette école, que le fait d'appartenir à une entité culturelle donnée impose aux associations, au choix et à la résonance des mots un cadre expressif, des lois stylistiques particulières. C'est ainsi qu'entre le français du Canada et le français européen il existe un écart et un faisceau de différences sémantico-affectives que les recherches de stylistique comparée sont éminemment aptes à montrer.

En conclusion, nous voyons une tâche urgente devant nous : celle d'élargir davantage le corpus de nos connaissances sur le français du Canada, en premier lieu par la détermination exacte de ses différents niveaux, ensuite [249] par l'intensification de la recherche, non seulement dans les domaines déjà mentionnés, mais aussi dans le vaste champ de la pédagogie et de l'application. La linguistique, qu'elle soit descriptive ou sociologique, devra descendre dans l'arène si elle désire enlever aux mains dangereusement inexpérimentées des amateurs le sort de notre langue. Les socio-linguistes, pour servir les masses populaires qui cherchent un appui et une direction - tout en échappant au purisme étroit et à la dictature des littéraires —, devront apprendre à les connaître dans toutes leurs manifestations linguistiques en relation avec une articulation sociologique. Il ne faut pas se leurrer : c'est par le consensus populaire que tout " remède ", toute mesure, tout précepte linguistiques prendront racine et agiront efficacement. Tout spécialiste de la langue chez nous, qu'il soit linguiste objectif ou grammairien normatif, devra se souvenir de la nécessité d'une connaissance la plus intégrale possible du milieu culturel et des parlers qu'il conditionne. Dans ce but, nous devrons nous attaquer, par la méthode de l'enquête monographique et régionale, aux idiomes des couches urbaines, prolétariennes, et non seulement rurales ; nous devrons recueillir les formes à la fois " correctes » et "incorrectes » de notre langue ; aucun dialecte, patois ou argot ne devra être négligé. Toute forme de langage, en relation avec un groupe ou des groupes sociaux donnés, 
devra être recueillie, classée, interprétée dans une synthèse qui reste encore à faire. Cette dernière s'amorcera une fois qu'on aura décidé de compléter nos connaissances sur nos parlers considérés dans une perspective sociologique, et non de façon purement grammaticale ou en référence avec le seul critère de la correction. De sorte que, au lieu d'opérer de manière négative une discrimination à propos de tel ou tel élément dialectal, « incorrect » ou soi-disant inutile, on oubliera de se voiler la face et on tiendra compte des différences de régions géographiques, de milieux culturels, d'influences et d'emprunts, de structures économiques, de types de contacts donnés. De cette manière, on approchera plus justement la réalité linguistique - la langue parlée et son miroir déformant, la langue écrite - que nous cherchons : l'état véritable de l'organe d'expression et de communication d'une collectivité d'origine provinciale française vivant, au milieu du $\mathrm{XX}^{\mathrm{e}}$ siècle, en milieu socio-culturel et économique de marque anglo-américaine. Réciproquement, sociologues et ethnologues trouveront dans les descriptions linguistiques des jalons et des éléments de solution.

Gilles-R. Lefebvre

Département de linguistique, Université de Montréal. 


\section{COMMENTAIRE}

\section{L'APPORT DE LA GÉOGRAPHIE LINGUISTIQUE}

Gaston Dulong

Département de linguistique, Université Laval.

[pp. 250-252.]

$\underline{\text { Retour à la table des matières }}$

Il est à mon sens très heureux que les organisateurs de ce colloque aient pensé à faire appel aux linguistes. Notre collègue Lefebvre vous a déjà laissé entrevoir ce que l'étude de la langue peut apporter. Je tiens ici à l'en remercier.

Si l'étude de la langue est si importante pour l'étude de groupes canadiens-français c'est que la langue, ce comportement verbal de l'homme dans la société, est la chose la plus humaine et la plus systématique qui soit.

De plus, l'étude de la langue est une science exacte. Nous sommes en présence d'un système fermé que nous pouvons cerner, dans lequel nous trouvons des unités, unités avec lesquelles nous pouvons faire des statistiques, unités que nous pouvons étudier sémantiquement, phonétiquement, aussi bien dans le temps que dans l'espace. 
L'étude de la langue française au Canada est commencée depuis longtemps. Il faut cependant reconnaître que les monographies, les dictionnaires ou les glossaires qui ont été faits, bien que éminemment précieux à bien des points de vue, n'ont pas cette rigueur scientifique que l'on exige aujourd'hui de tels travaux. La notation phonétique fut négligée tout à fait ou faite de façon approximative, la localisation ou la distribution géographique fut négligée ; on croyait ou, ce qui est plus grave, on voulait croire que la langue était uniforme.

Les enquêtes systématiques sur le français canadien, avec questionnaire et description phonétique, ont commencé il y a très peu de temps. À date une vingtaine d'enquêtes ont été faites dans les régions de Montréal, de Québec, du Bas du Fleuve, de la Gaspésie, sur la côte Nord, aux îles-de-la-Madeleine, au Nouveau-Brunswick, au CapBreton et dans le sud de la Nouvelle-Écosse. C'est très peu, c'est trop peu si l'on songe à l'étendue énorme de territoire à parcourir, mais c'est quand même suffisant pour laisser entrevoir les divergences phonétiques et dialectologiques de la langue.

Il s'agit en somme de géographie linguistique, c'est-à-dire de la transcription sur cartes de faits linguistiques recueillis sur le terrain. En reportant ainsi sur des cartes les résultats d'enquêtes, on voit tout de suite se dégager des zones communes, des îlots, qui s'expliquent sans doute par l'histoire du peuplement, par des zones anciennes ou actuelles à partir de tel ou tel centre, une ville la plupart du temps, zones linguistiques réelles, en tout cas, dont les sociologues devront sans doute tenir compte.

À titre d'exemples, voici quelques réalités désignées par des mots carto-graphiables.

\section{JABOT d'un oiseau}

Falle : région de Montréal, Yamachiche, Saint-Vallier (Bellechasse), Cacouna, Les Méchins, Sainte-Anne-des-Monts, Grande-Vallée, Grande-Rivière, Paspébiac, Carleton ; Grande-Digue, Cap-Pelé. 


\section{CLÔTURE}

Clôture : régions de Montréal et de Québec.

Bouchure: Paspébiac, Bonaventure, Gascons, Havre-SaintPierre, Natashquan ; Cap-Pelé, Grande-Digue ; Îles-de-laMadeleine ; Chéticamp, Pubnico.

[251]

BAISER (donner un...)

Bec : régions de Montréal et de Québec, Yamachiche, SaintVallier, Cacouna, Les Méchins, Sainte-Anne-des-Monts, Natashquan ; Chéticamp.

\section{PIS DE VACHE}

Pis : région de Montréal.

Per: région de Québec, Yamachiche, Saint-Vallier, Saint-Joseph-de-Beauce, Cacouna, Les Méchins, SainteAnne-des-Monts, Mont-Louis, Grande-Vallée, Paspébiac, Bonaventure, Havre-Saint-Pierre et à une vingtaine d'autres endroits.

Remeuil: Gascons, Carleton; Grande-Digue, Cap-Pelé ; Chéticamp, Pubnico ; Havre-Saint-Pierre, Natashquan.

\section{BOUILLOIRE}

Canard : région de Montréal.

Bombe: Yamachiche, île d'Orléans, Saint-Vallier, SaintJoseph-de-Beauce, Cacouna, Les Méchins, Sainte-Anne-desMonts, Mont-Louis, Grande-Vallée, Rivière-aux-Renards, Paspébiac, Bonaventure, Carleton, Grande-Rivière, Sept-Îles, Havre-Saint-Pierre, Natashquan.

Coquemar : Grande-Digue, Cap-Pelé ; Pubnico ; Havre-SaintPierre, Îles-de-la-Madeleine. 


\section{TOMBEREAU}

Tombereau : région de Montréal.

Banneau : Saint-Vallier, Saint-Narcisse, Lotbinière, Loretteville, Beauceville, Montmagny, île d'Orléans.

Charrette: Paspébiac, Bonaventure, Carleton, GrandeRivière, Îles-de-Ia-Madeleine ; Grande-Digue, Cap-Pelé ; Chéticamp.

\section{LOMBRIC}

Anchet : Cacouna, Rimouski, Matane, Les Boules, Les Méchins, Sainte-Anne-des-Monts, Mont-Louis, Rivière-auxRenards.

Lachet : Saint-Vallier, Saint-Joseph-de-Beauce.

Laiche : Îles-de-la-Madeleine ; Grande-Digue, Cap-Pelé ; Chéticamp, Pubnico.

\section{HAMEÇON}

Hameçon : région de Montréal.

Haim : Yamachiche, île d'Orléans, Saint-Raymond, SaintNarcisse, Cacouna, Trois-Pistoles, Les Boules, Bonaventure, Sainte-Anne-de-Madawaska.

Croc: Les Méchins, Sainte-Anne-des-Monts, Mont-Louis, Grande-Vallée, Havre-Saint-Pierre, Natashquan, Carleton, Gascons ; Cap-Pelé ; Chéticamp, Digby.

\section{PÉTRIN}

Huche : régions de Montréal et de Québec.

Maite : Gascons, Carleton, Bonaventure, Saint-Charles-deCaplan, Natashquan, Îles-de-la-Madeleine ; Grande-Digue, Cap-Pelé ; Chéticamp. 
Voilà, résumé très brièvement, ce qui a été fait jusqu'à aujourd'hui. Évidemment, ce n'est pas considérable. Cependant, l'échantillonnage permet déjà d'entrevoir ce que donnera un réseau d'enquêtes plus serré, centré cette fois dans les régions de Québec et de Montréal. On peut dès maintenant soutenir, avec preuves à l'appui, que plusieurs frontières linguistiques (mots ou prononciation) passent quelque part dans la région des [252] Trois-Rivières, frontières qui traversent le fleuve. Énumérons pour terminer les points variés sur lesquels devra porter le travail des chercheurs :

Enquêtes sur le terrain avec un questionnaire de façon à pouvoir comparer les réponses les unes avec les autres. Est-il besoin d'ajouter que toutes les réponses devront être transcrites phonétiquement pour être valables?

Dépouillement pour les $\mathrm{XVIII}^{\mathrm{e}}$ et $\mathrm{XIX}^{\mathrm{e}}$ siècles des documents (livres, journaux, manuscrits, inventaires) susceptibles de nous fournir des éléments sur la langue parlée au Canada. Il est sûr qu'on pourrait, à l'aide de ces faits, dresser des cartes de géographie linguistique que l'on pourrait comparer à celles que nous dresserons à l'aide des documents recueillis au cours d'enquêtes faites sur le terrain.

L'étude de la langue des citadins, sur laquelle nous possédons actuellement des données très minces.

Étude de la mentalité des francophones à travers les mots et les expressions, v.g. leur attitude vis-à-vis des sujets tabous, vis-à-vis de nos compatriotes de langue anglaise et de la langue anglaise, vis-à-vis aussi des aborigènes du Canada.

Tout cela constitue un programme énorme auquel nous devons nous atteler le plus tôt possible avant qu'il ne soit trop tard.

Gaston Dulong

Département de Linguistique, Université Laval. 
[253]

\title{
Situation de la recherche sur le Canada français \\ III. Perspectives sur l'étude de la structure sociale
}

\author{
Luc Lacourcière \\ Archives de folklore, \\ Université Laval

\section{“L’étude de la culture : le folklore.”}

[pp. 253-262.]

$\underline{\text { Retour à la table des matières }}$

Ce colloque et tous les congrès semblables qui réunissent un aussi grand nombre de conférenciers (présentement trente-six pour souligner un chiffre folklorique !) me font songer à l'étrange et cruel supplice auquel le fils de Poséidon soumettait les voyageurs entre les plaines d'Eleusis et d'Athènes. Procuste, si vous vous rappelez la légende, arrêtait les passants et les étendait sur un lit qui n'était jamais à leur taille. Quand leurs jambes étaient trop courtes, il les allongeait à coups de marteau. Mais si elles dépassaient, le brigand, il les coupait sans pitié. ${ }^{269}$ Ainsi en est-il de nous, itinérants des sciences sociographiques, naguère sciences de l'homme, dans cette plaine de Sainte-Foy ou de Québec que l'on a, je crois, déjà surnommée l'Athènes d'Amérique! II nous faut rogner de nos communications, sinon de notre

269 Nouvelle mythologie illustrée publiée sous la direction de Jean Richepin, Paris, s.d., vol. 1, 245. 
science, tout ce qui risque d'excéder les douze pages cubilaires exigées par le directeur du Département de sociologie et d'anthropologie de l'Université Laval. Mais la légende nous apprend encore qu'à la fin Procuste se vit infliger lui aussi le même supplice qu'il avait fait subir à tant de victimes. Et cela, monsieur Dumont, ce sera un peu la revanche de vos suppliciés à la fin de ces assises.

Quoi qu'il en soit, appliquée au folklore, la question thème de ce colloque sur la situation de la recherche sur le Canada français : «travail fait, travail à faire ", ne nous prend pas trop par surprise, puisque voilà bientôt vingt ans que nous travaillons à y apporter une réponse. Même je dirais que c'est en partant d'une question semblable, différemment formulée toutefois, que nous avons fondé en 1944 des Archives de folklore. Leur but était, précisions-nous alors, et demeure aujourd'hui l'étude scientifique des traditions populaires françaises d'Amérique dans leur état actuel, dans leurs créations et transformations, dans leurs rapports avec leurs sources européennes et les civilisations indiennes et saxonnes. 270

[254]

Aussi nous a-t-il paru facile, du moins à première vue, de répondre à la première partie de la question : " travail fait », par des statistiques sur le nombre de renseignements accumulés dans nos Archives. Et à cette fin nous repassions mentalement des chiffres qui nous sont familiers parce qu'au cours des années nous en avons suivi l'accroissement au jour le jour :

$1^{\circ}$ Une bibliothèque spécialisée de folklore canadien et comparé d'environ quatre mille volumes (y compris une centaine de collections de revues étrangères).

$2^{\circ}$ Dix-huit mille enregistrements inédits dont douze mille en exclusivité obtenus directement sur le terrain par une quarantaine de collecteurs travaillant, un petit nombre à temps plein et la majorité à temps partiel et parfois bénévolement pour les Archives. Les six autres mille sont des copies, très précieuses aus-

270 «Présentation », Les Archives de folklore, I, 1946, 7. Voir aussi le Mémoire présenté par les Archives de folklore de l'Université Laval à la Commission royale d'enquête sur les problèmes constitutionnels, mars 1954, 1. 
si, des enregistrements sur cylindres du Musée national du Canada, réalisés à partir de 1916 par M. Marius Barbeau et ses collaborateurs ; enregistrements tous repiqués dans nos laboratoires entre 1958 et 1961.

$3^{\circ}$ Plusieurs milliers de dossiers et de pièces manuscrites, vieux cahiers, vieux papiers de folkloristes disparus, journaux et notes d'enquêtes, transcriptions des enregistrements, etc., le tout classé aussi méthodiquement que possible.

$4^{\circ}$ Et surtout, pour retrouver la centaine dans une documentation complexe et multiforme, cent cinquante mille fiches de toutes sortes, réparties en bibliographies, catalogues analytiques et répertoires par sujets, classifications alphabétiques ou numériques selon le cas ; le tout complété par des index et concordances aux collecteurs, aux informateurs, aux lieux d'enquête ou de provenance des renseignements.

Ce bilan est assez impressionnant certes pour révéler l'ampleur de l'inventaire entrepris sur les traditions populaires, mais uniquement quant à l'aspect nombrable des renseignements accumulés. Et encore ces chiffres ne nous disent-ils que d'une façon globale et assez vague les catégories de faits populaires auxquels ils réfèrent.

Avant de définir davantage ces catégories ou plutôt d'en énumérer les parties constituantes, il nous faut nous arrêter sur ce mot folklore dont la fortune est assez curieuse. Créé en 1846 par l'Anglais William John Thoms pour remplacer des expressions trop vagues, trop particulières ou tendancieuses, comme l'étaient Popular antiquities, Popular literature, ou encore Vulgar and common errors alors en usage, ce mot folklore, dans l'esprit de son inventeur, devait désigner la science du peuple, «the Lore of the People ». Le mot s'imposa vraiment dans la deuxième moitié du $\mathrm{XIX}^{\mathrm{e}}$ siècle au fur et à mesure qu'il servit de nom à des sociétés comme English Folklore Society (1878), American Folklore Society (1889), etc., ou [255] de titre à des publications périodiques comme Folk-Lore Record (1878), Journal of American Folklore (1889), Zeitschrift für Volkskunde (1889), Bulletin de folklore (Bruxelles, 1892), ou à des recueils, traités et manuels, consacrés à l'étude des traditions populaires. Je ne retiens ici que deux titres qui, en raison de leur date, Folk-Lore (1885) du comte de Puymaigre et Le 
folklore de France de Paul Sébillot (4 vol., 1904-1907), ont consacré le mot en France. On peut affirmer qu'autour de 1900 le mot avait pénétré dans toutes les langues et faisait partie du vocabulaire international, mais sans avoir toutefois aboli toutes les résistances.

Au Canada français ou anglais, les premiers écrivains à employer le mot furent, croyons-nous, Honoré Beaugrand et Louis Fréchette, qui ont participé l'un et l'autre à la fondation, en 1892, d'une section canadienne de l'American Folklore Society. Dans leur esprit toutefois, beaucoup plus littéraire que scientifique, comme dans celui de quelques-uns de leurs contemporains, le mot paraît avoir eu à peu près exclusivement le sens de légendes. C'est pourquoi il n'est pas étonnant de voir, en 1907, figurer notre mot parmi une longue liste d'anglicismes à bannir de notre langue comme abusifs. L'abbé Émile Chartier propose alors de remplacer le terme par son équivalent français qui, d'après lui, était «légendes locales ». ${ }^{271}$ Mais cette opinion n'était certainement pas partagée par les autres rédacteurs du Bulletin du parler français au Canada, car quelques-uns d'entre eux, et le juge Rivard en tête, emploient couramment le mot folklore.

Il appartenait à E.-Z. Massicotte et à Marius Barbeau de vulgariser le mot tout en lui donnant une dimension plus étendue, celle qu'il avait acquise dans les autres pays pour désigner les traditions populaires elles-mêmes sous toutes leurs formes en même temps que l'étude scientifique de ces traditions. Ils ont prêché par l'exemple, Marius Barbeau surtout, en multipliant les enquêtes et les publications dans les domaines les plus divers, tant chez les Indiens que chez les Français de la vallée du Saint-Laurent. C'est dans le prolongement de leur œuvre française que s'inscrivent les Archives de folklore, comme nous l'avons déjà écrit maintes fois. Lors de leur fondation, nous n'avons aucunement hésité à employer ce mot tant pour définir notre centre d'archives et de documentation au sein de la Faculté des lettres de Laval, que pour désigner les publications qui en sont issues. Nous suivions en cela l'exemple d'organismes scientifiques de plusieurs pays. Le titre, croyions-nous, serait et de fait a été défini par les activités qu'il recouvre dans le domaine des traditions populaires. Le contenu a justifié l'étiquette même si celle-ci auprès de certains a le tort de ne

271 Abbé Emile CHARTIER, « La propriété de l'expression », Bulletin du parler français au Canada, 6, 1907, 10-11. 
pouvoir s'auréoler d'une étymologie gréco-latine, ce qui est un exemple à peu près unique dans le partage ou la terminologie des sciences de l'homme.

[256]

Cependant, si le vocable qui sert à la désigner ne crée pas nécessairement une science nouvelle, il peut arriver que son emploi quotidien en dehors de son application scientifique nuise à cette science et introduise de la confusion dans le langage. La sociologie elle-même n'at-elle pas eu à souffrir de l'emploi abusif de l'épithète " social », par exemple dans des expressions comme « carnet social » qui n'ont rien à voir avec la science de l'homme collectif ? De la même façon, le mot folklore est employé à toute sauce et sert à " couvrir des activités extra-scientifiques »: associations de loisirs, groupements de chanteurs, de danseurs, qui, en costumes de folklore plus ou moins authentiques, poursuivent depuis une quinzaine d'années - au Canada français, c'est surtout un phénomène d'après-guerre - des buts d'ordre récréatif, publicitaire, touristique et parfois esthétique. Pour cette jeunesse débordante, faire du folklore, cela signifie danser et s'amuser et rien d'autre. Grand bien lui fasse ! Il suffit que le public soit averti que le mot folklore tout comme le mot social n'est pas et ne peut être, en notre pays démocratique, une appellation contrôlée !

Mais depuis quelques années on fait un usage encore plus spécieux du mot folklore (et qui n'a, bien entendu, aucun rapport avec la science des traditions). Il subit une évolution sémantique dépréciative comme en ont connue certains mots tels que moyen âge, colon, habitant, concession. C'est ainsi qu'on en est venu à vouloir désigner par folklore tout ce qui est arriéré, tout ce qui est dépassé, et, bien plus, tout ce qui est blâmable, voire fautif et injurieux. Les journaux - estce un autre signe de leur pauvreté linguistique ? - nous donnent tous les jours des exemples de ces emplois tendancieux et cela dans les manchettes de nouvelles à sensation tout comme sous la plume de leurs meilleurs éditorialistes. J'ai collectionné, pour l'histoire du mot, un grand nombre de ces exemples qu'il faudrait bien sûr analyser dans leur contexte pour être juste. Ce serait trop long. Je vous en citerai cependant quelques-uns que j'emprunte à l'actualité pour vous faire saisir les nuances de ma pensée. Inutile de vous dire que, dans les phrases citées, seul m'intéresse l'usage du mot folklore et que je me garde bien de prendre parti pour ou contre l'opinion exprimée. 
1. Dans un éditorial du Devoir, 17 octobre 1959, sous la plume de Gérard Filion : «Cette politique folklorique [celle de déplacer les fonctionnaires après une élection] menaçait de ruiner toute l'administration fédérale $»$.

2. Dans Le Devoir encore, sous la plume de Pierre Laporte, le 20 juin 1961, en titre de trois colonnes : «Le travail des comités de la Chambre n’est plus du “folklore” comme sous le règne de Duplessis ». Folklore est ici entre guillements probablement pour souligner le caractère insolite de son emploi.

3. Sous la plume de Jean-Louis Gagnon, dans un premier-Montréal du Nouveau Journal du 10 février 1962: «Feu le docteur Paquette. Nommé au Conseil législatif dans un moment de distraction ou d'humeur, appartenant au folklore de l'ère duplessiste ... »

4. Manchette du Devoir, le 23 janvier 1962 : «Chaput : le Québec est la dernière colonie où le français soit folklorique ».

5. « Le séparatisme tient-il du folklore ? », titre d'un article de Gilles Hénault dans Le Nouveau Journal, 17 mars 1962, section des Arts.

6. « Nos manuels véhiculent le folklore, le mythe et la peur... » : Le Devoir, 19 mars 1962, compte rendu d'un débat sur les manuels scolaires.

7. Autre exemple emprunté à l'opinion d'André Langevin dans Le Nouveau Journal du 30 janvier 1962 : «... la culture française, dont nous étions dépositaires, s'est amoindrie pour n'être plus qu'un folklore. » Voilà comment la poule serait redevenue poussin, mais sans passer par l'œuf !

8. À ces citations tirées des journaux, j'ajouterai encore le slogan d'une pancarte étudiante lors d'une manifestation récente : «La Confédération survivance folklorique ».

[257]

Le dénominateur commun dans ces cas que je viens de citer est l'expression sans nuances d'un blâme et d'un mépris arrogant sur des catégories de faits politiques, pédagogiques ou simplement polémiques que l'on veut évoquer par le truchement du mot folklore. Cela est abusif et ne répond qu'à une notion confuse et des traditions populaires et de la science qui les étudie. Je me suis attardé à ce petit problème pour montrer que, par un retour curieux, ces contresens de l'argot journalistique nous reportent avant 1846, aux mêmes préjugés que William John Thoms voulait faire disparaître en inventant un mot tout à fait objectif. 
Est-ce à dire qu'il faille abandonner le mot aux profanes et lui substituer, comme certaine école l'a fait en France, le mot ethnographie pour décrire la démarche scientifique du spécialiste de la tradition ? Pour ma part, je ne le crois pas. Cela ne fait que déplacer le problème vers un autre mot dont l'évolution sémantique n'est pas non plus complètement fixé. Contentons-nous de constater qu'actuellement les mots folklore et ethnographie sont employés concurremment.

Ce qui importe davantage c'est la chose signifiée. En l'occurrence les traditions populaires. Mais lesquelles demandez-vous avec impatience ? Pour répondre à cette question, j'ai pensé utiliser le plan de la Bibliographie internationale des Arts et Traditions populaires, fondée en Suisse, en 1917, par John Meier et E. Hoffmann-Krayer, continuée par Paul Geiger et Robert Wildhaber avec l'assistance de nombreux collaborateurs, recrutés dans les organismes spécialisés en folklore des principaux pays occidentaux. Les derniers volumes ont paru avec le concours de l'UNESCO. Les Archives de folklore, par leur archiviste-bibliographe, M. Conrad Laforte, y collaborent, depuis 1950, pour le Canada.

Le plan de cette bibliographie est très vaste et sur plus d'un point déborde dans le domaine d'autres sciences de l'homme. Ou plutôt il fait valoir le point de vue du folklore en des sujets d'études qui sont communs à plusieurs disciplines. Il n'y a pas lieu, je crois, de s'en étonner et de chercher querelle à telle ou telle science sur les problèmes de frontières. Quoi qu'il en soit, ce plan répartit les matières à recenser en 22 chapitres dont quelques-uns ont à leur tour jusqu'à 12 et 14 subdivisions. En raison [258] de sa grande portée internationale et de sa diffusion trop limitée jusqu'ici en notre pays, je crois utile de le mettre sous vos yeux de façon un peu simplifiée, avant de faire quelques remarques sur les recherches en folklore au Canada français.

I. Folklore en général : A) Bibliographie. B) Histoire et étude du folklore (y compris musées, expositions, congrès) ; pays et nations ; chercheurs. C) Principes et méthodes ; rapports avec les autres sciences de l'homme. D) Synthèses et mélanges concernant des unités géographiques ou ethnographiques (y compris recueils de matériaux, questionnaires, atlas). Suit l'énumération des domaines linguistiques en Europe et hors d'Europe. 


\section{Habitat}

III. Bâtiments : A) Généralités. B) La ferme. C) La maison et ses dépendances. D) Églises, chapelles, cimetières.

IV. Objets : A) Études d'ensemble et divers. B) Biens immobiliers, monuments. C) Biens meubles, mobilier, outillage, objets variés.

\section{Marques et enseignes.}

VI. Technologie : métiers, arts et industries populaires : A) Études d'ensemble. B) Questions spéciales : arts et industries textiles ; imagerie populaire ; travail du bois ; poterie ; travail de la pierre et des métaux ; verrerie ; varia. C) Métiers et professions populaires, ouvriers saisonniers. D) Agriculture ; élevage ; sylviculture ; horticulture ; apiculture. E) Cueillette, chasse, pèche. F) Exploitation des mines. G) Commerce, voies de communication, transport, émigration. H) Marchés et foires. J) Calendrier, mesure du temps, mesures, monnaies.

VII. Caractères et types populaires.

VIII. Vêtement, parure.

IX. Alimentation.

X. Usages et coutumes, fêtes, jeux: A) Études d'ensemble et divers. B) Sujets particuliers : naissance, baptême, éducation ; initiation ; amour, fiançailles, mariage ; mort et enterrement ; vie domestique ; vie scolaire ; vie villageoise et urbaine ; usages agraires ; usages des classes et métiers ; sociétés, corporations, confréries, corps de métiers ; coutumes et fêtes ecclésiastiques ; coutumes saisonnières ; jeux et jouets ; varia.

XI. Coutumes sociales, droit populaire.

XII. Croyances populaires : A) Bibliographie. B) Religion, magie, superstition et mythologie en général. C) Études d'ensemble par groupes ethniques. D) Mythologie et culte : dévotion et hagiographie populaires ; pèlerinages ; objets en rapport avec la dévotion populaire : croix, crèches, ex-votos, etc. ; prières ; présages et oracles ; sorcellerie : sorciers ; amulettes, talismans et objets semblables ; formules d'incantation et de bénédiction ; livres de sorcellerie ; monographies.

XIII. Médecine populaire. 
XIV. Science populaire: A) En général. B) Flore. C) Faune. D) Météorologie et astronomie. E) Astrologie, divination, interprétation des songes. F) Alchimie.

XV. Folklore littéraire : généralités et travaux d'ensemble pour les chapitres XVI à XXI.

XVI. Poésie populaire: A) Questions générales et synthèses. B) Chansons populaires. C) Rimailles et formulettes. D) Enfantines et chansons d'enfants. E) Inscriptions.

XVII. Musique et danse : A) Instruments de musique. B) Yodler, etc. C) Danse.

[259]

XVIII. Conte, récit, mythe et légende : A) Bibliographie. B) Articles généraux.

C) Contes, thèmes et motifs. D) Anecdotes, récits facétieux, fables. E) Mythes et légendes non chrétiennes. F) Légendes chrétiennes.

XIX. Théâtre populaire.

XX. Autre littérature populaire.

XXI. Langage populaire : A) Généralités. B) Devinettes. C) Proverbes et dictons. D) Bons mots et moqueries, sobriquets et blasons populaires. E) Cris et formules. F) Mimologismes. G) Parler populaire, argots.

XXII. Onomastique : A) Généralités. B) Noms de lieux, de rues, de maisons. C) Prénoms, noms de famille.

Index divers.

Les détails de ce plan sont conçus dans une perspective plus européenne que nord-américaine, plus germanique que romane. Aussi les faits récoltés au Canada et en Amérique française ne s'ajustent-ils pas toujours très bien à ces divisions et subdivisions. Cependant les inconvénients, qu'ils proviennent d'une extension ou d'une limitation trop grandes, restent mineurs, parce qu'on peut toujours les corriger par les suppressions ou additions que les circonstances locales exigent. Au reste, il en est de même pour d'autres plans bibliographiques ou raisonnes de faits folkloriques, par exemple celui de la section 
« Ethnographie, coutumes, folklore » (chap. 39) de la Classification décimale universelle de l'Institut international de bibliographie, ${ }^{272}$ ou encore le Manuel bibliographique de folklore français contemporain d'Arnold Van Gennep, 273 et la Folklore Classification de Ralph Steele Beggs. 274

C'est en tenant compte de ces plans et de quelques autres qu'il serait trop long d'énumérer que nous avons nous-même réuni la matière de ce qui deviendra la Bibliographie raisonnée du folklore français en Amérique du Nord. Nous prévoyons qu'elle formera deux volumes. Le premier, virtuellement complété sur fiches, est consacré aux traditions orales proprement dites. C'est de beaucoup le plus considérable. Cela tient à plusieurs raisons. D'abord à l'abondance des matériaux recueillis, à l'excellence de leur conservation et dans certains cas, comme celui de la chanson populaire, à l'ancienneté de la recherche commencée il y a un siècle.

Au demeurant, voici un aperçu quantitatif des références aux livres, brochures et articles qui seront recensés dans ce premier tome.

I. Généralités, comprenant les dépôts d'archives, les principaux chercheurs, les bibliographies rétrospectives et courantes, l'historique des études de folklore, les essais théoriques ou de synthèse, les guides de recherches et questionnaires, les monographies générales, les compilations, revues [260] de folklore et ouvrages divers : environ 230 titres, classés dans un ordre à la fois géographique et chronologique.

II. Langue populaire. Cette section retiendra environ 250 titres des deux bibliographies publiées du parler français au Canada: celle de James Geddes et Adjutor Rivard, parue en 1906, et celle que la Société du Parler français au Canada a publiée dans la Revue de l'Université Laval, en 1961- 1962, par les soins de M. Gaston Dulong et de nousmême.

III. Onomastique. Une première partie a déjà paru sous le titre de « Bibliographie raisonnée de l'anthroponymie canadienne » dans les

272 Bruxelles, Institut international de bibliographie, tomes 1 à III, 1927-1929.

273 Paris, Picard, tomes II et III, 1937.

274 Parue dans le Southern Folklore Quarterly (Chapel Hill), XIII, 3, September 1949. 
Mémoires de la Société généalogique canadienne-française (IX, 3-4, juillet-octobre 1958, 153-174). 185 titres. Il y aura quelques additions. La seconde section consacrée à la toponymie sera de beaucoup plus considérable. Près de 400 titres.

IV. Proverbes et dictons. Une centaine de titres.

$\mathrm{V}$ et VI. Sciences populaires (superstitions) et légendes. C'est la partie la plus ardue à préparer, étant donné la dispersion des matériaux dans toutes sortes d'ouvrages. Nous essaierons autant que possible de suivre les principes du catalogue international dont on a jeté les bases au congrès sur les légendes tenu à Anvers, en septembre 1962. Un millier de titres environ.

VII. Contes populaires. Pour l'instant, nous renvoyons à la communication que nous avons présentée sur «Le conte populaire français en Amérique du Nord » au congrès de Kiel et Copenhague, en 1959, et publiée dans Internationaler Kongress der Volkserzählungsforscher in Kiel und Kopenhagen (Berlin, Walter de Gruyter \& Co., 1961, 142-151). Cette section du conte recense environ 325 titres. Mais pour les contes-types eux-mêmes il y a lieu de mentionner que la nouvelle édition du catalogue international de Antti Aarne et Stith Thompson (The Types of the Folktale, A Classification and Bibliography, second revision, Helsinki, Academia Scientiarum Fennica, 1961, 588 p.) contient les statistiques d'environ 4,000 versions compilées aux Archives de folklore en vue d'un catalogue complet du conte canadien. De plus, le «Catalogue raisonné » de Paul Delarue (Le conte populaire français, Paris, Éditions Érasme, vol. 1, 1957) contenait déjà une abondante documentation canadienne. Le second volume, qui paraîtra en 1963 par les soins de madame Marie-Louise Tenèze, continuatrice de l'œuvre de Paul Delarue, contiendra aussi pour chaque conte un aperçu de la documentation canadienne.

VIII. Chansons populaires. La chanson populaire représente non seulement le plus ancien domaine de la recherche folklorique au Canada, mais aussi le plus abondant. À l'heure actuelle, plus de 38,000 versions ont été analysées et classées dans nos Archives, les variantes étant regroupées sous un titre commun ou critique. La clef de cet 
immense [261] répertoire a été publiée par Conrad Laforte. ${ }^{275}$ Mais la bibliographie proprement dite est encore à paraître. Elle est si considérable avec ses 1,500 références qu'elle pourrait former à elle seule un ouvrage indépendant. En attendant, nous renvoyons à la bibliographie sommaire (une centaine de références) que M. Marius Barbeau a publiée à la suite de son article "Canadian Folk Songs", dans The Journal of International Folk Music Council (XIII, 1961, 23-31).

IX. Danse et musique instrumentale. Une centaine de références, comprenant la description de manuscrits (le plus ancien remontant à 1769) et une documentation éparse sur les danses populaires. Le tout complété par les airs notés. Cette documentation figurera dans le $R e$ cueil de danses folkloriques canadiennes en préparation par $\mathrm{M}^{\text {elle }} \mathrm{Si}$ monne Voyer (à paraître dans les Archives de folklore, probablement en 1963).

X. Devinettes. Une soixantaine de références tant à des collections manuscrites qu'à des imprimés.

XI. Formulettes et jeux. Plus de cent titres. Des notes bibliographiques sur ces sujets ont déjà paru dans notre étude sur les « Comptines canadiennes » Archives de folklore, 3, 1948, 109-157) et dans « Jeux, jouets et divertissements de la Beauce ", par Madeleine Doyon \{ibid., 159-207). Additions nombreuses à la suite de nouvelles enquêtes et publications depuis ces études.

Au total, la première partie de la bibliographie consacrée aux traditions orales comprend à l'heure actuelle un minimum de 4,300 titres. Mais ce serait une erreur de croire que tout est connu et qu'il n'y a plus rien à découvrir. D'ailleurs des enquêtes se continuent en bien des régions jusqu'ici inexplorées de notre immense pays. Des études aussi sont en préparation sous forme de thèses, de mémoires ou de monographies, interprétant les matériaux dans la perspective canadienne et parfois établissant la comparaison de façon plus vaste en l'étendant à tout le domaine roman et même occidental.

Les neuf chapitres que nous avons énumérés dans notre bibliographie des traditions orales correspondent en gros aux dernières parties

275 Le catalogue de la chanson folklorique française, préface de Luc Lacourcière, Québec, Les Presses Universitaires Laval, 1958, XXXIX+397 p. 
du plan de la Bibliographie internationale de John Meier et E. Hoffmann-Krayer : chapitres XII à XXII. Il restera donc pour notre second tome ce qui forme les onze premiers chapitres, c'est-à-dire tout ce qui a trait au folklore matériel (technologie, chapitres II à VI et VIII et IX) et à la vie de relation, soit les coutumes sociales, juridiques, les usages, etc. (chapitres VII, X et XI).

Ce n'est pas un simple caprice qui nous a fait inverser le plan de la Bibliographie internationale, mais une soumission aux faits euxmêmes, [262] récoltés et étudiés au Canada. Autant les traditions orales sont abondantes, comme on a pu le constater, autant les études de folklore matériel et social sont courtes et rares. La remarque nous en fut faite assez brutalement un jour de 1949 par Van Gennep lui-même que nous étions allé rencontrer dans son petit domaine de Bourg-laReine. Il était à rédiger le cinquième ou sixième volume de son $M a-$ nuel de folklore français contemporain et nous dit immédiatement sa déception de ne pouvoir étendre ses comparaisons provinciales au domaine français du Canada, faute de descriptions, de monographies et d'études appropriées.

Les conditions ont un peu changé depuis. Quelques études importantes ont été entreprises sur l'habitat rural, sur les objets et arts ancillaires, tels que la maison traditionnelle, l'outillage agricole, le costume, l'alimentation, et sur les coutumes et usages. Mais il est évident qu'en ces divers domaines un travail immense reste à faire, que de nombreuses enquêtes actuelles et rétrospectives sont à entreprendre, à poursuivre ou à étendre, et des classifications de faits bien contrôlés à établir. Ce n'est qu'à cette condition que l'on pourra parvenir à des analyses consistantes, à des interprétations valables et à une compréhension exacte des mentalités et de la psychologie traditionnelles vers laquelle doit tendre toute étude de folklore.

Luc Lacourcière

Archives de folklore, Université Laval. 


\title{
COMMENTAIRE
}

\author{
Historiographie et tradition orale
}

\author{
Benoît Lacroix, o.p. \\ Institut d'études médiévales, \\ Université de Montréal.
}

[pp. 263-266.]

Retour à la table des matières

En marge des travaux des Archives de folklore de l'Université Laval, permettez-moi de vous rappeler les points de vue de l'historiographie ancienne sur la tradition orale comme source de vérité. Ces points de vue d'historiens grecs et romains aussi bien que latins du Moyen Age sont les suivants.

L'histoire n'est utile, nous dit-on, ${ }^{276}$ que dans la mesure où elle est vraie. Elle est vraie dans la mesure où elle s'appuie sur ses sources. Trois catégories de sources font l'historiographie véridique : le témoin oculaire, celui qui a vu les faits ; la tradition orale, celui qui a entendu ; enfin, le texte écrit, le document à lire. La première des sources, la plus véridique, est d'avoir vu et même vécu les faits qu'on raconte.

276 Voir: J. T. Shotwell, The History of History, New-York, 1937 ; M. Schulz, Die Lehre von der historischen Methode bei den Geschichteschreibern des Mittelalters, Berlin, 1909. — Pour une vue panoramique de l'historiographie depuis ses origines jusqu'à maintenant: FITZsimons, PundT, NowELL, eds., The Development of Historiography, Harrisburg, 1954 (Stackpole Social Science Series). 
La seconde — il s'agit d'un ordre de mérite et de vérité — d'avoir entendu raconter. Enfin, le document écrit. ${ }^{277}$ Polybe, qui écrit en grec vers les 150/30 avant notre ère, résume bien la pensée de ses collègues sur le sujet :

Histoires XII, 27b : « ... des deux instruments... que nous possédons naturellement et qui nous servent à la connaissance et à l'information, l'ouïe et la vue, la vue est de beaucoup la plus véridique. » 278

Mais, XII, 25/ : «... la troisième forme de l'histoire, celle qu'il faut reléguer au troisième rang, est celle qui n'est fondée que sur l'étude des livres. » 279

Le meilleur guerrier est celui qui s'est battu le plus souvent, le meilleur avocat est celui qui a plaidé le plus de procès politiques. La plus petite indication fournie par un témoin oculaire conduit l'historien au cœur des événements. 280

Le même Polybe en veut surtout à ceux qui renoncent aux deux premières sources de vérité historique, témoin oculaire et tradition orale, pour s'en remettre aux documents seulement :

XII, 27, $4:$ «... c'est qu'on peut tirer des informations des livres sans péril et sans fatigue, pourvu qu'on ait pris la seule précaution de s'installer dans une ville possédant quantité d'ouvrages ou quelque part au voisinage d'une bibliothèque. Il ne reste plus qu'à faire des recherches, tout en restant couché et à collationner les erreurs des historiens antérieurs sans aucune espèce de fatigue. » 281

277 Témoignages grecs et romains groupés par B. LACROIX, L'histoire dans l'Antiquité, Paris et Montréal, 1951, 224-227. Heureux rappel — après notre communication - de M. Roch Valin, directeur du Département de linguistique de l'Université Laval : le titre même de l'ouvrage d'Hérodote nous laisse déjà penser que ce sont les histoires qui, en somme, font l'Histoire.

278 Polybe, Histoires, livre XII, texte établi et commenté par Paul Pedech, Paris ; Les Belles Lettres, 1961, 49.

279 Ibid., 41.

280 Ibid., 7-8, 50 ; cf. XII, 25e, 5-7 ; 25h, 5-6 ; 28a, 6-7.

281 Ibid., 50. 
[264]

En somme, la vérité de l'histoire est avant tout objective ; elle dépend de ses sources, c'est-à-dire, par ordre de dignité et d'efficacité, le témoin oculaire, la tradition orale et, enfin, le document écrit. Telle aussi fut la perspective du Moyen Âge latin 282 qui ajoute ici ses justifications théologiques, allant jusqu'à faire remarquer, par exemple, que Moïse et les évangélistes, piliers de la religion judéo-chrétienne, étaient avant tout témoins oculaires et représentants inspirés de la tradition orale de leur époque. (Devrais-je noter que les croisades ont favorisé la tradition orale au dépens même, un certain moment, du témoin oculaire ?)

Laissons passer les siècles. Consultons les grands travaux de méthodologie historique dont ont vécu nos ancêtres, soit le Lehrbuch der historischen Methode, de Ernst Bernheim, paru à Leipzig en 1889, ou la célèbre Introduction aux études historiques, de Langlois et Seignobos (Paris, 1898). Il faut ici convenir que le témoin oculaire est plutôt disparu de l'horizon. Quant à la tradition orale, elle n'est prise au sérieux 283 que pour encourager le retour aux documents. Autrefois, on écrivait ad succurendum memoriœe ; 284 maintenant la mémoire, le témoin oculaire, la tradition orale n'ont qu'une valeur secondaire. Tout

282 Voir l'ouvrage de M. Schulz ; texte capital sur la théologie de la tradition orale, Agnelus de Ravenne (IX ${ }^{\mathrm{e}}$ siècle). Liber Pontificalis, éd. Muratori, Rerum Italicarum Scriptores, I, 235.

283 Déjà, Jean BoDin 내1596 (La méthode de l'histoire, tr. P. Mesnard, Paris, Les Belles Lettres, 1941, 33-34) préfère l'histoire sur archives, contrairement à Lucien de Samosate $\frac{\mathrm{t}}{7175}$, dans Comment on écrit l'histoire, 39 (éd. Jacobitz, Teubner II, 37 ; Classiques Garnier, t. 2, p. 21) encore fortement attaché à l'information directe. Notons que Lucien et Jean Bodin sont les deux premiers auteurs de traités de méthodologie historique. Pour sa part, E. Bernheim écrit (p. 45) que la science historique commence bon gré mal gré avec le folklore, que ce dernier n'est cependant que le lot des peuples de basse culture (p. 100 : «Von diesen Disziplinen nimmt die Ethnographie in engeren Sinne der Geschichtswissenschaft geradezu einen Teil ihrer Arbeit $\mathrm{ab}$, insofern sie die Zustände und Leistungen bestimmter einzelner Volker niederster Kulturstufen zum Gegenstand hat... ») ; le folklore est un peu ce qui reste quand un historien a fini d'explorer le champ historique (cf. p. 571).

284 V.g. Thomas D'Aquin, Summa Theologiœ, I, Qu. 24, art. I, c. Éd. Léonine, t. I, 286. 
tourne autour de l'imprimé. Critique interne, critique externe, sciences " nouvelles » : archéologie, épigraphie, chronologie, géographie, siggilographie, numismatique, ethnologie et quoi encore ! sont mises au service de l'écrit. Le texte devient point de départ et point d'arrivée de toute vérité historique. Fustel de Coulanges écrit: l'histoire se fait avec les texte 285 Notre abbé Scott, jadis cité dans les Archives de folklore, ajoute : "L'on peut affirmer sans crainte que notre histoire est contenue tout entière dans les documents écrits ». ${ }^{286}$ Les soulignés sont de nous, évidemment.

Que s'est-il produit au juste entre la fin du Moyen Âge et notre historiographie moderne du $\mathrm{XIX}^{\mathrm{e}}$ siècle ? Comment rendre compte de cette dissociation des trois sources traditionnelles de l'histoire depuis le $\mathrm{XVI}^{\mathrm{e}}$ siècle ? Comment expliquer la dévalorisation de la tradition orale au dépens du texte écrit ? - Voici quelques explications. Elles pourront, j'ose l'espérer, vous aider à évaluer tout le mérite des folkloristes savants d'Amérique française.

1. En tout premier lieu, l'invention de l'imprimerie au milieu du XVe siècle. L'imprimerie accorde au mot écrit une importance presque [265] démesurée. Cette importance s'accroît souvent au dépens de la mémoire visuelle et de la mémoire orale. Celles-ci perdant leur utilité pratique, les historiens commencent à oublier et ils se fient de plus en plus aux livres. Devenus de moins en moins utiles, on a cru tout naturellement que le témoin oculaire et la tradition orale devenaient aussi de moins en moins véridiques. L'homme cultivé de ces derniers siècles est celui qui a lu beaucoup de livres et celui qui sait écrire beaucoup de mots, mais c'est aussi un homme qui a perdu la mémoire. La bibliographie assure la survie de sa mémoire. Imprimeries et bibliothèques valorisent l'homme du livre et dévalorisent, isolent plutôt l'homme sans livre. Celui-ci est l'illettré dont il ne faut pas trop s'occuper si on est sérieux et savant. Isolées de la culture officielle, culture écrite avant tout, la culture orale et la culture visuelle se trouvent bon gré mal gré associées aux sociétés dites archaïques, à la vie primitive, à tout ce qui regarde l'ignorance publique en général.

285 Avec la discussion de L. FeBVRE, Combats pour l'histoire, Paris, Colin, 1953, 4-5.

286 Archives de folklore, I, 1946, 19, note 5. 
Que nous sommes loin de la méthodologie grecque ! Et un exemple typique de cette dissociation, jusqu'à l'exclusion, des sources historiques au $\mathrm{XIX}^{\mathrm{e}}$ siècle, est le sort fait aux frères Grimm quand ils eurent l'idée d'expliquer les chansons de geste par la tradition orale. Ils exagéraient, bien entendu ; mais avaient-ils totalement tort ? Ce fut, comme on le sait, une réaction massive de la part des historiens des textes. Non ! il n'était pas possible qu'un texte aussi glorieux que la Chanson de Roland pût avoir été victime de la collectivité et de la tradition orale 287 ...

2. À la suggestion du professeur Fernand Grenier (Institut de géographie, Université Laval), j'ajouterai une seconde explication de la dépréciation des sources orales jusqu'en ces dernières années : l'abus même qu'en ont fait les chroniqueurs, les annalistes et les journalistes peut-être. Livrée à l'imagination du bon chroniqueur, l'histoire allait en certains cas regrettables devenir purement anecdotique. Déjà au $\mathrm{XII}^{\mathrm{e}}$ siècle, un Guillaume de Malbesbury réagissait contre l'abus de sources orales en historiographie.

3. Aussi, le succès non équivoque des sciences exactes a joué fortement sur la mentalité des historiens modernes. 288 Ceux-ci désirèrent une certitude égale à celle des sciences purement positives. Évidemment, on se devait de rejeter tout de suite contes, légendes, mythes, etc. Seuls les textes bien localisés, bien datés pouvaient conduire aux vraies certitudes, croyait-on.

287 Encore J. BÉDIER, Les légendes épiques, Paris, 1929, t. 3, 216-226, avec la mise au point de I. SiCILliANo, Les origines des chansons de geste, 1951.

288 Retour aux perspectives anciennes et grand souci technique en faveur de l'information visuelle et de la tradition orale dans L'Histoire et ses méthodes (Encyclopédie de la Pléiade) publié sous la direction de C. Samaran, Paris, 1961 ; aussi J. Garraghan, A Guide to Historical Method, Ed. Delanglez, New-York, 1946, ch. 4-5, 81-124. En rappelant à l'historien moderne ses limites, puisque le réel dépassera toujours le connu (cf. Marrou, De la connaissance historique, $4^{\mathrm{e}}$ édition, Paris, 1959, ch. 3, 68-95), en lui offrant de revenir à Xhomme comme sujet de connaissance (v.g. C. GALARNEAU, Revue d'Histoire d'Amérique française, IX, 1955, 3-14), à l'homme social, dira M. Trudel (symposium final du 7 avril), on s'oriente heureusement vers les perspectives humanistes des Grecs et on se dégagera peu à peu de la dictature du texte écrit. 
4. Enfin, le romantisme allemand, qui domina l'Europe à la même époque : comment un historien sérieux pourrait-il se fier au donné folklorique qui s'était trop souvent fait l'allié de la littérature d'imagination?

[266]

Il ne restait plus qu'un moyen de réhabiliter la tradition orale et le folklore en général comme source positive de vérité historique, c'était de les rendre à leur tour scientifiques et exacts grâce à des enquêtes objectives, grâce à des archives bien cataloguées, avec cinémathèques, photothèques, phonothèques et discothèques, etc. Tout ce travail de codification sera valable en histoire si les folkloristes savent se tenir en dehors de toutes les interprétations immédiates et s'ils veulent rester sévèrement fidèles à leur matière. Si la preuve est faite qu'une telle objectivité ne va pas toujours de soi, la preuve est faite aussi, aux Archives de folklore de l'Université Laval en particulier, que la tradition orale peut être science d'exactitude, et vraiment source d'histoire. En somme et en tant qu'historiens de la culture d'Occident, nous avons tout à espérer et rien à craindre des travaux de notre confrère Lacourcière et de sa vaillante équipe.

$$
\text { * * * }
$$

Enfin, il est significatif, à notre avis, qu'au nom de l'histoire de culture nous nous retrouvions tout à coup ici ensemble, historiens des traditions populaires, enquêteurs, et donc témoins oculaires de l'histoire qui se fait devant nous, sociologues et anthropologues. C'est tout naturellement que nous associons aujourd'hui ce que les trois derniers siècles d'historiographie avaient dissocié. Les Grecs n'avaient donc pas tellement tort de lier la connaissance historique globale à ces trois catégories de sources et Polybe avait peut-être raison de critiquer ceux qui, de son temps déjà, éprouvaient la tentation de réduire la connaissance historique à la seule connaissance des documents écrits.

Benoît LACROIX, o.p.

Institut d'études médiévales, Université de Montréal. 
Situation de la recherche sur le Canada français

III. Perspectives sur l'étude de la structure sociale

\author{
Marcel Rioux \\ Département de sociologie, \\ Université de Montréal

\section{"L'étude de la culture canadienne-française : aspects micro-sociologiques."}

[pp. 267-272.]

$\underline{\text { Retour à la table des matières }}$

Le titre de la communication que je dois présenter se lit ainsi : «L'étude de la culture canadienne-française: aspects microsociologiques ». Fernand Dumont et moi avons arrêté ce titre au cours d'une hâtive conversation téléphonique interurbaine ; ce titre me semble refléter une certaine ambiguité d'intention et de formulation : on semble se reporter, d'une part, à la culture globale et, d'autre part, aux aspects micro-sociologiques de la réalité sociale. Si j'essaie de déceler notre intention commune, j'y vois le désir que nous avions tous les deux de voir traiter l'étude de la culture au niveau des petites unités culturelles, c'est-à-dire des communautés qu'ont étudiées les anthropologues et les sociologues. Sans écarter tout à fait ce point de vue, je m'arrêterai surtout sur la triple complémentarité que recèle l'ambiguité de ce titre : 1 . sur les concepts de société et culture; 2 . sur les points 
de vue micro- et macro-socio-culturels ; et, enfin, 3. sur le fait que l'étude de la culture canadienne-française est surtout conduite par des spécialistes qui font eux-mêmes partie de cette culture ; ce qui pose le problème subjectivité-objectivité.

Ce n'est pas mon intention de traiter ces complémentarités séparément, mais plutôt d'essayer de faire voir comment elles sont au cœur même de la réalité et de la démarche du sociologue et de l'anthropologue. Pour ce qui est des deux concepts majeurs de nos disciplines anthropologiques, ceux de société et de culture, bien que je reconnaisse qu'il faille les distinguer au niveau analytique et que cette distinction puisse être extrêmement fructueuse, je n'essaierai pas de raffiner sur la distinction dont ont convenu Krœber et Parsons, au cours d'une fameuse rencontre au sommet, distinction qui pourrait se formuler ainsi : par culture, on entend désigner le contenu et l'agencement des valeurs, des idées et tout système symbolique significatif qui influent sur le comportement humain et les œuvres de civilisation ; par société, on désigne plus spécifiquement le système relationnel d'interaction des individus entre eux, des individus par rapport aux groupes ainsi que des groupes entre eux.

Mais plutôt que d'insister sur une théorie de la société et de la culture, il me semble plus fructueux pour l'étude d'une société en voie de transformation, [268] comme l'est le Canada français, d'insister sur l'aspect dynamique des relations entre ces deux séries de phénomènes et même sur le conflit que posent l'intégration sociale et l'intégration culturelle ; ce conflit tient, bien sûr, pour une large part à la conjoncture où nous nous trouvons, mais aussi à la nature même de ces deux intégrations. En plus de nous faire mieux comprendre la réalité proposée à notre observation, ce point de vue - conflit entre l'intégration sociale et l'intégration culturelle - recèle, selon moi, la possibilité de faire avancer la théorie du changement socio-culturel. De plus, en examinant les déterminants existentiels de la théorie structuralefonctionnelle, s'il est assez facile de repérer les raisons historiques générales - vestiges de la notion d'équilibre dans la théorie économique du libéralisme, par exemple,- - et, plus près de nous, la position et l'idéologie des sociologues américains au sein de la société capitaliste, il serait intéressant d'analyser ce qui au sein de notre société nous pousse à choisir le concept d'équilibre, plutôt que celui de conflit ; il serait bien surprenant que notre position de Canadiens français à l'in- 
térieur du Canada et que le système de sécurité que nous nous sommes bâti ne puissent se retrouver au fond de notre choix.

Dans la sorte d'inventaire que nous sommes en train de conduire ici, il ne me semble pas fructueux d'insister exclusivement sur ce que telle ou telle discipline a apporté et peut apporter à l'étude de la société canadienne-française, surtout quand il s'agit d'une réalité aussi vaste que la culture canadienne-française. On reconnaît dans les manuels que l'étude de la culture est l'objet propre de l'anthropologie ; si nous ne faisions que passer en revue les études anthropologiques sur le Canada français, notre bilan, faut-il l'avouer, serait fort mince. C'est pourquoi, au lieu d'insister sur les travaux proprement anthropologiques, j'essaierai de montrer pourquoi il y aurait lieu — à cause de la nature généralisante de l'anthropologie et de la totalité que représente la culture - de voir comment l'anthropologie et la culture débordent les frontières qu'elles s'assignent réciproquement. Ce qui me permettra d'esquisser une auto-critique de l'anthropologue et de sa culture.

Il semble que, du point de vue des relations entre l'histoire et les sciences anthropologiques, le balancier oscille de nouveau. L'exclusive qu'un grand nombre d'entre nous avaient prononcée contre l'aspect diachronique de nos études est, aujourd'hui, petit à petit rappelée. Pour toutes sortes de raisons qu'il serait trop long de détailler ici, les anthropologues ont eu tendance à minimiser les données historiques dont ils auraient pu disposer. Entre la société tribale, objet classique de l'anthropologie, et la société industrielle dont l'étude était réservée aux sociologues, il a longtemps existé un certain vide, du point de vue des praticiens de ces disciplines; ni les uns ni les autres ne s'étaient intéressés systématiquement aux sociétés agricoles pré-industrielles qui étaient demeurées l'apanage quasi-exclusif des historiens. Or, à cause des difficultés de communications d'une discipline à [269] l'autre, les travaux des historiens avaient tendance à rester lettre morte pour les spécialistes des sociétés tribales et des sociétés industrielles. Depuis quelque temps, des sociologues et des anthropologues réinterprètent de leurs propres points de vue les travaux des historiens sur ces sociétés-là. Je pense surtout à l'ouvrage de Sjoberg sur la ville pré-industrielle et la société agricole. Or, du point de vue de l'étude de la société et de la culture canadiennes-françaises, il apparaît que le modèle de la société pré-industrielle est plus fructueux que celui de la société industrielle développée et même celui de la folk-société — mé- 
lange de société tribale et de société paysanne. Le modèle de Sjoberg est particulièrement bien adapté pour rendre compte du passage de la société agricole à la société industrielle. D'autre part, sans modèle sociologique ou sans modèle, on risque de considérer les faits canadiens-français comme des faits uniques alors que leur spécificité est restreinte au conditionnement historique qui leur est propre.

En ce qui regarde l'histoire, notre qualité d'anthropologues et de sociologues canadiens-français nous impose d'autres limitations. Encore assez récemment, l'histoire qui s'écrivait chez nous avait moins pour fonction de nous faire connaître intégralement le passé que de nous donner des raisons de survivre comme peuple. Si, donc, à certains moments nous avons eu tendance à rejeter cette histoire, nous sommes peut-être allés trop loin et nous avons, comme disent les Anglais, jeté l'enfant avec l'eau sale de la cuvette.

Il en va de même pour les notions de société et culture globales. Parce qu'à un certain moment, une certaine idéologie voulait nous enfermer dans une culture et une société étriquée qu'elle avait pris soin de définir elle-même, plusieurs ont eu tendance à nier cette société et cette culture et à rechercher d'autres unités globales pour y intégrer notre réalité socio-culturelle. Il faut ici nous rappeler que les deux décennies d'existence de la recherche systématique en sociologie et en anthropologie coïncident avec la période où la plupart d'entre nous étaient en révolte contre cette idéologie-là.

D'une façon plus générale, on peut dire que l'oubli presque total dans lequel les notions de société et de culture globales sont tombées chez nos collègues canadiens-anglais et américains ne nous a pas aidés nous-mêmes à rattacher nos études micro-sociologiques et microculturelles à une totalité. Cela est particulièrement vrai de nos études de communautés qui sont, soit envisagées comme des unités fermées, ou encore comme des points de comparaison avec d'autres unités sans que, dans l'un et l'autre cas, elles soient insérées dans les sociétés globales dont elles font partie.

Un autre postulat que nous tenons de notre milieu, c'est celui de l'homogénéité du donné socio-culturel que nous étudions. Non seulement sommes-nous portés à penser que cette homogénéité existe aujourd'hui, mais qu'elle a toujours prévalu. Ce n'est que très récemment qu'historiens et sociologues ont mis en doute ce qu'on prenait aupara- 
vant largement pour acquis et ont commencé de démontrer qu'au contraire, cette homogénéité [270] ne datait que de la deuxième moitié du $\mathrm{XIX}^{\mathrm{e}}$ siècle. Allons plus loin et disons que cette homogénéité de la fin du $\mathrm{XIX}^{\mathrm{e}}$ siècle et d'une bonne partie du début du $\mathrm{XX}^{\mathrm{e}}$ siècle était surtout idéologique et correspondait beaucoup moins à une homogénéité de comportement ou de vision du monde ; ou pour mieux dire, disons que l'homogénéité culturelle postulée se rapportait surtout à l'aspect directif de la vision du monde des Canadiens, mais que ses aspects cognitifs et affectifs se différenciaient de plus en plus. En d'autres termes, nous étions tous sujets aux mêmes impératifs idéologiques et religieux, mais la vie quotidienne nous forçait à connaître une autre réalité et à lui affecter un coefficient d'affectivité différent de celui que lui donnaient les définisseurs de situation.

Ce postulat d'homogénéité n'est pas seulement culturel, mais aussi structurel. Et à ce sujet, la situation me semble encore plus complexe parce que, pour nous masquer la réalité, plusieurs facteurs sont en cause ; en plus de l'homogénéité directive de notre vision du monde, de l'homogénéité ethnique qui a longtemps été la nôtre, la réalité de la différenciation en classes sociales nous a longtemps été masquée. Il faut aussi dire qu'à cause du caractère monolithique de notre idéologie, les mots de classes sociales et de lutte des classes nous sont apparus comme renvoyant à des réalités quasi-démoniaques; à force de réfuter le marxisme dans nos manuels et de combattre verbalement le communisme, nous en sommes venus à nier la réalité à partir de laquelle Marx a construit sa théorie. Récemment, mon collègue Jacques Dofny et moi-même avons soutenu, lors d'un colloque à l'Université Carleton sur les classes sociales au Canada, 289 que la spécificité du problème des classes sociales au Canada français tient à ceci : le Canada français se considère, est considéré et est en réalité une classe sociale à base ethnique à l'intérieur du Canada ; cette classe sociale ethnique est nettement infériorisée par rapport au groupe anglais, et même du point de vue des immigrants. Selon Porter, de 1931 à 1951, la situation s'est encore aggravée. La prise de conscience sporadique du Canada français en tant que classe sociale ethnique et l'homogénéité culturelle relative qu'on lui prête, qu'elle se donne, et qu'elle a en

289 Jacques DofNy et Marcel Rioux, «Les classes sociales au Canada français ", Revue française de sociologie, III, 1962, 290-300. [Texte disponible dans Les Classiques des sciences sociales. JMT.] 
réalité, masquent l'hétérogénéité du Canada français qui, lui-même, se structure en classes sociales. En d'autres termes, parce que le Canada français se heurte souvent comme un tout aux autres classes ethniques, on oublie quelquefois qu'il se stratifié selon ses propres critères. Il est bien sûr que parce que les Canadiens français participent au système canadien et à cause de la présence d'Anglo-Canadiens dans le Québec, il peut exister une double échelle de stratification. Si les phénomènes de stratification présentent un terrain favori d'étude pour les sociologues, les classes sociales envisagées comme des sous-cultures intéressent vivement les anthropologues ; ce pourrait être là une aire de collaboration [271] entre ces spécialistes. Encore ici, notre appartenance à la société nord-américaine et l'influence qu'ont exercée sur nous ses sociologues ont eu tendance à masquer la réalité des classes sociales et à faire des praticiens des sciences de l'homme des fonctionnaires au service du gouvernement et des grandes entreprises. Les sociologues américains se proposent comme des spécialistes qui peuvent aider le système social à bien fonctionner. Ne leur ressemblonsnous pas quelque peu ? Dans un article récent des Archives européennes de sociologie, Raymond Aron a mentionné « la bienveillance intéressée » dont bénéficient les sociologues de l'U.R.S.S. et des U.S.A., les deux fonctionnant pour le plus grand bien de chaque système.

Avant de terminer ces remarques sur les conditionnements et les limitations que nous impose l'appartenance à la culture même que nous étudions, je voudrais mentionner une autre forme subtile d'aliénation qui n'est pas propre aux praticiens des sciences de l'homme, mais qui peut, toutefois, influencer certaines de leurs options. Il est bien sûr que si le Canada français est objectivement une classe sociale ethnique infériorisée à l'intérieur du Canada, la tentation sera grande pour plusieurs de valoriser la culture canadienne-française pour faire oublier la position structurelle inférieure du Canada français. Cette option, consciente ou inconsciente, fait bien l'affaire des deux principaux partis en cause ; la classe infériorisée économiquement et socialement se revalorise à ses propres yeux. Tandis que la classe ethnique supérieure peut se dire, dans le même temps, que l'ordre et la nature des choses restent ce qu'ils sont, ce qui m'amène à me demander jusqu'à quel point les objectifs de la recherche doivent-ils être influencés par la conjoncture ? Question qui n'est pas aussi électorale qu'elle peut paraître. C'est Gurvitch qui montre comment, au cours de l'histoire, la 
situation sociale que vivent les sociologues influe sur les problèmes qu'ils se posent. S'il existe une sociologie de la dépendance comme celle que notre collègue Georges Balandier a étudiée, il doit bien exister aussi une sociologie de l'indépendance ; quelle que soit celle que chacun choisira selon son tempérament et ses aptitudes, les études qui en découleront pourront non seulement nous renseigner sur notre société, mais influer peut-être sur la conjoncture même.

Au chapitre du conditionnement culturel de ceux qui étudient la culture canadienne-française, il faudrait mentionner les survivances scolastiques et les postulats de visions du monde qui continuent d'influencer même ceux qui étudient les variations de cette vision du monde. Si les premières sont assez facilement détectables à l'œil nu, les autres ne seront décelées qu'après des études systématiques.

Ces remarques répondent plutôt en zigzags à la question posée : ce qui a été fait, ce qui reste à faire. On admettra que les départements d'anthropologie et les spécialisations en anthropologie n'existant à Laval et à Montréal que depuis cette année ou l'an dernier, personne ne doit s'attendre à un bilan très riche. Ayant moi-même appartenu à une institution qui subventionne [272] des études dans les domaines de l'anthropologie, je puis dire qu'un grand nombre de documents, tant oraux que matériels, ont été recueillis sur la culture traditionnelle (folklore et culture matérielle) des Canadiens français. Un certain nombre de communautés rurales ont aussi été étudiées. Il faudrait aussi mentionner les quelques monographies ethnographiques qui ont été conduites par des universitaires d'ici ou d'ailleurs. Mais l'étude de la culture d'un groupe comme celui que forment les Canadiens français n'est pas exclusivement l'affaire des ethnographes et des anthropologues professionnels. À eux, peut-être, revient la tâche de systématiser les recherches et les documents accumulés par d'autres disciplines: histoire, linguistique, littérature, géographie humaine, sociologie, psychologie, droit, et j'en passe. Ce devrait être une des premières tâches des départements d'anthropologie ou, à tout le moins, de ceux des spécialistes qui s'intéressent à l'étude du Canada français de créer une schème de catégorisation et d'interprétation des œuvres culturelles des Canadiens français ; ce schème devrait être élaboré en fonction de la culture globale et, pour éviter l'aliénation culturaliste, ces efforts de systématisation devraient se conjuguer avec ceux des sociologues qui étudient la société globale ; aussi importante et peut-être, en l'occur- 
rence, plus importante devrait être l'étude des variations culturelles en fonction non seulement des époques, mais des classes sociales et des groupes sociaux. Peut-être que pour un certain temps et pour certains anthropologues, la tâche principale devrait être la création de techniques d'analyse et l'analyse des données qui, bien que non recueillies par des spécialistes en anthropologie, sont essentielles à l'étude de la culture. Il y a deux ou trois ans, un collègue de Laval et moi-même avions essayé d'établir une espèce de programme pour l'étude des variations des visions du monde chez les Canadiens français : or, dans ce programme, les travaux sur le terrain, exécutés par des spécialistes, ne constituaient qu'une partie des études projetées ; il aurait justement fallu, selon nous, analyser au moyen de techniques appropriées ce que les travaux d'autres disciplines recèlent de matériaux qu'il serait hautement important d'intégrer aux études anthropologiques proprement dites.

Quant aux études sur le terrain, il serait souhaitable que des équipes interdisciplinaires se forment pour étudier des communautés choisies en fonction des problèmes de la société globale et de la problématique contemporaine de nos disciplines. Ce que je recommande, en somme, c'est que nos disciplines ne se désintéressent pas des projets d'exister de notre communauté nationale, et qu'elles soient présentes dans les débats théoriques de l'heure. Je ne crois pas que ces deux souhaits soient antinomiques : ils se complètent plutôt.

\section{Marcel Rioux}

Département de sociologie, Université de Montréal. 


\title{
COMMENTAIRE
}

\author{
Claude Galarneau
}

Institut d'histoire, Université Laval.

[pp. 273-275.]

$\underline{\text { Retour à la table des matières }}$

Vous aurez entendu plusieurs exposés sur la culture dans ces " perspectives sur l'étude de la structure sociale », dont ceux de MM. Lacourcière, Falardeau, Lefebvre, Rocher, Mailhiot et Rioux. Comment un historien peut-il aborder ce problème de la culture au Canada français après des ethnographes, des linguistes, des sociologues et des anthropologues ? Il y a sans doute bien des façons de le faire ; aussi je me contenterai de soumettre à votre réflexion quelques aspects d'une nouvelle orientation de l'historiographie contemporaine. Cette nouvelle section, qui tend à regrouper des voies éparses, situées aux confins de la psychologie, de la sociologie, de l'anthropologie et de l'histoire connaît actuellement trois appellations : histoire de la psychologie collective, histoire des mentalités, histoire du mental collectif. ${ }^{290}$ Cette

290 Alphonse DupronT, « Problèmes et méthodes d'une histoire de la psychologie collective ", Comité International des sciences historiques, Stockholm, 21-28 août 1960. Résumé des communications. Göteborg-StockholmUpsala, Almquist et Wiksell, 1960, 26-28. M. Dupront a repris sa communication, sous le même titre, dans Annales. Économies. Sociétés. Civilisations, janvier-février 1961, 3-11, mais en proposant « histoire du mental collectif » au lieu « d'histoire de la psychologie collective ». M. L. Trénard avait employé « mental collectif » lors de la discussion, comme les Actes du Congrès le rapportent, à la page 50. Enfin, M. G. DuBY, dans L'histoire et ses métho- 
histoire se donne pour objet d'étude la psyché collective, l'âme collective, le mental collectif ; elle cherche à comprendre les attitudes mentales des groupes, à relier les représentations collectives et les conduites personnelles dans l'évolution totale d'un peuple ; histoire qui étudie encore la dialectique du groupe et de l'individu, histoire attentive aux « modèles » culturels comme aux réactions personnelles, histoire sociale et biographique, qui se développe selon les différentes cadences de la durée. ${ }^{291}$ Histoire qui se fonde évidemment sur l'existence de la psychologie collective elle-même, comme l'ont démontré Durkheim, Blondel et Halbwachs.

Quand je dis nouvelle section de l'histoire, cela ne signifie pas que cette discipline n'ait jamais été pratiquée. Histoire nouvelle en ce sens qu'elle précise ses méthodes, regroupe ses efforts et compte maintenant ses maîtres, ses chefs d'équipe en Europe occidentale, un peu selon les vœux d'Henri Berr et de Lucien Febvre. C'est M. Alphonse Dupront, professeur à la Sorbonne, qui en est l'un des animateurs les plus fervents.

Comme M. Dupront l'a formulé au XI ${ }^{\mathrm{e}}$ Congrès des sciences historiques en août 1960, le travail en histoire du mental collectif consiste à chercher dans trois grandes directions :

«Établir l'inventaire des formes, créations, images, valeurs, des expressions tant saines que morbides, par quoi se manifeste le mental collectif ; analyser les raisons de vivre, les forces de création, le « comment » de l'action ou de la passion des hommes d'une époque ou d'un pays, à un moment donné ; rendre manifeste l'existence de phénomènes périodiques, de rythmes, de retours, par rapport à certains besoins, idées, images mentales, mythes, archétypes, ou complexes de valeurs. » 292

des publiée sous la direction de Charles Samaran, Paris, Gallimard (Encyclopédie de la Pléiade, 1961, 965), propose, après Lucien Febvre, de garder « histoire des mentalités ». Voir : Lucien FEBVRE, Combats pour l'histoire, Paris, Colin, 1953.

291 Fernand BRAUDEI., « Histoire et sciences sociales ; la longue durée », Annales E.S.C., 1958, 725-753.

292 Alphonse Dupront, op. cit., 26-27. 
Cette histoire «n'existe que dans la mesure où elle se donne à ellemême sa matière » et " tout le donné historique est évidemment matière de l'histoire du mental collectif, qui doit justement rechercher dans le donné historique ce qui est collectif dans les besoins, les attitudes, les comportements ou les idéals. » 293

La méthode fondamentale de cette histoire, c'est celle de la description. La volonté de description doit être constante et ne doit pas se laisser attirer par la tentation d'établir des lois ou d'aboutir à une « systématique de la sociologie historique ».

Une fois les grandes voies de la recherche indiquées et la méthode signalée d'un mot, essayons d'indiquer quelques domaines plus précis où doit porter l'effort de l'historien. En ce qui concerne les idéesforces, étudier la naissance, la diffusion, le rôle des opinions, des « images » (par exemple la représentation qu'un peuple se fait d'un autre). En ce qui concerne les croyances, étudier les formes de la pratique religieuse aussi bien que les formes aberrantes de piété, les superstitions, la sorcellerie, les traditions ésotériques. Pour connaître l'outillage mental, étudier en tout premier lieu le langage, les moyens d'expression que l'individu reçoit du groupe et qui servent de cadre à toute sa vie mentale, dans le vocabulaire et la syntaxe ; étudier ensuite les autres procédés d'expression, ceux qui traduisent les quantités, les nombres, les mesures, les représentations du temps et de l'espace, supports sensibles de la pensée.

Histoire de l'éducation, au sens le plus large, qui comprend toutes les communications entre l'individu et son milieu, des moyens par lesquels il reçoit les modèles culturels, depuis l'enfance par la famille et les groupes d'écoliers, jusqu'à l'âge adulte par les propagandes ; histoire des institutions scolaires, de leurs structures, de leur contenu, des notions qu'elles veulent transmettre, de leur équipement, de leur implantation dans la société ; histoire de tous les moyens d'information, des véhicules de la culture tels que les livres, les bibliothèques, les journaux et périodiques.

Histoire des représentations que la collectivité canadiennefrançaise s'est faites du monde, de la vie, de la mort, de la religion, de

293 Ibid., 26. 
la politique ; histoire des thèmes de la création artistique dans les arts plastiques, la littérature écrite et orale.

Il faut s'arrêter là dans l'énumération des voies ouvertes à la recherche et se demander si cette histoire est possible, autrement dit, si elle compte déjà des ouvrages de valeur. Je n'en citerai que quelquesuns : le Rabelais 294 et le Martin Luther 295 de Febvre, la Grande Peur de George Lefebvre, ${ }^{296}$ Le temps de L'histoire de Philippe Ariès, ${ }^{297}$ le Déclin du moyen âge de Huizinga, 298 les Rois thaumaturges et la Société féodale de Marc Bloch 299 et la Chrétienté et l'idée de croisade d'Alphandery et Dupront, 300 le livre de Ferguson sur la Renaissan$c e^{301}$ et celui, plus récent, de Robert Mandrou sur l'Introduction [275] à la France moderne. 302 Tous ouvrages qui suffisent à prouver la validité de l'histoire du mental collectif.

De la recherche à Québec en ce domaine, quelques directions sont d'ores et déjà entreprises. En ce qui concerne l'histoire de l'enseignement, au point de vue de la transmission des connaissances, on peut lire dans le dernier numéro de la Revue d'Histoire de l'Amérique française la première tranche d'une étude sur les « Débuts de l'enseignement de l'histoire et de la géographie au Petit Séminaire de Québec (1765-1870) ». ${ }^{303}$ Un candidat au diplôme d'études supérieures est en train de rédiger sa thèse sur un sujet analogue dans un autre vieux collège du Québec.

Au plan de l'histoire des idées, un jeune collègue a commencé des recherches sur le mouvement de pensée ultramontaine au Canada français dans la dernière partie du $\mathrm{XIX}^{\mathrm{e}}$ siècle, courant de pensée rattaché à ses homologues américain, français et romain.

\footnotetext{
294 Paris, 1942.

295 Paris, 1928.

296 Paris, 1932.

297 Monaco, 1954.

298 Trad. fr., Paris, 1948.

299 Paris, $2^{\mathrm{e}}$ éd. 1962 et Paris, 1939-40.

300 Paris, 1954, 2 vol.

301 Trad. fr., Paris, 1950.

302 Paris, 1961.

303 Pierre SAVARD, « Débuts de l'enseignement de l'histoire et de la géographie au Petit Séminaire de Québec (1765-1870) », Revue d'Histoire de l'Amérique française, $X V$, 4, mars 1962, 509-525.
} 
Dans un autre secteur, je travaille pour ma part, depuis bientôt dix ans, sur les réactions de l'opinion canadienne-française devant la France et les Français depuis la Conquête jusqu'à la Capricieuse (1760-1855). ${ }^{304}$ Une telle recherche m'amène à étudier d'abord les relations entre les Français et les Canadiens sous tous leurs aspects, à analyser ensuite les représentations que se font les individus et les groupes canadiens-français de la France, des événements qui se passent en France. Je crois avoir réussi à cerner, entre autres résultats, à certains moments, le sentiment des classes populaires, alors que généralement, dans ce genre d'études, on ne réussit qu'à saisir l'opinion des élites, la seule qui se soit généralement exprimée et qui ait laissé des traces écrites. Je dois ces résultats aux ethnographes qui, dans leur cueillette des traditions orales, nous offrent une documentation d'une richesse unique en son genre.

Je terminerai ce commentaire en faisant remarquer qu'il ne faut pas trop se hâter en cette sorte d'histoire, qu'il convient d'aller lentement, avec de bons outils et des méthodes sûres, qu'on acquiert après une bonne quinzaine d'années d'études et de travaux personnels. Et, comme le dit monsieur Rioux, les historiens doivent se tenir en liaison étroite avec les autres spécialistes, ce qui est le cas déjà pour plusieurs d'entre nous et ce que peut favoriser davantage ce colloque. Nous avons maintenant, au Canada français, non seulement des individus, mais, pour la première fois, une équipe de chercheurs et de savants qui doivent travailler ensemble, autant que possible. Voilà comment un historien peut envisager l'étude de la culture en demeurant dans la dimension propre à sa discipline, qui est celle du passé.

\section{Claude Galarneau \\ Institut d'histoire, Université Laval.}

304 On pourra avoir une idée sommaire d'une partie de ce travail dans : «Les échanges culturels franco-canadiens depuis 1763 », dans : Le Canada français, aujourd'hui et demain, Paris, Fayard, 1961, 67-78 (Recherches et débats, cahier $n^{\circ} 34$ ). 
Situation de la recherche sur le Canada français

III. Perspectives sur l'étude de la structure sociale

Fernand Dumont

Département de sociologie et d'anthropologie, Université Laval

\section{"L'étude systématique de la société globale canadienne-française.”}

[pp. 277-292.]

\section{$\underline{\text { Retour à la table des matières }}$}

Je tiens à dissiper d'abord une équivoque. Le titre de mon exposé n'implique aucunement que je veuille m'attaquer à une sorte de synthèse des thèmes évoqués au cours de ce colloque. La plupart des auteurs de communications et de commentaires ont bien laissé entendre que l'ère des synthèses-résumés était close pour les chercheurs de cette génération. Parmi toutes les incertitudes dont nous avons fait le bilan au cours de ces journées, voilà un point qui, pour nous tous, est assuré. En parlant de l'étude de la société globale, je ne proposerai donc aucune voie - royale ou tortueuse - pour permettre au sociologue de survoler les lacunes énormes de nos recherches empiriques afin d'en arriver au plus vite à de nouvelles synthèses apaisantes. D'ailleurs, à mon sens (et cela apparaîtra, dans la suite, je l'espère), la notion de société globale n'indique qu'une voie de recherches empiriques parmi bien d'autres. 
Cependant, même si ce colloque se déroule sous les auspices de la sociographie, on n'aura aucune difficulté à convenir que le thème que j'ai à traiter appelle des précisions théoriques. La notion de "société globale » apparaît de plus en plus comme nécessaire. Nous savons bien que, le plus souvent, la dialectique de la recherche ne va pas de la monographie à la théorie ou inversement. Elle épouse plutôt le schéma : monographie - aire (ou société globale) — théorie. Pourtant, le concept de "société globale » est un des plus confus de la science contemporaine.

Ce n'est évidemment pas le lieu de proposer une discussion purement théorique à ce sujet. Nous ne saurions tout de même éviter de poser tout de suite une question qui s'impose d'emblée : à quels critères nous référer pour délimiter une société globale ? Ce sont de longs développements théoriques qu'il faudrait consacrer à ce problème. Mais nous en sommes dispensés par les impératifs que nous imposent les cadres de ce colloque : notre objet nous était donné au départ. Et, en fait, cela ne gêne pas trop nos idées sur la question. Chaque société globale présente une structure singulière dont le mode d'approche, dans la situation actuelle de la recherche, est à définir à chaque coup. On ne saurait généraliser à propos des sociétés globales comme on le fait, par exemple, en psychologie sociale [278] pour les petits groupes. Ceux-ci s'offrent à la perception comme des ensembles concrets : on est vite renvoyé alors à l'étude des traits généraux de structure. Il n'en est pas ainsi pour les sociétés globales : à première vue, diverses sociétés globales peuvent être délimitées à propos de la même réalité empirique. Nous devons alors fatalement recourir, du moins dans les premières démarches, aux représentations idéologiques où se marque, chez les agents sociaux eux-mêmes, l'appartenance à telle ou telle société globale. On pourra chercher ensuite les mécanismes spécifiques qui soutiennent ces représentations. De sorte que si la notion de société globale apparaît d'abord (nous le notions à l'instant) comme un palier nécessaire de l'observation sociologique, il se pourrait qu'elle corresponde aussi à des éléments concrets et spécifiques des sociétés.

Fidèle à ces remarques comme à l'esprit général du présent colloque, je ne commencerai donc pas par proposer une définition du concept, pour en chercher ensuite l'application à notre milieu. Dans la première partie de mon travail, qui sera consacrée à un bref inventaire, je voudrais plutôt partir avant tout des mécanismes par lesquels la so- 
ciété canadienne-française a tâché de se donner une représentation d'ensemble de ce qu'elle est ; l'étude systématique de la société globale nous apparaîtra ainsi en profonde continuité avec son objet. Dans une seconde section de cet essai, je voudrais ensuite proposer de brèves remarques théoriques pour dégager, enfin, quelques propositions de recherche.

\section{I}

J'avoue ne pas avoir relevé tous les essais — brefs ou élaborés où des auteurs ont tenté, depuis deux siècles, de définir le Canadien français. Je me bornerai à mentionner quelques échantillons typiques ordonnés autour d'une classification qui, je l'espère, n'est pas trop simplificatrice. Les tentatives diverses peuvent être rangées, me semble-t-il, en trois courants de pensée : $1^{\circ}$ les idéologies globales et leurs contestations ; $2^{\circ}$ l'historiographie; $3^{\circ}$ l'explication de notre société par une référence à ta société traditionnelle et à l'urbanisation. Dans notre milieu, ces trois voies de réflexion sont non seulement fort cohérentes pour leur part respective, mais elles se recoupent de façon à former un ensemble lui-même assez organique. J'essaierai, en tout cas, de le montrer.

1. Lorsque le sociologue ou l'anthropologue veut formuler une interprétation d'ensemble d'une société, il se heurte aux grandes idéologies du milieu en question. Avant que nous élaborions la science d'une société, celle-ci s'est déjà donné une vision cohérente de ce qu'elle est. Les idéologies qui nous occupent sont analogues aux rationalisations que doit affronter le psychanalyste. Cette situation comporte une conséquence, entre autres : toute interprétation scientifique d'une société globale sera fatalement en [279] continuité avec les idéologies dominantes du milieu. Parfois, elle en constituera simplement l'explication et la systématisation ; souvent, elle en sera une mise en question chargée plus ou moins de revendications et de jugements de valeur. Oublier cette condition très concrète, bien que gênante, de l'émergence de toute sociologie globale, ce serait nous accorder une objectivité de l'ordre du deus ex machina. On peut donc ainsi parler 
d'une sorte de polémique sous-jacente à toute interprétation sociologique d'ensemble d'une société globale. 305

Cette interpénétration des idéologies dominantes et des sociologies globales est tout particulièrement remarquable au Canada français. Dans une étude rédigée en collaboration avec Guy Rocher, 306 nous avons essayé de montrer que, chez nous, les idéologies s'étaient intégrées, surtout au cours du dernier demi-siècle, en un complexe unitaire. Nous avons aussi tâché de détecter les grandes antinomies qu'elles tendaient à concilier (le national et le social, la pluralité des allégeances politiques, le religieux et le profane) et les syncrétismes qui les caractérisaient. Devant ce monolithisme, la sociologie canadiennefrançaise ne pouvait éviter la confrontation - qui fut d'ailleurs, conformément à nos remarques de tantôt, le principal moteur de son développement. 307

305 On a toujours eu le sentiment plus ou moins confus de cette situation. Pour contourner la difficulté, on a parfois prétendu que toute société ne saurait être convenablement interprétée dans son ensemble que par un étranger. Solution bien précaire, à mon avis : une certaine identification avec l'objet est non seulement inévitable mais nécessaire dans nos sciences; tant que nous ne disposerons pas de techniques pour mesurer ou pour doser cette identification, le recours au «dépaysement » ne saurait être déterminant pour le problème qui nous occupe. D'autant plus que pour se sensibiliser à son objet, le sociologue étranger devrait bien recourir aux grandes représentations locales ou, tout au moins, les confronter avec sa propre explication.

306 Fernand DumonT et Guy RocHER, « Introduction à une sociologie du Canada français ", dans : Le Canada français aujourd'hui et demain (Recherches et débats, cahier n³4), Paris, Fayard, 1961, 13-39.

307 Si, pour donner en quelque sorte un pôle extrême à notre inventaire, nous voulions évoquer une « sociologie » canadienne-française qui ne soit pas autre chose qu'une systématisation de l'idéologie unitaire dont je parlais, c'est probablement l'œuvre de M. Esdras Minville qu'il faudrait retenir comme la plus typique. Retenons cette déclaration de l'auteur : « En fait, ce qui existe, c'est un problème canadien-français présentant un aspect économique comme il présente un aspect social, un aspect politique - aspects que l'on ne saurait s expliquer complètement si on ne les rattache au fait central » (Esdras Minville, Invitation à l'étude, Montréal, Fides, 1944, 126-127). Maurice TREMBLAY a consacré à M Minville une partie notable de son essai : «Réflexions sur le nationalisme », dans : Écrits du Canada français, V, Montréal, 1959. Comme autre exemple, nous aurions pu choisir aussi Notre question nationale du Père Richard ARES, S.J., surtout le tome premier (Montréal, Éditions de l'Action nationale, 1944). Mais nous ne pouvons 
Dans un échantillonnage forcément sommaire, il faut ici accorder la première place à l'étude que Maurice Tremblay a publiée dans les Essais sur le Québec contemporain sous le titre : "Orientations de la pensée sociale ». 308 [280]L'auteur y analyse d'abord les facteurs économiques et idéologiques de ce que l'on a considéré comme la vocation paysanne de la nationalité canadienne-française. Il évoque ensuite brièvement les principaux blocages à notre " pleine réintégration dans la vie économique du continent » : notre système particulier de classes sociales, nos collèges classiques, notre caractère français, notre philosophie catholique de la vie, nos frustrations collectives. Il termine en soulignant brièvement les contradictions du nationalisme économique. Je reconnais une sorte de priorité à ce bref essai parce qu'il est d'une facture remarquable, mais surtout parce qu'il a joué un rôle d'initiateur: on y retrouve, dans une formulation particulièrement nette, le schéma d'explication utilisé (souvent avec moins de bonheur) par les auteurs ultérieurs. 309

On ne saurait manquer de mentionner, à la suite de l'étude de Maurice Tremblay, l'essai passionné que Pierre-Elliott Trudeau a juxtaposé

nous attarder : ce genre d'entreprise mérite à peine le nom de « sociologie » puisque, pour reprendre notre analogie avec la psychanalyse, elle représente une sorte de sur-rationalisation. Nous nous attacherons plutôt aux tentatives pour constituer une sociologie par analyse, critique ou remplacement des idéologies dominantes.

308 Dans : Jean-C. FALARDEAU, éd., Essais sur le Québec contemporain, Québec, Les Presses Universitaires Laval, 1953, 193-208.

309 Je citerai ces quelques lignes qui disent l'essentiel : « Tout changement radical dans les conditions d'existence du groupe qui entraînera un examen critique de la tradition mettra en cause sa structure sociale aussi bien que les valeurs et les normes de son ethos. Pour autant, la résistance opposée au changement par un système culturel de pensée et par l'organisation sociale qui le supporte sera d'autant plus grande que le nouvel ordre de choses, au lieu d'être le résultat d'une évolution organique du groupe, aura été imposé à celui-ci de l'extérieur. Tel est le cas du groupe canadien-français dans le Québec. L'industrialisation qui transforme son milieu depuis une cinquantaine d'années prend le caractère d'un choc auquel ne l'avaient préparé aucune expérience, aucune évolution interne. Rien d'étonnant que les adaptations exigées par ce bouleversement aient trouvé dans la pensée et dans les institutions traditionnelles une opposition tenace. Cette opposition fut d'au tant plus vive que les Canadiens français étaient fortement intégrés dans un cadre social relativement anachronique » (193-194). 
aux monographies portant sur la grève de l'amiante. 310 On se souvient qu'après un bref rappel des transformations démographiques et économiques au cours de la première moitié de ce siècle, l'auteur y analyse longuement, pour la même période, la pensée nationaliste et ce qu'il appelle « notre doctrine sociale de l'Église » dont il essaie de montrer l'étroite collusion : il décrit ensuite les moyens par lesquels ces doctrines se proposaient de réaliser la restauration économique et sociale du milieu; enfin, il tâche de déceler les relations entre ce niveau de la pensée et celui des institutions. Trudeau est beaucoup plus polémique (ou plus " engagé », si l'on veut) que Maurice Tremblay. Il déclare : « Je ne me ferai pas scrupule de faire ressortir de la pensée nationaliste ces éléments qui encombrent maintenant le présent et nuisent à une action droite et libre ». 311 Il est souvent question de " nationalisme» sans que jamais on ne nous en propose quelque définition ou tout au moins quelques critères. Surtout, l'auteur pousse si loin l'intention polémique qu'il s'interdit, en définitive, le recours à des explications sociologiques. On ne peut, à la lecture de son essai, que conclure à l'absurdité [281] de la pensée qu'il analyse : mais justement, cela ne saurait satisfaire le sociologue pour qui, par définition, les idéologies ont toujours une signification, c'est-à-dire quelque fondement.

L'essai de Trudeau ne remonte guère au-delà du début du siècle. Bien qu'elle relève d'une tout autre inspiration, une étude de Michel Brunet, qui a eu aussi beaucoup de retentissement, lui fait en quelque sorte pendant. Elle s'intitule : «Trois dominantes de la pensée canadienne-française : l'agriculturisme, l'anti-étatisme et le messianisme ». ${ }^{312}$ L'auteur évoque toute l'évolution canadienne à partir des origines. Le constat n'est pas si éloigné du procès de Trudeau : M. Brunet nous parle d'« un faux spiritualisme », d'« angélisme »... Mais il revient à son explication familière — qu'il oppose d'ailleurs

310 Pierre-Elliott TrudeAu, « La Province de Québec, au moment de la grève », dans : La grive de l'amiante, Montréal, les Éditions de Cité Libre, 1956, 191.

311 Trudeau, ibid., 13

312 Publiée d'abord dans les Écrits du Canada français, III, Montréal, 1957, 33117. Réimprimée dans l'ouvrage de l'auteur : La présence anglaise et les Canadiens, Montréal, Beauchemin, 1958, 113-167. 
lui-même à celle de Trudeau. 313 « On ne doit jamais oublier, nous ditil, que la faiblesse fondamentale de la pensée canadienne-française vient de l'incapacité ou du refus de comprendre le sens véritable de la Conquête de 1760 ». ${ }^{314}$ On ne peut s'empêcher de regretter, chez M. Brunet, cette explication unilatérale. En fixant sans cesse l'attention sur 1760, l'auteur paraît s'interdire de considérer l'importance spécifique de l'histoire subséquente ; tout au moins, celle-ci n'apparaît-elle plus, à la limite, que sous le jour de la fatalité.

Parmi les tentatives pour constituer une sociologie globale de notre société par contestation des idéologies dominantes, je retiendrai un dernier exemple. Il s'agit d'un long article publié naguère dans Cité Libre par Marcel Rioux, sous le titre : "Idéologie et crise de conscience du Canada français. " 315 À beaucoup d'égards, cette étude de Rioux ajoute des nuances importantes à notre inventaire. Soulignons surtout que l'auteur se donne de longs préalables méthodologiques empruntés aux concepts-clés de l'anthropologie et de la sociologie : 316 il y définit, il est vrai, davantage la « culture » que l'idéologie. La thèse centrale peut être résumée ainsi : «L'idéologie 317 canadienne-française s'est toujours appuyée sur trois caractères [282] de la culture canadienne-française: minoritaire, catholique et française. C'est à partir de ces caractères, envisagés d'abord dans le concret, mais avec les années de plus en plus schématiquement, que l'idéologie a

313 Celui-ci « laisse croire, dit-il, que ces hommes de pensée et d'action ont erré parce qu'ils étaient nationalistes. L'explication serait trop simple ! Leur erreur ne vient pas du fait qu'ils ont voulu servir la collectivité canadiennefrançaise. On devrait, au contraire, louer leur sincère désir de travailler au progrès collectif du groupe ethnique dont ils se reconnaissent solidaires. Leur méprise, c'est de n'avoir pas compris la position tragique de la nationalité canadienne-française. Si leurs conceptions économico-sociales ont été fausses ou tronquées, c'est parce qu'ils étaient membres d'une nation démolie et asservie et non parce qu'ils étaient nationalistes. Ils ont eu le tort de croire que la collectivité canadienne-française avait conservé, malgré la Conquête, la pleine maîtrise de ses destinées. C'est ici que l'émotion nationaliste les a trompés » (op. cit., 116, en note).

314 Michel BRUNET, ibid., 117, en note.

315 Marcel Rioux, "Idéologie et crise de conscience du Canada français », Cité Libre, 14, décembre 1955, 1-29.

316 Ibid., 4 et suiv.

317 Remarquer l'emploi du singulier. 
formulé sa doctrine nationale et qu'elle en est arrivée à contrôler la pensée et la plupart des institutions éducationnelles et intellectuelles du Québec ». ${ }^{318}$ Ici encore, on le voit, la Conquête n'est pas oubliée. Mais la référence essentielle de l'auteur n'est pas là : c'est à partir de la crise de conscience provoquée par l'urbanisation et l'industrialisation que les questions sont, en définitive, posées. 319

2. L'historiographie d'une société donnée n'est jamais, dans sa structure, sans analogies profondes avec les idéologies dominantes. Cela s'entend facilement. Les hommes recourent à l'histoire pour des raisons semblables à celles qui les poussent à élaborer des idéologies. Dans un milieu social où les schémas de la tradition ne suffisent plus à définir les situations qui les confrontent et à déterminer les conduites à suivre, il faut bien que les hommes édifient consciemment une vision du milieu qui éclaire l'action. Le recours au passé joue alors fatalement un rôle crucial. Les sociétés complexes dont nous héritons prendraient fatalement la figure d'un pesant déterminisme si nous ne pouvions, grâce à l'histoire, en saisir la genèse et le sens et ainsi les restituer à la liberté de nos options. Le lien est ainsi profond et nécessaire entre l'histoire à faire et l'histoire qu'écrivent les historiens. Comme les idéologies, l'historiographie est ainsi une sociologie globale d'un milieu. Plus soucieuse de critique et d'exactitude que les idéologies, elle mérite évidemment d'être privilégiée.

Ces remarques qui valent pour toutes les sociétés s'appliquent tout particulièrement à la nôtre. Notre historiographie s'est constituée à un moment de crise très aiguë et elle a conservé, par la suite, dans ses schémas d'explication, une très remarquable cohérence. Plus encore peut-être que pour les idéologies dominantes, elle constitue le pôle

318 Marcel Rioux, ibid., 9-10. On mesurera la distance ainsi marquée par un pareil point de départ et la définition de «la culture » proposée par M. le chanoine Groulx : " le climat physique et spirituel du milieu national, un ensemble de formes ou d'influences qui façonnent un type d'humanité » (Directives, Montréal, Zodiaque, 1937, 142).

319 Voir Marcel Rioux, ibid., 3. Jean-C. FALARDEAu se place d'un point de vue ana logue dans son étude: "Les Canadiens français et leur idéologie ", dans : Mason WADE (sous la direction de), La dualité canadienne, Les Presses Universitaires Laval et University of Toronto Press, Québec et Toronto, 1960, 20-38. 
d'adhésion ou de contestation de toute tentative pour étudier systématiquement notre société globale.

La problématique de notre historiographie s'est fixée très tôt. On en trouve les motivations profondes chez François-Xavier Garneau lorsqu'il déclare, par exemple, dans sa lettre célèbre à Lord Elgin :

« J'ai entrepris ce travail dans le but de rétablir la vérité si souvent défigurée, et de repousser les attaques et les insultes dont mes compatriotes ont été et sont encore journellement [283] l'objet de la part d'hommes qui voudraient les opprimer et les exploiter tout à la fois. J'ai pensé que le meilleur moyen d'y parvenir était d'exposer tout simplement leur histoire. » 320

La structure d'ensemble du récit historiographique est fixée dès cette époque. Je citerai à nouveau Garneau :

«Si l'on envisage l'histoire du Canada dans son ensemble, depuis Champlain jusqu'à nos jours, on voit qu'elle se partage en deux grandes phases que divise le passage de cette colonie de la domination française à la domination anglaise, et que caractérisent, la première, les guerres des Canadiens avec les sauvages et les provinces qui forment aujourd'hui les États-Unis ; la seconde, la lutte politique et parlementaire qu'ils soutiennent encore pour leur conservation nationale. » 321

320 François-Xavier GARNEAU, «Lettre à Lord Elgin », dans : Histoire du Canada, éditée par Hector Garneau, 8 édition, Montréal, Éditions de l'Arbre, 1944, I, 20. - Et se situant, comme par avance, dans le contexte de nos discussions actuelles, il écrit aussi au sujet des Canadiens français : « Leur immobilité apparente tient à leurs habitudes monarchiques et à leur situation spéciale comme race distincte dans l'Amérique du Nord, ayant des intérêts particuliers qui redoutent le contact d'une nationalité étrangère. Ce sont ces deux puissants mobiles qui les ont fait revenir sur leurs pas en 1776, après avoir pour la plupart embrassé un instant la cause américaine ; qui les ont fait courir aux armes en 1812, et qui les ont retenus en 1837 » (ibid., 22).

321 GARNEAU, Discours préliminaire. Je cite d'après la première édition, Québec, Imprimerie de N. Aubin, 1845, 19-20. 
Ce schéma inspirera tous les manuels jusqu'à aujourd'hui et la recherche historique elle-même. Il est en effet extrêmement curieux de constater que la période de notre histoire qui suit les luttes constitutionnelles n'a pas donné lieu, chez nos historiens, à une structure propre d'explication. On a plutôt, dans certains cas, allongé, "étiré » (pour ainsi dire) la problématique antérieure : c'est pourquoi nous en savons davantage sur les luttes pour les écoles séparées que sur les processus d'urbanisation et d'industrialisation. En somme, les questions essentielles que notre société a posées et formule encore à son passé se sont fixées avant les transformations radicales que ce même milieu a vécues depuis la fin du siècle dernier. La conséquence la plus grave de ce phénomène, c'est que notre historiographie a pris un caractère systématique qui paraît l'éloigner fatalement de nos situations et de nos questions d'aujourd'hui. Un certain pluralisme des explications historiques est inhérent à la conscience historienne quand celleci est vraiment le remplacement de la tradition : or, ici, histoire et tradition coexistent en un syncrétisme fâcheux. 322

Il ne faudrait pas, cependant, exagérer ce monolithisme. On peut discerner des structures internes quelque peu antithétiques. Je signalerai les deux plus importantes à mes yeux.

La première nous renvoie à une dualité d'interprétation de nos relations avec les Anglais et les Canadiens anglais. Dès l'origine de notre historiographie, [284] n peut opposer l'attitude de Michel Bibaud favorable à l'extrême à la clique du gouverneur de l'époque - et celle de Garneau déjà nationaliste au sens où nous l'entendons aujourd'hui. Nous avons eu ensuite la dualité Chapais-Groulx : les deux auteurs se sont d'ailleurs opposés directement à propos de la thèse de Chapais sur

322 Voir Fernand DumONT, «De quelques obstacles à la prise de conscience chez les Canadiens français », Cité Libre, 19, 1958, 22-28 ; "La liberté a-telle un passé et un avenir au Canada français ? », dans : La liberté, Montréal, Institut canadien des Affaires publiques, 1959, 24-34. Voir aussi Léon Dion, « Le nationalisme pessimiste: sa source, sa signification, sa validité », Cité Libre, 18, nov. 1957, 3-19. 
le caractère " providentiel » de la Conquête anglaise. ${ }^{323}$ Plus récemment, on pouvait encore opposer l'École de Montréal et certains historiens comme l'abbé Maheux. Il serait tout à fait fallacieux d'y voir deux traditions rigoureuses : par exemple, on ne saurait, sans être ridicule, faire procéder d'un historien très médiocre et très partial comme Bibaud l'œuvre équitable, mesurée et bien construite de Chapais. Il s'agit plutôt d'une opposition d'attitudes qui renaît, pour ainsi dire, à chaque génération. De plus, l'antinomie n'est pas radicale. Nous l'avons vu : sur le fond de la problématique, sur la sélection des grands événements, la plupart des historiens paraissent profondément d'accord. Pour tous, la société canadienne-française peut être définie globalement sous la figure d'une minorité catholique et française.

Une autre coupure structurelle doit être signalée : celle que représente un groupe d'historiens de l'Institut d'histoire de l'Université de Montréal. Ils sont nationalistes, mais avec des colorations importantes qui les distinguent de leurs prédécesseurs - du chanoine Groulx, en particulier. Pour eux, la Nouvelle-France avait donné lieu à une structure sociale complète dont le dynamisme était représenté par une bourgeoisie locale importante. La Conquête est venue bouleverser ces assises de notre nationalité.

Considérée d'ailleurs à un autre niveau, l'école historique dont nous parlons a incontestablement apporté beaucoup à la définition de notre société. Alors qu'elle semble nous emprisonner tragiquement dans un passé assez lointain, les questions qu'elle pose sourdent de nos problèmes les plus actuels ; malgré les apparences, c'est encore le présent (et il en est toujours fatalement ainsi pour l'historien) qui est au départ de l'investigation. En effet, c'est une constatation des faillites des idéologies traditionnelles, de leur peu de réalisme devant nos problèmes d'aujourd'hui, qui différencie, en définitive, les travaux de M. Groulx et ceux de l'école dont nous parlons. On retrouve ainsi un accord profond avec d'autres courants de pensée non nationalistes : la dénonciation des mythes et du patriotisme sentimental n'est pas moins vive chez les uns que chez les autres. Tout en étant, sous bien des aspects, les héritiers de la problématique traditionnelle de notre historiogra-

323 Voir le parallèle entre Chapais et Groulx tracé naguère par Henri D'ARLES, Nos historiens, Montréal, Bibliothèque de l'Action française, 1921, 193 et suiv. 
phie, ces jeunes historiens témoignent, à leur manière, de sa faillite. Enfin, il serait tout à fait ridicule de nier la très grande importance de la Conquête dans l'édification de notre société : en ce sens, pour reprendre une expression familière aux sociologues, on peut dire que cette école a construit un modèle partiel parfaitement valable de notre société globale.

[285]

3. Poursuivant notre inventaire, nous abordons maintenant le troisième courant de pensée qui a tenté de définir, ici, la société globale : celui qui est axé sur le passage de la société traditionnelle à la société urbanisée et industrialisée. À première vue, nous sommes alors devant un mode de réflexion tout à fait hétéroclite par rapport aux deux précédents : mais la liaison de ce troisième courant avec les deux autres se constate aisément. La contestation des idéologies dominantes et les remaniements de la problématique historiographique procèdent largement, chez nous, de la prise de conscience d'une distance considérable entre la situation présente et les vieilles définitions de la société globale : et ce sont les concepts d'« urbanisation » et d'« industrialistion » qui définissent, le plus souvent, chez les auteurs, cette reconnaissance d'une nouvelle situation. ${ }^{324}$ L'étude de l'industrialisation et de l'urbanisation de notre milieu est ainsi, à la fois, la source de l'interrogation sur les représentations idéologiques traditionnelles, en marquant le décrochage subit d'avec les situations d'hier, et la mise en forme des questions à poser au présent comme au passé de notre so-

324 Marcel Rioux écrit, par exemple : « Aujourd'hui, avec l'urbanisation et l'industrialisation de notre province, une partie toujours grandissante de notre prolétariat sent et comprend que l'idéologie l'a enfermé dans un univers bien mesquin. La crise de conscience du Canada français se reflète chez ces individus ; ils voient qu'un profond décalage existe entre l'idéologie et l'existence de tous les jours, entre la théorie et la pratique » (« Idéologie et crise de conscience du Canada français », 3). Pour M. Brunet, les nouvelles générations « attendent des chercheurs en sciences sociales les données, les faits et les conclusions dont elles ont un urgent besoin pour éclairer leur action au moment où elles se sentent bouleversées et bousculées par la marche rapide des événements " (La présence anglaise et les Canadiens, 118). Le même auteur oppose «le réalisme de ces nouvelles générations » à l'idéalisme de naguère. 
ciété. Pour ce troisième courant de recherches, les aspects polémiques sous-jacents à l'investigation ne sont donc pas négligeables.

La société traditionnelle, qui constitue le premier pôle de référence, a été fortement valorisée par nos idéologies dominantes. On peut dire qu'avant même que les sociologues ou les anthropologues s'y attachent, les idéologies en avaient déjà fait un « type idéal » (ce dernier terme étant pris à la fois dans le sens de Weber et dans celui plus courant où nous parlons, par exemple, de « l'épouse idéale »!). Il serait donc surprenant, à première vue, que les élaborations proprement sociologiques n'aient pas été contaminées par le contexte idéologique.

Fort heureusement, l'œuvre de celui que l'on peut considérer, par rapport à ce courant d'idées, comme un initiateur, dénote un esprit remarquablement positif. Je veux parler évidemment de Léon Gérin. 325 Je me bornerai à souligner quelques aspects, que je considère comme importants, de sa sociologie globale.

[286]

Dans l'œuvre de Gérin, considérée comme interprétation d'ensemble de notre société, on constate, au départ, une curieuse discontinuité. Le premier sociologue canadien n'a pas négligé l'histoire : plusieurs de ses travaux sur le régime français fournissent encore aujourd'hui à la recherche de stimulantes hypothèses. C'est alors la structure sociale globale qui intéresse Gérin. Et on se rappelle qu'il l'interprète du point de vue d'un phénomène qui lui apparaît comme privilégié : l'entrepreneurship. Mais, sauf pour un article (qui, d'ailleurs, porte sur les premières années après la Conquête), ${ }^{326}$ Gérin n'a pas poursuivi, pour les périodes ultérieures de notre histoire, ses études de structures globales. La continuité véritable de son cadre de référence se situe à un autre plan : la famille paysanne, qu'il a étudiée aussi bien à l'origi-

325 Jean-C. FALARDEAU, «Léon Gérin : une introduction à la lecture de son œuvre ", Recherches sociographiqu.es, I, 2, avril-juin 1960, 123-160 et "Analyse sociale des communautés rurales », Revue de l'Université Laval, IV, 3, novembre 1949, 210-218 ; Hervé CARRIER, s. j. Le sociologue canadien Léon Gérin, Montréal, Éditions Bellarmin, 1960. L'étude de Falardeau comporte une excellente bibliographie des travaux de Gérin ; elle nous dispensera de les énumérer ici à notre tour.

326 Léon GÉRIN, "L'intérêt sociologique de notre histoire au lendemain de la conquête ", Revue trimestrielle canadienne, I, mai 1915. 3-14. 
ne, en Nouvelle-France, que par observation directe, à la fin du XIX ${ }^{\mathrm{e}}$ siècle et au début de ce siècle. Je ne saurais m'empêcher de penser qu'ici, l'École de Le Play — où Gérin, on le sait, avait trouvé ses maîtres - lui a joué quelque mauvais tour par la primauté (appuyée surtout sur des postulats idéologiques) qu'elle accordait à la famille et au groupement.

Malgré la valeur des diverses recherches sur notre société traditionnelle, on ne niera pas qu'elles sont bien insuffisantes : 327 il nous manque, en particulier, trop d'études historiques régionales sur le peuplement. La même constatation s'applique, avec plus de force encore, à l'autre pôle : celui de l'urbanisation et de l'industrialisation. Nous vivons encore largement des constatations faites et des hypothèses formulées par Hugues dans ce classique de nos études sociologiques que constitue French Canada in Transition. ${ }^{328}$ Il faut rappeler aussi, bien entendu, la moisson de faits et de suggestions que constituent les Essais sur le Québec contemporain. ${ }^{329}$ Mais on admettra que [287]

327 Signalons pourtant deux articles de Marcel Rioux où celui-ci, en marge d'une critique des thèses de $\mathrm{P}$. Garigue, apporte peut-être à l'étude de notre société traditionnelle les plus précieux éléments depuis Gérin : «Sur le développement socio-culturel du Canada français », Contributions à l'étude des sciences de l'homme, 4, 1959 ; "Remarques sur les concepts de folksociété et de société paysanne », Anthropologica, 5, 1957, 147-162.

328 Traduit en français par Jean-C. Falardeau sous le titre : Rencontre de deux mondes : la crise d'industrialisation du Canada français, Montréal, Parizeau, 1945. Hugues, on le sait, se réfère expressément comme arrière-plan, à Miner et surtout à Gérin. C'est peut-être Hugues — bien davantage que Gérin lui-même - qui a privilégié, parmi les monographies de Gérin, celle qui porte sur Saint-Justin. Ce passage paraît l'indiquer : «La société rurale du Québec est composée de familles propriétaires de domaines. C'est ainsi que l'a décrite, avec une précision parfaite, le pionnier de nos sociologues, Léon Gérin : «La campagne canadienne-française, écrit-il, se présente comme une simple juxtaposition de familles qui sont à peu près toutes égales ; à peu près toutes engagées dans la culture ; qui presque toutes se suffisent à ellesmêmes, mais dont aucune n'a plus haute ambition que de transmettre intact le bien de famille à quelqu'un de ses enfants, tout en favorisant dans la mesure de ses ressources l'établissement des autres hors du foyer ». La citation renvoie à l'ouvrage de Gérin, Le type économique et social des Canadiens, Montréal, Éditions de l'A.CF., 1938,84-85. — Or il s'agit justement d'un passage où Gérin parle de Saint-Justin...

329 En particulier, les deux études qu'y a insérées Jean-Charles FALARDEAU : « The Changing Social Structures » et « Perspectives » (101-122, 239-257). 
c'est trop peu. La référence à l'urbanisation et à l'industrialisation est devenue si courante dans les propos de nos essayistes et de nos sociologues qu'elle risque de nous conduire à des clichés simplistes plutôt qu'à une problématique complexe. La grande lacune est, ici aussi, du côté des travaux historiques (d'histoire économique, en particulier) qui sont préalables à toute interprétation sociologique un peu complexe de ce genre de phénomènes. ${ }^{330}$ Ce n'est d'ailleurs pas dans cette direction qu'il faut chercher, à mon sens, la voie d'une sociologie globale de notre milieu.

\section{II}

J'ai souligné, au cours de l'inventaire qui précède, l'unité profonde des trois voies de recherches que nous avons successivement envisagées. Plus directement que je ne l'ai fait jusqu'ici dans mes remarques critiques, je voudrais maintenant formuler quelques propositions au sujet des recherches à entreprendre dans le secteur qui relève de mon exposé. J'aurai alors l'occasion de marquer que rien n'est à renier, pour l'essentiel, dans l'effort déjà accompli ; pourtant, pour indiquer des orientations, on ne saurait éviter de prendre une certaine distance visà-vis les démarches poursuivies. Sous forme de réflexions forcément très rapides, il faudra bien dire ce qu'on peut entendre, à un niveau un peu théorique, par le concept de « société globale ».

De façon superficielle il est vrai, ce concept semble supposer une sorte de fonctionnalisme intégral — dont Merton a fort bien dénoncé les postulats. Mais les analyses de Merton ne font, à mon sens, que reporter le problème un peu plus loin. Même si nous admettons, sans peine, que tous les éléments d'une société ne sont pas nécessaires à sa structure globale, qu'il y a, au surplus, des fonctions manifestes et des fonctions latentes, la difficulté décisive se pose toujours : comment

330 Je me permets ici de souligner la très grande importance des recherches de mon collègue Albert Faucher sur la période pré-confédérative — tout en regrettant qu'elles ne soient pas d'un accès plus facile aux chercheurs. Rappelons, en particulier : (en collaboration avec Maurice Lamontagne), " History of Industrial Development », dans : Essais sur le Québec contemporain, 2337. 
pourrons-nous identifier, dans un ensemble social un peu complexe, toutes ces dysfonctions, toutes ces fonctions manifestes ou latentes ? Nous sommes renvoyés à un inventaire indéfini.

S'il est impossible (et cela paraît incontestable) de montrer, en détail, l'intégration ou la dysfonction de tous les éléments d'une société, le concept serait-il purement gratuit ? Ou tout au plus, n'est-il pas qu'une simple "idée directrice », au sens où Kant parle de la finalité biologique ? Alors — comme Kant l'a aussi montré — une pareille idée a peut-être un sens épistémologique, mais ne saurait prétendre au statut de concept scientifique ; [288] elle orienterait notre pensée mais il nous serait interdit de l'utiliser explicitement dans nos travaux.

Des solutions de remplacement ont été, en fait, envisagées. On a suggéré, par exemple, que l'analyse globale d'une société renvoie à un angle privilégié où la société globale apparaîtrait dans sa spécificité. Et on évoque souvent, en ce sens, les " phénomènes sociaux totaux » de Mauss - que lui-même définissait comme des phénomènes-clefs « où s'expriment à la fois et d'un coup toutes sortes d'institutions ». 331 Dans une voie toute proche de recherche, on a aussi pensé que certaines crises décisives des sociétés pouvaient permettre d'en atteindre les structures spécifiques. Les analyses de Georges Balandier sur les Fang et les Ba-Kongo sont une fort belle illustration de cette intention. ${ }^{332}$

331 Marcel MAuss, Sociologie et anthropologie, Paris, Presses Universitaires de France, 1950, 274. [Livre disponible dans Les Classiques des sciences sociales. JMT.]

332 L'auteur l'a définie lui-même en ces termes : «Ces crises concernent la quasi-totalité de la société, les institutions comme les groupements et les symboles sociaux. Les désajustements constituent autant d'issues permettant à l'analyse de s'insinuer et de saisir non seulement les phénomènes de contact entre société coloniale et société colonisée, mais encore de mieux comprendre cette dernière dans ses formes traditionnelles en manifestant certaines faiblesses caractéristiques ou certaines structures et représentations collectives irréductibles » (Georges BALANDIER, Sociologie actuelle de l'Afrique noire, Paris, Presses Universitaires de France, 1955, 28). Le même auteur écrit aussi, dans une étude plus récente, à propos du bilaba des Fang : «Ces manifestations totales servent non seulement à « exposer » le système social - en le jouant en quelque sorte sur la place publique - elles ont aussi une efficacité thérapeutique " "Phénomènes sociaux totaux et dynamique sociale », Cahiers internationaux de sociologie, $X X X, 1961,34)$. 
Malgré l'intérêt tout particulier de cette perspective, on ne saurait y voir une solution à notre problème. Car la question essentielle se pose toujours : on ne peut parler de " phénomène social total » ou de " crise concernant la quasi-totalité de la société » qu'en se référant, explicitement ou implicitement, à une vision globale de la société en question. Cette vision globale, peut-on en construire une image scientifique explicite?

Il ne reste qu'une voie praticable, à mon sens, pour arriver à une solution. Il n'est pas possible, nous l'avons dit, de supposer que tous les éléments d'une société sont fonctionnels; le relevé de toutes les dysfonctions, de toutes les fonctions manifestes ou latentes est exclu ; la détection des phénomènes ou des crises privilégiés où apparaîtrait la figure d'ensemble de la société suppose quelques précisions sur les caractéristiques de cet ensemble. Tout cela nous conduit à cette conclusion - qui est aussi une observation assez simple : si une société n'est pas totalement intégrée, on ne peut manquer de constater que chacune possède des mécanismes particuliers et concrets qui travaillent spécifiquement à son intégration globale. La tâche de la recherche théorique et empirique, dans le secteur qui nous occupe, se réduirait alors à identifier ces mécanismes, à mesurer leur efficacité et les obstacles qu'ils rencontrent. Entre ces éléments sociaux, dont le nombre serait relativement réduit, il faudrait établir une analyse fonctionnelle « de second degré », pour ainsi dire.

[289]

Sans prétendre établir un inventaire exhaustif de ces mécanismes, je proposerais la liste suivante: les idéologies et l'historiographie (dont je ferais une première catégorie), le système d'éducation scolaire, le pouvoir, les classes sociales. On se rappellera, à ce point, nos remarques initiales. Nous ne prétendons aucunement que cette liste des mécanismes d'intégration convient à toute société globale. Elle nous paraît s'appliquer à la société globale canadienne-française dans le contexte singulier où celle-ci s'offre à l'appréhension du sociologue : c'est justement ce contexte que l'analyse critique de notre première partie visait à définir. Mais, en même temps, dans la ligne d'une théorie générale qui reste à élaborer, cet inventaire nous paraît s'ouvrir à la comparaison avec d'autres cas particuliers. 
Il faudrait commenter longuement chacun de ces thèmes ; pour faire bref, je me contenterai de proposer quelques remarques sur chacun en les situant dans le contexte des recherches à faire sur la société globale canadienne-française.

1. En premier lieu, j'ai isolé les idéologies et, subsidiairement, l'historiographie. Je crois en avoir suffisamment souligné tantôt la signification du point de vue de la structuration des sociétés globales : ce sont là les représentations que les sociétés élaborent elles-mêmes pour se donner une vue globale de leur existence et de leur avenir. J'ai noté aussi le nombre et l'importance des essais récents portant sur les idéologies canadiennes-françaises. En plus des critiques que j'ai déjà faites, une lacune essentielle peut être relevée — et qui indique, à mon sens, l'orientation que devraient prendre des investigations prochaines. On n'a pas considéré suffisamment les idéologies comme des phénomènes spécifiques ; le plus souvent, la définition explicite ou implicite des auteurs s'élargit à toute la culture canadienne-française ou encore désigne un ensemble d'attitudes diffuses. Ainsi, Marcel Rioux écrit : " Au Canada français, il y a toujours eu une idéologie ; depuis 1760, elle n'a guère varié... » 333 Beaucoup d'auteurs parlent ainsi de " l'idéologie » canadienne-française. ${ }^{334} \mathrm{M}$. Brunet, dans son étude déjà citée, confond visiblement la notion d'idéologie avec la reconstitution plus ou moins large d'un complexe d'attitudes. C'est ainsi qu'après avoir traité longuement d'«agriculturisme », il écrit en conclusion : "Une nouvelle génération de sociologues, de militants syndicalistes et d'écrivains semble convaincue que notre progrès collectif dépend maintenant de la classe ouvrière... L'ouvriérisme a remplacé l'agriculturisme ». 335 On voit le danger de subjectivité : la perception du chercheur risque de se substituer à la structure propre du phénomène ; les « ismes » sont peut-être autant des auteurs que de la société à étudier. Il n'y a pas là seulement une [290] source de confusion, mais le chercheur s'interdit ainsi de détecter bien des facteurs d'explication. C'est ainsi que M. Trudeau, dans l'étude déjà citée, après

333 Marcel Rioux, « Idéologie et crise de conscience au Canada français », 9.

334 Rappelons le titre même d'un essai de Jean-C. FAlARdEAU, « Les Canadiens français et leur idéologie ", dans : La dualité canadienne, 20-38.

335 Michel BRunET, La présence anglaise et les Canadiens, 162. 
avoir composé un système idéologique rigide, ne sait trop comment expliquer l'émergence d'un syndicalisme qu'il reconnaît comme étant dynamique. Le recours au hasard qu'il nous propose explicitement provient sans doute d'un vice initial de méthode, d'une conception trop large de l'idéologie qui ne laisse plus guère de place aux tensions au sein même des idéologies et aux facteurs non idéologiques d'explication.

Les études de nos idéologies devraient donc réduire beaucoup plus strictement leur objet. L'analyse consisterait alors, avant tout, à retrouver des mécanismes de rationalisation : c'est-à-dire les tensions, les antinomies, entre les sources idéologiques diverses, entre les sources idéologiques et les autres éléments du social définis par ailleurs, entre le groupe ou l'institution support de l'idéologie et les autres groupes ou institutions. En somme, après s'être surtout - et un peu prématurément - attachée aux relations entre idéologies et milieux, c'est vers la configuration interne des idéologies que notre attention devrait se tourner. La même réflexion s'applique, à mon sens, à notre tradition historiographique : un travail d'ensemble, mettant en évidence les schèmes d'explication, devrait lui être consacré au plus tôt.

2. Je l'ai suggéré : la restriction du champ d'analyse des idéologies nous conduirait fatalement à nous interroger sur les porteurs de ces idéologies. Celles-ci ayant été définies jusqu'à maintenant de façon trop large, on a été porté à supposer qu'elles étaient partagées soit par les Canadiens français en général, soit par les intellectuels (cette catégorie n'ayant d'ailleurs jamais été, ici, cernée ni historiquement ni sociologiquement avec précision) — ou encore, qu'elles se confondaient avec des attitudes reliées logiquement sans que l'on s'attardât sur leurs titulaires. C'est pourquoi, dans un programme de recherches sur la société globale, le système d'éducation scolaire devrait occuper la première place après les idéologies. Bien entendu, parce que c'est le mécanisme par excellence de transmission des idéologies. Mais ne cédons pas à un fonctionnalisme trop facile : ce qui devrait nous intéresser avant tout, dans le système d'éducation scolaire, du point de vue de la société globale, c'est la différence entre ce que l'on appelle couramment « le développement de l'enfant » et la scolarisation considérée comme processus spécifique. Il est curieux de constater que, dans les ouvrages d'ensemble sur la psychologie de l'enfant, on ne marque 
pas davantage la coupure radicale qu'y produit l'entrée à l'école. Sans doute parce que cela introduirait une dualité gênante dans les sources de l'explication : lorsqu'il commence à fréquenter l'école, l'enfant ne quitte pas seulement l'univers restreint des relations profondément affectives pour entrer dans une société plus anonyme, il est surtout soumis aux normes les plus générales et les plus codifiées de sa société. Les normes qui, dans cette situation exceptionnelle, [291] sont transmises à l'enfant révèlent, de façon très directe, les mécanismes d'intégration de la société globale.

Peu d'investigations ont été menées dans cette perspective. Le groupe de recherches de l'École de pédagogie de l'Université Laval s'y est attaché parmi d'autres thèmes. Une thèse dont j'ai eu la responsabilité cette année nous offre déjà une première coupe dans les données de la question, pour ce qui est de l'enseignement secondaire. ${ }^{336} \mathrm{De}$ toute manière, dans le contexte canadien-français, une hypothèse décisive me paraît s'imposer : dans notre société, la distance entre les normes vécues dans les comportements quotidiens et celles qui sont transmises par les écoles est tellement grande qu'elle vaut tout particulièrement d'être considérée de près. On se souviendra que, par rapport à une situation de ce genre, Mead et Bateson proposaient de voir là une cause privilégiée d'évolution sociale rapide : 337 il vaudrait la peine d'essayer de le vérifier dans notre société. 338

3. En troisième lieu, on n'aura pas de peine à admettre qu'il faut introduire le pouvoir - à condition d'en diversifier largement les mécanismes fonctionnels. Il faudrait en distinguer, pour le moins, trois niveaux : l'État proprement dit, les partis, le pouvoir social. ${ }^{339}$ Au pre-

336 Nicole GAGNON, L'idéologie humaniste dans la revue «L'enseignement secondaire », thèse de maîtrise, Département de sociologie et d'anthropologie, Université Laval, 1962.

337 Voir Gregory BATESON et Margaret MEAD, Balinese Character : A Photographie Analysis, New-York, 1942.

338 Sur l'analyse sociologique d'un système d'éducation, en particulier du nôtre, je me permets de renvoyer à mon étude : "Scolarisation et socialisation : pour un modèle général d'analyse en sociologie de l'éducation », à paraître dans Contributions à l'étude des sciences de l'homme (Montréal).

339 Voir mon article à paraître dans un prochain numéro de Recherches sociographiques, « De la sociologie politique en général à celle du Canada français. » 
mier plan, nous disposons déjà d'une bonne esquisse de Maurice Lamontagne 340 qui, pour être contestable sur certains points, n'en est pas moins extrêmement suggestive. Au niveau des partis, des hypothèses fort plausibles ont déjà été formulées : le caractère abstrait et incolore des partis canadiens qui doivent tenir compte, à la dimension du pays, de problèmes non seulement très divers, mais antinomiques ; la longue emprise du même parti sur le pouvoir, aussi bien au gouvernement fédéral que dans notre province ; l'interférence - abstraite elle aussi - des conflits ethniques et de la politique fédérale ; etc. Cette « abstraction » nous renvoie, ici plus qu'ailleurs peut-être, à l'étude des mécanismes fonctionnels qui relient le pouvoir politique et le pouvoir social. Le " patronage » doit sans doute, en ce sens, retenir notre attention : si nous pouvions déterminer en quoi il répond aux besoins locaux et régionaux qui, dans notre milieu, ne reçoivent pas une réponse d'autre manière, nous saurions du même coup beaucoup de choses sur notre société. Mais, la liaison précise du pouvoir politique et du pouvoir [292] social resterait encore à chercher, me semble-t-il, dans une direction que paraît indiquer clairement notre histoire : je veux parler de la politisation traditionnelle, dans notre milieu, des voies d'accès au monde des élites. Edmond de Nevers a naguère (1896) donné sur ce point des observations qui sont encore éclairantes. Mais des élites nouvelles apparaissent dans notre société : dans le milieu syndical et dans le mouvement coopératif, en particulier. Ces leaders élaborent de nouveaux éléments idéologiques encore mal intégrés, mais qui révèlent sans doute les colorations diversifiées des attitudes des Canadiens français. Ce phénomène donne lieu à des spéculations sociologiques et politiques bien générales ; une sociologie globale de notre société devrait, il me semble, s'y attacher minutieusement.

4. Cette recherche sur les élites nous amène naturellement aux classes sociales. Sur ce point particulièrement, la recherche est, ici, dans l'enfance. Beaucoup de préjuges, hérités des classes moyennes - comme aux États-Unis, - nous empêchent de poser facilement le problème. Et, par surcroît, nous sommes souvent obsédés encore par

340 Maurice Lamontagne, Le fédéralisme canadien : évolution et problèmes, Québec, Les Presses Universitaires Laval, 1954. 
la nostalgie d'une activité économique qui serait aux mains des Canadiens français. Pourtant, c'est bien par rapport aux classes sociales que les questions que nous nous posons au sujet des idéologies dominantes prennent toute leur signification. Toutes nos discussions - chez les sociologues comme chez les historiens - sur le "nationalisme », « l'agriculturisme » et autres denrées de ce genre devraient nous amener à une question décisive : quelles strates sociales ont eu intérêt à fabriquer de pareilles productions intellectuelles ? Je ne parle évidemment pas seulement d'intérêts économiques. C'est pourquoi j'élargirais ma question : aux difficultés et aux angoisses de quels groupements ont répondu nos productions idéologiques? Nous retrouvons ainsi le noyau du programme de recherche que j'ai essayé d'esquisser très superficiellement. C'est pourquoi, à mon avis, les recherches sur le leadership et les classes sociales délimitent, particulièrement dans notre milieu, la problématique d'une étude de la société globale.

Fernand Dumont

Département de sociologie et d'anthropologie, Université Laval. 


\title{
COMMENTAIRE
}

\author{
Maurice Tremblay \\ Département de science politique, \\ Université Laval.
}

[pp. 293-294.]

$\underline{\text { Retour à la table des matières }}$

J'avoue que je suis un peu gêné d'avoir à amorcer la discussion sur la communication de monsieur Dumont. D'abord, parce que la place qu'il a accordée, dans son inventaire, à mon étude des Essais sur le Québec contemporain me donne la troublante impression d'être réduit à la catégorie inerte des objets qu'on analyse et qu'on dissèque et où l'on se sent un peu comme paralysé. Et le fait que cette place soit privilégiée, au-delà même du mérite facile de mon essai d'être l'un des premiers du genre, ne fait qu'ajouter à ma gêne.

Pour ma part, je vois dans cette espèce de traitement de faveur qu'il m'accorde une bonne illustration de la difficulté qu'a tout sociologue d'atteindre à la parfaite objectivité, biaisé qu'il peut être par ses sentiments personnels relatifs à son objet d'étude et, entre autres, par ses sentiments d'amitié. Et cette illustration de l'influence insidieuse de la subjectivité est d'autant plus probable que l'auteur de la communication m'a avoué avoir eu des scrupules à donner une appréciation objective de mon essai, alors qu'il me savait invité à faire le premier mes commentaires et qu'il ne voulait ni m'influencer ni même avoir l'air de m'influencer en sa faveur. 
Je ne doute pas de la sincérité de ces propos ; mais vous reconnaîtrez avec moi qu'ils n'avaient rien qui puisse dissiper mon malaise ni atténuer le conditionnement dont M. Dumont se faisait scrupule. Mais comme je ne pouvais plus me récuser, il ne me restait, en préparant mes remarques, qu'à tâcher de dominer ma gêne et à rechercher à mon tour l'objectivité, en essayant de résister à la tentation de la condescendance reconnaissante.

La chose m'est d'ailleurs facilitée, car je crois m'être rendu compte qu'en plus du préjugé favorable inconscient un défaut de méthode expliquait la place avantageuse que je partageais avec Marcel Rioux dans l'analyse comparative des quatre essais choisis par l'auteur comme échantillons, dans la catégorie des études portant sur les idéologies traditionnelles.

Si nous avons trouvé grâce devant l'auteur ne serait-ce pas parce que nos études étaient les deux seules de l'échantillon qui aient eu des prétentions sociologiques et qui aient eu quelque chance de répondre aux exigences « d'une interprétation scientifique » et de constituer une contribution à « la sociologie globale » de notre milieu ?

On peut être justifié de considérer que l'essai de Pierre-Elliott Trudeau constitue une étrange introduction aux autres études contenues dans La grève de l'amiante, mais peut-on légitimement lui appliquer rigoureusement les canons de la sociologie alors que l'auteur se situe d'emblée au plan normatif de la contestation des idéologies traditionnelles, considérées non pas comme objets à analyser spéculativement, mais uniquement dans la mesure où, comme le dit Trudeau, et comme le note d'ailleurs l'auteur, certains de leurs éléments « encombrent maintenant le présent et nuisent à une action droite et libre ».

Sans doute, ce genre de contestation ne dispense-t-il pas de toute analyse objective et peut-être que Pierre-Elliott Trudeau ne s'est pas appliqué assez systématiquement à faire ressortir tous les facteurs qui ont pu conditionner nos "définisseurs de situation » traditionnels et qui pouvaient même leur donner en partie raison à l'époque ; en sorte qu'il a pu les faire paraître moins lucides qu'ils ne l'avaient été en réalité. Mais peut-être importe-t-il de rappeler que l'explication la plus exhaustive n'équivaut jamais à une [294] justification et que le but de Trudeau, dans son essai, était précisément de montrer que nos maîtres à penser s'étaient largement trompés dans l'évaluation de la situation 
qu'ils prétendaient définir ; ce qui est parfaitement légitime, mais ce qui implique nécessairement, comme tout jugement de valeur, un engagement de l'auteur beaucoup plus explicite qu'une simple analyse sociologique qui, elle, peut se permettre d'être beaucoup plus détachée et plus sereine.

Dans la mesure où l'essai de Michel Brunet se présente lui aussi comme une contestation de certaines idéologies traditionnelles, je considère qu'il est normal qu'il prenne à son tour l'allure d'un procès et qu'il devient alors difficile de le comparer, sans transgression de genres, à des essais qui se réclament exclusivement de la sociologie et de ses méthodes. Ce qui ne m'empêche pas, bien sûr, de reconnaître le bien-fondé des critiques formulées par l'auteur au sujet des explications ou plutôt de l'explication sociologique sur laquelle Michel Brunet appuie ses jugements de valeur.

Il existe par ailleurs un essai proprement sociologique concernant nos idéologies qui se prêtait de soi à la comparaison dans ce contexte et qui l'aurait supportée très avantageusement. Il s'agit, vous l'avez facilement deviné, de l'essai en tous points remarquable publié par Fernand Dumont et Guy Rocher sous le titre d'« Introduction à une sociologie du Canada français » dans le numéro de la collection $R e$ cherches et débats consacré au Canada français. L'auteur de la communication y a fait une allusion discrète, mais il pouvait difficilement en faire la recension qui s'imposait. Il me revient donc de combler cette lacune, ce qui me permettra de payer ma dette envers Fernand Dumont avec la meilleure conscience du monde.

L'intérêt de cet essai est d'abord méthodologique en ce qu'il suit de très près l'approche féconde que propose Fernand Dumont à la fin de sa communication pour l'étude des sociétés globales par l'analyse fonctionnelle de leurs idéologies.

Quant au contenu, l'essai complète très heureusement sous plusieurs aspects les études antérieures. D'abord en ce qu'il étudie plus systématiquement qu'on ne l'avait jamais fait la genèse de l'idéologie dominante dans la première partie du $\mathrm{XX}^{\mathrm{e}}$ siècle, en montrant tout particulièrement, par des références à ce grand méconnu qu'est notre $\mathrm{XIX}^{\mathrm{e}}$ siècle, que son monolithisme ne devait pas être projeté trop en arrière dans l'histoire. Mais la principale contribution des auteurs est d'avoir analysé l'éclatement de ce bloc idéologique qui est en train de 
s'opérer sous le pouvoir des forces nouvelles par la dissociation du social et du national, du religieux et du profane et même, à l'intérieur de l'Église, par l'affranchissement du laïcat du contrôle clérical.

Je clos là mes remarques laissant à d'autres le soin de les compléter, et en particulier aux historiens celui de commenter les considérations de l'auteur sur l'évolution de notre historiographie.

Je me contenterai en conclusion de souhaiter, dans le prolongement des vœux de Fernand Dumont, que celui qui dans dix ans aurait à recenser les contributions à une analyse de notre société globale, puisse faire état d'études substantielles et non plus seulement d'une courte série d'essais.

Maurice Tremblay

Département de science politique, Université Laval.

Fin du texte 